\title{
Studies on the Closed-Loop Digital Control of Multi-Modular Reactors
}

Report No. MITNRL-049

by

John A. Bernard ${ }^{1}$, Allan F. Henry ${ }^{2}$, David D. Lanning ${ }^{2}$, and John E. Meyer ${ }^{2}$

MIT Nuclear Reactor Laboratory ${ }^{1}$ and MIT Department of Nuclear Engineering ${ }^{2}$

November 1992

Prepared for

United States Department of Energy

and

Oak Ridge National Laboratory

Dr. John W. Lewellen

Division of University and Industry Programs

Office of Energy Research

Contract No. DE-FG07-90ER12930

and

Mr. James D. White

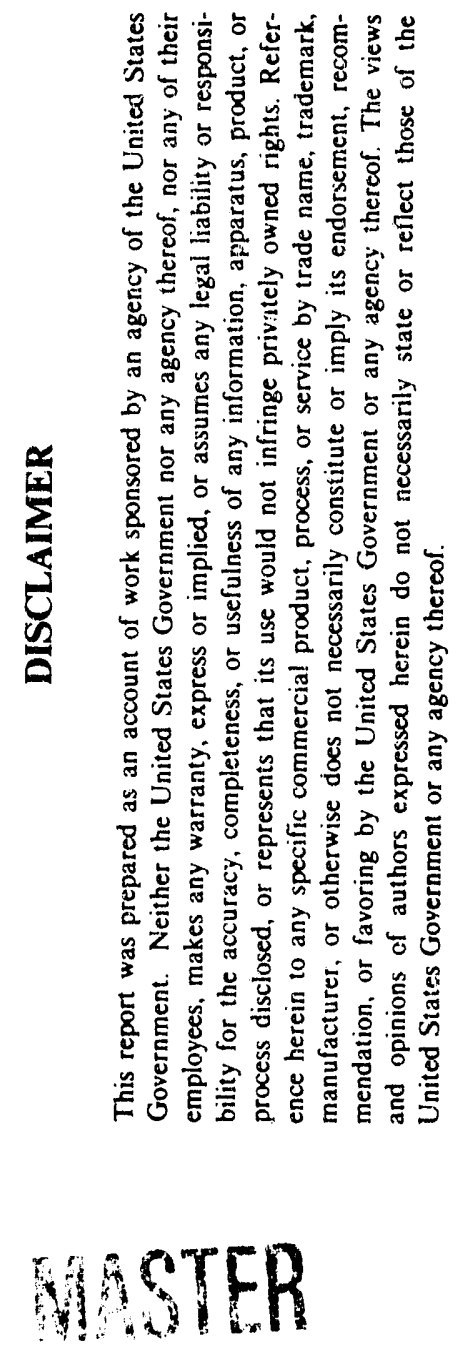

Instrumentation and Controls Division

Martin Marietta Energy Systems, Inc. 
With appreciation to Keung Koo, Kwan, Matt, and Shing Hei. 


\section{Executive Summary}

This report describes the theoretical development and the evaluation via both experiment and simulation of digital methods for the closed-loop control of power, temperature, and steam generator level in multi-modular reactors. The major conclusion of the research reported here is that the technology is currently available to automate many aspects of the operation of multi-modular plants. This will in turn minimize the number of required personnel and thus contain both operating and personnel costs, allow each module to be operated at a different power level thereby staggering the times at which refuelings would be needed, and maintain the competitiveness of U.S. industry relative to foreign vendors who are developing and applying advanced control concepts. The technology described in this report is appropriate to the proposeci multi-modular reactor designs and to present-generation pressurized water reactors. Its extension to boiling water reactors is possible provided that the commitment is made to create a real-time model of a BWR. The work reported here was performed by the Massachusetts Institute of Technology (MIT) under contract to the Oak Ridge National Laboratory (ORNL) and to the United States Department of Energy (Division of Industry and University Programs, Contract No. DEFG07-90ER12930.)

The work reported here was undertaken with the objective of developing and, to the maximum extent possible, demonstrating ao $v$ anced control technologies for the operation of nuclear power plants in which several reactors, each with its own steam generator, provide steam to a common turbine. Such plants are attractive because the size of each reactor can be relatively small (a few hundred megawatts) thereby enabling the incorporation of passive safety features. Yet, because only one turbine is used, the economies of scale that are associated with large present-day reactors will not be entirely lost. These advantages not withstanding, the operation of a multi-modular plant does pose certain challenges. First, if operating costs are to be contained, then it will not be possible to have a full crew for each module. This implies that a high degree of automation will be needed. Second, plant economic performance will be degraded if it becomes necessary to shut a! modules down simultaneously for refueling. Hence, operation under conditions of unbalanced loads will be necessary. The research reported here was conducted, in large measure, to identify control concepts that would resolve these two concerns.

This report is organized as follows. Chapters One-Three provide material on the desigr. of multi-modular reactors, an overview of the MIT program for the advanced instrumentation and control of nuclear reactors, and summaries of earlier MIT work including both the reactivity constraint approach and period-generated control. The former is a supervisory technique that precludes challenges to the reactor safety system as the result of an automatic control action. The latter is a method for the trajectory tracking of reactor power. Chapter Four describes the Advanced Control Computer System which embodies a computer architecture suitable for the digital operation of a nuclear plant. Chapter Five reports on the 'perturbed reactivity method' which is a technique for the online estimation of a reactor's initial degree of subcriticality. When combined with a trajectory-tracking strategy such as period-generated control, this method allows automated reactor startups. Chapter Six provides an experimental comparison of various techniques for the trajectory-tracking of reactor power. Chapter Seven focuses on the design of a numerical model of a multi-modular plant. Chapter Eight addresses the control of steam generator level and in particular the use of model-based compensators to offset the effects 
of shrink and swell. Chapter Nine reports the development of strategies for the adjustment of reactor power under conditions of unbalanced loads. Chapter Ten discusses the challenges associated with the development of intelligent support tools for reactor operators. The report concludes with an overview of the relation between the work completed here and those tasks needed for full automation of a multi-modular plant. Practical considerations in the operation of these plants are also enumerated.

The major finding of this report is, as noted above, that the technology currently exists to automate many aspects of a multi-modular plant's operation. Specific findings are as follows:

1. A multiple computer/single task system is an appropriate architecture for the closed-loop digital control of a nuclear reactor. This approach has the advantage that generic safety-related software that remains invariant can be separated from the control law software that is updated as plant procedures change. This facilitates a fault-tolerant design, software validation, real-time operation, and high numerical throughput.

2. Automated reactor startups are possible provided that the reactor's initial degree of subcriticality is known accurately. This can be estimated on-line during the initial stages of a startup through the use of the perturbed reactivity method. Under this approach the net reactivity present in the core is treated as two separate entities. The first is that associated with a known perturbation. This quantity, together with the observed period and the reactor's describing parameters, are the inputs to the method's algorithm. The second entity, which is the algorithm's output, is the sum of all other reactivities including those resulting from inherent feedback and the initial degree of subcriticality. During an automated startup, feedback effects will be minimai. Hence, when applied to a shutdown reactor, the output of the perturbed reactivity method will be a constant that is equal to the initial degree of subcriticality. This is a major advantage because repeated estimates can be made of this one quantity and signal smoothing techniques can be applied to enhance accuracy.

3. Automated power increases are possible provided that accurate trajectorytracking techniques are available. Many such techniques have been developed for purposes of robot control. Experimental comparisons show that superior performance is achieved if an error defined by comparison of the observed and specified paths is fed back through a system model to obtain the signal to the actuator. The use of feedback allows for modeling errors and unforeseen perturbations while the presence of the model corrects for non-linearities.

4. The sophistication of advanced reactor physics and thermal-hydraulic methods is now such that accurate, real-time models of multi-modular plants can be run on present-generation mini-computers. Such a model was constructed here and it was then used both to conduct simulation studies and to design non-linear control strategies.

5. The control of steam generator level is especially important in the operation of multi-modular plants because, in addition to plant power changes, there will be 
module power changes as individual modules are brought on and taken off line. The deleterious effects of shrink and swell can be greatly mitigated through the use of model-based compensators. To this purpose, a controller was developed that employed compensators for feedwater flowrate, steam flowrate, and primary coolant temperature. Simulation studies of this controller showed it to be effective.

6. The economic operation of a multi-modular plant will require that each module be refueled at a different time. Hence, operation under conditions of unbalanced loads will be necessary so that each module's rate of fuel depletion will be different. Strategies to permit this were developed and demonstrated via simulation. These are complicated because, even though each module is at a different power level, the pressures in their steam generators must all be the same. Otherwise steam will not flow. Hence, the average coolant temperature of each module must be different. The strategies developed entail coordinating changes in steam flow to the turbine with changes in reactor power that are initiated by control rod movement so as to cause both power and temperature to change as desired. One other finding of this effort was that the 'sliding $\mathrm{T}_{\text {ave }}$ control programs that are employed for present-generation pressurized water reactors can only be used for the highest-powered module in a multi-modular plant. This is a further result of the need for each module to maintain a common steam generator pressure.

7. A major advantage of proposed multi-modular reactor designs is their simplicity. Nevertheless, work is in progress to improve th $\&$ man-machine interface even further through the use of expert systems an $\downarrow$ other forms of artificial intelligence. It was concluded here that expert systems are making a contribution to reliable reactor operation in such areas as plant design, facility management, maintenance planning, and interactive diagnostics. However, it remains an open question as to whether intelligent support systems can be successfully developed for real-time diagnosis and operator guidance. It is evident that the needed technology will not result from the mere extension of conventional, expert systems which encode experiential knowledge in production rules. The need for real-time performance imposes too many difficulties. Intelligent support systems will have knowledge bases derived from temporally-correct plant models, inference engines that permit revisions in the search process as information is updated, and non-interactive man-machine interfaces. Also, their knowledge bases will require internal organization so as to facilitate diagnostics and possibly control activities.

In summary, the objectives of the research described in this report were to investigate, develop, and where possible experimentally demonstrate generic techniques for the closed-loop digital control of power, temperature, and steam generator level in multimodular plants. Specific topics that were addressed included the design of a computer architecture in which invariant supervisory constraints are kept separate from plantdependent control laws, the identification of a method for automated startups with the online estimation of subcriticality, a comparison of trajectory-tracking techniques, the development of a multi-modular plant model, the design and evaluation of compensators for the proper control of steam generator level despite shrink and swell effects, the 
enumeration and evaluation of strategies for the adjustment of reactor power under conditions of unbalanced loads, and an assessment of the challenges involved in the provision of intelligent support tools to reactor operators. When these techniques are combined with others including existing methods for automating turbine run-ups and yetto-be developed methods for automated diagnostics, it should be possible to operate each module of a multi-modular plant from a manned central control room.

This report is the sixth in the series prepared by MIT on advanced instrumentation and control for nuclear reactors. Earlier ones addressed the reactivity constraint approach as applied to space-independent reactors, the time-optimal trajectory control of neutronic power, applications of expert systems within the nuclear industry, the use of the trajectorytracking technique for automated power increases from subcritical, and the extension of the reactivity constraint approach to the control of reactors characterized by spatial dynamics. The fundamental premise of this MIT program is that digital technology can make a significant contribution to the safe, efficient operation of nuclear reactors provided that it is applied in a planned systematic manner. For this to occur, digital controllers should be designed on a theoretically rigorous basis and extensively evaluated at the research and test reactor level. On-line testing is particularly important because it is only through repeated, incremental usage that the confidence will develop within both industry and the regulatory agencies to apply digital sytems to large reactors. 


\section{Preface}

The research reported here was perforned by the Massachusetts Institute of Technology (MIT) under contract to the Oak Ridgo: National Laboratory (ORNL) and the U.S. Department of Energy's Division of Industry and University Programs (Contract No. DE-FG07-90ER 12930). Support was also received for one of the graduate students working on this project from the Stone \& Webster Engineering Corporation. The project began in October 1988 and ran for almost four years. The thrust of the project was to investigate a variety of the tasks needed for the automated operation of multi-modular plants. Thus, much new ground was broken. In addition, accomplishments from previous MIT studies on reactor control were revisited and the theoretical foundation for that work was significantly strengthened. For example, period-generated control emerges as a general means of trajectory-tracking rather than a technique specific to nuclear reactors. It should also be noted that the work reported here was performed with a larger and very impressive effort at the Oak Ridge National Laboratory. While these two programs both concerned multi-modular reactors, they differed in focus and thus complemented each other. One possible point of confusion is that both MIT and ORNL used the term 'supervisory control' to mean different things. At MIT it refers to constraints developed as part of the predictive aspect of the control process while at ORNL it describes automated planning.

The success of the research described in this report was due in large measure to the efforts of our graduate students. The technical contributions of each are noted in the report itself. Noted here is their dedication, persistence, and curiosity. Keung Koo Kim and Kwan S. Kwok each earned their Ph.D. degrees for work reported here. Shing Hei Lau received the degree of Nuclear Engineer and Mather K. Waltrip received his M.S. degree. Each is now pursuing his career in the nuclear industry.

The experimental studies that were conducted as part of this research reflect the contributions of many people. These include Mr. Paul T. Menadier, Mr. William McDermott, Mr. Mark R. Anderson, Ms. Susan Tucker, Mr. Frank Warmsley, Ms. Toni Korzan, and Mr. Gerald Bolton, all of the MIT Nuclear Reactor Laboratory. One regret ihat must be noted is that it was not possible to repeat and extend the experimental work at the Annular Core Research Reactor that is operated by Sandia National Laboratories. However, the possibility of doing this in 1993 is under discussion.

This report was typed by Ms. Carolyn Hinds and Ms. Georgia W'oodsworth. Their skill in layout of the numerous equations contained in this report was mosi appreciated. Computer-generated figures were prepared by Mr. Shing Hei Lau, Mr. Ara Sanentz, and Dr. Keung Koo Kim. Other figures were done by Mr. Leonard Andexler. Assistance with layout and editing was received, as always, from Mr. Ara Sanentz.

Finally we wish to express our deep appreciation to Mr. James D. White of the Oak Ridge National Laboratory and to both Dr. John W. Lewellen and Dr. Richard E. Stephens of the United States Department of Energy for their unflagging enthusiasm for this project in particular and for nuclear engineering research and education in general.

November 1992

John A. Bernard

Allan F. Henry
David D. Lanning

John E. Meyer 
-viii-

\section{Notice of Patent Protection}

Certain of the control concepts described in this report are protected under United States and foreign patents. These include:

(a) Apparatus and method for the Closed-Loop Control of Reactor Power: Standard Dynamic Period Equation. (U.S. Patent No. 4,637,911 to Dr. John A. Bernard with rights assigned to the Massachusetts Institute of Technology; foreign patents pending.)

(b) Apparatus and Method for the Closed-Loop Control of Reactor Power: Alternate Dynamic Period Equation. (U.S. Patent No. 4,710,341 to Dr. John A. Bernard, Dr. Allan F. Henry, Dr. David D. Lanning, and Dr. Kwan S. Kwok with rights assigned to the Massachusetts Institute of Technology; foreign patents pending.)

(c) Apparatus and Method for the Closed-Loop Control of Reactor Power in Minimum Time: The MIT-SNL Period-Generated Minimum Time Control Laws. (U.S. Patent No. 4,781,881 to Dr. John A. Bernard with royalty-free rights to the U.S. Department of Energy, all other rights reserved; foreign patents pending.)

Those desiring further information should contact either Dr. Bernard or the MIT Technology Licensing Office, Building E32-300, Massachusetts Institute of Technology, Cambridge, MA 02139. 


\section{Table of Contents}

Page

Cover Sheet...................................................................

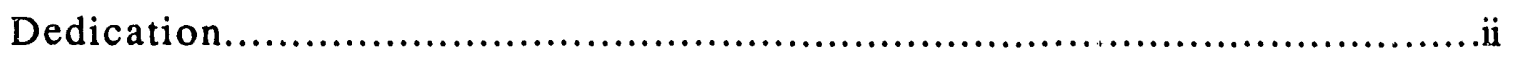

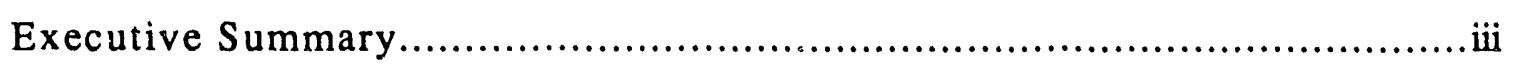

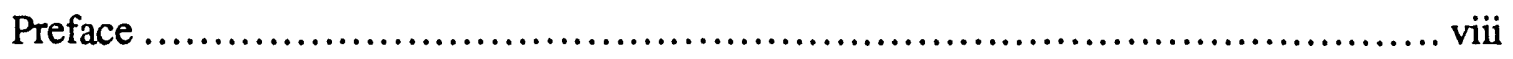

Notice of Patent Protection ...................................................... vii

Table of Contents................................................................

List of Figures ..........................................................

List of Tables ........................................................... xxvii

1. Scientific and Engineering Objectives ....................................

1.1 Multi-Modular Reactors ...............................................2

1.1.1 Liquid Metal Multi-Modular Power Plants......................3

1.1.2 Gas-Cooled Multi-Modular Power Plants .......................3

1.1.3 PWR-Type Multi-Modular Power Plants .........................4

1.2 Rationale for the Closed-Loop Digital Control of Multi-Modular Reactors ...........................................................4

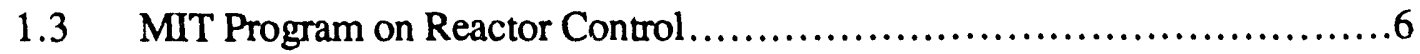

1.4 Rationale for Experimental Research .....................................

1.5 Experimental Facilities..............................................

1.5.1 MIT Research Reactor (MITR-II) ........................7

1.5.2 Annular Core Research Reactor (ACRR) ...................... 10

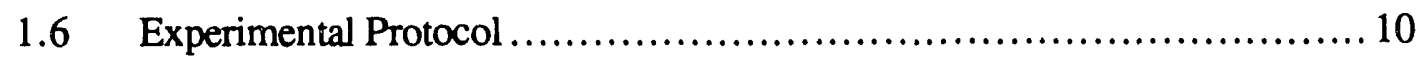

1.7 Definitions of Neutronic and Thermal Power.......................... 12

2. Scope of this Report ................................................. 13

3. Methodology for the Closed-Loop Digital Control of Nuclear Reactors ............. 15

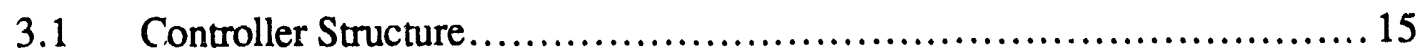


3.2 Overview of Reactor Dynamics ................................... 20

3.2.1 Manual Operation ...................................... 22

3.2.2 Closed-Loop Operation .................................. 22

Controller Design ............................................... 26

3.3.1 Non-Linear Reactor Model .................................. 26

3.3.2 Selection of the Actuator $u$.gnal.............................29

3.3.3 Supervisory Control..................................... 30

3.3.3.1 Feasibility of Control ........................... 30

3.3.3.2 Reactivity Constraint Approach................... 30

3.3.3.3 Demonstration of the Reactivity Constraint Approach ........................................ 32

3.3.4 Control Laws.......................................... 34

3.3.4.1 Period-Generated Control ........................ 35

3.4 Summary of MIT Work on the Control of Reactors .................... 41

4. Advanced Control Computer System................................... 45

4.1 Statement of Problem............................................ 45

4.2 Advanced Control Computer System................................ 46

4.3 Data Acquisition and System Interfaces .............................. 49

4.3.1 Data Acquisition and Interface Software ...................... 49

4.3.2 Hardware Interface to Reactor ...........................50

4.3.3 Broad-Range Power Sensor.............................. 50

4.3.4 Safety and Interlock Circuits .............................52

4.4 Demonstration of Advanced Control Computer System................ 52

4.5 Assessment of Advanced Computer Control System..................... 52

5. Automared Reactor Startup with On-Line Estimation of Subcriticality .............55

$5.1 \quad$ Statement of Problem........................................... 56 
$5.2 \quad$ Perturbed Reactivity Method ........................................ 56

$5.3 \quad$ Implementation Issues.......................................... 58

5.3.1 Control Device Movement .................................... 59

5.3.2 Source Term Estimation ..................................60

5.3.3 Techniques for Signal Smoothing ............................60

5.4 Experimental Evaluation of the Perturbed Reactivity Method.................. 61

5.5 Assessment of Perturbed Reactivity Method..............................69 69

6. Experimental Comparison of Trajectory Tracking Techniques .................... 72

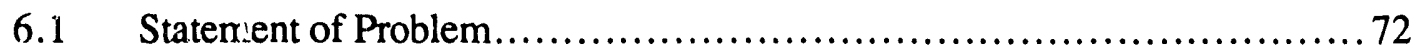

6.2 Rationale for Feedforward Control .................................. 72

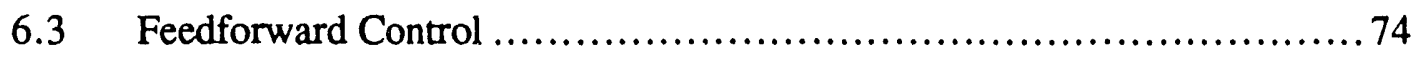

6.4 Experimental Evaluation........................................ 82

6.5 Assessment of Feedforward Control Techniques ...................... 82

7. Simulation of PWR-Type Multi-Modular Power Plants...................... 89

7.1 Statement of Problem............................................ 89

7.2 Basis of PWR-Type Multi-Modular Power Plants....................... 90

7.3 Models Used in Multi-Modular Plant Simulator....................... 90

7.3.1 Neutron Kinetics Model ..................................... 94

7.3.2 Fuel Temperature Model ................................. 95

7.3.3 Primary Coolant System Model.............................100

7.3.4 Steam Generator Secondary Side Model........................ 104

7.3.5 Main Steam Line Common Header Model......................106

7.3.5.1 Matrix Representation of MSLCH State

Equations...

$7.4 \quad$ Numerical Solution Methods ........................................ 112

7.4.1 Finite Difference Approximation to System Equations...........112 
-xii-

7.4.1.1 Neutron Kinetics Model ...........................112

7.4.1.2 Fuel Temperature Model........................113

7.4.1.3 Primary Coolant System Model ..................113

7.4.1.4 Steam Generator Secondary Side Model ..............115

7.4.1.5 Main Steam Line Common Header Model .............115

7.4.2 Numerical Solution Procedures ...............................115

7.4.2.1 Steady-State Simulation ........................117

7.1.2.2 Transient Simulation ............................117

7.5 Evaluation of Multi-Modular Plant Simulator.............................120

7.5.1 Steady-State Simulation..................................120

7.5.2 Transient Simulation.....................................124

7.5.2.1 Null Transient Simulation .......................124

7.5.2.2 Symmetry Test............................... 124

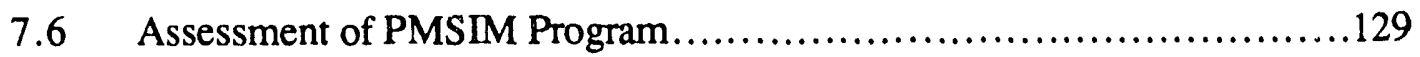

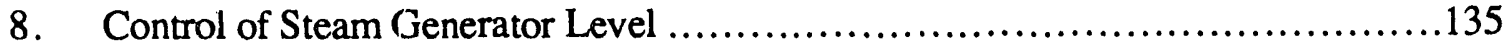

8.1 Statement of Problem............................................. 135

8.2 Steam Generator Design, Dynamics, and Modeling ..........................

8.2.1 Steam Generator Design and Operation .......................136

8.2.1.1 Feedwater Flow Control System ..................138

8.2.2 Steam Generator Water Level Dynamics........................139

8.2.2.1 Shrink and Swell Effects ........................141

8.2.2.2 Inverse Response...........................151

8.3 Simplified Steam Generator Model ................................. 151

8.3.1 Simplified Transfer Function...............................152

8.3.2 Identification of Model Parameters ..........................154

8.3.3 Evaluation of the Simplified Steam Generator Model .............162 
8.4 Modeling and Analysis of Current-Generation Steam Generator Level Controllers

8.4.1 Model of Current-Generation Steam Generator Level Controllers

8.4.2 Analysis of Current-Generation Steam Generator Level Controllers

8.4.2.1 Frequency-Domain Analysis..........................168

8.4.2.2 Time-Domain Analysis

8.4.3 Summary of Control Problems Associated with CurrentGeneration Steam Generator Level Controllers

8.5 Design of Proposed Steam Generator Level Controller

8.5.1 Solutions Previously Proposed to the Inverse Response Issue.

8.5.2 Compensator Design and Evaluation ............................182

8.5.2.1 Design of the Feedwater Flowrate Compensator ......183

8.5.2.2 Stability Analysis of the Closed-Loop System with the Feedwater Flowrate Compensator 188

8.5.2.3 Design of the Load Parameter Compensators.

8.5.2.4 Incorporation of Feedforward Action. 196

8.5.3 Controller Synthesis. 196

8.6 Evaluation of Proposed Steam Generator Level Controller .................199

8.6.1 Feedwater Flowrate Perturbation Transients ...................179

8.6.2 Power Transients .......................................201

8.6.3 Application to Multi-Modular Power Plants .....................206

8.6.4 Sensitivity Study of the Compensation Parameters...............211

8.7 Assessment of Compensator-Based Steam Generator Level Controller.....213

9. Control of Power in PWR-Type Multi-Modular Reactors ......................214

9.1 Statement of Problem..............................................214

9.2 Control of Power and Temperature in PWRs ......................215 
9.2.1 PWR Control Strategies ......................................

9.2.2 PWR Analog Controller Control Logic ........................217

9.3 Operation of PWR-Type Multi-Modular Power Plants....................220

9.3.1 Operating Characteristics...................................220

9.3.2 Operational Modes and Operating Strategies .....................224

9.3.3 Control of Power and Temperature............................227

9.3.3.1 Adjustment of Turbine Valve Position -- Power Change............................................227

9.3.3.2 Adjustment of Control Rod Position Temperature Change ................................227

9.3.3.3 Adjustment of Multi-Modular Reactor Power and Temperature........................................230

9.4 PWR-Type Multi-Modular Reactor Controller ..........................235

9.4.1 Controller Architecture and Components .......................235

9.4.1.1 Plant Power Controller ..............................235

9.4.1.2 Module Power Controller ............................238

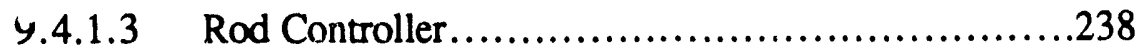

9.5 Evaluation of PWR-Type Multi-Modular Reactor Controller..............240

9.5.1 Evaluation of the Rod Controller................................243

9.5.2 Evaluation of Module Power Controller .......................245

9.5.3 Evaluation of Plant Power Controller ..........................245

9.5.3.1 Case One: Power Increase in the Specified Power Demand Mode.

9.5.3.2 Case Two: Power Increase in the Arbitrary Power Demand Mode...............................251

9.5.3.3 Case Three: Power Decrease in the Specified Power Demand Mode.

9.5.3.4 Case Four: Power Decrease in the Arbitrary Power Demand Mode 
9.5.3.5 Case Five: Power Transient When Demand

Allocation Method is Changed...

9.6 Assessment of PWR-Type Multi-Modular Power Plant Controller .268

10. Issues Regarding the Design and Acceptance of Intelligent Support Systems for Reactor Operators

10.1 Statement ố Problem..................................................269

10.2 Operator Needs......................................................272

10.3 Expert System Capabilities .........................................273

10.4 Design $e_{i}$ Reai-Time Intelligent Support Systems ......................275

10.4.1 Source of Knowledge ......................................275

10.4.2 Content of Knowledge Base .............................275

10.4.3 Mode of Interaction.......................................277

10.4.4 Organization of Knowledge Base............................277

10.4.5 Inference Engine .........................................278

10.4.6 Man-Machine Interface....................................279

10.5 Acceptance of Real-Time Intelligent Support Systems ....................279

10.5.1 Training ..................................................280

10.5.2 Signal Validation..........................................280

10.5.3 Alarm Filtering and Prioritization ...........................280

10.5 .4 Predictive Displays ......................................281

10.5.5 Operator Assistanc's......................................281

10.6 Assessment of Potential for the Use of Expert Systems in MultiModular Plants .283

11. Issues Relevant to the Digital Control of Multi-Modular Reactors .................285

11.1 Automated Operation of Multi-Modular Plants .........................285

11.2 Suggestions on the Operation of Multi-Modular Plants ..................285

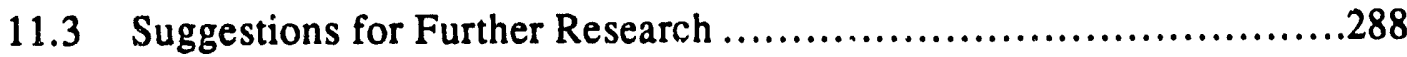


References 290

Appendix A Steam Generator Water Level Predictive Display Program. Al

A.1 Statement of Problem...........................................A1

A.2 Predictive Display Program .................................A2

Appendix B Smith's Dead-Time Compensator.................................. 1

B.1 Statement of Problem ................................................

B.2 Compensator Function and Design............................B2 
-xvii-

\section{List of Figures}

Eigure

Title

Page

Schematic Diagram of PW'R-Type Multi-Modular Power

Isometric View of MIT Research Reactor .5

Vertical Cross Section of MIT Research Reactor

Isometric View of Annular Core Research Reactor Facility.

(Source: Sandia National Laboratories).

Structure of Digital Closed-Loop Controller.

Importance of Signal Validation

Strip Chart Recording of Power and Rod Bank Height:

Dropped Rod Experiment ...

Fission Irocess Shorving Prompt, Delayed, and PhotoNeutron Production

Reactor Transient Illustrating Need for Supervisory Control

Power Prediction Using a Linearized Model....

Power Increase from $15.2 \mathrm{~kW}$ to $170 \mathrm{~kW}$ on the Annular Core Research Reactor Using the Alternate Reactivity

Constraint Approach

Period-Generated Control

Configuration and Functions of MITR-II Advanced Control

Computer System ....

Power Increase from 100 to $500 \mathrm{~kW}$ on a 100 -s Period

Using Alternate MIT-SNL Law with Proportional-Integral

Feedback ....

Sinusoidal Power Variation from 100 to $200 \mathrm{~kW}$ on

Frequency of One Cycle Every $90 \mathrm{~s}$ Using Alternate MrT-

SNL Law....

5.4-1 Demonstration of Perturbed Reactivity Method for an

Automated Startup with Reactor Initially Subcritical by 100 mß; Static Subcriticality Conditions Assumed.

Use of Perturbed Reactivity Method to Estimate Initial Reactivity Present for Automated Startup Shown in Figure 1

$5.4-3$

Use of Perturbed Reactivity Method to Estimate Initial Reactivity Present for an Automated Startup from Full Shutdown Conditions (Initial Phase) 
List of Figures (Continued)

Figure

Title

Page

$5.4-4$

Use of Perturbed Reactivity Method to Estimate Initial

Reactivity Present for an Automated Startup from Full

Shutdown Conditions (Intermediate-I Phase).

Use of Perturbed Reactivity Method to Estimate Initial

Reactivity Present for an Automated Startup from Full

Shutdown Conditions (Intermediate-II Dhase).

Use of Perturbed Reactivity Method to Estimate Initial

Reactivity Present for an Automated Startup from Full

Shutdown Conditions (Final Phase)...

Demonstiation of Perturbed Reactivity Method for an

Automated Startup with Reactor Initially Subcritical by

$1000 \mathrm{~m} \beta$; Dynamic Subcriticality Conditions Assumed

Use of Perturbed Reactivity Method to Estimate Initial

Reactivity Present for Automated Startup Shown in

Figure 5.4-7....

Error-Driven Feedback Control

Pure Feedforward Control

Hybrid Feedforward/Feedback Control

Pure Feedforward Controller Demonstrated on MIT

Research Reactor. 78

$6.3-4$

Hybrid Feedforward/PD-Feedback Controller Demonstrated

on MIT Research Reactor ....

Period-Generated Controller Demonstrated on MIT Research

Reactor.

$6.4-1$

Pure Feedforward Controller

Feedforward/PD-Feedback Controller.

Feedforward/PD-Feedback Controller with Perturbation .85

Feedforward/PID-Feedback Controller with Perturbation

Period-Generated Controller with Perturbation........................ 87

Schematic Diagram of PWR-Type Multi-Modular Power

Contrul Rod Worth Versus Control Rod Position [77]

Fuel Temperature Reactivity Coefficient [77] .96 
$-x i x-$

\section{List of Figures (Continued)}

Figure

7.3.3-2

7.3.4-1

7.3.4-2

7.3.5-1

7.4.2-1

7.4.2.1-1

7.4.2.1-2

7.4.2.2-1

7.5.1-1

7.5.1-2

7.5.1-3

7.5.2.1-1

7.5.2.1-2

7.5.2.1-3

7.5.2.1-4

7.5.2.1-5

7.5.2.1-6

7.5.2.1-7

7.5.2.2-1

7.5.2.2-2

7.5.2.2-3

7.5.2.2-4

7.5.2.2-5
Title

Page

Typical Control Region for the Primary Coolant System

Model

Steam Generator Secondary Site Model [68]

Feedwater Temperature as Function of Module Power

MSLCH Simulation Model ...

Information Flow Between the System Submodels.

Steady-State Simulation Procedure 118

Flow Chart for Steady-State Calculation of Reactor Primary and Secondary Conlant System State Variables

Transient Simulation Procedure

Average Primary Coolant Temperature at Steady-State

.122

Steam Flowrate from Each Steam Generator at Steady-State

Steam Generator Internal Pressure at Steady-State

Calculated Steam Flowrates of Module \#1 for Two Different

Time Step Sizes

Calculated Average Coolant Temperature and Steam

Generator Pressure of Module \#1 for Two Different Time

Step Sizes

Neutron Power During Null Power Transient

Average Primary Coolant Temperature During Null Power

Transient.....................................................126

Steam Generator Pressures During Null Power Transient

Steam Flowrate from Each Power Module During Null

Power Transient.

Pressure and Quality at the Main Steam Line Header During

Null Power Transient.

Neutron Power During Symmetry Test. (Only Module \#2's

Power is Changed).

Neutron Power During Symmetry Test. (Only Module \#4 's

Power is Changed).

Average Primary Coolant Temperature During Symmetry

Test. (Only Module \#2's Power is Changed).

Average Primary Coolant Temperature During Symmetry

Test. (Only Module \#4's Power is Changed).

Steam Flowrate from Each Power Module During Symmetry

Test. (Only Module \#2's Power is Changed). 


\section{List of Figures (Continued)}

Eigure

Title

Page

7.5.2.2-6

7.5.2.2-7

7.5.2.2-8

7.5.2.2-9

7.5.2.2-10

8.2.1-1

8.2.1.1-1

8.2.2-1

8.2.2.1-1

8.2.2.1-2

8.2.2.1-3

8.2.2.1-4

8.2.2.1-6

8.2.2.1-7

8.2.2.1-8

8.2.2.2-1

8.2.2.2-2

8.3.2-1

Steam Flowrate from Each Power Module During Symmetry

Tesi. (Only Module \#4's Power is Changed)

Steam Generator Level During Symmetry Test. (Only

Module \#2's Power is Changed)

Steam Generator Level During Symmetry Test. (Only

Module \#4's Power is Changed)

Pressure and Quality at the Main Steam Line Common

Header during Symmetry Test. (Only Module \#2's Power is

Changed)

Pressure and Quality at the Main Steam Line Cornmon

Header during Symmetry Test. (Only Module \#4's Power is

Changed)

Schematic of U-Tube Stearn Generator [83]

Steam Generator Water Leve: Control [83]

Steady-State Recirculation Flowrate

Schematic View of Steam Generator Level Shrink

Phenomenon

Steam Generator Level Shrink Effect as Result of a Sudden

Feedwater Flowrate Increase

Steam Generator Level Shrink Effect as Result of a Sudden

Steam Flowrate Decrease.

Steam Generator Level Shrink Effect a Result of a Sudden

Primary Coolant Temperature Decrease

Schematic View of Steam Generator Level Swell

Phenomenon

Steam Generator Level Swell Effect as Result of a Sudden

Feedwater Flowrate Decrease

Steam Generator Level Swell Effect as Result of a Sudden

Steam Flowrate Increase

Steam Generator Level Swell Effect as Result of a Sudden

Primary Coolant Temperature Increase

Typical Inverse Response of a System Consisting of an

Integral Term and a First Order Lag Term

Non-Inverse Response of a System Consisting of an Integral

Term and a First Order Lag Term

Steam Generator Simplified Transfer Function Coefficient,

$\mathrm{G}_{2 \mathrm{f}}$ 


\section{List of Figures (Continued)}

Eigure

Title

Page

Steam Generator Simplified Transfer Function Coefficient,

$\mathrm{T}_{2 \mathrm{f}}$.....

Steam Generator Simplified Transfer Function Coefficient, $\mathrm{G}_{3 i}$

Steam Generator Simplified Transfer Function Coefficient, $\mathrm{T}_{3 \mathrm{f}}$.

Steam Generator Simplified Transfer Function Coefficient, $\omega_{3 f}$.

Steam Generator Simplified Transfer Function Coefficient, $\mathrm{G}_{2 \mathrm{~s}}$

Steam Generator Simplified Transfer Function Coefficient, $\mathrm{T}_{2 \mathrm{~s}}$

Steam Generator Simplified Transfer Function Coefficient,

$\mathrm{G}_{1 \mathrm{~T}}$.

Steam Generator Simplified Transfer Function Coefficient, $\mathrm{G}_{2 \mathrm{~T}}$.

Steam Generator Simplified Transfer Function Coefficient, $\mathrm{T}_{1 \mathrm{~T}}$

Steam Generator Simplified Transfer Function Coefficient,

$\mathrm{T}_{2 \mathrm{~T}}$

Steam Generator Level after Feedwater Flowrate Increase in Step Fashion at $20 \%$ FP

8.3.3-2 Steam Generator Level after Steam Flowrate Increase in Step

Fashion at $20 \% \mathrm{FP}$.

8.3.3-3 Steam Generator Level after Primary Coolant Temperature

Increase in Step Fashion at $20 \%$ FP

8.4.1-1 Block Diagram of Current-Generation Steam Generator

Water Level Controller Configured for High Power

Operation

8.4.1-2 Block Diagram of Current-Generation Steam Generator

Water Level Controller Configured for Low Power

Operation

8.4.2.1-1 Pole and Zero Map of the Single-Element Steam Generator Level Transfer Function at $10 \%$ FP.

8.4.2.1-2 Pole and Zero Map of the Single-Element Steam Generator Level Transfer Function at $100 \% \mathrm{FP}$. 
Root Locus Plot of the Clpsed-Loop Transfer Function with a Proportional Controller $\left(\mathrm{T}_{1}=\infty\right)$ at $10 \% \mathrm{FP}$

Root Locus Plot of the Clpsed-Loop Transfer Function with a Proportional Controller $\left(\mathrm{T}_{1}=\infty\right)$ at $50 \% \mathrm{FP}$

Root Locus Plot of the Closed-Loop Transfer Function with

Maximum Feedback Control Gain as a Function of Power

Root Locus Plot of the Closed-Loop Transfer Function with a Proportional plus Integral Controller with $\mathrm{T}_{1}=228 \mathrm{~s}$

(Tuned by Ziegler-Nichols Method) at $10 \%$ FP

Steam Generator Level Response to $5 \mathrm{~kg} / \mathrm{s}$ Step Feedwater Flowrate Perturbation. (Single-Element PI Controller at $10 \% \mathrm{FP}$ ).

Effect of Tuning on Controller Performance During the Feedwater Jowrate Perturbation. (PI Controller at $10 \% \mathrm{FP}$ ).

Steam Generator Level Response to $5 \mathrm{~kg} / \mathrm{s}$ Step Feedwater

Flowrate Perturbation. (Single-Element PI Controller at $100 \%$ FP)

8.4.2.2-4 Steam Generator Level Response to $5 \mathrm{~kg} / \mathrm{s}$ Step Feedwater Flowrate Perturbation. (Three-Element PI Controller at $10 \% \mathrm{FP}$ )

Block Diagram of Feedwater Flowrate Compensator

Compensated Level After Feedwater Perturbation Without

Level Control Action.

Equivalent Block Diagram of Feedback Type Feedwater

Flowrate Compensator.

8.5.2.2-2 Root Locus Plot of the Closed-Loop Transfer Function Associated with Feedwater Flowrate Compensator $\left(\alpha_{\mathrm{f}}=10 \mathrm{~s}\right)$ at $10 \% \mathrm{FP}$.

8.5.2.2-3 Root Locus Plot of the Closed-Loop Transfer Function Associated with Feedwater Flowrate Compensitor $\left(\alpha_{\mathrm{f}}=100 \mathrm{~s}\right)$ at $10 \% \mathrm{FP}$

8.5.2.3-1 Steam Generator Level During Power Ramp Transients from $10 \% \mathrm{FP}$ to $15 \% \mathrm{FP}$ at a $5.0 \% \mathrm{FP} /$ minute Ramp Rate

Block Diagram of Load Parameter Compensators. 
-xxiii-

List of Eigures (Continued)

Eigure

Title

Page

8.5.3-1

8.5.3-2

8.6.1-1

8.6.? -1

8.6.2-2

8.6.2-3

$8.6 .2-4$

8.6.3-1

8.6.3-2

8.6.3-4

8.6.3-6

8.6.4-1

9.2.1-1

9.2.1-2

Schematic of the Overall Proposed Steam Gencrator Water Level Control System

Effect of Inverse Response Compensation During Power

Ramp Transients from $10 \% \mathrm{FP}$ to $15 \% \mathrm{FP}$ at a 5.0

\%FP/minute Ramp Rate

Steam Generator Level During Feedwater Flowrate

Perturbation Transient at $10 \%$ FP

Steam Generator Level During Power Ramp Transients from $10 \% \mathrm{FP}$ to $15 \% \mathrm{FP}$ at a $5.0 \% \mathrm{FP} /$ minute Ramp Rate ..........203

Steam and Feedwater Flowrate Variation During Power

Ramp Transients from $10 \% \mathrm{FP}$ to $15 \% \mathrm{FP}$ at a 5.0

\%FP/minute Ramp Rate.

Steam Generator Narrow Range Level During Power Ramp

Transients from $15 \% \mathrm{FP}$ to $10 \% \mathrm{FP}$ at a $5.0 \% \mathrm{FP} /$ minute

Ramp Rate

Steam Generator Narrow Range Level During Power Ramp

Transients from $95 \% \mathrm{FP}$ to $100 \% \mathrm{FP}$ at a $5.0 \% \mathrm{FP} /$ minute

Ramp Rate.

Steam Generator Narrow Range Level During Power Ramp

Transients from $10 \% \mathrm{FP}$ to $15 \% \mathrm{FP}$ at a $5.0 \% \mathrm{FP} /$ minute

Ramp Rate on Module \#1 Only. .208

Steam Flowrate During Unbalanced Power Ramp Transients from $10 \% \mathrm{FP}$ to $15 \% \mathrm{FP}$ at a $5.0 \% \mathrm{FP} /$ minute Ramp Rate on Module \#1 Only .208

Steam Generator Narrow Range Level During Balanced and Unbalanced Power Ramp Transients

Steam Generator Narrow Range Level During Unbalanced Power Ramp Transients from $10 \%$ FP to $15 \% \mathrm{FP}$ at a $5.0 \% \mathrm{FP} /$ minute Ramp Rate on Module \#1 Only ....

Steam Flowrate During Unbalanced Power Ramp Transients with PI Controller Tuned to Lower Feedback Control Gain $(2.0 \mathrm{~kg} / \mathrm{s} / \%)$.

Steam Flowrate During Unbalanced Power Ramp Transients.

(Module \#1: from 15\%FP to 20\%FP; Module \#2: from 15

$\%$ FP to $10 \%$ FP; Module \#3 and \#4: held at $15 \%$ FP)

Steam Flowrate During Power Ramp Transients with Incorrect Compensation

Constant Average Temperature Control Program

Constant Steam Pressure Control Program 


\section{List of Figures (Continued)}

Eigure

Title

Simplified PWR Analog Control System

(Source: Shearon-Haris I\&C Manual [100]) .....................218

9.2.2-2 Simplified PWR Rod Control System

(Source: Shearon-Haris I\&C Manual [100]) ......................219

Operation Under Sliding Average Temperature Control

Program with Unbalanced Loads ....

9.3.1-2

PWR-Type Multi-Modular Power Plant Operation Map.

(Steady-State Average Primary Coolant Temperature)

PWR-Type Multi-Modular Power Plant Operation Map.

(Steady-State Steam Generator Pressure)

Behavior of PWR Following Increase in Turbine Valve

Position

(Continued)

Behavior of PWR Following Rod Withdrawal

(Continued)

Behavior of Primary Temperature and Steam Generator

Pressure in Multi-Modular Plant upon Shift to Unbalanced

Load Conditions.

Behavior of Power and Steam Flowrate in Multi-Modular

Plant upon Shift to Unbalanced Load Conditions

Hierarchical Structure of PWR-Type Multi-Modular

Controller. (Source: After ORNL [102]) .236

9.4.1.1-1

Relationship Between Plant Power Controller and Other

Systems. (Source: After ORNL [102]) ......................237

9.4.1.3-1

Rod Speed and Direction Control Program ....

Schematic of Proposed Rod Controller. .242

9.5.1-1

Simulation Study of Rod Controller .244

9.5.3.1-1

Module and Turbine Power. (Case 1 Transient) .248

9.5.3.1-2

Average Primary Coolant Temperatures. (Case 1 Transient).. .248

9.5.3.1-3

Equivalent Control Rod Positions. (Case 1 Transient) .249

9.5.3.1-4

Steam Flowrates from Individual Steam Generators.

(Case 1 Transient).

9.5.3.1-5

Steam Generator Levels. (Case 1 Transient). .250

9.5.3.1-6

Steam Generator Pressures. (Case 1 Transient) .250

Module and Turbine Power. (Case 2 Transient). .252 
$-X X V$

\section{List of Figures (Continued)}

Eigure

Title

Page

9.5.3.2-2

Average Primary Coolant Temperatures. (Case 2 Transient)..........252

9.5.3.2-3

Control Rod Positions. (Case 2 Transient)

.253

9.5.3.2-4

Steam Flowrates from Individual Steam Generators.

9.5.3.2-5

(Case 2 Transient)....

9.5.3.2-6

Steam Generator Levels. (Case 2 Transient)

.254

Steam Generator Pressures. (Case 2 Transient) ....................254

9.5.3.3-1

Module and Turbine Power. (Case 3 Transient)

9.5.3.3-2

Average Primary Coclant Temperatures. (Case 3 Transient)

9.5.3.3-3

Steam Flowrates from Individual Steam Generators.

(Case 3 Transient)..... .258

9.5.3.3-4

Steam Generator Levels. (Case 3 Transient) .258

9.5.3.4-1

Module and Turbine Power. (Case 4 Transient) .260

9.5.3.4-2

Average Primary Coolant Temperatures. (Case 4 Transient). .260

9.5.3.4-3

9.5.3.4-4

Steam Flowrates from Individual Steam Generators.

(Case 4 Transient).

Steam Generator Levels. (Case 4 Transient) .261

9.5.3.5-1

Module and Turbine Power in Specified Power Demand

Mode. (Case 5 Transient). 264

9.5.3.5-2 Average Primary Coolant Temperatures in Specified Power Demand Mode. (Case 5 Transient) 264

9.5.3.5-3

Steam Flowrates from Individual Steam Generators in Specified Power Dernand Operational Mode. (Case 5 Transient) 265

9.5.3.5-4 Steam Generator Levels in Specified Power Demand Operational Mode. (Case 5 Transient) 265

9.5.3.5-5

Module and Turbine Power in Arbitrary Power Demand

Mode. (Case 5 Transient). 266

9.5.3.5-6 Average Primary Coolant Temperatures in Arbitrary Power Demand Mode. (Case 5 Transient).

Steam Flowrates from Individual Steam Generators in Arbitrary Power Demand Operational Mode. (Case 5 Transient). .267

9.5.3.5-8 Steam Generator Levels in Arbitrary Power Demand Mode. (Case 5 Transient)....................................................267

10.1-1 Application of Expert Systems Within the Nuclear Industry in 1989 [5] .270 


\section{List of Figures (Continued)}

Figure

Title

Page

A-2

Steam Generator Level Display.

A4

B-1

Schematic of Control System with Smith's Dead-

Time Compensator

B-2

Equivalent Block Diagram of the Control System with Smith's Dead-Time Compensator 
-xxvii-

\section{List of Tables}

Table

Title

Page

MIT Thesis Research on the Closed-Loop Digital Control of

Multi-Modular Reactors

PWR-Type Multi-Modular Power Plant Parameters Used in PMSIM

8.5.2.3-1 Control Performance Indices During Power Ramp Transients from $10 \% \mathrm{FP}$ tr. $15 \% \mathrm{FP}$ on a $5.0 \% \mathrm{FP} /$ minute Ramp Rate

8.6.1-1 Control Performance Inu'ces During Feedwater Flowrate Perturbation Transients at $10 \%$ FP.

Control Performance Indices During Power Ramp

Transients from $10 \% \mathrm{FP}$ to $15 \% \mathrm{FP}$ at a $5.0 \% \mathrm{FP} /$ minute

Ramp Rate.

8.6.2-2 Control Performance Indices During Power Ramp

Transients from $15 \% \mathrm{FP}$ to $10 \% \mathrm{FP}$ at a $5.0 \% \mathrm{FP} /$ minute

Ramp Rate.

8.6.2-3 Control Performance Indices During Power Power Ramp Transients from $95 \% \mathrm{FP}$ to $100 \% \mathrm{FP}$ at a $5.0 \% \mathrm{FP} /$ minute R.amp Rate.

8.6.4-1 Control Performance Indices During Power Ramp

Transienis with Incorrect Parameters

9.4.1.1-1 Static Time Delays for Module Power and Turbine Power

Demand Allocation

9.5.3.1-1 Sequence for Power Increase in Specified Power Demand

Mode.

9.5.3.3-1 Sequence for Power Decrease in Specified Power Demand

Mode.

9.5.3.5-1 Sequence for Power Transient with Change of Demand

Allocation Miethod.

Major Functions Associated with the Startup and Operation of a Multi-Modular Reactor Power Plant 


\section{Studies on the Closed-Loop Digital Control of}

\section{Multi-Modular Reactors}

\section{Scientific and Engineering Objectives}

This report describes the theoretical and experimental study of certain of the tasks needed for the closed-loop digital control of multi-modular reactors. Emphasized is the architecture of the digital control system, methods for automated startup with on-line estimation of the degree of initial subcriticality, a comparative assessment of trajectory tracking techniques, the numerical modeling of multi-modular plants, steam generator level control, the adjustment of module power and temperature in the presence of unbalanced loads, and the prospects for achieving automated diagnostics. The work reported here should contribute to the automated operation of multi-modular reactors and thereby (1) minimize required operating personnel and thus contain operating and training costs, (2) permit individual units of a multi-modular plant to be run at different power levels thereby avoiding the need to refuel all units simultaneously, and (3) maintain the competitiveness of U.S. industry relative to foreign vendors who are developing and applying advanced control concepts. The progress made relative to each of the aforementioned objectives is described in the ensuing chapters of this report.

The work reported here was performed jointly by the MIT Nuclear Reactor Laboratory (NRL) and the MIT Department of Nuclear Engineering (NED). The research was funded by the U.S. Department of Energy's Office of Energy Research (Division of University and Industry Programs) under Contract \#DE-FG07-90ER 12930 and by the Oak Ridge National Laboratory (ORNL) under a separate contract. The period covered by these two consecutive grants was 1 October 1988 - 30 June 1992.

The control research reported here is but one component of an on-going MIT program to develop and experimentally evaluate an integrated, fault-tolerant methodology for the closed-loop, digital control of power and temperature in nuclear reactors. In particular, the overall controller should provide signal validation, instrument fault detection, a supervisory algorithm that precludes challenges to the safety system, a number of selectable control laws, a means of reconfiguring those control laws depending on the mission, a method for verifying signal implementation, and some means for on-line performance assessment such as automated reasoning. In addition, there should of course be an independent safety system. Details of this approach, as applied to nuclear reactors, are given in four earlier reports. The first was prepared by MIT for the National Science Foundation under grant \#CPE-8317878 [1]. It describes the formulation and experimental evaluation of the 'reactivity constraint approach' which is a means of precluding challenges to a reactor's safety system as the result of any action initiated by an automated controller. The second report was prepared by MIT for Sandia National Laboratories (SNL) as part of the U.S. Department of Energy's Multi-Megawatt Reactor Program [2]. It describes the derivation and experimental assessment of the 'MT-SNL Period-Generated Minimum Time Control Laws' which are a trajectory tracking technique suitable for the rapid maneuvering of ieactor power. The third report, which was also prepared for Sandia National Laboratories, concerns applications of the MIT-SNL laws including their use for automated power increases from subcritical [3]. The fourth report, which was prepared for 
the U.S. Department of Energy, concerns the extension of the reactivity constraint approach to the closed-loop digital control of reactors characterized by spatial dynamics [4]. In addition to these four reports on reactor control, MTT has also conducted an extensive study of the use of expert systems within the nuclear industry [5]. That material bears on the issue of autonomous control.

\section{$1.1 \quad$ Multi-Modular Reactors}

The commercial generation of electricity from nuclear energy is a global reality. The 1992 edition of the World Nuclear Industry Handbook [6] cites the following figures: The United States, where the technology originated, leads the world with 106,905 MW(e) of installed nuclear generating capacity as of August 30,1991. This corresponds to $20.6 \%$ of the U.S. electricity supply. In Canada, $14.4 \%$ of the electricity supply is generated by nuclear facilities. Many European nations are also making significant use of nuclear energy. For example, in France, $75.0 \%$ of the electricity supply was generated from nuclear energy in 1990. In the neighboring nations of Switzerland and Belgium, the figures were 42.6 and $60.2 \%$, respectively. For the United Kingdom, the figure was $20.0 \%$; for Germany and Spain, 33.1 and $35.7 \%$, respectively; and for the Scandinavian nations of Finland and Sweden, nuclear electricity was 35.0 and $46.0 \%$ of the total, respectively. Among the nations of Eastern Europe, the use of nuclear generated electricity is also substantial. The figures for Hungary, Bulgaria, and Czechoslovakia for 1990 were $50.0,35.7$, and $28.5 \%$, respectively. The figure for the Soviet Union was $12.2 \%$. The situation is similar in Asia with 1990 usage figures for Japan, Korea, and Taiwan being $27.1,49.1$, and $38.3 \%$, respectively. The use of nuclear energy is also becoming significant in South America; Argentina, for example, is at $16.9 \%$.

Fission reactors offer many advantages when compared to alternate sources of energy. Specifically, they constitute an already available technology, the fuel supply can be made virtually inexhaustible through the use of breeder technology, and minimal pollutants are released during normal operation. These are significant considerations that are too often ignored by industry critics. Nevertheless, many nations are reevaluating the nuclear option and in some countries, the United States among them, there have been no new plant orders for many years. As a result, the industry's attention is currently focused on new powergeneration concepts that offer both greater safety and cost-effectiveness. One such possibility is a multi-modular arrangement in which several power modules (each with its own reactor core and steam generator) supply steam to a common header. There are several potential advantages to this concept. First, the small size of the reactor core may enable it to ride out a loss of coolant accident without either active decay heat removal or safety systems of any kind. Thus, the multi-modular concept has the potential to make the overall plant more reliable and less demanding of the control system than is a large, singlereactor plant. Second, the individual modules of a multi-modular power plant are to be sized so that components related to nuclear safety can be factory-fabricated. This is an advantage because quality can be more readily controlled in a factory than in the field. After the major components are made, they are to be transported to the site for rapid installation. This manufacture and construction method is expected to reduce the licensing effort because the module will be pre-licensed and only site-specific issues will have to be considered in the final licensing procedures. Third, a multi-modular power plant has the potential to provide a higher capacity factor over hat of existing, 'arge, single reactors because the modular make-up of the plant will ensure partial power output from unaffected 
units whenever any one unit or module is off-line for refueling and/or maintenance. Also, the small size of the components and the simplicity of the power loop should reduce maintenance.

Several multi-modular reactors have been designed in order to realize the above advantages. These are summarized here [7].

\subsubsection{Liquid Metal Multi-Modular Power Plants}

Liquid metal-cooled multi-mouular power plant designs include PRISM (Power Reactor Inherently Safe Module), proposed by General Electric Corporation, and SAFR (Sodium Advanced Fast Reactor), proposed by Rockwell International. The PRISM plant concept was subsequently selected by the U.S. Department of Energy (DOE) as the basis for an Advanced Liquid Metal Reactor. Accordingly, a brief summary of PRISM is given here.

Each PRISM reactor is a single loop, pool-type 134-MWe fast breeder reactor with annular flow. Three such reactors and steam generators together with one turbine form a single power block. Primary coolant (low-pressure liquid sodium) circulates through the core to remove heat generated by the fission process. The advantage of sodium is that it has excellent heat transfer characteristics. Its disadvantage is that it becomes activated by the neutron flux in the core and therefore may represent a hazard to personnel. In order to isolate the radioactive coolant, the sodium is fumped through an intermediate heat exchanger. That is, heat is transferred from the primary loop to the secondary loop by intermediate heat exchangers and a secondary sodium coolant loop then transports the thermal energy from the intermediate heat exchanger to a steam generator. Steain that is generated in each power module is supplied to the turbine through a common steam header. Feedwater to the three steam generators in the power block is also supplied through a common header.

The PRISM modules are grouped so that they share the major balance of plant components and thus capture the economies of scale of a larger plant without incurring the penalties associated with a larger core, such as the need for active emergency core cooling systems. A 1205-MWe plant consists of three power blocks (nine modules).

\subsubsection{Gas-Cooled Multi-Modular Power Plants}

Another multi-modular design is the Modular High Temperature Gas-Cooled Reactor (MHTGR). Two designs have been proposed, one jointly by Germany and GA Technologies and the other by the U.S. Department of Energy (DOE) $[8,9]$. Each power module is a pebble-bed reactor with four in-line steam generators located above or to the side of the reactor core. German pebble-bed core designs are graphite-reflected cylinders and are operated at 200 to $250-\mathrm{MWt}$ per module. Another possibility is the MHTGR that was designed under the U.S. DOE MFITGR Program. The DOE design is a $350-\mathrm{MWt}$ annular core with a steam generator located below and to the side of the core. In addition to these, MIT designed a direct-cycle gas turbine coupled MHTGR that gives high thermal efficiencies, up to $\sim 45 \%$ [10]. Because it does not use a steam generator or a secondary coolant loop, the MIT design has less potential for water ingress, a less complex balance of plant, simpler operation and maintenance, and greater cost-effectiveness. 
The MHTGR is a passively safe reactor with the design goal of essentially zero risk to the health and safety of the public. The key element in the MHTGR's passive safety concept is the fuel. The fissile material is encapsulated in multiple layers of pyrolytic carbon (PyC) and silicon carbide (SiC). These particles will not release fission products at burnups of well over $100,000 \mathrm{MWD} / \mathrm{tc}$ nne and they can sithstand temperatures greater than $1600^{\circ} \mathrm{C}$ without significant release of fission products.

\subsubsection{PWR-Type Multi-Modular Power Plants}

Figure 1.1.3-1 is a schematic of a possible Pressurized Water Reactor (PWR)-Type Multi-Modular Power Plant. This design was the basis for the models developed in the course of the research reported here. It was chosen for study because previous research at MIT on the digital control of nuclear reactors was oriented to PWRs and hence many data bases and benchmarked codes were available. This allowed the focus of the research to be directed more towards reactor control issues and less towards matters of model verification. It should be noted that the resulting control concepts are generic and can be applied to many types of multi-modular reactors.

\subsection{Rationale for the Closed-Loop Digital Control of Multi-Modular Reactors}

Arguments in support of the closed-loop digital control of nuclear reactors have been previously enumerated $[1-4,11]$. Accordingly, the discussion here is limited to factors that are directly relevant to the economical operation of multi-modular reactors:

1. Containment of Operating Costs: Automation, if properly implemented, may allow the same number of operating personnel to control two or three reactors as are now necessary to operate a single unit.

2. Reduction in Training Costs: The cost of a licensed operator is not merely that individual's salary. A far greater cost is usually the support staff and equipment needed to first train and then provide continual upgrading of each operator. If the number of operators can be limited to that now required for a large plant, then training costs can at least be kept constant.

3. Fuel Management: One of the advantages of the modular design concept is that only one of the clustered units would be out of service at any time. Hence, the capacity factor for the station as a whole would remain high and the need for expensive replacement electricity would be minimized. A prerequisite to the achievement of this advantage is that it be possible to deplete the fuel in each modular unit at a different rate. Otherwise, all units would require refueling at the same time. This in turn creates a complicated control problem because each reactor is supplying steam to a common header. Thus, for each unit, the pressure of the steam being generated must be equal even though it is desired that the thermal power outputs be different. This might be accomplished by operating each reactor at a different temperature. That is, the average primary coolant temperatures would be different. To do so will require a sophisticated control strategy. 


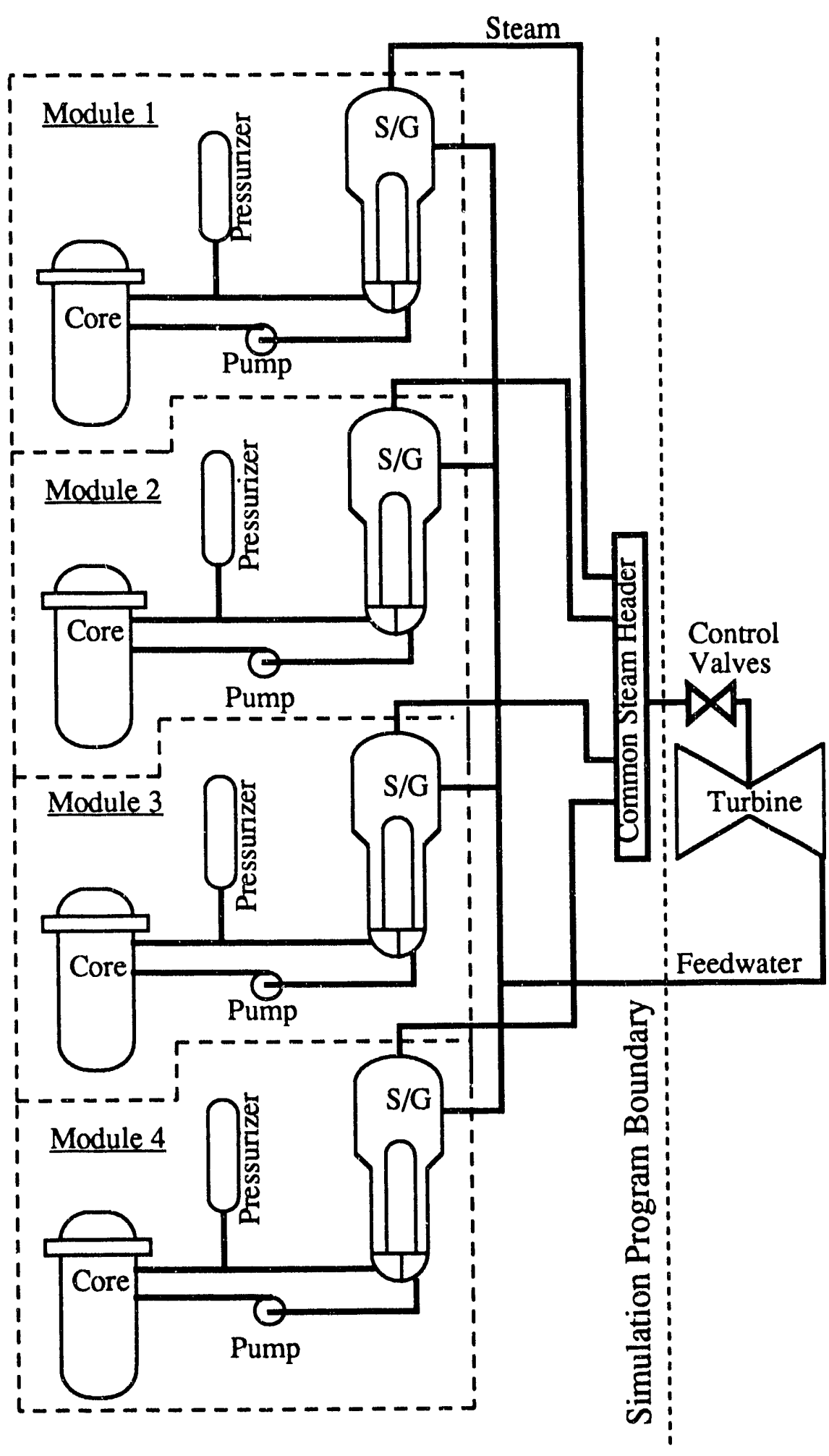

Figure 1.1.3-1 Schematic Diagram of PWR-Type Multi-Modular Power Plant. 
Another factor relevant to the consideration of digital technologies for the operation of multi-modular plants is the competitiveness of the U.S. nuclear industry in international markets. European, Asian, and Canadian vendors are all incorporating sophisticated digital designs in their products. The United States needs to do likewise or risk losing market share.

\section{$1.3 \quad$ MIT Program on Reactor Control}

The MIT program on advanced instrumentation and reactor control originated in the late 1970s under the direction of Professor David D. Lanning of the Nuclear Engineering Department. Studies at that time principally concerned the development of accurate, realtime models of various plant components such as pressurizers, steam generators, and condensers. The existence of accurate models is of course fundamental to the construction of a controller. In 1980, Dr. John Deyst, Dr. John H. Hopps, and Dr. Asok Ray, who were all from the Charles Stark Draper Laboratory (CSDL), initiated an experimental program at the 5-MWt MIT Research Reactor (MITR-II) to demonstrate signal validation and instrument fault detection. The 'parity space approach,' which was subsequently used on both test and commercial reactors, was one result of this effort [12]. Once both validated signals and real-time models were available, it became possible to consider closed-loop digital controllers. A supervisory algorithm that precluded challenges to the reactor's safety system was developed and demonstrated on the MITR-II by Dr. John A. Bernard in February 1983. Designated as the 'MIT-CSDL Non-Linear Digital Controller' or NLDC, this methodology uses reaciivity constraints to determine if a change should be made to the present control signal in order to avoid a power overshoot at some future time [1,13-15]. The 'reactivity constraint approach' was licensed by the U.S. Nuclear Regulatory Commission for general use on the MIT Research Reactor in April 1985 and soon became the basis of an experimental protocol for the on-line testing of novel control strategies under conditions of closed-loop digital control [16]. Funding for the MIT control program was provided by the National Science Foundation (Dr. Royal Rostenbach, Division of Energy and Energetics) from 1984 to 1986. Other significant results achieved with NSF Support included the design and implementation of a rule-based controller, an on-line demonstration of control law reconfiguration, and the derivation by Professor Allan F. Henry of the alternate formulation of the dynamic period equation [17-20]. In July 1986, a project was initiated with Sandia National Laboratories (SNL) to develop control strategies for reactor-powered spacecraft. One result of that effort was the derivation by Dr. Bernard of the 'MIT-SNL Period-Generated Minimum Time Control Laws' which are closed-form expressions that permit a reactor's neutronic power to be raised by many orders of magnitude both without overshoot and within a few seconds [2,3,21-23]. With the support of Dr. Kwan S. Kwok and Mr. Paul T. Menadier of MTT and Mr. Frank V. Thome and Mr. Francis J. Wyant of SNL, both these laws and the NLDC were subsequently demonstrated experimentally on SNL's Annular Core Research Reactor [2426]. In September 1986, a project was begun under the sponsorship of the U.S. Department of Energy to extend the reactivity constraint approach to large, spatiallydependent reactors. That work, which involved the development of 'faster-than-real-time' reactor models that use supernodal methods to describe the neutron flux behavior and fast running thermal-hydraulic codes to characterize reactivity feedback effects, is the subject of this report. In April 1988, a program was begun under the sponsorship of Sandia National Laboratories to investigate use of the MIT-SNL laws for the rapid startup and control of reactor-powered spacecraft [3]. That collaboration remains on-going and is now focused 
on the operation of spacecraft reactors that use thermionics. Also, in 1988, joint research was initiated with the Oak Ridge National Laboratory and the U.S. Department of Energy concerning the closed-loop digital control of power and temperature in multi-modular reactors. The results of that effort are the subject of this report. A complete, yet concise, summary of the MIT approach toward reactor control was given at the ANS/NIST Conference 'Fifty Years with Nuclear Fission' in April 1989 [27] and in several other, more recent publications $[28,29]$.

\subsection{Rationale for Experimental Research}

A distinguishing and perhaps unique characteristic of the MIT program on reactor control and also of the joint MIT-SNL research has been the performance of on-line experiments. Whenever possible, each control concept has been evaluated experimentally on either the 5-MWt Research Reactor (MITR-II) or the Annular Core Research Reactor (ACRR) that is operated by Sandia National Laboratories, or both. This was done in the firm conviction that true engineering progress can only be achieved through extensive experimentation and prototypic demonstration. Simulation studies are, of course, of importance. They serve to validate software, to provide initial evaluation of concepts, and to evaluate techniques beyond the allowed range of operation of actual systems. However, simulation is not a substitute for experiment. Simulations are successful only insofar as the process being studied can be modeled. If something is not known or not fully understood, it can not be simulated. Hence the need for well-designed and rigorously monitored experiments. Moreover, by performing on-line tests, confidence is built in the approach. It is unrealistic to expect either industry to adopt or regulators to accept advanced digital technology without there first being a history of reliable operation at the prototype level.

\section{$1.5 \quad$ Experimental Facilities}

Experiments to support the research reported here were conducted on the 5-MWt MIT Research Reactor (MITR-II). Accordingly, a brief description of that facility is given in the following subsection. Also described is the Annular Core Research Reactor (ACRR) that is operated by Sandia National iaboratories. The ACRR has figured prominently in several past MIT studies on reactor control concepts.

\subsubsection{MIT Research Reactor (MITR-II)}

The MITR-II is a 5-MWt, light-water cooled and moderated, heavy-water reflected, tank-type reactor that uses plate-type, uranium-aluminide fuel. The fuel is enriched to $93 \%$ U-235. Energy is continuously removed by forced circulation of the primary coolant. The maximum permitted operating temperature is $55^{\circ} \mathrm{C}$. Figures $1.5 .1-1$ and 1.5.1-2 are isometric and vertical cross-section views respectively of the facility.

The nuclear instrumentation used for the research described in this report consisted of three neutron flux sensors and a gamma-ray sensor that correlated neutron power with the radioactivity $(\mathrm{N}-16)$ of the primary coolant. All four sensors were directly proportional to the power over the range of interest. Measurements were also available of the coolant flow, coolant temperature, and control mechanism position. Four independent measurements of primary coolant flow were obtained from the pressure differences across orifices. Primary coolant temperatures were measured as follows: two sensors for the hot 


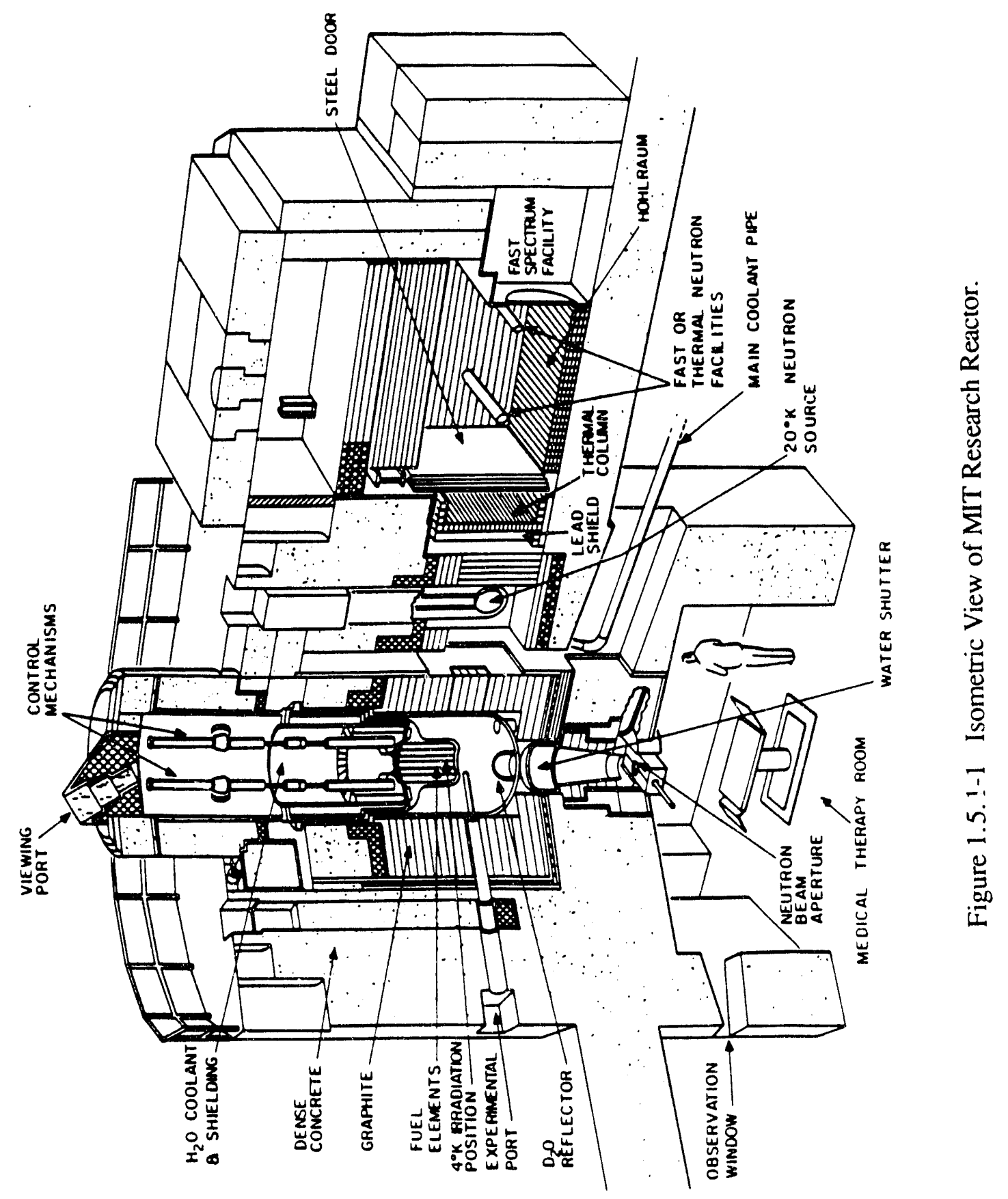




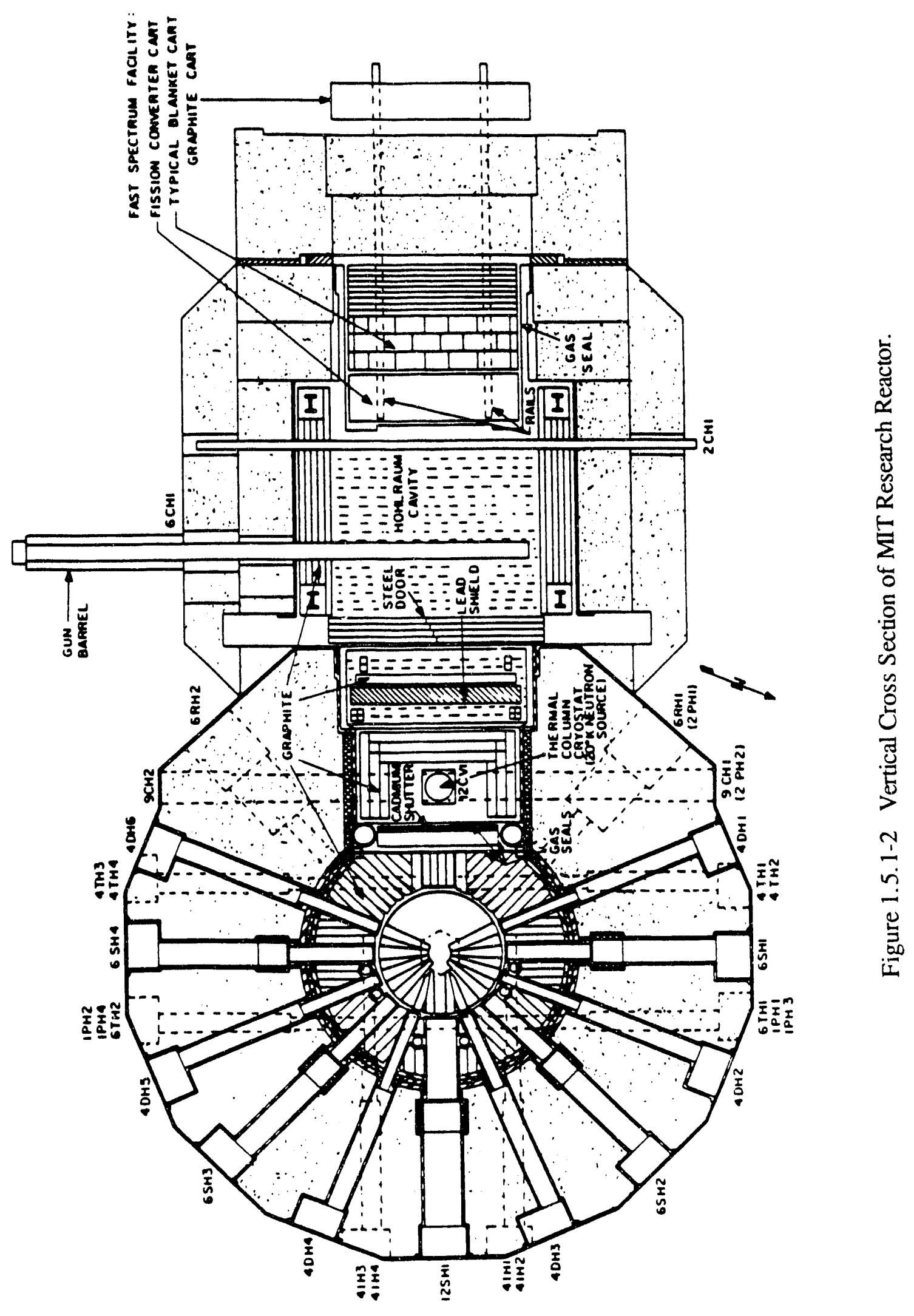


leg, two sensors for the cold leg, and one sensor for the temperature difference between the legs. In effect, three measurements were available for the temperature difference. None of the sensors that form the MITR-II's safety system were used for this research. Also, it should be noted that the noise and statistical characteristics of the MITR-II's flow, temperature, and neutron flux instrumentation are similar to those in commercial reactors.

Coarse control of the power in the MITR-II is achieved by positioning a bank of six shim blades. Once critical, the neutron flux is normally maintained constant by adjusting the position of a fine-control regulating rod. Both the regulating rod and one of the shim blades were made available to the experimental program described in this report. Each is normally moved at a fixed speed of 4.25 inches per minute. However, for the research reported here, each was specially equipped with a variable speed stepping motor so that the rate of change of reactivity could be made to vary as specified by the control laws. The minimum allowed periods on the MITR-II are 50-seconds steady and 30-seconds dynamic. There is a negative coefficient of reactivity associated with the fuel, coolant, and reflector temperatures. However, its magnitude is rather small, averaging $-8 \cdot 10^{-5} \Delta \mathrm{K} / \mathrm{K} / \mathrm{C}$. The MITR-II's effective delayed neutron fraction and prompt neutron lifetime are 0.00786 $\Delta \mathrm{K} / \mathrm{K}$ and 100 microseconds respectively.

\subsubsection{Annular Core Research Reactor (ACRR)}

The ACRR is a modified TRIGA that uses $\mathrm{UO}_{2}-\mathrm{BeO}$ fuel elements, enriched to $35 \%$ U-235. Its annular-shaped core is formed by 236 of these elements arranged in a hexagonal grid around the $23-\mathrm{cm}$ diameter, dry, central irradiation cavity. The reactor operates in either a steady-state or a pulsed mode. For steady-state operation, the maximum allowed power level is $2 \mathrm{MWt}$. For pulsed operation, there is no restriction on the power. Rather, there is a limit of $500 \mathrm{MJ}$ total energy per pulse and one of $1800^{\circ} \mathrm{C}$ on the fuel temperature.

The ACRR is controlled by two fuel-followed safety rods, three poison transient rods, and six fuel-followed control rods. The transient rods, which are operated as a bank and which are driven by variable speed stepping motors, were used for the conduct of the experiments. There is an exceptionally large negative coefficient of reactivity associated with the fuel which is, in units of $\Delta \mathrm{K} / \mathrm{K} / \mathrm{C}$, given by the expression $(-3.85-730 / \mathrm{T}) \cdot 10^{-5}$ where $\mathrm{T}$ is the temperature of the fuel in $\mathrm{K}$. The ACRR' $s$ effective delayed neutron fraction and prompt neutron lifetime are $0.0073 \Delta \mathrm{K} / \mathrm{K}$ and 24 microseconds respectively. Figure 1.5.2-1 is a vertical cutaway view showing the major components of the ACRR.

\subsection{Experimental Protocol}

The testing of novel control strategies on the MIT Research Reactor is permitted if the following protocol is observed. First, the heat removal and the reactor safety systems are prepared for operation at full power, $5 \mathrm{MW}$. Second, the control strategy that is to be tested is permitted to raise or lower the power over some portion of the normal operating range, usually 1-4 MW. Third, the decisions of the novel controller are reviewed by the MIT-CSDL Non-Linear Digital Controller (NLDC) prior to their being implemented. The NLDC is based on the 'reactivity constraint approach' [1]. The NLDC is programmed to intervene if a decision made by the novel controller could result in the power exceeding some fraction of the maximum allowed power, usually 4.5 MW. This arrangement 


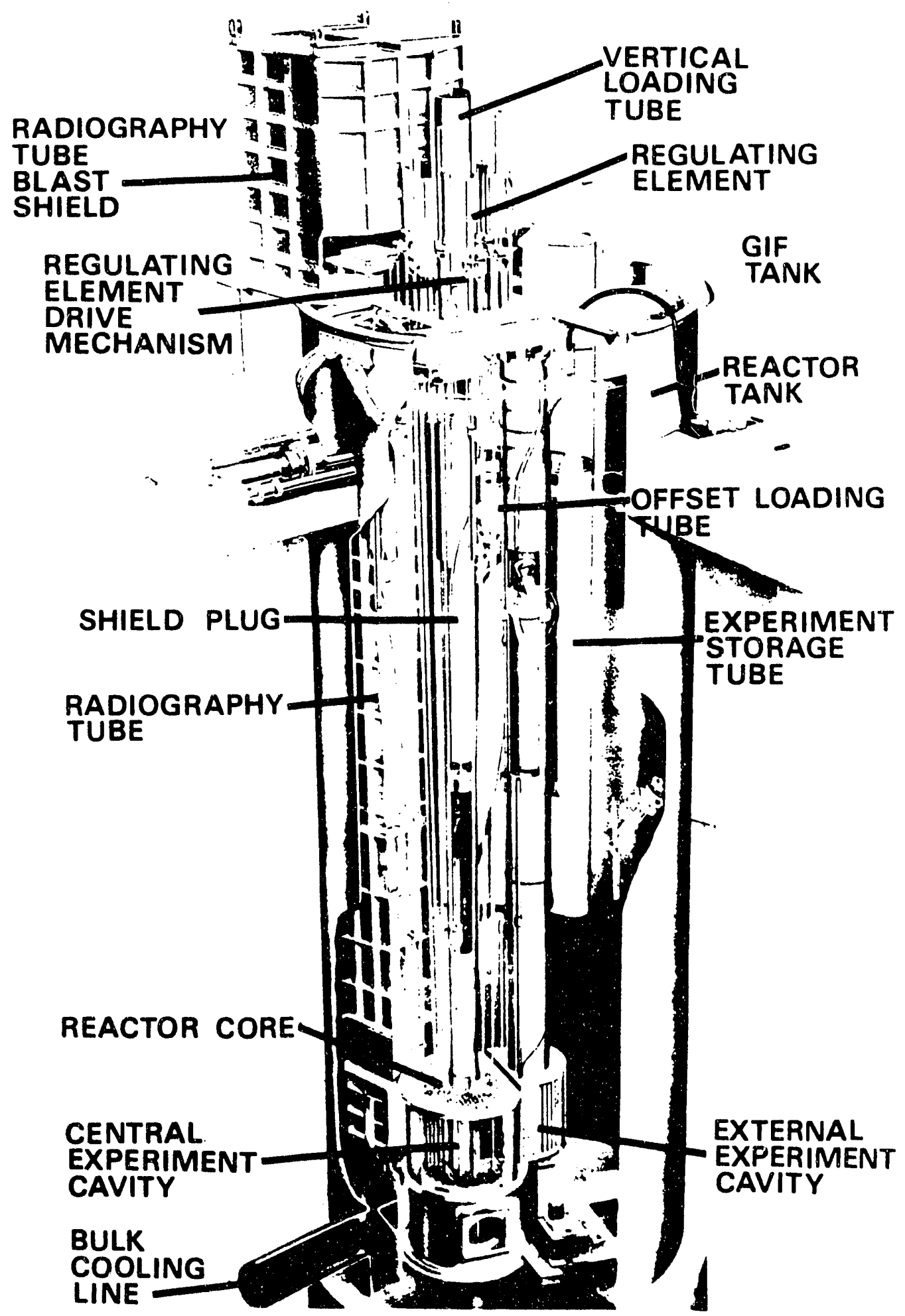

Figure 1.5.2-1 Isometric View of Annular Core Research Reactor Facility. (Source: Sandia National Laboratories) 
guarantees that the novel controller will not challenge the safety system while permitting it to act as if it had full control. Hence, when examining the experimental results shown in this report, it should be realized that the fact that power overshot the targeted value in some cases is significant to the experiment, but never to the reactor which was at all times operated conservatively.

It was necessary to modify this protocol for use on the Annular Core Research Reactor (ACRR). The limiting condition for the ACRR is not a specific power level but rather the total energy produced during the transient. Accordingly, it was originally thought necessary to develop an energy constraint which would assure that the reactor would be at or below its allowed steady-state operating power prior to the limit on integrated power production being exceeded. Such a constraint was developed and used for the initial experiments performed on the ACRR [2]. However, as confidence grew in the technology and as experience was gained by the experimenters on the operation of the ACRR, it was realized that an energy constraint was not necessary. The final protocol adopted for ACRR control experiments contained three provisions. First, limits were imposed through software on the allowed power, net energy production, fuel temperature, startup rate, and stepper motor drive frequency. Second, hard-wired circuits were employed to preclude conditions such as overspeed of the stepper motors. Third, the ACRR's safety system was maintained as a separate entity.

\subsection{Definitions of Neutronic and Thermal Power}

Both the terms 'neutronic power' and 'thermal power' are used in this report. The former is defined here as the instantaneous core power associated with the fission reaction rate within the core. The latter is the rate at which energy is removed from the primary system by the steam generators. The two are equal only under equilibrium conditions. During transients, the effects of plant heat capacity and transport lags will cause the two to differ. 


\section{Scope of this Report}

This report covers the scientific and engineering progress achieved as a result of the DOE-supported MIT project "Studies of the Closed-Loop Digital Control of Multi-Modular Reactors." The period covered is 1 October 1988 - 30 June 1992. This report builds on several earlier ones. These were "Fault-Tolerant Systems Approach Toward Closed-Loop Digital Control of Nuclear Power Reactors" that was prepared by MIT for the National Science Foundation, "Formulation and Experimental Evaluation of Closed-Form Control Laws for the Rapid Maneuvering of Reactor Neutronic Power" that was prepared for the Sandia National Laboratories and the U.S. Department of Energy, "Startup and Control of Nuclear Reactors Characterized by Space Independent Kinetics" that was prepared for the Sandia National Laboratories and the U.S. Air Force, and "Closed-Loop Digital Control of Nuclear Reactors Characterized by Spatial Dynamics" that was prepared for the U.S. Department of Energy [1-4].

It is intended that this report on the control of multi-modular reactors be a selfcontained entity. Accordingly, material developed in earlier MIT research programs that is relevant to the understanding of the results presented in this report has been summarized as a courtesy to the reader. This includes an overview of the MIT-SNL approach to reactor control as well as the theoretical basis for both the reactivity constraint approach and the MIT-SNL Period-Generated Minimum Time Control Laws. Certain other relevant material has, for reasons of space, been omitted. For example, those desiring information on the control of reactors characterized by spatial dynamics should refer to the earlier reports. Much of the work that forms the basis of this report was performed as student thesis research. Summarized in Chapters Four through Ten are the principal methods and conclusions of those efforts. Readers who are especially interested in a particular topic should refer to the individual thesis. These are listed in Table 2-1.

Chapter Three of this report provides the aforementioned review of results from previous MIT research on reactor control. Those already familiar with that material may wish to skim it or else omit it altogether. Each of the chapters that follows concerns a specific aspect of the operation of a multi-modular reactor. Chapter Four describes the design, implementation, and initial testing of a multiple-computer/single task system for the closed-loop, digital control of a reactor. This system was used for some of the experimental work reported in subsequent chapters. Chapter Five reports the development and experimental evaluation of a method for performing automated reactor startups with online determination of the initial degree of subcriticality. Chapter Six is a comparative study of various cc itrol methods for the trajectory-tracking of reactor neutronic power. Chapter Seven report the development and numerical testing of simulation program for a PWRtype multi-modular plant. Chapter Eight addresses the issue of steam generator level control with emphasis on the design of model-based compensators for shrink and swell effects. Chapter Nine describes the design and assessment of strategies for the adjustment of power and temperature in a multi-modular plant. This chapter's focus is operation with unbalanced loads. Chapter Ten explores another area, the challenges involved in the development of real-time intelligent suppcrt tools for reactor operators. The report concludes with a discussion of research needed for the further application of digital technology to the control of multi-modular plants. 
Table 2-1

MIT Thesis Research on the Closed-Loop Digital Control of Multi-Modular Reactors

1. Lau, S. H., "Experimental Evaluation of Trajectory Tracking Methodologies for Control of Reactor Power," NE Thesis, Deptartment of Nuclear Engineering, Massachusetts Institute of Technology, Cambridge, MA, Feb. 1991.

2. Kim, K. K., "Design and Simulation of a Digital Control System for a PWR-Type Multi-Modular Power Plant," Ph.D. Thesis, Deptartment of Nuclear Engineering, Massachusetts Institute of Technology, Cambridge, MA, Aug. 1992.

3. Kwok, K. S., "Automated Startup of Nuclear Reactors: Reactivity Estimation, Computer System Development, and Experimental Evaluation," Ph.D. Thesis, Deptartment of Nuclear Engineering, Massachusetts Institute of Technology, Cambridge, MA, June 1991.

4. Waltrip, M. K., "Multi-Modular Nuclear Reactor Plant Simulation and Control," M.S. Thesis, Deptartment of Nuclear Engineering, Massachusetts Institute of Technology, Cambridge, MA, Nov. 1988. 


\section{Methodology for the Closed-Loop Digital Control of Nuclear Reactors}

This chapter summarizes the results of previous MIT research concerning the closed-loop digital control of reactor neutronic power. Included is an overview of the MIT philosophy regarding the design of controllers for nuclear reactors, a discussion of reactor dynamics, use of the dynamic period equation as a reactor model, and the theoretical basis of both the reactivity constraint approach and the MIT-SNL Period-Generated Minimum Time Control Laws. For those not familiar with the MIT program on reactor control, the 'reactivity constraint approach' is the supervisory technique developed at MIT in the early 1980s to preclude challenges to the safety system as tie result of an automatic control action. Its implementing algorithm is the MIT-CSDL Non-Linear Digital Controller. CSDL stands for 'Charles Stark Draper Laboratory' which, together with the National Science Foundation, funded that aspect of the MIT program. The MIT-SNL laws are the trajectory racking technique developed jointly by MIT and Sandia National Laboratories (SNL) in 1986. The material presented here is drawn from previous MIT reports on reactor control [1-3], from the paper "Progress Toward a Generic Methodology for the Closed-Loop Digital Control of Nuclear Reactor Power" that was presented at the ANS/NIST Conference 'Fifty Years with Nuclear Fission' [27], and from the paper "Considerations in the Design and Implementation of Control Laws for the Digital Operation of Research Reactors" [28].

\subsection{Controller Structure}

Figure 3.1-1 shows current MIT thinking concerning the structure of a controller for the automated operation of a nuclear reactor. Major features of this controller are:

- A separate safety system.

- A means for signal validation and instrument fault detection.

- A supervisory algorithm that precludes challenges to the safety system.

- A set of predictive control laws.

- A reconfiguration logic to select the most appropriate law.

- A means for the verification of signal implementation.

- A module for automated reasoning.

- A man-machine interface.

Of special significance is the hierarchical structure of this controller. The actions of the predictive control law chosen by the reconfiguration logic are reviewed by the supervisory algorithm so as to ensure the absence of a challenge to the safety system. This 'defense-indepth' approach is desired even though the safety system is an independent entity. Note that, in accordance with this hierarchical structure, there are four distinct closed pathways or loops within this diagram. The first is the safety system which operates independently of the other loops. The second is the supervisory logic which consists of sensors, the signal validation and fault detection routine, the supervisory algorithm, and a comparator that results in a supervisory override should the potential exist for a challenge to the safety system. The third loop generates the actual control signal. Its components include sensors, the signal validation and fault detection routine, and modules for automated reasoning and state identification. These modules permit determination of the reactor's 


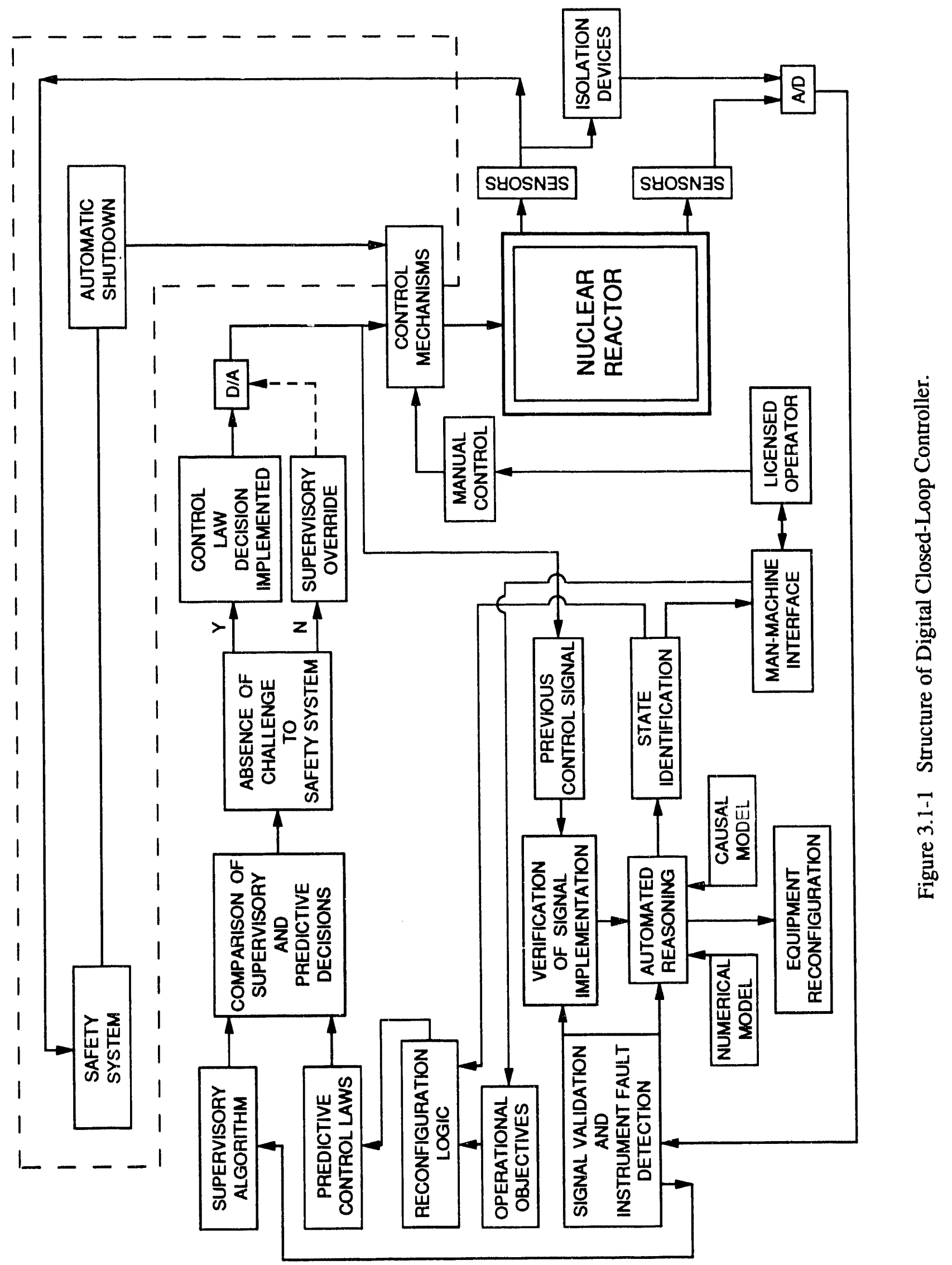


status. That information together with operational objectives is then provided to a reconfiguration logic which selects the control law. Prior to implementation, the decision of the control law is sent to the comparator for determination that it will not create a challenge to the safety system. The fourth of the loops shown in the diagram functions as a check on the overall operation of the controller. It serves to verify that the previously generated control signal was in fact implemented and that it did have its intended effect. The following specific features of the controller are of significance:

Separation of safety and control systems: The nuclear safety system is separate from the closed-loop controller. The word 'separate' is defined as meaning that the output of an instrument used in the safety system must not be influenced by interaction with the control system. Thus, if an instrument is common to both systerns, its signal must be passed through an isolation device, such as an optical transformer, to preclude any possibility of feedback from the control system. The purpose of keeping the two systems separate is to ensure that the capability of the safety system to perform its intended function will never be compromised.

Signal validation and instrument fault detection: All sensor information is processed by signal validation and fault detection routines. There are several methods for accomplishing this. The simplest is to verify that each reading is within the range expected for a given plant condition. A more sophisticated approach is to identify the largest consistent subset of signals and to reject any that is not a member of that set. A further refinement is to incorporate a real-time system model that generates an analytic value for the measured parameter. Sensor readings are then checked for consistency both with each other and with the calculated value. This latter method has been demonstrated at MIT as part of a numerical technique known as the 'parity space approach' [12]. In addition to validating sensor readings, this methodology performs instrument fault checks in which the weighting factor for each sensor is adjusted in proportion to the frequency with which its readings are judged to be valid. Thus, reliance on a failing sensor is gradually reduced, thereby assuring a 'bumpless' transition when complete failure actually occurs.

Figure 3.1-2 illustrates the importance of using validated signals. Shown is a strip chart recording of the logarithm of two power signals obtained during a power increase of three orders of magnitude that was accomplished on SNL's Annular Core Research Reactor (ACRR using the Standard MIT-SNL Period-Generated Minimum Time Control Law. The specified period was $1.0 \mathrm{~s}$. Power was increased from $0.57 \mathrm{~kW}$ to $500 \mathrm{~kW}$ in $6.73 \mathrm{~s}$. Initially, the sensor on the right was on-scale while that on the left was off-scale low with the reactor power at $500 \mathrm{~W}$. As the power increased, the sensor on the right saturated and failed while that on the left became functional. The software was programmed to recognize this. As a result, the power increase was completed properly. Had some means of signal validation not been available, the controller would have withdrawn the ACRR's transient rod bank continuously in a vain effort to raise the power as seen by the saturated sensor.

Supervisory algorithm, reconfiguration logic, and predictive control laws: The controller contains a supervisory algorithm, a number of selectable control laws, and a reconfiguration logic. The supervisory algorithm establishes the limiting conditions within which control will always be feasible and thereby guarantees that there will not be a challenge to the safety system as the result of any automatic control action. It does this by requiring a change in the present value of the control signal if conditions are such that an 


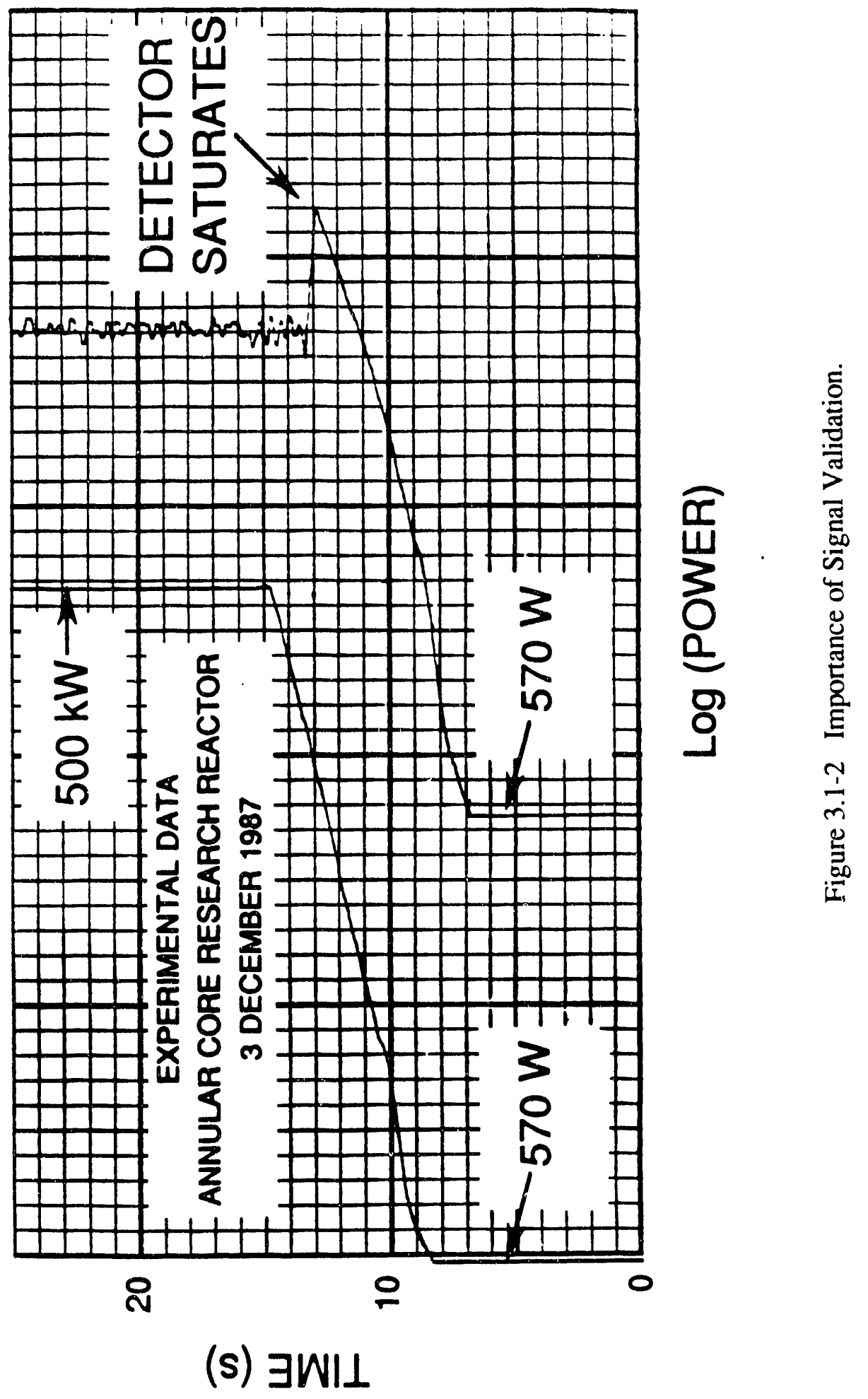


overshoot could occur at some future time. Among the selectable control laws are ones suited for each operational mode. The MIT-SNL minimum time control laws, which were developed for the trajectory control of reactor neutronic power, would be one option within this group of control laws. Others might include a rule-based controller and the conventional proportional-integral-derivative approach. The reconfiguration logic selects the law that is most applicable to the current plant condition [18]. The decision of the selected control law and that of the supervisory algorithm are then compared and the more conservative is implemented. This approach has the advantage that it can combine a general-purpose, supervisory algorithm that precludes challenges to the safety system with a plant-specific predictive control law. Detailed discussions of both the supervisory algorithm and the MIT-SNL minimum time control laws are given in Sections 3.3.3 and 3.3.4 of this chapter and in subsequent chapters of this report.

Command validation: The implementation of the control signal should be verified because there could be a failure in either the computer interface or in the actuators. Verification should be performed in the broadest possible manner. For example, if the controller's decision was to reduce the reactivity, then it should be determined both that a control device was inserted and that the period did actually lengthen.

Automated reasoning: The purpose of the automated reasoning module is to replicate those functions that would normally be performed by a human operator. Studies of the process by which humans exercise control suggest that four tasks are involved [30-34]. These are planning, prediction, implementation, and assessment. Planning involves determining both the desired plant state and the most efficient means of achieving that state given the overall operational objectives and the confines of engineering limitations. The planning process is therefore basically a search procedure in which operational goals are matched against available options. Moreover, the search space is essentially closed. That is, the number of possible options is finite. Expert systems technology is now being used to assist the managers of terrestrial reactors with such planning functions as preparation of work orders, verification of technical specification compliance, and the determination of rod withdrawal sequences in boiling water reactors [35]. It is therefore anticipated that this technology could be incorporated in an autonomous controller to perform the planning function. Prediction, which is the second of the four control subtasks, entails formulating projections of the effect that any of the available control options will have on the plant's behavior. For a control system to perform the prediction subtask autonomously, accurate numerical models of the plant will be required. The third subtask, implementation, is generally already automated and is therefore not discussed here. The last of the four control subtasks is assessment. This is perhaps the most difficult because it involves first distinguishing abnormal from normal behavior and then, if the former is present, diagnosing it as to cause. The automated performance of the assessment subtask will probably require the use of several different approaches. For example, numerical models could be used to establish normal system behavior and pattern recognition techniques might serve to identify abnormalities. Also, real-time analysis is now being attempted with expert systems. For example, within the nuclear industry, several very successful systems are being used for turbine generator diagnostics [36-37]. However, this success may not be typical. The problem is that, unlike the planning subtask, the field of search is extremely large and all possible rules may not be identified. The potential for utilizing expert systems for diagnostics has been previously discussed $[5,38]$. 
Figure 3.1-3 illustrates the importance of automated diagnostics. Shown are the reactivity and power profiles from a run in which the ACRR's neutronic power was raised from $3 \mathrm{~kW}$ to $3 \mathrm{MW}$, three orders of magnitude, on a period of $1.0 \mathrm{~s}$. Reactivity was estimated using the parity space approach with inverse kinetics being the default in the event of indecision. Note that power is shown on a logarithmic scale. The transient was completed in the allotted time of $6.7 \mathrm{~s}$ with essentially no overshoot. The slope of the power profile is straight indicating that power was indeed rising on the specified period of $1.0 \mathrm{~s}$. Upon attaining the desired power level, $3 \mathrm{MW}$, the power was held at that value. Of special significance is that at about $24 \mathrm{~s}$, one of the ACRR's three transient rods was deliberately dropped back into the core. This caused a reactivity insertion of -400 millibeta. The controller was not 'told' of this perturbation. Yet, excepting only a minor downward blip in the power trace, it held the power constant at $3 \mathrm{MW}$. Specifically, the remaining two rods were withdrawn to provide the necessary compensation. The above action occurred during a carefully monitored experiment and was most impressive. However, suppose that the controller acted in the same manner for a situation in which the loss of reactivity was only temporary. Should that reactivity return after the controller had provided compensation, a serious power excursion might occur. Hence, the challenge to the designers of autonomous controllers for safety-constrained systems is not merely to devise a control law that can compensate for perturbations but also to identify the cause of all such perturbations.

Man-Machine Interface: User acceptance of a digital controller may well depend on whether or not the man-machine interface is designed to support human cognitive needs. In this respect, the display should reinforce both the operator's understanding of the plant and his or her mental approach to the analysis of plant behavior. Displays that show trends and predictions satisfy the first of these two criteria because such information will assist operators in anticipating plant response. As for the second criteria, graphics should be emphasized so that an operator need only look at a display to comprehend it. This approach allows experienced operators to continue using their pattern recognition skills. In contrast, were text to be displayed, an operator would have to switch to a deductive mode of reasoning in order to make sense of the information. A discussion of factors bearing on the design of a man-machine interface is given in Chapter 11 of a previous report [4] and elsewhere [28].

\subsection{Overview of Reactor Dynamics}

A reactor's multiplication factor is defined as the ratio of the neutrons produced from fission to those losi by either leakage or absorption. The reactor is subcritical, critical, or supercritical depending on whether the multiplication factor is less than, equal to, or greater than unity. The quantity most fundamental to the control of power in a nuclear reactor is the reactivity. It is defined as the fractional departure of the multiplication factor from unity. A reactivity of zero therefore corresponds to the critical condition. Adjustments of power in reactors described by space-independent kinetics are generally accomplished by positioning neutron-absorbing control rods or rotating drums so as to temporarily alter the neutron multiplication rate. If a power increase is desired, a control rod would be withdrawn (or a drum rotated) so as to insert positive reactivity and thereby place the reactor on a period. (Note: Period is defined as the power level divided by the rate of change of power. Thus, a period of infinity corresponds to steady-state, while one equal to a small positive number indicates a rapid power increase.) Having established a 


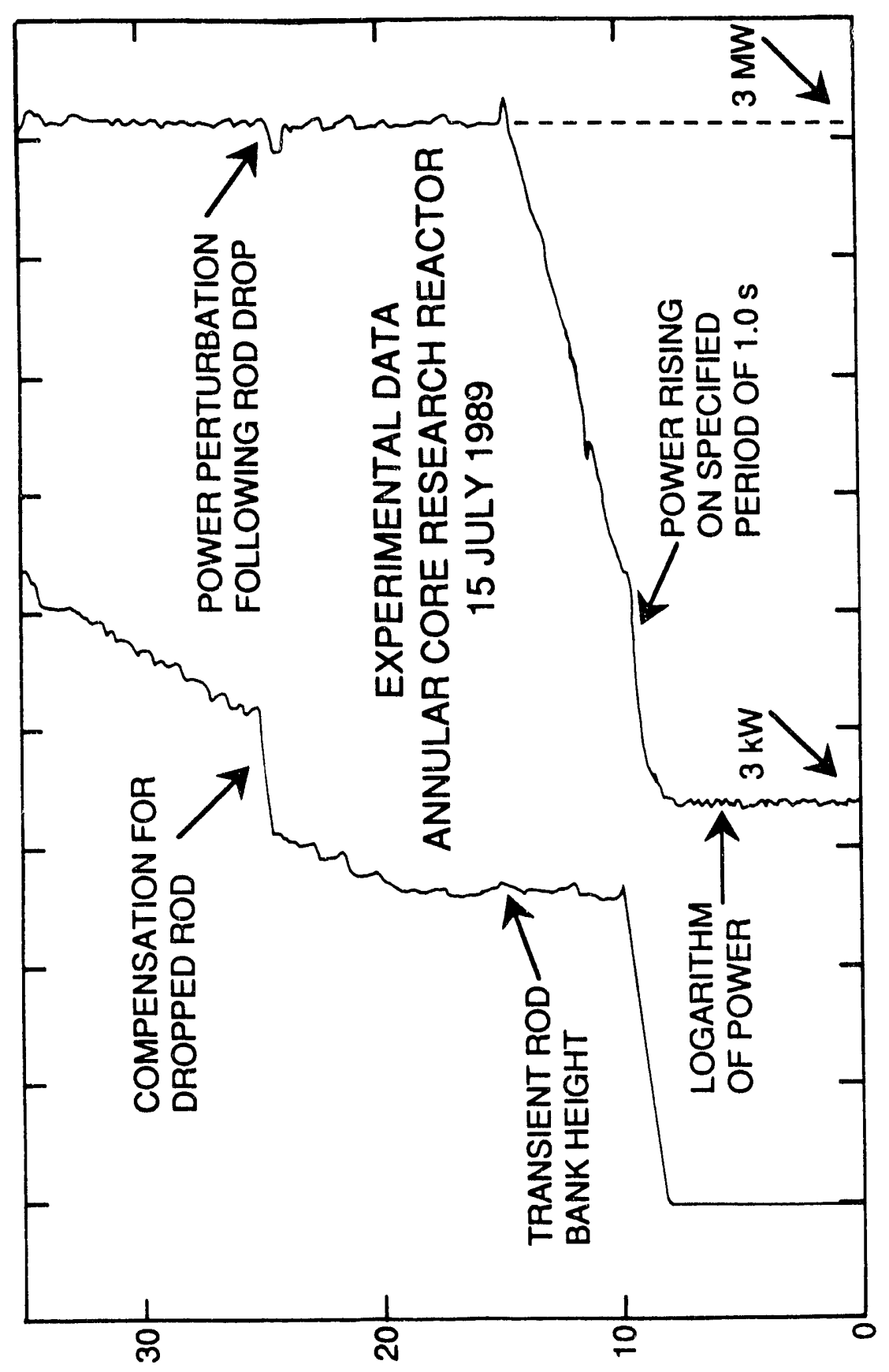

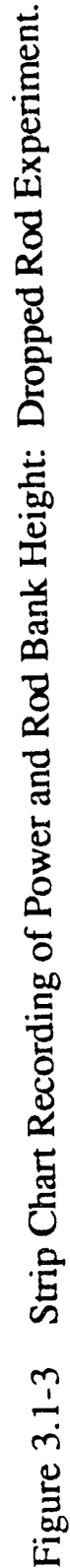

(s) $\exists W I \perp$ 
period, the power is allowed to rise. Once the power level approaches the desired value, the control device is gradually returned to its original position in order to reduce the reactivity to zero and to level the power without overshoot. Reactor operation is characterized by several reactivity feedback mechanisms. One of the most important is that a rise in fuel temperature will result in the increased absorption of neutrons in reactions that do not lead to fission. Known as the 'Doppler' effect, this is an inherent safety feature that limits the potential for an accident. Specifically, an increase in the reactor power will cause the fuel temperature to rise which will in turn decrease the number of neutrons available to sustain the fission chain reaction. The net result is a negative reactivity insertion followed by either a lessening of the rate of rise of power or, for some reactors, an actual power decrease.

\subsubsection{Manual Operation}

Licensed MITR and ACRR operators rely on their understanding of reactor dynamics and their past experience to perform power changes. This requires considerable skill because reactor dynamics are governed by non-linear relations. In particular, the reactor period and hence the rate of change of power depend on both the rate of change of reactivity and on the net reactivity present. Also, the rate at which reactivity can be removed under normal control conditions is a function of the insertion speed of the control rods and therefore finite. This means that merely halting rod withdrawal, or in some situations even reversing the direction of rod motion, may not immediately stop a power increase. Some degree of preplanning is necessary in order to level the power at the desired value without overshoot. Additional complications are that reactivity is not directly measurable, that the differential reactivity worths of the control rods are usually non-linear functions of position, that the reactivity is altered by thermal feedback effects resulting from power changes, and that the relation between power and period is exponential, not linear.

\subsubsection{Closed-Loop Operation}

A control algorithm should fulfill two major functions. First, it should specify the desired plant trajectory and, if the actual state of the plant differs from the specified one, generate a feedback signal that reduces the error. Second, it should both define the envelope of conditions under which it will be possible to halt the transient and preclude operation beyond that envelope. These two functions are referred to here as 'predictive' and 'supervisory' respectively. Supervisory control is generally only required if, as is the case with a nuclear reactor, a system exhibits either non-linear or time-delayed behavior. In order to appreciate the nature of this problem, it is useful to consider further the dynamics of a nuclear reactor. Figure 3.2.2-1 is a simplified schematic of the fission process. Relative to reactor control, the most significant feature is that there are three parallel but separate mechanisms for the production of neutrons. Prompt neutrons appear directly following the fission event and have lifetimes that are quite short. A typical value is 100 microseconds. (Note: Neutron lifetime is considered here to be the time required for the neutron to appear, thermalize, and be reabsorbed.) Delayed neutrons are produced following the decay by beta particle emission of certain fission products. Those fission products that undergo this process are referred to as 'precursors.' The delay in the appearance of a delayed neutron relative to the fission event is the result of the precursor half-life. There are six recognized groups of precursors with half-lives ranging from 0.23 to 55 seconds. The average value is 12.2 seconds. The third mechanism for neutron 


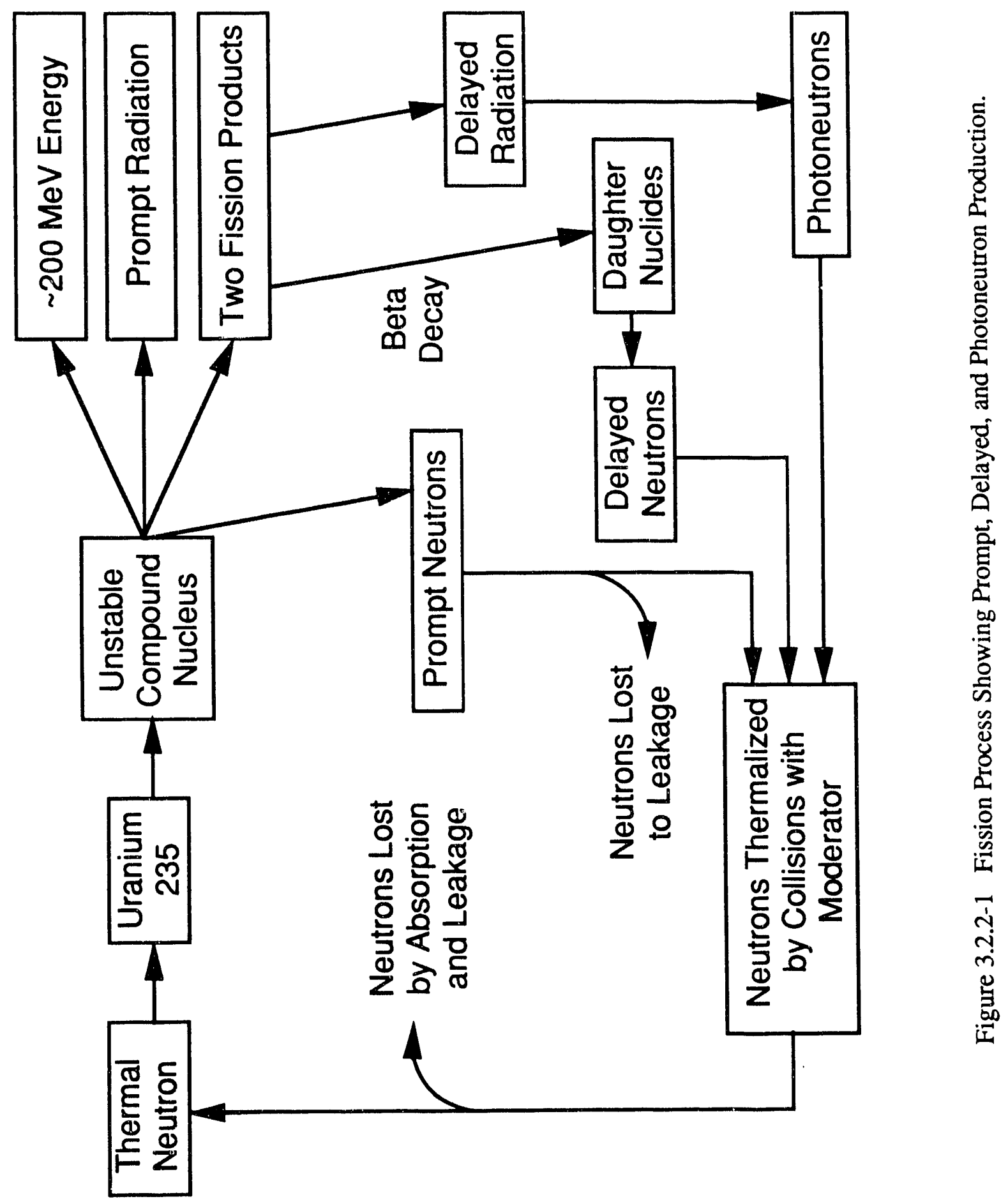


production is the interaction of fission product gamma rays with certain moderating materials, most notably heavy water and beryllium. The appearance of photoneutrons is delayed relative to the fission event because of the time required for the fission products to undergo radioactive decay and emit the needed gamma rays. Prompt, delayed, and photoneutrons are all produced at high energies. In order to sustain the fission reaction, these neutrons must be slowed down or thermalized. This is accomplished by designing the re tor so that the neutrons will collide with the nuclei of a moderating material, thereby giving up much of their kinetic energy. (Note: Photoneutrons may be represented mathematically in the same way as delayed neutrons. Hence, the term 'delayed' is subsequently used in this report to refer to both neutrons resulting from precursor decay and photoneutrons.)

The importance of delayed neutrons to reactor safety is well recognized. They lengthen the average neutron lifetime so that changes in a reactor's dynamic behavior will occur over intervals that are long relative to human response times. The significance of delayed neutrons to reactor control is less well understood and is therefore discussed here at some length. A problem originates in that the respective appearances of prompt and delayed neutrons following fission occur on very different time scales. As a result, at any given moment, the prompt neutron population will be proportional to the current power level while the delayed neutron population will be a function of the previous power levels. This difference has no significance during extended steady-state operation because the previous and current power levels are the same. However, such will not be the case during transient operation. Specifically, because of the interval that elapses between the creation of precursors and the appearance of delayed neutrons, the delayed neutron population will not be in direct proportion to the transient power. This non-equilibrium condition of the delayed neutrons has several implications. First, during power increases, the contribution of the delayed neutrons will always be less than it would be at equilibrium and the contribution of the prompt neutrons will therefore be correspondingly greater. Similarly, during power decreases, the delayed neutron population will be greater than its equilibrium value. Second, upon attaining the desired power level, the rate of increase of the delayed neutrons, unlike that of their prompt counterparts, can not be immediately halted. Rather, the delayed neutron population will continue to rise until it attains equilibrium with the precursor population that corresponds to the reactor's power level. Hence, if power overshoots are to be averted, it is essential to limit the delayed neutron contribution so that, upon attainment of the desired power, the insertion of the control mechanism will make the rate of change of the prompt neutrons sufficiently negative so as to offset the continued increase in the delayed neutrons. Assuming that these two rates can be balanced, the reactor period can be made infinite and the power kppt constant while equilibrium of the neutron populations at steady-state is achieved. Physically, this condition of balance can be readily achieved because the prompt and delayed neutron populations are proportional to the rate of reactivity change and to the net reactivity respectively. Figure 3.2.2-2 illustrates the process. Shown is a transient obtained from the MIT Research Reactor. Initially, there is positive reactivity present, the reactor is on a positive period, and the power is rising. At approximately 95 seconds, the operator was told to halt the transient. The control mechanism is driven in causing the reactivity to decrease and the period to lengthen. There is a change in the slope of the power curve, but the power continues to rise. Delayed neutrons are appearing at a faster rate than the prompt ones are being cut back. The power does momentarily level off at 112 seconds. This occurs when the positive reactivity (i.e., 


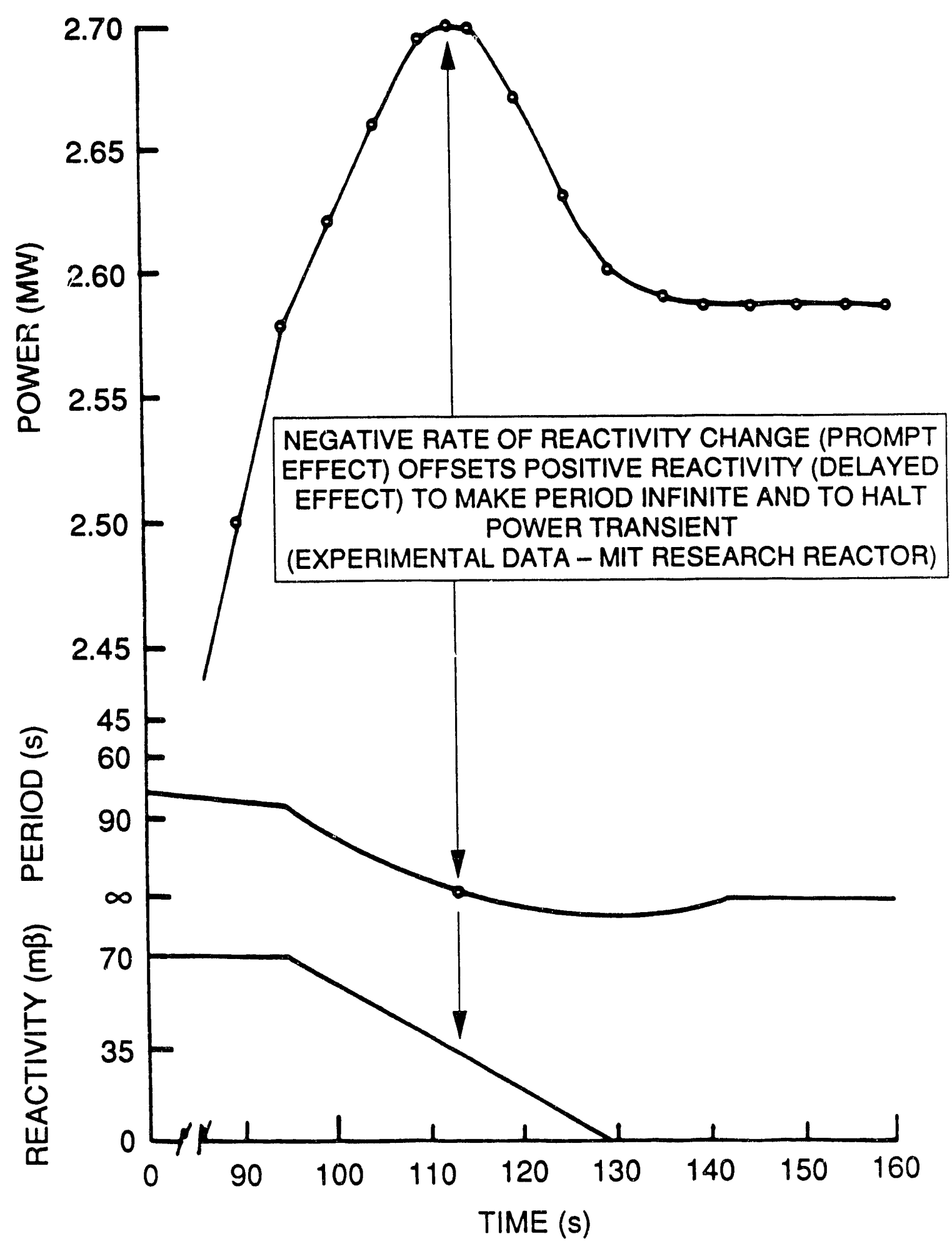

Figure 3.2.2-2 Reactor Transient Illustrating Need for Supervisory Control. 
the rising delayed neutron contribution) is balanced by the negative rate of change of reactivity (i.e., the decreasing prompt neutron contribution). The ability to identify and maintain this balance between reactivity and its rate of change is central to the control of reactor power. Controllers exhibiting this capability are designated as incorporating the property of 'feasibility of control' $[1,19]$. Subsequent to the 112 second mark, the reactivity decrease was continued. The period becomes negative and the power decreases, eventually being leveled at the desired value.

The above understanding of reactor dynamics explains the need for supervisory control in a nuclear reactor. Mere prediction of the anticipated power trajectory is not enough. The envelope of conditions under which a transient can be halted must also be continuously defined.

\subsection{Controller Design}

The MIT approach to the design of a controller for reactor power has been guided by two premises. The first is that reactor dynamics are non-linear and that reactor controllers should therefore be designed on a non-linear basis. The second is that theoretical advances in controller design should be verified through experiment. Both of these premises are evident in the discussion that follows.

\subsubsection{Non-Linear Reactor Model}

The basis of most reactor control methodologies is the space-independent kinetics equations. One approach to controller design would be to linearize those equations about an intended operating point and then write the resulting relations in state-space form. This is an appealing option because it would facilitate the application of the many excellent design techniques that have been developed for the control of linear systems. However, were this option to be exercised, much would be lost. Figure 3.3.1-1 is from a simulation study in which power profiles obtained using linearized and non-linear models were compared. The transient shown was rather mild, involving a ramp reactivity addition of 100 mbeta over 20 seconds. Yet the error in the linearized system's response was substantial.

The MIT approach to model development is to combine the space-independent kinetics equations through a process of differentiation and substitution to obtain the dynamic period equation $[2,19,39]$. This relation, which can be written in either a standard or an alternate form, gives the instantaneous reactor period as a function of the rate of change of reactivity, the reactivity, and the rate of redistribution of the delayed neutron precursors. The alternate version is:

$$
\tau(t)=\frac{\bar{\beta}-\rho(t)+l^{*}\left[\frac{\dot{\omega}(t)}{\omega(t)}+\omega(t)+\lambda_{e}^{\prime}(t)\right]}{\dot{\rho}(t)+\lambda_{e}^{\prime}(t) \rho(t)+\sum \bar{\beta}_{i}\left(\lambda_{i}-\lambda_{e}^{\prime}(t)\right)}
$$




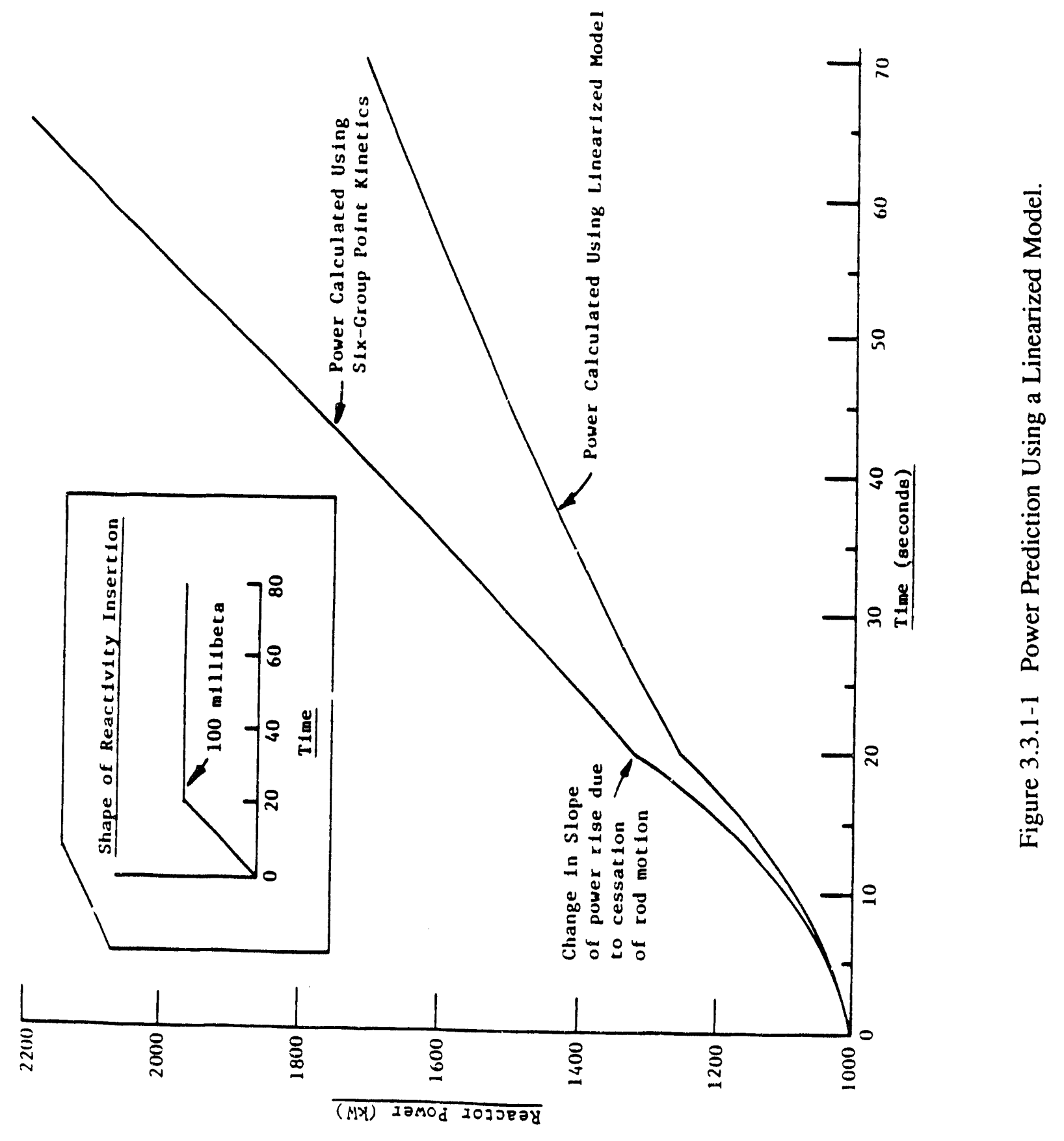


where the alternate, effective, multi-group decay parameter is defined as:

$$
\lambda_{e}^{\prime}(t) \equiv \sum \lambda_{i}^{2} C_{i}(t) / \sum \lambda_{i} C_{i}(t) \text { for } i=1, N
$$

and where other symbols are defined as:

$\tau(t)$ is the instantaneous or dynamic reactor period,

$\bar{\beta} \quad$ is the effective delayed neutron fraction,

$\rho(t) \quad$ is the net reactivity,

$l^{*} \quad$ is the prompt neutron lifetime,

$\dot{\omega}(t)$ is the rate of change of the inverse of the dynamic reactor period,

$\omega(t)$ is the inverse of the dynamic reactor period,

$\dot{\rho}(t) \quad$ is the rate of change of the net reactivity,

$\bar{\beta}_{i} \quad$ is the effective fractional yield of the ith group of delayed neutrons,

$\lambda_{i}$ is the decay constant of the ith precursor group,

$\mathrm{C}_{\mathrm{i}}(\mathrm{t}) \quad$ is the concentration of the ith precursor group normalized to the initial power, and

$\mathrm{N}$ is the number of groups of delayed neutrons, including photoneutrons.

The standard version of the dynamic period equation is:

$$
\tau(t)=\frac{\left.\bar{\beta}_{-i} t\right)+l^{*}\left[\frac{\dot{\omega}(t)}{\omega(t)}+\omega(t)+\lambda_{e}(t)-\frac{\dot{\lambda}_{e}(t)}{\lambda_{e}(t)}\right]}{\dot{\rho}(t)+\lambda_{e}(t) \rho(t)+\frac{\dot{\lambda}_{e}(t)}{\lambda_{e}(t)}(\bar{\beta}-\rho(t))}
$$

where the standard, effective decay parameter is defined as:

$$
\lambda_{e}(t) \equiv \sum \lambda_{i} C_{i}(t) / \sum C_{i}(t) \text { for } i=1, N
$$

and the quantity $\dot{\lambda}_{e}(t)$ is the rate of change of the standard, effective, multi-group decay parameter. Other symbols are as previously defined.

Derivations of the standard and alternate dynamic period equations both with and without source terms are given in previous reports [2,3]. Relative to these equations, it is worth noting that the well-known inhour relation is a special case of the dynamic period equation [40]. 
Advantages to the use of the dynamic period equation are that it preserves the nonlinear aspects of a reactor's ${ }^{\prime}$ 'namics, it is an exact relation that is rigorous even for spacedependent kinetics, it appli s to all reactor operating regimes, and it explicitly shows each of the physical processes that can affect the instantaneous reactor period. The dynamic period equation is not without its limitations. It is a form of space-independent kinetics and hence its use is restricted to transients for which the shape of the neutronic flux remains unchanged. Also, for space-dependent kinetics, the reactivity must be defined and measured so as to account for changes in the shape of the neutron distribution.

Relative to the theory of process control, the use of a single relation, such as the dynamic period equation, to describe a system's behavior is a significant departure from current practice which is to model an $n$th order system as a set of $n$ first-order differential equations. This is referred to as a 'state-space' representation. Its advantage is that the internal dynamics of the system become accessible. Thus, instead of feeding back merely the output of the process, each system parameter can be assigned a feedback coefficient. Hence, for an nth order system, $n$ degrees of freedom are introduced. This provides the control engineer with enormous flexibility. In particular, by the judicious choice of the gain coefficients associated with each of the system parameters, the shape of the system's response as well as its stability can be specified. Disadvantages to the state-space approach are that it is restricted to linear systems and that physical understanding of the process dynamics is often lost. Use of the dynamic period equation avoids these drawbacks and, as is shown in the discussion of time-optimal control, also provides a means for shaping system response.

\subsubsection{Selection of the Actuator Signal}

The control laws described in this report are formulated in terms of the rate of change of reactivity. This means that the signal sent to the actuator is the speed at which the control device should be moved. This choice contrasts with the traditional approach to the design of controllers for nuclear reactors which is to specify a control action in terms of the magnitude of the reactivity. Use of this latter practice means that the signal sent to the actuator is the desired position of the control device. There are a number of reasons for selecting the rate of change of reactivity as the actuator signal. First, specification of the appropriate rate of reactivity change means that both the direction and speed of the control device are uniquely determined. In contrast, if only the reactivity were specified, then the desired final position of the control device would be known but the speed at which the device should be moved to attain that position would be undetermined. Second, as is evident from the dynamic period equation, the response of a reactor depends on both the magnitude and the rate of change of the reactivity. Failure to allow for the latter means that sudden variations will occur in the rate at which power is being raised whenever rod motion is started or stopped. Third, a major requirement in the design of controllers for safety-constrained processes such as nuclear reactors is that it be possible to alter the controi signal on demand and thereby have an immediate effect on the process in question. Reactivity does not fulfill this requirement because it is a function of the distance that a control rod has been moved beyond the critical position and adjustments in a device's position can only be made over a finite interval. In contrast, the rate of change of reactivity can be immediately altered by merely initiating movement of a control device. Moreover, a wide range of rates of change is achievable through the use of variable speed stepper motors. Fourth, the rate of change of reactivity corresponds to the effect of a changing 
prompt neutron population while the reactivity itself reflects other effects including changing delayed neutron precursor populations and changing distributions of delayed neutron precursors within the defined groups. Precursor populations are a function of the power history and therefore can not be altered on demand. In contrast, the prompt neutron population is essentially a function of only the current power level and is therefore immediately controllable. Hence, if an immediate change is required in a reactor period, an adjustment should be made in the rate of change of reactivity rather than in the reactivity itself.

\subsubsection{Supervisory Control}

The traditional function of a control algorithm is to specify the desired plant trajectory and, if the actual state of the plant differs from the specified one, generate a feedback signal that reduces the error. For safety constrained systems, the control algorithm should also both define the envelope of conditions under which it will be possible to halt the transient and preclude operation beyond that envelope. The need for supervisory control is often unrecognized because it is generally only required if, as is the case with a nuclear reactor, a system exhibits either non-linear or time-delayed behavior.

Data from a power increase that was intentionally performed on the MITR-II to illustrate the need for supervisory control was shown in Figure 3.2.2-2. The data shows that there are only certain combinations of reactivity and rate of change of reactivity for which it will be possible to halt a transient. The capability to identify and maintain a balance between these two quantities is the basis of the reactivity constraint approach which is the supervisory control methodology developed at MIT and successfully implemented on both the MITR-II and the ACRR.

\subsubsection{Feasibility of Control}

On a formal basis, a reactor together with a specified control mechanism is defined here as constituting a system that is 'feasible to control' if the system can be transferred from a given power level and rate of change of power (i.e., period) to a desired steady-state power levil without overshoot (or conversely, undershoot) beyond specified tolerance bands, if any. This concept has two important attributes. First, it applies to a reactor and to the specific control mechanism designated for use in accomplishing a given transient. Second, not all states (combinations of reactivity and available rate of change of reactivity) are allowable intermediates through which the system may pass while transiting from some initial to some final power. Excluded are both those states that represent actual overshoots and those from which overshoots could not be averted by manipulation of the specified control mechanism. (Note: It should be recognized that the concept of feasibility of control is distinct from the more general property of 'controllability' which has a specialized meaning in that a system is said to be controllable if "any initial state can be transferred to any final state in a finite time by some control sequence." This definition does not place any restrictions on intermediate states.)

\subsubsection{Reactivity Constraint Approach}

The objective of the reactivity constraint approach is to provide a means for the closed-loop digital control of a reactor's neutronic power during transients so that there will 
not be a challenge to the reactor's safety system. Currently, the technique has been implemented for situations in which the prompt-jump approximation is valid. Under this condition, the standard dynamic period equation becomes:

$$
\tau(t)=\frac{\bar{\beta}-\rho(t)}{\dot{\rho}(t)+\lambda_{e}(t) \rho(t)+\frac{\dot{\lambda}_{e}(t)}{\lambda_{e}(t)}(\bar{\beta}-\rho(t))} .
$$

The avoidance of safety system challenges can be achieved if it is possible to make the instantaneous reactor period infinite upon attainment of the desired power level. This goal can be realized if the net reactivity is constrained so that:

$$
\left[\lambda_{e}(t) \rho(t)+\left(\dot{\lambda}_{e}(t) / \lambda_{e}(t)\right)(\bar{\beta}-\rho(t))+\dot{\rho}_{f}(t)\right] \leq\left|\dot{\rho}_{c}\right|
$$

where the term $\rho(t)$ is the net reactivity, both that added deliberately by the control mechanisms and that present indirectly from feedback effects. The quantity $\dot{\rho}_{\mathrm{f}}$ denotes the rate of change of reactivity due to thermal-hydraulic feedback effects, and the symbol $\left|\dot{\rho}_{c}\right|$ denotes the maximum available rate of change of reactivity that could be obtained were a control mechanism to be moved. As such, $\left|\dot{\rho}_{c}\right|$ is always a non-zero finite number regardless of whether or not the mechanism is actually being moved. If the above inequality is observed, then upon the insertion of the control devices, the quantity $\dot{\rho}_{c}$ will be negative and the sum of the terms in the denominator of the standard dynamic period equation will be zero. As a result, the reactor period will be driven to infinity. Equation (3.3.3.2-2) is basically a statement that delayed neutron effects, which are not subject to direct control, must be restricted to that which can be offset by an induced change in the prompt population.

For reasons that have been previously discussed [1], it is preferable from a control viewpoint to rewrite equation (3.3.3.2-2) as:

$$
\left[\lambda_{\mathrm{e}}(\mathrm{t}) \rho(\mathrm{t})\right] \leq\left|\dot{\rho}_{\mathrm{c}}\right|
$$

Equation (3.3.3.2-3) is an 'absolute reactivity constraint.' If observed, it will be possible to level the reactor power at any time during a transient by reversing the direction of movement of the control devices. The quantity $\left.\dot{\rho}_{\mathrm{c}} \mid \lambda_{\mathrm{e}}(\mathrm{t})\right)$ is therefore said to be the amount of reactivity for which control is 'continuously feasible.' A less stringent constraint can be written that specifies that there should be sufficient time available to eliminate whatever reactivity is present beyond the amount that can be immediately negated by reversal of the direction of motion of the designated control mechanism before the desired power level is attained. This requirement, a 'sufficient reactivity constraint' can be written for power increases as:

$$
\left[\rho(t)-\mid \dot{\rho}_{d} / \lambda_{e}(t)\right] / \mid \dot{\rho}_{d} \leq \tau(t) \ln \left(P_{F} / P(t)\right)
$$


where $P_{F}$ and $P(t)$ are the desired and current power levels, respectively, and $\tau(t)$ is either the observed (instantaneous) reactor period or the asymptotic period that corresponds to the net reactivity, whichever results in a more conservative decision. The quantity on the left of equation (3.3.3.2-4) is the time that will be required to reduce the net reactivity to the amount that can be offset by continuous insertion of the control mechanism. It is designated as the 'required time.' The quantity on the right is a measure of the time remaining to attain the specified power. It is designated as the 'available time.' If the inequality represented by the constraint is satisfied, then control mechanism movement as specified by an associated control law is permitted. If the constraint is not satisfied, then the control device is inserted. A major advantage of the reactivity constraint approach is that it determines if a change should be made to the present control signal in order to avoid an overshoot at some future time. Moreover, it does this without the use of predictive models.

Reactivity constraints may also be derived from the alternate dynamic period equation. These are of the form:

$$
\begin{aligned}
& {\left[\lambda_{e}^{\prime}(t) \rho(t)\right] \leq\left|\dot{\rho}_{c}\right|} \\
& {\left[\rho(t)-\left|\dot{\rho}_{c}\right| / \lambda_{e}^{\prime}(t)\right] /\left|\dot{\rho}_{c}\right| \leq \tau(t) \ln \left(P_{P} / P(t)\right)}
\end{aligned}
$$

where all symbols are as previously defined except that $\lambda_{e}^{\prime}(t)$ is the alternate, effective, multi-group decay parameter. Unlike the MIT-SNL minimum time laws, the standard and alternate forms of the reactivity constraints are mathematically different. For example, using the alternate approach, the amount of reactivity for which control is 'continuously feasible' is given by the relation $\left(\dot{\rho}_{c} \mid / \lambda_{e}^{\prime}\right)$. Because of the different definitions of the multi-group decay parameters, this quantity is not the same as when calculated using the standard version. In fact, it differs by roughly a factor of five. (Note: Refer to equations (3.3.1-2) and (3.3.1-4) for the definitions of the multi-group decay parameters.) This point is often a source of confusion. Why should the amount of reactivity for which control is feasible depend on whether it is calculated using the standard or the alternate form of the dynamic period equation? The reason is that both the quantities $\left.\int \dot{\rho}_{c} \mid / \lambda_{e}(t)\right)$ and $\left(\left|\dot{\rho}_{c}\right| / \lambda_{e}^{\prime}(t)\right)$ are approximations and that the approximation obtained from the alternate equation is the more conservative. The selection of a standard or an alternate constraint is, as discussed in $[1,20]$, a matter of the user's preference.

\subsubsection{Demonstration of the Reactivity Constraint Approach}

Figure 3.3.3.3-1 shows a power increase of a decade, $15.2 \mathrm{~kW}$ to $170 \mathrm{~kW}$, conducted on SNL's Annular Core Research Reactor (ACRR) using the alternate sufficient constraint as given by equation (3.3.3.2-6). The control device was the ACRR's transient rod bank which provided an available rate of reactivity change of $320 \mathrm{mbeta} / \mathrm{second}$. The control law consisted of a directive to withdraw the transient rod bank continuously. This poor control law was deliberately chosen so as to ensure testing of the constraint. Shown in the upper portion of the figure are the power and reactivity profiles. Shown in the lower portion are the required available times. The transient was completed without overshoot in about 4 seconds. The dynamic effect of the rod bank's insertion pernitted power to be leveled despite the presence of 434 mbeta of positive reactivity. Specifically, the quantity 

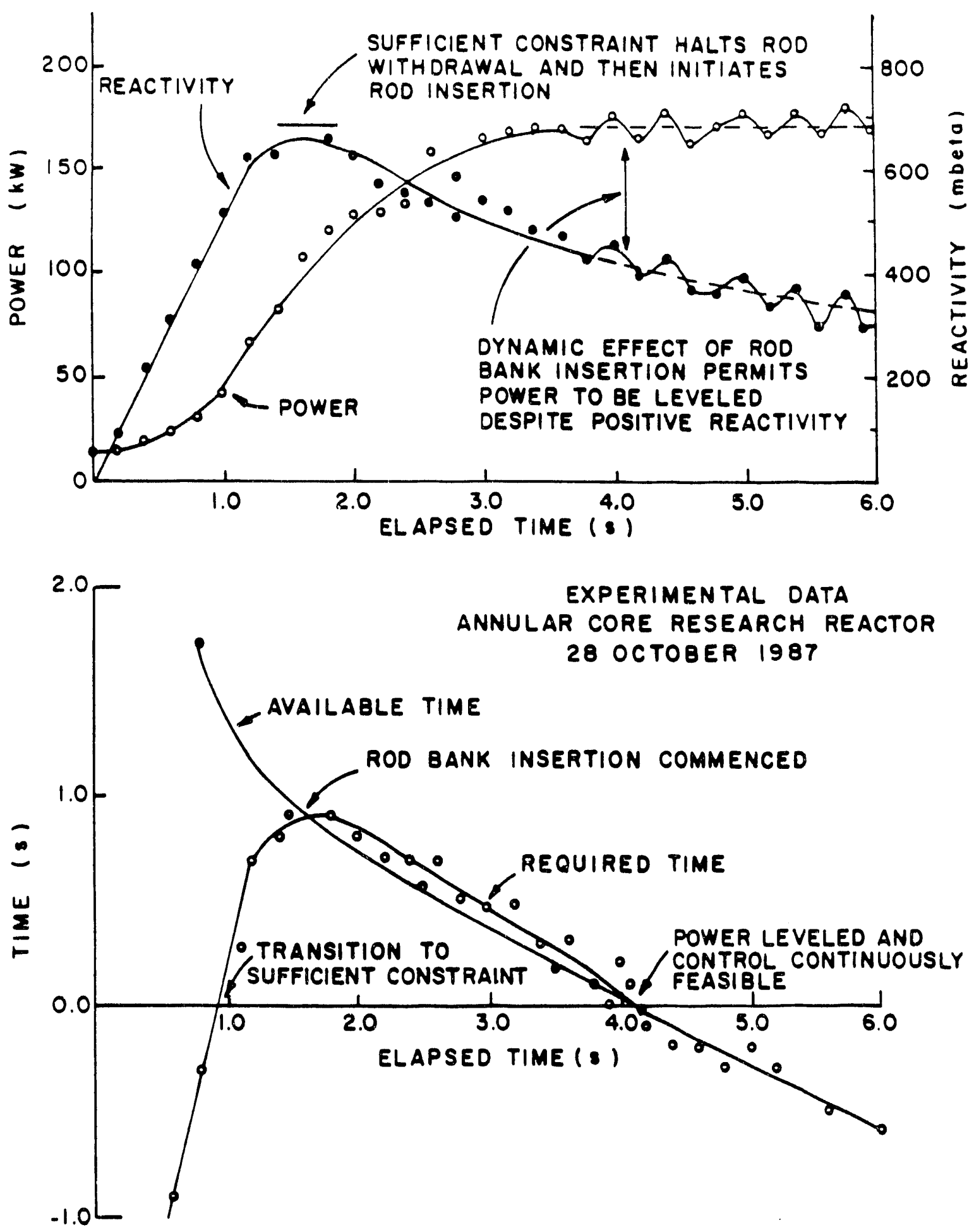

Figure 3.3.3.3-1 Power Increase from $15.2 \mathrm{~kW}$ to $170 \mathrm{~kW}$ on the Annular Core Research Reactor Using the Alternate Reactivity Constraint Approach. 
$\left(\left|\dot{\rho}_{c}\right| / \lambda_{e}^{\prime}\right)$ was $(320 / .75)$ or 427 mbeta which, within the limit of the experiment's accuracy, negated the positive reactivity that was present.

The role of the available and required times warrants examination. At the outset of the transient, the reactivity is zero. Given that there is some rate of change of reactivity available were the transient rod bank to be moved, the term $\left|\dot{\rho}_{c}\right|$ is non-zero. Hence, the required time is negative and equal to $\left(-1 / \lambda_{e}^{\prime}\right)$ or -2.5 seconds. This indicates that control is already continuously feasible. The available time is initially zero because the desired power equals the actual power. Once the change is made in the demanded power, the available time becomes infinite because the period in the previously steady-state reactor was infinite. Control mechanism motion then commences and the reactivity becomes positive. The required time becomes less negative, passes through zero, and then becomes positive indicating that some finite interval must now elapse before the transient can be halted. The transition from the absolute to the sufficient constraint occurs when the required time is zero. The available time tends towards zero because the period is becoming shorter and the power is rising. Once the time required to restore continuous feasibility of control equals the time remaining to attain full power, continued control mechanism withdrawal is prohibited and insertion is begun. This occurs regardless of the signal from the associated control law. The required time is continuously bounded by the available time indicating that the control mechanism is being more or less constantly inserted. The required and available times eventually both become zero. When this occurs, the reactor power can be leveled because control is again within the range of the absolute constraint. There is still positive reactivity present in the core at this time and the dynamic effect of control mechanism insertion is required to counter this positive reactivity. Hence, the control mechanism must be driven in continuously at this time. As the reactor power settles out, the available time remains zero. The required time becomes negative, eventually resuming its original value of $\left(-1 / \lambda_{e}^{\prime}\right)$.

It is important to recognize the complementary roles of the supervisory algorithm and the control law. The former's contribution is that it reviews the decision of the control law in terms of current reactor conditions and intervenes as necessary. Its deficiencies are that it lacks both the ability to project values of power and reactivity forward in time and the capability to cause the shape of the transient power curve to conform to a particular set of specifications. The predictive portion of the control law can provide those functions in which the supervisory program is deficient. However, it can not guarantee the safety of its own actions unless the reactor is operating within the envelope of conditions for which that law was designed. Both components are necessary.

\subsubsection{Control Laws}

Complementing the supervisory function of the reactivity constraint approach are predictive control laws that determine the actual control signal. These laws generally incorporate a reactor model and hence can project the reactor power forward in time. That is their advantage. Their deficiency is that they contain no provision for determining if it will be possible to halt the transient upon attaining the desired power level. A further problem is that, being model-based, laws of this type may yield erroneous results if there are errors in the model. Hence, the rationale for implementing the decision of these laws subject to review by a supervisory algorithm. 
As part of the MIT program on advanced instrumentation and control, a number of control laws have been developed and evaluated. These include a rule-based controller, a law obtained using state-space methods, and a technique based on set-theoretic control. Of the various laws that have been tried, the most successful has been the MIT-SNL PeriodGenerated Minimum Time Control Laws which were developed for the time-optimal control of reactor neutronic power $[2,3]$.

\subsubsection{Period-Generated Control}

Period-generated control was developed at MIT for the purpose of adjusting reactor neutronic power in a rapid yet safe manner [24]. It is a method for tracking trajectories that are defined in terms of a demanded rate and, as is discussed in Chapter Six of this report, it has been shown through experiment to offer superior performance as compared to other forms of model-based feedforward/feedback control. There are four major steps in its implementation. First, an error signal is defined by comparison of the observed process output with that which was specified. Second, a demanded inverse period (a velocity) is generated in terms of the error signal. Third, the demanded inverse period is processed through a system model to obtain the requisite control signal. Fourth, the control signal is applied to the actual system. Advantages to period-generated control are that it is readily implemented, that it is model-based and hence can be applied to non-linear systems, and that the resulting control laws may approach time-optimal behavior for the special case of rate-constrained processes.

The application of period-generated control to a nuclear reactor is as follows. It is desired that the reactor power, $n(t)$, conform to a certain trajectory. Accordingly, some measure of the rate of rise of the power is needed. A logical choice is the inverse reactor perioci which, it will be recalled, is defined as:

$$
\omega(t) \equiv \dot{n}(t) / n(t)
$$
such that:

The first step in applying period-generated control is to define an error signal, $e(t)$,

$$
e(t)=\ln \left(n_{d}(t+j \Delta t) / n(t)\right)
$$

where $n_{d}(t)$ is the demanded trajectory, $n(t)$ is the observed trajectory, and $j$ is a positive integer. A Taylor series expansion of this logarithmic expression reveals the rationale for selecting this particular arithmetic form for the error signal:

$$
\begin{aligned}
& e(t)=\ln \left(n_{d}(t+j \Delta t)\right)-\ln \left(n_{d}(t)\right)+\ln \left(n_{d}(t)\right)-\ln (n(t)) \\
& \approx \ln \left(n_{d}(t)\right)+j \Delta t \frac{d}{d t}\left(\ln \left(n_{d}(t)\right)\right)-\ln \left(n_{d}(t)\right)+\ln \left(n_{d}(t)\right)-\ln (n(t)) \\
& =j \Delta t \frac{d}{d t}\left(\ln \left(n_{d}(t)\right)\right)+\ln \left(n_{d}(t) / n(t)\right)
\end{aligned}
$$




$$
=j \Delta t \omega_{t}(t)+\ln \left(n_{d}(t) / n(t)\right)
$$

where $\omega_{t}(t)$ is the inverse period that corresponds to the power trajectory, $n_{d}(t)$. Thus, the error signal used in period-generated control is the sum of a feedforward action from the inverse period associated with the demanded trajectory, and a proportional action from the quotient of the demanded and observed system outputs. The former defines the system path. The latter provides corrective action against deviations. For nuclear reactors, it has been shown that the value of $j$ should be at least 2 in order to ensure stability against oscillations [41].

The second step in the application of period-generated control is to define a demanded inverse period in terms of the error signal. Thus,

$$
\omega_{d}(t)=\left(e(t)+\left(1 / T_{i}\right) \int e(t) d t+T_{d} \dot{e}(t)\right) / j \Delta t
$$

where the parameters $T_{i}$ and $T_{d}$ correspond to the integral and derivative times in a conventional feedback expression. That is, $\omega_{d}(t)$ equals $\omega_{1}(t)$ when the observed power is on the demanded trajectory, Otherwise, the two differ with $\omega_{\mathrm{d}}(\mathrm{t}) \mathrm{dr}: \mathrm{i}$ : $\mathrm{ng}$ the system to the demanded trajectory.

The third step is to develop an inverse dynamics model that translates the demanded period into the requisite control signal which, for a reactor, is the rate of change of reactivity. This is readily achieved by rearranging terms in the dynamic period equation so that the quantity $\dot{\rho}(t)$ becomes the dependent variable. Thus,

$$
\begin{aligned}
\dot{\rho}_{c}(t)= & (\bar{\beta}-\rho(t)) \omega(t)-\lambda_{e}^{\prime}(t) \rho(t)-\sum \bar{\beta}_{i}\left(\lambda_{i}-\lambda_{e}^{\prime}(t)\right)-\dot{\rho}_{f}(t)+ \\
& l^{*} \dot{\omega}(t)+l^{*}\left((\omega(t))^{2}+\lambda_{e}^{\prime}(t) \omega(t)\right)
\end{aligned}
$$

where the alternate form is used because it is the easier of the two to program on a digital computer. (Note: The subscript ' $c$ ' is used to denote the control signal.) It remains to address the term $\dot{\omega}(t)$ which represents the system acceleration. It is treated using the relation:

$$
\dot{\omega}(t)=\left(\omega_{d}(t)-(t)(t)\right) / k \Delta t
$$

where $\omega_{d}(t)$ is the demanded inverse period, $\omega(t)$ is the observed inverse period, $\Delta t$ is the time step, and $\mathrm{k}$ is the number of time steps over which it is desired that the system attain the specified trajectory. The quantity $k$ should be chosen to be small because the objective of period-generated control is to cause the controlled parameter to begin rising (or falling) quickly at the demanded rate. For this to occur, the acceleration term must rapidly die out. However, as a practical matter, there is a lower limit to the value of $\mathbf{k}$. Should it be made too small, $\dot{\omega}(\mathrm{t})$ will be quite large and an excessive rate of change in the control signal will be needed for transient initiation. 
The desired control law can now be constructed by substituting $\omega_{d}(t)$ for $\omega(t)$ in Equation (3.3.4.1-5) and then substituting Equation (3.3.4.1-6) for $\dot{\omega}(t)$. The term $\dot{\rho}_{c}(t)$ is then the control signal which, on application to the actual process, will cause the system output to track the demanded trajectory.

Figure 3.3.4.1-1 is a block diagram of the period-generated technique as applied to a nuclear reactor. The observed reactor power, $n(t)$, is compared to that which is demanded, $n_{d}(t+j \Delta t)$. The difference between the two results in an error signal, $e(t)$, which is used to generate the demanded inverse period, $\omega_{d}(t)$, that is needed to drive the reactor to the specified power level. This demanded inverse period is processed through a system model to obtain the appropriate actuator signal which, in this case, is a rate of change of the reactivity, $\dot{\rho}_{c}(t)$. Shown in Figure 3.3.4.1-2 are the power and reactivity profiles obtained during a trial of period-generated control on the ACRR in which the power was to be raised from $3 \mathrm{~kW}$ to $12 \mathrm{MW}$ on a 0.60 -s period. This corresponds to a power ascension rate of more than forty decades per minute. The period-generated control law raised the power to the demanded 12-MW level and held it at that level despite negative reactivity feedback from the Doppler effect. Moreover the transient was completed within the expected time of $4.98 \mathrm{~s}$. Also apparent in the figure are the rapid insertions and removals of reactivity needed to initiate and terminate the transient.

The capability of the period-generated control law to determine the control signal needed to make the reactor power conform to the demanded trajectory is of course a major asset. However, it should also be realized that the control law does not check to determine if the required control signal can in fact be generated. That is the role of the constraint. For example, the control law calculates the rate of reactivity removal needed to halt the transient, but it has no means of verifying that the actuator can actually be inserted at the requisite speed. The constraint ensures that capability by limiting the net reactivity to that which can be offset by the available rate of reactivity insertion.

One of the strengths of the period-generated approach is that the combination of a model-based feedforward action with feedback facilitates the control of non-linear systems. This is best illustrated by example. Denote the quantities $(\bar{\beta}-\rho)$ and $\left[\lambda_{e}^{\prime} \rho+\Sigma \bar{\beta}_{i}\left(\lambda_{i}-\lambda_{e}^{\prime}(t)\right]\right.$ by the symbols $R$ and $r$ respectively. A functional description of period-generated control can be written as:

$$
\begin{aligned}
& e(t)=\ln \left[n_{d}(t+j \Delta t) /(n(t)]\right. \\
& \omega_{d}(t)=\left[e(t)+\left(1 / T_{i}\right) \int e(t) d t+T_{d} \dot{e}(t)\right] / j \Delta t \\
& \dot{\rho}_{c}(t)=\hat{R}(t) \omega_{d}(t)-\hat{r}(t)+\left[\omega_{d}(t)-\omega(t)\right] / k \Delta t \\
& \omega(t)=(R(t))^{-1}[\dot{\rho}(t)+r(t)-\dot{\omega}(t)]
\end{aligned}
$$




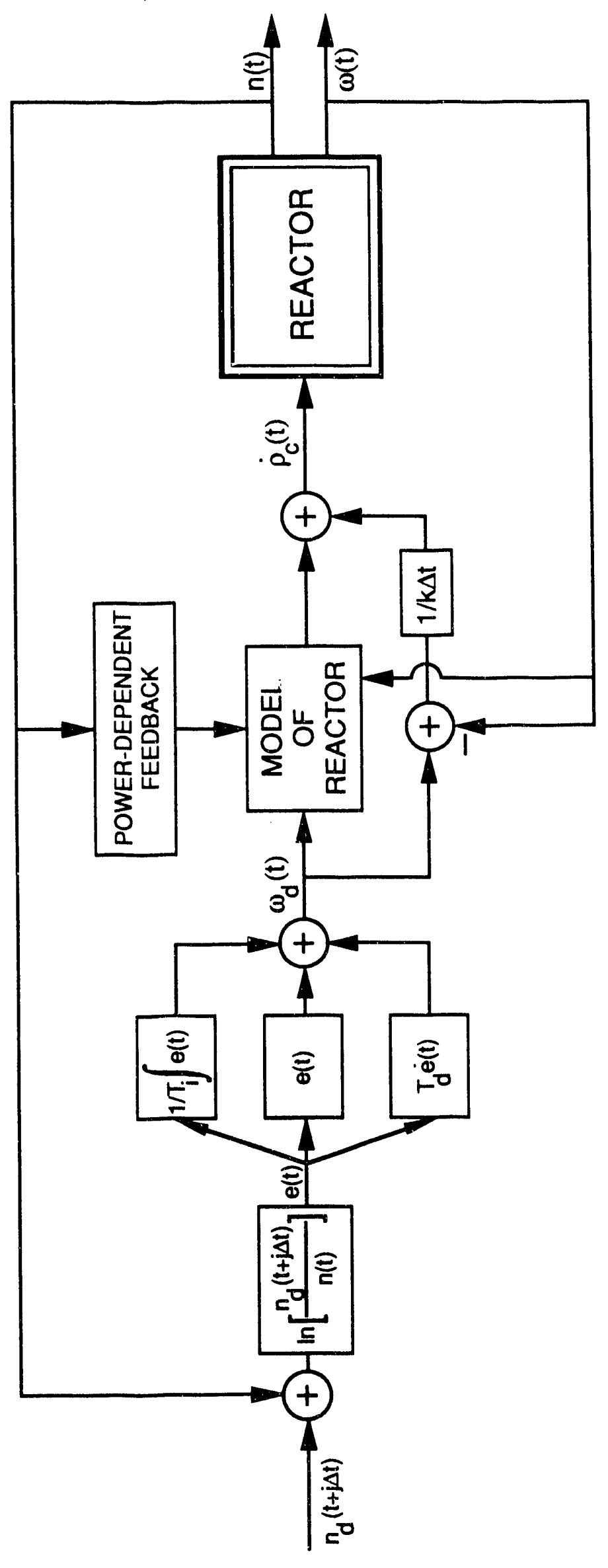

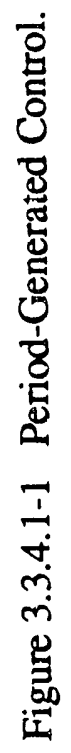


(s)!un) $\perp H פ I \exists H ~ Y ् र N \forall G ~ O O Y ~ \perp N \exists I S N \forall Y \perp$

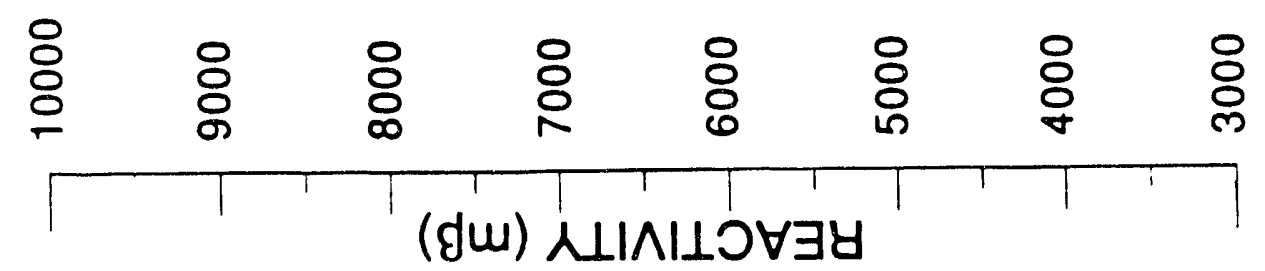
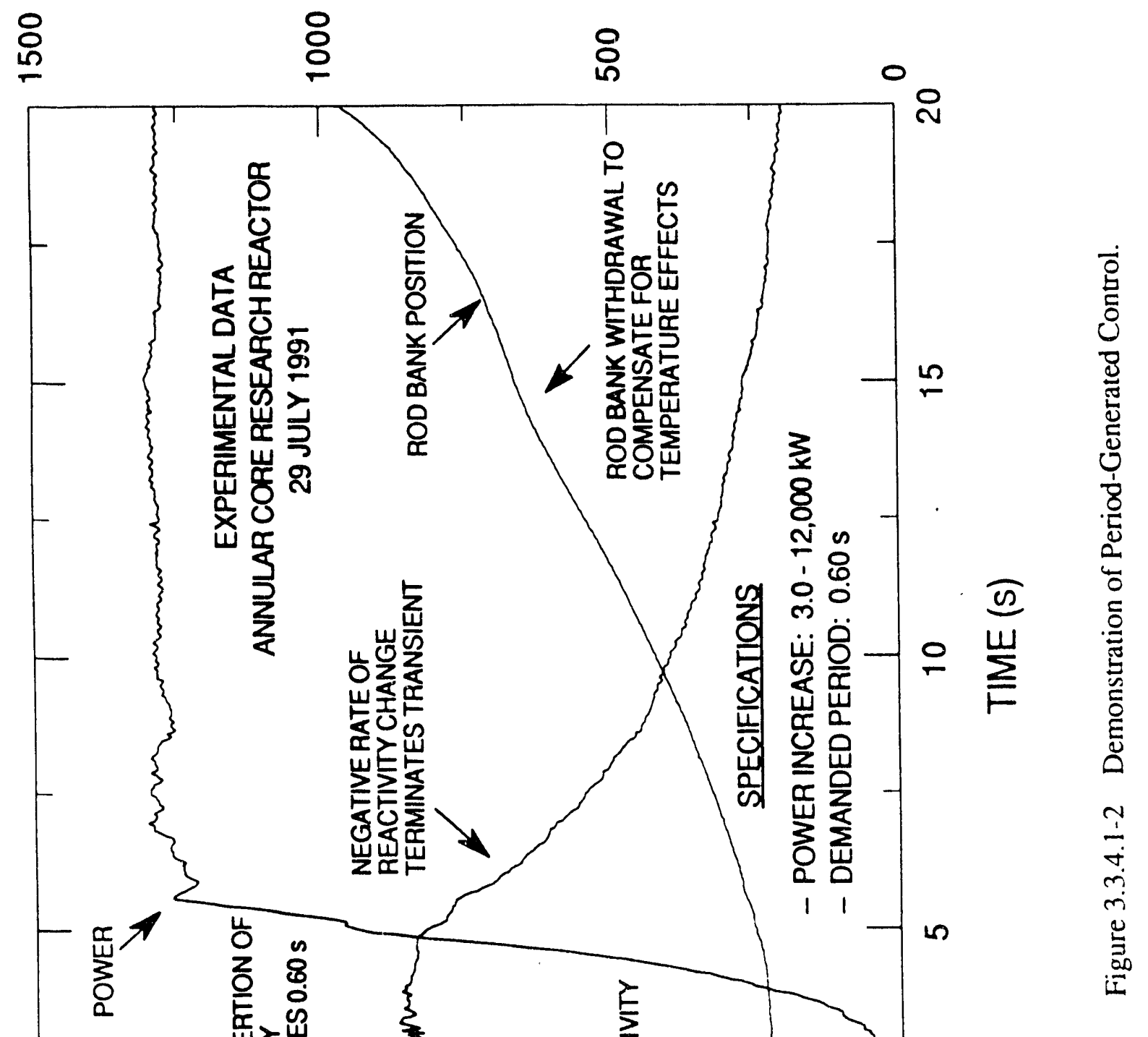
where for clarity of illustration the prompt neutron lifetime has been taken as unity and several terms of small order have been omitted. The above equations show the four basic steps in the implementation of period-generated control including definition of the tracking error, determination of the demanded inverse period, the inverse dynamics model, and the actual system. The superscript $\left({ }^{\wedge}\right)$ is used here to denote an estimated parameter. Substitution of $\dot{\rho}_{c}(t)$ for $\rho(t)$ in Equation (3.3.4.1-10) results in the feedforward control action. So doing yields:

$$
\begin{aligned}
\omega(t) & =(R(t))^{-1}\left[\hat{R}(\hat{t}) \omega_{d}(t)-\hat{r}(t)+\left[\omega_{d}(t)-\omega(t)\right] / k \Delta t+r(t)-\dot{\omega}(t)\right] \\
& \left.=(R(t))^{-1} \hat{R}(t) \omega_{d}(t)-(\hat{R}(t))^{-1} \hat{r}(t)-r(t)\right]+(R(t))^{-1}\left[\left[\omega_{d}(t)-\omega(t)\right] / k \Delta t-\dot{\omega}(t)\right] \\
& =\omega_{d}(t)+(R(t))^{-1}\left[\left[\omega_{d}(t)-\omega(t)\right] / k \Delta t-\dot{\omega}(t)\right] .
\end{aligned}
$$

It is evident from Equation (3.3.4.1-11) that if the quantities $\hat{R}$ and $\hat{\mathbf{r}}$ are accurate, then the combination of the inverse dynamics calculation and the feedforward action will result in the canceling of the system dynamics. Hence, once the acceleration term has been driven to zero, the actual and demanded inverse periods will be equal. This behavior is the strength of the period-generated approach and is of special importance for the trajectory control of non-linear systems. In particular, the result of the cancelation is that Equation (3.3.4.1-8), which is the standard P-I-D expression, is the determining factor in the system's response. Its use here results in accurate tracking because the incorporation of a system model in the period-generated method causes the observed inverse period to equal that which is demanded once acceleration effects have died out. This will occur regardless of whether the process being controlled is linear or non-linear. In contrast, were that same P-I-D expression to be applied directly to a non-linear system with no use being made of a model, the tracking would not be accurate except for the specific trajectory for which the controller had been tuned.

Another advantage of the period-generated technique is that it results in closed-form control laws that can be implemented in real time and which may approach a time-optimal response. Specifically, for systems that are subject to a rate constraint, the time-optimal trajectory will be the one that moves the system along that constraint. Hence, rather than identify the optimal control by the computationally-intensive process of solving the system's describing equations subject to both the constraint and a performance index, it is more direct to define the physical conditions that correspond to system movement along that limiting constraint. Period-generated control can achieve this by taking the demanded period to be that associated with the limiting constraint. For exampic, many nuclear research reactors are operated subject to limits on the power, temperature, coolant flowrate, and rate of rise of power. Suppose that the limit on the latter quantity for the ACRR were a period of $0.60 \mathrm{~s}$. In that case, the power and reactivity profiles shown in Fig. 3.3.4.1-2 are those of the time-optimal trajectory.

The degree to which a period-generated control law approaches a time-optimal response depends on the treatment of the acceleration term. In the ideal case, the trajectory would be instantly switched to and from the limiting path. The presence of the acceleration 
term makes this scenario physically impossible. However, the impact of the acceleration term can be made quite small provided that the control signal can be rapidly changed. Under such circumstances, period-generated control laws can closely approximate imeoptimal responses for rate-constrained systems.

\subsection{Summary of MIT Work on the Control of Reactors}

The MIT Program on the closed-loop digital control of neutronic reactors has now been in place for more than a decade. Moreover, it remains a major on-going activity. The summary below gives the accomplishments of this effort as of 30 June 1992. In addition to the references cited, additional information can be found on each item in [1-4].

1. Two models of the 5-MWt MIT Research (MITR-II) were developed. One described both the core and the heat removal systems (primary, reflector, shield, and secondary) while the other described only the core [42].

2. A simulator was designed to permit the testing of control strategies on the MITR-II models [42].

3. Methods for validating power, flow, and temperature signals from the MITR-II were investigated. The parity space approach with analytic redundancy was selected $[12,43]$.

4. Upon completion of extensive safety evaluations, equipment for the direct digital control of the MITR-II's regulating rod was installed. This included signal transmitters, isolation devices, analog-to-digital converters, an LSI11/23 mini-computer, and digital-to-analog converters. This was made possible by the Charles Stark Draper Laboratory.

5. Open-loop trials were conducted of the signal-validation routine.

6. Closed-loop trials in which the MITR-II was operated at steady-state using validated signals were performed $[44,45]$.

7. The standard dynamic equation (without prompt terms) was derived for use as a reactor model [42].

8. The 'reactivity constraint approach' was formulated and verified via simulation [42].

9. Closed-loop trials in which power was raised and lowered using the reactivity constraint approach were conducted [42].

10. The experimental protocol described in Section 1.6 of this report was formulated.

11. A variety of control techniques including proportional, heuristic, and statespace were investigated [42]. 
12. Approval was requested of and received from the U.S. Nuclear Regulatory Commission for the direct digital control of the MITR-II's shim blades. This approval was subject to the provision that the reactivity constraint approach be used in a supervisory capacity [16].

13. The alternate form of the dynamic period equation was derived [19].

14. A rule-based controller that used fuzzy logic was designed and demonstrated [17].

15. Comparative studies were performed of supervisory controllers based on the standard and alternate forms of the dynamic period equation [20].

16. The on-line reconfiguration of control laws was accomplished on the MITR-II. This was done with support from the Charles Stark Draper Laboratory [18].

17. It was recognized that the rate of change of reactivity, rather than the reactivity itself, should be used as the control signal (the signal sent to the actuator) for eactor power controllers $[21,22]$.

18. The standard form of the dynamic period equation (with prompt terms) was derived [39].

19. It was realized that time-optimal trajectories of systems bounded by a constraint could be achieved by designing a control law that caused the system to track the consiraint. This observation and the understanding that the rate of change of reactivity should be used as the signal to the actuator lead directly to the MIT-SNL minimum time laws [21,22].

20. The 'MIT-SNL Period-Generated Minimum Time Control Laws,' which are closed-form expressions for the time-optimal control of reactor neutronic power were derived $[21,22]$.

21. The MIT-SNL laws were studied extensively via simulation.

22. A simulator was constructed at Sandia National Laboratories for testing the MIT-SNL laws. It consisted of two separate computers, one for the controller and one for a model of SNL's Annular Core Research Reactor (ACRR). The use of two computers enabled testing of the hardware-to-software interface and the signal acquisition equipment.

23. The hardware and hardware-to-software interface needed for testing closedloop digital controllers on the ACRR was designed, built, and installed. Thic followed extensive safety evaluations [46].

24. A method for the accurate, real-time estimation of precursor concentrations was devised and verified via simulation [47]. 
25. On-line, experimental trials of the MIT-SNL Period-Generated Minimum Time Control Laws on both the Annular Core Research Reactor and the MIT Research Reactor were conducted. Power increases of five orders of magnitude were achieved on the ACCR without overshoot on demanded periods of $0.5 \mathrm{~s}$ [23-25].

26. The generic nature of both the 'MIT-SNL Control Laws' and the 'Reactivity Constraint Approach' was established by using each technique on both the MITR-II and the ACRR. These two reactors are very different in design [23-25].

27. Conditions for global stability and siability against oscillations about a specified trajectory were devised for the MIT-SNL laws [41].

28. Predictive displays were developed and tested on the MITR-II. These allow a reactor operator to visualize the consequences of a planned control action before actually having to implement it [30].

29. Proportional-integral-derivative feedback was incorporated in the MIT-SNL Period-Generated Minimum Time Control Laws $[48,49]$.

30. Techniques for reducing controller sensitivity to noise were developed and demonstrated on the ACRR [50].

31. Algorithms based on the dynamic period equation for use in measuring reactivity were devised and evaluated on the MITR-II [51].

32. The MIT-SNL laws were modified for use in raising reactor power from subcritical conditions [52].

33. Power cycling (sinusoids) and rapid power decreases were demonstrated [53].

34. Experiments were performed to evaluate a method for the control of core temperature [54].

35. The concept represented by the MIT-SNL laws was generalized as 'periodgenerated' control and applied through simulation to several non-nuclear systems [55].

36. Comparative evaluations of several trajectory control techniques were made on the MITR-II. Included were proportional-integral-derivative, feedforward, sliding mode, and period-generated control [56].

37. The theoretical basis for the use of the MIT-SNL laws as a general method for the trajectory control of reactor power was described [28,29].

38. A near real-time, three dimensional model of a PWR's neutronic and thermalhydraulic behavior was developed and benchmarked [57-59]. 
39. The reactivity constraint approach was extended to the control of reactors described by spatial dynamics [60].

The above list is impressive. Nevertheless, much remains to be done including automated diagnostics, coordinated control of temperature and power, identification of core designs that optimize controller performance, the enumeration of quantitative criteria for determining the reasonableness of the point kinetics approximation, and practical methods for command validation. In the following chapters of this report, certain aspects of the work listed above are extended to multi-modular reactors. 


\section{Advanced Control Computer System ${ }^{(1)}$}

This chapter describes the design, implementation, and initial testing of a multiplecomputer/single-task system for the closed-loop, digital control of the MIT Research Reactor (MITR-II). A major advantage of the multiple-computer approach is that generic safety-related software that remains invariant can be separated from the control law software that is updated as plant procedures change. This facilitates software validation. Also, this approach allows both real-time operation and high numerical throughput. System compatibility was achieved through design of a special passive back plane which enabled the otherwise incompatible components to be operated in an integrated system. This multiple-computer system was designated as the Advanced Control Computer System (ACCS). In addition to a description of both the system and its associated hardware and software interfaces, experimental results are presented from its initial trials.

\subsection{Statement of Problem}

Since the late 1970s, the Massachusetts Institute of Technology has been engaged in a program to develop and demonstrate advanced techniques for the instrumentation and control of nuclear reactors. In 1981, with the support and partnership of the Charles Stark Draper Laboratory, an LSI-11/23 minicomputer was purchased and installed in the control room. The availability of this system made possible on-line demonstrations of signal validation. Once validated signals were shown to be effective, efforts were focused on closed-loop control. This led to the 'reactivity constraint approach' which is a generic supervisory method that ensures the absence of challenges to a reactor's safety system as the result of any automated control action $[1,19]$. In 1985, the reactivity constraint approach became the basis of a license approval from the U.S. Nuclear Regulatory Commission for the general use of digital systems on the MITR-II [16]. This in turn made possible the evaluation of a rule-based controller, closed-form control laws that operate in minimum time, and comparative evaluations of predictive displays [30]. The LSI-11/23 system consisted of a single computer that performed all required functions including data acquisition, signal validation, supervisory control, calculation of the actuator signal, and signal output.

The Advanced Control Computer System is a major improvement in that it consists of five inter-connected computers, each responsible for a $r^{\prime}$ :fferent set of tasks. In addition to permitting more flexible operation and allowing testi.s of computation-intensive concepts, this new system enhances safety because safety-related functions such as supervisory control can now be run separately from control law calculations. Software for the former is well-established, is based on fir: principles, and is invariant. In contrast, software for the latter will probably always be under development because the objective of the MIT program is to identify new methods of control and make their use a practical reality. A further advantage of this segregation of functions important to safety from other types of calculations is that it limits the amount of software for which verification and validation is needed. Other noteworthy features of the ACCS are that it combines real-time operation with high numerical throughput, utilizes a no-moving-part hard disc for greater reliability, includes the means to perform simulation studies, and employs a passive back

(1) This chapter is based on work performed by Kwan S. Kwok under the direction of Dr. John A. Bernard and Professor David D. Lanning. 
plane to permit non-interactive operation of the bus structure. This latter feature in turn allows the coexistence of otherwise incompatible components.

\subsection{Advanced Control Computer System}

The primary function of the MITR-II's digital control system is to permit research on control strategies for the automated operation of nuclear reactors. Among the problems currently under investigation are automated startups, coordinated control of power and temperature, and applications of artificial intelligence. The performance of these tasks requires real-time operation, high numerical throughput, on-line access to large amounts of memory, and auto-ranging signal acquisition whereby both the level and scale of an instrument is read. An immediate difficulty arose in that, at the time of the ACCS's design, there was no single, commercially-available product that offered all of the above features at a reasonable price. Accordingly, the solution adopted for the Advanced Control Computer System was to design a multiple-computer system in which an IBM-PC compatible computer would perform the real-time functions and a VAX computer would perform the computations and data collection. Additional computers could be added as necessary for specific applications.

As ultimately configured, the Advanced Control Computer System consists of five separate computers, all linked in a multiple-computer/single-task architecture. Figure 4.2-1 is a block diagram showing the configuration and purpose of each component. These are:

1. Rack-Mount 80386: This data acquisition IBM-AT computer is assigned three major tasks. First, it collects data from a maximum of thirty-two sensors, performs signal validation on the collected data, outputs the validated information to up to four other computers, and displays the validated information on the console CRT monitor. Second, it computes the maximum allowed control signal using the supervisory reactivity constraint algorithm $[1,19]$ as well as limits of other MITR technical specifications, receives the requested control signal from the other computers, compares that signal with the one calculated by the supervisory algorithm, outputs the more conservative signal to the control rod motors, and displays the control decision on the screen. The computer's third function is to write the desired data to the permanent disk. Changes to the computer's software are not required except for routine calibrations and adjustments of the scaling and zero offset constants used in the data acquisition routines. Changes may be made to the software in other computers when the testing of novel control concepts is desired. But the cor. on this IBM-AT remains unchanged and unperturbed. This is one of the miyot advantages to the new digital control system. Another advantage to this data acquisition computer is that it uses a no-moving-part, permanent hard disc as its primary storage device. Also, the signal from any instrument that is common to both the safety and control systems is sent through a signal isolation device such as an ptical transformer. This is done to ensure that a fault in the control system can not propagate backwards through the instrumentation and affect the proper functioning of the safety system.

2. MicroVAX-II: The VAXstation II/GPX is a machine dedicated for intensive floating-point computations. Engineering and control calculations such as are 


\section{REACTOR}

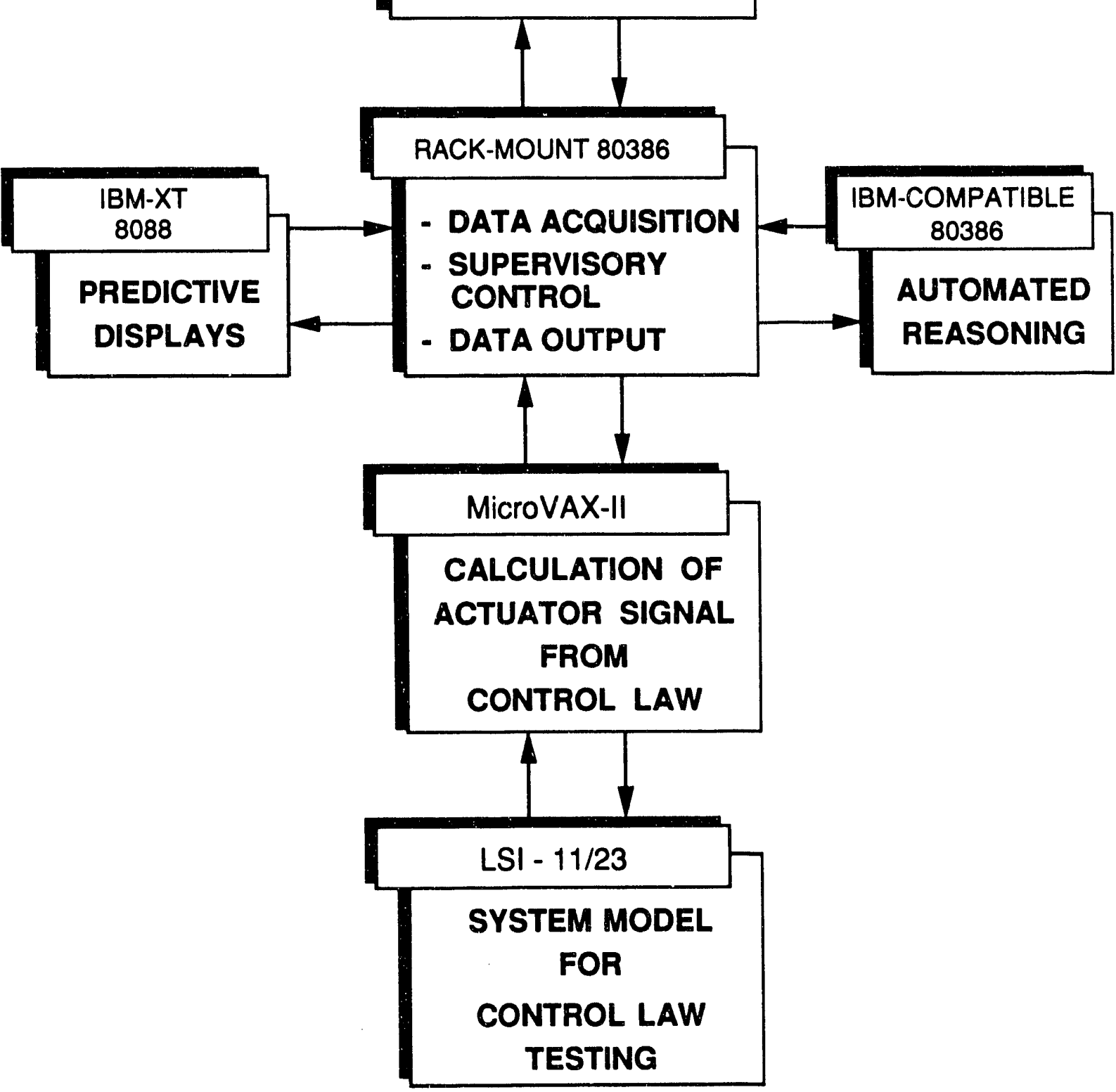

Figure 4.2-1 Configuration and Functions of MITR-II Advanced Control Computer System. 
required for the MIT-SNL minimum time control laws [2,3], are performed on this machine. The MicroVAX-II receives validated information on the reactor from the data acquisition system (IBM-AT). It then calculates the demanded control signal from whatever control law is being tested, and exports that signal to the data acquisition system for output to the control rod motors. Changes in this system's software are expected to be frequent, especially when new control concepts are identified. However, because this computer is a separate entity, these changes will not affect the experimental envelope prescribed by the reactor's technical specifications.

This VAXstation-II/GPX computer is equipped with a MicroVAX-II CPU which operates at about one million instructions per second (1 MIPS). The back plane is a full-size 16-slot enclosure with the 22-bit Q-bus firmware. There are $9 \mathrm{Mb}$ of RAM and $159 \mathrm{Mb}$ of permanent storage. A cartridge tape system is available for backup and file transfer purposes. Eight serial ports provide support for a printer and for communications with other computers. There is a 256-color 8-plane color graphics GPX controller with a large color monitor and a mouse form the main console available to the computer operator.

3. IBM-Compatible 80386: This is a high-speed machine on which computer programs are first edited, compiled, and finally linked to form an executable module. This machine is capable of supporting automated reasoning using PROLOG, LISP, or C. It is designed to be compatible in all details with the Rack-Mount 80386 data acquisition system. Sub-components within the two computers are exactly identical whenever possible. Unavoidable hardware differences are, as a minimum, compatible at the register level. That is the register calls, which control all interrupt services for the sub-system, are identical. This means that any differences are not visible to the operating system when the computers are accessed through the use of the vendor-supplied BIOS (Basic Input and Output Services) routines. The advantage of the compatibility between these two machines is that computer codes that run on one machine will also run on the other. This facilitates software development because the necessary tools are not on the Rack-Mount 80386 system.

The 80386 architecture was selected for both the data acquisition system and this computer. The 80386 is a true 32-bit microprocessor with 32-bit internal registers, a 32-bit data bus, and a 32-bit address bus. The size of the address bus provides a maximum addressable memory of $2^{32}$ bytes or 4 gigabytes. The 32-bit internal and 32-bit data bus capabilities allow 4 bytes of data to be read from, written to, or fetched from the main memory at a time. These features plus a high operating frequency (16 and $20 \mathrm{MHz}$ for the two computers respectively), and other features including an expanded instruction set, a new register set, memory management, page translation, and task management functions made the 80386 the best choice as the CPU.

4. IBM-XT 8088: This computer's role is to receive validated signals from the data acquisition computer and to display model-based predictive information $[30,31]$ or a safety parameter display on its screen. 
5. LSI-11/23: This unit is connected to the MicroVAX-II for the purpose of providing an independent machine on which a model of a reactor can be run. This permits new controllers to be programmed on the VAXstation II/GPX and tested against a simulation model running on the LSI-11/23 prior to the performance of actual closed-loop runs on the reactor. This approach has the advantage that new programs are tested under realistic conditions. In particular, signals must be passed between two computers as is done for actual implementations. Previously, the new control law and the model ran on the same unit.

Integration of components within the data acquisition computer was accomplished through a passive back plane which is basically a non-intelligent bus that allows only lines such as data, status, and timing to be passed. Integration of the five separate computers was achieved through use of RS-232 serial communication. In spite of the inherent limitation on the data transfer rate of the RS-232 serial communication, it is adequate for applications at the MIT Research Reactor. If a faster data transfer rate is desired, the techniques discussed here are equally applicable to RS-422 serial communication which provides a data transfer rate that is significantly faster that that of the RS-232.

\subsection{Data Acquisition and System Interfaces}

Implementation of the Advanced Computer Control System required much more than the mere assembly of the five computers described in the preceding section. Data acquisition routines, a software interface, a hardware interface, and special instrumentation as well as interlock circuits were also required. These are described here.

\subsubsection{Data Acquisition and Interface Software}

The data acquisition system installed on the Rack-Mount 80386 computer consists of two DT-2821 boards manufaciured by Data Translation. These are analog and digital input/output (I/O) boards designed either for the IBM-AT personal computer or for other IBM-compatible computers. The primary features of the DT-2821 board are analog-todigital (A/D) conversion rates of $40 \mathrm{kHz}$, digital-to-analog (D/A) conversion rates of 130 $\mathrm{kHz}, 16$ digital $\mathrm{I} / \mathrm{O}$ lines, and an onboard pacer clock for real-time operation. The resolution of both the $A / D$ and $D / A$ converters is 12 bits.

Input voltages to the $A / D$ converters are read and converted into a 12-bit binary word which provides 4096 possible discrete levels. This represents a $0.002-\mathrm{V}$ resolution for a 0 to $10 \mathrm{~V}$ uni-polar signal. In other words, the data acquisition system is able to resolve voltages as small as $0.002 \mathrm{~V}$ for uni-polar inputs and $0.004 \mathrm{~V}$ for bi-polar (-10 to $10 \mathrm{~V}$ ) inputs. The binary word that represents the voltage reading of the selected channel is passed to the executing program via the operating system (MSDOS-3.3) and a set of machine language instructions that reside in a vendor-supplied program called a device driver. The device driver contains machine language instructions that interact between the computer hardware and the operating system. Information is then passed to the executing program through the operating system.

The Rack-Mount 80386 data acquisition computer's executing program contains subroutines that serve several different purposes. Subroutines that perform low level system functions are written in assembly language. Those that interact with devices and 
those that facilitate general engineering calculations are written in high level languages such as C or FORTRAN. The compilers used were Microsoft Macro Assembler Version 5.1, Microsoft C Version 5.1, and Microsoft FORTRAN Version 4.1. The main program was written in $\mathrm{C}$ because of that language's flexibility. Specifically, it allows interfacing with both assembly languages and with FORTRAN.

A set of software routines were written to control the hardware, collect data, maintain the system in real time, select the proper range on the instruments, relay the pertinent information to the control laws, and transmit the control law decision. Diagnostic functions were designed in the software so that any significant hardware or software errors would be detected and displayed on the screen to warn the computer/console operator. Storage of the collected data on a permanent device was also supported by these routines. The organization of these routines is shown in Figure 4.3.1-1.

\subsubsection{Har!'ware Interface to Reactor}

There are twenty sensors connected to the analog input channels of the data acquisition computer. These are the reactor neutronic power (4), primary flow (4), primary hot-leg temperature (2), primary cold-leg temperature (2), primary delta-temperature (1), pcsition of the regulating rod (2), positions of shim blades number one (1) and four (1), output of the thermal power indicator (1), reactor period generated by the period network of MITR-II's nuclear channel one (1), and demanded power as input by the console operator (1). These sensors were connected to the data acquisition computer via a master terminal strip and two breakout junctions. The latter were necessary to permit regrouping and a change of cable type. The type of cable had to be changed from ribbon-type to the roundtype for routing purposes and regrouping was necessary so as to connect the twenty sensors to two different DT-2821 boards.

\subsubsection{Broad-Range Power Sensor}

One of the reasons for constructing the Advanced Control Computer System was to permit automated startups of the MITR-II. For this to occur, the digital system would have to monitor power levels that ranged from a few watts at source-level to $5 \mathrm{MW}$ at full power. The span is about seven decades. Accordingly, a boron-lined, gammacompensated ion chamber which correctly indicated the reactor's neutron flux level from full shutdown to full power was made available. This chamber was equipped with a KEITHLEY Model 485 auto-ranging picoammeter with a built-in Model 4853 IEEE-488 interface. This unit is basically a 4-1/2 digit (4 significant digits with sign) auto-ranging picoammeter with seven DC current ranges. The heart of the Model 485 is a transresistance amplifier (current-to-voltage converter) followed by an $A / D$ converter that translates the conditioned analog input signals into a form usable by its internal microcomputer. The microcomputer records and executes the user-selected functions through the main panel on the instrument. Additionally, it communicates with a host computer via the IEEE-488 interface. The host computer can send commands through the IEEE-488 bus and either interrogate the status or control the response of the Model 485.

The design of this system allows the Rack-Mount 80386 data acquisition system to receive an on-scale reading of the output of the neutron-sensitive compensated ion-chamber in a digitized form. A further advantage is that the output of the Model 485 is digitized. 


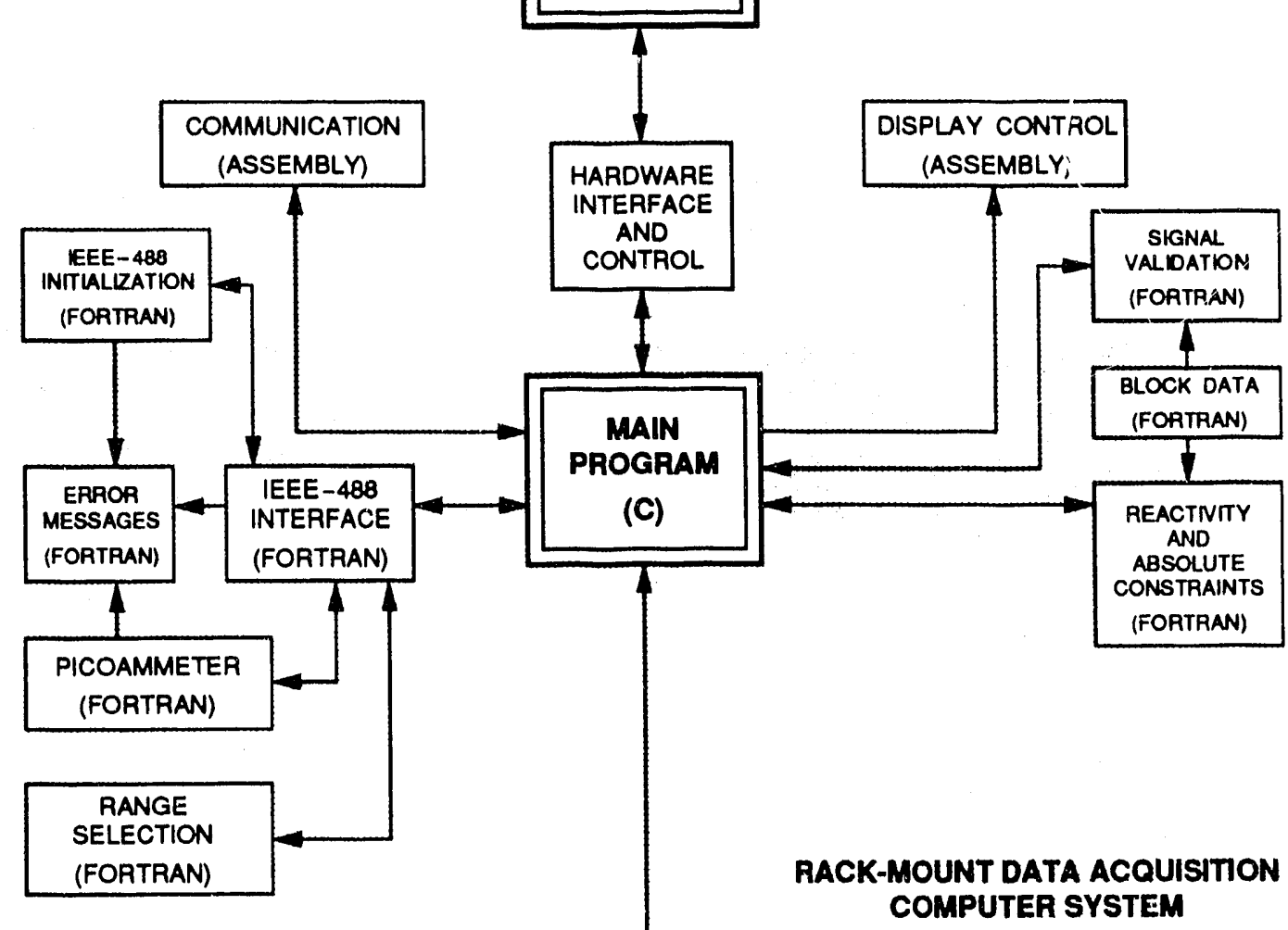

REACTOR

MIcroVAX II

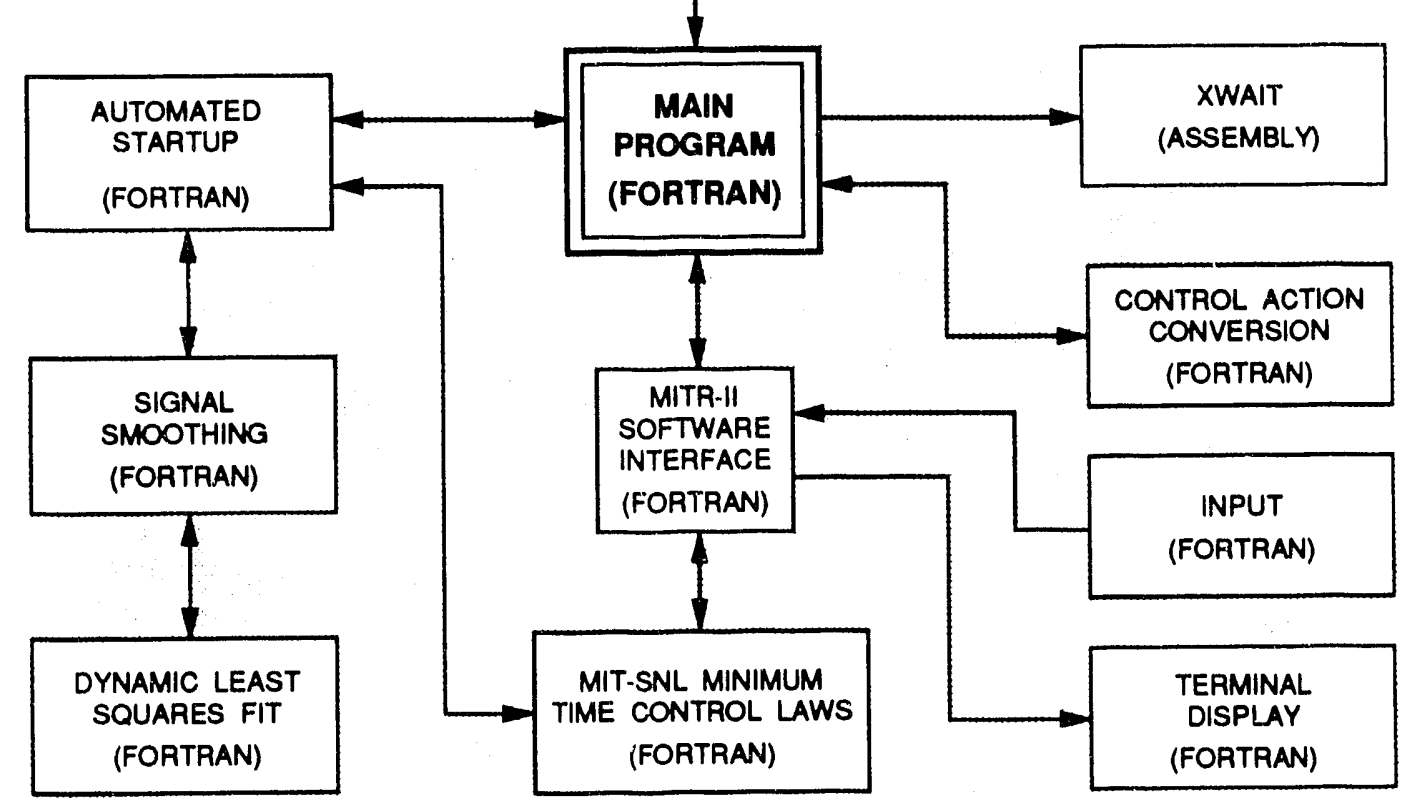

Figure 4.3.1-1 Organization of Software. 
This makes the system less prone to electrical interference or noise than if a signal were first obtained in analog form and then converted for digital operation.

\subsubsection{Safety and Interlock Circuits}

The Advanced Control Computer System was equipped with both safety and interlock circuits such as a 'watchdog timer' that guards against common computer faults including divide checks, infinite loops, and failure of the software to execute sequentially. These circuits had been developed for the original MITR-II digital control system, the LSI$11 / 23$ minicomputer [1]. For the most part, those designs were retained here.

\subsection{Demonstration of Advanced Control Computer System}

The Advanced Control Computer System was subject to extensive preoperational testing. This included the use of electronically-generated signals to test the data acquisition computer for proper processing by the software and for transmission to the hardware's output device. Similarly, electronic test signals were used to check the response of the broad-range power sensor and the auto-ranging picoammeter for both operability and linearity. Also, all safety and interlock functions were verified to operate at their proper setpoints. Finally, the system as a whole was evaluated by inserting a series of electronic signals that simulated a rise in reactor power on a demanded period. The actuator signal was observed to vary in the expected manner.

Closed-loop trials of the Advanced Control Computer System on the MITR-II were begun on 28 December 1990 . Figure 4.4-1 shows the power and reactivity profiles from a transient in which the power was raised from $100 \mathrm{~kW}$ to $500 \mathrm{~kW}$ on a 100 -s period. The Alternate MIT-SNL Period-Generated Minimum Time Control Law with proportionalintegral feedback was used. The transient was completed successfully. Figure 4.4-2 is from another trial in which the same control law was used to cycle the power between 100 $\mathrm{kW}$ and $200 \mathrm{~kW}$ on a frequency of one cycle every $90 \mathrm{~s}$. Again the test was successful.

\subsection{Assessment of Advanced Computer Control System}

The major contribution of the Advanced Control Computer System (ACCS) is that a computer architecture was envisioned, designed, and implemented that promotes the safe application of digital control techniques on nuclear reactors. Specifically, the selected architecture permitted separation of software important to safety from software important to reactor control. Examples of the former include algorithms that implement reactivity constraints and software statements that limit allowed reactor parameters such as the power, period, and temperature. Examples of the latter include the control law algorithms that are used to determine the appropriate actuator signal. Software essential to safety is usually generic in nature and invariant. Changes to this software are infrequent. In contrast, changes to the control software may be required on a fairly routine basis as reactor conditions change to reflect modifications in reactor systems, updates to operating procedures, and progress in control engineering. From a safety point of view, it is highly desirable that it be possible to alter the control software without there being any possibility of affecting the safety software. The Advanced Control Computer System architecture that was implemented here facilitates this objective by providing a multiple-computer/single-task computing environment. Specifically, separate but interconnected computers are used for 


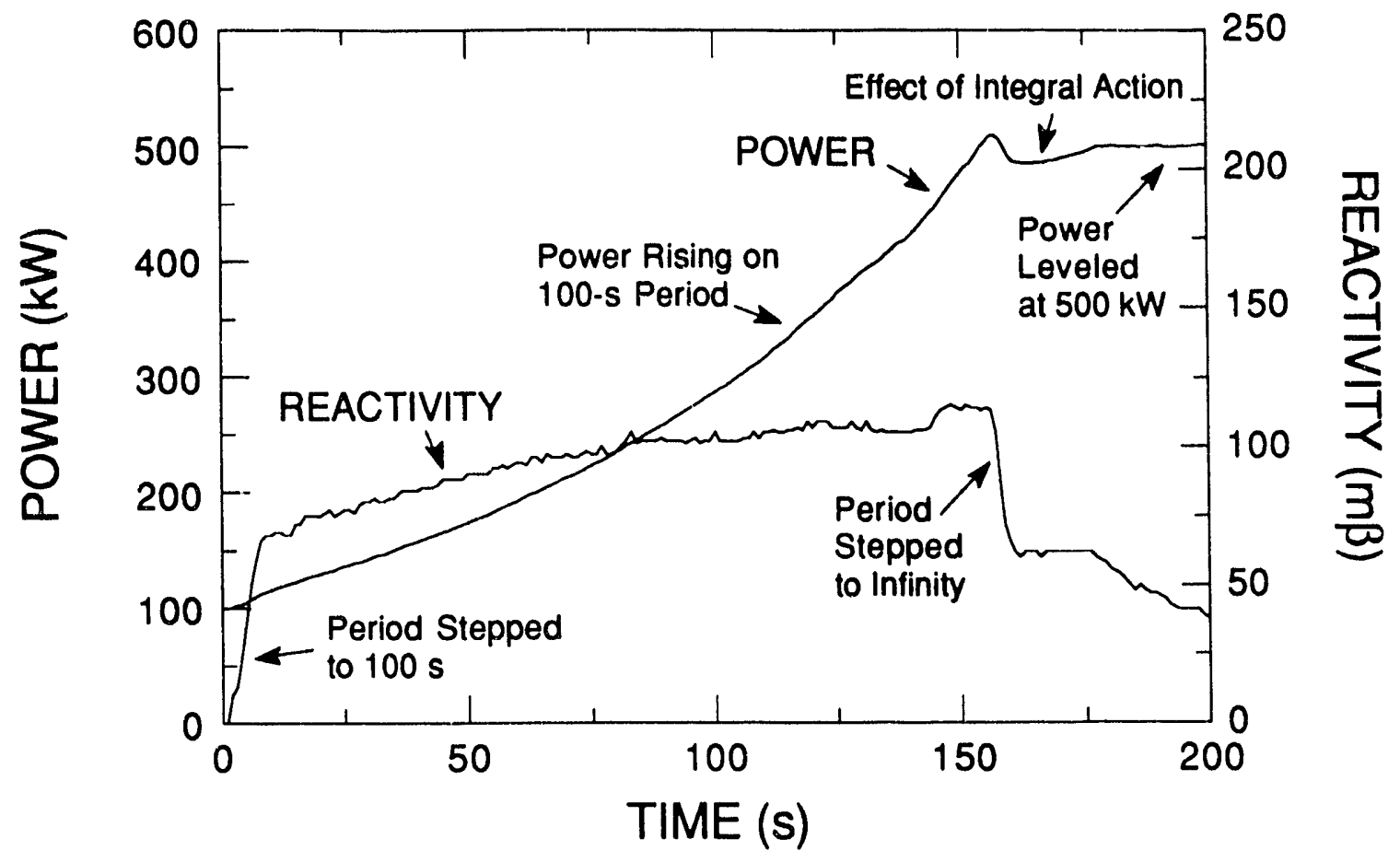

Figure 4.4-1 Power Increase from 100 to $500 \mathrm{~kW}$ on a 100-s Period Using Alternate MIT-SNL Law with Proportional-Integral Feedback.

Experimental Data Obtained 12/28/90 on the MIT Research Reactor.

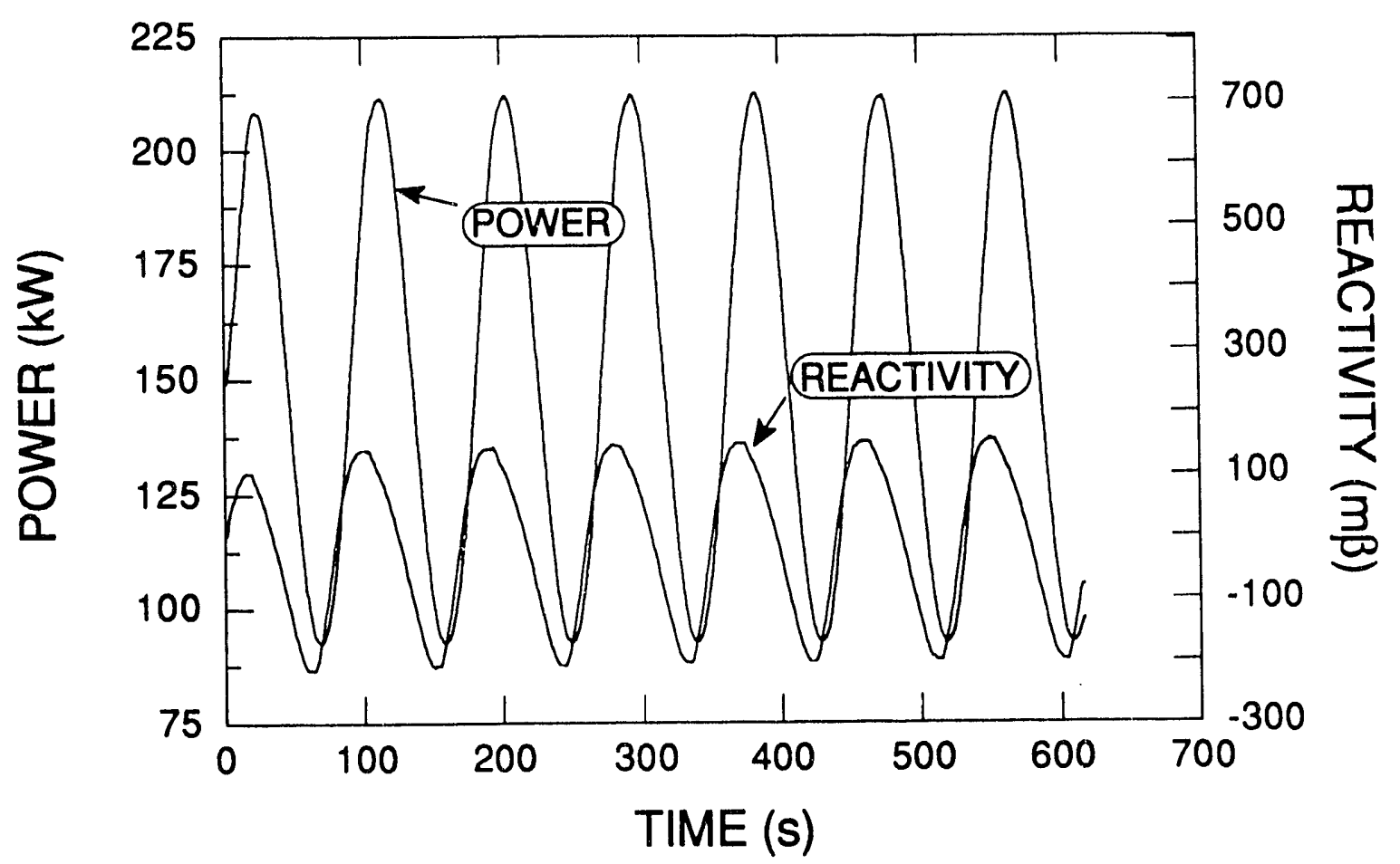

Figure 4.4-2 Sinusoidal Power Variation from 100 to $200 \mathrm{~kW}$ on Frequency of One Cycle Every $90 \mathrm{~s}$ Using Alternate MIT-SNL Law.

Experimental Data Obtained 12/28/90 on the MIT Research Reactor. 
data acquisition, implementation of software related to safety, control law computations, the man-machine interface, and automated reasoning.

Other advantages of the ACCS are as follows. First, it uses an .luto-ranging picoammeter in conjunction with a compensated ion chamber. This means that the data acquisition system will transmit the scale as well as the instrument reading. This capability is essential to the performance of automated startups because power increases of many orders of magnitude are involved. Second, the ACCS takes a digitized signal directly from the neutron instrument (the auto-ranging picoammeter) as opposed to taking an analog signal and then converting it to digital form. The ACCS approach significantly reduces noise in the signal and hence in the controller. Third, simulations can be run in an interactive manner because a dedicated computer is available that interfaces with the actual hardware that is used to operate the digital controller. Thus, the simulations test the hardware, the software, and their associated interfaces. Moreover, safety is not compromised because, in this mode of operation, the output of the data acquisition computer is physically directed to the computer on which the simulation is run and not to the actual reactor. In summary, the Advanced Control Computer System offers many improvements relative to the original MITR-II digital control system and it is now being used routinely to conduct research in support of the MIT program on advanced instrumentation and control of nuclear reactors. Additional information has been previously given [61]. 


\section{Automated Reactor Startup with On-Line Estimation of Subcriticality (1)}

This chapter reports the use of the 'perturbed reactivity method' in conjunction with the MIT-SNL Period-Generated Minimum Time Control Laws for the conduct of automated reactor startups. The performance of such startups was one of the objectives of the MIT program on the control of multi-modular reactors because of the need to coordinate control of reactor neutronic power with that of the steam production system (turbines and steam generators) during low power operation. Specifically, the availability of some means for automating reactor startups and the subsequent initiation of steam flow might reduce the incidence of plant trips caused by malfunctions associated with steam generator level and/or feedwater flow. In addition to this motivation, there were two other reasons for developing an automated startup methodology. First, the capability to conduct automated power increases from subcritical conditions is of extreme importance to nuclear-powered spacecraft, where, for reasons of safety, the reactor will be launched in a shutdown cordition and only made critical once a 'nuclear-safe' orbit has been achieved. Second, an automated startup capability would be desirable for reactors used on board naval and maritime vessels where rapid recovery of the reactor plant may be necessary in order to quickly restore propulsion power and thereby prevent damage to the vessel itself. MIT, in conjunction with Sandia National Laboratories, had previously demonstrated use of the MIT-SNL laws for the conduct of power increases from subcritical [3]. However, for those trials, the initial degree of subcriticality had been known. The perturbed reactivity method eliminates the need for the a priori specification of the amount by which the reactor is shutdown and hence permits a startup that is automated in all respects.

The perturbed reactivity method is a general technique for the estimation of reactivity. It is particularly suited to the determination of a reactor's initial degree of subcriticality and was developed to facilitate the automated startup of both spacecraft and multi-modular reactors using model-based control laws. It entails perturbing a shutdown reactor by the insertion of reactivity at a known rate and then estimating the initial degree of subcriticality from observation of the resulting reactor period. While similar to inverse kinetics, the perturbed reactivity method differs in that the net reactivity present in the core is treated as two separate entities. The first is that associated with the known perturbation. This quantity, together with the observed period and the reactor's describing parameters, are the inputs to the method's implementing algorithm. The second entity, which is the algorithm's output, is the sum of all other reactivities including those resulting from inherent feedback and the initial degree of subcriticality. During an automated startup, feedback effects will be minimal. Hence, when applied to a shutdown reactor, the output of the perturbed reactivity method will be a constant that is equal to the initial degree of subcriticality. This is a major advantage because repeated estimates can be made of this one quantity and signal smoothing techniques can be applied to enhance accuracy. In addition to describing the theoretical basis for the perturbed reactivity method, factors involved in its implementation such as the movement of control devices other than those used to create the perturbation, source estimation, and techniques for data smoothing are presented. Also, experimental results are shown in which the concept was used in conjunction with periodgenerated control laws to perform automated startups of the 5-MWt MIT Research Reactor.

(1) This chapter is based on work performed by Kwan S. Kwok under the direction of Dr. John A. Bernard and Professor David D. Lanning. 


\subsection{Statement of Problem}

Conventional methods for reactivity determination include reactivity balances and inverse kinetics [62]. The use of a balance c: 'culation is, by definition, not possible for the performance of an automated startup when tite degree of subcriticality is unknown. That leaves inverse kinetics. Bernard reported two variations of the inverse kinetics approach based on the dynamic period equation [51]. These were 'inverse dynamics' and the 'hybrid method.' These, and the original inverse kinetics approach, yield the net reactivity that is present in the core. The perturbed reactivity method, which is developed in this report, also gives the net reactivity. However, the method of calculation is somewhat different. Specifically, rather than providing sequential estimates of the continuouslyvarying net reactivity, the perturbed reactivity method yields repeated calculations of a reactor's initial degree of subcriticality.

The perturbed reactivity method, like inverse kinetics, is based on the spaceindependent kinetics equations. Its distinguishing feature is that it differentiates between reactivity that is deliberately inserted as part of a planned perturbation and that which was either initially present or the result of feedback. This is a major advantage. For example, consider an automated startup in which reactor power is to be raised on a demanded trajectory. Inputs to the perturbed reactivity method's algorithm will be the magnitude of the planned reactivity perturbation, the observed reactor period, and the reactor's describing parameters such as precursor yields and decay constants. The output will be the aggregate of all other reactivities including feedback effects and the initial degree of subcriticality. In the case of an automated startup, feedback effects will be minimal and the output of the perturbed reactivity method will therefore reduce to the reactor's initial degree of subcriticality. This quantity is invariant and repeated calculations can therefore be obtained of it. Hence, statistical methods can be applied to produce a very reliable estimate of the reactivity that was present upon initirting the startup. This will in turn facilitate estimation of the net reactivity and therefore make practical the use of model-based control laws for both the conduct of the startup and the subsequent tracking of the demanded trajectory. Such laws offer superior performance, but require accurate characterization of all reactor parameters including net reactivity [28].

\subsection{Perturbed Reactivity Method}

When utilized as part of an automated startup, the perturbed reactivity method entails perturbing a shutdown reactor by the insertion of reactivity at a known rate and then estimating the initial degree of subcriticality from observation of the resulting reactor response. For the purpose of this report, the time-dependent response of the subcritical reactor will be characterized by the instantaneous inverse period $\omega(t)$ where $d n / d t=$ $\omega(t) n(t)$, and $n(t)$ is proportional to the total neutron population. The insight that led to this method was the realization that comparison of the observed reactor period in a perturbed shutdown reactor with the period calculated to exist in a similarly perturbed critical reactor would provide a means of estimating the degree by which the real reactor was actually shutdown. The mathematical method used to implement the perturbed reactivity method does not explicitly make this comparison. But it does depend on making a perturbation of known magnitude to the subcritical reactor and observing the resulting instantaneous period. 
The essential feature of the perturbed reactivity method is that the net reactivity, $\rho(t)$, present in the reactor is represented separately using superposition as:

$$
\rho(t)=\rho_{u k n}(t)+\rho_{k n}(t)
$$

where $\rho_{u k n}(t)$ is the reactivity rresent in the core excluding the reactivity associated with the known perturuation, and

$\rho_{\mathrm{kn}}(\mathrm{t}) \quad$ is the reactivity associated with the known perturbation.

In a shutdown reactor, the quantity $\rho_{u k n}$ will be the initial degree of subcriticality and it will remain constant during the startup. The quantity $p_{k n}$ would normally be generated by moving a calibrated control device connected to a digital controller. Also, it would normally be computed by means of a balance using data from previously performed calibrations.

The perturbed reactivity method requires a model of the reactor dynamics for its implementation. Thus far, two have been considered. These are the point kinetics equations and the alternate dynamic period equation [19]. Both accurately describe reactors characterized by space independent kinetics. The difference between the two is that the rate of change of reactivity is explicitly represented in the latter. The advantage of the second approach is therefore that it results in a more accurate estimation of the unknown reactivity during transient conditions under which reactivity is inserted at a substantial rate. This occurs because the effect of the perturbation is immediately apparent in the numerical implementation of the model based on the dynamic period equation.

When the perturbed reactivity method is implemented with the point kinetics model, the unknown reactivity is given by:

$$
\rho_{u k n}(t)=l^{*} \omega(t)+\bar{\beta}-\rho_{k n}(t)-\frac{l^{*}}{T(t)} \sum_{i=1}^{N} \lambda_{i} C_{i}(t)-\frac{l^{*}}{T(t)} Q(t)
$$

When implemented with the alternate dynamic period equation as the model, the unknown reactivity is given by:

$$
\begin{aligned}
\rho_{u k n}(t)= & \left\{\omega(t)\left(\bar{\beta}-\rho_{k n}(t)\right)+l^{*}\left[\dot{\omega}(t)+(\omega(t))^{2}+\lambda_{e}^{\prime}(t) \omega(t)\right]-\right. \\
& \dot{\rho}_{u k n}(t)-\dot{\rho}_{k n}(t)-\lambda_{e}^{\prime}(t) \rho_{k n}(t)-\sum \bar{\beta}_{i}\left(\lambda_{i}-\lambda_{e}^{\prime}(t)\right)- \\
& \left.l^{*}\left[\lambda_{e^{\prime}}^{(t) Q(t)}+\dot{Q}(t)\right] / T(t)\right\} /\left[\lambda_{e^{\prime}}^{(t)}+\omega(t)\right]
\end{aligned}
$$


where the quantity $\lambda_{\mathrm{e}}^{\prime}$ is the alternate, multi-group decay parameter which is defined as:

$$
\lambda_{e}^{\prime}(t) \equiv \sum \lambda_{i}^{2} C_{i}(t) / \sum \lambda_{i} C_{i}(t)
$$

and where other symbols are defined as:

$l^{*} \quad$ is the prompt neutron generation time,

$\omega(t)$ is the inverse of the dynamic reactor period,

$\bar{\beta} \quad$ is the effective delayed neutron fraction,

$T(t) \quad$ is the amplitude function and is a weighted integral of all neutrons present in the core,

$\mathrm{N}$ is the number of groups of delayed neutrons, including photoneutrons,

$\lambda_{i} \quad \mathrm{i}$ the decay constant of the ith precursor group,

$\mathrm{C}_{\mathrm{i}}(\mathrm{t}) \quad$ is the concentration of the ith precursor group normalized to the initial power,

$Q(t) \quad$ is the effective source strength,

$\dot{\omega}(t) \quad$ is the rate of change of the inverse of the dynamic reactor period,

$\bar{\beta}_{i} \quad$ is the effective fractional yield of the ith group of delayed neutrons,

$\dot{\rho}_{k n}(t) \quad$ is the rate of change of the known reactivity, and

$\dot{Q}(t) \quad$ is the rate of change of the effective source strength.

The method used for the on-line estimation of precursor concentrations was developed by Myung H. Kim and Professor Allan F. Henry and is described in [47]. It is emphasized that Ec uations (5.2-2) and (5.2-3) are numerically equivalent in that both yield the same quantity. However, Equation (5.2-3), with its explicit representation of rate-dependent effects, was found experimentally to offer superior performance during transients that involved sudden or large changes in reactivity.

\subsection{Implementation Issues}

The computer system utilized for the real-time implementation of the dynarnic perturbation method in conjunction with the automated startup of the 5-MWt MIT Research Reactor (MITR-II) has been previously described [63]. Discussed here are other factors that affect the method's implementation including movement of control devices other than those used to create the perturbation, source term estimation, and techniques for signal smoothing. 


\subsubsection{Control Device. Movement}

Implementaticn of the perturbed reactivity method is as follows. First, a known reactivity change is made to the reactor by moving a designated control element. This change is the required perturbation. The quantities $\rho_{\mathrm{kn}}$ and $\dot{\rho}_{\mathrm{kn}}$ are calculated using data from previous calibrations where, at any instant, $\rho_{\mathrm{kn}}$ is the total reactivity that has been inserted by the control element since the initiation of the transient and $\dot{\rho}_{k n}$ is the rate at which the reactivity is currently being changed. As the perturbation progresses, the reactivity inserted by the designated control element continues to be tracked as $\rho_{\mathrm{kn}}$. Reactivity inserted by other means, such as feedback or movement of other control elements, is summed together with any reactivity that was initially present in the core. These combined effects constitute the term $\rho_{u k n}$. This quantity will therefore be invariant (and hence $\dot{\rho}_{\text {ukn }}$ zero) only if there are no reactivity feedback effects such as those associated with temperature and voids and if no control devices other than those utilized to generate the intended perturbation are moved. The first of these conditions is almost a ceriainty during a reactor startup. However, the second will be true only if the reactor is initially subcritical by an amount such that withdrawal of the control device used to generate the needed perturbation is sufficient to attain criticality. This may or may not be the case. Accordingly, for purposes of implementing the dynamic perturbation method, it is convenient to define conditions of 'static' and 'dynamic' subcriticality. Thus,

1. Static Subcriticality: This condition is said to exist if the reactor is subcritical by less than the worth of the control device that is to be used to create the perturbation or if it is acceptable to reshim the reactor every time that this device attains its out-limit. If the former is true, then the quantity $\dot{\rho}_{u k n}$ is zero. If the latter is true, the quantity $\dot{\rho}_{u k n}$ can still be set to zero. However, the startup must be performed in a stepwise manner. That is, the reactor's other control devices are moved but not during the time intervals that measurements are made to obtain data for the perturbed reactivity method. In particular, movement of the control devices for the startup and that for the perturbation must be done at separate times. This approach allows the perturbed reactivity method to be used in cases where the reactor is deeply subcritical. (Note: A general limitation to such use is that the shape of the neutron flux be constant during the startup. This restriction is inherent in the point kinetics relations and is not specific to the perturbed reactivity method [64].)

2. Dynamic Subcriticality: This condition is said to exist if simultaneous movement of both the control device used to create the perturbation and other control devices will be necessary. The $\dot{\rho}_{\mathrm{ukn}}$ term can therefore not be set to zero. Under these circumstances, Equations (5.2-2) and (5.2-3) are solved by calculating $\dot{\rho}_{u k n}$ using either a backwards difference or the slope calculated by a dynamic least squares fit. The $\dot{\rho}_{\mathrm{ukn}}$ calculated using the backwards difference method lags real time by two time steps whereas the method based on the slope of the dynamic least squares fit is in real time. Both methods give excellent results.

Numerical details of both approaches are given elsewhere [61]. Experimental results that demonstrate the efficacy of the perturbed reactivity method under conditions of both static and dynamic subcriticality are given later in this chapter. 


\subsubsection{Source Term Estimation}

The source term in Equations (5.2-2) and (5.2-3) is expressed as a function of time so as to represent the most general case. The neutron source utilized for reactor startup in the MITR-II is a distributed photoneutron source that originates from $(\gamma, n)$ reactions within the reactor's $\mathrm{D}_{2} \mathrm{O}$ reflector. That is, the fission product inventory in the core emits gamma rays with various half-lives and some of these are sufficiently energetic to cause neutrons to be ejected from deuterium nuclei, thus forming photoneutrons. The relevant fission products decay with an effective half-life on the order of days while an automated startup is completed on the order of minutes. It is therefore valid to neglect the time-varying rature of the photoneutron source in both Equations (5.2-2) and (5.3-3). A relation between the source term, the shutdown power level, and the reactivity can then be found from the point kinetics equations. It is:

$$
\mathrm{Q}_{0}=-\frac{\mathrm{T}_{\mathrm{o}}}{l^{*}} \rho_{\mathrm{o}}
$$

where the subscript (o) denotes the shutdown condition. This relation was used to calculate source strengths for a number of power histories. Once sufficient data was accumulated, empirical correlations were developed that gave the source strength as a function of each particular power history. This approach obviated the need to know the initial degree of subcriticality $\left(\rho_{o}\right)$ in order to estimate the source that was in turn required to implement the perturbed reactivity method.

\subsubsection{Techniques for Signal Smoothing}

Signal smoothing techniques were investigated for the purpose of improving the estimates of both the initial degree of subcriticality and the net reactivity. The two methods that were found to give the best results were a 'moving average' technique and a 'dynamic least squares fit.'

1. Moving Average Method: The concept underlying the moving average method is the performance of an averaging calculation over a fixed number of data points. If a total of $\mathbf{N}$ data points are to be used, then the set selected consists of the data point for the current time step and those for the $(\mathrm{N}-1)$ previous time steps. Thus, the average is constantly 'moving' because the set of data points used in the average calculation moves forward in time as the sample number increases. In addition to the calculated average, this method provides the associated standard deviation. Advantages of the moving average method are that it is simple to implement, that it is ideal for the application of the perturbed reactivity method to 'static' cases in which $\rho_{u k n}$ is constant, and that, once the desired power level is attained, it generates smoother power profiles than competing methods. The primary disadvantage of the moving average method is that it reacts slowly to changes because the full effect of a change in $\rho_{u k n}$ cannot be realized until all previous values of $\rho_{\text {ukn }}$ have 'moved' out of the set of data points used in the calculacion. Another disadvantage is that it responds poorly to unknown reactivity perturbations. 
2. Dynamic Least Squares Fit Method: The dynamic least squares fit method of signal smoothing is based on the least squares fit linear regression technique. The method is 'dynamic' because it is capable of responding to change. A predetermined number of data points are selected in reverse chronological order beginning with the value for the current time step. A slope and a y-intercept for a fitted straight line are computed. A standard deviation based on the difference between indiviaual data points and their respective values predicted by the equation of the fitted straight line is also calculated. This standard deviation is then used to eliminate any data points for which the deviations are unusually large. For example, it might be appropriate to discard those with differences larger than three standard deviations. However, in order to allow for abrupt or sudden changes, the current data point is always retained in the calculation. Advantages of the dynamic least squares fit method are that it responds rapidly to changes in the measured data and that it provides a direct means of estimating $\dot{\rho}_{u k n}$. Relative to this second advantage, $\dot{\rho}_{u k n}$ is the rate of change of the unknown reactivity and the slope given by the least squares fit represents the rate of change of $\rho_{\text {fit }}$. Given that the dynamic least squares fit method provides $\rho_{\text {fit }}$ as an estimate of $\rho_{u k n}$, the slope of $\rho_{\text {fit }}$ is an estimate of $\dot{\rho}_{u k n}$. Disadvantages of the dynamic least squares fit method are that the method may lead to numerical instability in the calculation of $\rho_{\mathrm{ukn}}$ if the $\dot{\rho}_{\mathrm{ukn}}$ term is retained, that it causes time delays which are proportional to the number of data points used in the least squares fit calculation, and that it is a more complex algorithm than the moving average method.

\subsection{Experimental Evaluation of the Perturbed Reactivity Method}

The perturbed reactivity method was initially evaluated by simulation and then through open-loop trials. Once judged to be effective, it was used for the automated startup of the 5-MWt MIT Research Reactor under conditions of closed-loop, digital control. A total of 175 separate runs were performed. First, sensitivity studies were conducted to compare signal smoothing techniques, to evaluate numerical methods, and to assess the efficacy of the reactor models used to implement the perturbed reactivity method. Automated startup experiments were then performed in which reactor power was increased from source level to $50 \mathrm{~kW}$ on a 50 -s period. Experiments were initiated with the reactor exactly critical and then repeated with the reactor subcritical by a greater amount for each successive run. Automated startups were also done with the reactor initially in a fully shutdown $(-8350 \mathrm{~m} \beta)$ state. The technique was shown to be both effective and reliable. Selected results are shown here.

Figures 5.4-1 and 5.4-2 are from one of the experiments that was performed to evaluate the perturbed reactivity method for automated startups under conditions of static subcriticality. Shown in Figure 5.4-1 are the power and reactivity profiles obtained during an automated startup in which the reactor was initially subcritical by 1000 mbeta. The reactivity plotted is that inserted by the control device rather than the net reactivity. The power was leveled at the demanded value of $50 \mathrm{~kW}$ and the demanded trajectory, a $50-\mathrm{s}$ period, was attained. Figure 5.4-2 shows the same power profile together with the estimate of the initial degree of subcriticality. As is evident from this figure, the perturbed reactivity method correctly determined that quantity throughout the transient. The control law utilized for these runs was the alternate MIT-SNL Period-Generated Minimum Time 

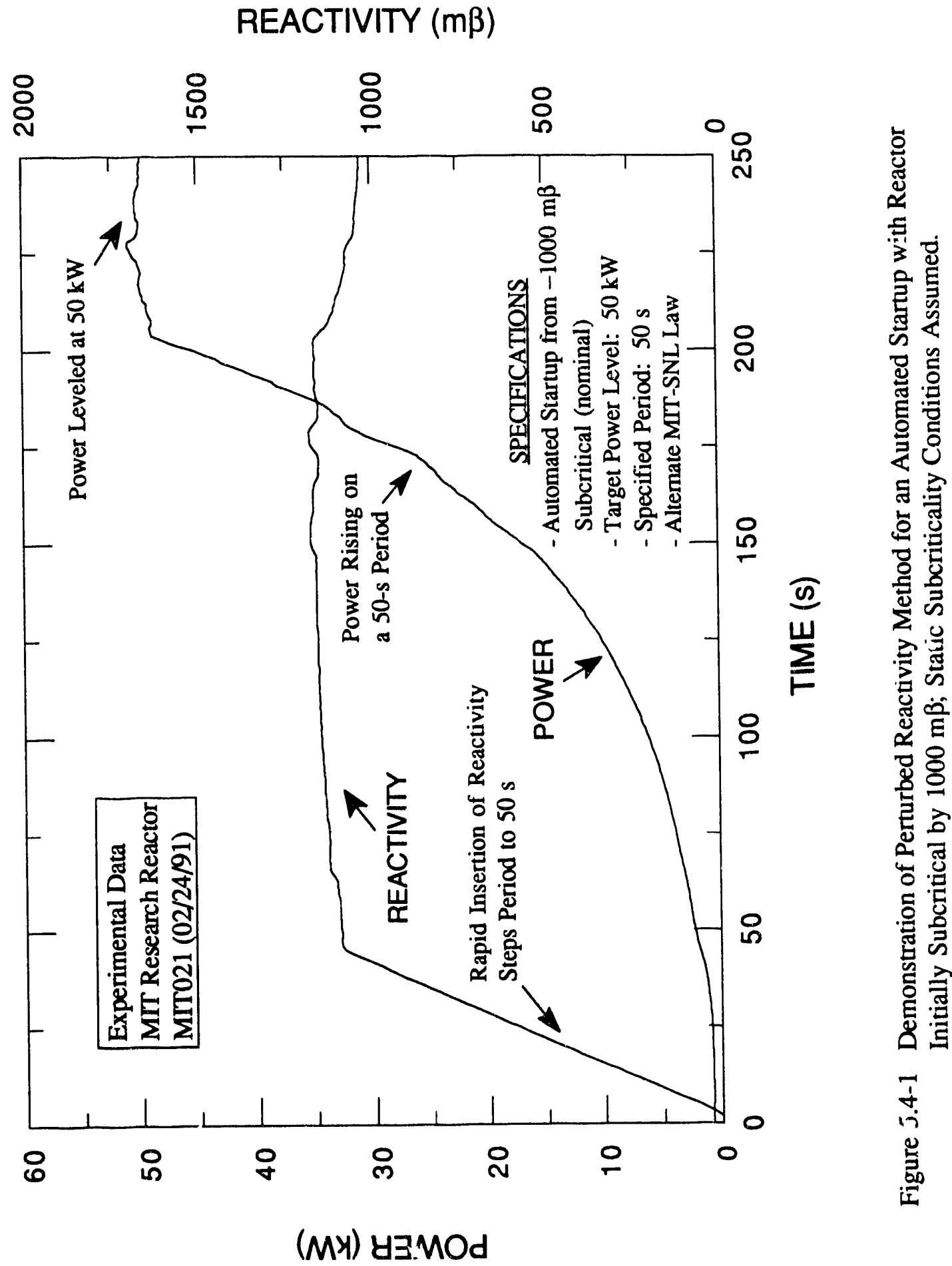
INITIAL REACTIVITY $(\mathrm{m} \beta)$

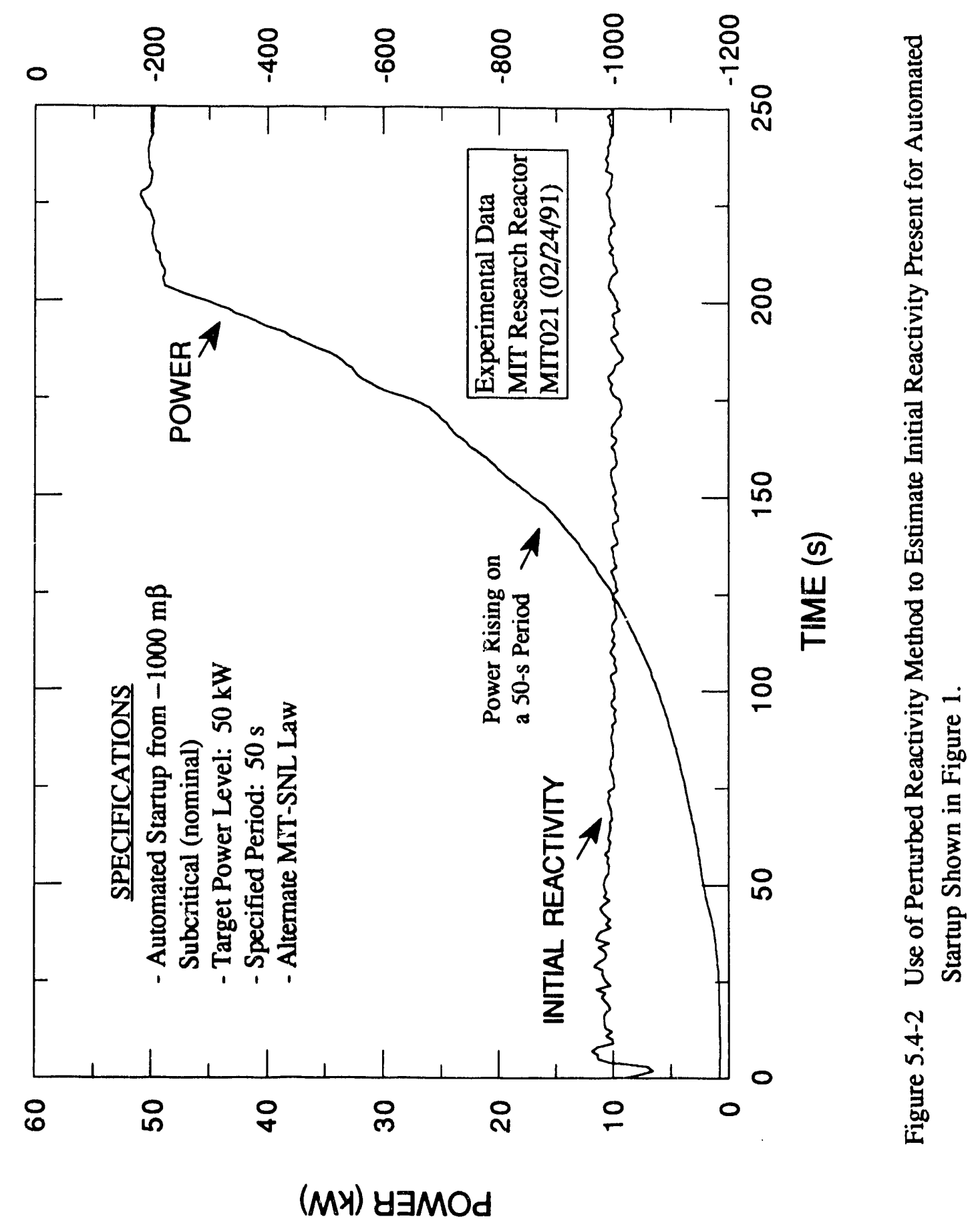


Control Law. This law, which is a form of period-generated control, is excellent for trajectory tracking. The rapid insertion and removal of reactivity upon both transient initiation and termination is a characteristic feature.

Figures 5.4-3 to 5.4-6 show the power and reactivity profiles obtained during an automated startup from deep subcriticality. The version of the perturbed reactivity method utilized was that associated with the direct use of the point kinetics relations for the model. For this particular experiment, the MITR-II had been operated at full power, $4.9 \mathrm{MW}$, for 277 hours and then left in a fully shutdown condition for 1 hour. The reactor is equipped with six shim blades, each of which is worth about 2 Beta. One of these may be connected to the digital controller. The following protocol was observed. Upon satisfaction of all administrative and license requirements [28], the digital controller was activated and directed to raise the reactor power from the shutdown level, which was a few watts, to 50 $\mathrm{kW}$ on a 50-s period. It was, of course, impossible for the controller to achieve this because the automated blade was only worth $2020 \mathrm{~m} \beta$ and the reactor was shutdown by several times that amount. Accordingly, the controller fully withdrew the automated blade. At this time, the digital control action was halted and the licensed console operator directed to reshim the reactor in such a manner that there was no net change in the criticality condition. The result of the reshim process was that the automated control blade was again fully inserted and the remaining five blades were uniformly withdrawn by a distance corresponding to the reactivity worth of the automated blade. The digital controller was again activated and the above process repeated until the degree of subcriticality became less than the worth of the automated control blade. The digital controller then took the reactor critical and achieved the demanded power level. The four figures should therefore be interpreted as a single series with Figure 5.4-3 corresponding to automated control action prior to the first reshim, Figure 5.4-4 corresponding to automated control action prior to the second reshim, and so on. As determined by the perturbed reactivity method, the total reactivity inserted was $8350 \mathrm{~m} \beta$. Of this, $7850 \mathrm{~m} \beta$ was inserted by the automated control blade and $500 \mathrm{~m} \beta$ manually. The latter action was taken to avoid a fifth reshim. The 8350 $\mathrm{m} \beta$ figure was verified upon completion of the automated startup by performing a reactivity balance calculation. This calculation gave a figure of $8340 \mathrm{~m} \beta$ which, within the limits of experimental accuracy, was in excellent agreement with that provided by the perturbed reactivity method.

Figures 5.4-3 to 5.4-6 show the degree of subcriticality that was estimated by application of the perturbed reactivity method to the above startup. If the method was accurate, then the value shown in Figure 5.4-3 which was for the initial phase of the startup, would be $8340 \mathrm{~m} \beta$. For Figure 5.4-4, which shows the calculated data obtained after the first reshim, the correct value for the initial reactivity was $(8340-2020)$ or 6320 $\mathrm{m} \beta$. For the next figure, the correct value was $4300 \mathrm{~m} \beta$. At the beginning of the final figure, the correct value for the initial reactivity was 2280 and at the end of that figure (i.e., after the manual addition of $500 \mathrm{~m} \beta$ ) it was $1780 \mathrm{~m} \beta$.

Examination of the above figures shows that the calculated value of the initial reactivity was accurate at the outset of the startup. This was to be expected given that the source strength had been accurately determined. The accuracy of the calculation subsequently deteriorated somewhat and then recovered. In general, the calculation was most accurate during the latter stages of each segment of the startup. 
INITIAL REACTIVITY $(\mathrm{m} \beta)$

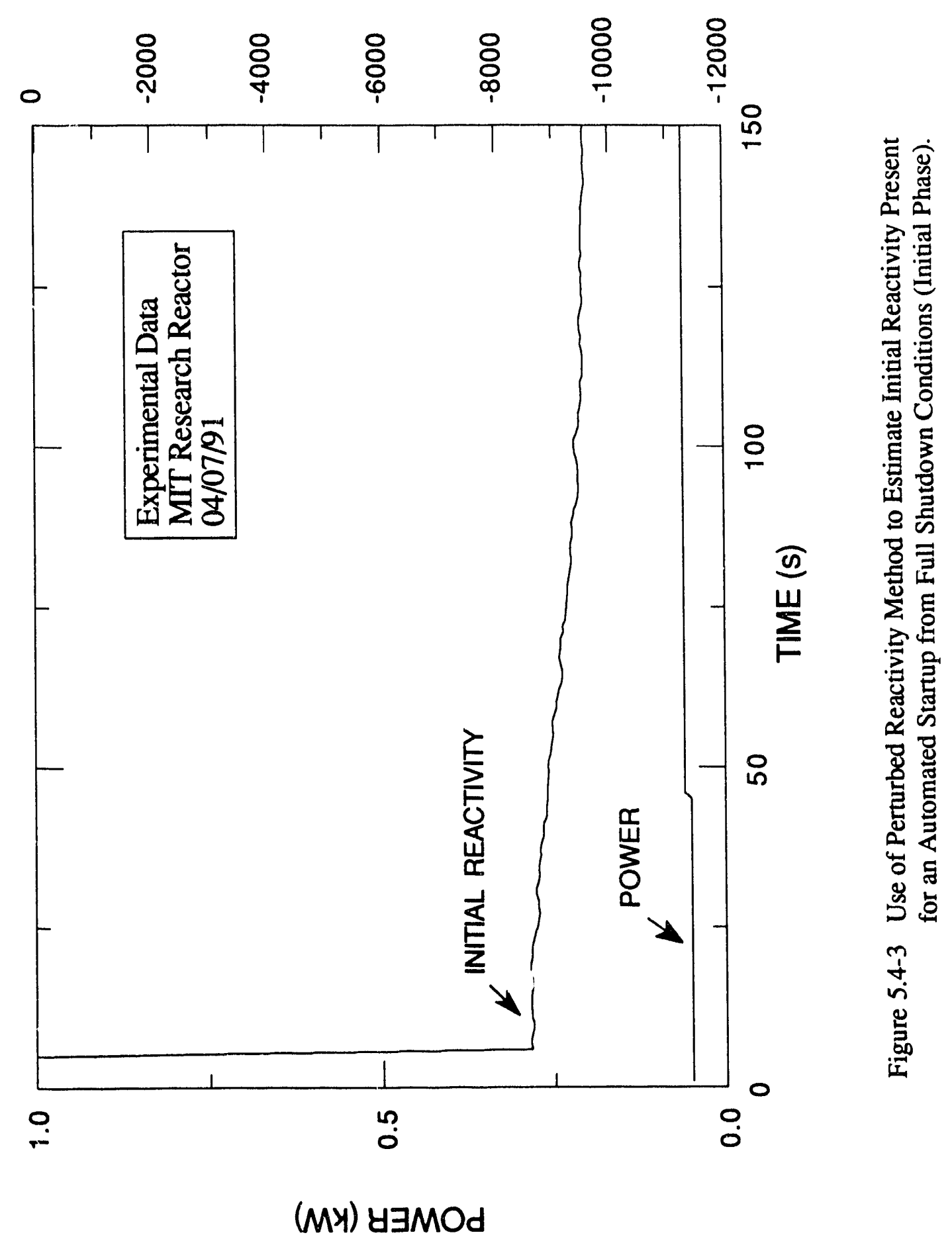


INITIAL REACTIVITY (m $\beta)$

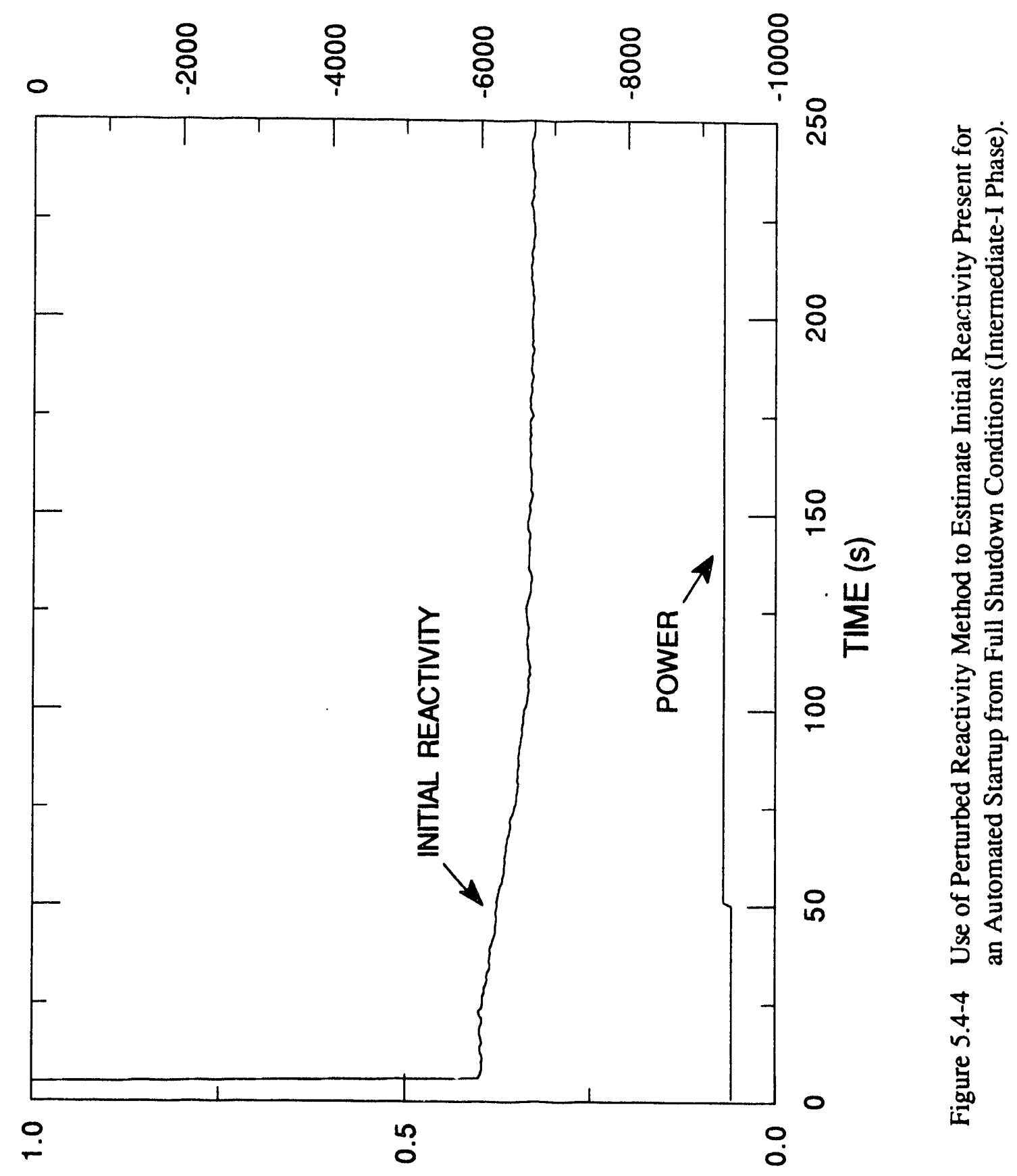

(MY) y 


\section{INITIAL REACTIVITY (m $\beta)$}

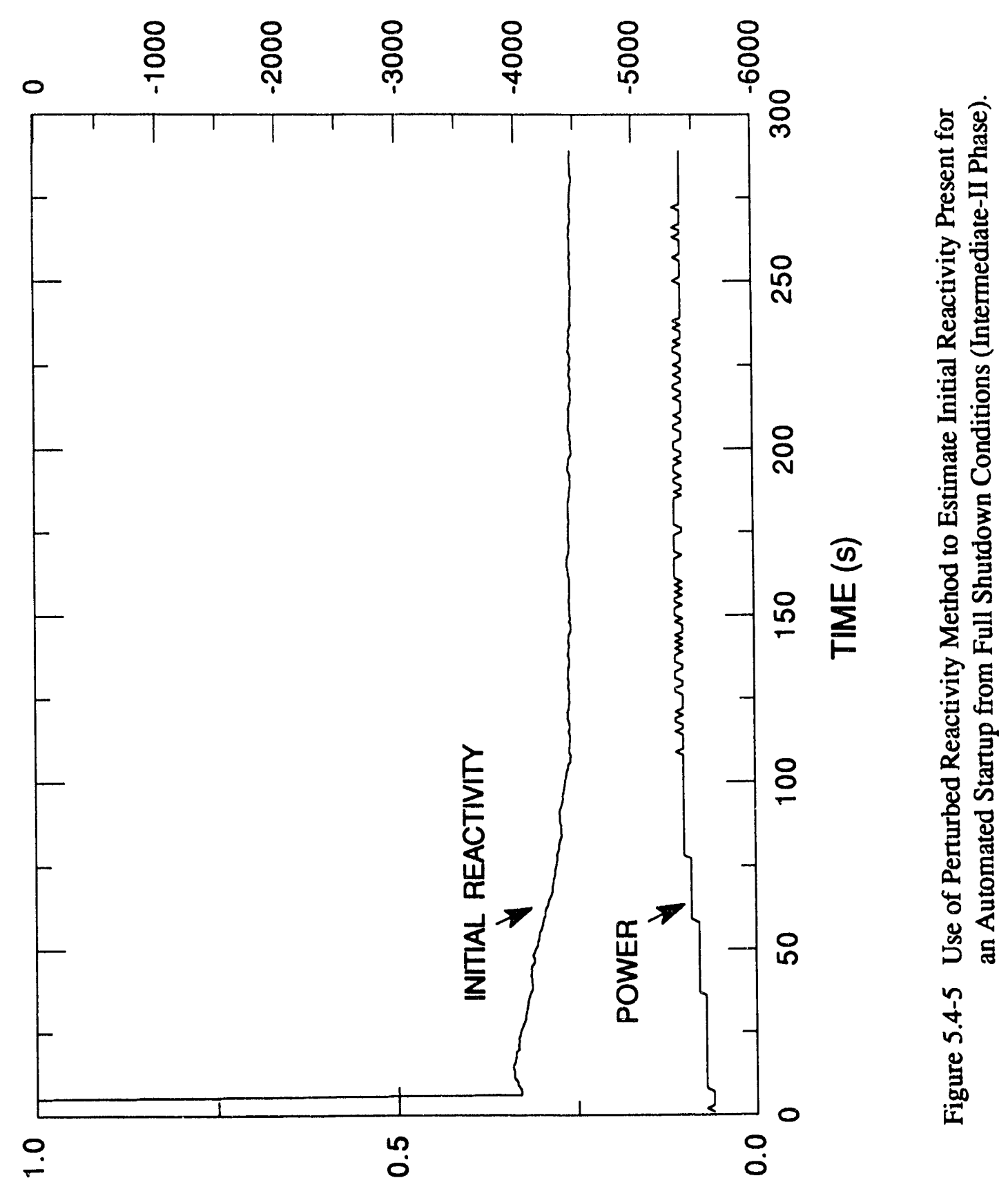

(MY) $8 \exists M O d$ 
INITIAL REACTIVITY (mß)
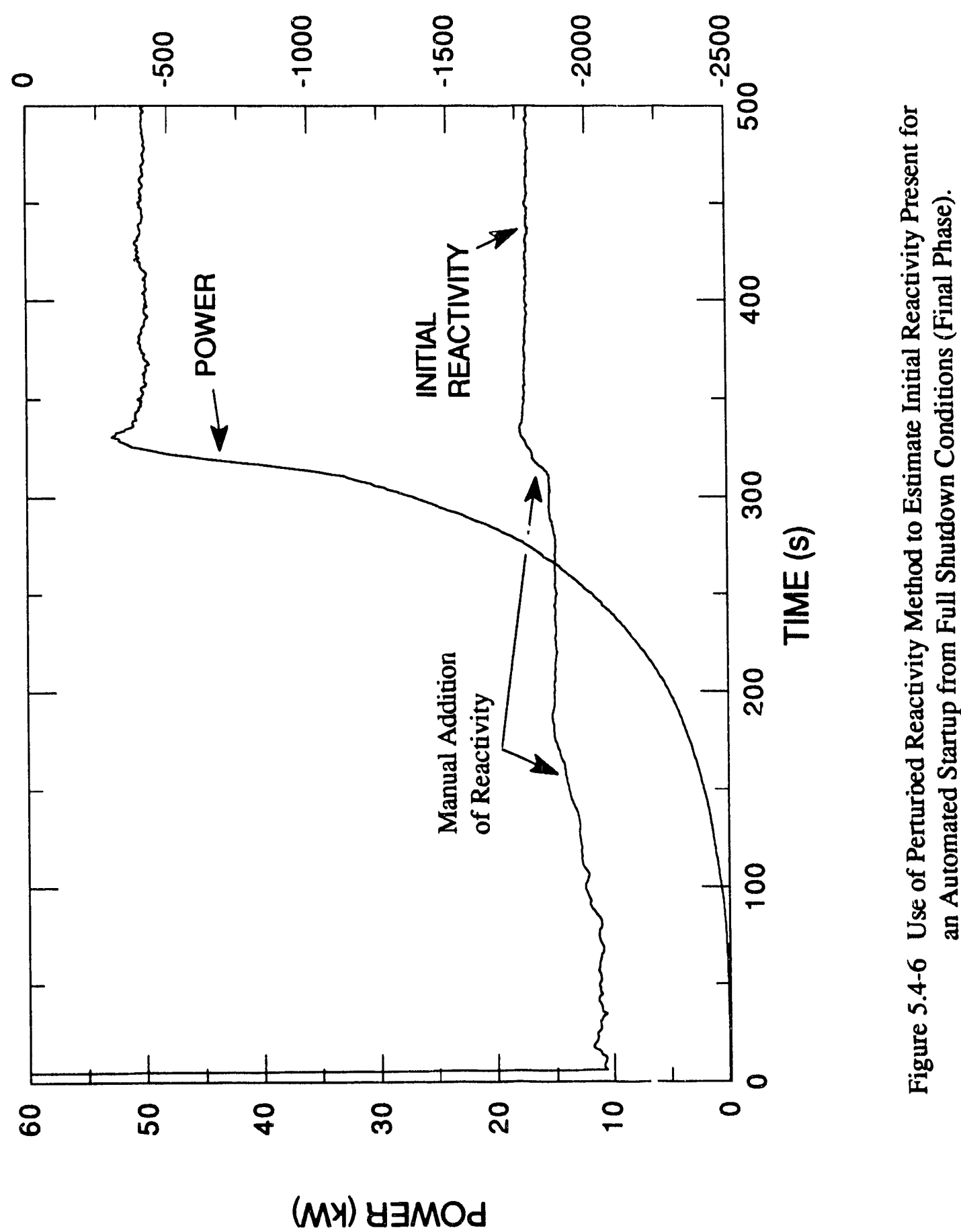
Figures 5.4-7 and 5.4-8 are from a test series that was conducted to demonstrate the efficacy of the perturbed reactivity method when implemented under conditions of dynamic subcriticality. That is, the quantity $\dot{\rho}_{u k n}$ was retained and solved for using the aforementioned least squares fit approach. Shown are the power, net reactivity, and initial degree of subcriticality obtained for an automated startup that was performed with the reactor nominally subcritical by $1000 \mathrm{~m} \beta$. The digital controller was directed to raise the reactor power to $50 \mathrm{~kW}$ on a 50 -s period and the perturbed reactivity method was used to estimate the initial degree of subcriticality. As is evident, the power maneuver was completed satisfactorily.

\subsection{Assessment of Perturbed Reactivity Method}

A technique for the estimation of a reactor's initial degree of subcriticality has been derived and experimentally demonstrated. This concept, which has been designated as the perturbed reactivity method, functions by separating the net reactivity that is present in the reactor into that associated with a planned perturbation and that associated with all other effects including feedback, movement of control devices not used to create the perturbation, and the initial degree of subcriticality. The first of these two reactivities is referred to as 'known' while the other is termed 'unknown.' Knowledge of the magnitude and rate of insertion of reactivity associated with the known perturbation and observation of the resulting reactor period allows calculation of the unknown reactivity. If this is done for a reactor startup, the unknown reactivity will be the initial degree of subcriticality which is a constant. Repeated calculations can therefore be made of this one quantity and the accuracy of those calculations improved through use of signal smoothing techniques, such as the moving average and the dynamic least squares fit methods. The efficacy of the perturbed reactivity method was demonstrated through hoth simulation studies and on-line experimental trials conducted under conditions of clcsed-loop, digital control on the 5-MWt MIT Research Reactor.

The significance of the perturbed reactivity method is that it allows a reactor's initial degree of subcriticality to be determined on-line during an automated startup. This information can then be supplied to a model-based control law which in turn permits power ascension to be achieved on a demanded trajectory. Potential applications of the concept include the automated startup of both spacecraft and milti-modular terrestrial reactors. 
REACTIVITY ( $m \beta)$
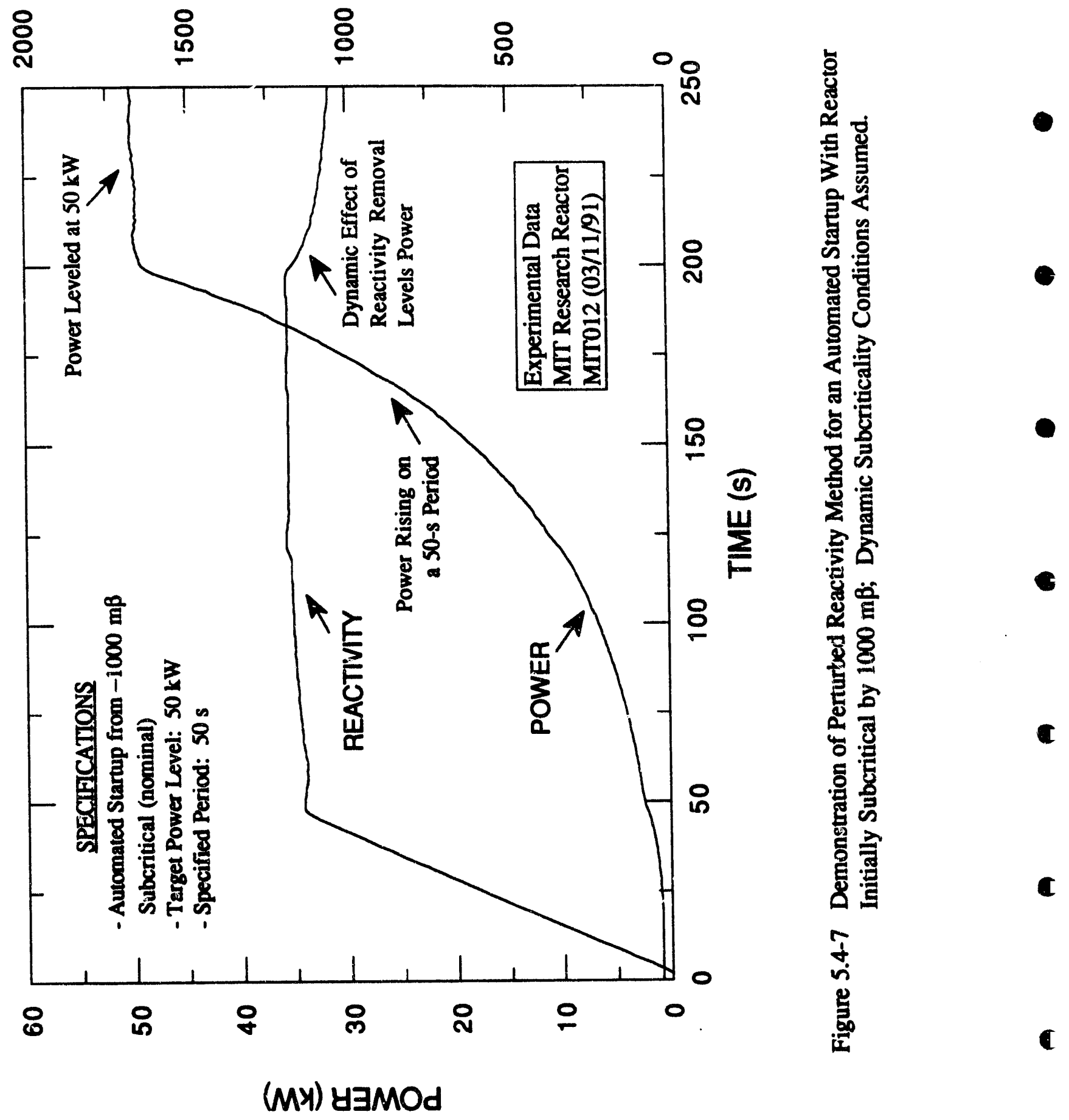
INITIAL REACTIVITY (m $\beta)$

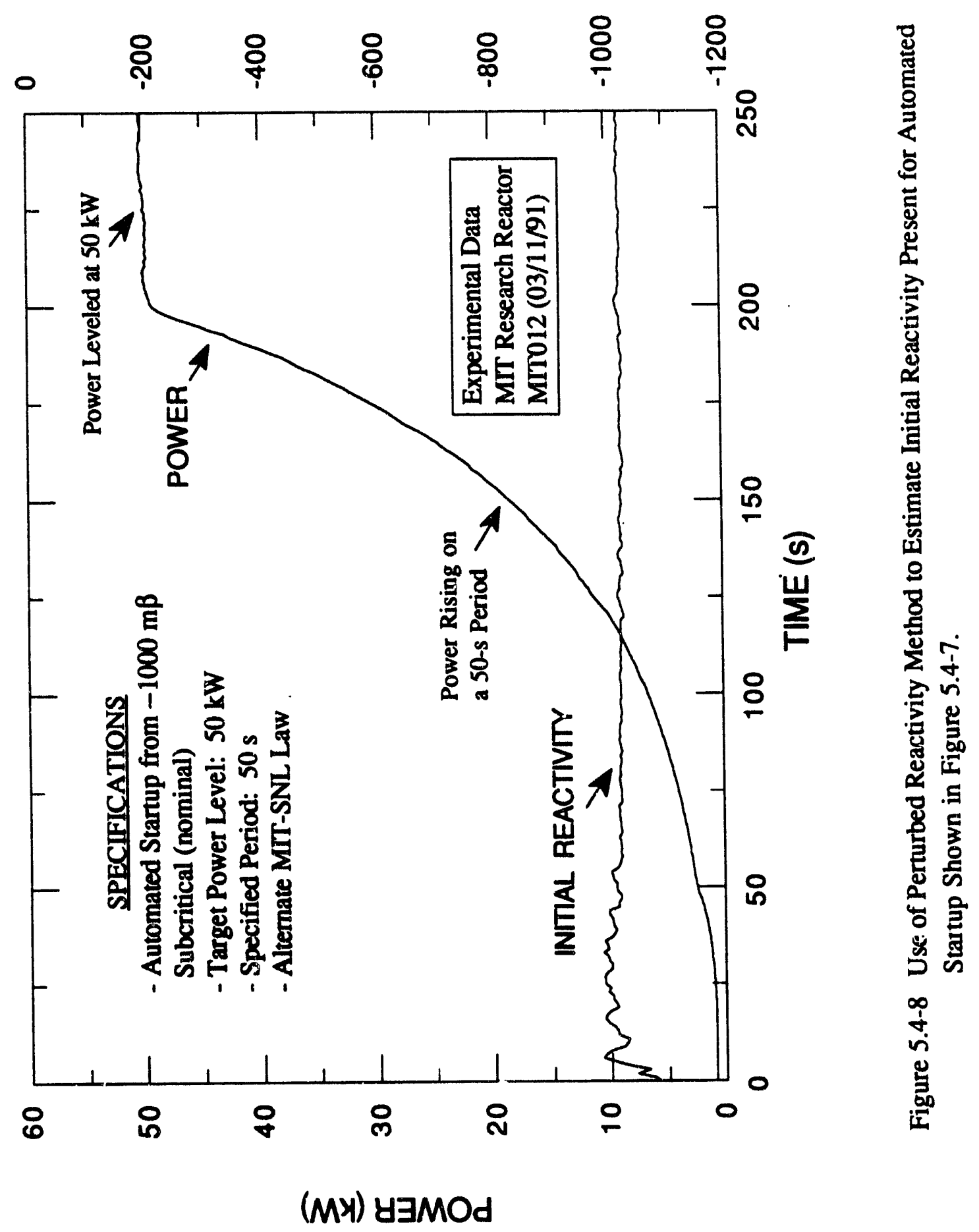




\section{Experimental Comparison of Trajectory Tracking Techniques ${ }^{(1)}$}

This chapter presents the results of an experimental comparison of feedforward control techniques for the trajectory tracking of reactor neutronic power. This research was undertaken as part of a systematic effort to assess the potential for applying robotic control concepts to the operation of multi-modular reactors. For example, the resulting control law could be used to adjust power in a slow but deliberate manner so as to limit thermal stress during plant heatups and cooldowns. Included in the comparison were pure feedforward control in which the actuator signal is found solely by processing a demanded output through a system model, hybrid feedforward/feedback control in which the actuator signal is obtained by summing feedforward and feedback components, and periodgenerated control in which feedback is used to update the demanded trajectory prior to its being processed through the system model for calculation of the actuator signal. This latter approach was found to be the most effective. In addition to the experimental results, discussions are given of both the rationale for model-based, feedforward control and the designs of the various controllers.

\subsection{Siatement of Problem}

One of the major accomplishments of the MIT program on the development and experimental evaluation of advanced control concepts for nuclear reactors has been the MIT-SNL Period-Generated Minimum Time Control Laws, which combine a rigorous, non-linear model of a reactor's dynamics with proportional-integral feedback to achieve the desired system response [2,3]. While period-generated control had been shown experimentally to function exceptionally well, it was recognized that it was only one of many techniques that could be used for the tracking of a demanded trajectory. Many of the other options are being explored as part of on-going research in robotics [65]. Hence the intent here to identify the relative merits of those concepts through experimental comparison.

\subsection{Rationale for Feedforward Control}

The most commonly employed method of control is error-driven feedback. Figure 6.2-1 illustrates this approach. An error signal, $e(t)$, is obtained by comparing the output of the process, $n(t)$, to a reference, $\bar{n}(t)$. This signal is then sent to the controller which multiplies it by a grin coefficient to obtain the control signal, $u(t)$. The resulting control action can be improved through the addition of terms that reflect both the integral and the derivative of the error signal. The resulting controller is of the form:

$$
u(t)=k_{p} e(t)+k_{i} \int e(t) d t+k_{d} \dot{e}(t)
$$

where $k_{p}, k_{i}$, and $k_{d}$ are the gain constants for the proportional, integral, and derivative terms. This mode of mntrol is termed proportional-integral-derivative (P-I-D). Its most salient feature is that no use is made of n system model. In fact, Equation (6.2-1) is so general that it could be applied equally well to control reactor power, the location of a robot, the level in a tank, or virually any process requiring positional control.

(1) This chapter is based on work performed by Shing Hei Lau under the direction of Dr. John A. Bernard and Professor David D. Lanning. 


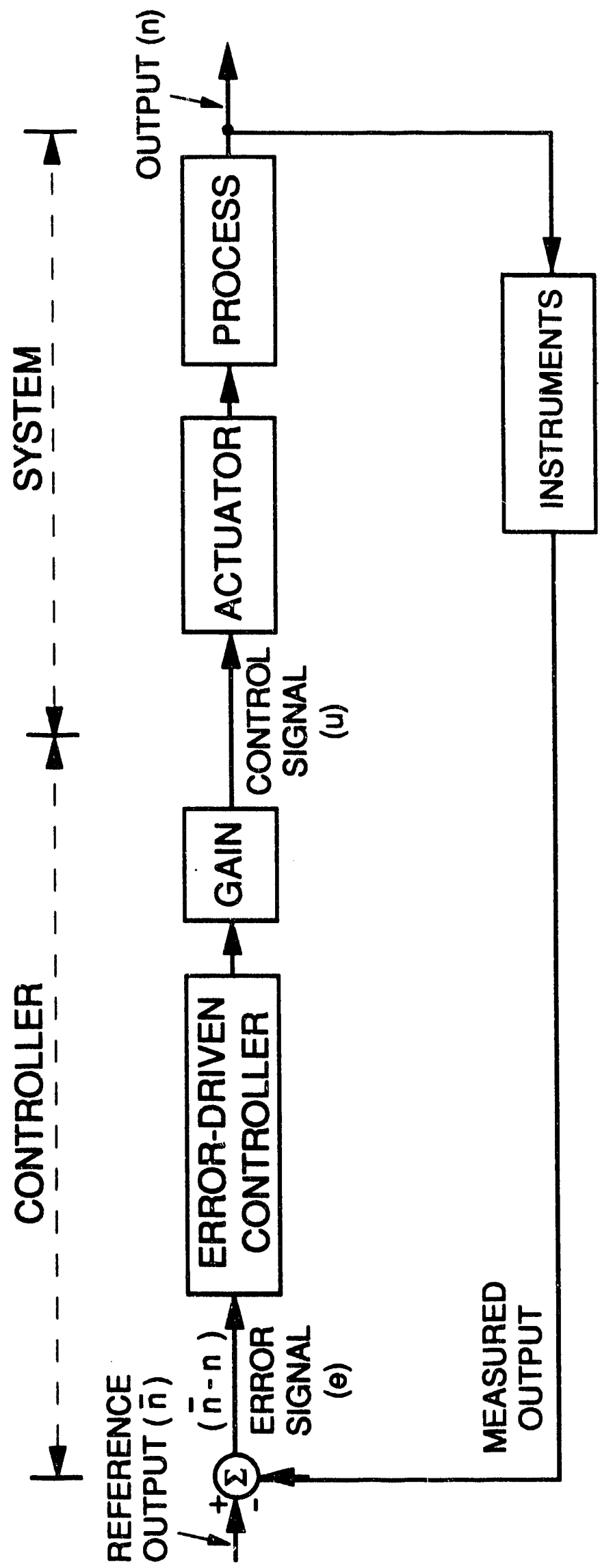

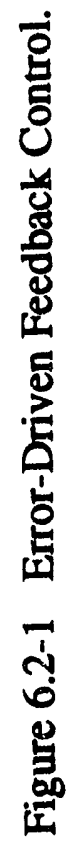


Pure feedback control, as represented by Equation (6.2-1), often exhibits certain deficiencies. First, an error must exist between the ubserved and demanded system outputs in order for a corrective control signal to be generated. Thus, the perfect match of an actual to a demanded trajectory is not possible. Second, because no use is made of a system moxiel, a feedback controller has no means of recognizing the correct actuator signal until the error signal is made zero. This can result in an oscillatory response. Third, high gains are often desired so that perturbations can be rapidly overcome. However, there are physical limits to the speed at which an actuator can respond and, even if achievable, the use of high gains to offset poor performance may be unsafe because high gains will amplify inaccuracies in parameter estimates and noise. Also, their use may lead to instability. Fourth, controller gains can not be treated as constants for non-linear systems. A set of gains chosen to properly execute one type of transient may result in poor performance for another. Fifth, the selection of the gains is empirical and hence timeconsuming.

Feedforward control, which incorporates a rigorous system model in the controller, offers the possibility of eliminating some of the deficiencies associated with the pure errordriven approach. For example, the presence of the model allows calculation of the proper actuator signal for a given system load. Thus corrective action can be initiated as soon as a load change occurs. For many processes, the cost of developing and calibrating a model is not justified. However, the use of a model is a necessity if proper control is to be accomplished of systems, such as nuclear reactors, that are time-delayed and non-linear.

\subsection{Feedforwand Control}

The characteristic feature of feedforward control is that a model of the system's dynamics is used to determine the actuator signal needed to achieve a given demand. This process is often referred to as an 'inverse' calculation because the system response, which is the parameter being controlled, is the input to the model and the actuator signal is the output. Feedforward control is an effective strategy for reducing the influence of planned or measurable disturbances on the controlled variable of a process system and, in theory, can result in perfect trajectory tracking. Figure 6.3-1 illustrates the concept. Note tha:, while the controlled variable is shown as being measured, it is not fed back. This is emphasized by referring to the mode of control shown in the figure as 'pure' feedforward. A major advantage of feedforward control is that corrective action can be initiated at the outset (or even in advance) of a known disturbance. There is no need to wait until an error signal attains appreciable magnitude. Another advantage is that the presence of a model in the controller makes it possible to determine the correct actuator signal for a given load. The searching and oscillatory behavior that often accompanies the use of pure feedback control is avoided. Also, the model provides a rational basis for selecting system gains and system stability can be analyzed. The disadvantages of pure feedforward control are, of course, that the system's dynamics must be modeled with great accuracy and that all disturbances must be known in advance. Such requirements are unrealizable. Hence, it is common practice to combine feedforward and feedback action. Controllers of this type are referred to here as 'hybrid' feedforward/feedback. Figure 6.3-2 illustrates the general concept. The basic premise to this method of control is that the feedforward calculation compensates reasonably well for the system dynamics and that feedback action is therefore only needed to correct for small modeling errors and perturbations of unknown origin. 


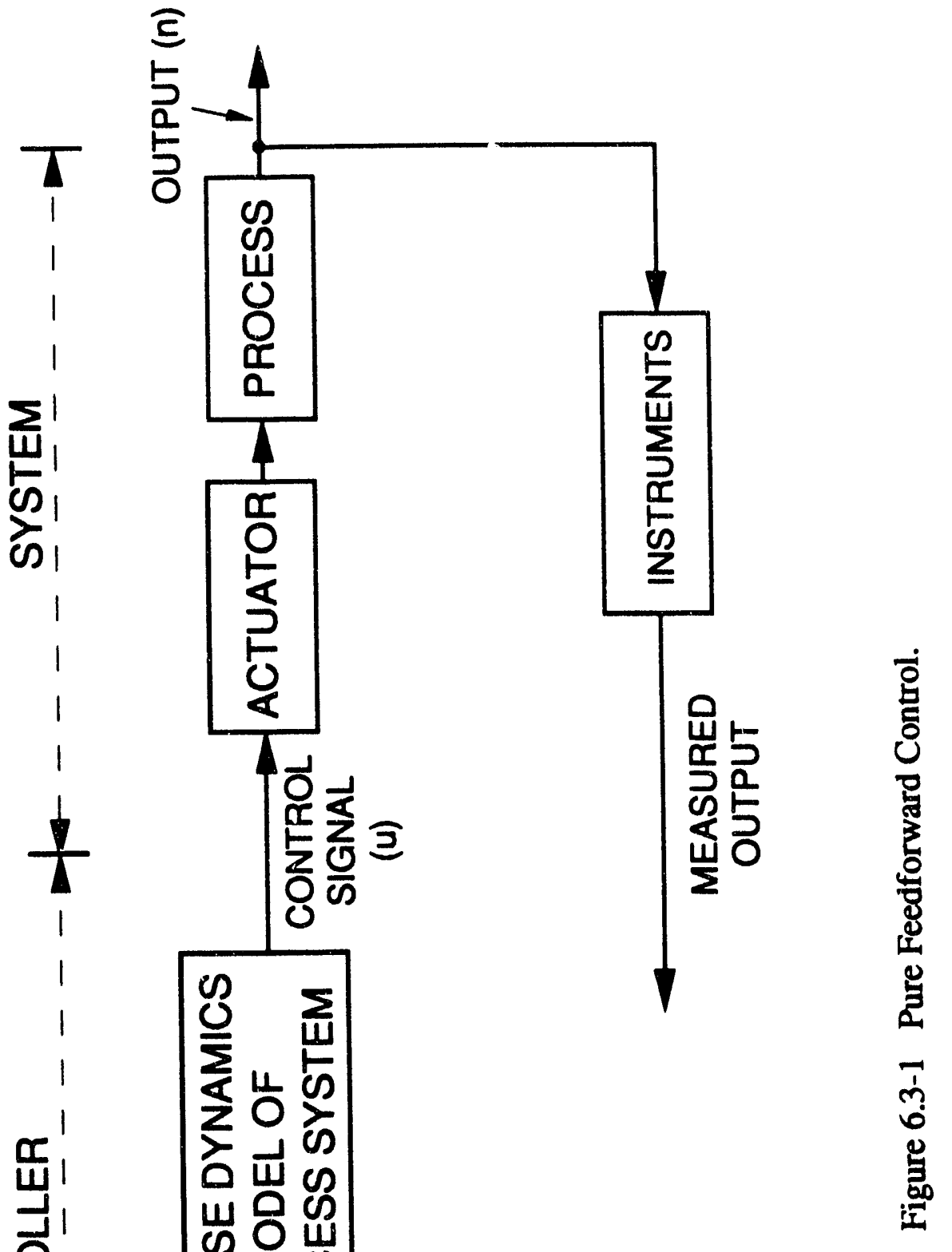




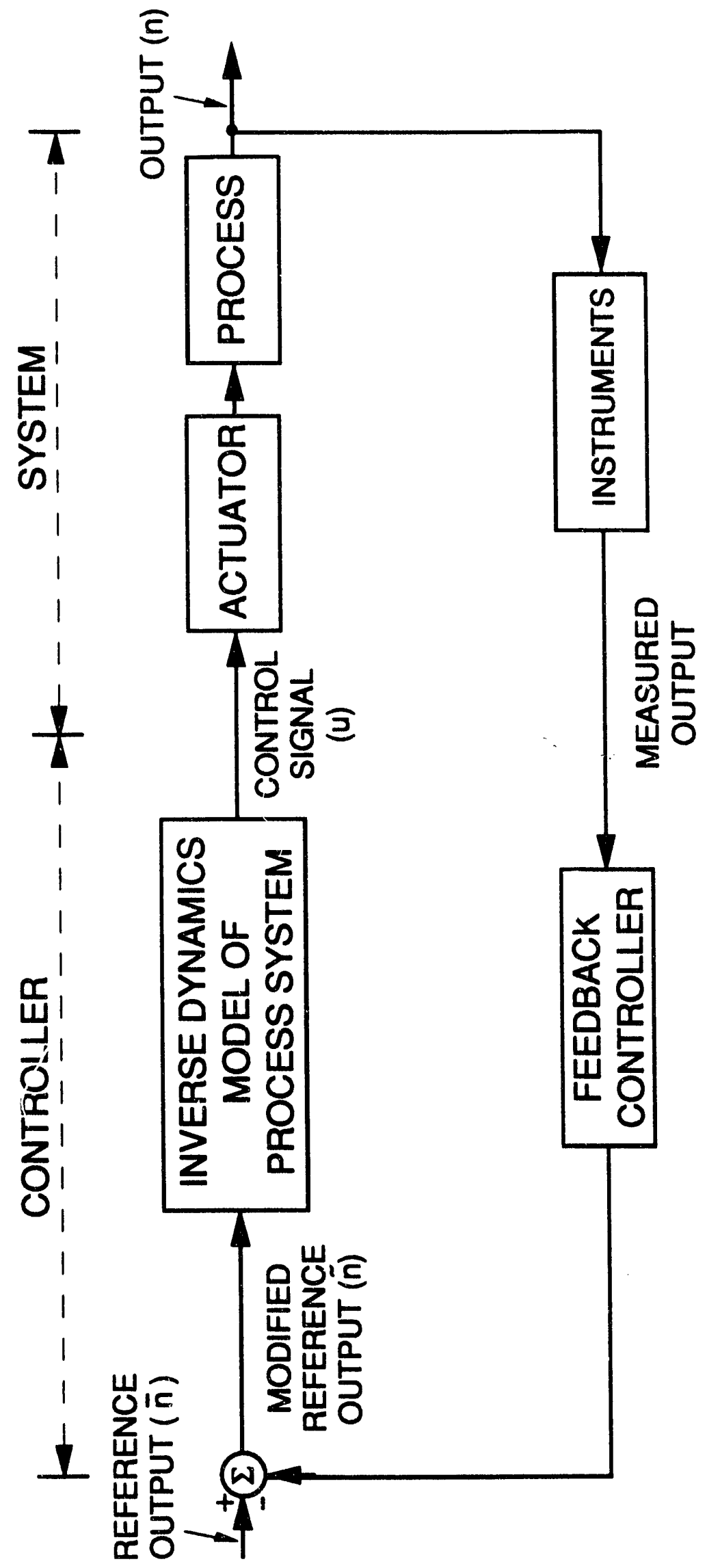


The architectures shown in Figures 6.3-1 and 6.3-2 were both used to design feedforward controllers for implementation on the 5-MWt MIT Research Reactor (MITRII). This reactor's core is compact and can therefore be described by space independent kinetics. Accordingly, the model chosen for incorporation in the feedforward controller was the alternate dynamic period equation. The parameter chosen for the actuator signal was the rate of change of reactivity. This choice was advantageous because it meant that both the speed and direction of the reactor's control device were determined by the controller.

Figure 6.3-3 shows the structure of MITR-II's neutronic power pure feedforward controller. Its purpose is to cause the observed reactor power, $n(t)$, to track a particular trajectory, $n_{d}(t)$. This is done by translating the desired trajectory into a demanded inverse period, $\omega_{d}(t)$, and then processing that demand through a system model to obtain the appropriate actuator signal. Application of this signal to the reactor's control devices should cause reactivity to be adjusted in such a way that the observed power does in fact track that demanded. Note that no use is made of feedback in this controller. The observed reactor power and inverse period are measured, but these are used only to update the model. No corrective control action is generated. The governing equation for this controller is:

$$
\dot{\rho}_{c}=\hat{R}^{-1}\left(n_{d}, \omega_{d}, n, \omega\right)
$$

where all quantities are time-dependent and symbols are defined as:

$\dot{\rho}_{c} \quad$ is the rate of change of reactivity to be supplied by the actuator,

$\hat{\mathbf{R}}^{-1}$ is the reactor model,

$n_{d}$ is the demanded power,

$\omega_{d}$ is the demanded inverse period,

n is the observed power level, and

$\omega \quad$ is the observed inverse reactor period.

The reactor model is denoted as an inverse because it is being used to solve for the actuator signal. Upon substitution of the alternate dynamic period equation for the model, Equation (6.3-1) becomes:

$$
\begin{aligned}
\dot{\rho}_{c}(t)= & (\bar{\beta}-\hat{\rho}(t)) \omega_{d}(t)-\hat{\lambda}_{e}^{\prime}(t) \hat{\rho}(t)-\sum \bar{\beta}_{i}\left(\lambda_{i}-\hat{\lambda}_{e}^{\prime}(t)\right)- \\
& \hat{\rho}_{f}(t)+l^{*} \dot{\omega}(t)+l^{*}\left[(\omega(t))^{2}+\hat{\lambda}_{e}^{\prime}(t) \omega(t)\right]
\end{aligned}
$$

where the superscript $(\sim)$ denotes a modeled parameter and symbols not previously defined are:

$\bar{\beta} \quad$ is the effective delayed neutron fraction,
$\lambda_{e}^{\prime} \quad$ is the effective, multi-group decay parameter, 

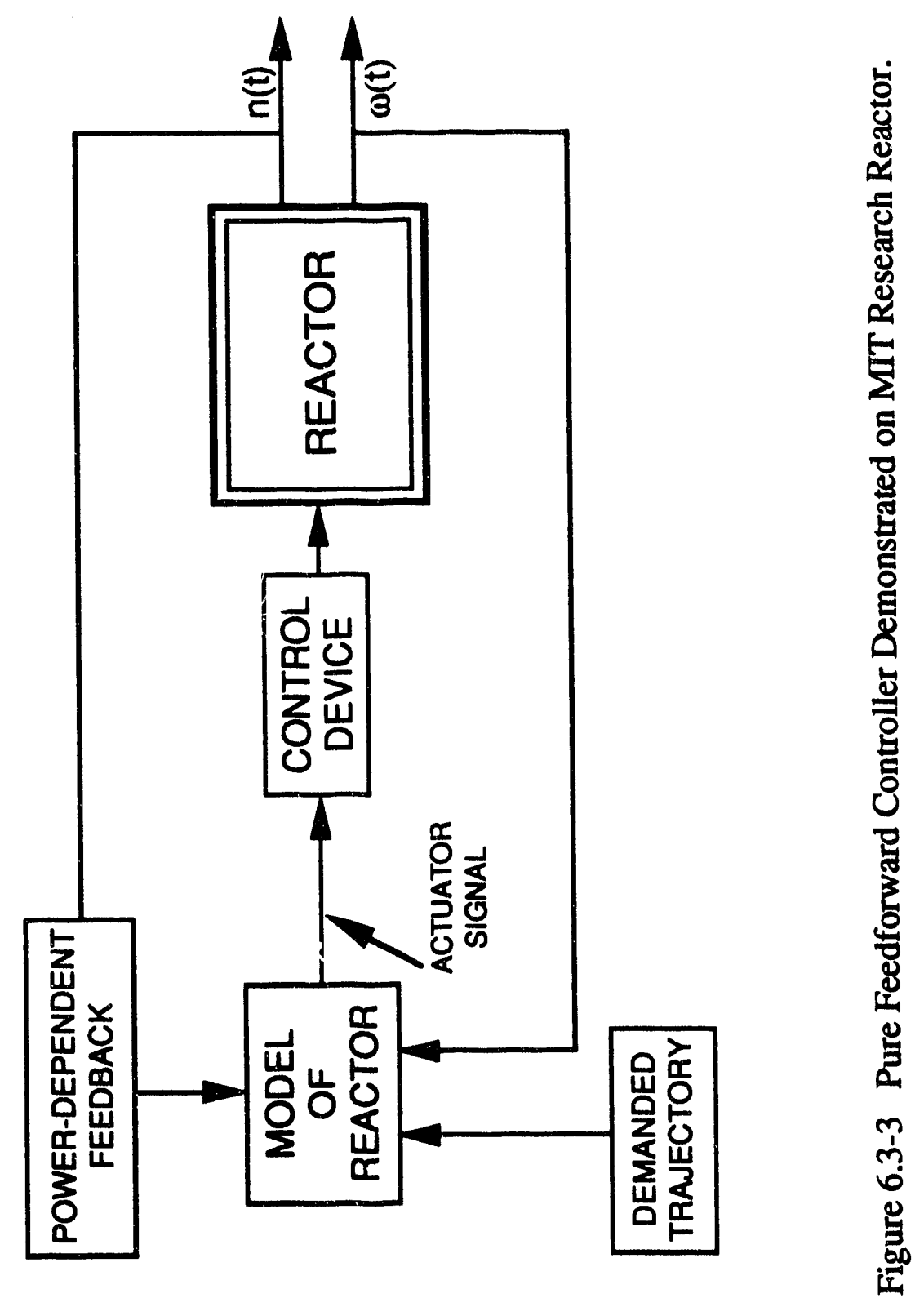


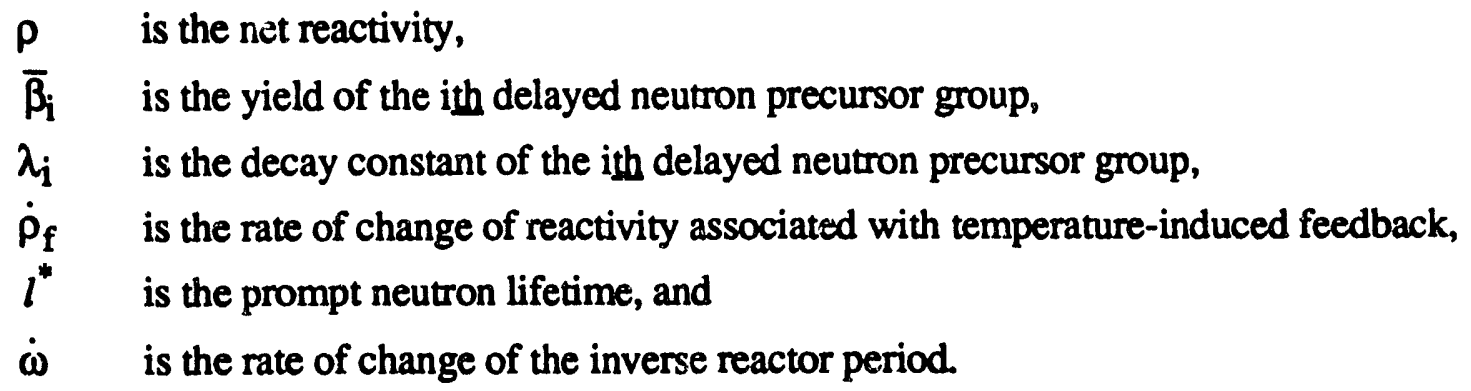

The controller architecture shown in Figure 6.3-2 was also used to design a control system for the MIT Research Reactor. However, this process was somewhat ambiguous because the manner in which the feedforward and feedback actions are to be combined is not specified. A typical approach, and one that has been explored for robotics [65], is simply to add the output of the feedforward and feedback components. This concept, as applied to the MITR-II, is shown in Figure 6.3-4. The governing equation is:

$$
\dot{\rho}_{c}=\hat{R}^{-1}\left(n_{d}, \omega_{d}, n, \omega,\right)+k_{p}\left(n_{d}-n\right)+k_{d}\left(\omega_{d}-\omega\right)
$$

where all symbols are as previously defined. Equation (6.3-3) is referred to here as a 'hybrid feedforward/PD-feedback controller.'

Ther 13 no theoretical reason for combining the feedforward and feedback components through simple addition and, as observed during the experimental evaluations, this approach is not particulariy effective. A better idea is, as advocated by Shinskey [66], to use the feetback controller to update the feedforward action. This is the approach embodied by the MIT-SNL period-generated minimum time control laws, the structure of which is shown in Figure 6.3-5. The basic idea is as follows. A comparison of the observed and desired system outputs is used to update the demanded trajectory at every sampling interval. This modified trajectory is then processed through an inverse model of the system dynamics to obtain the appropriate actuator signal. This mode of control, which originated at MIT, has been designated as 'period-generated' because it was first formulated in terms of a demanded reactor period. More recently, it has been extended to systems that are to be controlled according to a demanded velocity [67]. The governing equations are:

$$
\begin{aligned}
e(t)=\ln \left[n_{d}(t+j \Delta t) / n(t)\right] \\
\omega_{d}(t)=\left[e(t)+\left(1 / T_{i}\right) \int e(t) d t+T_{d} \dot{e}(t)\right] / j \Delta t \\
\dot{\rho}_{c}(t)=(\bar{\beta}-\hat{\rho}(t)) \omega_{d}(t)-\hat{\lambda}_{e}^{\prime}(t) \hat{\rho}(t)-\sum \bar{\beta}_{i}\left(\lambda_{i}-\hat{\lambda}_{e}^{\prime}(t)\right)- \\
\\
\hat{\rho}_{f}(t)+l^{*} \dot{\omega}(t)+l^{*}\left[(\omega(t))^{2}+\hat{\lambda}_{e}^{\prime}(t) \omega(t)\right]
\end{aligned}
$$

where all symbols are as previously defined except that $\mathrm{j}$ is the number of sample intervals by which the calculation of the demanded power level should be advanced in order to satisfy stability criteria [41]. Also, $T_{i}$ and $T_{d}$ are the integral and derivative times 

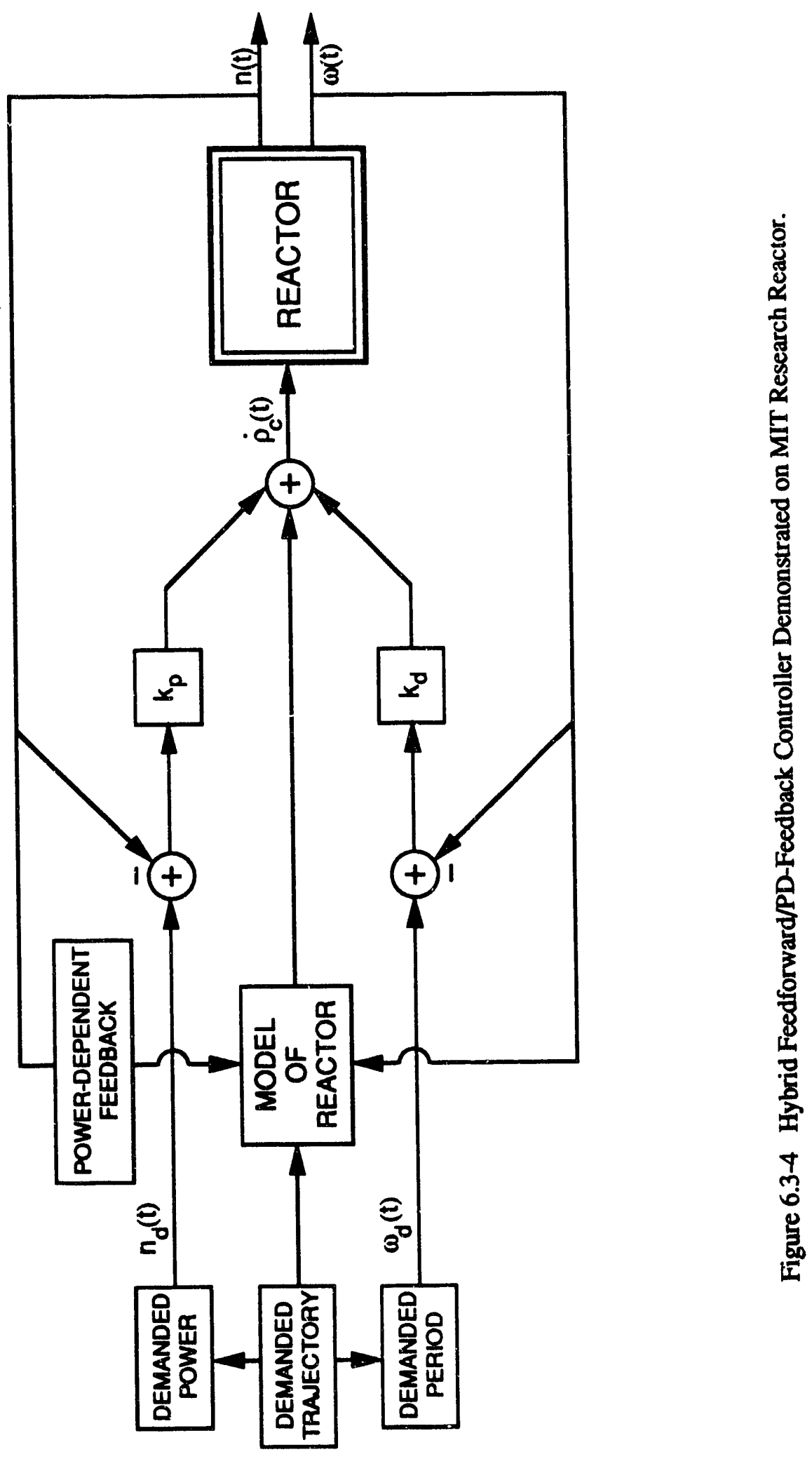


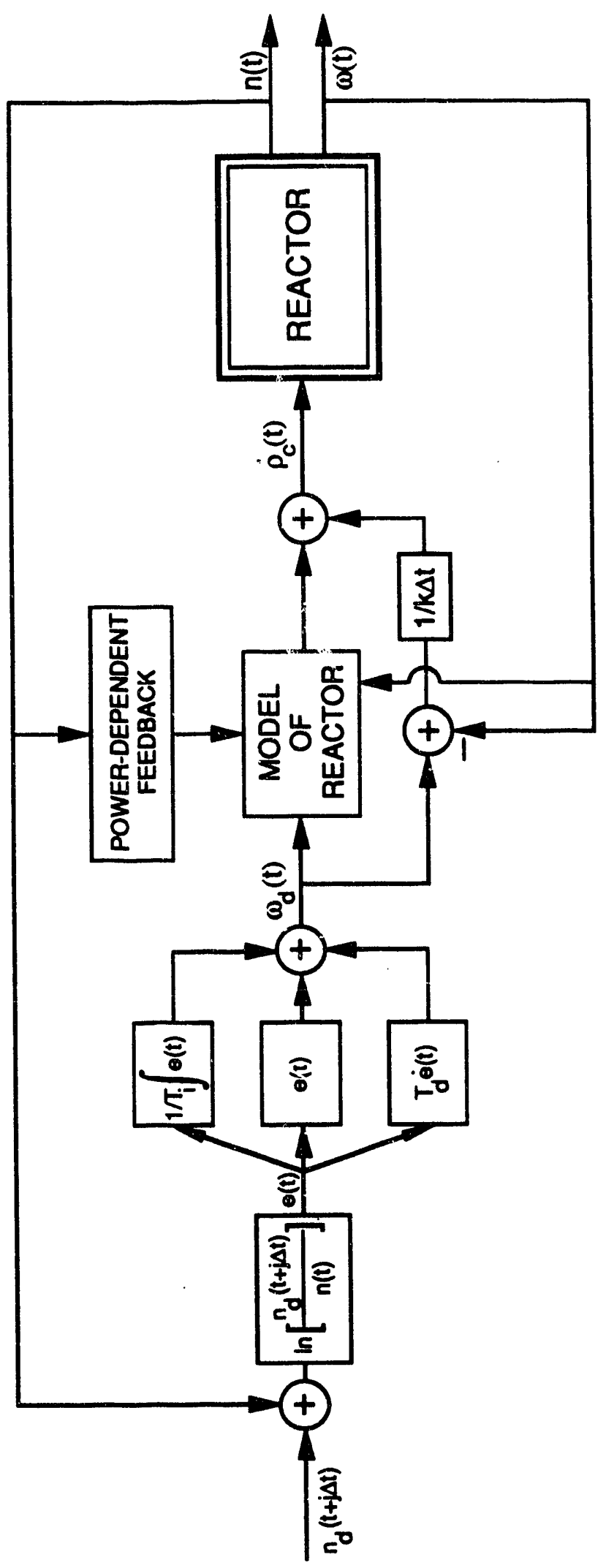

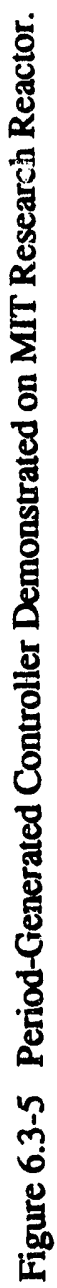


respectively. Equation (6.3-4) defines the tracking error by comparison of the demanded and observed power levels. Equation (6.3-5) uses this error signal to modify the demanded trajectory. If the power is being raised on the desired path, the quantity $\omega_{\mathrm{d}}$ will remain as originally specified. However, if a deviation has occurred, $\omega_{\mathrm{d}}$ will be updated so as to drive the observed power back to the demanded trajectory. Equation (6.3-6) is the feedforward model from which the actuator signal is computed. The difference between pure feedforward (Equation (6.3-2)) and period-generated control (Equations (6.3-4 to 6.3-6)) should be clearly understood. In the former, the demanded inverse period is never altered. In the latter, it is updated at every sample step by means of a feedback action that compares the demanded and observed power profiles.

\subsection{Experimental_Evaluation}

Experiments to evaluate the various feedforward controllers were conducted under conditions of closed-loop digital control on the 5-MWt MIT Research Reactor. A previously approved protocol was observed in order to ensure safety [1]. Figure 6.4-1 is from a trial of the pure feedforward coritroller, in which the reactor power was to be increased from $1000 \mathrm{~kW}$ to $1500 \mathrm{~kW}$ on a demanded period of $100 \mathrm{~s}$. The transient should have been completed in $41 \mathrm{~s}$. As is evident from the figure, the power was raised to 1500 kW but $55 \mathrm{~s}$ were required. This indicates that while the model was basically valid, it was not perfect. Further tests were made of this controller in which reactivity perturbations of unknown origin were deliberately introduced. As was expected, the controller's performance was poor. Figure 6.4-2 shows the performance of the hybrid feedforward/PD-feedback controller under the same conditions. This controller's gains had been optimized and, as would be expected, performance was much improved with the transient being completed in $35 \mathrm{~s}$. However, the controller's response to perturbations was marginal. Figure 6.4-3 shows this for a run in which power was to be raised from 1000 $\mathrm{kW}$ to $1200 \mathrm{~kW}$ on a $100-\mathrm{s}$ period. A negative reactivity perturbation was deliberately introduced at $40 \mathrm{~s}$. The controller compensated for this by further withdrawing the control device. However, this compensation was insufficient and the power drifted below the setpoint. The addition of integral feedback action improved the situation. This is shown in Figure 6.4-4 which presents the performance of a hybrid feedforward/PID-feedback controller with a negative reactivity perturbation of unknown origin introduced at $50 \mathrm{~s}$. Figure 6.4-5 shows the results from a trial of the period-generated controller in which power was to be raised from $1000 \mathrm{~kW}$ to $3000 \mathrm{~kW}$ on a $50-\mathrm{s}$ period. Positive and negative reactivity perturbations of unknown origin were introauced at $100 \mathrm{~s}$ and $200 \mathrm{~s}$ respectively. The controller did quite well, with the demanded power 8 ttained in $50 \mathrm{~s}$ as compared to the expected $55 \mathrm{~s}$. Also, the recovery from both perturbations was quite rapid.

\subsection{Assessment of Feedforward Control Techniques}

Feedforwand control refers to the prosess whereby an actuator signal is calculated by processing a demanded system output through an inverse model of the system's dynamics. If the model is accurate and if there are no unknown perturbations, application of this calculated actuator signal to the actual process should then cause the observed output to conform to that demanded. The procedure can be improved by combining error-driven feedback with the model-based feedforward calculation. Methods for implementing this mode of control include total reliance on the system model, the summation of feedforward and feedback components, and the use of feedback to modify the feedforward action. 
REACTIVITY (m $\beta)$

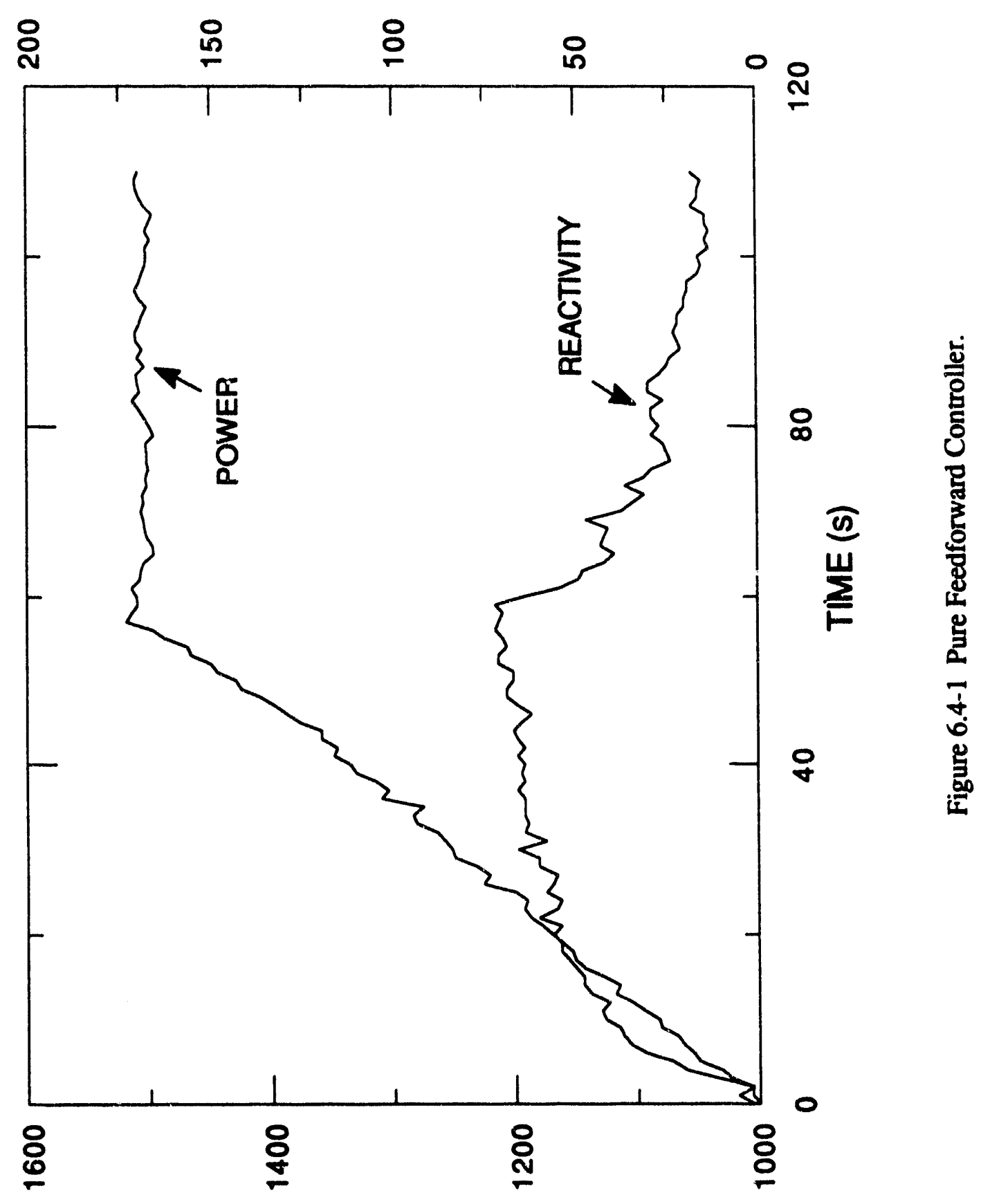

(MY) y 8 MOd 
REACTIVITY (m $\beta)$

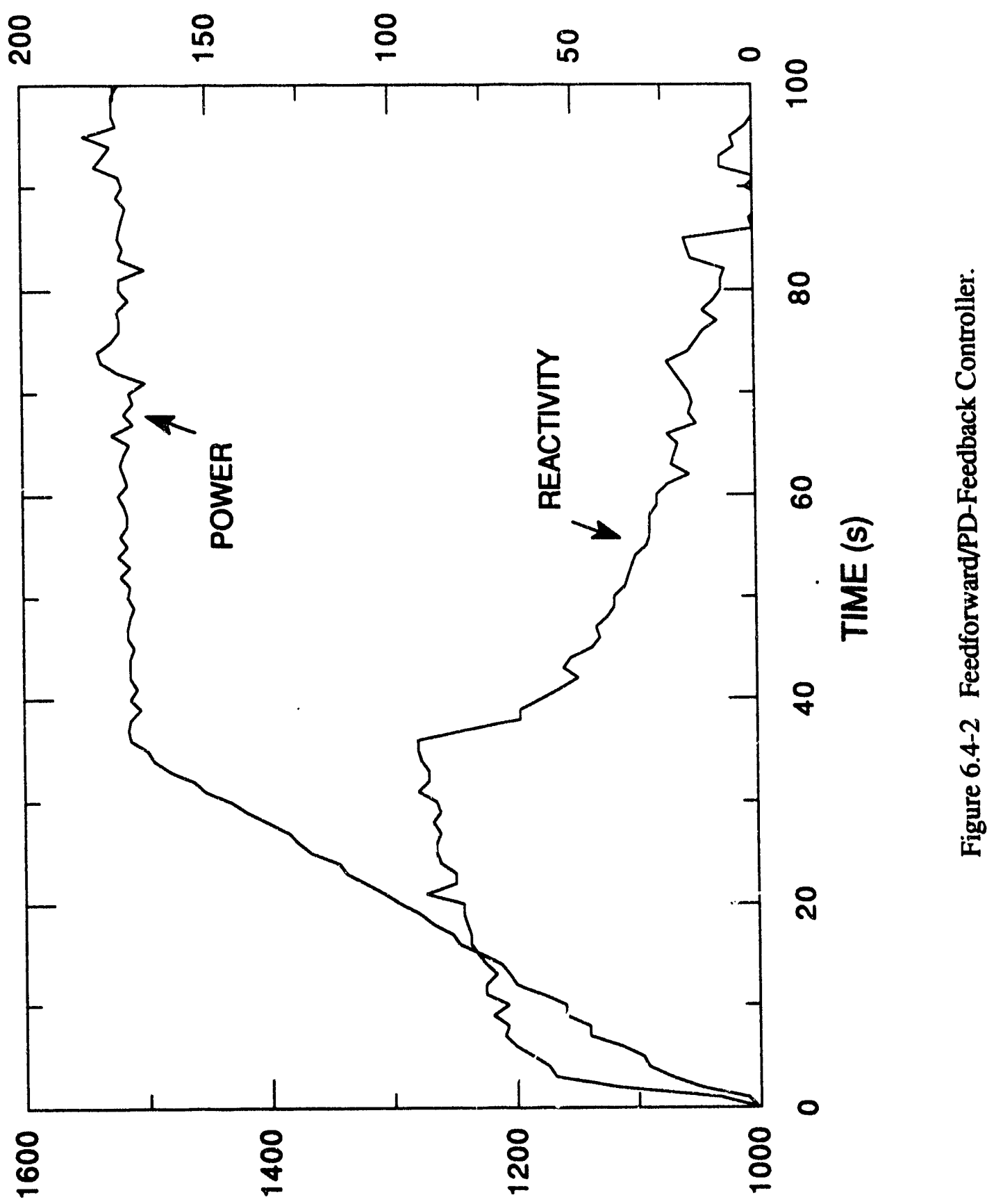

(MY) $8 \exists M O d$ 
REACTIVITY $(m \beta)$

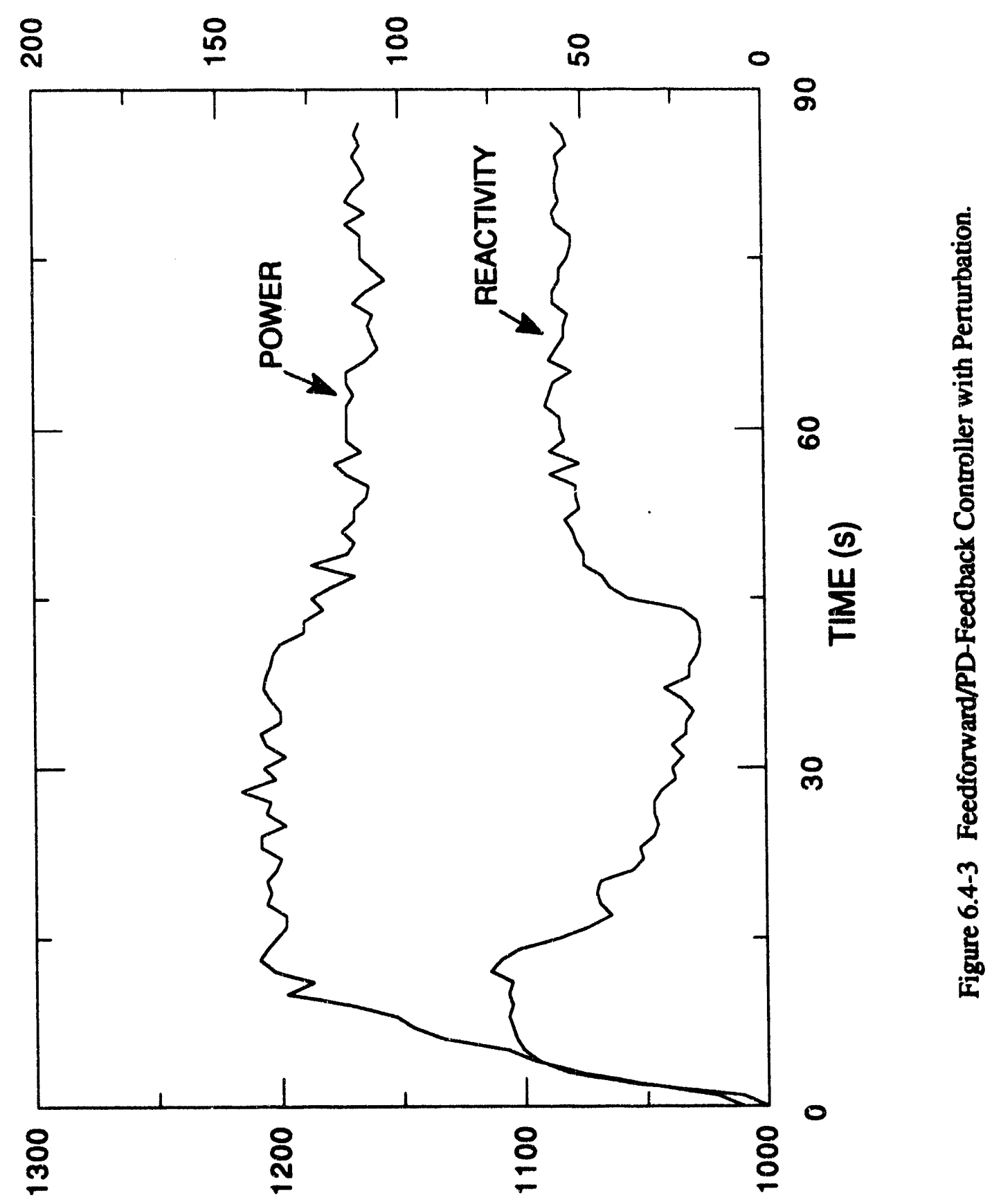

(MY) $4 \exists M O d$ 
REACTIVITY (mß)

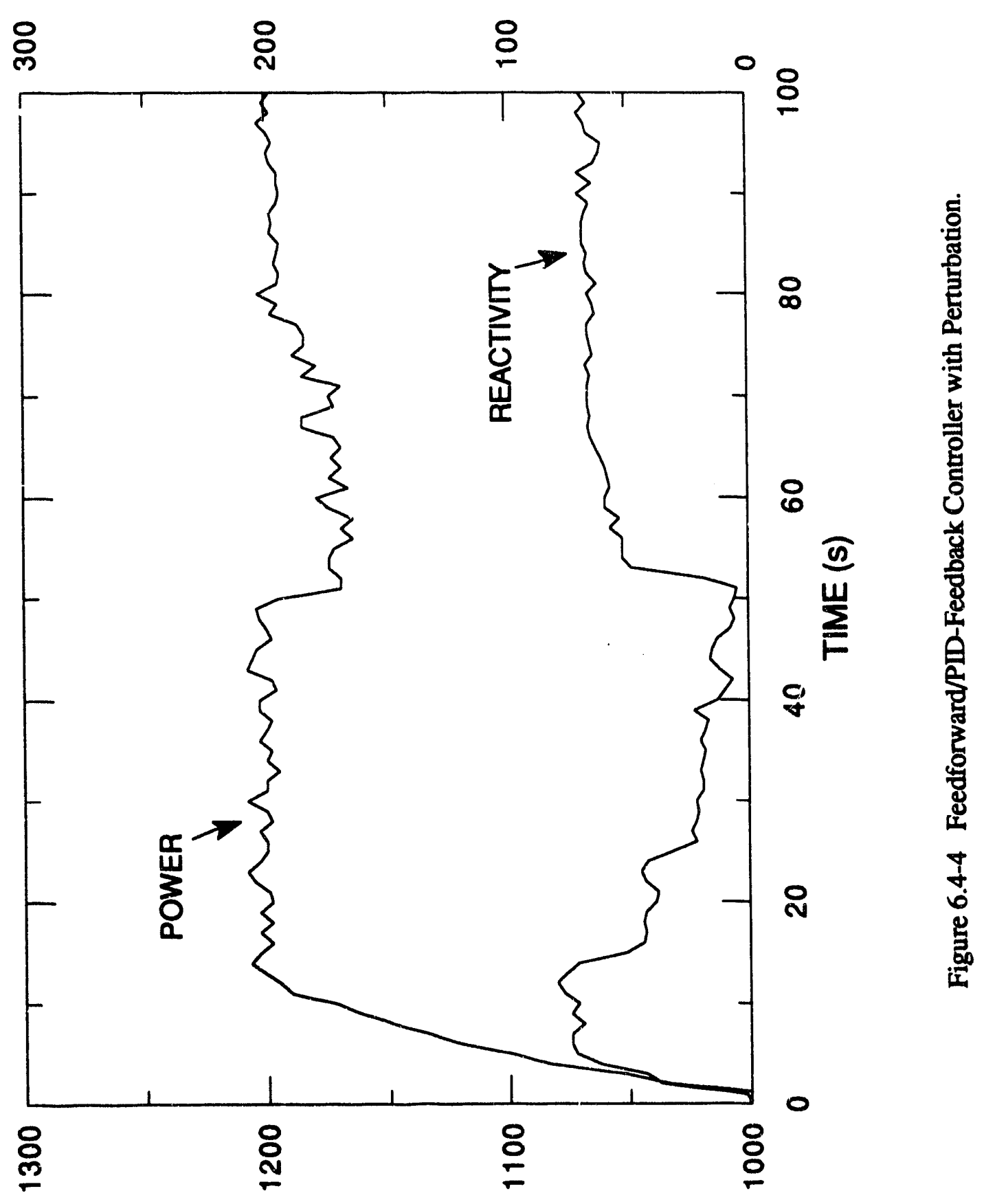

(MY) y 8 MOd 
REACTIVITY $(m \beta)$

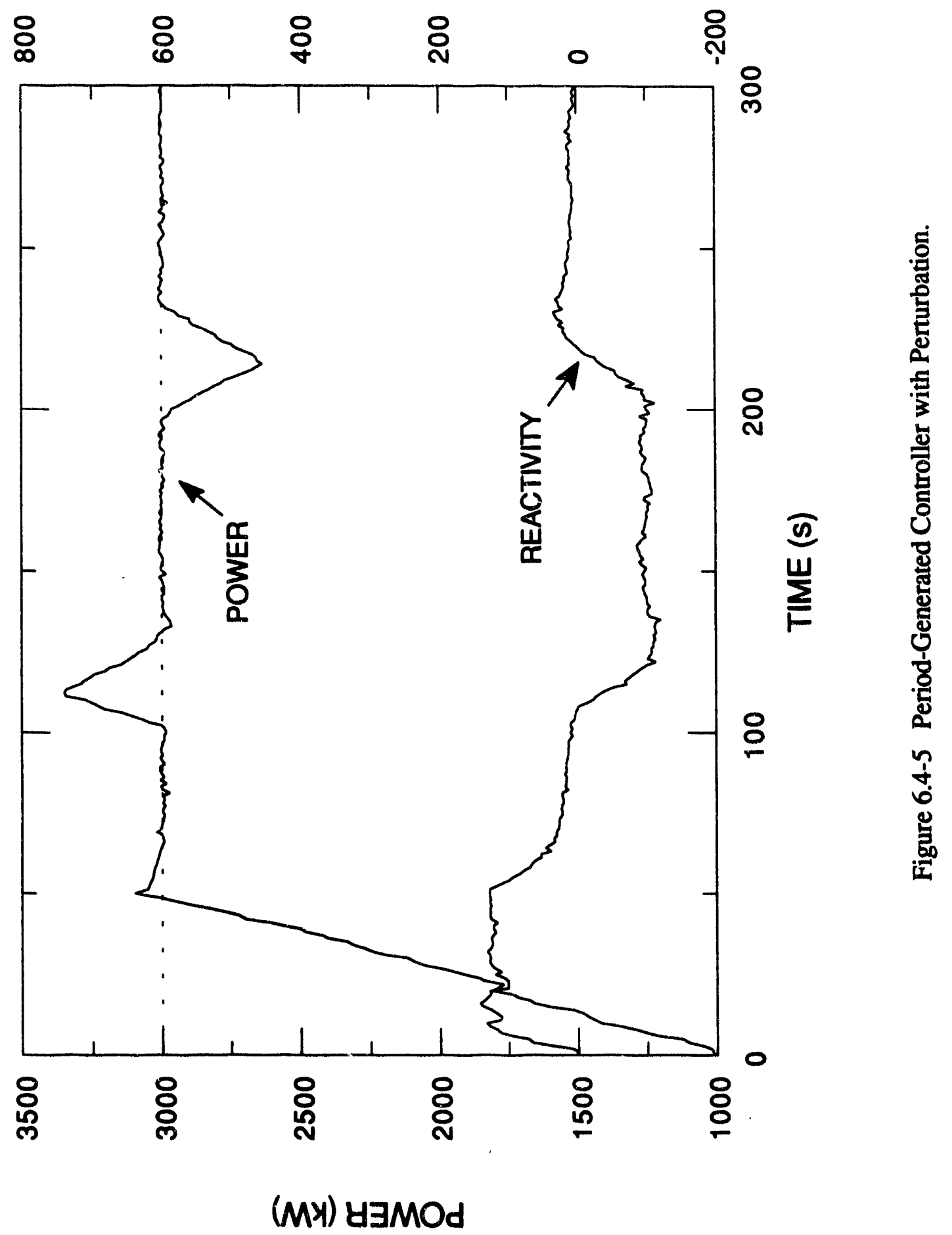


Experiments were conducted on the 5-MWt MIT Research Reactor in which a comparative assessment of each of these options was made. These experiments involved increases of reactor neutronic power under conditions of digital, closed-loop control. It was found that superior performance is obtained when feedback is used to update the demanded trajectory at every sampling interval. The advantage of this approach is that the model-based feedforward action allows for non-linear dynamics while the feedback corrects for modeling errors and perturbations of unknown origin. 


\section{Simulation of PWR-Type Multi-Modular Power Plants ${ }^{(1)}$}

This chapter describes the development and numerical testing of a simulation program for a PWR-type multi-modular power plant. As many as four modules may br included in the simulations. This simulator has been designated as PMSIM which stands for PWR-type Multi-modular power plant SIMulation program. Presented here is a summary of the governing equations for the plant subsystem models, the corresponding numerical solution methods, and the results of numerical evaluations.

\subsection{Statement of Problem}

The principal objective in developing PMSIM was to facilitate studies on the control of multi-modular power plants. Of particular interest was the maintenance of steam generator level within allowed bands and the coordination of neutronic power between modules. This meant that PMSIM had (1) to replicate steam generator level dynamics including 'shrink and swell' effects; (2) to simulate major plant and module parameters including module powers, primary coolant temperatures, and steam flowrates during both power increases and decreases; and (3) to model the main steam line common header.

In multi-modular plants, the theimal-hydraulic behavior of both the steam generator and the main steam line header determines the steam flowrate from individual steam generators. Thus, each module in PMSIM consists of a neutron kinetics routine, a model of the heat transfer from fuel to coolant, a one-dimensional primary coolant loop model, a steam generator model, and controller simulation model. Each of the is combined with the main steam line header model. A total of four modules are simulated.

Some features were not modeled in PMSIM. For example, models of instruments and measuring devices are not included. Instead it is assunied that plant variables are measured without any time delay. (Note: Measurement noise on the princiral plant variables is considered.) Also excluded are the dynamics of the various actuators. Thus, it is assumed that control rod drives and feedwater regulating valves act perfectly and without delay. Finally, pressurizer, turbine-generator, and condenser models are not included. Instead, steam flowrate from the main steam line header through the turbine control valves is specified on the basis of energy conservation and the temperature of the feedwater is specified as a function of turbine power.

The development of PMSIM benefitted from previous work done both at MIT and elsewhere to develop PWR simulation programs. In the early 1980s, Strohmayer developed a dynamic simulation program for a vertical, U-tube steam generator [68]. This program uses one-dimensional conservation equations of mass, momentum, and energy and predicts the steam generator level dynamics, especially 'shrink and swell' effects. Choi modified this model to improve the simulation of steam generator level dynamics during low power operation and used it to design a model-based digital steam generator level controller [69].

In Korea, Auh developed a PWR transient and accident simulation program that runs on a micro-computer [70]. This program includes a two-fluid pressurizer model, a

(1) This chapter is based on work performed by Keung Koo Kim under the supervision of Professors John E. Meyer and David D. Lanning. 
boiling pot steam generator model, and a point-kinetics core model. At MIT, S. P. Kao developed a multiple-loop primary system model for a PWR power plant. This was the first 'whole-plant' simulation model [71]. It has since been extended to a version designated as Pressurized Reactor Interactive Simulation Model (PRISM) [72]. PRISM includes neutron kinstics, heat transfer from fuel to coolant, a multiple-loop primary coolant system, a pressurizer, and a steam generator. In Japan, a real-time, accidenttracking PWR simulation program has been developed to investigate small break loss-ofcoolant accidents [73]. Cabral developed a three-dimensional, thermal-hydraulic simulation model for a reactor core [59]. This simulation program consisted of a three-dimensional heat transfer and fluid flow model of the reactor core with neutron kinetics, a primary coolant loop model, and steam generator models similar to those of S. P. Kao. P. W. Kao developed a three-dimensional neutronics model using analytical nodal methods [58]. Aviles combined the Cabral and P. W. Kao programs to create a space-dependent simulation program [60]. However, because he focused on the plant's primary sid's, especially the reactor core, his simulation program does not simulate the secondary side except for a boiling pot steam generator model.

Although of value, the previous MIT work was focused on PWRs and therefore could not be used directly to construct PMSIM. For example, Strohmayer and Choi do not address primary-side simulations and the others use a boiling pot steam generator model which cannot describe variations in steam generator level. As regards previous MIT work on the multi-modular power plants, Waltrip had developed a simple simulation program [74] for application to a multi-modular liquid metal cooled reactor. However, it could handle only one power module and therefore could not be used for the study of unbalanced load operation. Also, a simulation program for LMR-type multi-modular power plants is under development at the Oak Ridge National Laboratory (ORNL) [75]. It uses a parallel computer.

\subsection{Basis of PWR-Type Multi-Modular Power Plants}

Figure 7.2-1 is a schematic of the PWR-type multi-modular plant on which PMSIM is based. For the most part, data for a typical four-loop PWR were used. Specifically, for the fuel rod structure, PWR fuel rod geometry data was used. For the steam generators, Westinghouse type ' $F$ ' units were assumed. Typical plant and steam generator data were taken from Kao [72] and Choi [76] respectively. Table 7.2-1 lists many of the characteristic values used in the multi-modular power plant simulation program.

Two assumptions are made in modeling the reactor core and primary coolant system. First, because a module's power is only one fourth that of a typical 1100-MWe PWR, the core volume is treated by dividing it by four and placing it in four identical modules. Second, the momentum equation is not solved in the primary coolant system model because primary pressure and coolant flowrate remain nearly constant over the range of transients studied.

\subsection{Models Used in Multi-Modular Plant Simulator}

Described here are the various models used to construct PMSIM. Included are those for the neutron kinetics, the fuel temperature, the primary coolant system, the steam generator secondary side, and the main steam line cominon header. The latter two are 


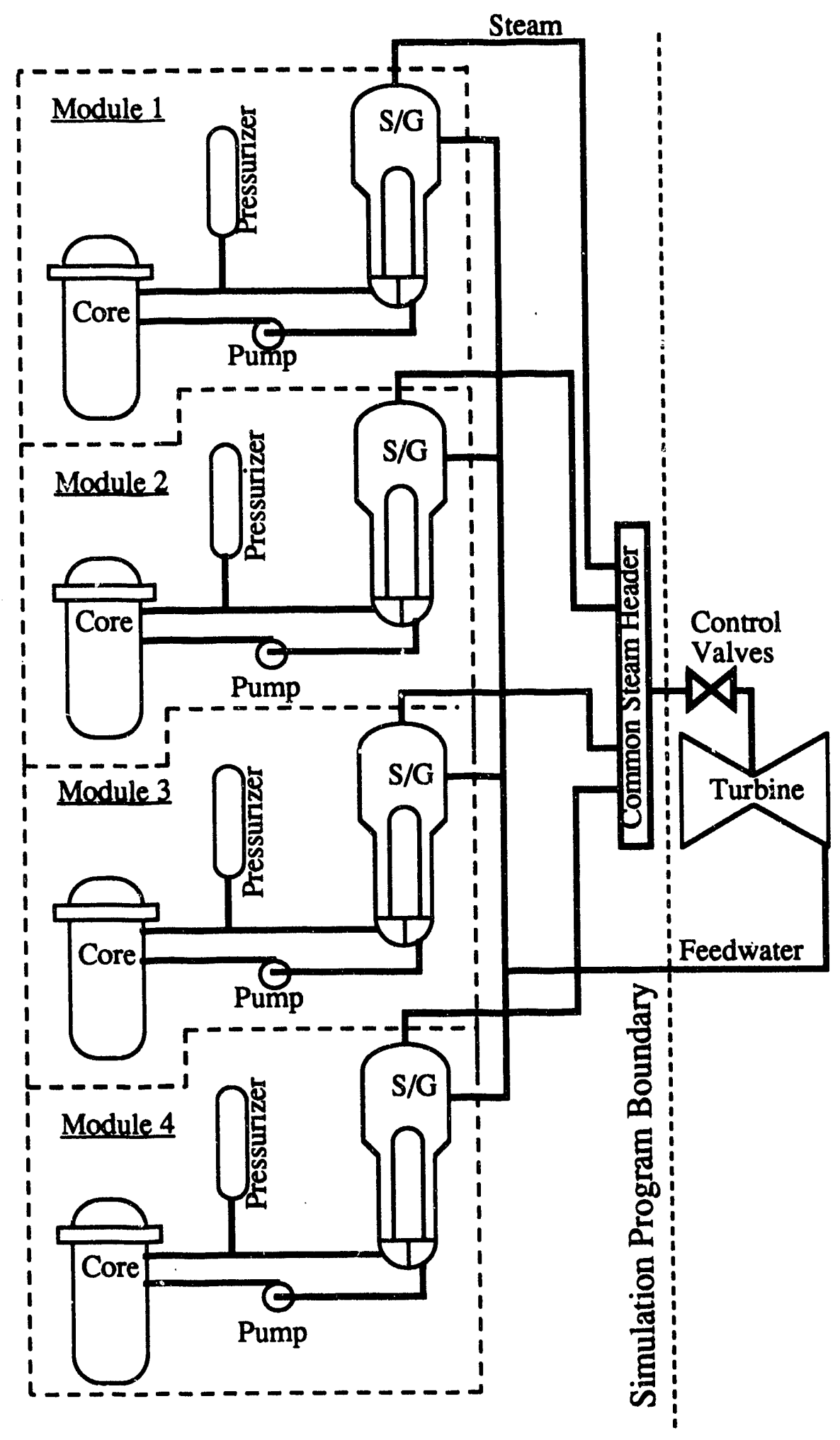

Figure 7.2-1 Schematic Diagram of PWR-Type Multi-Modular Power Plant. 
Table 7.2-1

PWR-Type Multi-Modular Power Plant Parameters Used in PMSIM

1. Plant

Number of power modules

Total power of multi-modular power plant (MWt)

Power of individual module (MWt) 853.

Heat generated in the fuel (\%)

97.0

Heat generated in the moderator (\%)

Prin_ary heat per pump (MWt)

2. Core

Volume of coolant $\left(\mathrm{m}^{3}\right)$

Mass of fuel (Mg)

Mass of cladding (Mg)

3. Primary Coolant System

System pressure (MPa)

Coolant flowrate $(\mathrm{Mg} / \mathrm{s})$

Upper plenum volume $\left(\mathrm{m}^{3}\right)$

Inlet plenum volume $\left(\mathrm{m}^{3}\right)$

10.14

Downcomer volume $\left(\mathrm{m}^{3}\right)$

19.61

Lower plenum volume $\left(\mathrm{m}^{3}\right)$

23.7

Hot leg volume $\left(\mathrm{m}^{3}\right)$

3.07

Suction leg volume $\left(\mathrm{m}^{3}\right)$

5.13

Cold leg volume $\left(\mathrm{m}^{3}\right)$ 


\section{Fuel Rods}

Total number

12738

Fuel material $\mathrm{UO}_{2}$

Density (\% of theoretical $\mathrm{UO}_{2}$ density)

Pellet diameter (mm)

Cladding inside diameter $(\mathrm{mm})$

Rod outside diameter (mm)

Rod height (m)

Total heat transfer area $\left(\mathrm{m}^{2}\right)$

Gap heat transfer coefficient $\left(\mathrm{W} / \mathrm{m}^{2} \mathrm{~K}\right)$

\section{Reactivity Parameters}

Doppler temperature coefficient $(\mathrm{pcm} / \mathrm{K})$ $-5.2 \sim-1.8$

Moderator temperature coefficient $(\mathrm{pcm} / \mathrm{K})$

$$
\text { . } \quad 0 \sim-63
$$

Boron reactivity coefficient ( $\mathrm{pcm} / \mathrm{ppm}$ )

$-12.5 \sim-7.5$

Delayed neutron fraction

0.0075

Prompt neutron lifetime ( $\mu s)$

19.4

\section{Steam Generator}

Type

U-tube

Full load pressure (MPa)

6.89

Heat transfer area $\left(\mathrm{m}^{2}\right)$

5110

Primary side flow area $\left(\mathrm{m}^{2}\right)$

1.05

Tube outside diameter (mm)

17.48

Tube inside diameter (mra)

15.44

Tube metal mass $(\mathrm{Mg})$

39.69 
given in some detail because major portions of them were developed as part of the research reported here.

\subsubsection{Neutron Kinetics Model}

A point kinetics model is often sufficient to simulate reactor power for control studies and hence such a model was assumad here. Should the need arise to consider spatial effects, the more complex model described in the report entitled, "Closed-Loop Digital Control of Nuclear Reactors Characterized by Spatial Dynamics," could be substituted [4]. The point kinetics equations are:

$$
\begin{aligned}
& \frac{d}{d t} T(t)=\frac{\rho(t)-\bar{\beta}}{l^{*}} T(t)+\sum_{i=1}^{N} \lambda_{i} C_{i}(t) \\
& \frac{d}{d t} C_{i}(t)=\frac{\bar{\beta}_{i}}{l^{*}} T(t)-\lambda_{i} C_{i}(t) \quad i=1,2, \ldots, N
\end{aligned}
$$

where $T(t)$ is the amplitude function and is a weighted integral of all neutrons in the core, $\rho(t)$ is the net reactivity, $\bar{\beta}_{i}$ is the fractional yield of the ith precursor group, $\bar{\beta}$ is the effective delayed neutron fraction, $C_{i}$ is the concentration of the ith preciursor group, $\lambda_{i}$ is the decay constant of the ith precursor group, $l$ is the prompt neutron lifetime, and $\mathbf{N}$ is the number of delayed neutron precursor groups. The above equations were simplified by taking the amplitude function to be the reactor neutronic power.

The total reactivity present in a reactor is produced from several different mechanisms and includes the following:

1. Control Reactivity: In a PWR, changes in power can be instigated either by moving control rods or by altering the concentration of the boron that is dissolved in the primary coolant. Movement of the control rods can be done more rapidly than can adjustment of the soluble boron concentration. Hence, rod movements are used here to initiate transients.

2. Eeedback Reactivity: Neutron levels affect heat and xenon production. Consequently fuel and moderator temperature changes and variations in xenon concentration affect reactivity.

Provided that the shape of the neutron distribution remains constant, each of these reactivities can be added linearly as shown by the following expression:

$$
\rho=\Delta \rho_{\text {rod }}+\Delta \rho_{f}+\Delta \rho_{m}+\Delta \rho_{\text {Xe }}+\Delta \rho_{B}
$$

where $\Delta \rho_{\text {rod }}$ is the control rod reactivity, $\Delta \rho_{\mathrm{f}}$ and $\Delta \rho_{\mathrm{m}}$ are the fuel and moderator feedback reactivities respectively, $\Delta \rho_{X e}$ is the xenon reactivity, and $\Delta \rho_{B}$ is the soluble boron reactivity. The symbol $\Delta$ means difference between current value of each variable and its initial value. 
Because a control rod's reactivity worth depends on the rod's position, a predetermined reactivity curve is incorporated in the model. A detailed, space-dependent reactor physics code was used to generate this relation from a typical PWR and the resulting data was fitted to a parabolic equation. Figure 7.3.1-1 shows this reactivity worth curve as a function of equivalent rod position.

In power reactors, fuel and coolant feedback reactivity changes are determined from temperature coefficients that are defined as the ratio of the total reactivity change to the fuel (or coolant) temperature change. Thus,

$$
\begin{aligned}
& \Delta \rho_{\mathrm{f}}=\int_{\mathrm{T}_{\mathrm{fo}}}^{\mathrm{T}} \alpha_{\mathrm{Tf}}(\mathrm{T}) \mathrm{dT} \\
& \Delta \rho_{\mathrm{m}}=\int_{\mathrm{T}_{\mathrm{mo}}}^{\mathrm{T}} \alpha_{\mathrm{Tm}}(\mathrm{T}) \mathrm{dT}
\end{aligned}
$$

where $\alpha_{\mathrm{Tf}}$ and $\alpha_{\mathrm{Tm}}$ are the fuel and moderator temperature coefficients, and $T_{\mathrm{fo}}$ and $T_{\mathrm{mo}}$ are the reference fuel and moderator temperatures, respectively. As shown in the above equations, the fuel temperature coefficient is a function of the fuel temperature. Similarly, the moderator temperature coefficient depends on the moderator temperature and also perhaps on the boron concentration in the moderator. Typical PWR fuel and moderator temperature coefficients, shown in Figures 7.3.1-2 and 7.3.1-3 respectively, were fitted to a polynomial and used in the simulation program.

Boron reactivity can be calculated from the measured boron concentration and a 'dissolved boron reactivity' coefficient. A typical dissolved boron reactivity coefficient is shown in Figure 7.3.1-4. Feedback reactivity resulting from changes in xenon concentration was also included in the simulation.

\subsubsection{Fuel Temperature Model}

The average fuel temperature was needed in order to estimate both the fuel temperature feedback reactivity and the thermal power transferred from the fuel rods to the coolant. In a typical PWR primary coolant system, the thermal transport path proceeds from a point of fission energy deposition within solid fuel, through layers of fuel, through gas at the interface of the fuel and cladding, and then through the cladding to the interface with the light-water coolant. In order to simulate these processes, a doubly-lumpedparameter model was adopted as shown in Figure 7.3.2-1 [79]. The thermal energy balance equations of the fuel and cladoing regions were written as:

1. Fuel Region:

$$
M_{f} C_{f} \frac{d \bar{T}_{f}}{d t}=\dot{Q}-\dot{Q}_{g}
$$

where $M_{f}$ is the fuel mass, 

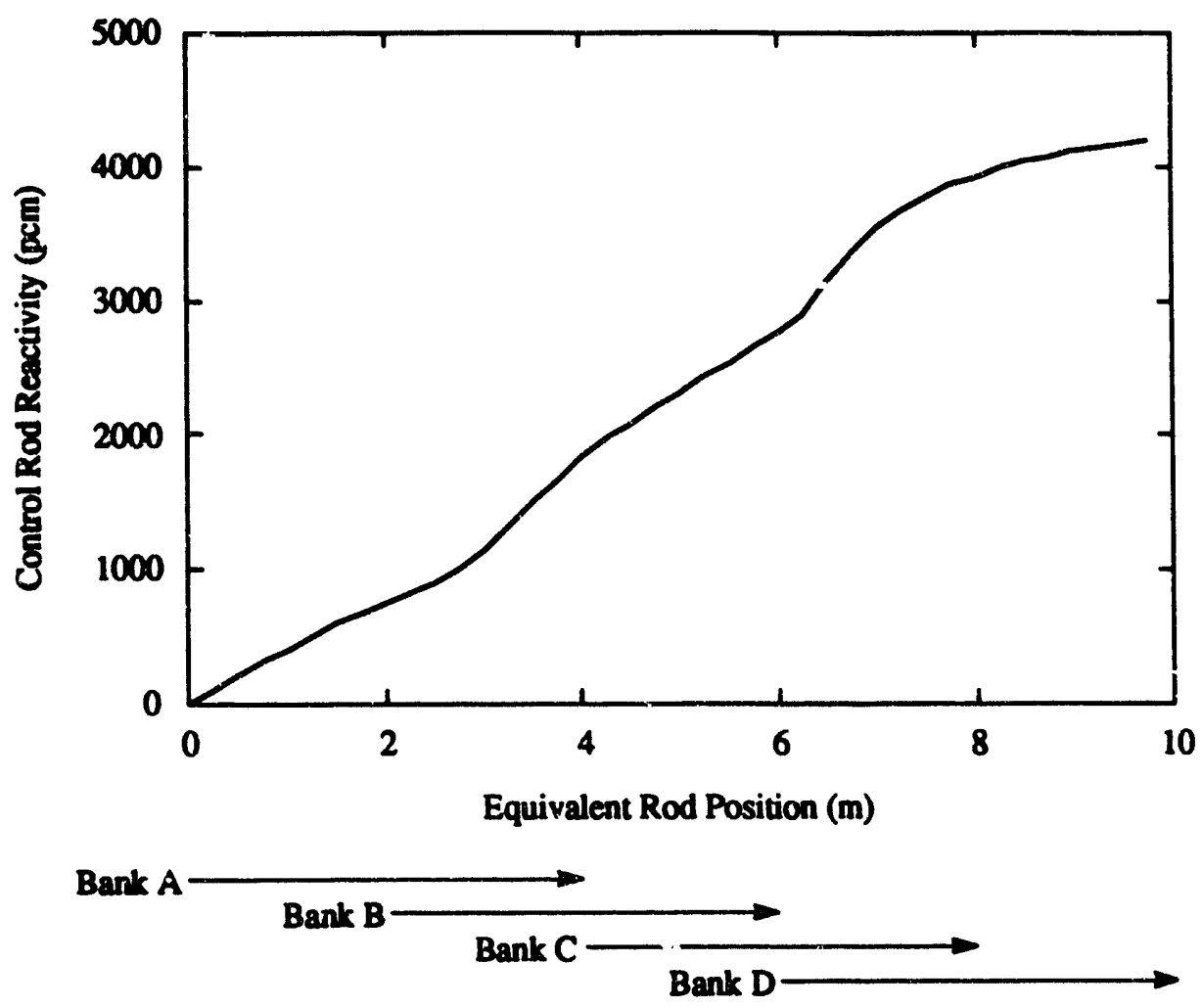

Figure 7.3.1-1 Control Rod Worth Versus Control Rod Position [77].

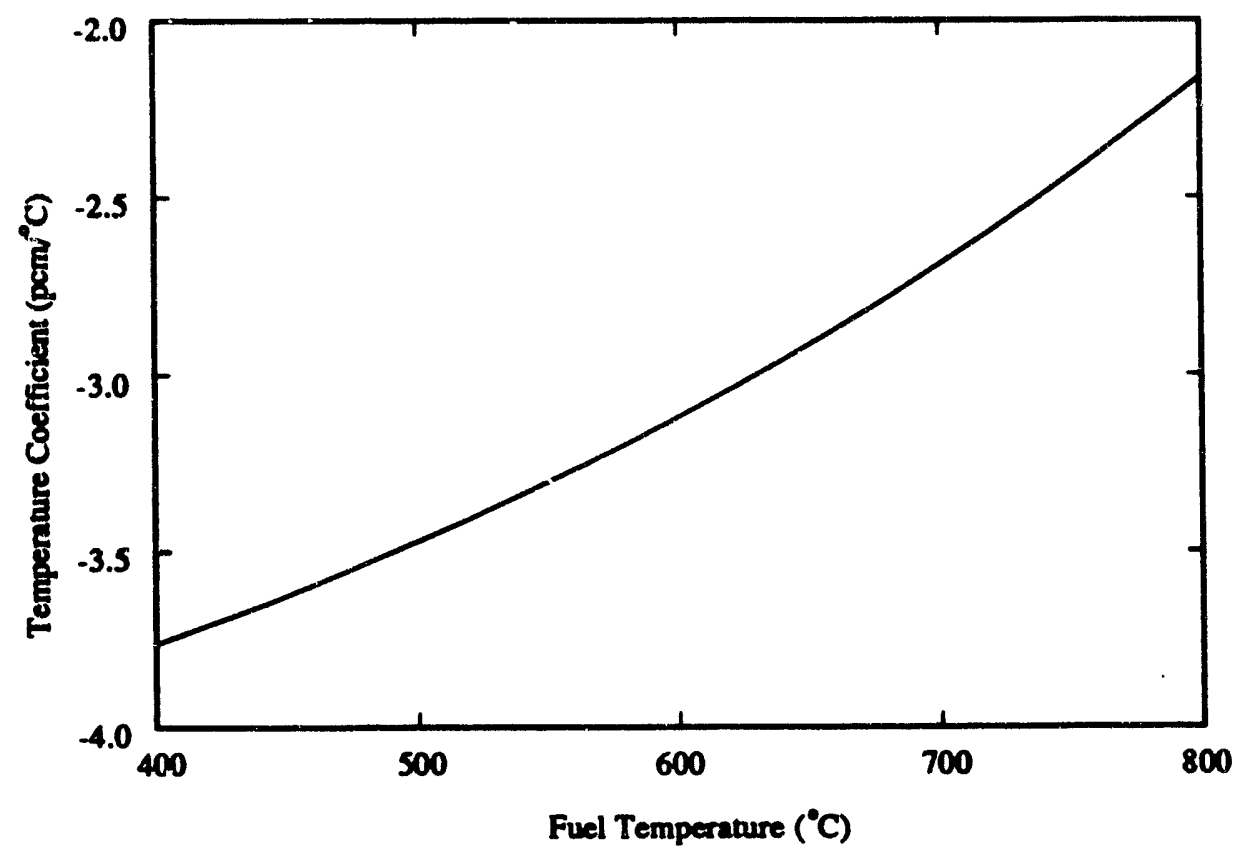

Figure 7.3.1-2 Fuel Temperature Reactivity Coefficient [77]. 


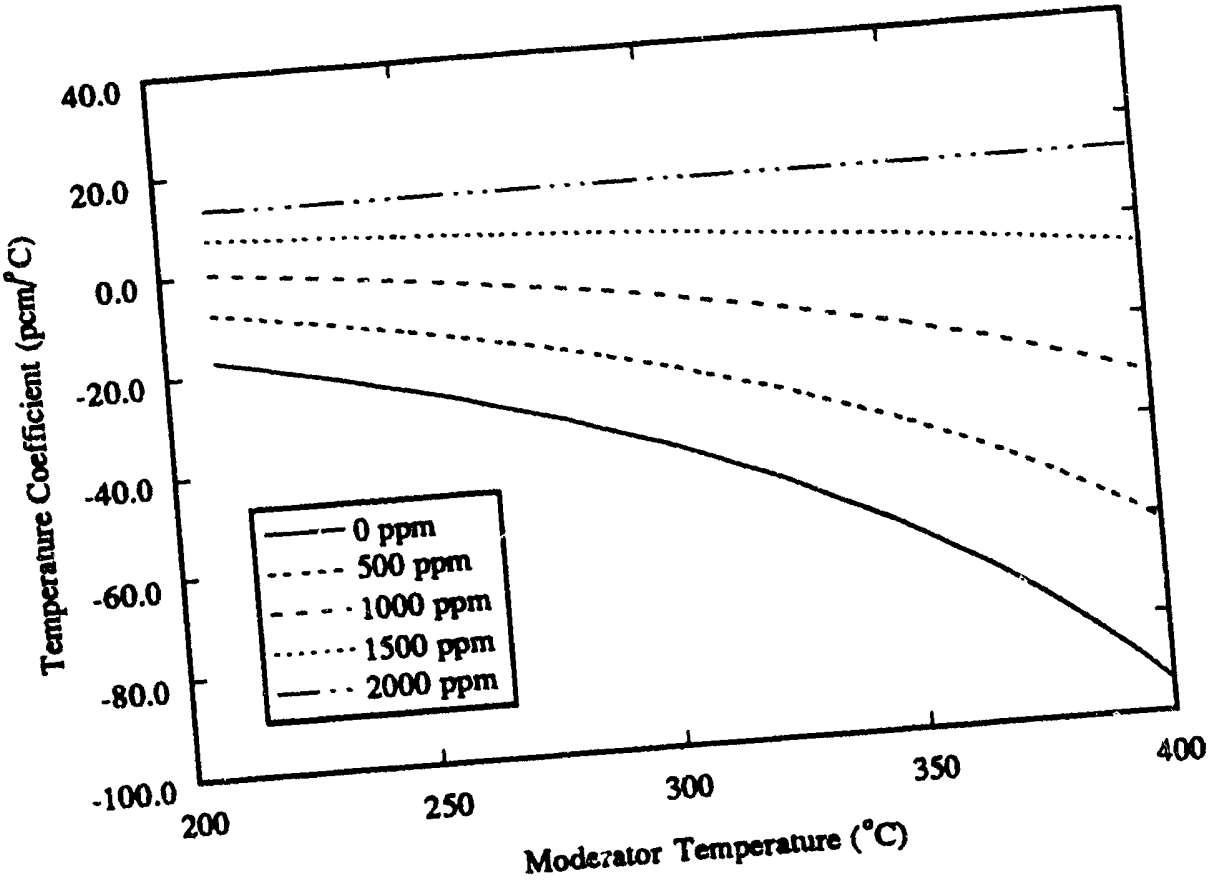

Figure 7.3.1-3 Moderator Temperature Reactivity Coefficient [77].

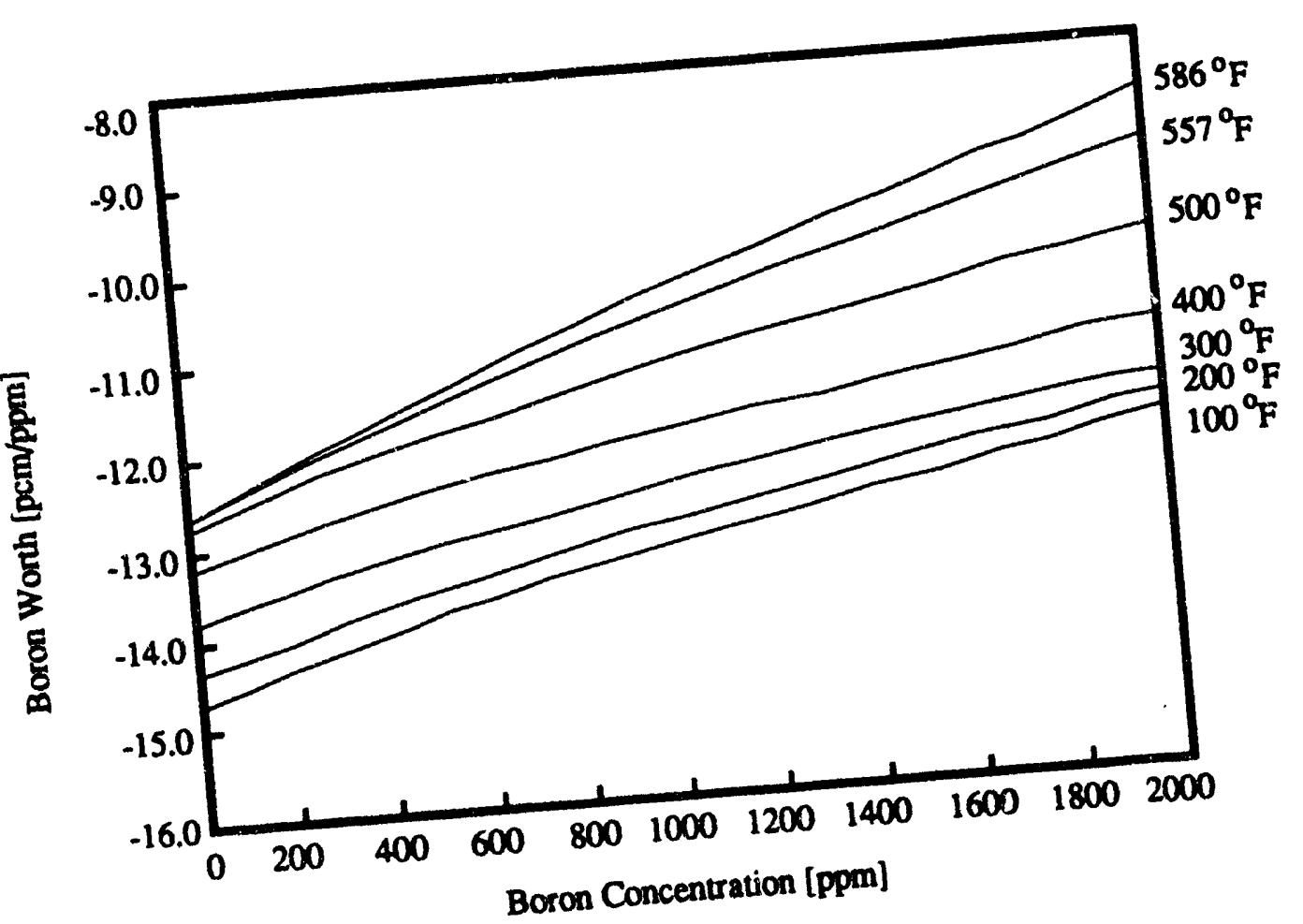

Figure 7.3.1-4 Dissolved Boron Reactivity Coefficient [78]. 


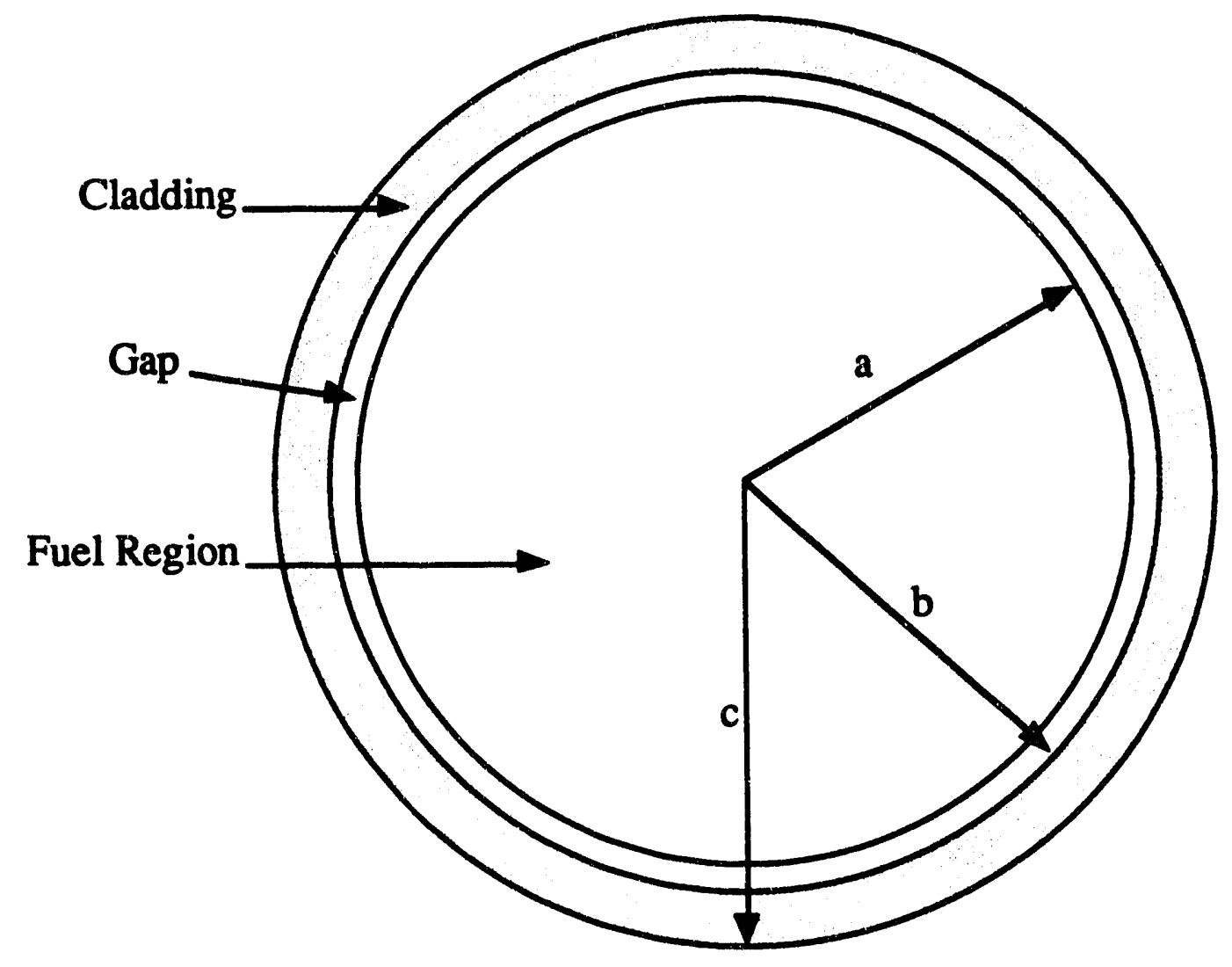

Figure 7.3.2-1 Typical Fuel Rod Cross Section. 
$\mathrm{C}_{\mathrm{f}}$ is the specific heat capacity of the fuei region,

$\overline{\mathrm{T}}_{\mathrm{f}}$ is the average fuel temperature,

$\dot{Q}$ is the heat generation rate within the fuel region, and

$\dot{\mathrm{Q}}_{\mathrm{g}} \quad$ is the heat transfer rate from fuel to cladding.

\section{Cladding Region:}

$$
M_{c l} C_{c l} \frac{d \bar{T}_{c l}}{d t}=\dot{Q}_{g}-\dot{Q}_{t h}
$$

where $\mathrm{M}_{\mathrm{cl}}$ is the cladding mass,

$\mathrm{C}_{\mathrm{cl}}$ is the specific heat capacity of the cladding region,

$\overline{\mathrm{T}}_{\mathrm{cl}}$ is the average cladding temperature, and

$\dot{Q}_{t h}$ is the heat transfer rate from the cladding to coolant.

To solve Equations (7.3.2-1) and (7.3.2-2), it is assumed that heat transfer coefficients derived from steady-state relationships between the radial average temperatures of fuel, cladding, and coolant are maintained during transients. These heat transfer coefficients, which are designated as $\mathbf{R}_{\mathbf{g}}$ and $\mathbf{R}_{\mathrm{c}}$, are defined as through the relations:

$$
\dot{\mathrm{Q}}_{\mathrm{g}} \equiv \frac{\mathrm{NL}}{\mathrm{R}_{\mathrm{g}}}\left(\overline{\mathrm{T}}_{\mathrm{f}}-\overline{\mathrm{T}}_{\mathrm{cl}}\right)
$$

and

$$
\dot{\mathrm{Q}}_{\mathrm{th}} \equiv \frac{\mathrm{NL}}{\mathrm{R}_{\mathrm{c}}}\left(\overline{\mathrm{T}}_{\mathrm{cl}}-\mathrm{T}_{\mathrm{c}}\right)
$$

where $\mathbf{N}$ is the total number of fuel rods and $L$ is the length of a fuel rod. $R_{g}$ and $R_{c}$ can then be expressed as:

$$
R_{g}=\frac{1}{8 \pi k_{f}}+\frac{1}{2 \pi h_{g}}+\frac{1}{2 \pi k_{c l}}\left\{\frac{c^{2}}{c^{2}-b^{2}} \ln \left(\frac{c}{b}\right)-\frac{1}{2}\right\}
$$

and

$$
R_{c}=\frac{1}{2 \pi k_{c l}}\left\{\frac{1}{2}-\frac{c^{2}}{c^{2}-b^{2}} \ln \left(\frac{c}{b}\right)\right\}+\frac{1}{2 \pi c h_{c}}
$$


where $\mathbf{k}_{\mathrm{f}}$ and $\mathbf{k}_{\mathrm{cl}}$ are the thermal conductivities of fuel and cladding, respectively; $a$ is the outer radius of the fuel pellet; and $b$ and $c$ are the inner and outer radii of the cladding, respectively. The quantities $h_{g}$ and $h_{c}$ are the heat transfer coefficients of the gap and the fuel rod outer surface, respectively.

\subsubsection{Brimary Coolant System Model}

The primary coolant system transports heat deposited in the fuel to the steam generator. Accordingly, major concerns in the development of a primary coolant system model are that time delays associated with heat transfer and fluid transport be accurately represented and that the model interface properly to those of the fuel rods and the steam generators. S. P. Kao developed a one-dimensional primary loop model for a typical multi-loop PWR plant simulation [71]. Cabral also developed a one-dimensional primary loop model [59]. However, it only solves the mass and energy equations. These primary loop models were modified for the multi-modular coolant system model that is described here.

The number of control regions in each module's primary coolant system was minimized so as to represent only the major components. These were the reastor core, the reactor vessel upper plenum, the hot leg and steam generator inlet plenum, the steam generator tube bundle, the steam generator outlet plenum and the cold leg, and the reactor vessel downcomer region and the lower plenum. Figure 7.3.3-1 shows the control regions assigned in the primary coolant system model. Because the pressure of the primary coolant does not change significantly during operational transients, pressurizer operation does not affect the primary coolant temperature. Therefore, instead of including a pressurizer model, the primary system pressure is given as a user-specified boundary condition. Similarly, a momentum balance equation was not needed and changes in the primary coolant flowrate are input as a boundary condition. The primary coolant pump in the cold leg is modeled as an energy source within the coolant. Pump dynamics are not considered and the pump power input is assumed to be constant.

A typical control region for the primary coolant system model is shown in Figure 7.3.3-2. The donor cell method is used and the 'mixing-cup' enthalpy, $h_{j}$, is defined in terms of the total mass and energy content inside the ith control region cell. The governing equations for a given control volume are those of mass and energy conservation. These are:

1. Mass Conservation:

$$
\frac{d M_{i}}{d t}=\dot{m}_{i}-\dot{m}_{i-1}
$$

2. Energy Conservation:

$$
\frac{\mathrm{d} \mathrm{U}_{\mathrm{i}}}{\mathrm{dt}}=(\dot{\mathrm{m} h})_{\mathrm{i}}-(\dot{\mathrm{m} h})_{\mathrm{i}-1}+\dot{Q}_{\mathrm{i}}
$$




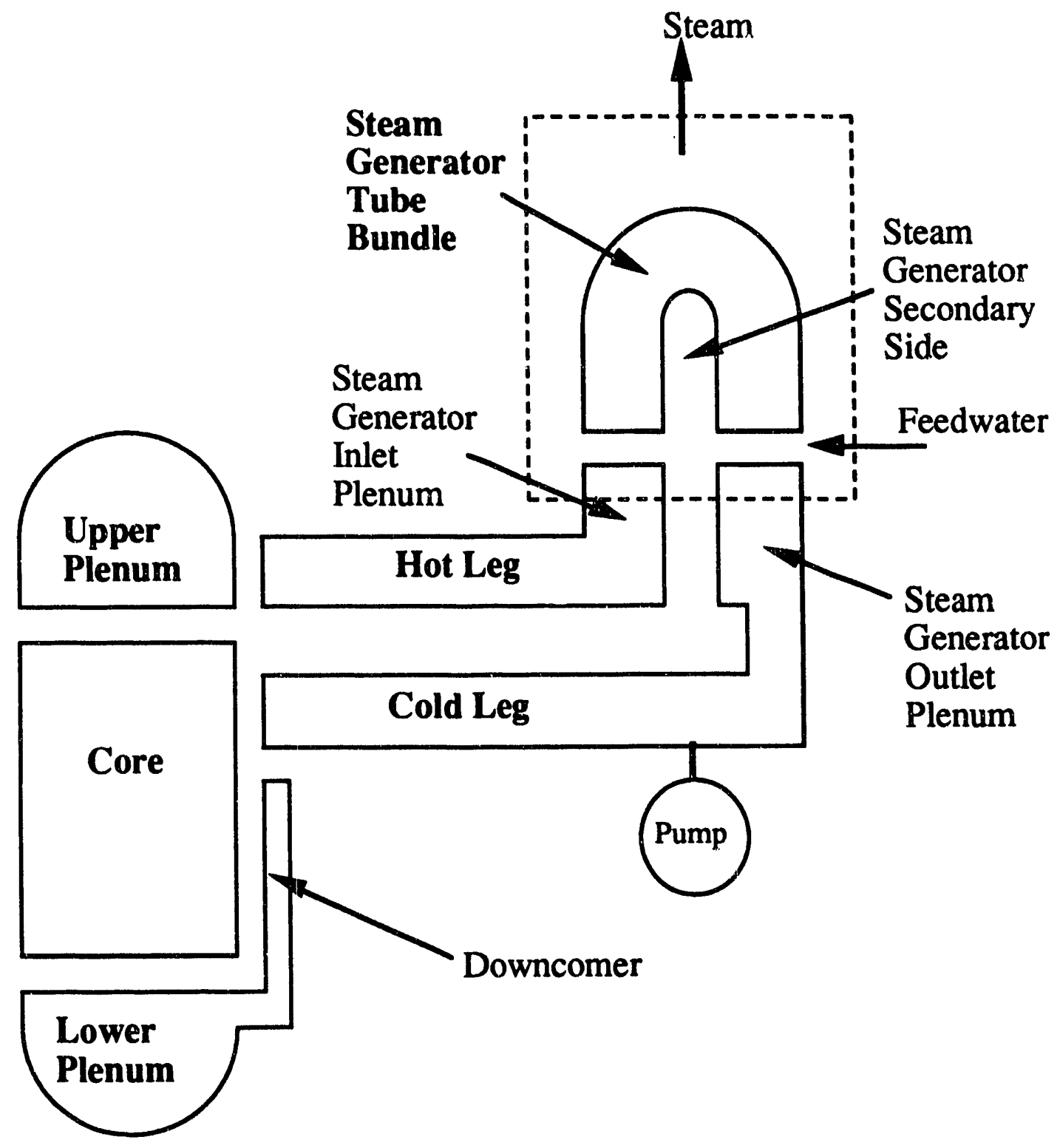

Figure 7.3.3-1 Control Regions for the Primary Coolant System Model. 


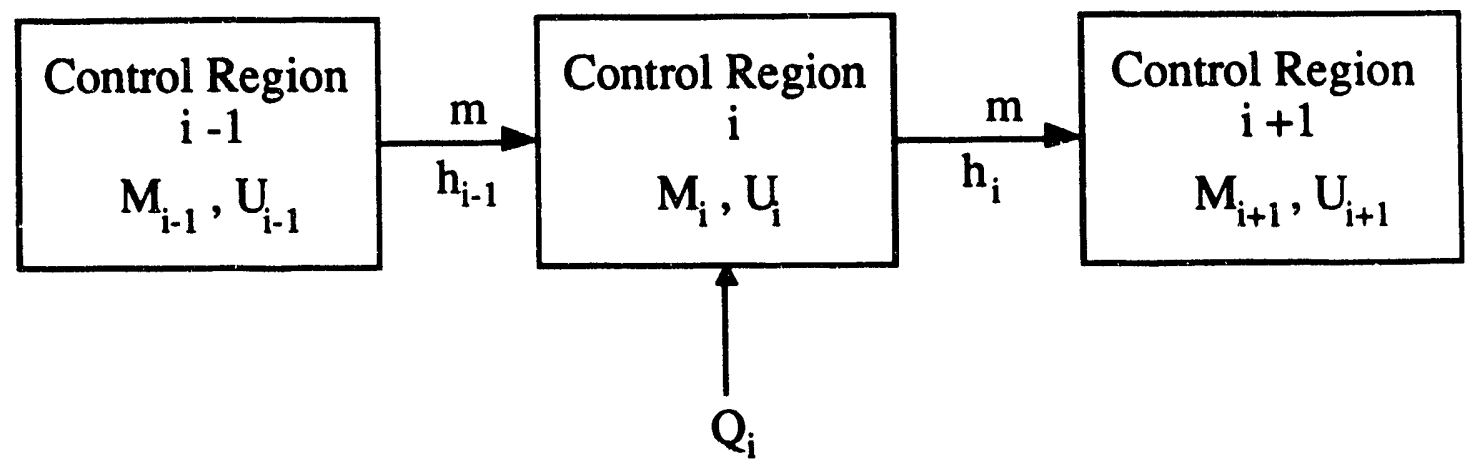

Figure 7.3.3-2 Typical Control Region for the Primary Coolant System Model. 
where $\mathrm{M}_{\mathrm{i}}$ is the total mass of the primary coolant in the ith control region,

$\dot{\mathrm{m}}_{\mathrm{i}}$ is the mass flowrate leaving the ith control region,

$U_{i}$ is the total internal energy of the primary coolant in the ith control region,

$h_{i} \quad$ is the mixing cup enthalpy leaving the ith control region, and

$\dot{Q}_{i}$ is the heat input rate for the ith control region.

Strohmayer suggested that the mass and energy equations could be combined because the mass flowrate through the primary coolant system is approximately uniform during normal operational transients [68]. Therefore, a single mass flowrate in the primary coolant loop was assumed and treatment of the mass conservation equation was not needed. This assumption is valid for transients in which the temporal variation of a control volume's mass is too small to affect the spatial mass flowrate distribution. A further assumption, and a very valid one under normal operational conditions, is that the coolant in the primary system is always single phase. Any vapor that might be produced in the hottest channel of the core would be condensed in the reactor vessel upper plenum before traveling to the hot leg. Under the above assumptions, Equations (7.3.3-1) and (7.3.3-2) can be expressed as follows:

$$
V_{i} \rho_{i} \frac{d u_{i}}{d t}=\dot{m}\left(h_{i}-h_{i-1}\right)+\dot{Q}_{i}
$$

where $V_{i}$ is the volume ith control region, $\rho_{i}$ is the coolant density of the ith control region, $u_{i}$ is the specific internal energy of the ith control region, and $\dot{m}$ is the primary coolant system flowrate. the relation:

Internal energy $(u)$ is related to the enthalpy $(h)$, pressure (p), and density $(p)$ by

$$
u=h-\frac{p}{\rho}
$$

Thus, for constant density, the temporal variation of the internal energy can be written as:

$$
\rho \frac{d u}{d t}=\rho \frac{d h}{d t}-\frac{d p}{d t}
$$

For slow pressure changes, the temporal variation of the pressure can be neglected. Also, it is assumed that the temporal variation of the static enthalpy of the ith control region equals that of the mixing cup enthalpy. Under these assumptions, the combined mass and energy equation can be expressed as follows: 


$$
M_{i} \frac{d h_{i}}{d t}=\dot{m}\left(h_{i}-h_{i-1}\right)+\dot{Q}_{i}
$$

where $h_{i}$ does not represent the average enthalpy of the ith control region, but rather the mixing cup enthalpy of the coolant leaving that region.

\subsubsection{Steam Generator Secondary Side Model}

U-tube steam generator water level dynamics are complex. In particular, there are counterintuitive effects known as 'shrink' and 'swell.' For example, upon increasing steam flowrate from a generator, the void fraction in the tube bundle region will increase. This will cause a temporary rise in level in the downcomer region and hence give the false impression that mass inventory is actually increasing. Accordingly, a detailed steam generator model is required. For this purpose, an existing steam generator model, that developed by Strohmayer [68] and improved by Choi [69], was adopted for the steam generator secondary side simulation.

This model is low-order, non-linear, and fast-running. Two salient features of the model are the incorporation of an integrated secondary recirculation loop momentum equation and the retention of all non-linear effects. This model has been validated over a wide range of steady-state and transient conditions by comparing results calculated with the model to experimental data or to other calculated results. Choi modified this model to improve the simulation of the shrink and swell effects. The modified model uses a different specific volume profile in the tube bundle region. The original and modified steam generator models are described in detail by Choi [69].

In this model, the steam generator secondary side is divided into four regions. These are those of the tube bundle, the riser, and the steam dome-downcomer which in turn is divided into a saturated and a subcooled region as shown in Figure 7.3.4-1. The steam generator secondary side equations consist of mass and energy conservation relations for each steam generator region and momentum conservation relations for the recirculation loop. The system equations were expressed as six, first-order, coupled, differential equations of the form:

$$
\dot{\mathbf{x}}=\mathbf{f}\left(\mathbf{x}, \dot{\mathrm{Q}}_{\mathrm{sg}}, \dot{\mathrm{m}}_{\mathrm{s}}, \dot{\mathrm{m}}_{\mathrm{fw}}, \mathrm{T}_{\mathrm{fw}}\right)
$$

where $x$ is a state vector whose elements are $U_{0}, V_{v},\left\langle\alpha_{r}\right\rangle,\left\langle\alpha_{n}\right\rangle, P_{s g}$, and $\dot{m}_{r}$, and where these elements are defined as follows:

$\mathrm{U}_{0}$ is the internal energy of the steam dome downcomer,

$V_{v}$ is the void volume in the steam dome downcomer,

$\left\langle\alpha_{p}\right\rangle$ is the void fraction at the riser exit,

$\left\langle\alpha_{n}\right\rangle$ is the void fraction at the tube bundle exit,

$P_{\text {sg }} \quad$ is the saturation pressure inside the steam generator, 


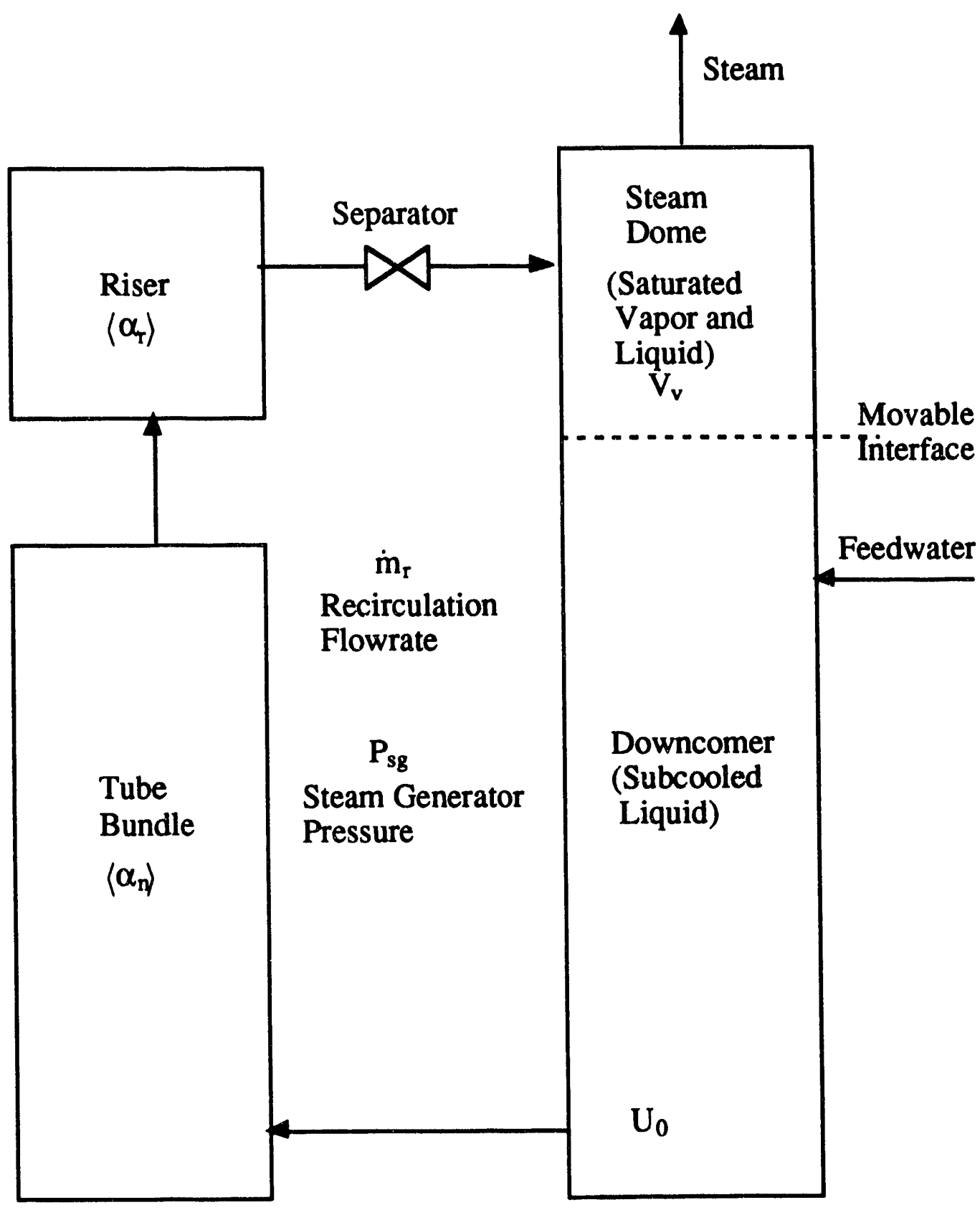

Figure 7.3.4-1 Steam Generator Secondary Side Model [68]. 
$\dot{\mathrm{m}}_{\mathrm{r}}$ is the recirculation flowrate in the steam generator,

$\dot{\mathrm{m}}_{\mathrm{s}}$ is the steam flowrate,

$\dot{\mathrm{m}}_{\mathrm{fw}}$ is the feedwater flowrate,

$\mathrm{T}_{\mathrm{fw}} \quad$ is the feedwater temperature, and

$\dot{\mathbf{Q}}_{\mathrm{sg}} \quad$ is the heat transfer rate from the primary to secondary side.

In order to increase thermal efficiencies and to reduce inverse response effects, the feedwater is often preheated by the steam extracted from the turbine. Thus, the feedwater temperature changes as a function of plant power. In this research, it is assumed that the feedwater temperature is a known function of the total plant power. Figure 7.3.4-2 shows the feedwater temperature as a function of a module power.

\subsubsection{Main Steam Line Common Header Model}

The main steam line common header (MSLCH) receives steam from each power module and discharges it to the turbine. Because the steam flowrate from each steam generator to the MSLCH depends on the hydraulic conditions that exist between that power module and the MSLCH, the MSLCH model must include momentum conservation equations. Figure 7.3.5-1 shows the MSLCH simulation model. The mass, energy, and momentum conservation equations that are solved consist of:

i) Mass Conservation Equation:

$$
\frac{d M_{m s}}{d t}=\sum_{i=1}^{N M} \dot{m}_{s, i}-\dot{m}_{m s}
$$

where $\mathbf{M}_{\mathrm{ms}}$ is the mass of steam in the MSLCH,

$\dot{\mathrm{m}}_{\mathrm{s}, \mathrm{i}}$ is the steam flowrate from the steam generator of the ith power module,

NM is the number of power modules, and

$\dot{\mathrm{m}}_{\mathrm{ms}}$ is the steam flowrate from the MSLCH to the turbine.

In Equation (7.3.5-1), the total mass in the MSLCH can be replaced by a product of volume and density. Thus,

$$
V_{m s} \frac{d \rho_{m s}}{d t}=\sum_{i=1}^{N M} \dot{m}_{s, i}-\dot{m}_{m s}
$$

where $\rho_{m s}$ is the density of the steam and moisture mixture in the MSLCH. It is calculated as follows: 


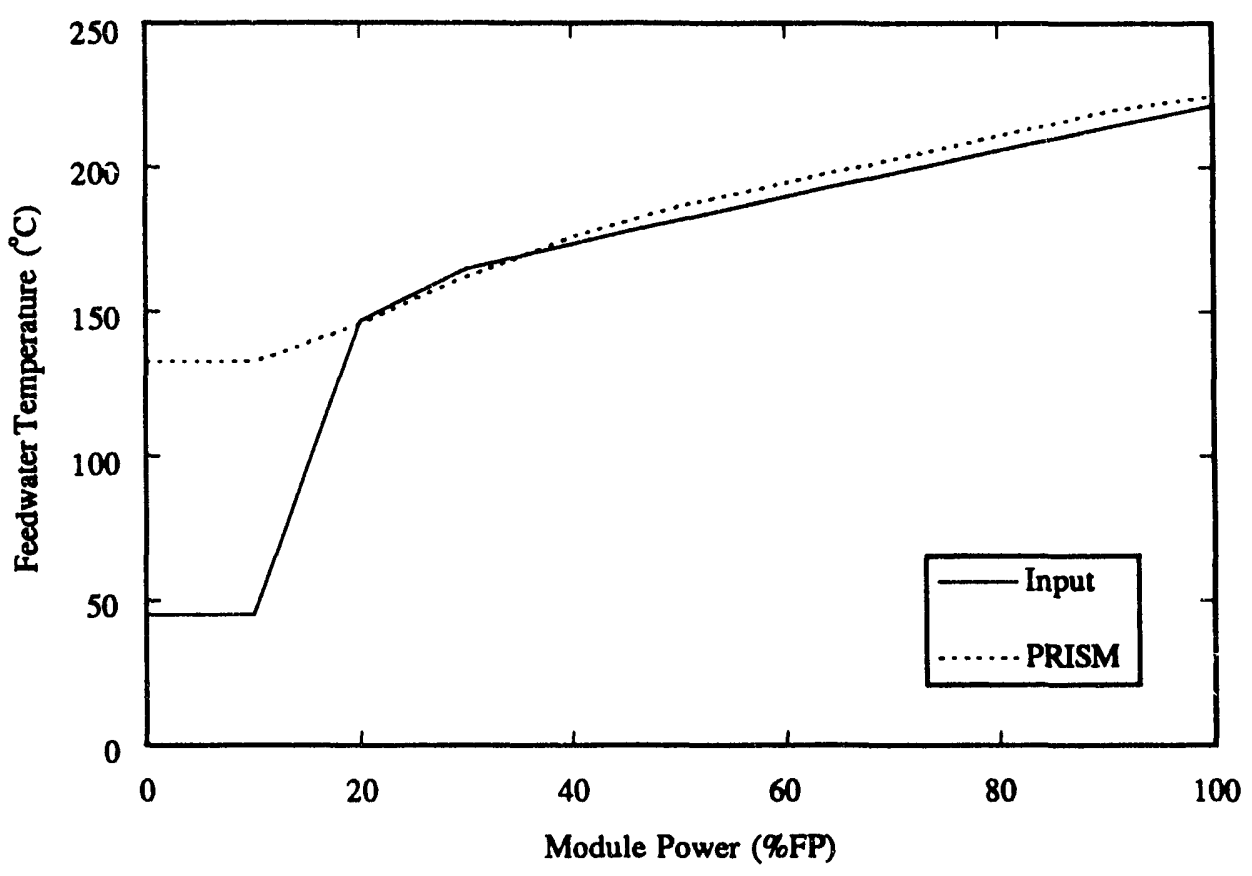

Figure 7.3.4-2 Feedwater Temperature as Function of Module Power. 


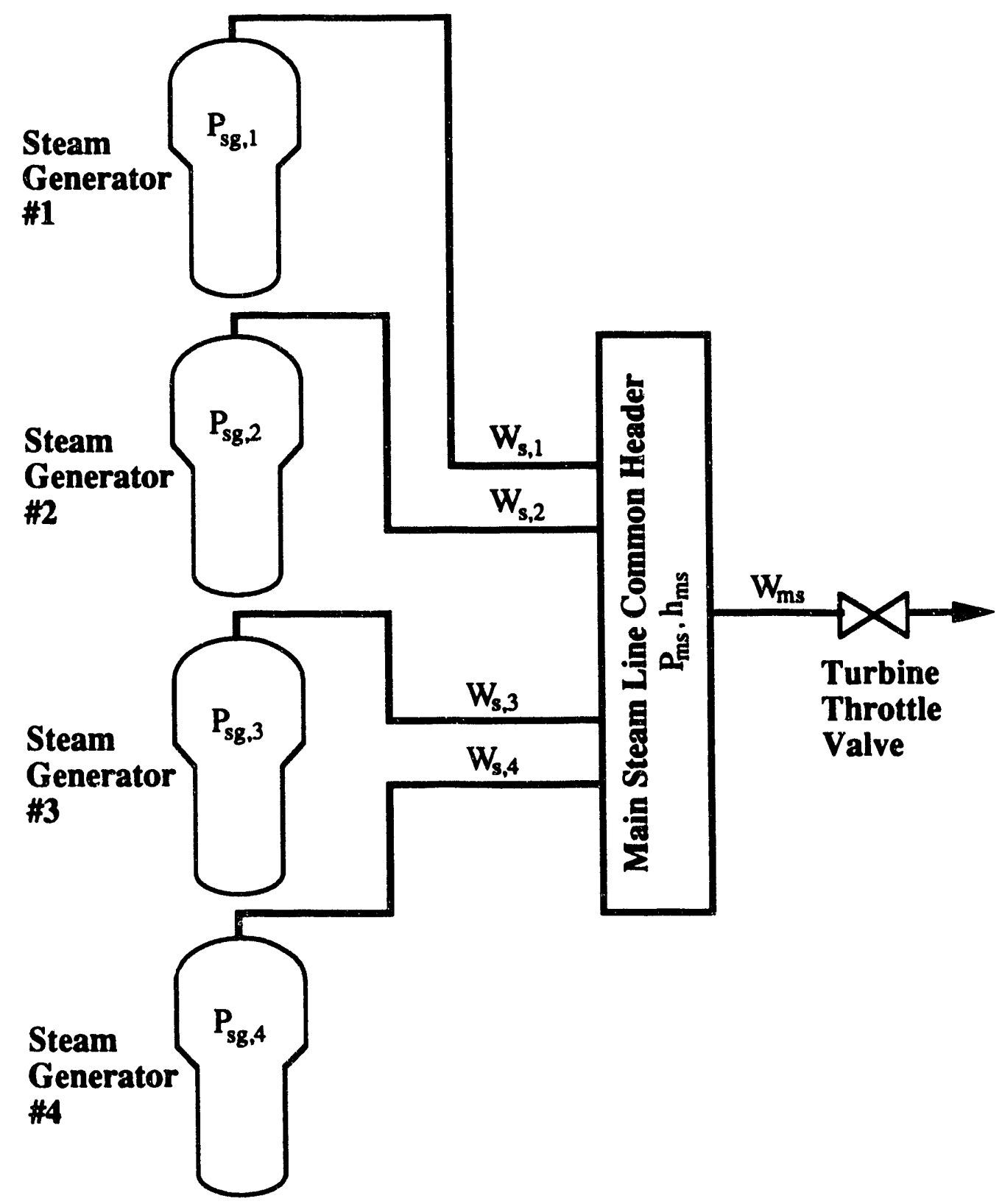

Figure 7.3.5-1 MSLCH Simulation Model. 


$$
\frac{1}{\rho_{m s}}=V_{f}+X_{m s} V_{f g}
$$

where $\mathrm{X}_{\mathrm{ms}}$ is the steam quality, $\mathrm{V}_{\mathrm{f}}$ is the specific volume of liquid, and $\mathrm{V}_{\mathrm{fg}}$ is the increase in specific volume upon evaporation. In the simulation model, steam quality is greater than unity if the steam is superheated. The quantity $\mathrm{d} \rho_{\mathrm{ms}} / \mathrm{dt}$ can be expanded in terms of state variables as:

$$
\frac{d \rho_{m s}}{d t}=\frac{\partial \rho_{m s}}{\partial P_{m s}} \frac{d P_{m s}}{d t}+\frac{\partial \rho_{m s}}{\partial X_{m s}} \frac{d X_{m s}}{d t}
$$

where:

$$
\begin{aligned}
& \frac{\partial \rho_{m s}}{\partial P_{m s}}=-\frac{1}{\rho_{m s}^{2}}\left(\frac{\partial V_{f}}{\partial P_{m s}}+X_{m s} \frac{\partial V_{f g}}{\partial P_{m s}}\right) \text { and } \\
& \frac{\partial \rho_{m s}}{\partial X_{m s}}=-\frac{1}{\rho_{m s}^{2}} V_{f g} .
\end{aligned}
$$

Substitution of Equation (7.3.5-4) into Equation (7.3.5-2) yields the final mass conservation equation:

$$
V_{m s}\left(\frac{\partial \rho_{m s}}{\partial P_{m s}} \frac{d P_{m s}}{d t}+\frac{\partial \rho_{m s}}{\partial X_{m s}} \frac{d X_{m s}}{d t}\right)=\sum_{i=1}^{N M} \dot{m}_{s, i}-\dot{m}_{m s}
$$

ii) Energy Conservation Equation:

$$
\frac{\mathrm{dU}}{\mathrm{ms}}=\sum_{\mathrm{i}=1}^{\mathrm{NM}} \dot{\mathrm{m}}_{\mathrm{s}, \mathrm{i}} \mathrm{h}_{\mathrm{sg}, \mathrm{i}}-\dot{\mathrm{m}}_{\mathrm{ms}} \mathrm{h}_{\mathrm{ms}}
$$

where $U_{m s}$ is the internal energy of the steam in the MSLCH,

$h_{\text {sg, } i}$ is the enthalpy of the steam flowrate from the steam generator of the ith power module, and

$h_{m s}$ is the enthalpy of steam flowrate to the turbine.

Similarly, the internal energy of the steam in the MSLCH can be represented by:

$$
\mathrm{U}_{\mathrm{ms}}=\mathrm{M}_{\mathrm{ms}} \mathrm{h}_{\mathrm{ms}}-\mathrm{V}_{\mathrm{ms}} \mathrm{P}_{\mathrm{ms}}
$$

where:

$$
h_{m s}=h_{f}+X_{m s} h_{f g} .
$$


Insertion of Equation (7.3.5-9) into Equation (7.3.5-8) yields:

$$
M_{m s} \frac{d h_{m s}}{d t}-V_{m s} \frac{d P_{m s}}{d t}=\sum_{i=1}^{N M} \dot{m}_{s, i}\left(h_{s g, i}-h_{m s}\right)
$$

where:

$$
\begin{aligned}
& \frac{d h_{m s}}{d t}=\frac{\partial h_{m s}}{\partial P_{m s}} \frac{d P_{m s}}{d t}+\frac{\partial h_{m s}}{\partial X_{m s}} \frac{d X_{m s}}{d t}, \\
& \frac{\partial h_{m s}}{\partial P_{m s}}=\frac{\partial h_{m s}}{\partial P_{m s}}+X_{m s} \frac{\partial h_{m s}}{\partial P_{m s}} \text {, and } \\
& \frac{\partial h_{m s}}{\partial X_{m s}}=h_{f g} .
\end{aligned}
$$

Substitution of Equation (7.3.5-11) into Equation (7.3.5-10) yields an energy conservation equation of the form:

$$
M_{m s}\left(\frac{\partial h_{m s}}{\partial P_{m s}} \frac{d P_{m s}}{d t}+\frac{\partial h_{m s}}{\partial X_{m s}} \frac{d X_{m s}}{d t}\right)-V_{m s} \frac{d P_{m s}}{d t}=\sum_{i=1}^{N M} \dot{m}_{s, i}\left(h_{s g, i}-h_{m s}\right)
$$

\section{iii) Momentum Conservation Equation:} module is:

The momentum conservation equation through the steam line of the ith power

$$
\left(\frac{L}{A}\right)_{i} \frac{d \dot{m}_{s, i}}{d t}=P_{s s, i}-P_{m s}-F_{i} \quad i=1,2, \ldots, N M
$$

where $\left(\frac{L}{A}\right)_{i}$ is a geometric parameter that relates the steam flowrate to the inertia of the steam in the ith power module,

$\mathbf{P}_{\mathbf{s g}, \mathrm{i}}$ is the pressure inside the steam generator of the ith power module,

$P_{m s}$ is the pressure at the MSLCH, and

$F_{i} \quad$ is the resistance through the steam line of the ith power module.

Pressure losses in the line from each steam generator to the main steam header occur as both the result of friction losses within the pipe itself and specific losses resulting from components within the pipe including valves, restrictors, and several curvatures. 
Given that these steam lines are typically 30 " pipes, it was assumed that friction pressure losses were negligible when compared to those resulting from the contained components. Also, while each line may be different in its length and structure, these differences were not considered. Thus, the resistance to flow through the steam line of the ith power module was expressed as:

$$
F_{i}=\frac{f_{r}}{2 \rho_{m s}} \frac{\dot{m}_{s, i}^{2}}{A_{s}^{2}}+\left(\frac{1}{\rho_{m s}}-\frac{1}{\rho_{s g, i}}\right) \frac{\dot{m}_{s, i}^{2}}{A_{s}^{2}}
$$

where $f_{r}$ is the flow resistance, $A_{s}$ is the cross-sectional area of the steam line, and $\rho_{s g, i}$ is the steam density in the steam generator of the ith power module.

\subsubsection{Matrix Representation of MSLCH State Equations}

The state equations of the MSLCH consist of mass, energy, and momentum relations. The state variables are the pressure and quality at the MSLCH and the steam flowrate from each power module. Equations (7.3.5-7), (7.3.5-14), and (7.3.5-15) can be expressed in matrix form as:

$$
A \dot{x}=f\left(x, P_{s g, i}, \dot{m}_{m s}\right)
$$

where:

$$
\begin{aligned}
& x=\left[P_{m s}, x_{m s}, \dot{m}_{s, i}\right]^{T} \quad i=1,2, \ldots, N M \\
& A=\left[\begin{array}{ll}
A_{1} & O_{1} \\
\mathbf{O}_{2} & A_{2}
\end{array}\right] \\
& A_{1}=\left[\begin{array}{cc}
V_{m s} \frac{\partial \rho_{m s}}{\partial P_{m s}} & V_{m s} \frac{\partial \rho_{m s}}{\partial X_{m s}} \\
-V_{m s}+M_{m s} \frac{\partial h_{m s}}{\partial P_{m s}} & M_{m s} \frac{\partial h_{m s}}{\partial X_{m s}}
\end{array}\right] \text {, } \\
& A_{2}=\text { diagonal }\left\{\left(\frac{L}{A}\right)_{i}\right\} \quad i=1,2, \ldots, N M
\end{aligned}
$$

$f$ is a super matrix whose element matrices are $f_{1}$ and $f_{2}$. The former is defined as: 


$$
f_{1}=\left[\begin{array}{l}
\sum_{i=1}^{N M} \dot{m}_{s, i}-\dot{m}_{m s} \\
\sum_{i=1}^{N M} \dot{m}_{s, i}\left(h_{s g, i}-h_{m s}\right)
\end{array}\right]
$$

and the latter $\left(f_{2}\right)$ is an (NM $\left.\times 1\right)$ matrix whose elements are:

$$
f_{2, i}=P_{m s}-P_{m s}-F_{i}, \quad i=1,2, \ldots, N M
$$

\subsection{Numerical Solution Methods}

Described here are both the numerical representations and procedures used to solve the models that were developed as part of the multi-modular plant simulator.

\subsubsection{Einite Difference Approximation to System Equations}

The describing equations for each system model were approximated using finite differences. The resulting relations are given here.

\subsubsection{Neutron Kinetics Model}

In order to solve the point kinetics equations without undue numerical stability restrictions, an implicit procedure was employed. For this purpose, a method used by Cabral was adopted because it could be executed quickly and because it gave a solution that was sufficiently accurate for simulation and control studies [59].

Equations (7.3.1-1) and (7.3.1-2) are transformed into finite difference equations through the use of first-order backwards differences. Thus,

$$
\begin{aligned}
& \frac{T^{n}-T^{n-1}}{\Delta t}=\frac{\rho^{n}-\bar{\beta}}{l^{n}} T^{n}+\sum_{i=1}^{N} \lambda_{i} C_{i}^{n} \\
& \frac{C^{n}-C^{a-1}}{\Delta t}=\frac{\bar{\beta}}{l^{l}} T^{n}-\lambda_{i} C_{i}^{n} \quad i=1,2, \ldots, N
\end{aligned}
$$

where the superscript $\mathbf{n}$ denotes the time step and $\Delta t$ is the time step size. In Equations (7.4.1.1-1) and (7.4.1.1-2), all state variables are unknown at the current time step. Therefore, these equations define a set of linear equations in $\mathrm{N}+1$ unknowns. These are of the form:

$$
\frac{x^{n}-x^{n-1}}{\Delta t}=f\left(x^{n-1}, p^{n}\right)
$$


where $\mathrm{x}^{\mathrm{n}}$ is a column vector of the neutron power and the precursor concentrations at each time step. The reactivity at the current time step is determined from the power demand and the reactor state variables at that same time step. Thus,

$$
\rho^{n}=f\left(T_{f}^{n}, T_{c}^{n}, C_{B}^{n}, X e^{n}, 1^{n}\right)
$$

where $T_{f}^{n}$ and $T_{c}^{n}$ represent the fuel and coolant average temperatures, $C_{B}^{n}$ and $X e^{n}$ are the boron and xenon concentrations, and $l^{n}$ is the control reactivity at the current time step.

\subsubsection{Fuel Temperature Model}

The heat conduction equations, Equations (7.3.2-1) and (7.3.2-2), are solved explicitly by applying first-order forwand differences to the time derivatives. Thus,

and

$$
M_{f} C_{f}\left(\frac{\vec{T}_{f}^{n}-\vec{T}_{f}^{n-1}}{\Delta t}\right)=Q^{n-1}-\frac{N L}{R_{g}}\left(\vec{T}_{f}^{n-1}-\vec{T}_{c l}^{n-1}\right)
$$

$$
M_{c l} C_{c l}\left(\frac{\bar{T}_{c l}^{n}-\bar{T}_{c l}^{n-1}}{\Delta t}\right)=\frac{N L}{R_{g}}\left(\bar{T}_{f}^{n-1}-T_{c l}^{n-1}\right)-\frac{N L}{R_{c}}\left(\bar{T}_{c l}^{n-1}-\bar{T}_{c}^{n-1}\right) .
$$

Equations (7.4.1.2-1) and (7.4.1.2-2) form a linear equation set in two unknowns, the average fuel and cladding temperature. Thus, they can be written as:

$$
\frac{x^{n}-x^{n-1}}{\Delta t}=f\left(x^{n-1}, T_{c}^{n-1}, \dot{Q}^{n-1}\right)
$$

where $x^{n}$ is a column vector of the average fuel and cladding temperatures at the current time step, $T_{c}^{n-1}$ is the coolant average temperature at the previous time step, and $\dot{Q}^{n-1}$ is the neutron power at the previous time step. The property correlations for the fuel and cladding materials as a function of fuel and cladding temperatures are taken from the THERMIT program which was developed at MIT [80].

\subsubsection{Primary Coolant System Model}

The difference equation set for the primary coolant system is obtained by specifying a mass-energy relation of the form of Equation (7.3.3-6) for each control region. Explicit finite difference equations are then obtained by approximating the time derivatives in those equations as first-order backwards differences. Thus, the following is obtained for each node in the model:

Core:

$$
M_{c}\left(\frac{h_{c}^{n}-h_{c}^{n-1}}{\Delta t}\right)=\dot{m}\left(h_{l p}^{n}-h_{c}^{n-1}\right)+\dot{Q}_{t h}^{n-1}
$$


Reactor Vessel Upper Plenum:

$$
M_{u p}\left(\frac{h_{u p}^{n}-h_{u p}^{n-1}}{\Delta t}\right)=\dot{m}\left(h_{c}^{n-1}-h_{u p}^{n-1}\right)
$$

Hot Leg and Steam Generator Inlet Plenum:

$$
M_{h l}\left(\frac{h_{h l}^{n}-h_{h l}^{n-1}}{\Delta t}\right)=\dot{m}\left(h_{u p}^{n-1}-h_{h l}^{n-1}\right)
$$

Steam Generator Tube (Primary Side):

$$
M_{\text {sgp }}\left(\frac{h_{s g p}^{n}-h_{s g P}^{n-1}}{\Delta t}\right)=\dot{m}\left(h_{h 1}^{n-1}-h_{s g p}^{n-1}\right)-\dot{Q}_{s g}^{n-1}
$$

Cold Leg and Steam Generator Outlet Plenum:

$$
M_{\mathrm{cl}}\left(\frac{h_{\mathrm{cl}}^{\mathrm{n}}-\mathrm{h}_{\mathrm{cl}}^{\mathrm{n}-1}}{\Delta \mathrm{t}}\right)=\dot{\mathrm{m}}\left(\mathrm{h}_{\mathrm{sgp}}^{\mathrm{n}-1}-\mathrm{h}_{\mathrm{cl}}^{\mathrm{n}-1}\right)+\dot{\mathrm{Q}}_{\mathrm{pump}}
$$

Reactor Vessel Lower Plenum:

$$
M_{l p}\left(\frac{h_{l p}^{n}-h_{l p}^{n-1}}{\Delta t}\right)=\dot{m}\left(h_{c l}^{n-1}-h_{l p}^{n-1}\right)
$$

where following subscript nomenclature is used:

c denotes reactor core,

up denotes reactor vessel upper plenum,

hl denotes hot leg,

sgp denotes steam generator primary side coolant,

cl denotes cold leg, and

Ip denotes reactor vessel lower plenum.

$\dot{\mathrm{Q}}_{\text {pump }}$ is the energy transferred to the coolant by the coolant pump and it is assumed to be a known constant at all times. $\dot{Q}_{\text {sg }}^{n-1}$ denotes the heat transfer rate from primary to the secondary side of the steam generator at the previous time step. Therefore, the above form a linear set in six unknowns. These are the enthalpies of each control region. Thus, 


$$
\frac{x^{n}-x^{n-1}}{\Delta t}=f\left(x^{n-1}, \dot{Q}_{t h}^{n-1}, \dot{Q}_{s g}^{n-1}\right) .
$$

In order to solve Equation (7.4.1.3-2), the heat transfer rate from the primary to the secondary side of the steam generator must be known. In order to do this, Strohmayer's heat transfer model was adopted [68]. In this model, the heat transfer rate to the secondary side is a function of the log mean temperature difference between the primary coolant and the secondary saturated temperatures. Thus,

$$
\dot{\mathrm{Q}}_{\text {sg }}=f\left(\mathrm{~T}_{\text {sgpin }}, \mathrm{T}_{\text {sgpout }}, \mathrm{T}_{\text {sgs }}\right)
$$

where $T_{\text {sgpin }}$ and $T_{\text {sgpout }}$ are the primary coolant temperatures at the steam generator tube bundle inlet and outlet respectively, and $T_{\text {sgs }}$ is the saturated temperature of the steam generator secondary side.

\subsubsection{Steam Generator Secondary Side Model}

Finite difference equations are obtained from Equation (7.3.4-1) by applying first order forward finite differences to the time derivatives. Thus,

$$
\frac{x^{n}-x^{n-1}}{\Delta t}=f\left(x^{n-1}, \dot{Q}_{s g}^{n-1}, \dot{m}_{s}^{n-1}, \dot{m}_{f w}^{n-1}, T_{f w}^{n-1}\right)
$$

where $x^{n}$ is a column vector whose elements are the internal energy at the bottom of the steam dome downcomer, the void volume in the steam dome downcomer, the void fraction at the tube bundle exit, the void fraction at the riser exit, the saturation pressure inside the steam generator, and the recirculation flowrate in the steam generator. Each of these variables is at the current time step.

\subsubsection{Main Steam Line Common Header Model}

The finite difference version of Equation (7.3.5.1-1) is:

$$
A^{n-1}\left(\frac{x^{n}-x^{n-1}}{\Delta t}\right)=f\left(x^{n-1}, P_{s g, i}^{n-1}, \dot{m}_{m s}^{n-1}\right)
$$

This matrix equation is a linear equation set in $(\mathrm{NM}+2)$ unknowns. These are the pressure and quality of the MSLCH and the steam flowrates from the power modules. The steam flowrate from the MSLCH at the current time step is calculated from the current load demand.

\subsubsection{Numerical Solution Procedures}

In Section 7.4.1, five subsets of finite difference equations were obtained. Four were for the power modules and one was for the main steam line common header. Figure 7.4.2-1 shows the information flow among them. The overall plant can be now simulated by specifying the demanded load and a controller logic. In this section, solution procedures for both steady-state and transient operations are explained. 


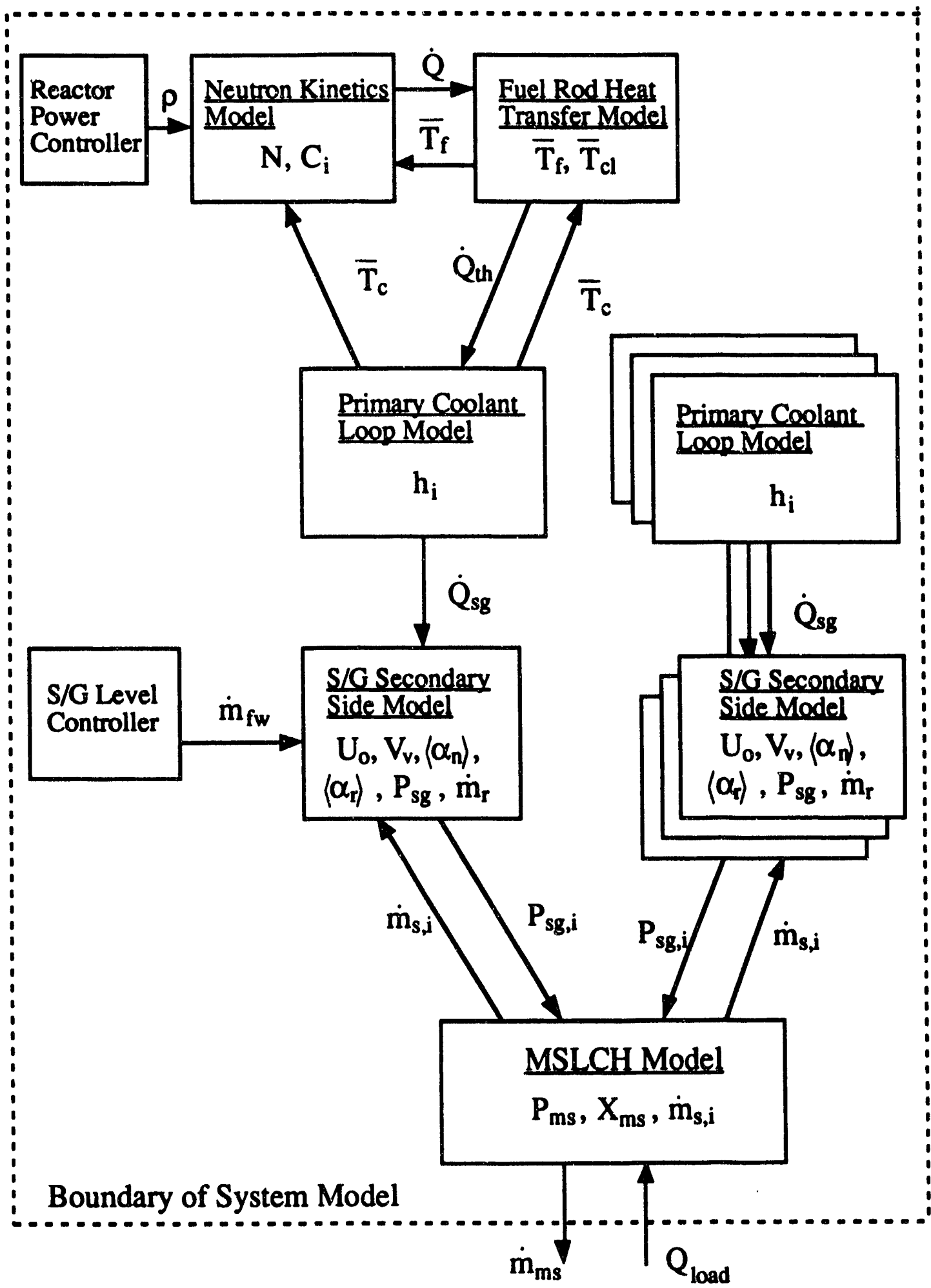

Figure 7.4.2-1 Information Flow Between the System Submodels. 


\subsubsection{Steady-State Simulation}

Initialization of the model requires that the module power and coolant average temperatures for the module that is operating at the highest power level be specified. All other state variables can then be determined as described below.

Figure 7.4.2.1-1 is a flowchart that shows the steady-state solution procedure. First, the input data is read and all geometries, variables, and arrays are initialized. Second, the steady-state system equations are solved. For the primary and secondary reactor coolant systems, a special procedure is required to allow each power module to operate at a different load. Figure 7.4.2.1-2 shows the procedure used to solve for the steady-state, thermal-hydraulic state variables. It is as follows:

1. Solve the primary loop mass-energy equation for the highest-power module using the specified reactor power and coolant average temperatures.

2. Estimate the steam generator pressure in the highest-power module using the heat transfer constraint from the primary to secondary coolant.

3. Initialize the steam generator state variables using the power and steam generator pressure.

4. For the MSLCH, calculate both the pressure of the MSLCH and those of the steam generators for each power module from the pressure drop through each steam line.

5. Calculate the steam flowrate for each of the other power modules using each module's power and steam generator pressure.

6. Initialize the steam generator state variables in the other power modules using the power and steam generator pressures.

7. Determine the primary coolant temperatures at the steam generator inlet and outlet using the heat transfer constraint from the primary to secondary coolant.

8. Determine the core inlet temperature and all other coolant temperatures for the other power modules.

Fuel and cladding average temperatures are calculated from the average coolant temperature and power. The xenon concentration equations are solved to obtain the initial xenon concentrations. The point kinetics equations are also initialized to calculate the initial concentrations of the delayed neutron precursors. Initial reactivity is assumed to be zero (steady-state).

\subsubsection{Transient Simulation}

Once the steady-state solution has been determined, the transient simulation is begun. The transient simulation routine consists of a simulation routine for the plant controller as well as the five subsets of system difference equations (one for each power 
Input and Initialization

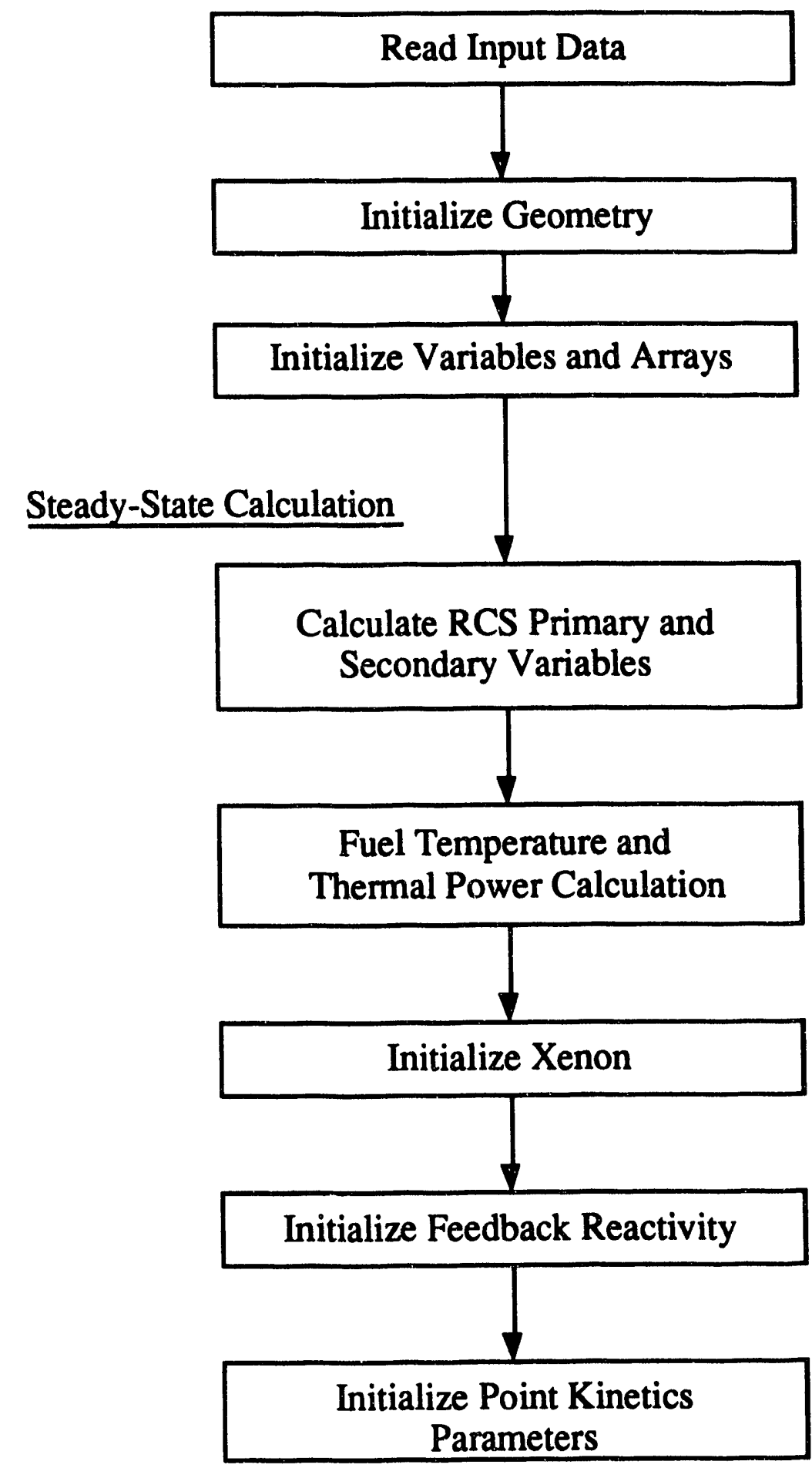

Figure 7.4.2.1-1 Steady-State Simulation Procedure. 
Highest-Power Module
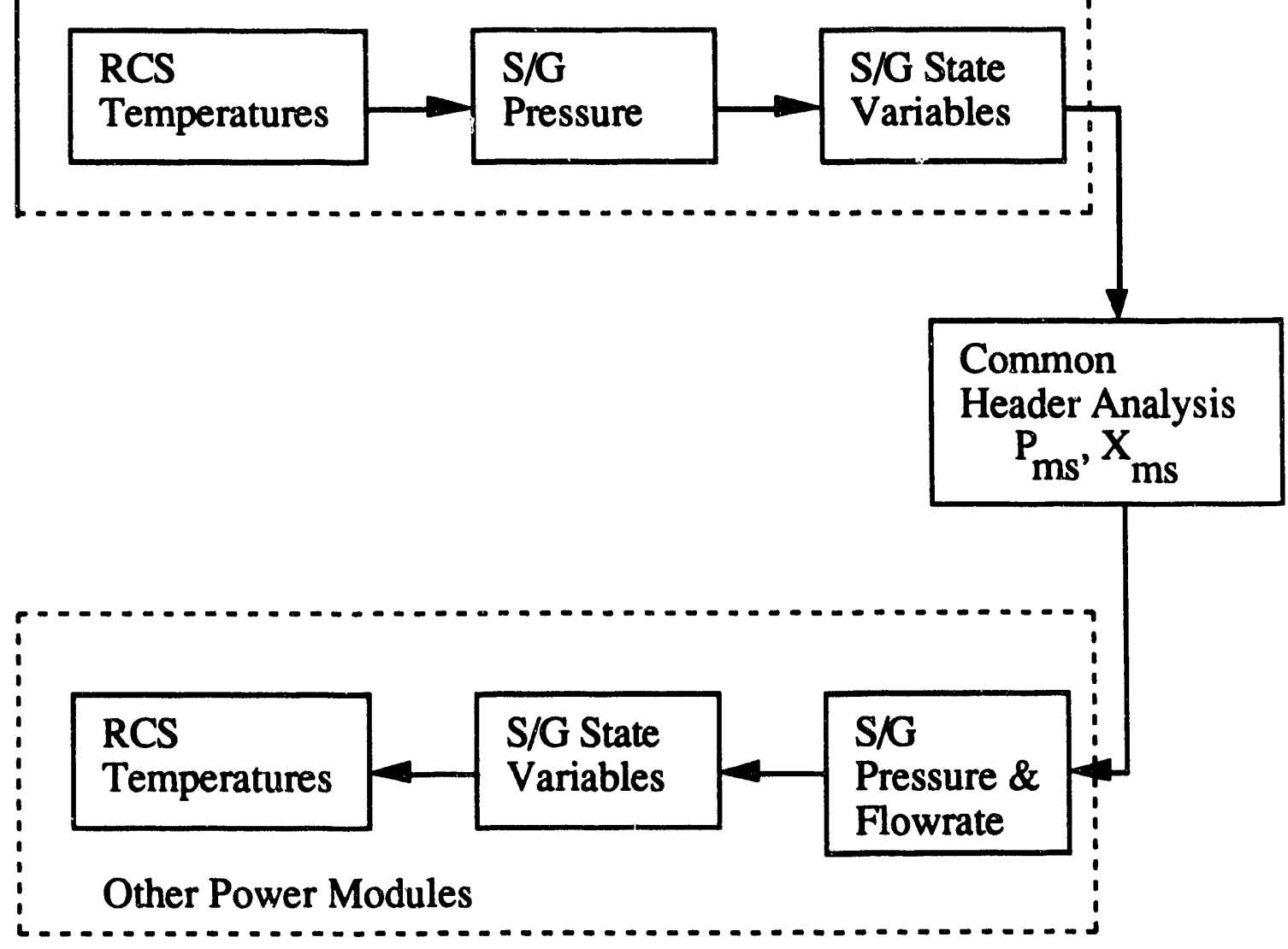

Figure 7.4.2.1-2 Flow Chart for Steady-State Calculation of Reactor Primary and Secondary Coolant System State Variables. 
module and one for the MSLCH). A tandem approach is used to advance the transient solution. Figure 7.4.2.2-1 illustrates the transient simulation procedure.

1. The boundary conditions for each time step consist of the demanded load and the non-modeled parameters in the simulation program including the primary coolant flowrate and pressure, charging flowrate, and soluble boron concentration in the charging flowrate.

2. The ractor coolant system and steam generator secondary side equations for each power module are solved simultaneously to give coolant average temperatures and steam generator pressures.

3. The MSLCH equations are solved to determine the steam flowrate from each power module.

4. The fuel and coolant average temperatures are calculated for each power module.

5. The control action to change control rod motion and feedwater flowrate is simulated.

6. The point kinetics equations are solved to calculate the reactor power.

The above six steps are then repeated for the duration of the simulation.

\subsection{Evaluation of Multi-Modular Plant Simulator}

In this section, the results of the validation and testing of the developed simulation program are described. The validation and testing were accomplished by simulating static and transient cases using typical Westinghouse PWR plant data. This was necessary because neither reference plant design data nor a reference simulation program for a PWRtype, multi-modular power plant exist. Simulation results were compared with either actual plant operational data or with the results of other reference programs. It is important to note that primary and secondary coolant conditions for PWR-type multi-modular power plants are essentially the same as those for a PWR power plant provided that all the modules are operated at the same demanded load. Some of the subsystem simulation models, including those for the steam generator secondary side, steam generator heat transfer, point kinetics, and primary loop had been previously validated [59,71]. Therefore, the focus of attention here was the validation of the overall integrated performance of the simulation program, PMSIM.

\subsubsection{Steady-State Simulation}

The validation of the steady-state simulation was accomplished by analyses of PWR steady-state characteristics. Simulation results were compared with those of the Pressurized Reactor Interactive Simulation Model (PRISM)[72]。 For these simulations it was assumed that all modules were operated at the same load. Figures 7.5.1-1 through 7.5.1-3 show comparisons of the average primary coolant temperature, steam flowrate, and steam generator pressure as a function of module power. These show that the PMSIM program was functioning properly. 


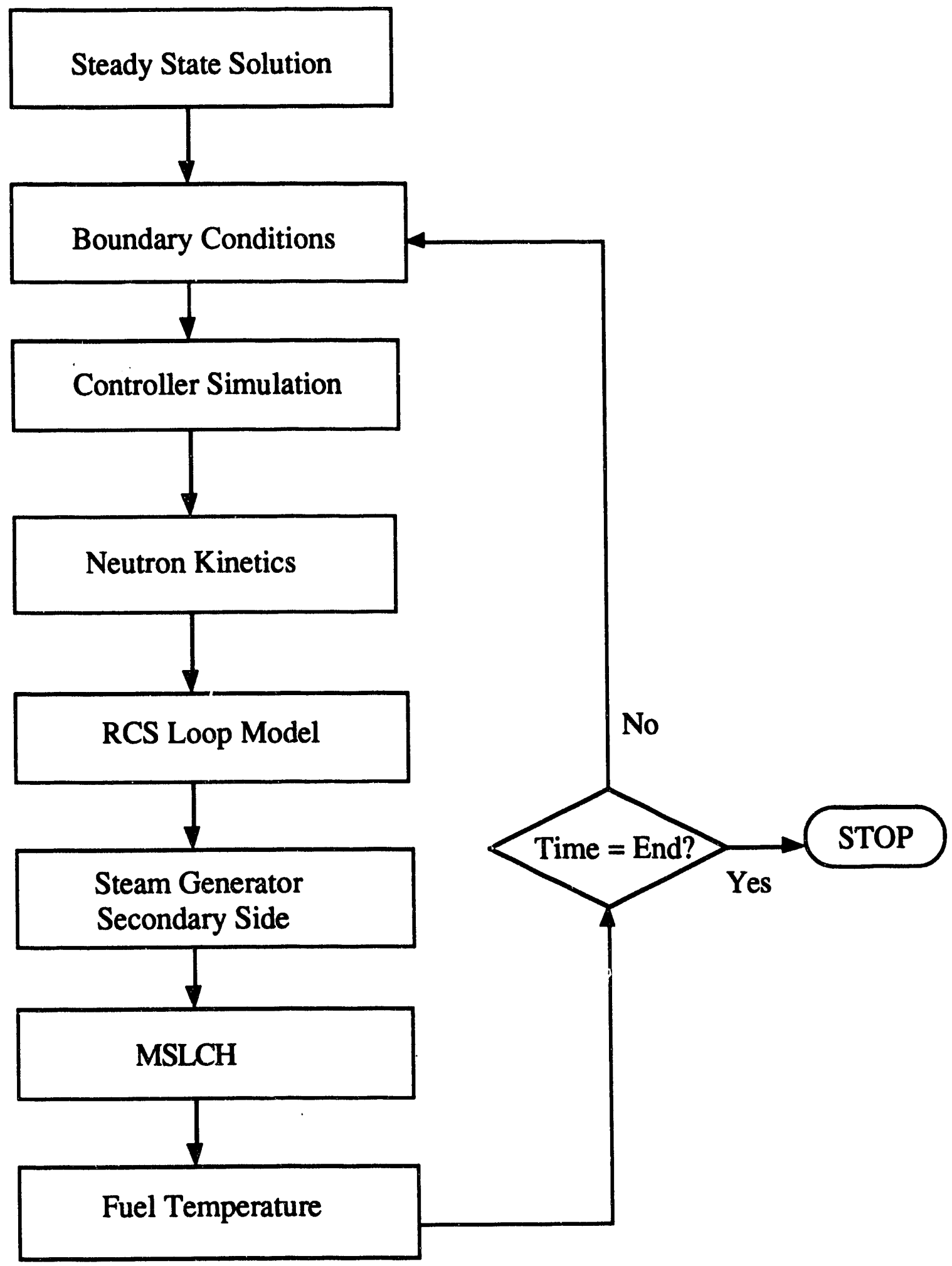

Figure 7.4.2.2-1 Transient Simulation Procedure. 


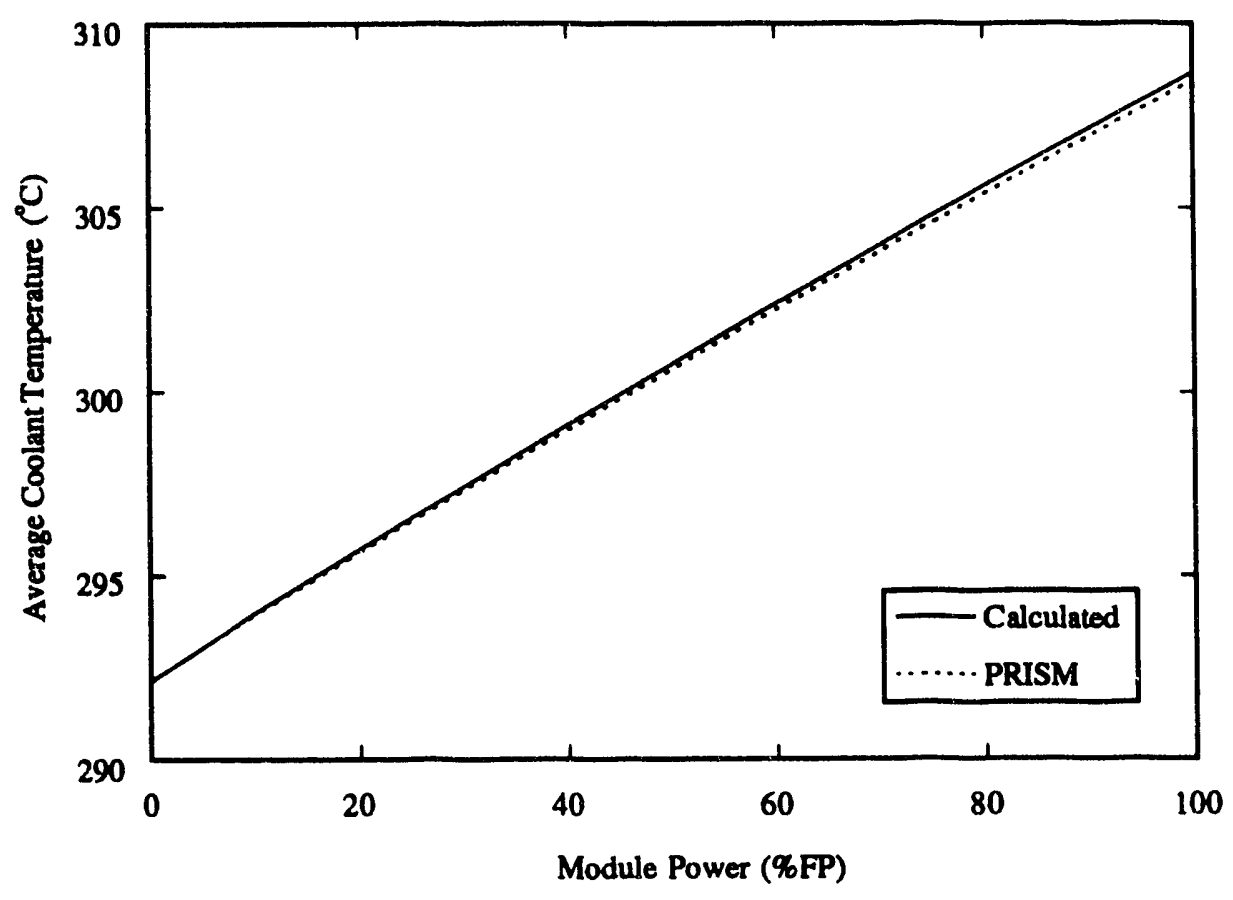

Figure 7.5.1-1 Average Primary Coolant Temperature at Steady-State.

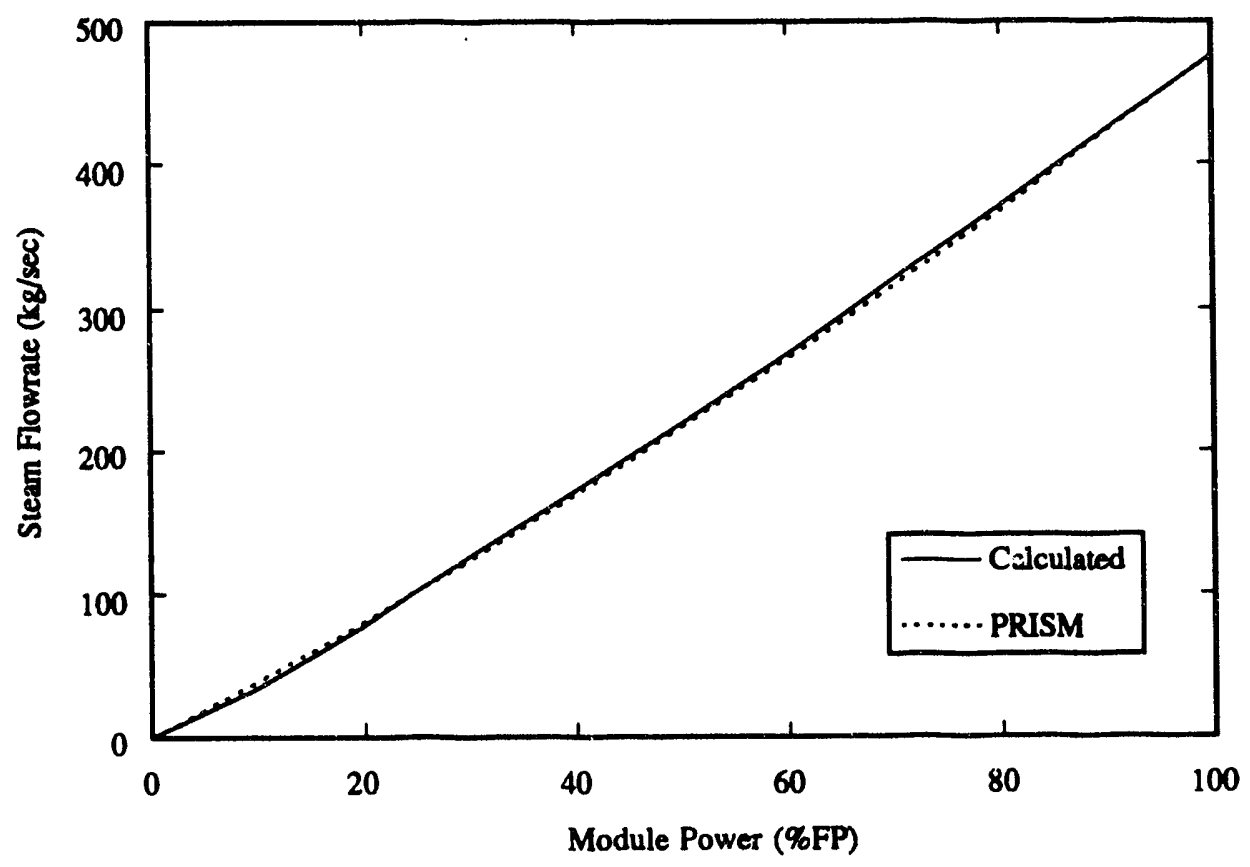

Figure 7.5.1-2 Steam Flowrate from Each Steam Generator at Steady-State. 


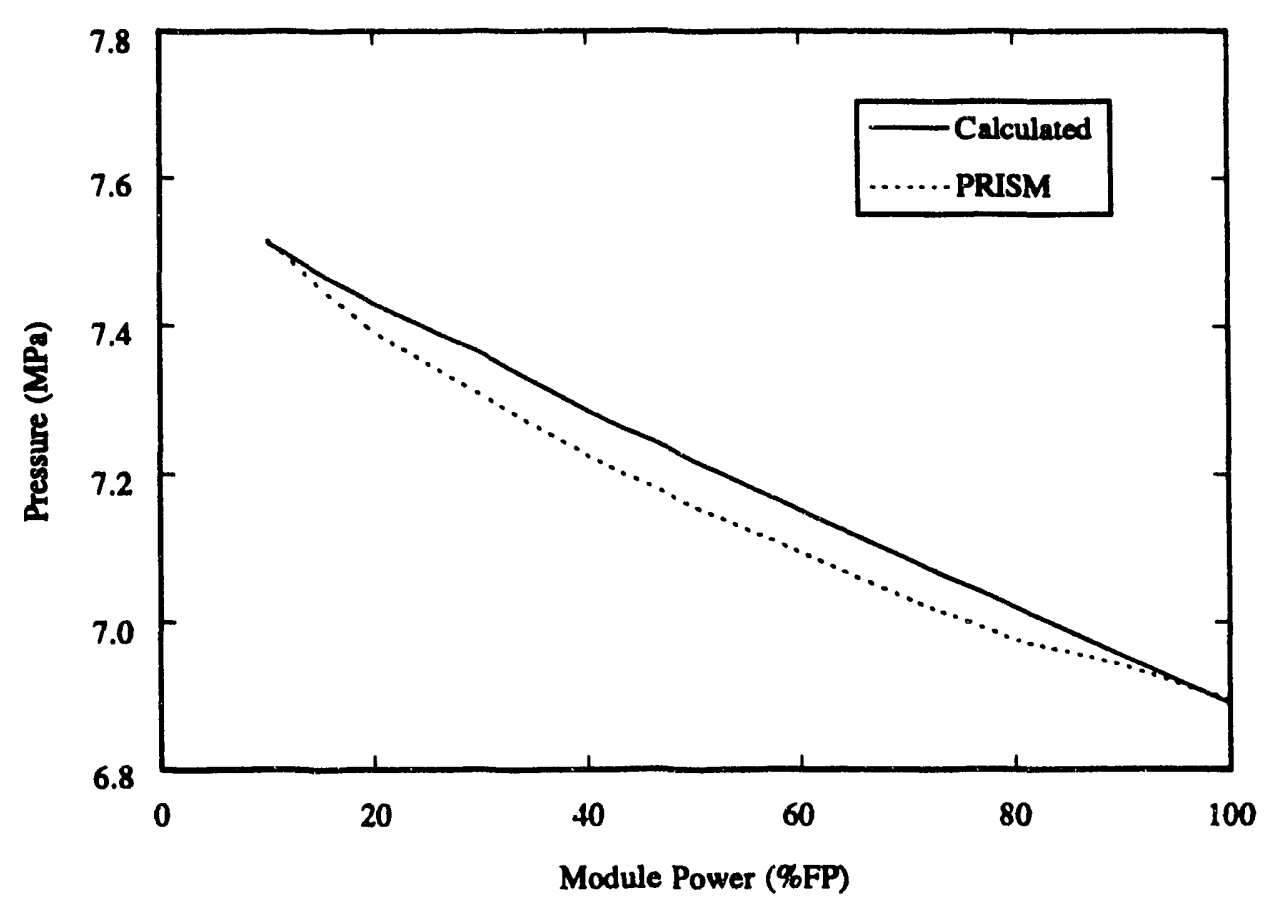

Figure 7.5.1-3 Steam Generator Internal Pressure at Steady-State. 


\subsubsection{Transient Simulation}

In order to validate the transient simulation capability of the PMSIM program, the following cases were analyzed:

1. Null Transient Simulation: Simulate transients with no perturbations and no control action and compare the results with steady-state cases.

2. Symmerry Test: Perturb only one of the power modules and compare the transient behavior of each of the other modules. The initial configuration for these tests was that all modules were at a uniform power level.

\subsubsection{Null Transient Simulation}

The purpose of this test was to establish the numerical stability of the simulation program. Given that some of the system differential equations are solved using explicit numerical approximation methods, it was expected that there would be a limit on the time step size. If time step sizes in excess of this limit were attempted, numerical instabilities would appear. It would have been desirable to calculate this limit from theory. However, because the simulation program consisted of many complex differential equations, it was not possible to do this. Therefore, the allowable time step size was found by performing a series of transients, each with a different time step size. For these simulations, the initial power levels of modules \#1, \#2, \#3, and \#4 were assumed to be $100 \%$ MFP (Module Full Power), $95 \%$ MFP, $90 \%$ MFP, and $85 \%$ MFP, respectively. The null transient was simulated up to $700 \mathrm{~s}$ and calculation time step sizes were increased by $0.01 \mathrm{~s}$ until numerical instability appeared.

Figure 7.5.2.1-1 shows the steam flowrate of module \#1 when time step sizes of $0.18 \mathrm{~s}$ and $0.19 \mathrm{~s}$ were used. If time step sizes of less than $0.18 \mathrm{~s}$ were used, neither oscillation nor divergence of the solutions occurred. However, if a $0.19-\mathrm{s}$ time step size was used, the solutions oscillated and diverged. Based on these results, it was concluded that the simulation prog $\mathrm{m}$ had a time step size limit between $0.18 \mathrm{~s}$ and $0.19 \mathrm{~s}$ and that time step sizes less thari $0.18 \mathrm{~s}$ should be used to avoid numerical instability. Figure 7.5.2.1-2 shows the time behavior of the average coolant temperature and steam generator pressure for module \#1 when $0.18 \mathrm{~s}$ and $0.19 \mathrm{~s}$ time step sizes were used. Even though the steam flowrate oscillated dramatically, variations in the average coolant temperature and steam generator pressure were neglizsble. This means that the determining factor in the overall time step limit of the PMSIM program is the MSLCH simulation model. According to Strohmayer's calculation, the limiting time step size of the steam generator secondary side model is $\sim 0.7 \mathrm{~s}$ [68]. This is larger than the MSLCH time step size limit. Therefore, the numerical solution method for the MSLCH model should, as part of a future project, be made implicit. A 0.1-s time step size was used in all subsequent null transient simulations. Figures 7.5.2.1-3 through Figure 7.5.2.1-7 show the null transient results when a 0.1-s time step was used. All plant parameters remained at their steady-state values.

\subsubsection{Symmerry Test}

As mentioned previously, each module can be operated at a different power level. Symmetry tests were carried out to verify this capability. The initial power of all modules was assumed to be $100 \%$ MFP and the following two cases were simulated: 


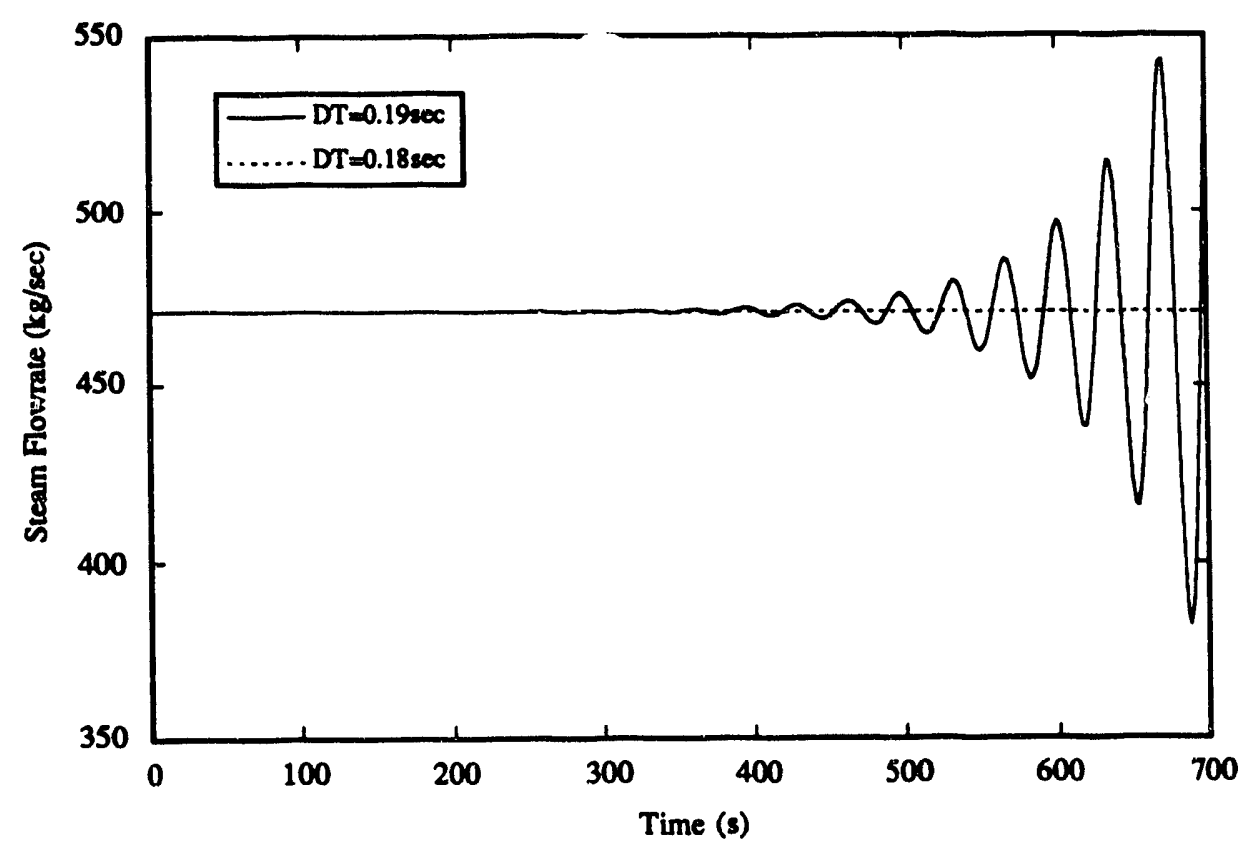

Figure 7.5.2.1-1 Calculated Steam Flowrates of Module \#1 for Two Different Time Step Sizes.

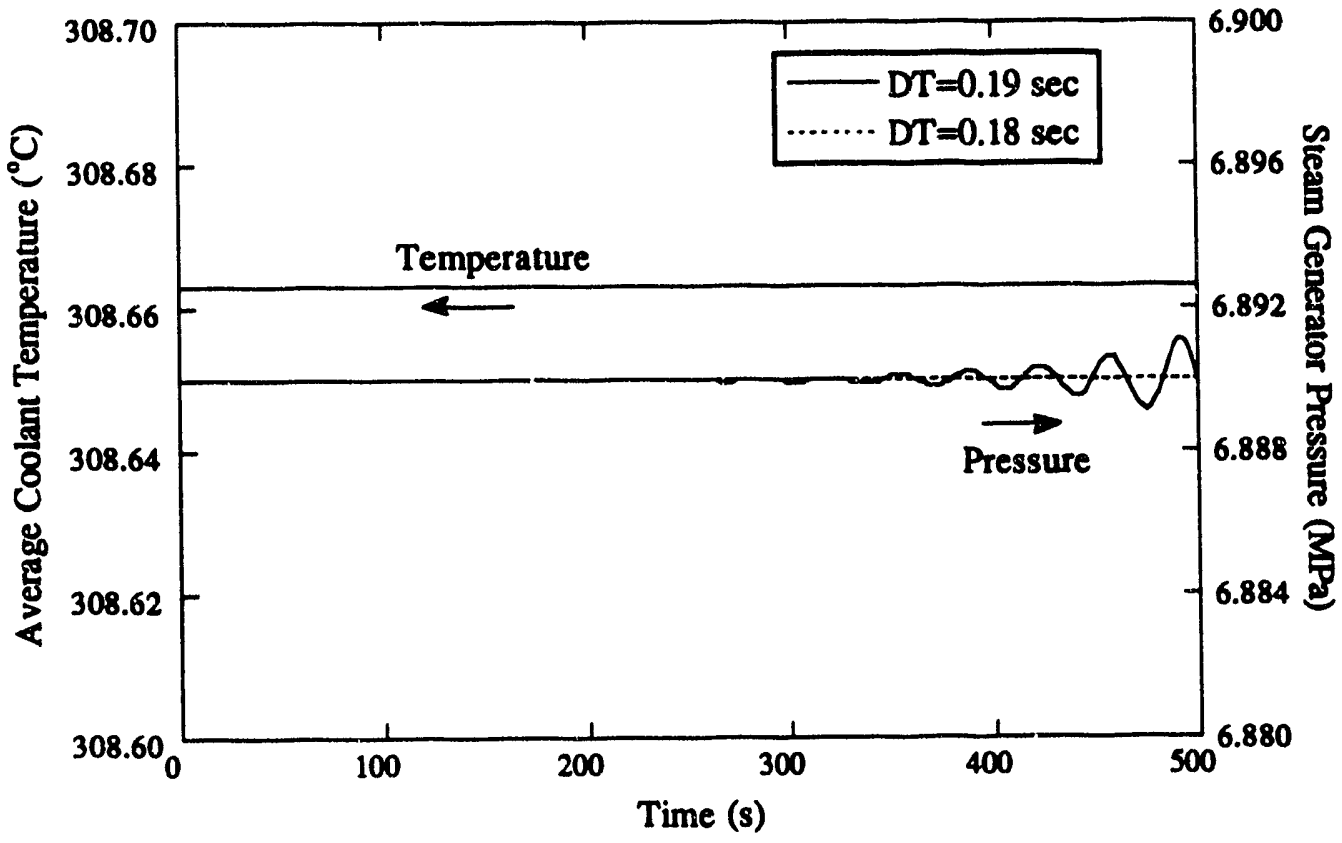

Figure 7.5.2.1-2 Calculated Average Coolant Temperature and Steam Generator Pressure of Module \#1 for Two Different Time Step Sizes. 


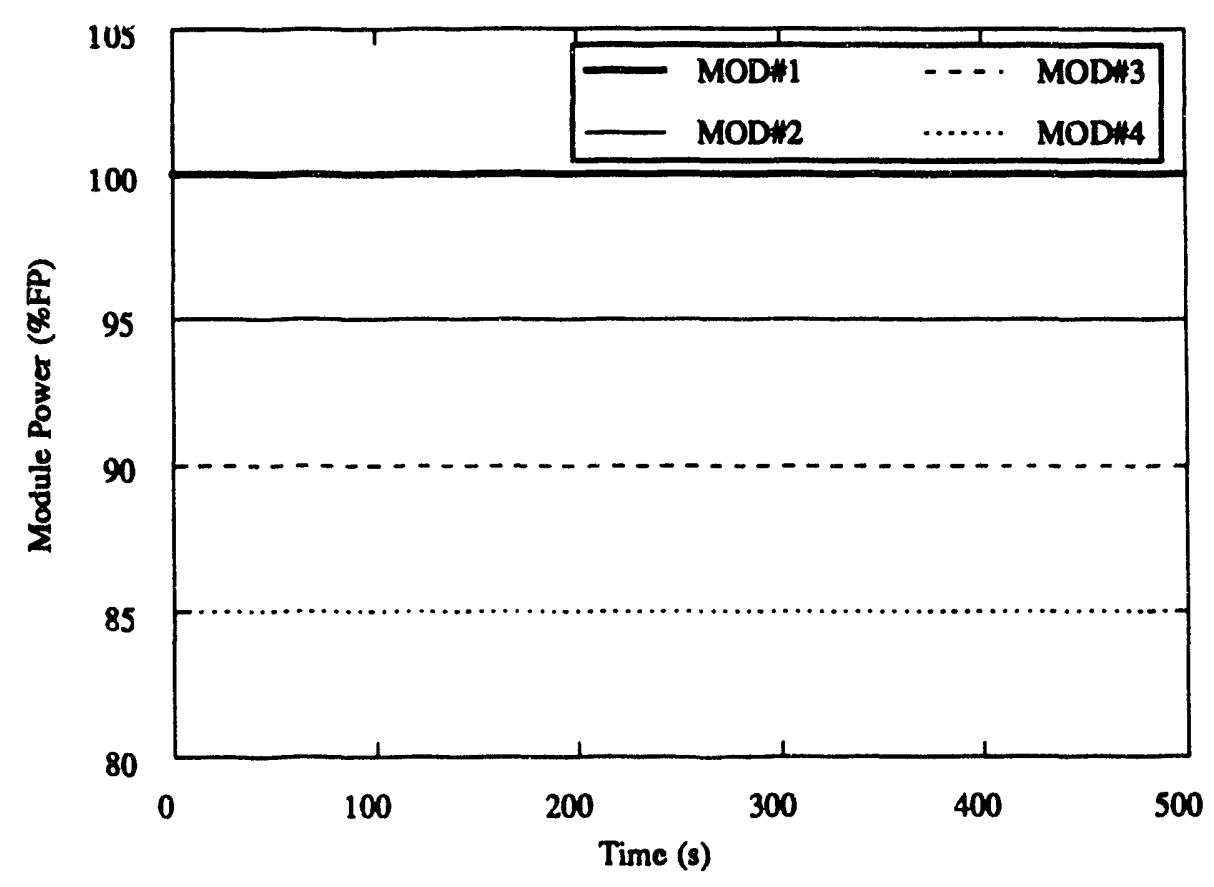

Figure 7.5.2.1-3 Neutron Power During Null Power Transient.

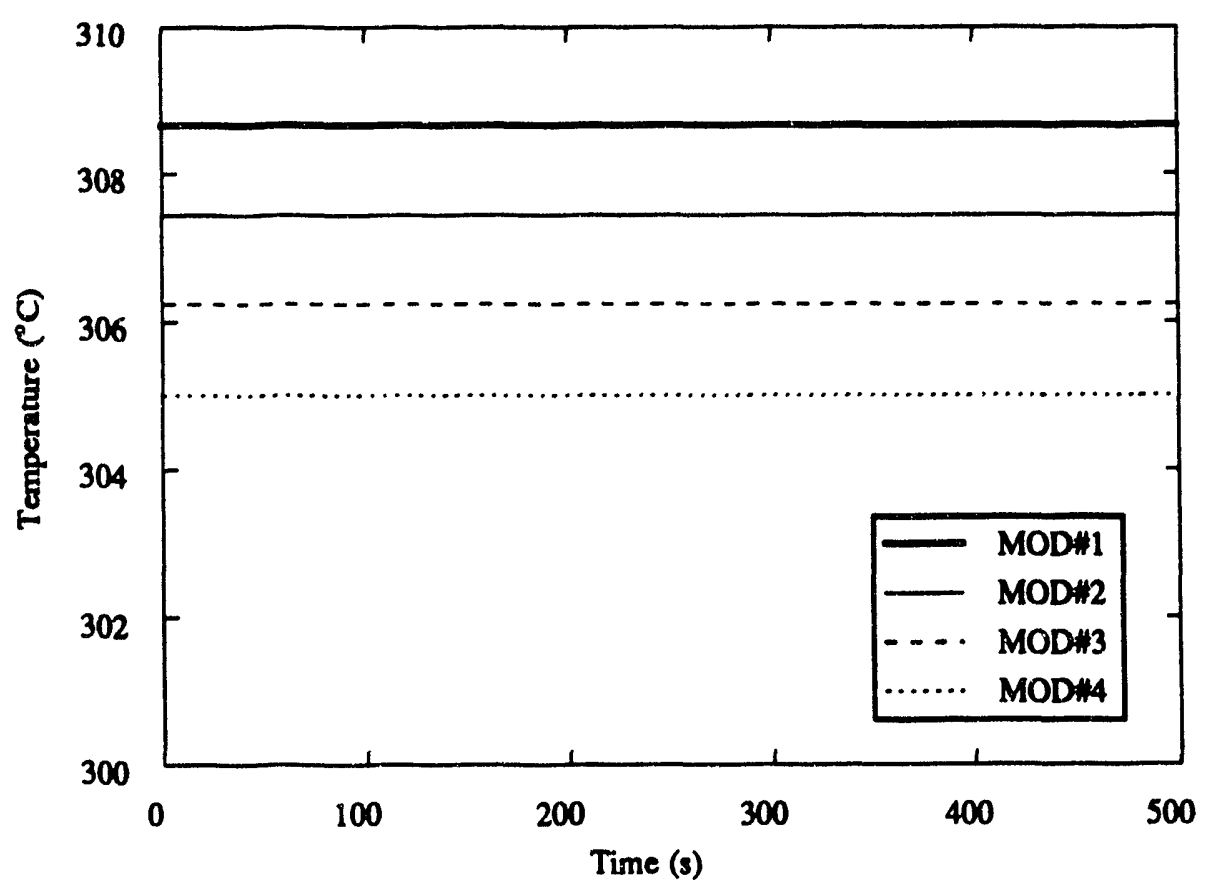

Figure 7.5.2.1-4 Average Primary Coolant Temperature During Null Power Transient. 


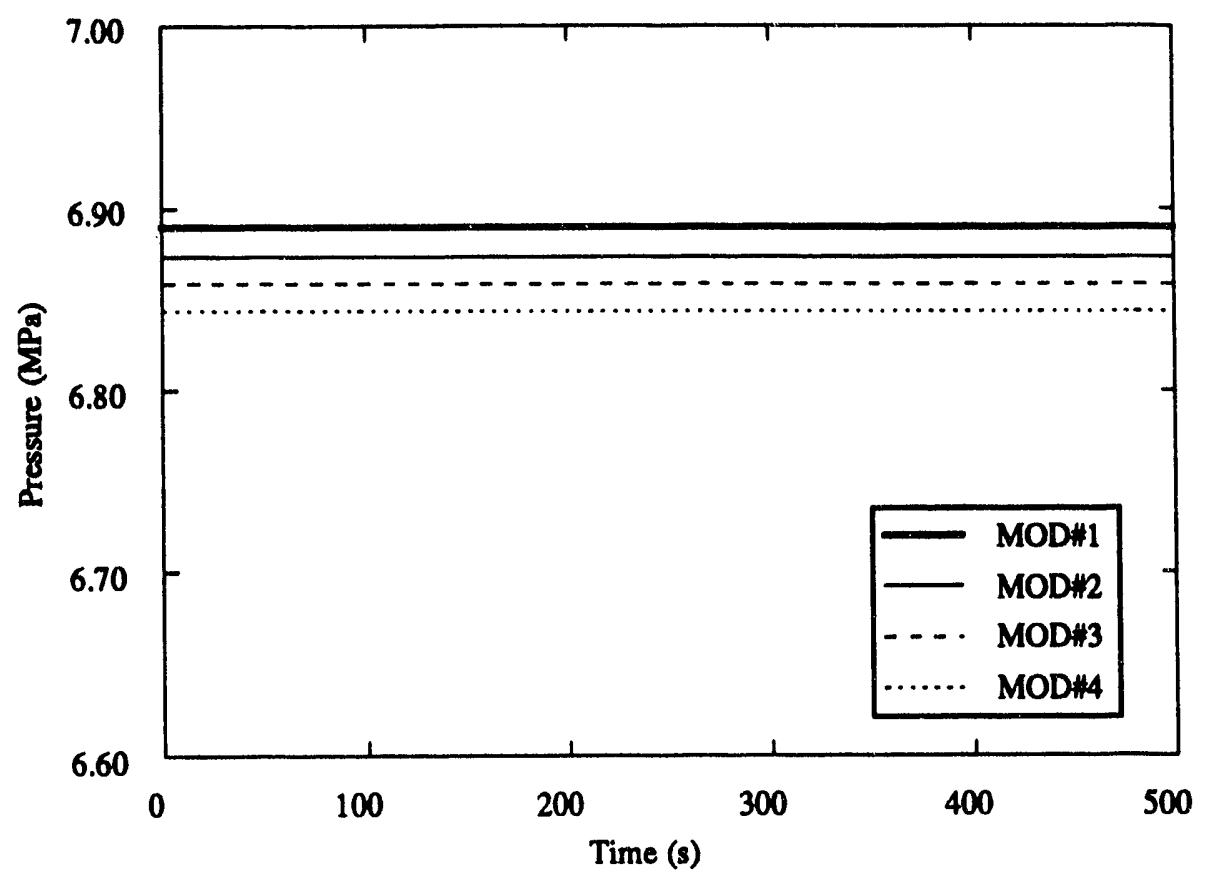

Figure 7.5.2.1-5 Steam Generator Pressures During Null Power Transient.

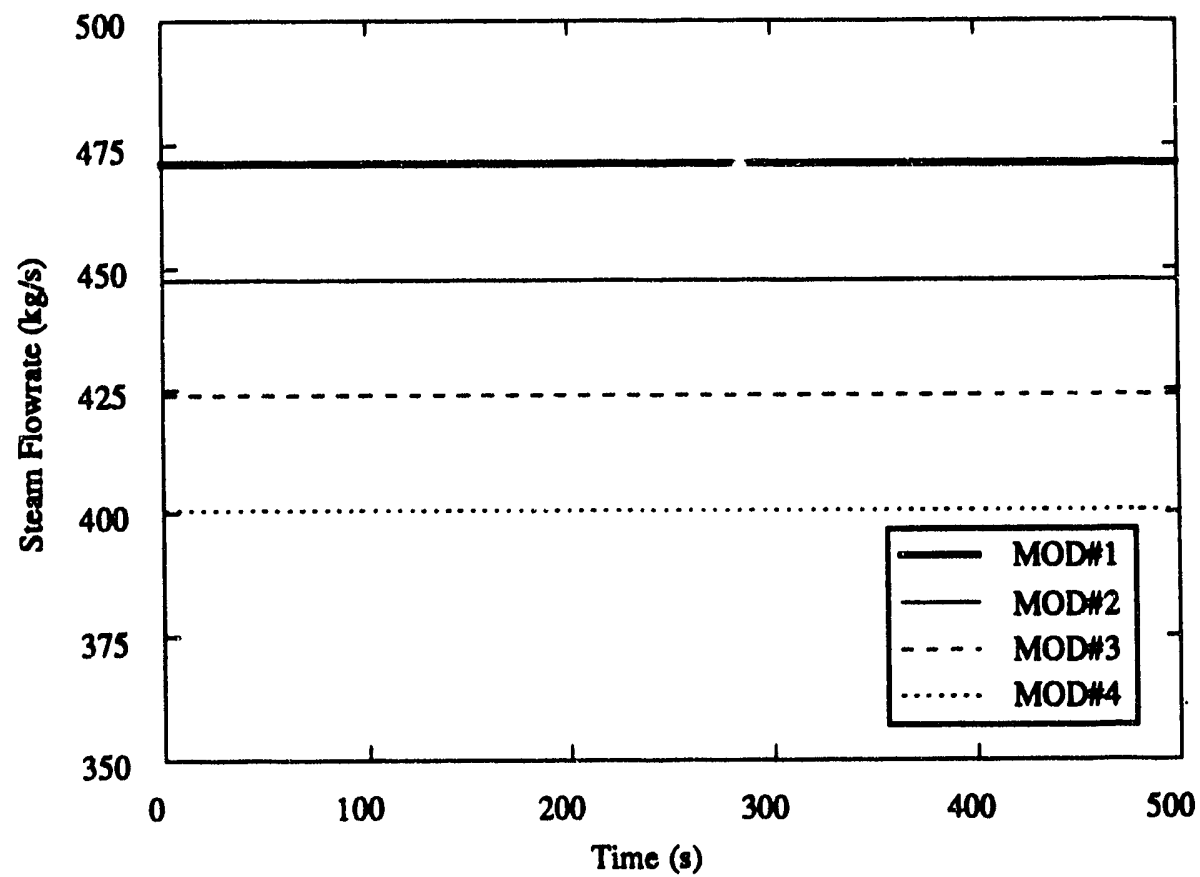

Figure 7.5.2.1-6 Steam Flowrate from Each Power Module During Null Power Transient. 


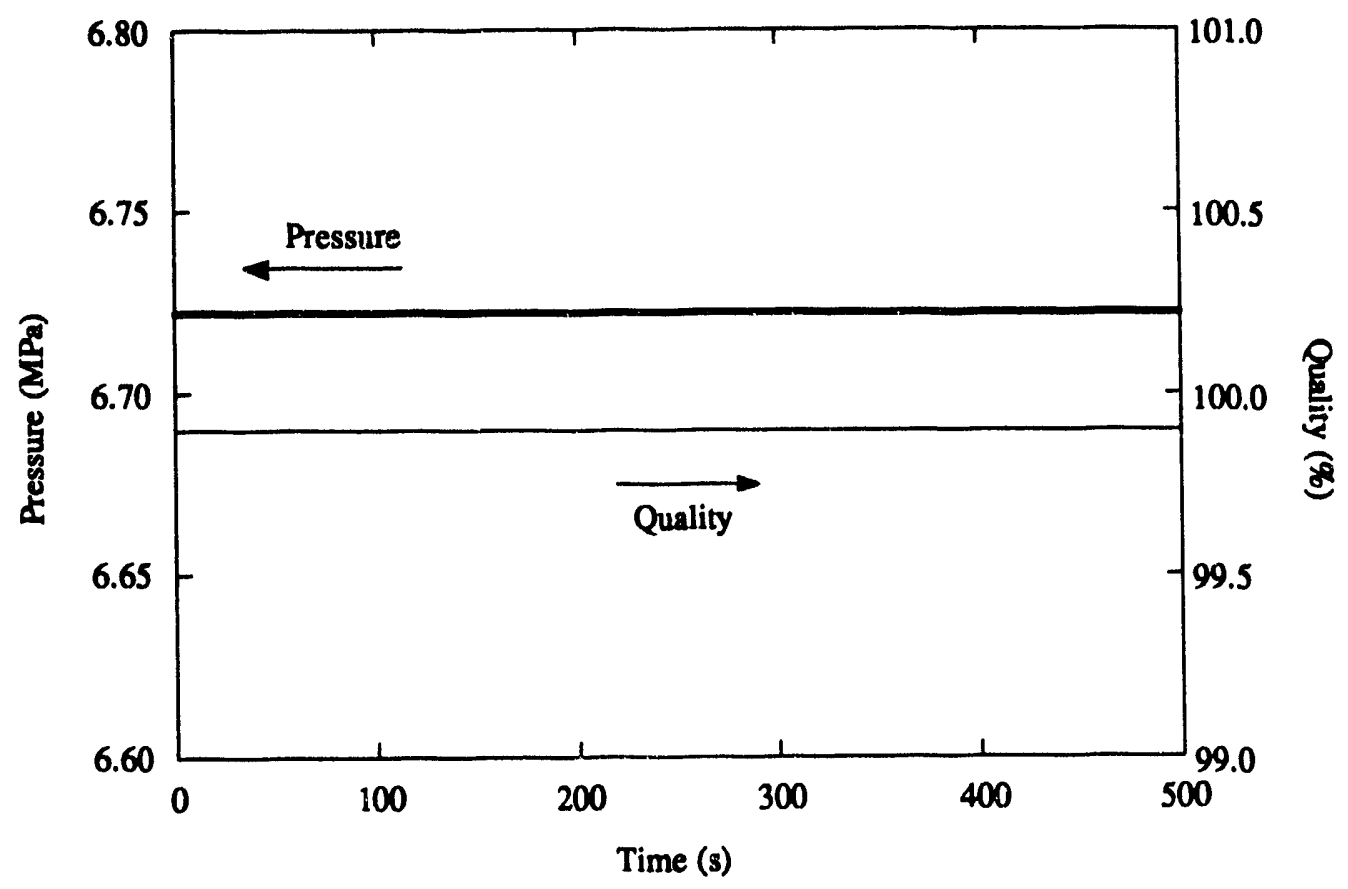

Figure 7.5.2.1-7 Pressure and Quality at the Main Steam Line Header During Null Power Transient. 
1. Case 1: Only module \#2's power was changed to meet the variation in plant demand. No perturbations except thermal-hydraulic feedback effects were allowed.

2. Case 2: Same as Case \#1, but power was perturbed in module \#4 instead of module \#2.

The simulation results are shown in Figures 7.5.2.2-1 through 7.5.2.2-10. As shown in these figures, all plant parameters for the three uncontrolled modules behaved in the same way in both cases. Also, the controlled modules, module $\# 2$ in Case 1 and module $\# 4$ in Case 2, showed the same transient behavior. Based on these results, it was concluded that the PMSIM program could simulate multi-modular power plant transients.

\subsection{Assessment of PMSIM Program}

A simulation program for a PWR-type multi-modular power plant has been developed. This program, which is designated as PMSIM, can simulate up to four PWR power modules and their associated main steam line common header. A point kinetics model and a single coolant-loop model are used for the primary system of each module. Fuel and coolant thermal-hydraulic feedback, fission product poisons, control rod motion, and chemical shim are included in the reactivity calculations. A U-tube steam generator model was adapted to simulate steam generator level variations. The MSLCH simulation model incorporates the moisture content at the MSLCH.

The PMSIM program can reproduce typical PWR steady-state operational data. However, because explicit numerical approximations are used in some of the subsystem models, the time step size is limited to $0.18 \mathrm{~s}$ during transient simulations. Verification tests, in which a symmetric response of the associated modules was observed, were used to determine that this program can simulate a PWR-type multi-modular power plant during transient conditions. In summary, the PMSIM program is more than adequate for its intended purpose which is to facilitate the study of control methods for multi-modular power plants when operating under conditions of unbalanced loads. 


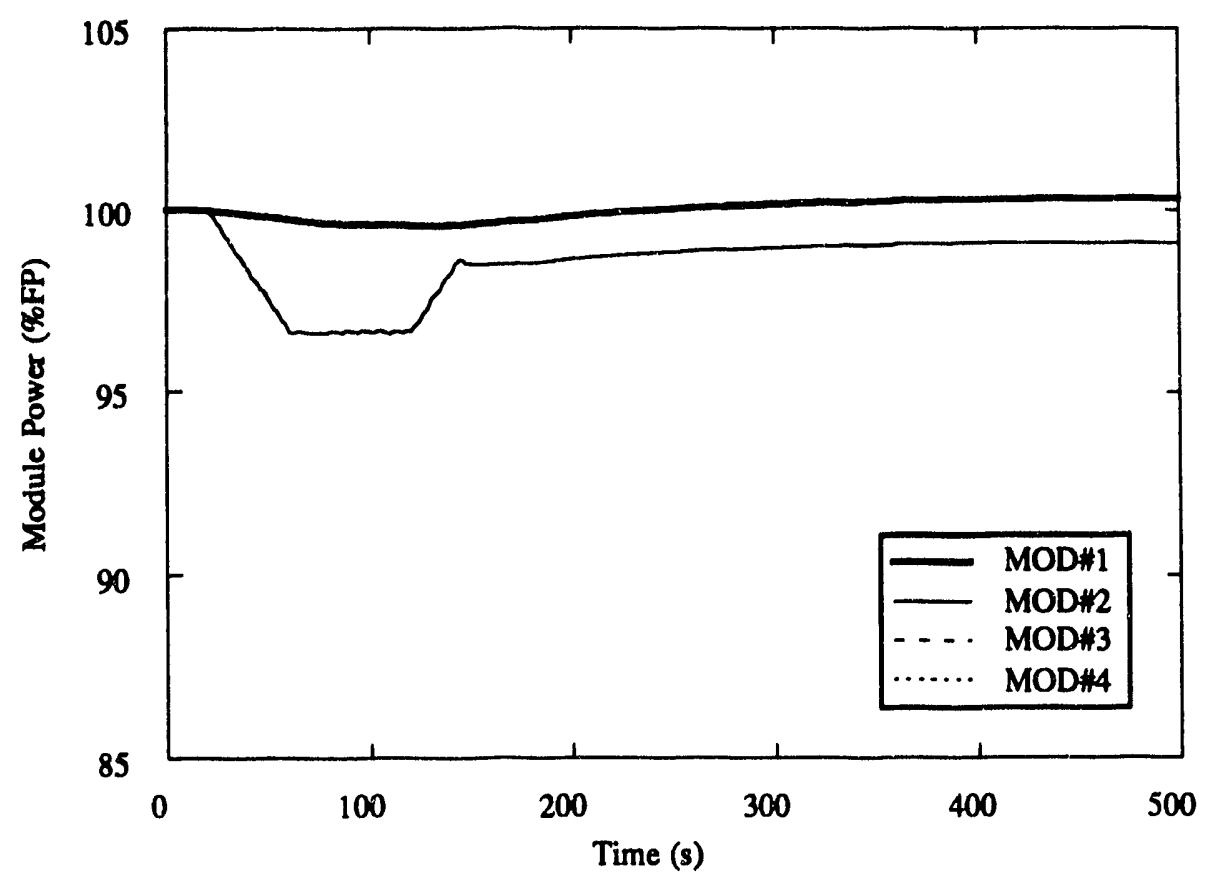

Figure 7.5.2.2-1 Neutron Power During Symmetry Test. (Only Module \#2's Power is Changed)

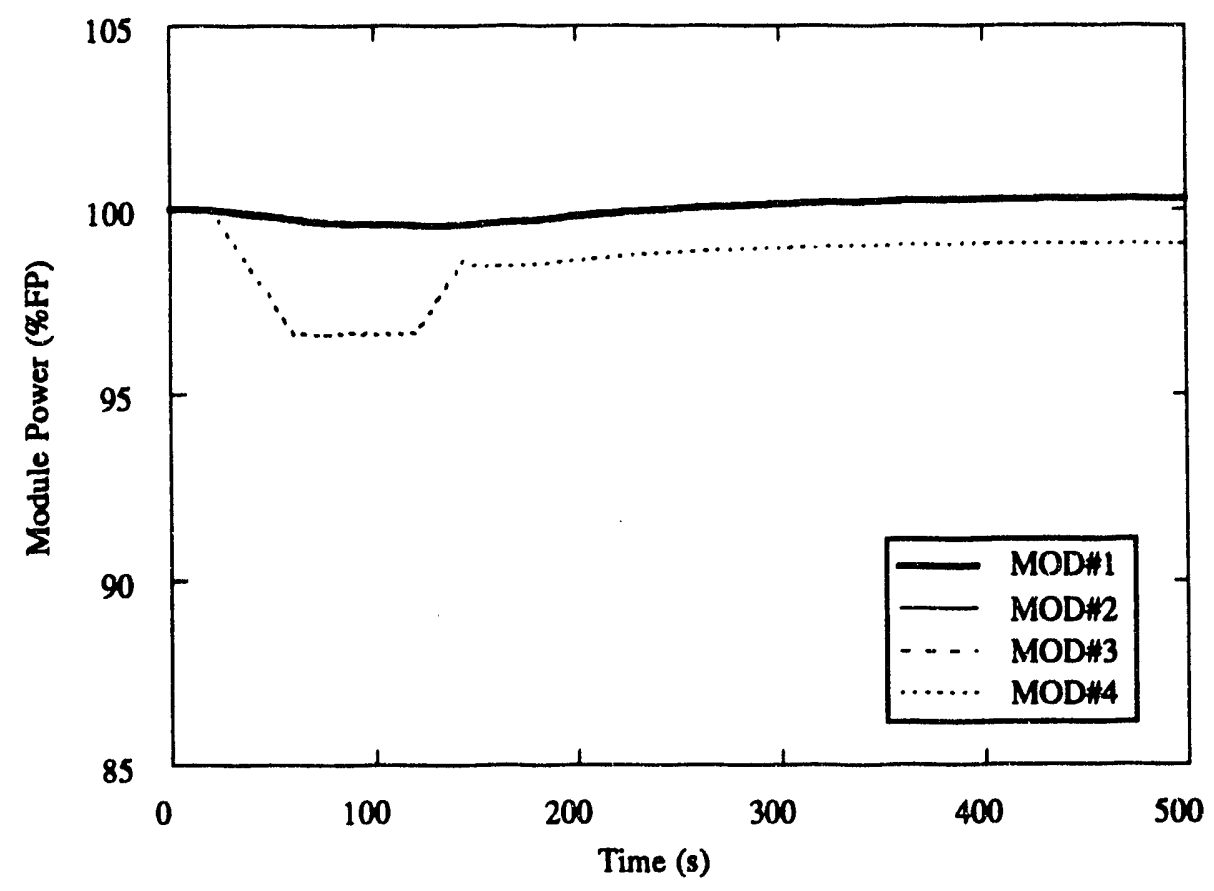

Figure 7.5.2.2-2 Neutron Power During Symmetry Test. (Only Module \#4 's Power is Changed) 


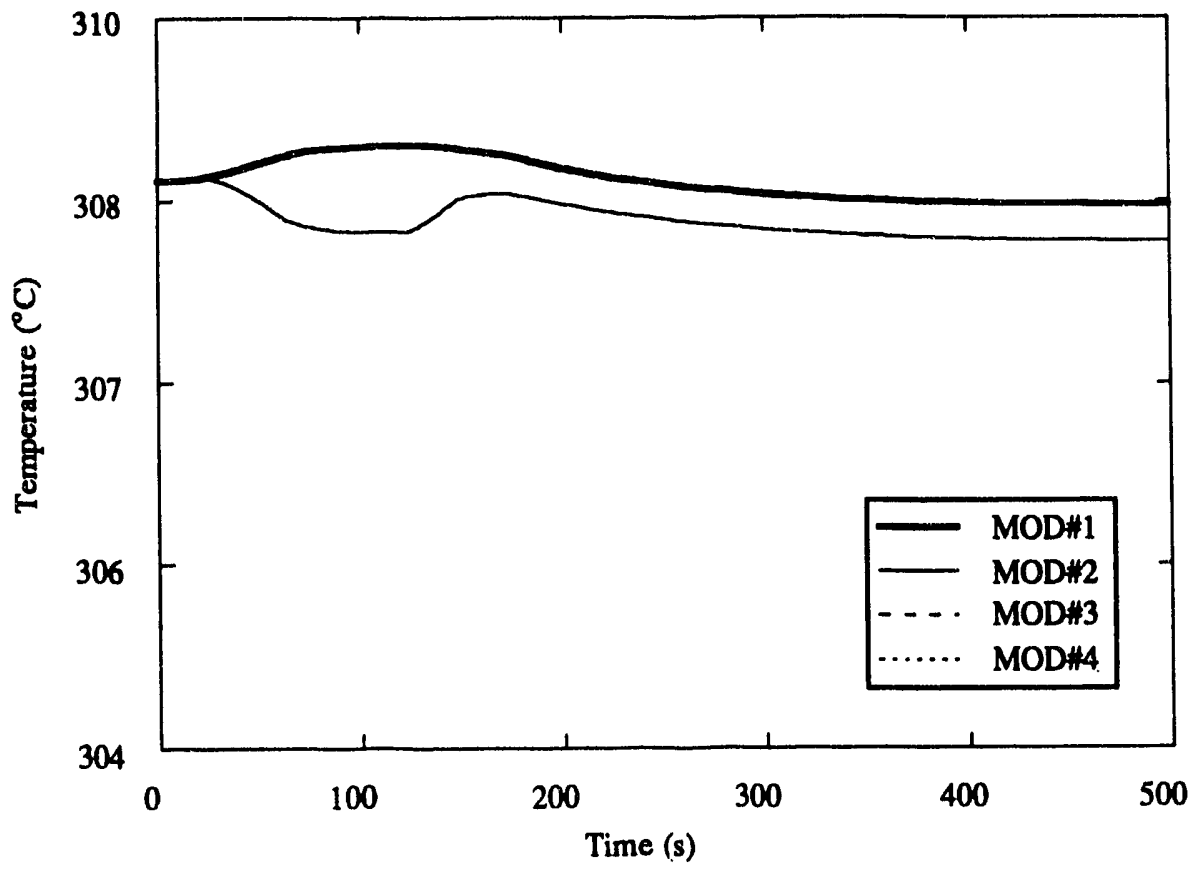

Figure 7.5.2.2-3 Average Primary Coolant Temperature During Symmetry Test. (Only Module \#2's Power is Changed)

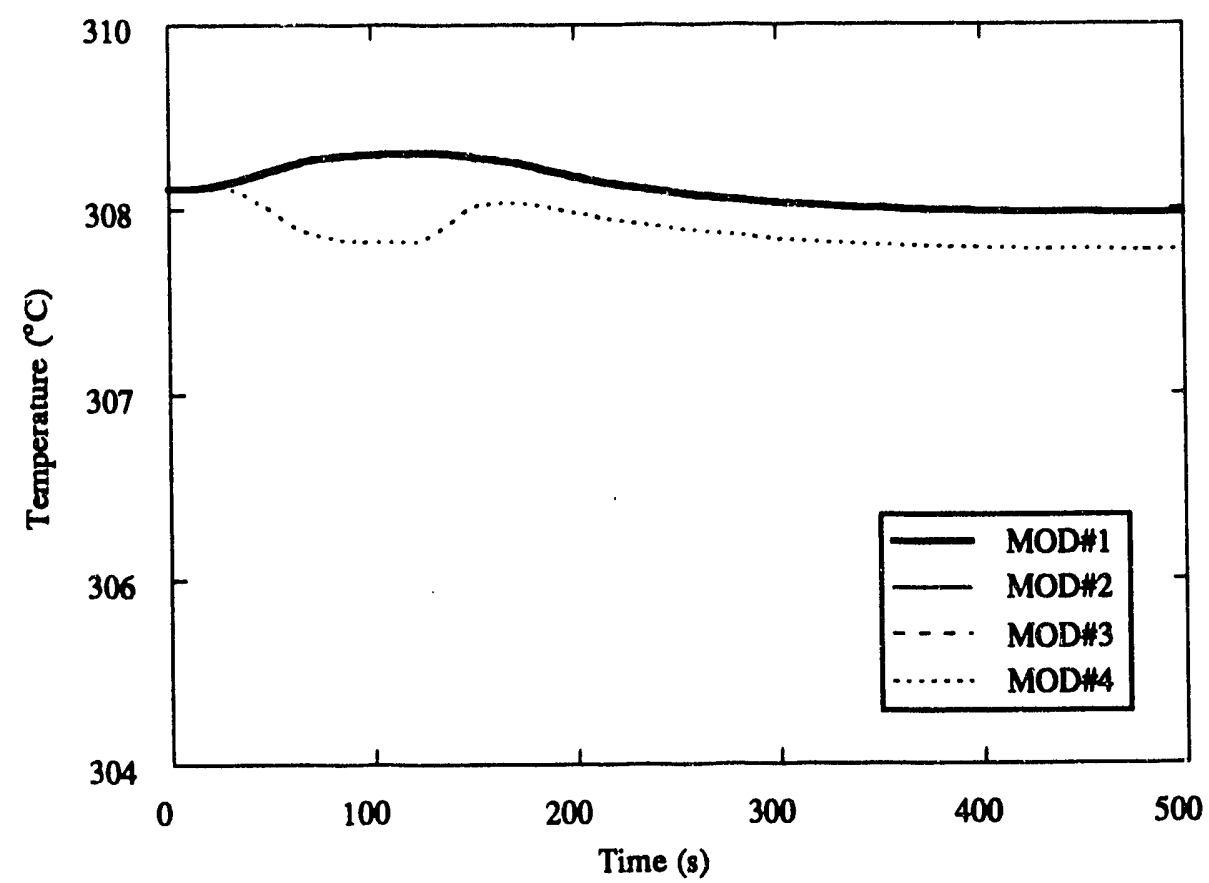

Figure 7.5.2.2-4 Average Primary Coolant Temperature During Symmetry Test. (Only Module \#4's Power is Changed) 


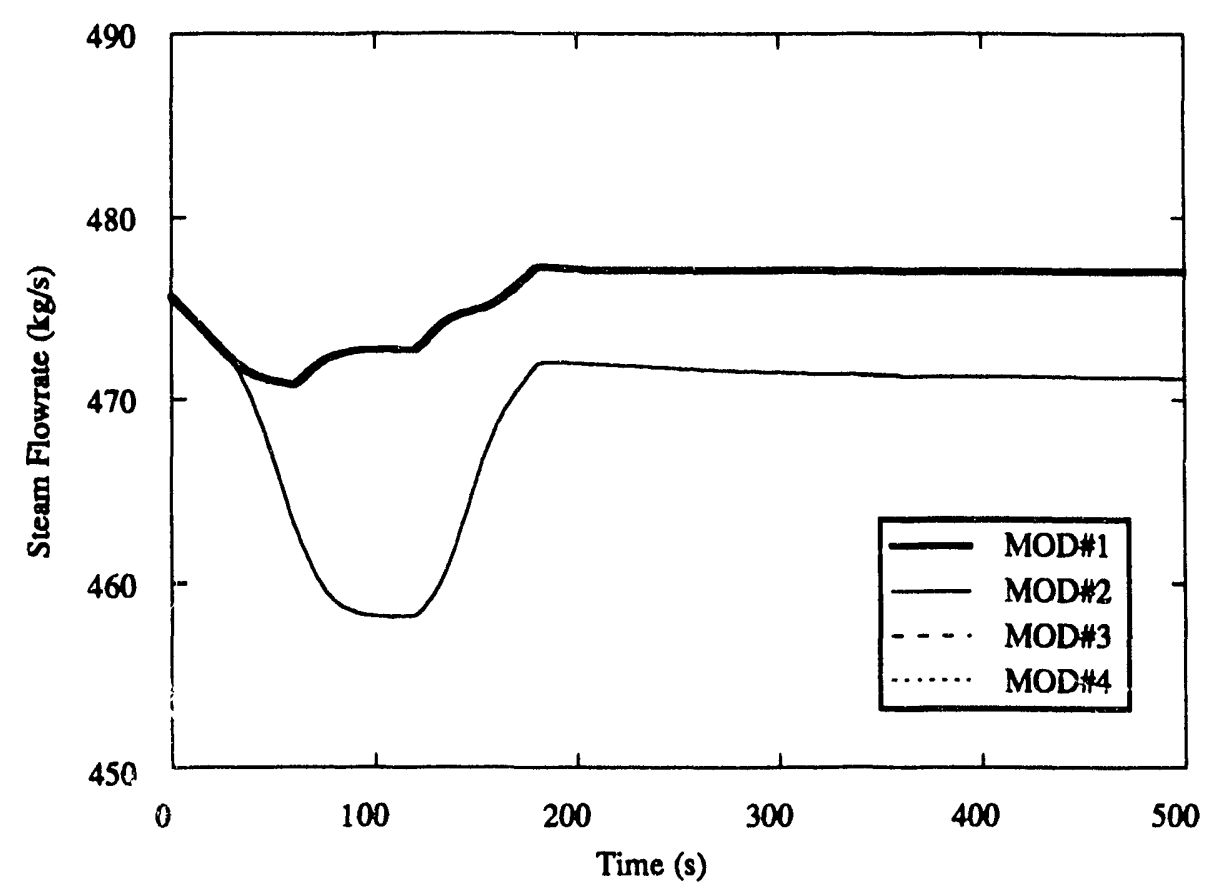

Figure 7.5.2.2-5 Steam Flowrate from Each Power Module During Symmetry Test. (Only Module \#2's Power is Changed)

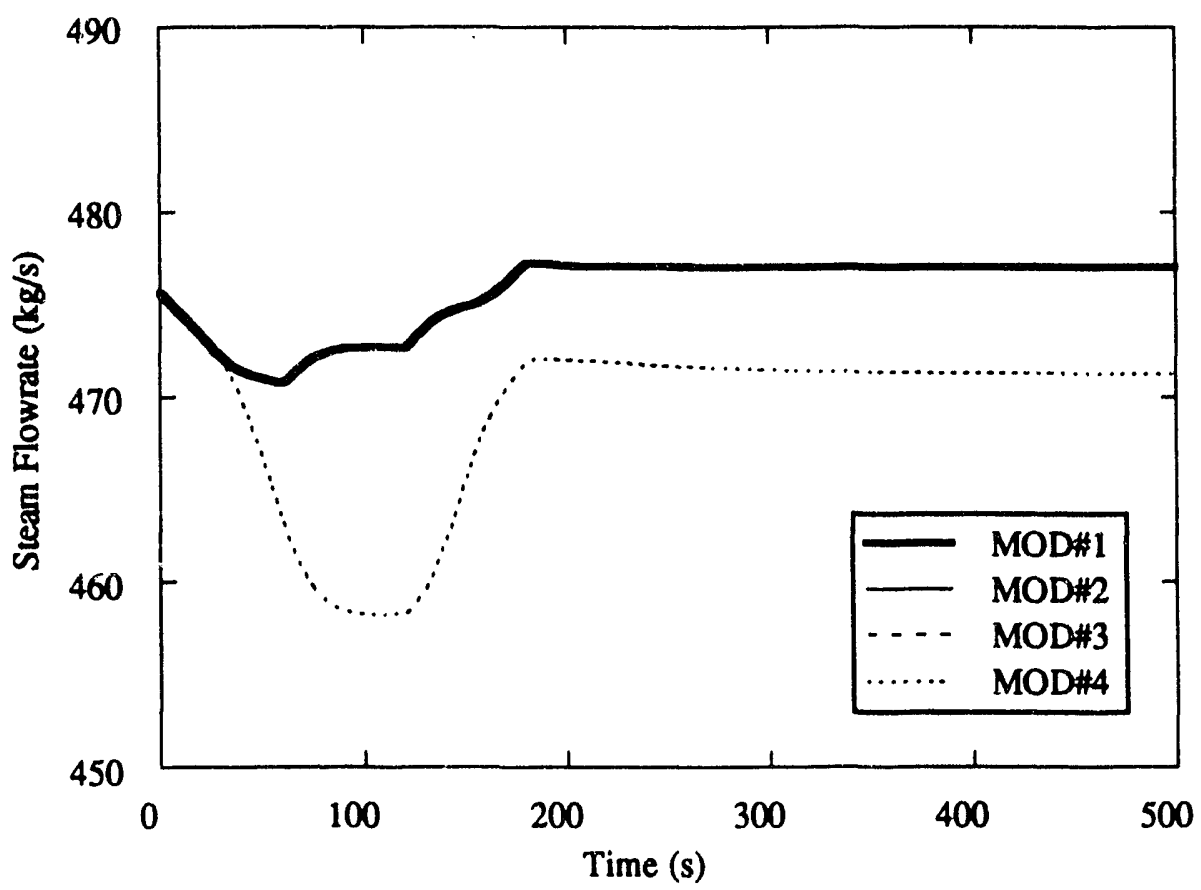

Figure 7.5.2.2-6 Steam Flowrate from Each Power Module During Symmetry Test. (Only Module \#4's Power is Changed) 


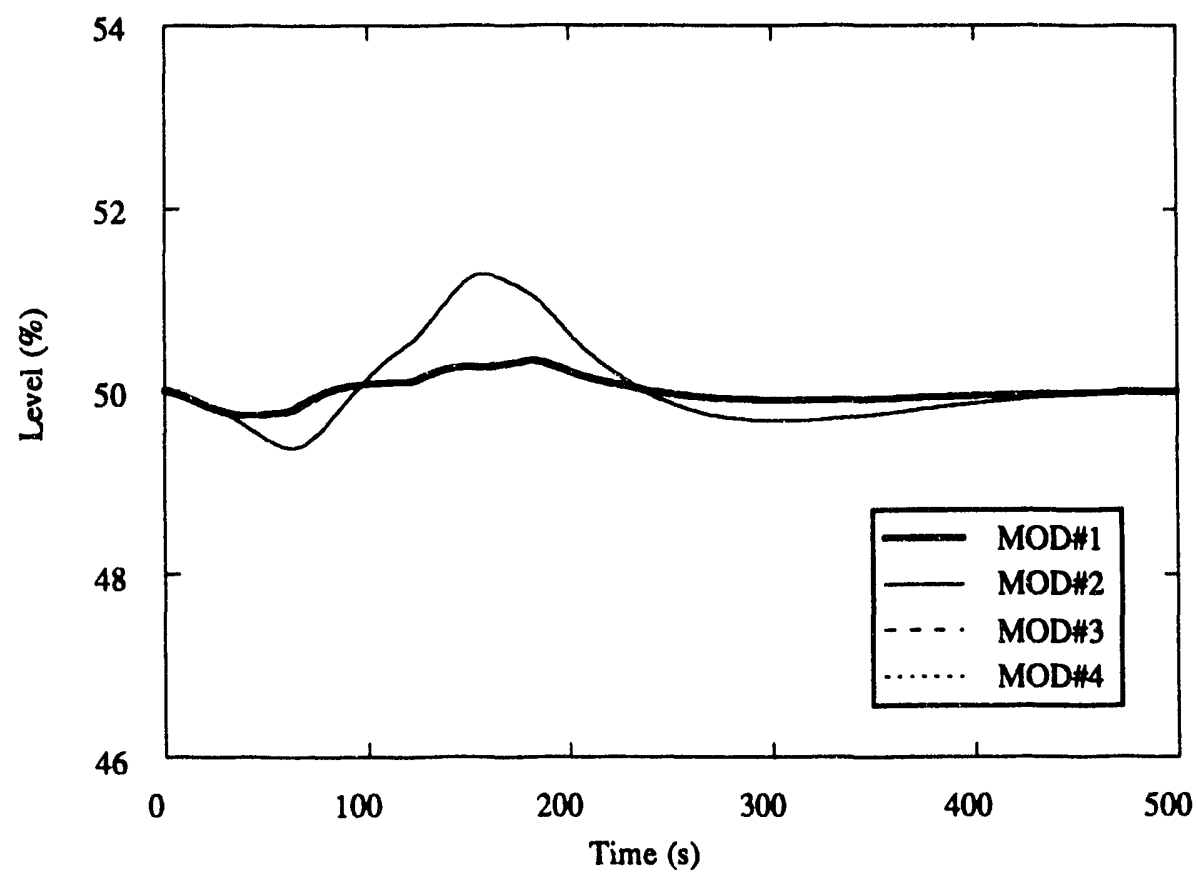

Figure 7.5.2.2-7 Steam Generator Level During Symmetry Test. (Only Module \#2's Power is Changed)

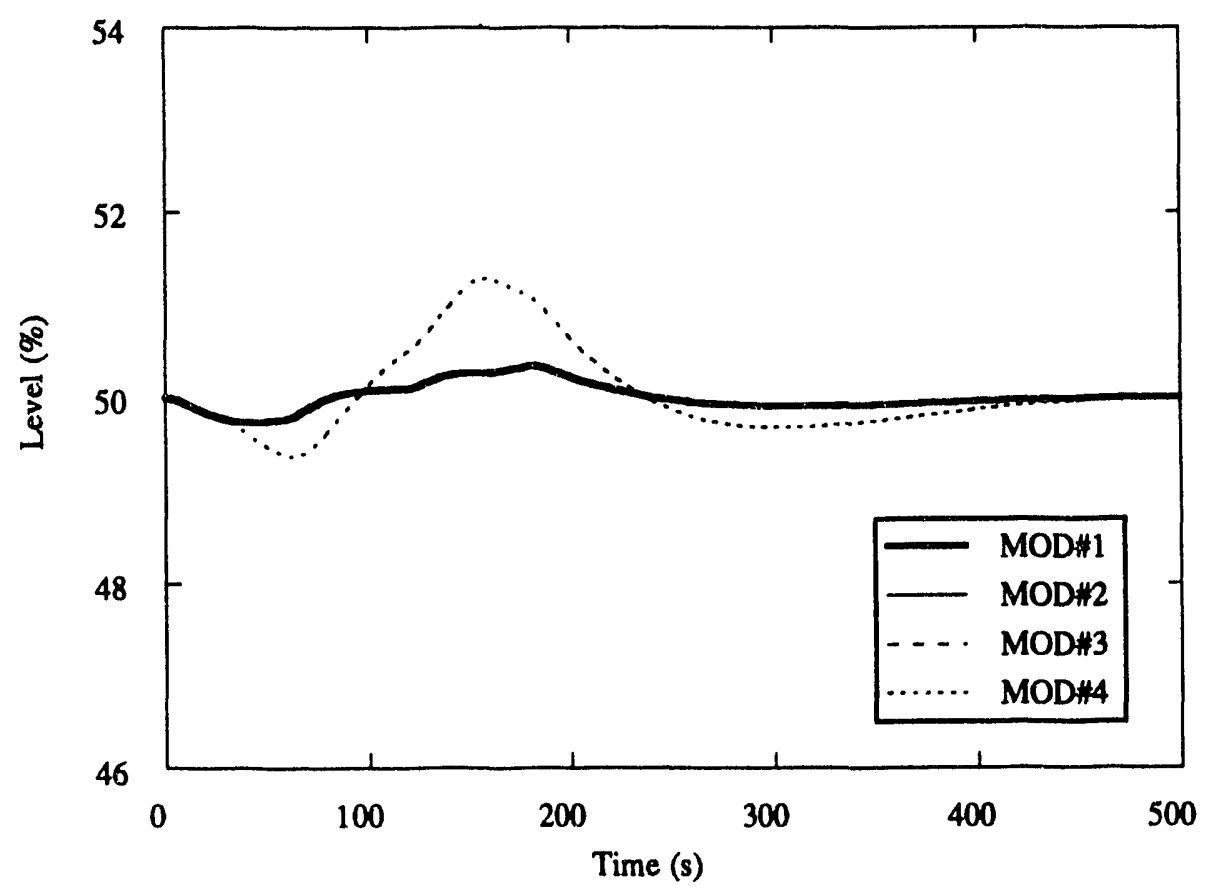

Figure 7.5.2.2-8 Steam Generator Level During Symmetry Test. (Only Module \#4's Power is Changed) 


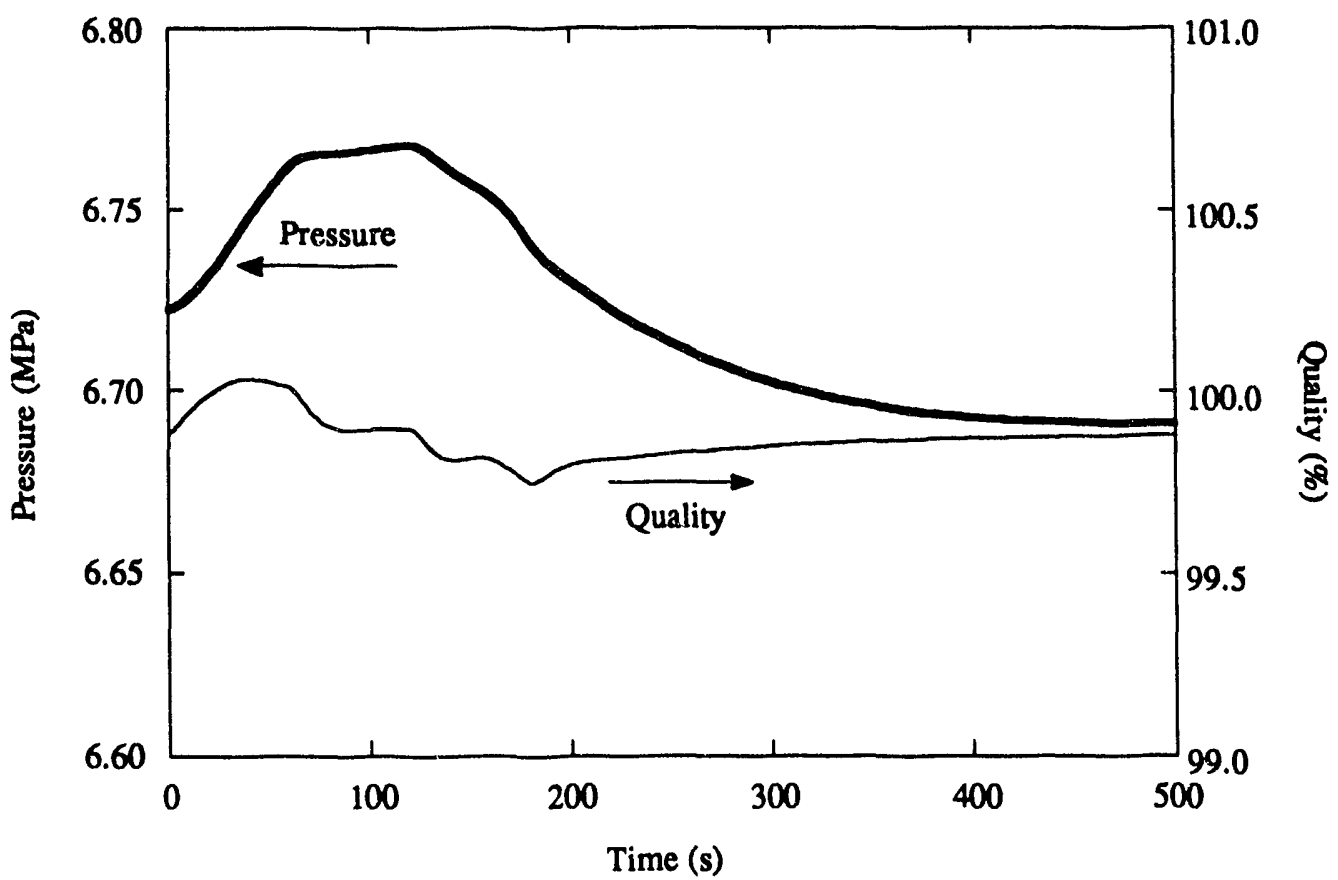

Figure 7.5.2.2-9 Pressure and Quality at the Main Steam Line Common Header during Symmetry Test. (Only Module \#2's Power is Changed)

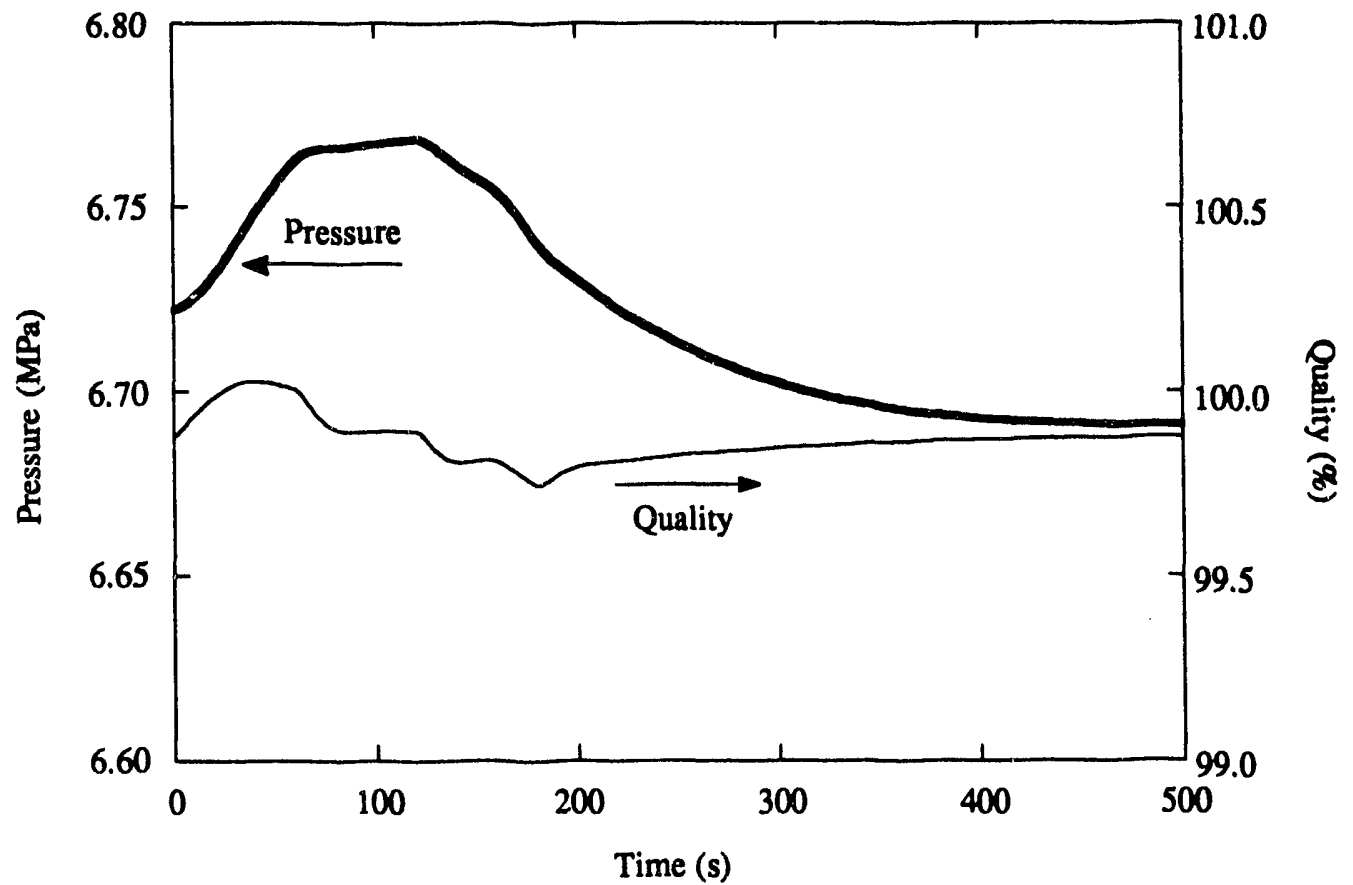

Figure 7.5.2.2-10 Pressure and Quality at the Main Steam Line Common Header during Symmetry Test. (Only Module \#4's Power is Changed) 


\section{Control of Steam Generator Level(1)}

This chapter describes the design and evaluation of an automatic steam generator water level controller that ensures satisfactory performance over all power regions. This controller is applicable to both conventional PWR and PWR-type multi-modular power plants. The chapter begins with a review of the difficulties associated with steam generator operation. Steam generator design, conventional level control systems, and level dynamics are then summarized. Next, a simplified transfer function model of a steam generator is developed and characterized. This is followed by material on the modeling and analysis of current-generation steam generator level controllers. The proposed controller, which uses compensators for each parameter that can perturb performance, is then presented together with the results of an extensive series of simulations in which the new controller's efficacy is demonstrated.

\subsection{Statement of Problem}

Steam generators function by transferring energy from the primary coolant to the turbines where it is converted to electricity. As such, they serve as a heat sink for the reactor core. For proper performance, the steam generator water level must be held within predetermined operating bounds. Too low a level may portend inadequate heat removal from the core while too high a level may degrade steam quality in the separators, dryers, and steam outlets. This will in turn cause erosion of the turbine blades. Steam generator level control is complicated by counterintuitive dynamics. Specifically, an inverse response effect known as 'shrink and swell' causes the water level to respond initially in manner a opposite to its long-term asymptotic behavior. This phenomenon is accentuated during start-up and low power operation. As a result, it is not uncommon for a human operator to initiate an incorrect control action when bringing a plant on-line.

Existing analog steam generator level controllers use both level and flow measurements to generate the appropriate control signal. The former provides feedback based on the actual level error while the latter is a feedforward action that anticipates a level error. However, flow measurements are too uncertain for reliable use during low power operation. Therefore, at low power, automatic control action is a function of only steam generator level measurements. The result is that current-generation level controllers may not provide adequate performance at low power. Unfortunately, manual control may also result in unsatisfactory performance because even skilled operators may have difficulty in reacting properly to an inverse response. In particular, operators sometimes overcompensate when restoring level and, in so doing, cause a reactor trip.

The need for a new method of steam generator level control when operating at low power has been previously verified [81]. This need is even more accute for multi-modular piants because operation under conditions of unbalanced load will require careful coordination of steam flowrates. This chapter explores one approach, lead/lag compensation, for the improved control of steam generator level. Other digital approaches have been tried at some nuclear facilities and found to yield significant improvements [82].

(1) This chapter is based on work performed by Keung Koo Kim under the supervision of Professors John E. Meyer and-David D. Lanning. 


\subsection{Steam Generator Design. Dynamics, and Modeling}

The design, dynamics, and modeling of U-tube steam generators are reviewed here.

\subsubsection{Steam Generator Design and Operation}

Each of the three PWR manufacturers (Westinghouse Electric Corp., Combustion Engineering Inc., and Babcock and Wilcox Co.) designs and manufactures its own steam generators. Westinghouse Electric Corp. and Combustion Engineering Inc. use a U-tube version while Babcock and Wilcox Co. offers a 'once-through' unit. The features described here are those of a Westinghouse Model-F steam generator that is used in a fourloop, 1150-MWe Westinghouse PWR. These are shown in Figure 8.2.1-1.

The primary coolant enters the steam generator through the primary inlet nozzle, flows within the U-tubes where it transfers heat to the secondary coolant, and then leaves through the outlet nozzle. The steam generator secondary side consists of two integral sections: an evaporator and a steam drum. The evaporator section is the U-shaped tube bundle. The steam drum section consists of the riser, moisture separators, and dryers. Subcooled feedwater is introduced into the steam generator via the feedwater nozzle and is distributed throughout the feedwater mixing region by the feedwater ring. There, it mixes with saturated liquid that is being returned from the steam separation devices. This is termed the 'recirculation flow.' The resulting subcooled liquid flows downward through the annular downcomer region formed by the wrapper and the steam generator's outer shell. At the bottom of the dowincomer, the water is turned and flows upward through the shell side of the tube bundle region where it is heated to saturation and boils. The secondary fluid exits the tube bundle region as a saturated two-phase mixture and then flows upward through the riser into the steam-separating equipment. Steam separation is achieved by using a combination of centrifugal steam separators for bulk liquid-vapor separation and chevron-type steam dryers for the removal of any residual moisture. The relatively dry steam, with a moisture content of less than $0.25 \%$, flows through the steam outlet nozzles at the top of the steam generator, while the saturated water is directed downward to mix with the entering feedwater.

The secondary fluid path described above constitutes a natural circulation loop. The driving head for this recirculating flow is the density difference between the subcooled column of liquid in the downcomer region and the two-phase mixture in the tube bundle and riser regions. This driving head is counterbalanced by the various pressure losses in the loop, such as frictional losses in the tube bundle and losses within the steam separators.

Upon exiting the generator the steam passes through a flow restrictor prior to entering the main steam line. This restrictor has two purposes. First, it limits steam flow during an unisolable steam line rupture. Second, the differential pressurs, across it determines the steam flow signal that is in turn used in current-generation steam generator water level control systems. Steam from each steam generator then flows into the main steam line header which serves as a cross-connect. The pressure at the exit of this header is therefore common to all steam generators. Hence, the pressures at the individual inlets to the header should all be the same. Otherwise, steam will not flow from the low-pressure 


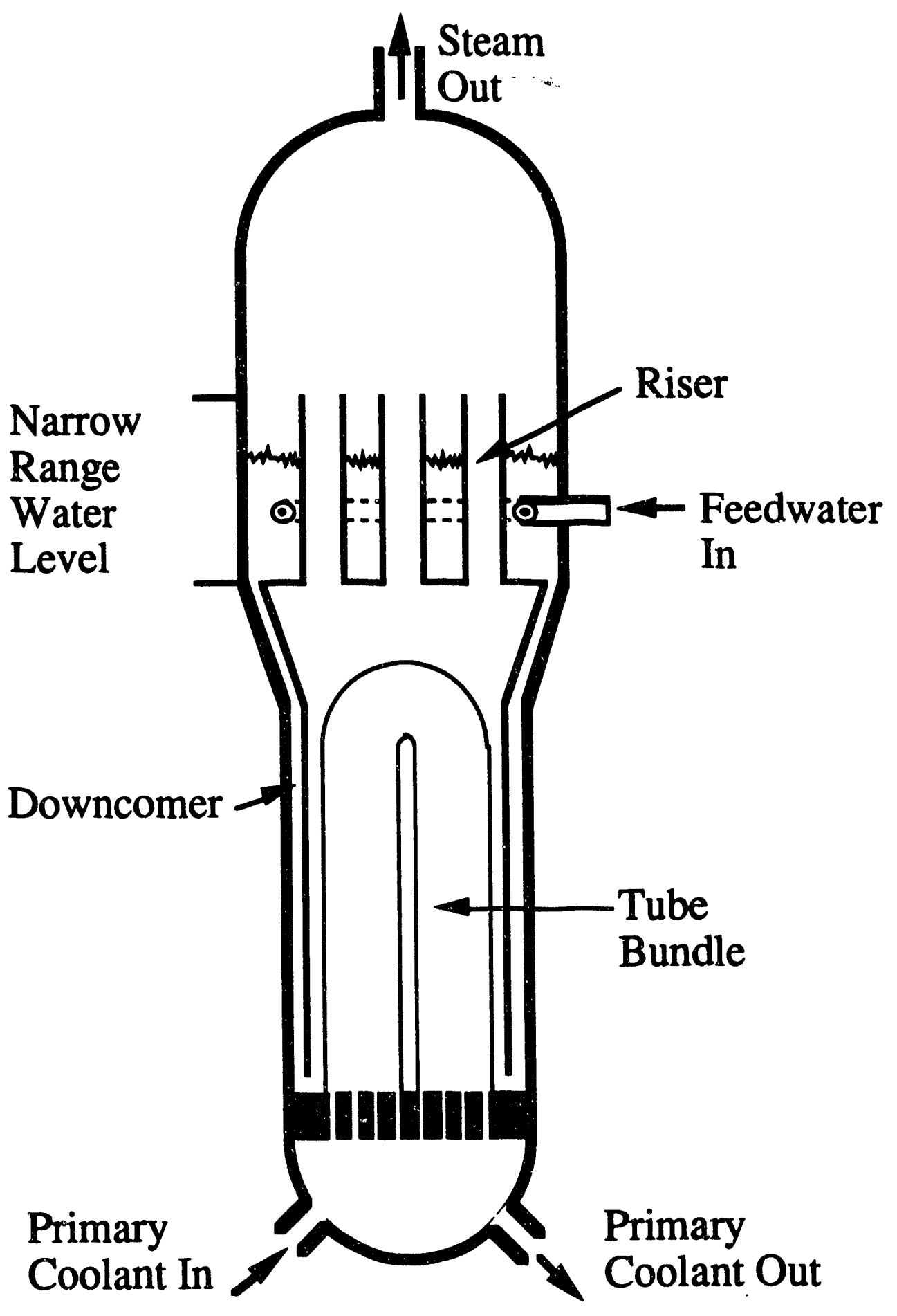

Figure 8.2.1-1 Schematic of U-Tube Steam Generator [83]. 
units and the desired power distribution will not be maintained among the on-line modules. This observation is central to the coordinated control of reactor power and temperature in a multi-modular plant.

The heat energy of the steam is converted to directed kinetic energy during passage through the turbine. Exhaust steam from the main and feedwater pump turbines is then condensed to water under a vacuum condition. The condensate is collected and pumped by condensate and booster pumps through feedwater heaters to the suction of the main feed pumps. Feedwater heating increases plant efficiency and decreases the temperature difference between the incoming feedwater and the saturation temperature in the steam generator. Minimization of this temperature difference is beneficial because it reduces shrink and swell effects, and hence facilitates control of steam generator level. Three feedwater pumps with a common suction and discharge header are provided. Two of these pumps are turbine-driven variable speed units that are used during power operation. The third is electric-driven and is used both for startup and as a reserve or backup unit. These pumps discharge through the high pressure feedwater heaters into a common header. This header is monitcred for pressure and that signal is transmitted to the feed pump speed control input. The feedwater header has several branch lines, one for each of the steam generators. There is one control valve and flowrate measurement venturi in each of these lines. The valve for a particular generator is operated by an automatic control system which regulates feedwater flow in order to maintain the desired water level in the associated steam generator. There is an independent feedwater control system for each steam generator. At low loads, a small bypass valve is used to control feedwater flow in lieu of the large main control valve. These valves are installed in a bypass that goes around the feedwater regulating valve. These bypass valves have both an automatic and a manual flow control capability.

\subsubsection{Feedwater Flow Control System}

The feedwater control system is used to adjust the feedwater flowrate to the steam generator so as to maintain the steam generator water level within the allowed operational band. It consists of two interacting sub-systems. These are the steam generator water level control and the feed pump speed control systems. The latter is not discussed here except to note that it varies the speed of the feed pumps so as to maintain a certain pressure drop across the feedwater regulating valves. Hence, it responds to whatever change is made in the position of the feedwater regulating valves by the level controller.

The steam generator water level control system computes the steam generator water level setpoint from a program that is based on the turbine load. For the Westinghouse model F-type steam generators, the programmed water level is set at $50 \%$ for all power levels. However, for the smaller generators of some other PWR plants, water level is programmed to vary linearly from 33\% at zero power to $44 \%$ for power levels of $20 \%$ FP or greater. In this research, the Westinghouse model-F type steam generator was selected as the basis of study and a constant steam generator level (50\%) control program was therefore adopted. There are several factors that bear on the choice of setpoint for steam generator water level. These include: 
1. The effects of shrink which may cause loss of level indication;

2. The effects of swell which may cause poor moisture separation performance and subsequent turbine blade damage; and

3. The influence on the magnitude of the peak containment building pressure attained as a result of the complete blowdown of a steam generator's inventory during a steam-line break accident.

The first factor establishes a lower bound for the programmed level. If operating above this bound, the probability that a sudden load rejection will cause so much shrink so as to necessitate a reactor trip because of a 'low-low' steam generator water level is minimized. The second factor establishes an upper bound for the programmed level. If the level is kept below this bound, then the swell that results from a sudden load increase should not cause the downcomer level to back up into the moisture separators, thereby degrading their performance. The third factor also sets an upper bound on the programmed level because a steam line break at hot zero power (mass inventory inside the steam generator is maximum) establishes a limit on the maximum allowable steam generator fluid inventory. Specifically, if a steam line were to break, the subsequent vapor release within the containment would cause building pressure to rise with the magnitude of that rise being a function of the amount of steam released, and hence proportional to fluid inventory of the steam generator.

In order to maintain steam generator level at the programmed setpoint, the steam generator water level control system, which is shown in Figure 8.2.1.1-1, produces an electrical control signal that positions the pneumatically-operated feedwater regulating valves. This signal has two components. The first is the difference between the measured and programmed water levels. The second is the difference between the steam and feed flowrates. The former provides feedback based on the actual level error while the latter is a measure of feedforward action because it anticipates changes in level. These two error signals (level and flow) are then processed via a proportional-integral or PI controller. At high power, both signals are used. However, at low power, the flow measurements are unreliable and only a single element (steam generator water level) is used. Further information is given in Section 8.4 of this report.

\subsubsection{Steam Generator Water Level Dynamics}

At high power, steam generators operate in the recirculation mode. Feedwater enters the steam generator through the feedwater sparger and flows into the downcomer where it mixes with the recirculating saturated liquid that is being returned from th: moisture separators. The combined flow moves through the downcomer and enters the tube bundle region at the bottom of the steam generator. As fluid rises through the tube bundle region, it absorbs heat from the primary coolant. This causes steam formation. The resulting two-phase mixture rises until it reaches the separators which remove liquid from the steam, return the liquid to the downcomer for further recirculation, and allow the steam to rise to the dryers. The recirculation process is sustained by an imbalance in the hydraulic head of the fluid between the downcomer and the tube bundle regions. During power operation, this driving force is significant and recirculation flow is dominant. It is 


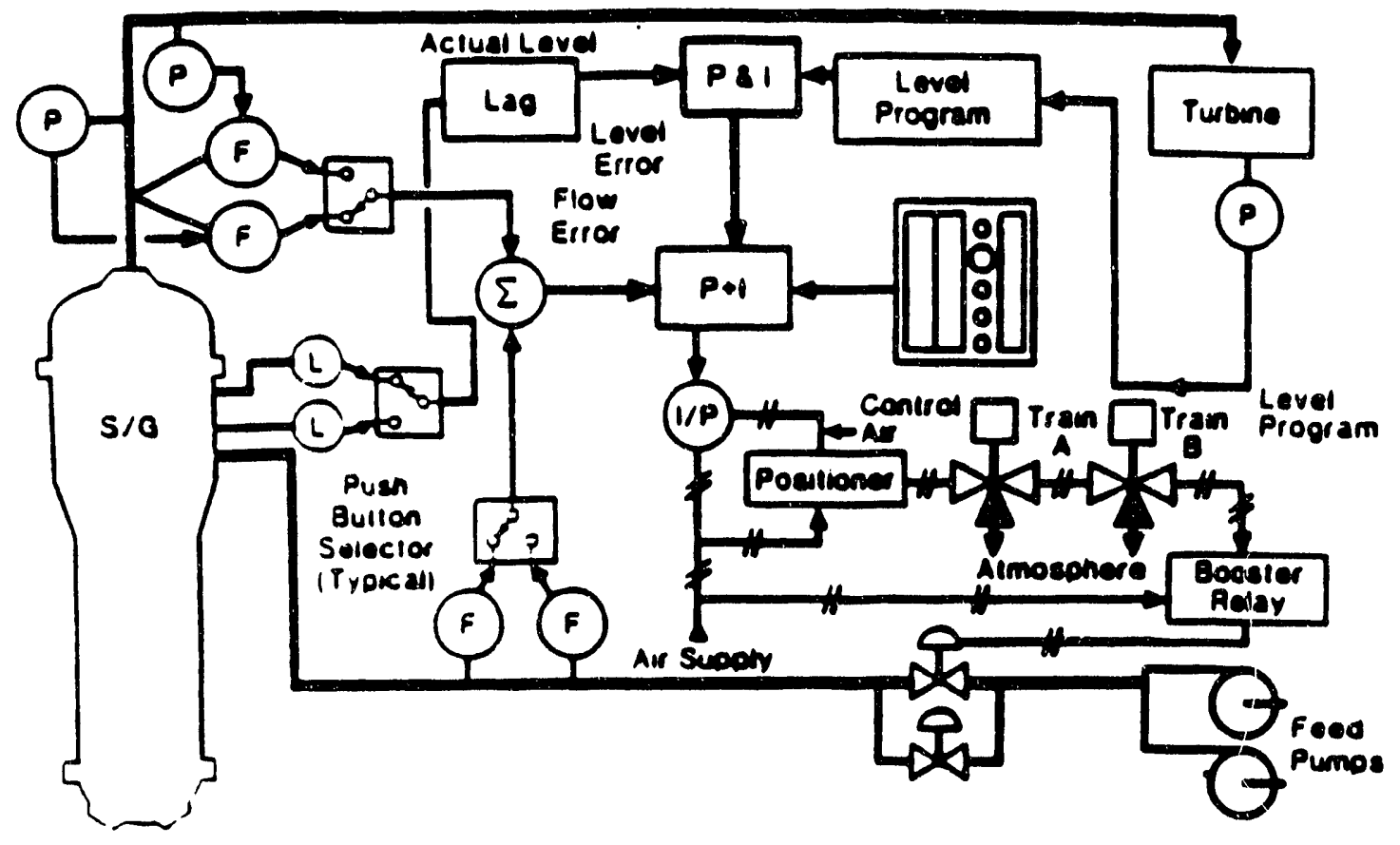

Figure 8.2.1.1-1 Steam Generator Water Level Control System [83]. 
important to note that any perturbation in the amount of vapor in the tube bundle region will cause the driving head to change and that in turn will alter the recirculation flow and ultimately the downcomer level.

The situation is different at low power levels because the amount of boiling in the tube bundle region is insufficient to create a driving head for recirculation flow. Hence, as the power level decreases, the recirculation flowrate also decreases and finally stops because the hydraulic head difference can no longer provide the needed driving force. Under this condition, the steam generator behaves like a boiling pot. Feedwater simply enters the downcomer, passes through the tube bundle region, and exits as steam.

Figure 8.2.2-1 shows the steady-state recirculation flowrate as a function of power. As shown in this figure, the recirculation is very large except at extremely low power levels.

\subsubsection{Shrink and Swell Effects}

Steam generator level shrink refers to the temporary reduction of the water level in the downcomer region that results from steam bubble collapse in the tube bundle region. With the collapse of steam bubbles, the volume taken by the two-phase mixture suddenly decreases and is filled by liquid from the downcomer region. Hence, the indicated level, which is obtained from the downcomer, drops even though the mass of fluid in the steam generator has risen. Figure 8.2.2.1-1 illustrates this phenomenon. Swelling is essentially the reverse effect.

Shrink and swell may be caused by either a change in the feedwater or steam flowrates or by a change in primary coolant temperature. However, the mechanisms involved are different. First, consider the effect of changing the feedwater flowrate. Introduction of feedwater at a temperature below saturation in the tube bundle region will cause internal condensation. Thus, a sudden increase in feedwater flowrate will momentarily reduce the boiling rate and cause an outflow from the downcomer. Hence, the downcomer level will initially decrease even though the long-term effect of the increased feedwater flow will be to raise the level. Next consider the effect of a changing steam flowrate. A decrease in steam flowrate will cause steam generator pressure to rise which will collapse the existing steam bubbles in the tube bundle region. "igher pressure also will cause an increase in the saturation temperature which in turn will reduce heat transfer from primary coolant. Therefore, the boiling rate will decrease following a steam flowrate decrease. The long-term effect will be a level increase because the rate of mass removal from the steam generator is reduced. However, the immediate impact will be a loss of level as water flows out of the downcomer and into the volume formerly occupied by the collapsed steam bubbles. Another initiator of shrink and swell effects is the primary coolant temperature. Specifically, a primary coolant temperature decrease that results from a reactor power drop will also cause a decrease in both the heat transfer rate from the primary coolant and the boiling rate in the tube bundle region. Hence, once again the immediate effect is outflow from the downcomer even though the long-term trend will be for the downcomer level to rise. 


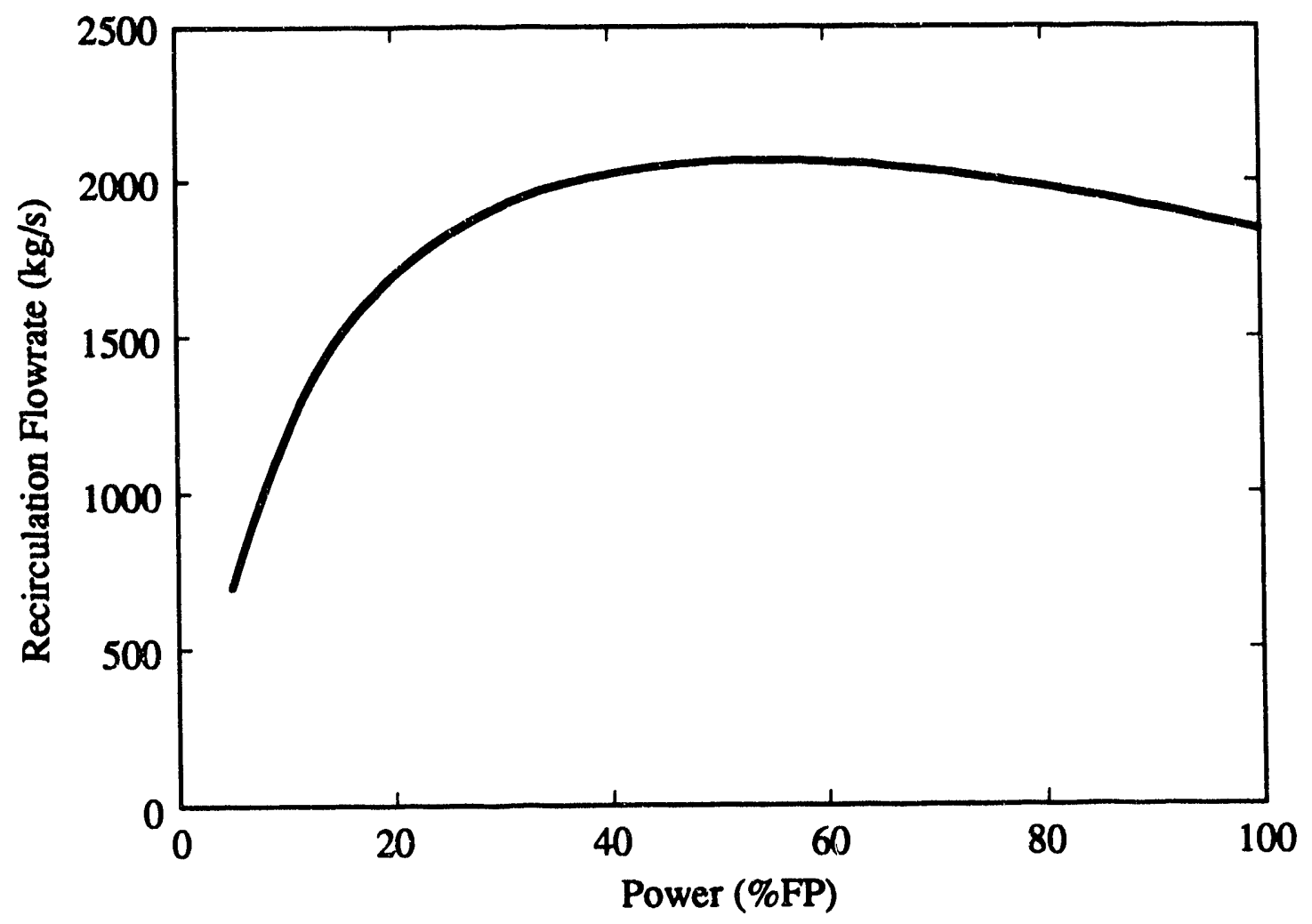

Figure 8.2.2-1 Steady-State Recirculation Flowrate. 


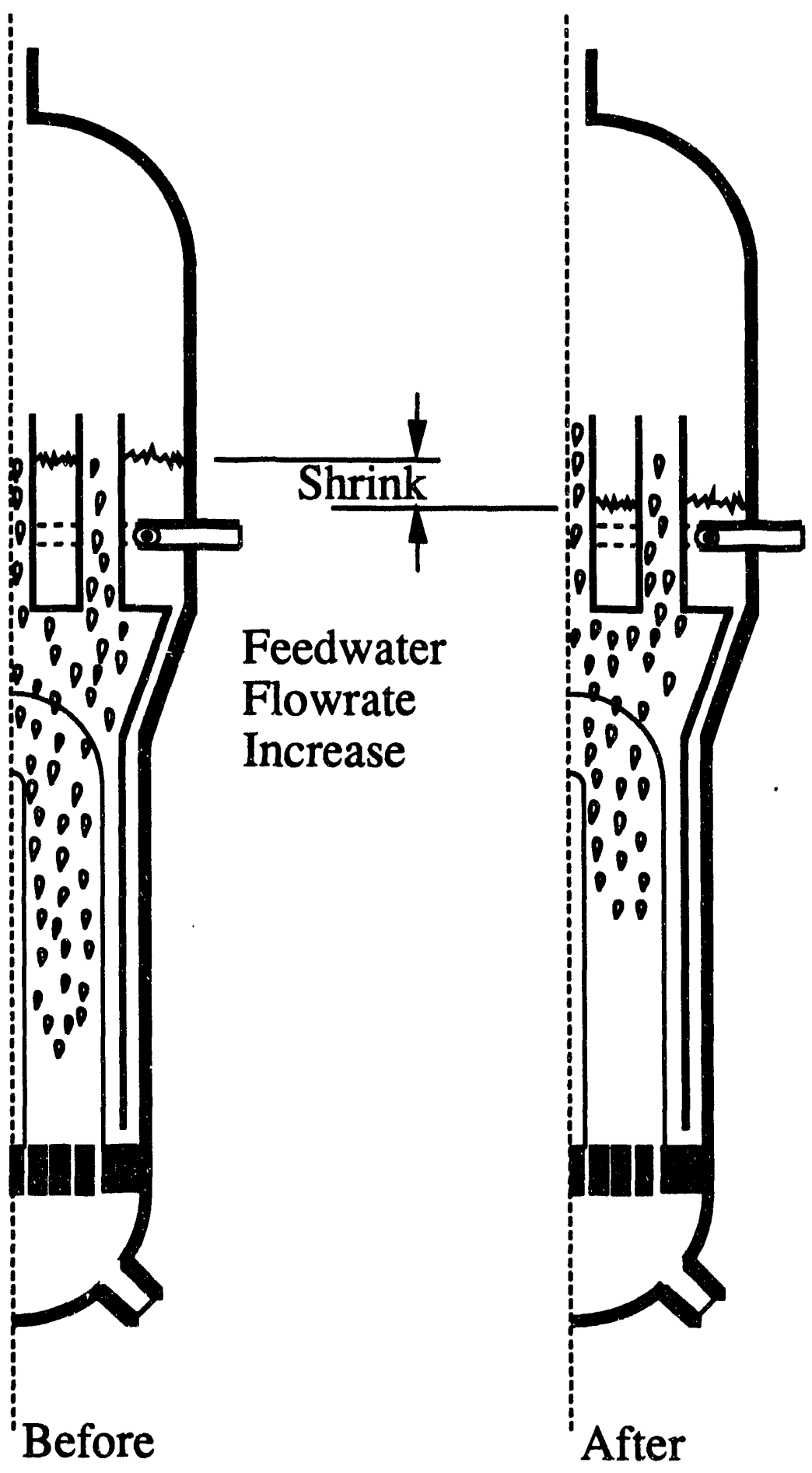

Figure 8.2.2.1-1 Schematic View of Steam Generator Level Shrink Phenomenon. 
Figures 8.2.2.1-2, 8.2.2.1-3, and 8.2.2.1-4 show steam generator level shrink as a function of these three different perturbation parameters at different power levels. For these simulations, the detailed non-linear model described in Chapter Seven of this report was used. In the first figure, the effect of a step increase in feedwater flowrate is shown. The level first rises, then drops (shrink effect), and then again rises with the rise rate related to the increase in feedwater flowrate. The initial small rise in level is the result of a mass increase in the downcomer region. However, the increase in downcomer hydraulic head soon causes an increase of flow into the tube bundle region and this causes the level to shrink.

Changes in steam flowrate cause a greater shrink effect than do ones in the feedwater flowrate because the saturation pressure increase occurs immediately after the steam flowrate decrease. The increased pressure results in significant vapor collapse in the tube bundle region and hence, a major shrink in level. This is shown in the second of the three figures.

As shown in the third figure, the level response to a primary coolant temperature perturbation differs from those of the feedwater or steam flowrate perturbations in that the mass inventory remains constant (i.e., constant feedwater flowrate and steam flowrate) and the level therefore stabilizes at a new equilibrium value. There are two factors that determine this final level. First, the lower primary coolant temperature causes a decrease in vapor production in the tube bundle region and hence a level decrease. Second, a lower primary coolant temperature causes a decrease in steam generator pressure that results in an increase of the vapor fraction in the tube bundle region and a level increase. Which of these two conflicting effects is dominant depends on the power level. At low power, the level ultimately attained will be less than the initial one because the vapor fraction in the tube bundle region decreases substantially with the primary coolant temperature decrease. However, at high power, a lot of vapor exists in the tube bundle region with the result that the second effect is dominant and the ultimate level is increased.

Steam generator water level swell is the reverse of the shrink effect. The expansion of the steam volume in the tube bundle region displaces liquid which backs up into the downcomer region thereby causing the indicated steam generator water level to rise. Figure 8.2.2.1-5 shows the steam generator swell phenomenon. A decrease in the feedwater flowrate causes the fluid into the tube bundle region to become hotter. Thus, for the same amount of heat transfer, there is an increase in the boiling rate and consequently in the steam bubble void fraction. This causes water to back up in the downcomer region and hence gives a transitory indication of a rising level. Similarly, an increase in steam flowrate causes an increase in the steam bubble volume and a decrease in steam pressure. This causes the saturated water to flash to steam and hence creates an increase in tube bundle steam volume with a concomitant temporary rise of fluid in the downcomer. Finally, an increase in primary coolant temperature creates an increased heat transfer rate from primary coolant. This in turn increases the boiling rate and creates the level swell effect.

Figures 8.2.2.1-6 to 8.2.2.1-8 show steam generator level swell as a function of the three different perturbation parameters at different power levels. The explanations for 


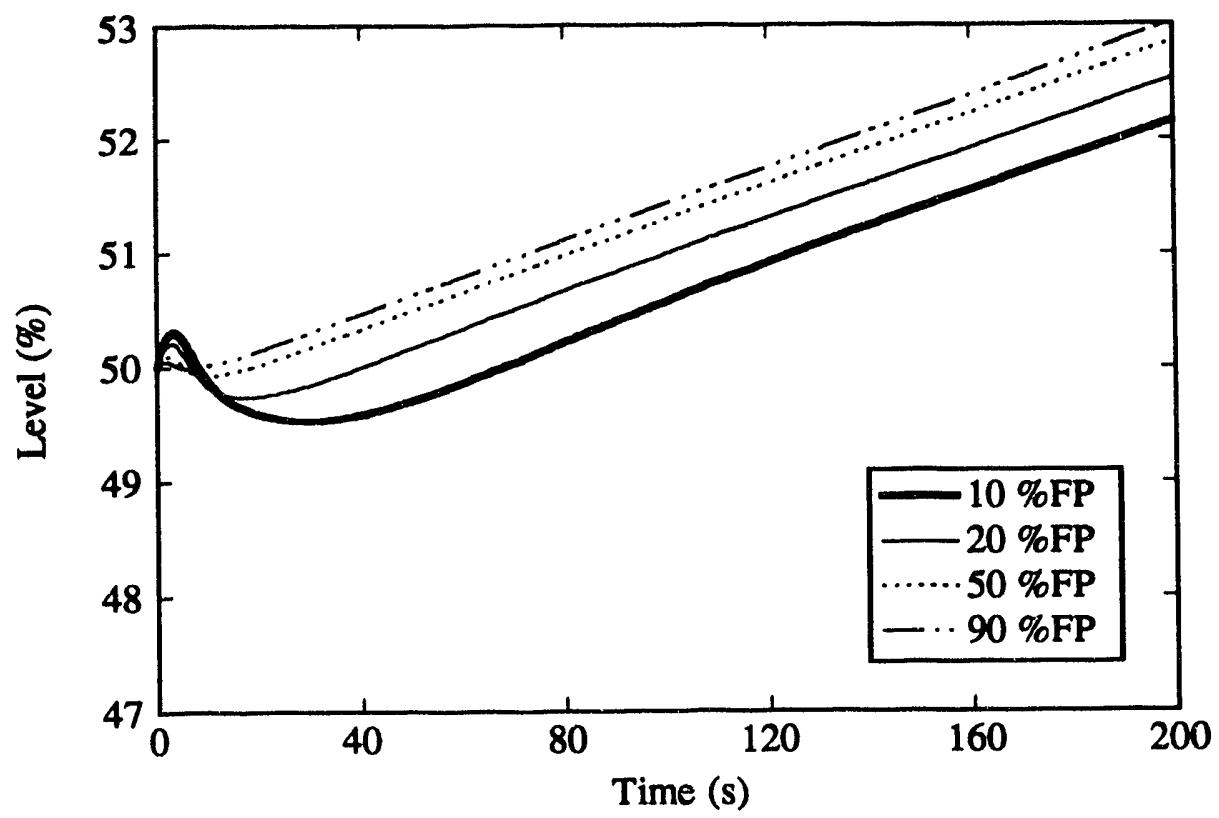

Figure 8.2.2.1-2 Steam Generator Level Shrink Effect as Result of a Sudden Feedwater Flowrate Increase.

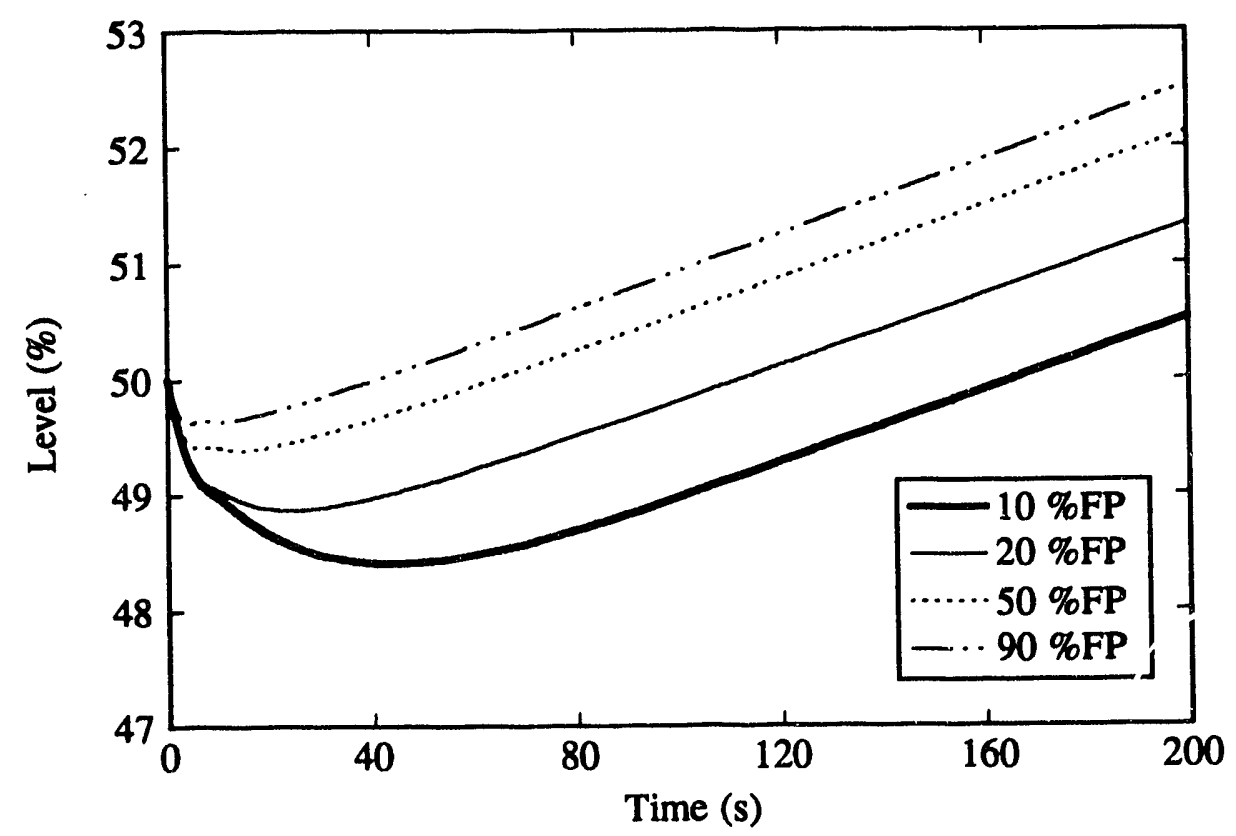

Figure 8.2.2.1-3 Steam Generator Level Shrink Effect as Result of a Sudden Steam Flowrate Decrease. 


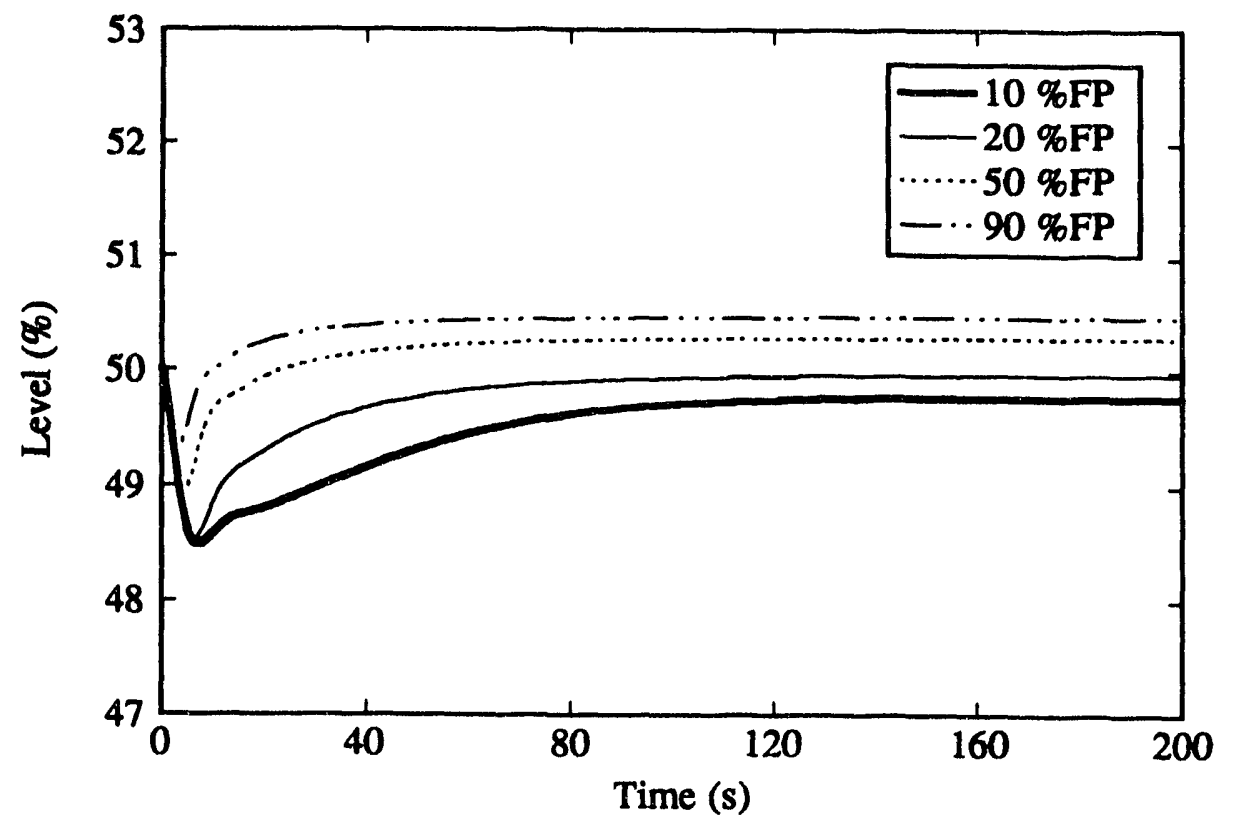

Figure 8.2.2.1-4 Steam Generator Level Shrink Effect a Result of a Sudden Primary Coolant Temperature Decrease. 


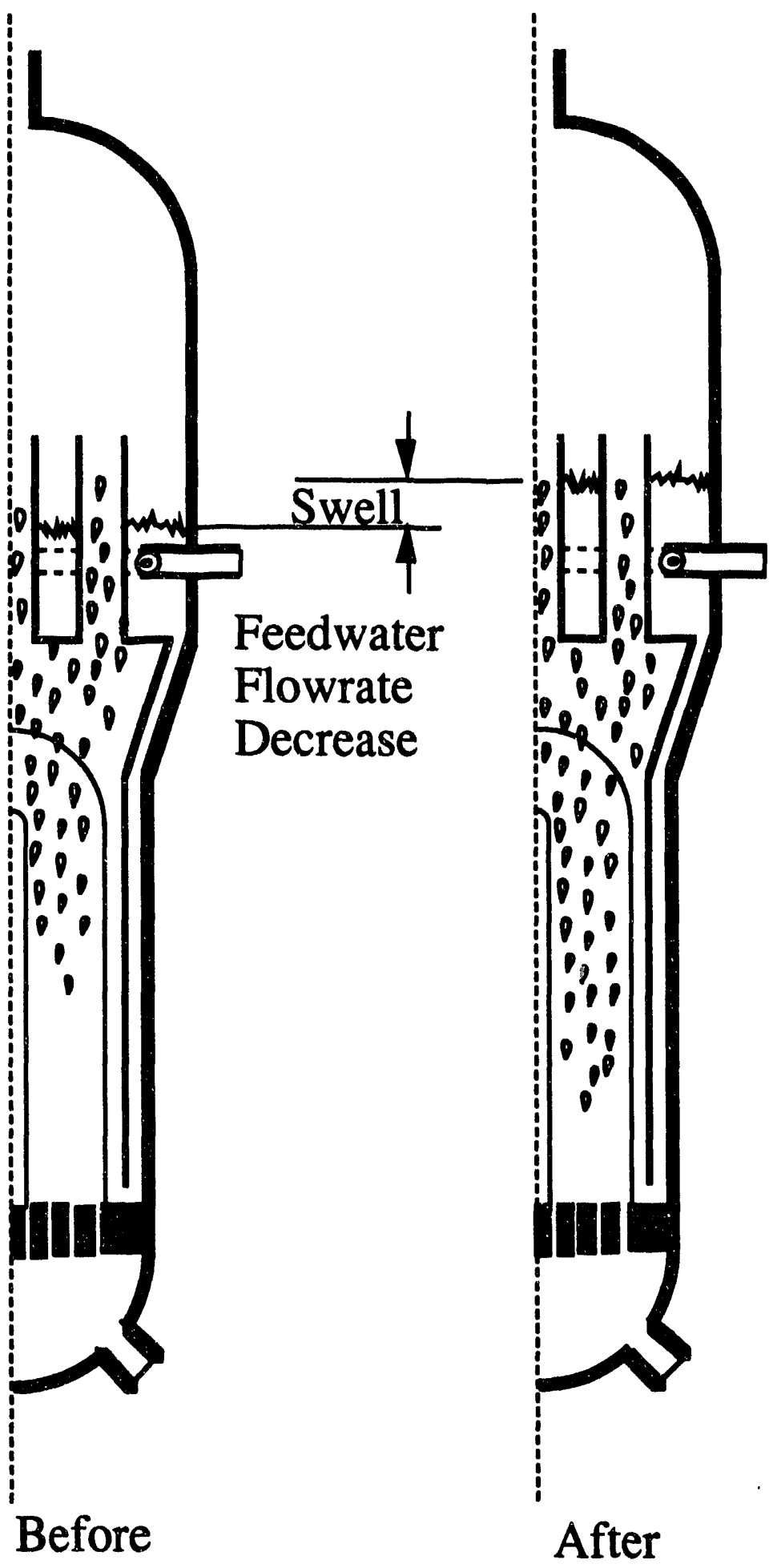

Figure 8.2.2.1-5 Schematic View of Steam Generator Level Swell Phenomenon. 


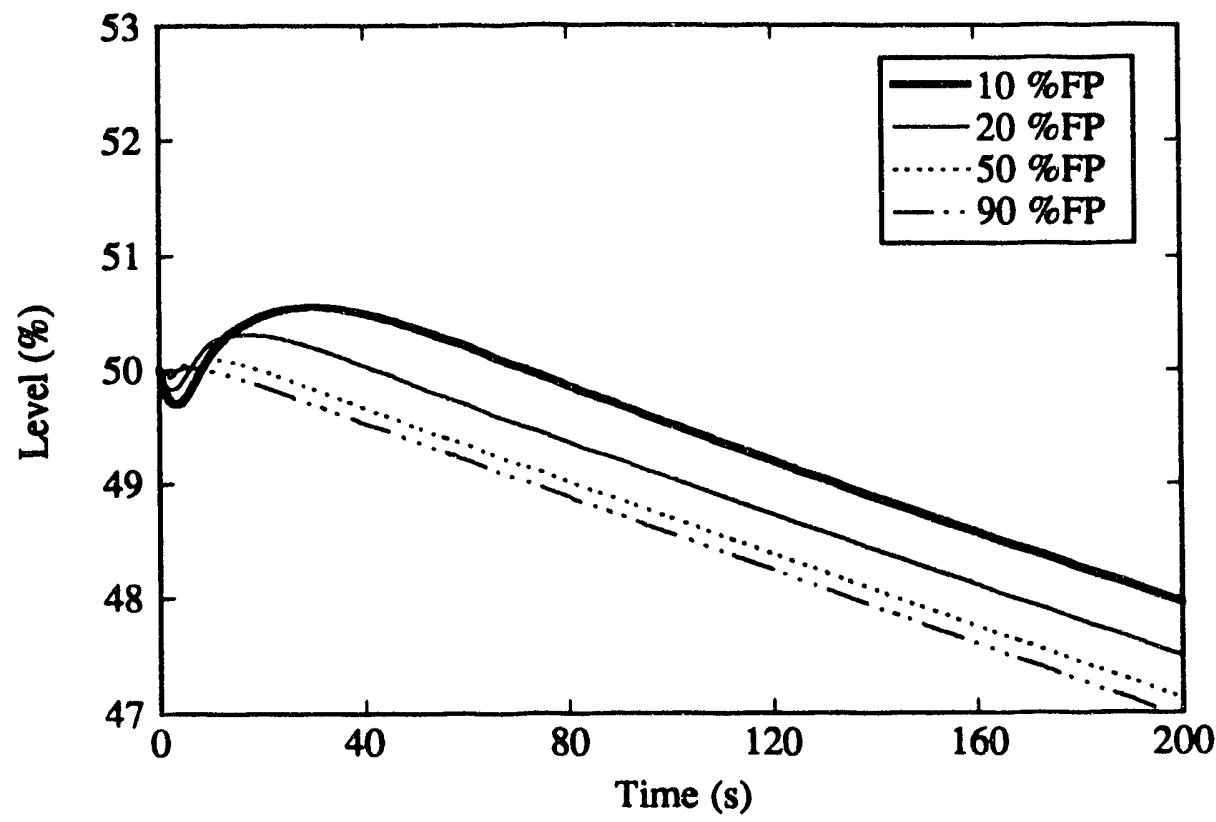

Figure 8.2.2.1-6 Steam Generator Level Swell Effect as Result of a Sudden Feedwater Flowrate Decrease.

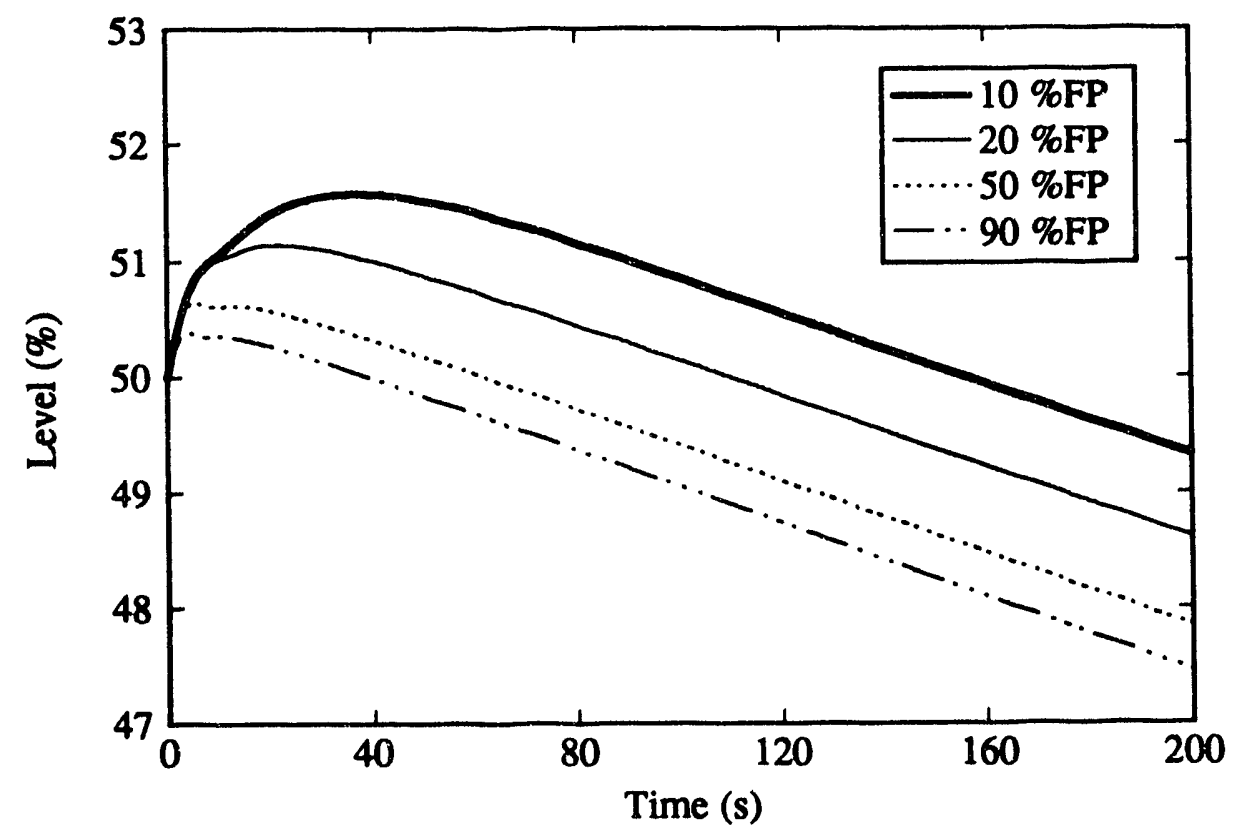

Figure 8.2.2.1-7 Steam Generator Level Swell Effect as Result of a Sudden Steam Flowrate Increase. 


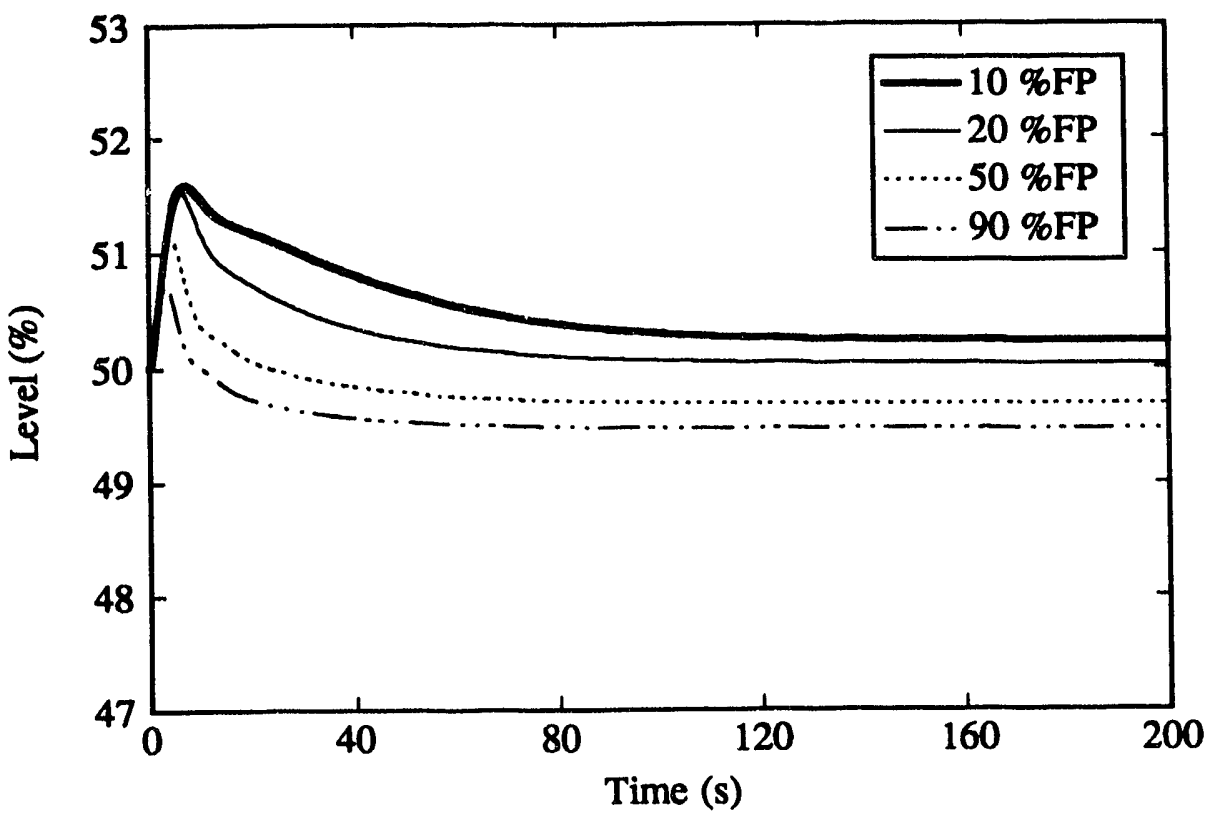

Figure 8.2.2.1-8 Steam Generator Level Swell Effect as Result of a Sudden Primary Coolant Temperature Increase. 


\subsubsection{Inverse Response}

Steam generator water level ultimately follows changes in the mass inventory. However, as was described in Section 8.2.2.1, the initial behavior of the steam generator's level following a feedwater or a steam flowrate change will be in a direction opposite to its asymptotic behavior because of shrink and swell effects. In the case of a change in primary coolant temperature, the initial steam generator water level response will also be in the opposite direction to its final behavior if at high power. This behavior is termed inverse response' or 'non-minimum phase response' $[84,85,86]$. Such behavior is exhibited by certain other processing units including drum boilers (level) [84] and distillation tower columns (temperature) [87].

Ilinoya and Altpeter list several transfer functions that exhibit inverse responses and which represent or approximate a number of physical processes [88]. In all cases, it is evident that if a system is characterized by an inverse response, then its transfer function contains a zero in the right-half plane of the Laplace domain. An illustrative example is given here. The transfer function of the boiler level response in a drum boiler system consists of an integral term and a negative first order lag [85]. The combined transfer function is expressed as follows:

$$
\frac{\bar{y}(s)}{\bar{f}(s)}=\frac{K_{2}}{s}-\frac{K_{1}}{\tau s+1}
$$

This system has poles at 0 and $-1 / \tau$. It has a zero at $K_{2} /\left(K_{1}-K_{2} \cdot \tau\right)$. If $\left(K_{2} \cdot \tau\right)$ is less than $\mathrm{K}_{1}$, then the zero is positive and the system response to a step change in input is inverse in nature. Figure 8.2.2.2-1 shows the output after such an input. (Note: This illustration is based on $K_{1}=10, K_{2}=0.2$ and $\tau=10 \mathrm{~s}$.) Because the first order term dominates initially, the overall response is temporarily opposite to its asymptotic one. However, if the condition, $K_{2} \cdot \tau$ is less than $K_{1}$ is not satisfied, then an inverse response does not occur. This is shown in Figure 8.2.2.2-2. (Note: This illustration is based on $\mathrm{K}_{1}=1, \mathrm{~K}_{2}=\mathbf{0 . 2}$, and $\tau=10 \mathrm{~s}$.)

\subsection{Simplified Steam Generator Model}

As described in Chapter Seven of this report, the steam generator simulation model consists of six coupled differential equations and many additional equations. These accurately describe steam generator level dynamics. Because this set of equations is of a high order and non-linear, it is not easy to obtain an analytic solution. This in turn makes it difficult to evaluate the performance of a steam generator water level controller. Therefore, a reasonably accurate but low-order steam generator model is preferred.

The complicated dynamics of a steam generator is approximated here by a simplified mathematical model that is deduced through system identification from transient simulation results obtained from a detailed, high-order, non-linear model. Fortunately, it is not necessary to model the entire steam generator for control system design. Only level dynamics including shrink and swell effects are of interest. 


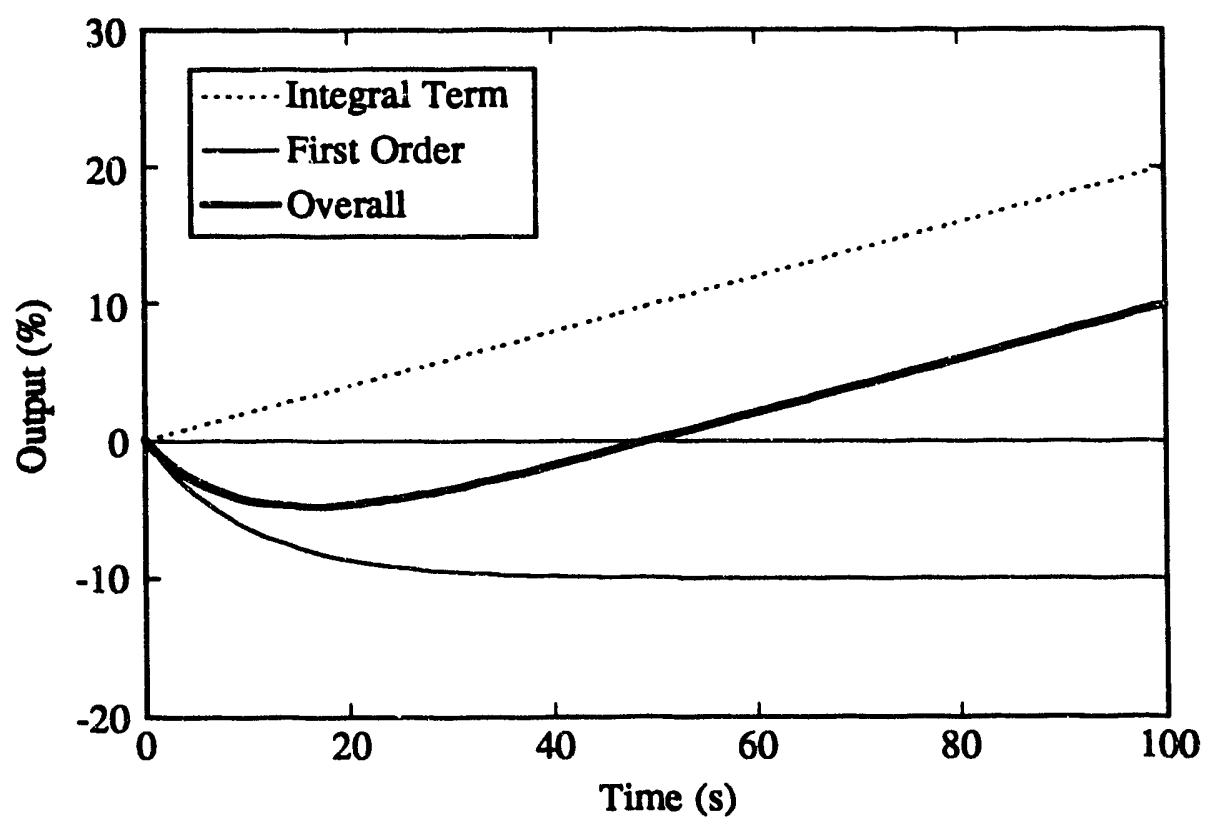

Figure 8.2.2.2-1 Typical Inverse Response of a System Consisting of an Integral Term and a First Order Lag Term.

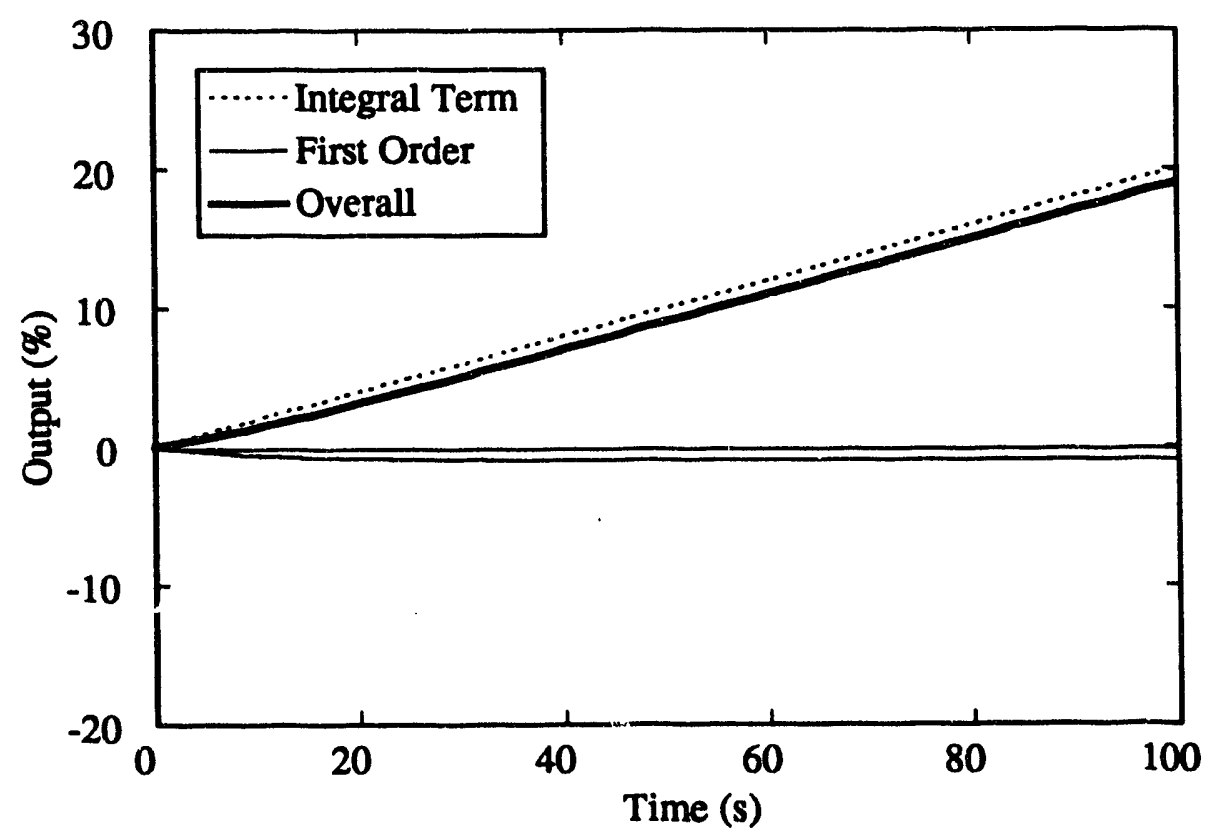

Figure 8.2.2.2-2 Non-Inverse Response of a System Consisting of an Integral Term and a First Order Lag Term. 
The simplified model that is described here was developed to facilitate control studies. However, because it functions in real time, other uses are possible. For example, predictive displays could be developed that allow $t^{2} \cdot e$ operator to visualize the consequences of a proposed manual control action before impler inting it. Such displays were developed in the course of the research reported here. They are described in Appendix A to this report.

\subsubsection{Simplified Transfer Function}

The mathematical description of the dynamic characteristics of a system is called a model. For control purposes, such models may be written in either the time domain (differential equations) or the frequency domain (Laplace transform). The latter approach is used here with a transfer function diffined as the ratio of the Laplace transform of the system output to that of the input. If the model is non-linear, then it must first be linearized about an operating point and expressed in terms of deviation variables. In cases where the system dynamics are very complicated, the mathematical model will also consist of complicated differential equations and it will not be trivial to obtain the Laplace transform. Therefore, it is often useful to approximate a complicated physical system by a simplified mathematical model that is based on either simulation analyses of a detailed mathematical model or experimental study of the physical system.

Irving and Bihoreaux suggested a simple transfer function that successfully describes the shrink and swell effects that are created by feedwater or steam flowrate changes [89]. The inputs to the equation are feedwater and steam flowrates. The output is the downcomer water level. The equation is:

$$
\begin{aligned}
L_{w}(s)= & {\left[\frac{G_{1}}{s}-\frac{G_{2 f}}{\left(\tau_{2 f} s+1\right)}+\frac{G_{3 f} s}{\left(s+1 / \tau_{3 f}\right)^{2}+\omega^{2}}\right] \dot{m}_{f w}(s)+} \\
& {\left[-\frac{G_{1}}{s}+\frac{G_{2 s}}{\left(\tau_{2 s} s+1\right)}\right] \dot{m}_{s}(s) }
\end{aligned}
$$

where $s$ is the Laplace variable, $L_{w}(s)$ is the Laplace transform of the steam generator level change due to feedwater or steam flowrate changes, and $\dot{\mathbf{m}}_{\mathrm{fw}}(\mathbf{s})$ and $\dot{\mathrm{m}}_{\mathrm{s}}(\mathbf{s})$ are the Laplace transforms of the changes in feedwater and steam flowrate respectively. Each of these quantities represents a deviation. Thus, they are $\left[L_{w}(t)-L_{w}(0)\right],\left[\dot{m}_{f w}(t)-\dot{m}_{f w}(0)\right]$, and $\left[\dot{\mathrm{m}}_{\mathrm{s}}(\mathrm{t})-\dot{\mathrm{m}}_{\mathrm{s}}(0)\right]$ respectively.

Each term of Equation (8.3.1-1) has physical significance. $G_{1} / s$ is the mass capacity term of the steam generator where $G_{1}$ is a measure of the steam generator's height to volume ratio. This mass capacity term represents changes in the steam generator level caused by mass influx or efflux from the volume of the particular steam generator. If the $G_{1} / s$ term were the only quantity in the level equation, then the steam generator level indicator would be an accurate representation of the contained mass. 
The $G_{2 f} /\left(\tau_{2 f} s+1\right)$ and $G_{2 s} /\left(\tau_{2 s} s+1\right)$ terms are first order and represent the shrink and swell effects. $G_{2 f}$ and $G_{2 s}$ are variables that describe the magnituile of the shrink and swell effects that result from changes in feedwater and steam flowrate respectively. These two values depend on the operating power of the reactcr. The quantities $\tau_{2 f}$ and $\tau_{2 s}$ are the characteristic decay times for the shrink and swell effects caused by the feedwater and steam flowrate changes, respectively.

The remaining term in Equation (8.2.3.1-1) describes the mechanical oscillation that results from the direct addition of feedwater to the steam generator. This quantity only appears in response to a feedwater change and it decays rapidly.

Equation (8.3.1-1) does not include the effect of a change in primary coolant temperature. As described in Section 8.2.2, primary coolant temperature changes can cause shrink or swell effects. These effects can be represented by the addition of two opposing first order terms:

$$
L_{T}(s)=\left[\frac{G_{1 T}}{\left(1+\tau_{1 T} s\right)}-\frac{G_{2 T}}{\left(1+\tau_{2 T} s\right)}\right] T_{p}(s)
$$

where $\mathrm{L}_{\mathrm{T}}(\mathrm{s})$ is the Laplace transform of the steam generator level change that results from a primary coolant temperature change and $T_{p}(s)$ is the Laplace transform of the change in primary coolant temperature.

The steam generator level response is therefore the sum of the individual responses to the three different inputs and is as follows:

$$
L(s)=G_{f w}(s) \dot{m}_{f w}(s)+G_{s}(s) \dot{m}_{s}(s)+G_{T}(s) T_{p}(s)
$$

where:

$$
\begin{aligned}
& G_{f w}(s)=\frac{L_{f w}(s)}{\dot{m}_{f w}(s)}=\frac{G_{1}}{s}-\frac{G_{2 f}}{\left(\tau_{2 f} s+1\right)}+\frac{G_{3 f} s}{\left(s+1 / \tau_{3 f}\right)^{2}+\omega^{2}} \\
& G_{s}(s)=\frac{L_{s}(s)}{\dot{m}_{s}(s)}=-\frac{G_{1}}{s}+\frac{G_{2 s}}{\left(\tau_{2 s} s+1\right)} \\
& G_{T}(s)=\frac{L_{T}(s)}{T_{p}(s)}=\frac{G_{1 T}}{\left(1+\tau_{1 T} s\right)}-\frac{G_{2 T}}{\left(1+\tau_{2 T} s\right)}
\end{aligned}
$$

and where $\mathrm{L}_{\mathrm{fw}}(\mathrm{s}), \mathrm{L}_{3}(\mathrm{~s})$, and $\mathrm{L}_{\mathrm{T}}(\mathrm{s})$ are the Laplace transforms of the steam generator level changes that result from perturbations in feedwater flowrate, steam flowrate, and primary coolant temperature respectively. The quantities $G_{f w}(s), G_{S}(s)$ and $G_{T}(s)$ are called the process transfer functions of the feedwater flowrate change, steam flowrate change, and primary coolant temperature change respectively. In reality, all of the so-called 'constants' in the above equations are functions of the operating power and must be determined for the 
specific reactor and steam generator. The system identification method that was used for this purpose is explained in the following section of this report.

\subsubsection{Identification of Model Parameters}

Transfer functions are often determined by means of experimental analysis, usually frequency response methods [86]. Specifically, if the amplitude ratio and phase shift between the input and output are measured at a sufficient number of frequencies, then they may be plotted on a Bode diagram and the transfer function determined from asymptotic approximations. For the research conducted here, it was of course impossible to use an actual steam generator for experimental analyses. Accordingly, simulation results from the detailed non-linear model were used in lieu of experimental data. Also, as described in the preceding sections of this report, the forms of the transfer functions were already given. Hence, only the coefficients in those equations needed to be determined. The method employed here for the identification of those coefficients was to minimize the error between the level response of the simplified transfer function and that of the detailed non-linear model.

To obtain an equation for the steam generator level, it is necessary to solve Equation (8.3.1-3) by taking the inverse Laplace transform of each term. All changes of perturbation parameters were in the form of a step. Therefore, the Laplace-transformed inputs of the perturbation parameters are expressed as:

$$
\begin{aligned}
& \dot{\mathrm{m}}_{\mathrm{fw}}(\mathrm{s})=\delta \dot{\mathrm{m}}_{\mathrm{fw}} \cdot(1 / \mathrm{s}) \\
& \dot{\mathrm{m}}_{\mathrm{s}}(\mathrm{s})=\delta \dot{\mathrm{m}}_{\mathrm{s}} \cdot(1 / \mathrm{s}) \\
& \mathrm{T}_{\mathrm{p}}(\mathrm{s})=\delta \mathrm{T}_{\mathrm{p}} \cdot(1 / \mathrm{s})
\end{aligned}
$$

where $\delta \dot{m}_{f w}, \delta \dot{m}_{s}$, and $\delta T_{p}$ are multipliers that are directly proportional to the magnitude of the feedwater flowrate, steam flowrate, and primary coolant temperature respectively. Substitution of these relations into Equation (8.3.1-3) allows the latter to be solved. In order to identify coefficients, one of the perturbation parameters is selected as the input and the others are kept constant. For example, if feedwater flowrate alone is changed in a step fashion, then $\dot{m}_{\mathrm{fw}}(\mathrm{s})=\delta \dot{\mathrm{m}}_{\mathrm{fw}} / \mathrm{s}, \dot{\mathrm{m}}_{\mathrm{s}}(\mathrm{s})=0$, and $\mathrm{T}_{\mathrm{p}}(\mathrm{s})=0$. Hence, the transient response is:

$$
\begin{aligned}
L_{f w}(s) & =G_{f w}(s) \cdot \dot{m}_{f w}(s) \\
& =\left[\frac{G_{1}}{s}-\frac{G_{2 f}}{\left(\tau_{2 f} s+1\right)}+\frac{G_{3 f} s}{\left(s+1 / \tau_{3 f}\right)^{2}+w^{2}}\right] \frac{\delta \dot{m}_{f w}}{s} .
\end{aligned}
$$

Upon taking the inverse Laplace transform of Equation (8.3.2-4), the steam generator water level response in the time domain is obtained. Thus, 


$$
L_{f w}(t)=\left[G_{1} t-G_{2 f}\left\{1-\exp \left(-\frac{t}{\tau_{2 f}}\right)\right\}+\frac{G_{3 f}}{\omega} \exp \left(-\frac{t}{\tau_{3 f}}\right) \sin (\omega t)\right] \delta \dot{m}_{f w} .
$$

The level transient response to the steam flowrate and the primary coolant temperature perturbations can be calculated in a similar manner. For steam flowrate, the result is:

$$
\begin{aligned}
L_{s}(s) & =G_{s}(s) \cdot \dot{m}_{s}(s) \\
& =\left[-\frac{G_{1}}{s}+\frac{G_{2 s}}{\left(\tau_{2 s} s+1\right)}\right] \cdot \frac{\delta \dot{m}_{s}}{s} .
\end{aligned}
$$

or in the time domain:

$$
L_{s}(t)=\left[-G_{1} t+G_{2 s}\left(1-\exp \left(-\frac{t}{\tau_{2 s}}\right)\right\}\right] \cdot \delta \dot{m}_{s} .
$$

For primary coolant temperature, the result is:

$$
\begin{aligned}
\operatorname{LT}_{T}(s) & =G_{T}(s) \cdot T_{p}(s) \\
& =\left[\frac{G_{1 T}}{\left(1+\tau_{1 T} s\right)}-\frac{G_{2 T}}{\left(1+\tau_{2 T} s\right)}\right] \cdot \frac{\delta T_{p}}{s} .
\end{aligned}
$$

or in the time domain:

$$
L_{T}(t)=\left[G_{1 T}\left(1-\exp \left(-\frac{t}{\tau_{1 T}}\right)\right)-G_{2 T}\left(1-\exp \left(-\frac{t}{\tau_{2 T}}\right)\right)\right] \cdot \delta T_{p} .
$$

The next step in the parameter identification process was to obtain an exact level response from the detailed non-linear steam generator model. Many detailed simulations at different power levels (three different simulations, one each for changes in feedwater flowrate, steam flowrate, and primary coolant temperature) were performed for this purpose. A MATLAB [90] software package was then used to calculate the unknown coefficients by minimizing the quadratic error between the level responses to a step change of a specific perturbaiion parameter obtained from the simplified model and that obtained from the detailed non-linear model. The coefficients were then fitted as polynomial functions of power. The results are shown in Figures 8.3.2-1 to 8.3.2-11. (Note: The coefficients are functions of both the power and the feedwater temperature. However, because the feedwater temperature is itself a function of power, only power dependence was considered.) An interesting feature of these coefficients is that their magnitudes tend to increase dramatically as power decreases. There are several reasons for this. First, ai low power, the driving head for the recirculation flow is reduced and hence, the recirculation 


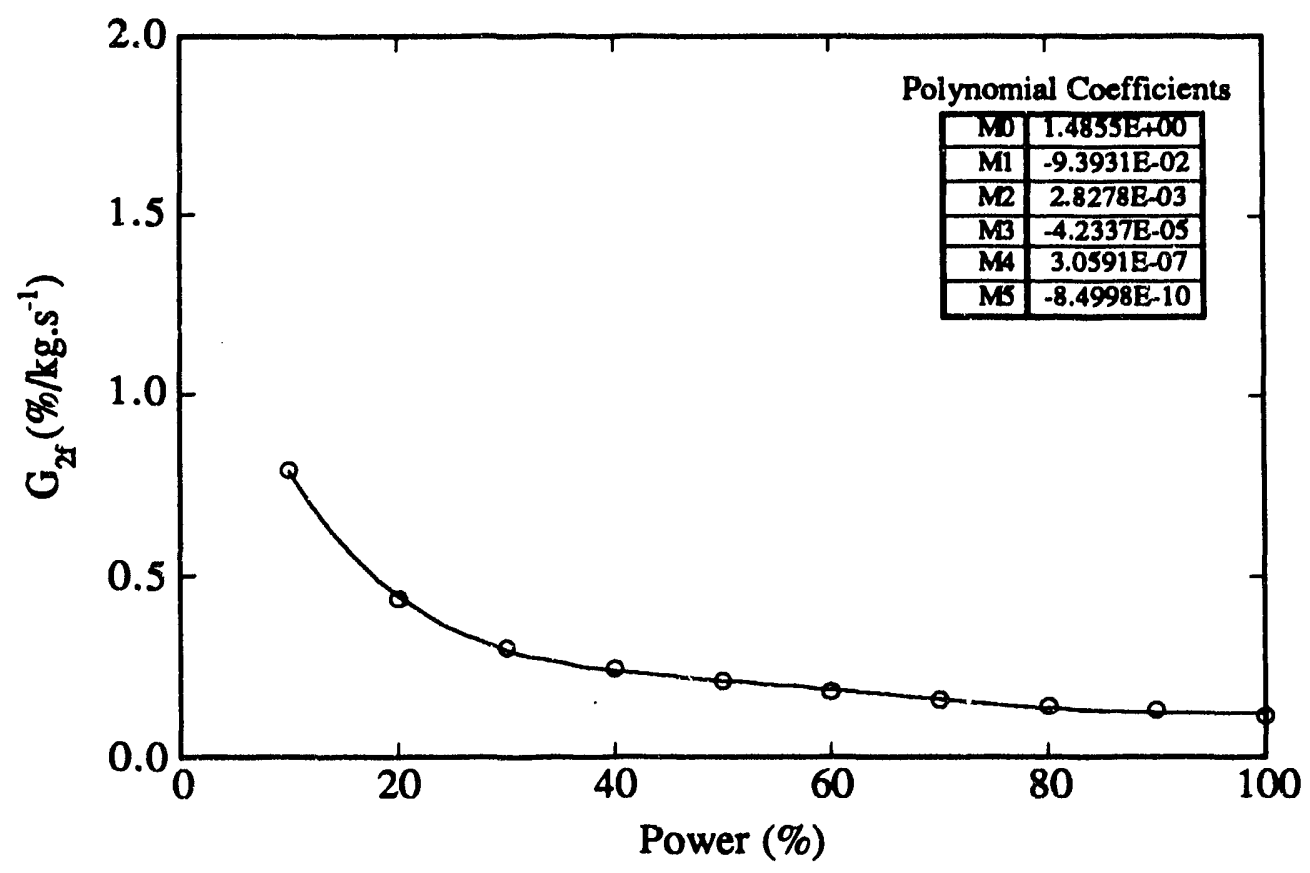

Figure 8.3.2-1 Steam Generator Simplified Transfer Function Coefficient, $\mathbf{G}_{2 f}$.

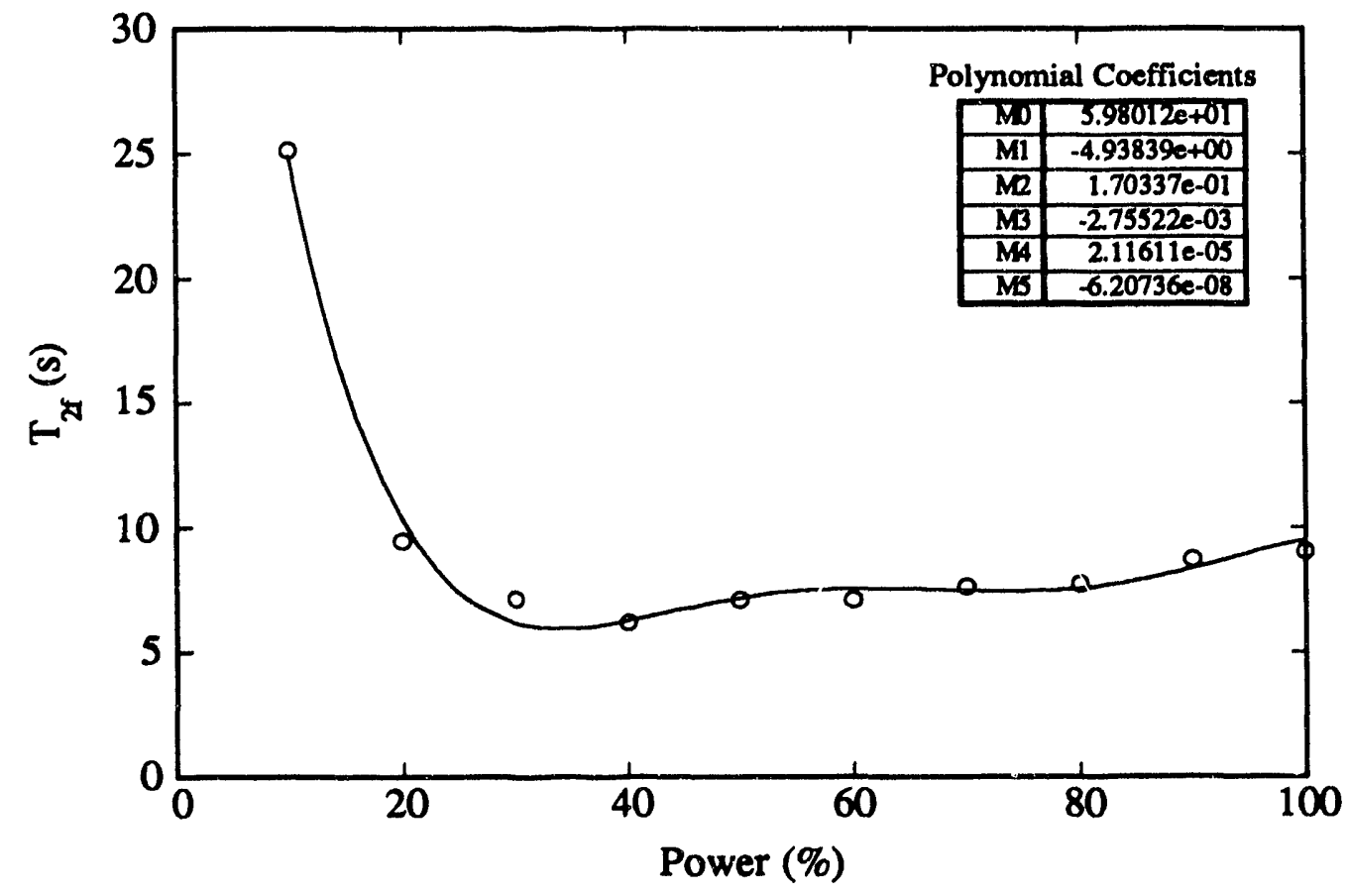

Figure 8.3.2-2 Steam Generator Simplified Transfer Function Coefficient, $T_{2 f}$. 


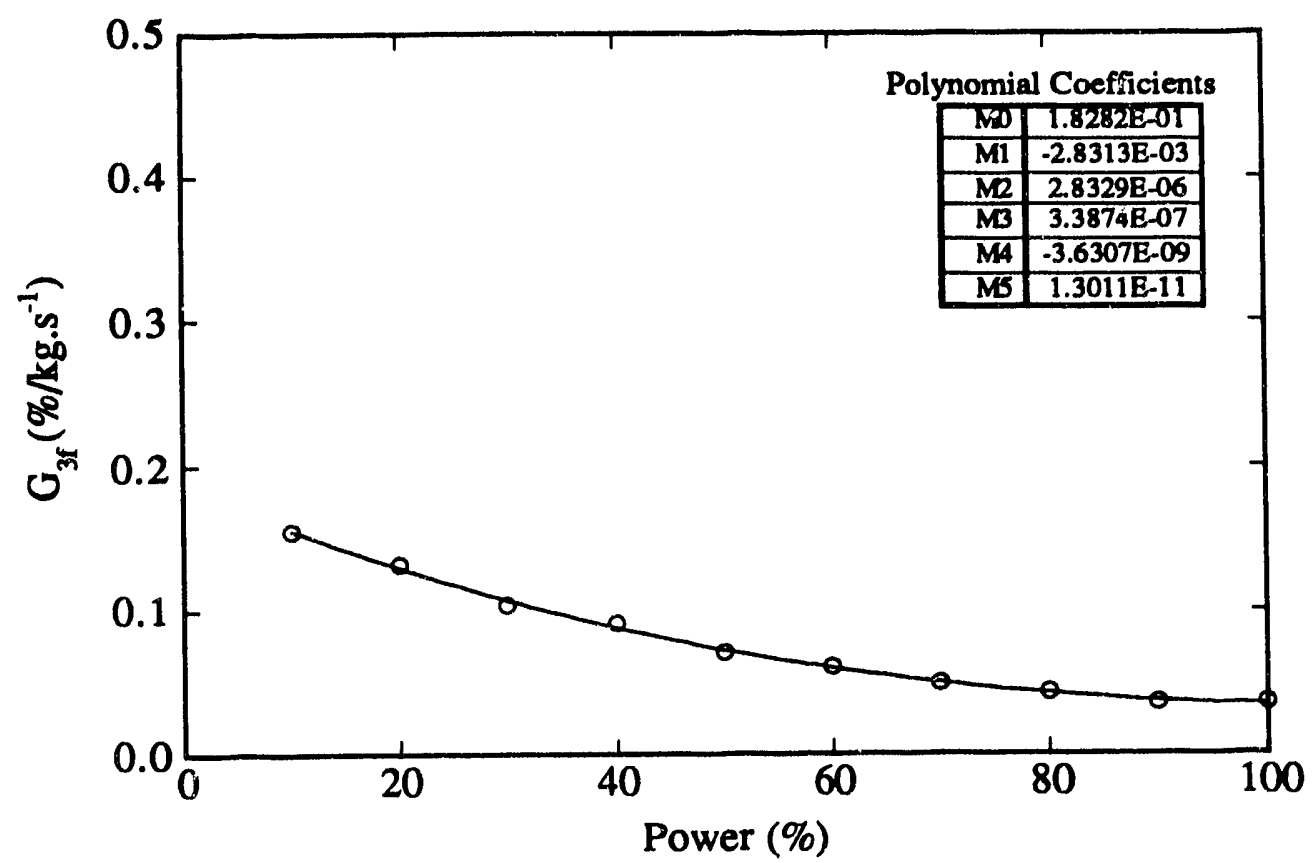

Figure 8.3.2-3 Steam Generator Simplified Transfer Function Coefficient, $G_{3}$.

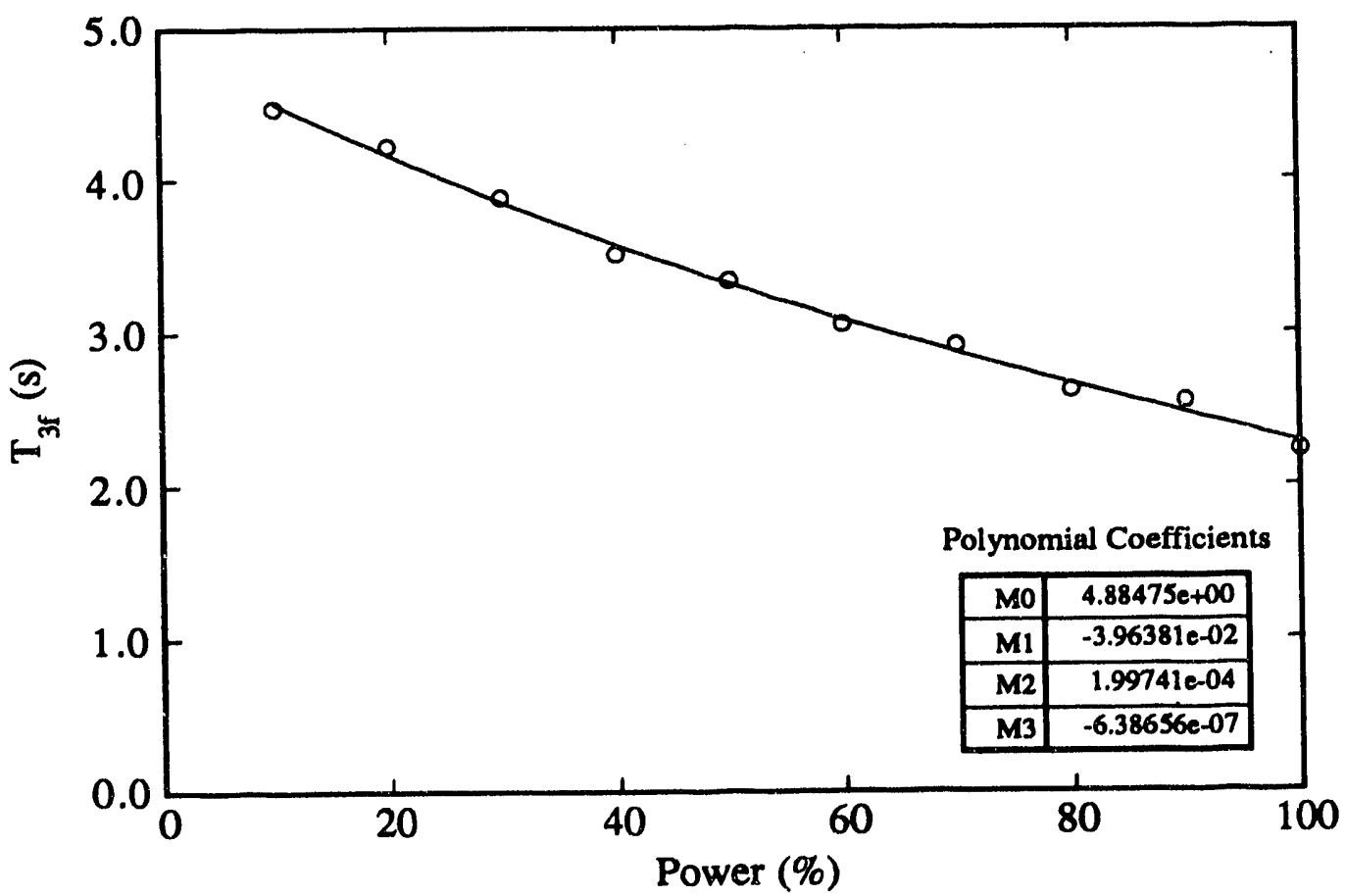

Figure 8.3.2-4 Steam Generator Simplified Transfer Function Coefficient, $T_{3 f}$. 


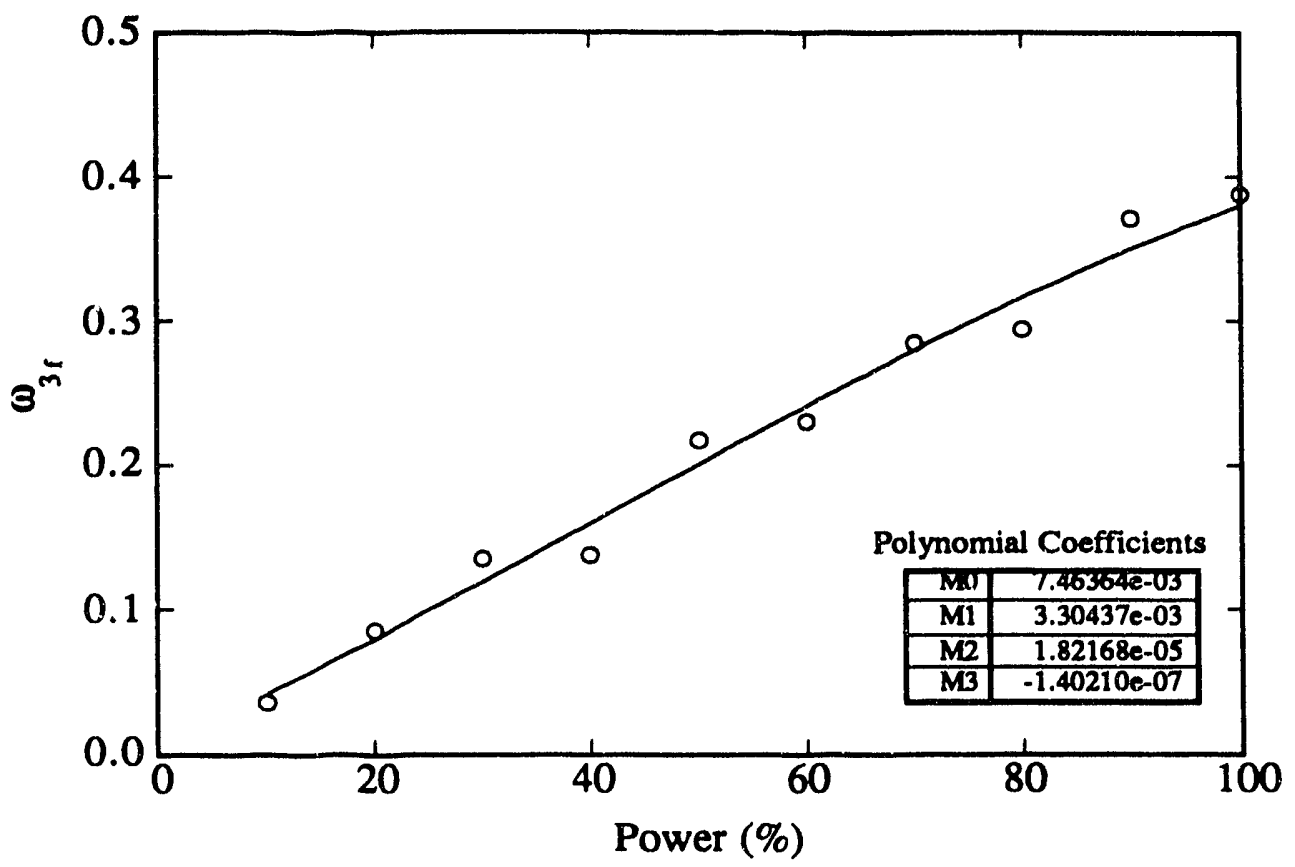

Figure 8.3.2-5 Steam Generator Simplified Transfer Function Coefficient, $\omega_{3 \mathrm{f}}$.

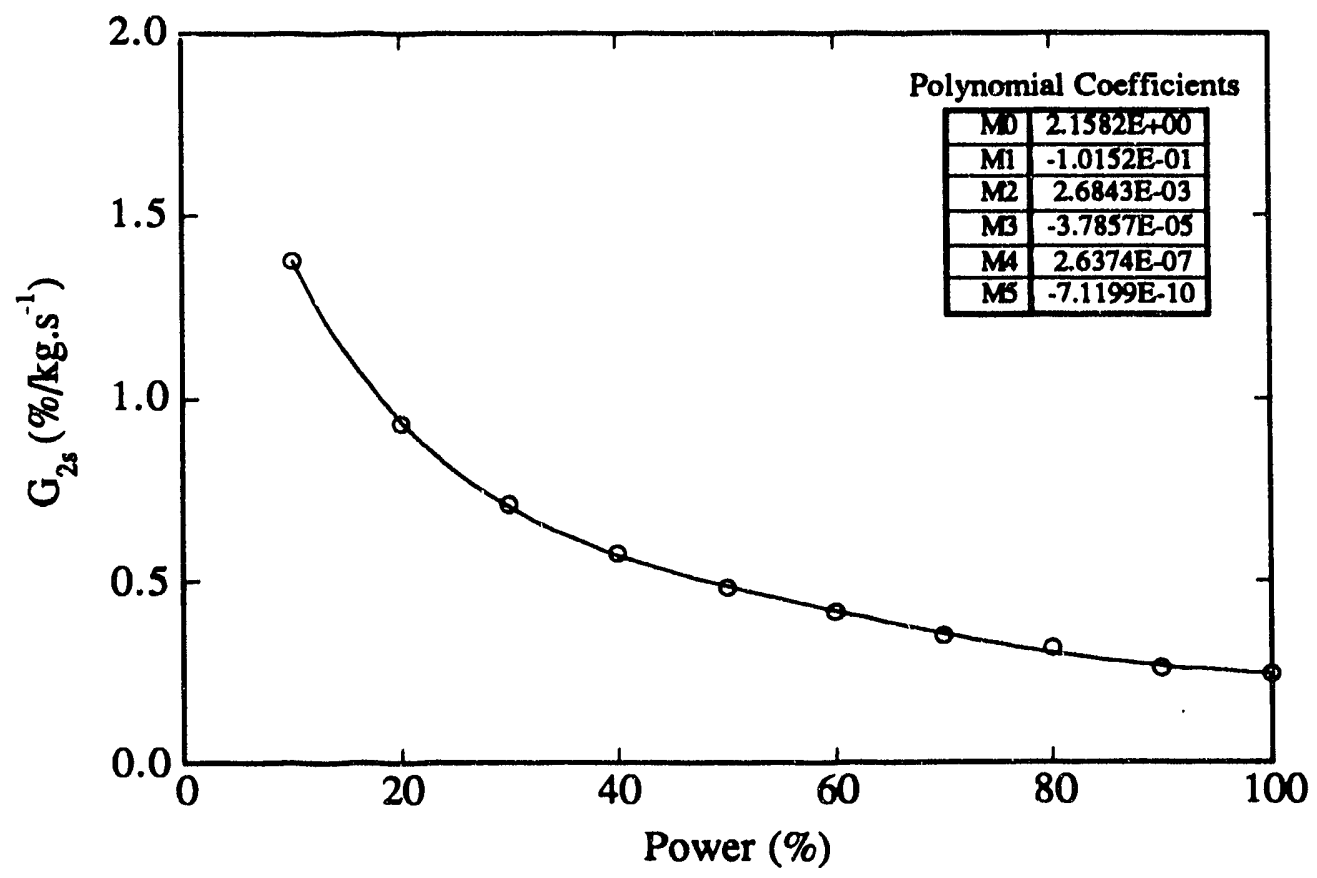

Figure 8.3.2-6 Steam Generator Simplified Transfer Function Coefficient, $\mathrm{G}_{2 \mathrm{~s}}$. 


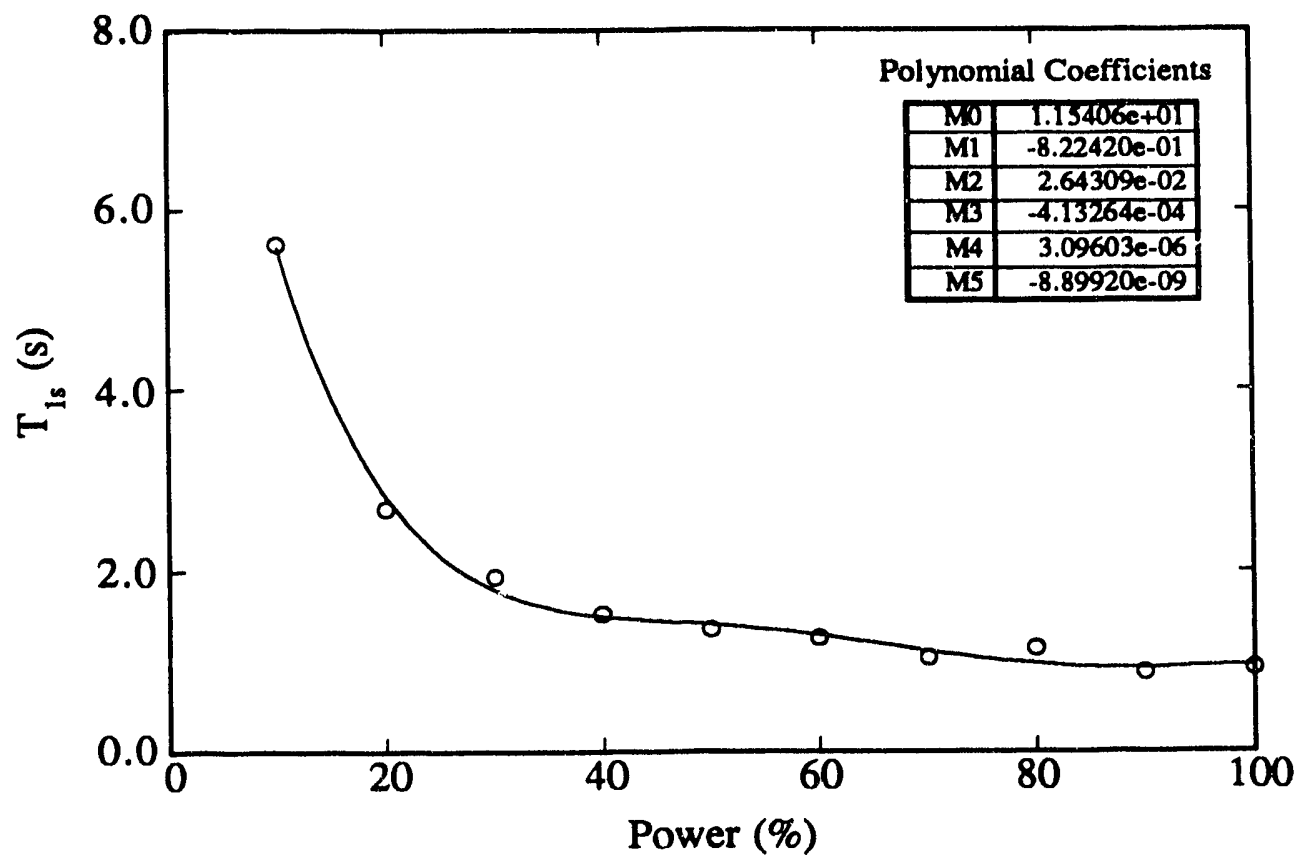

Figure 8.3.2-7 Steam Generator Simplified Transfer Function Coefficient, $\mathrm{T}_{2 \mathrm{~s}}$.

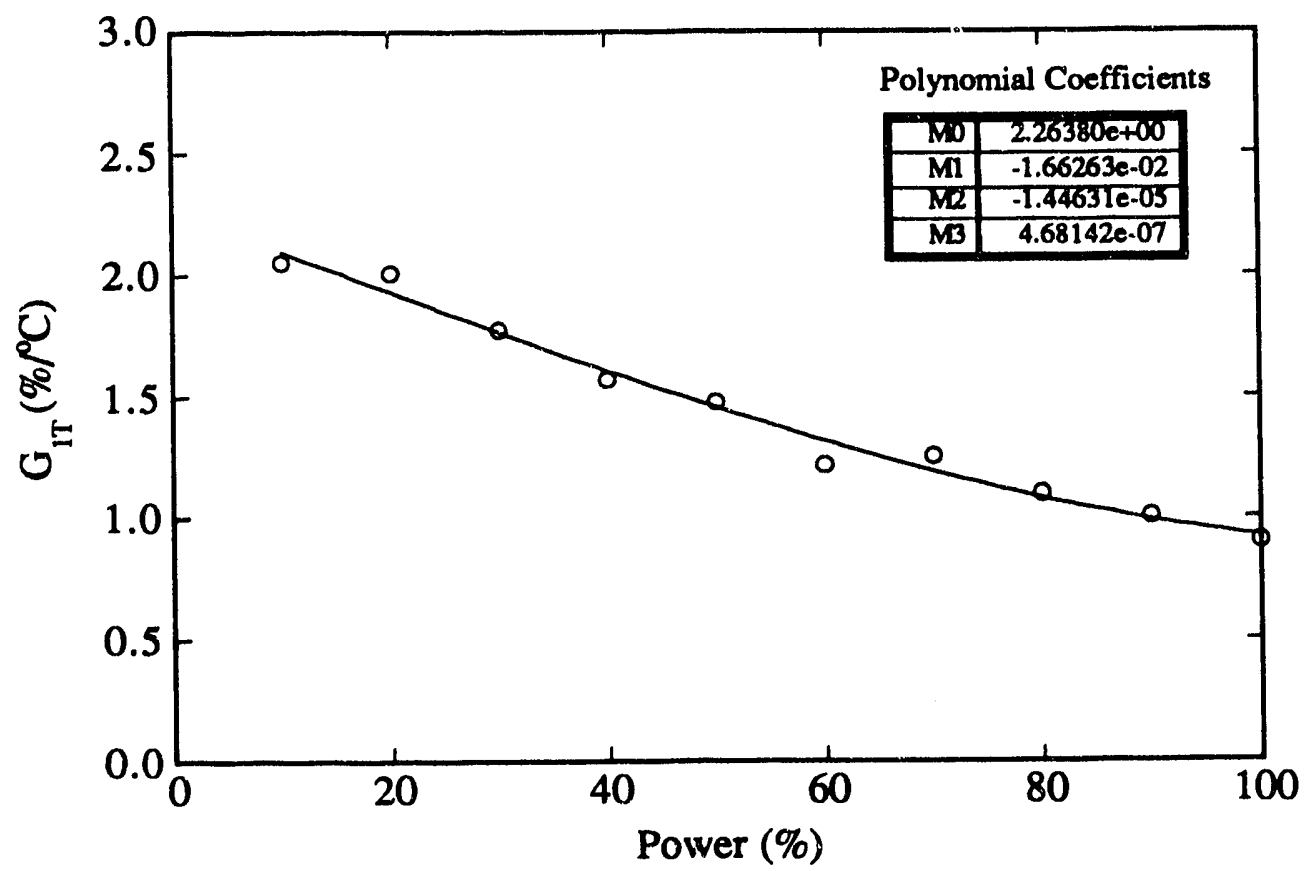

Figure 8.3.2-8 Steam Generator Simplified Transfer Function Coefficient, $\mathrm{G}_{1 \mathrm{~T}}$. 


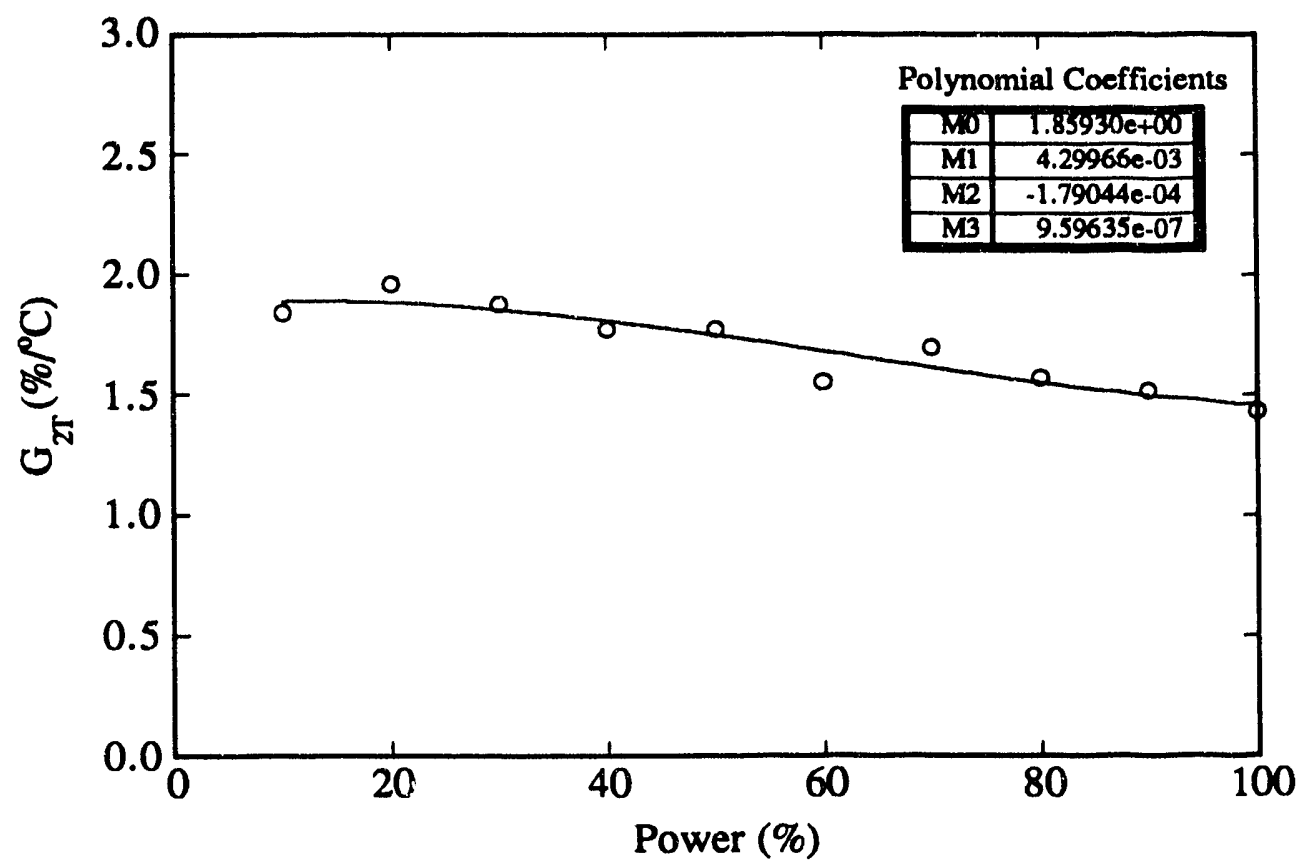

Figure 8.3.2-9 Steam Generator Simplified Transfer Function Coefficient, $\mathrm{G}_{2 \mathrm{~T}} \mathrm{~T}$.

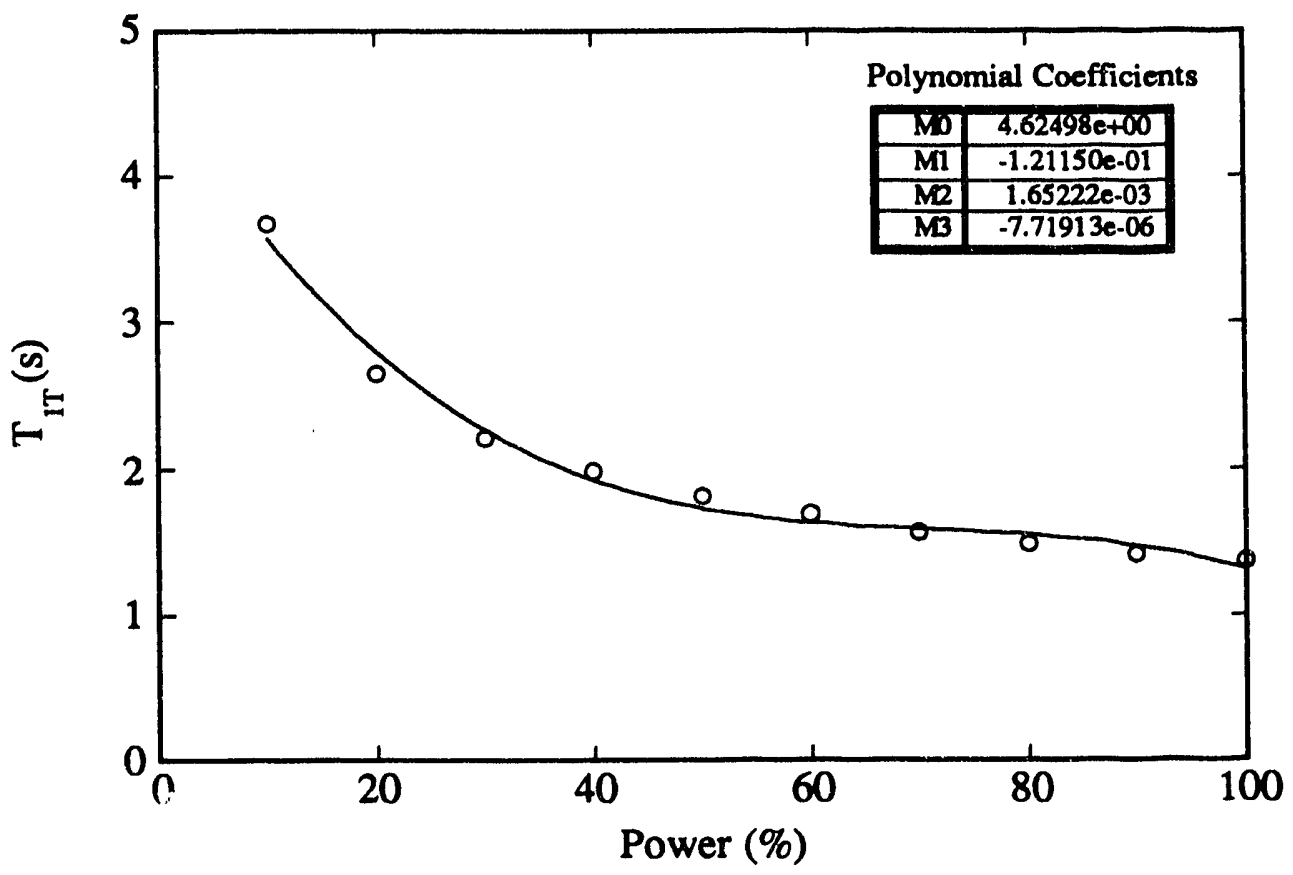

Figure 8.3.2-10 Steam Generator Simplified Transfer Function Coefficient, $\mathrm{T}_{1 \mathrm{~T}}$. 


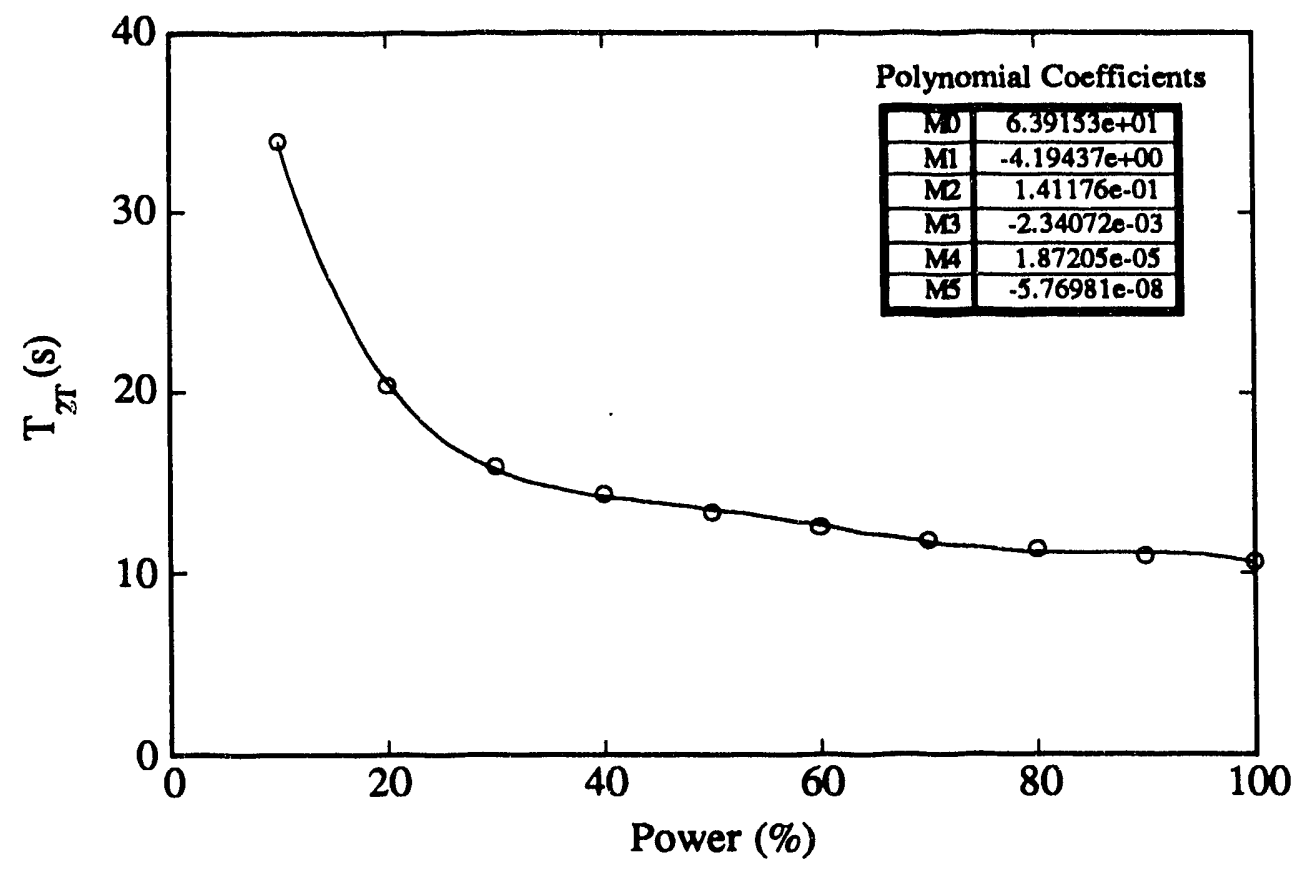

Figure 8.3.2-11 Steam Generator Simplified Transfer Function Coefficient, $T_{2 T}$. 
flowrate decreases. Second, the amount of saturated water in the tube bundle region is large and the steam bubble volume is, as a result, smaller. Third, small changes in steam generator pressure due to steam flowrate changes can cause the flashing of a lot of nearly saturated water. Therefore, a small perturbation can create a large change in the steam volume. This is especially true for changes in steam flowrate. Also, small changes in feedwater flowrate can result in large deviations from equilibrium in the steam generator at low power because the feedwater is colder at low power.

\subsubsection{Evaluation of the Simplified Steam Generator Model}

Various steam generator water level transients were simulated using the simplified model and the results are shown in Figures 8.3.3-1 to 8.3.3-3. The simplified model predicts the steam generator water level response almost exactly for the case of a feedwater perturbation. When the steam flowrate or primary coolant temperature is changed, the simplified model shows less than a $0.5 \%$ difference when compared to the detailed non-linear model.

\subsection{Modeling and Analysis of Current-Generation Steam Generator Level Controllers}

In this section a current-generation steam generator level controller is described together with analyses of existing control problems. Included are both a mathematical model and stability analyses.

\subsubsection{Model of Current-Generation Steam Generator Level Controllers}

Figure 8.4.1-1 depicts a current-generation steam generator level controller. The unit is a three-element proportional-integral (PI) design and is configured for high power operation. It can be regarded as combining two types of control action: feedback based on a level error and a feedforward action based on a flowrate error. For the former, the error is generated by comparing the level setpoint and the measured level. For the latter, the error is computed as the difference between steam and feedwater flowrates. Each of these subcontrollers is of the PI design.

The overall control law for this three-element st am generator level controller is expressed in the frequency domain as:

$$
\dot{\mathrm{m}}_{\mathrm{fw}}(\mathrm{s})=\mathrm{K}_{\mathrm{l}}\left(1+\frac{1}{\mathrm{~T}_{\mathrm{l}} \mathrm{s}}\right) \varepsilon_{\mathrm{l}}+\mathrm{K}_{\mathrm{w}}\left(1+\frac{1}{\mathrm{~T}_{\mathrm{w}} \mathrm{s}}\right) \varepsilon_{\mathrm{w}}
$$

where $K_{1}$ is the proportional level gain,

$T_{1}$ is the level error reset time,

$\varepsilon_{1}$ is the level error $\left(L_{\text {ref }}-L\right)$,

$\mathbf{K}_{\mathbf{w}}$ is the proportional flowrate gain, 


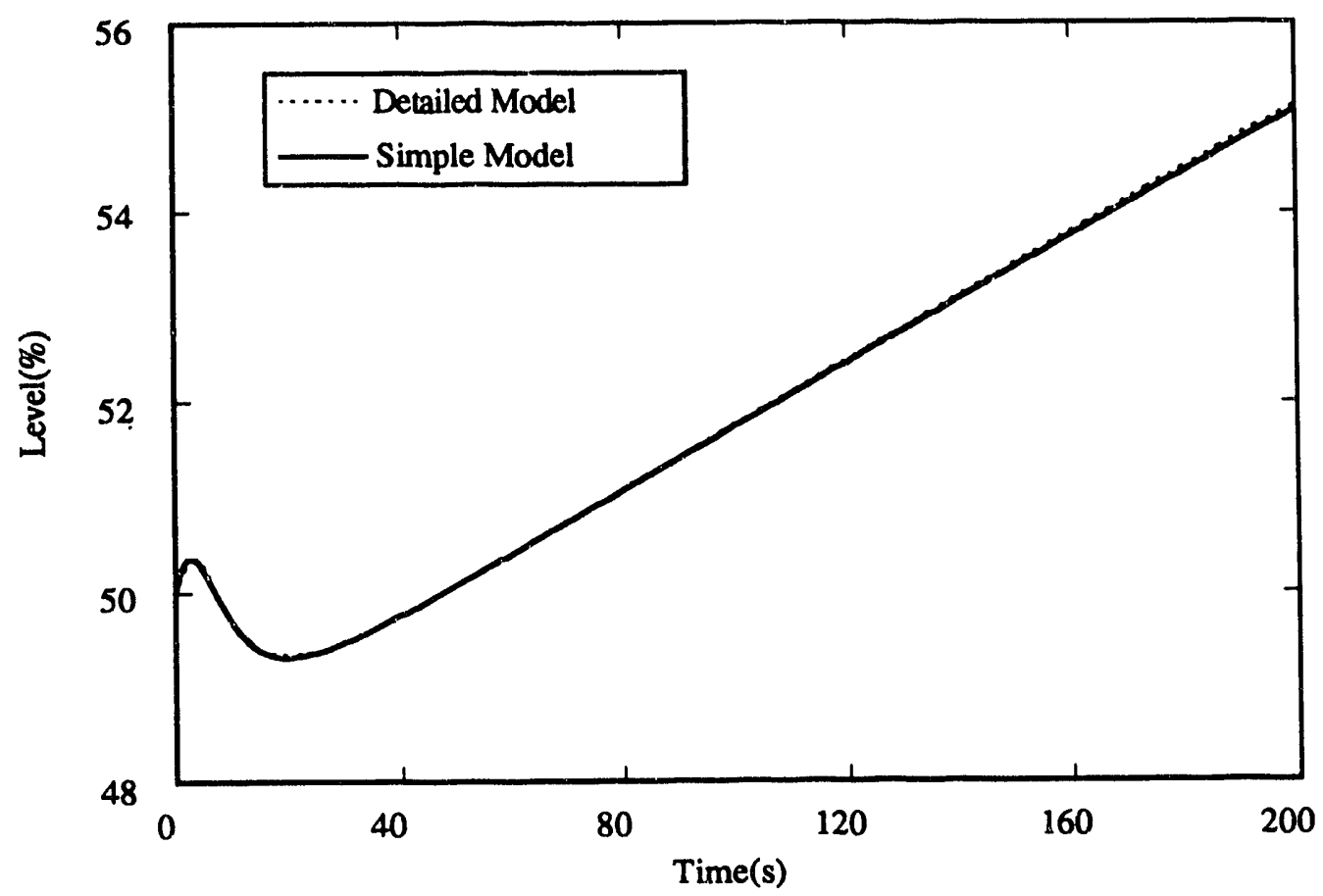

Figure 8.3.3-1 Steam Generator Level after Feedwater Flowrate Increase in Step Fashion at $20 \%$ FP.

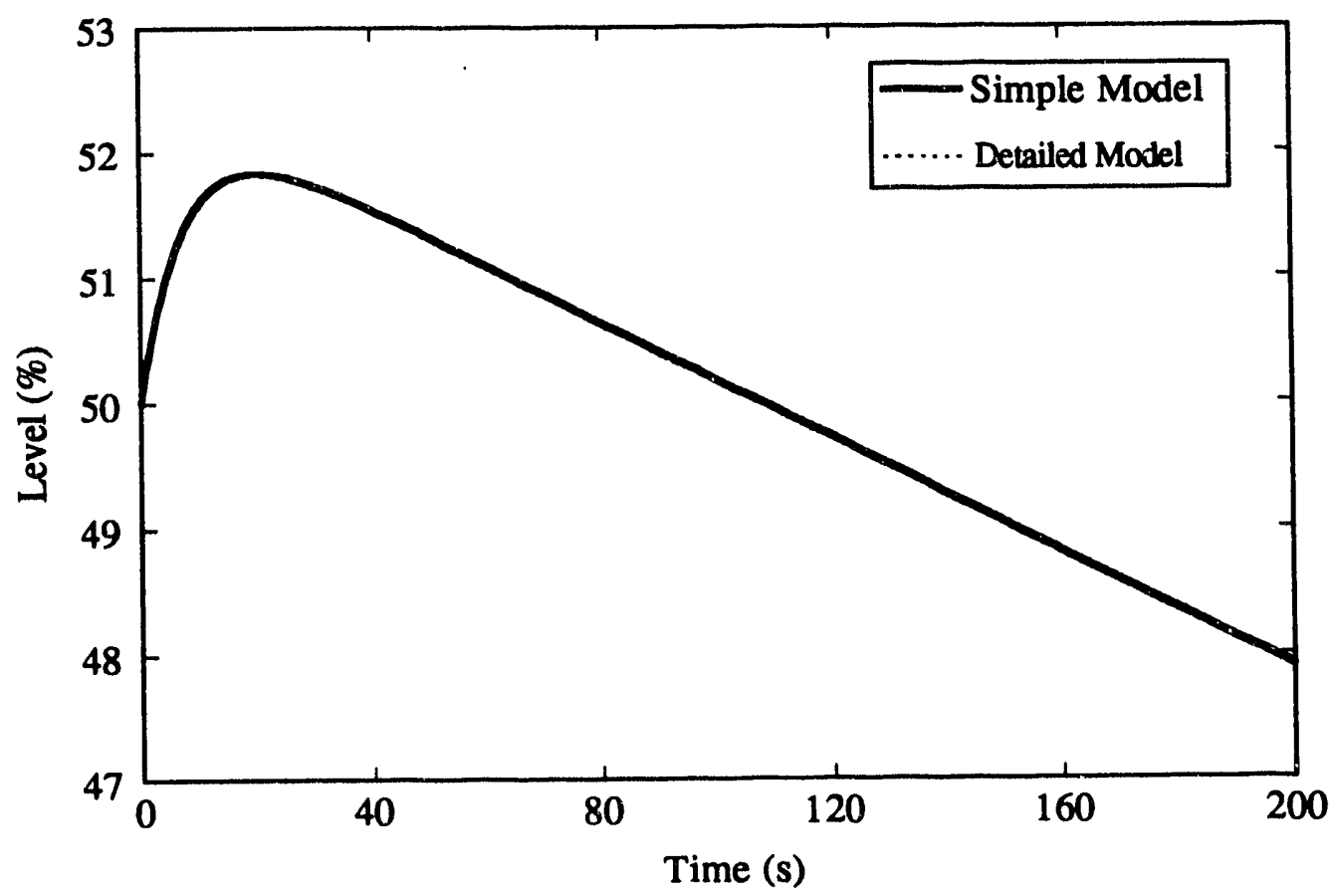

Figure 8.3.3-2 Steam Generator Level after Steam Flowrate Increase in Step Fashion at $20 \%$ FP. 


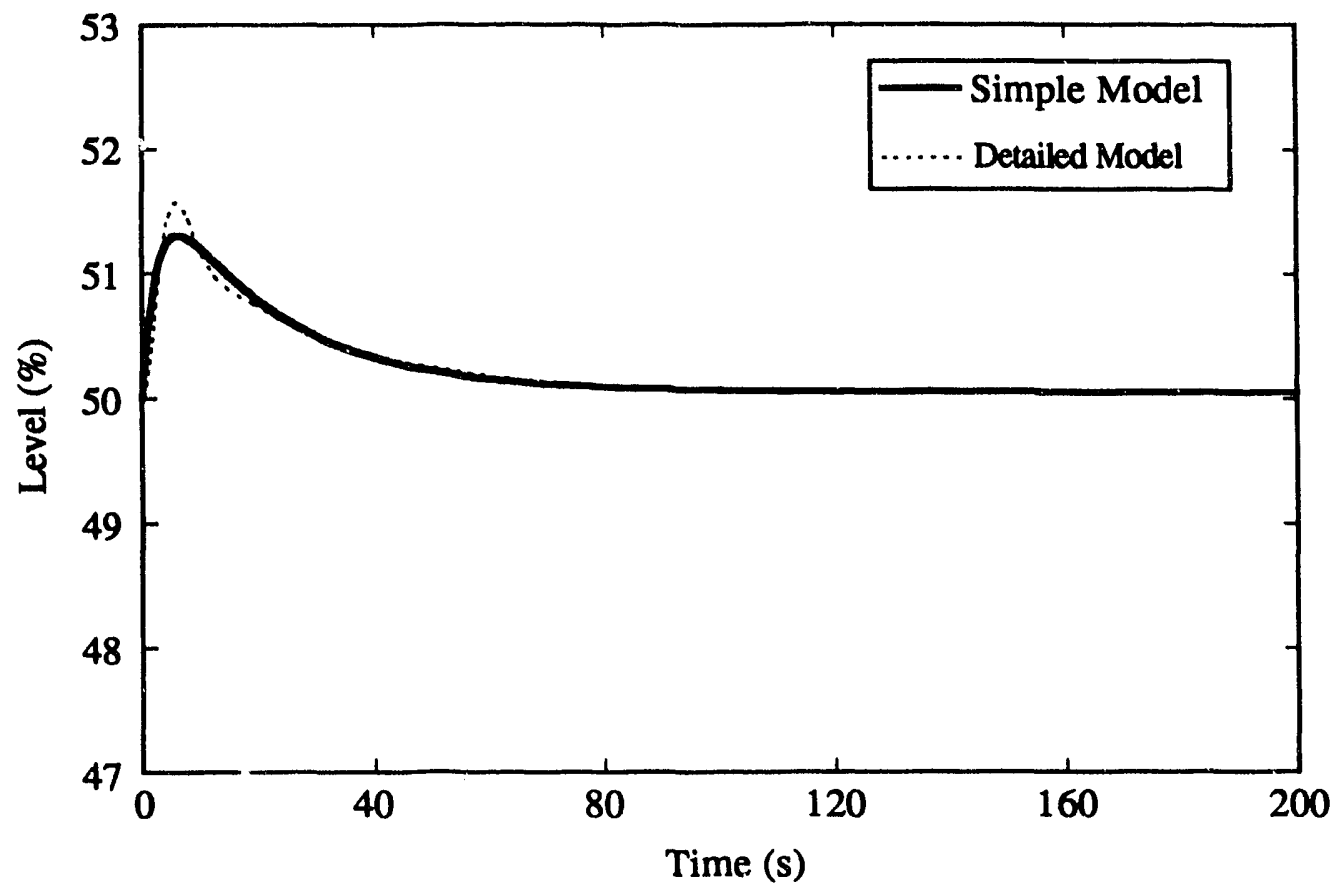

Figure 8.3.3-3 Steam Generator Level after Primary Coolant Temperature Increase in Step Fashion at $20 \%$ FP. 


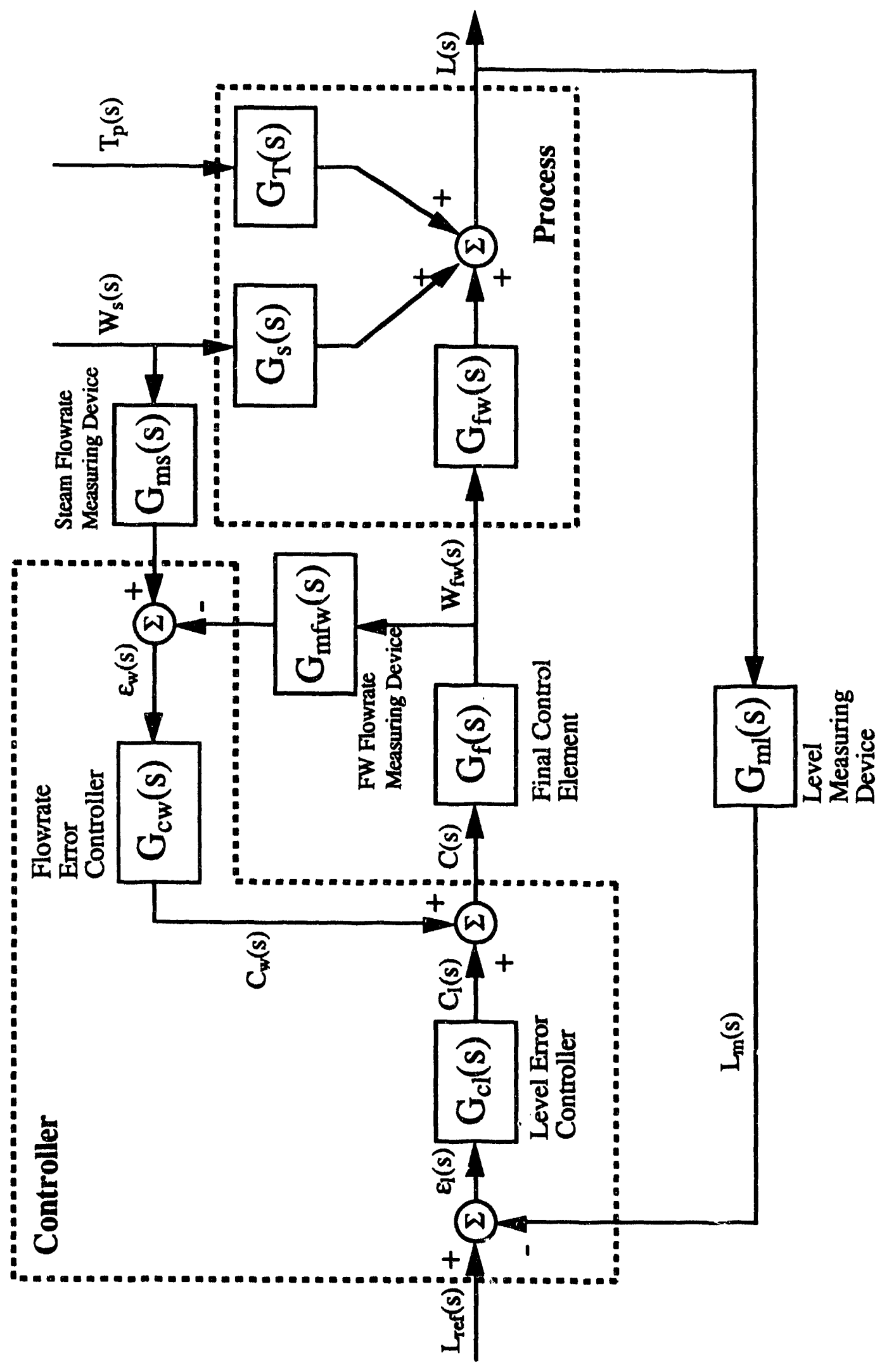

몽 
$T_{w}$ is the flowrate error integral time,

$\varepsilon_{w}$ is the flowrate error $\left(\dot{m}_{3}-\dot{m}_{f w}\right)$, and

$L_{\text {ref }}$ is the reference steam generator water level ( $\left.50 \%\right)$.

In order to simulate contro' action in the time domain, the level response can be calculated by expressing Equaison (8.4.1-1) as a time-differential equation:

$$
\frac{d\left(\dot{m}_{f_{w}}(t)\right)}{d t}=K_{l}\left(\frac{d \varepsilon_{1}}{d t}+\frac{\varepsilon_{l}}{T_{l}}\right)+K_{w}\left(\frac{d \varepsilon_{w}}{d t}+\frac{\varepsilon_{w}}{T_{w}}\right) .
$$

As described in Section 8.2.1.1 of this report, steam and feedwater flowrate measurements are not used in the steam generator level controller when operating at low power. This means that several changes must be made to Equation (8.4.1-2) in order for it describe the functioning of a steam generator level controller at low power. First, feedforward control action is excluded because the flowrate error is not used. Second, the proportional level gain is decreased because the inverse response gain (the term $G_{2 f}$ in Figure 8.3.2-1) is greatly increased.

Figure 8.4.1-2 is a block diagrann of the steam generator water level controller when configured for low-power operation. The numerical model for this single-element controller is obtained by setting the flowrate gain to zero. Thus, the control laws for the single-element controller in the frequency and the time domains are given by Equations (8.4.1-3) and (8.4.1-4) respectively:

$$
\begin{aligned}
& \dot{\mathrm{m}}_{\mathrm{fw}}(\mathrm{s})=\mathrm{K}_{1}\left(1+\frac{1}{\mathrm{~T}_{1} s}\right) \varepsilon_{1} \\
& \frac{d \dot{\mathrm{m}}_{\mathrm{fw}}(\mathrm{t})}{\mathrm{dt}}=\mathrm{K}_{1}\left(\frac{\mathrm{d} \varepsilon_{1}}{\mathrm{dt}}+\frac{\varepsilon_{1}}{\mathrm{~T}_{1}}\right) .
\end{aligned}
$$

Implementation of the level control signal during low-power operation is achieved through use of the bypass valves which provide much finer regulation than do the main feedwater regulating valves.

\subsubsection{Analysis of Current-Generation Steam Generator Level Controllers}

The steam generator level controller can be analyzed in either the frequency s, the time domain. Both approaches were used here. The former entails first using Lapl'ace transform techniques to find the transfer function, then specifying the desired performince of the closed-loop system and controller, and finally determining stability criteria. This mode of analysis offers significant computational advantages because time-depencient differential equations are converted to frequency-dependent algebraic ones. Time-dom in analyses are often more difficult to perform. Their advantage is that the result is sometimes more meaningful given that it can be related directly to observations. The single-element 

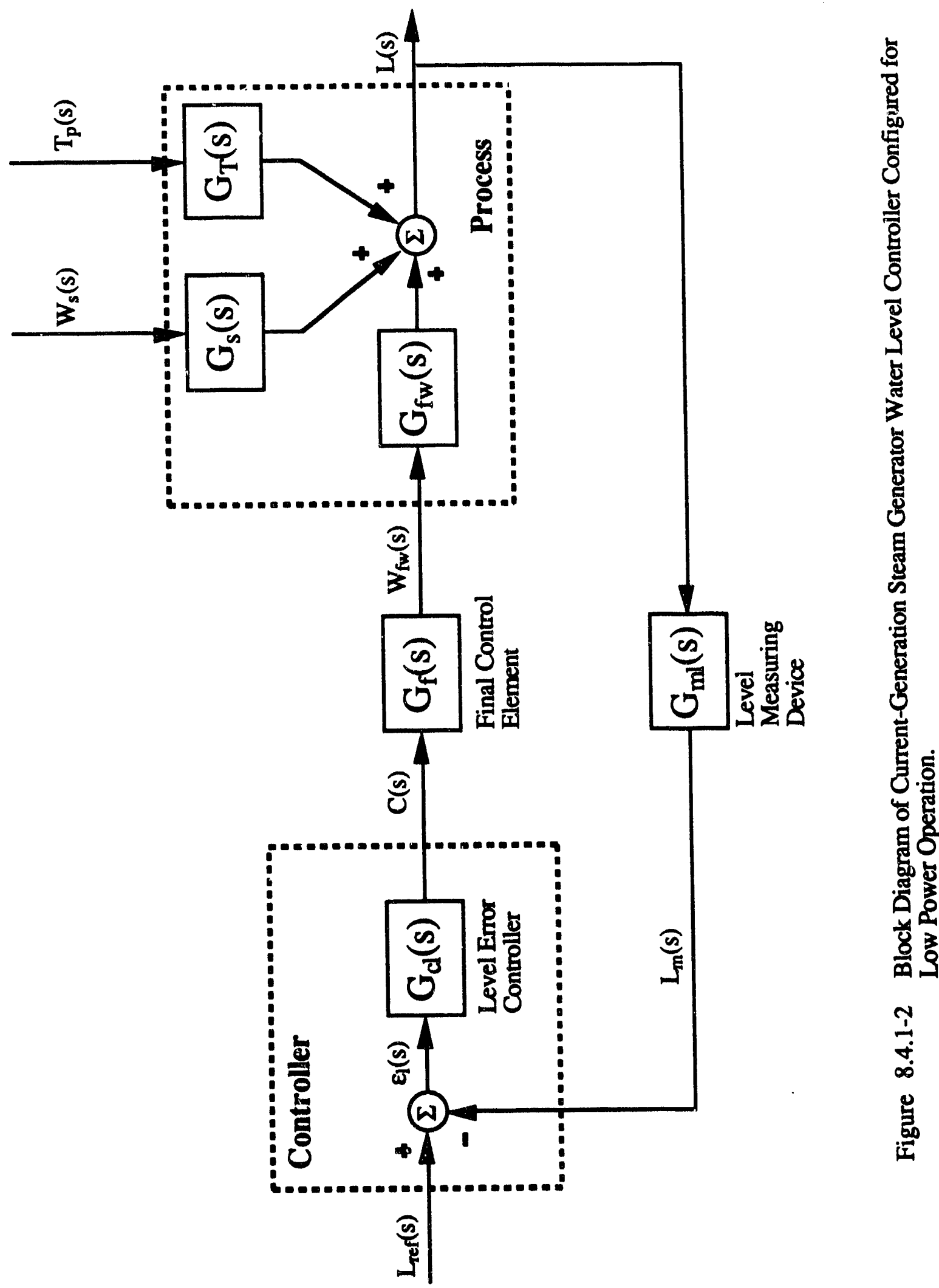
controller, that is shown in Figure 8.4.1-2, is emphasized in the ensuing analysis because most steam generator level control problems occur at low power.

\subsubsection{Erequency-Domain Analysis}

Transfer function analysis in the frequency domain makes it possible to determine whether or not a system is intrinsicaily stable Mathematically, if a system is unstable, its transfer furction will have at least one pole in the right-half of the s-plane. If one were to consider the same problem in the time domain, the presence of a pole in the right-half of the s-plane corresponds to a growing exponential term. Hence, system response to a perturbation is unbounded and therefore unstable. Insight can be gained about the stability of the closed-loop system by studying the characteristics of the open-loop transfer function. Accordingly, the open-loop behavior is examined first. Figures 8.4.2.1-1 and 8.4.2.1-2 show the poles and zeroes of the open-loop steam generator level transfer function (Eq. 8.3.1-3) at two power levels (10\%FP and $100 \% \mathrm{FP})$. Because zeroes lie in the right-half s-plane at each power level, the system is non-minimum phase and, as was discussed in Section 8.2.2.2 of this report, its response will exhibit inverse behavior. It is important to note that the positive zeroes do not make the open-loop system unstable. Stability depends only on the poles of the transier function. However, when the open-loop system is made closed-loop by combining it with the feedback controller, these positive zeroes may cause instability, especially at high feedback gains. Specifically, the effect of increasing the feedback gain is to cause the poles of the now closed-loop system to move to the zeroes of the previous open-loop system.

The closed loop transfer function can be obtained from either of the block diagrams shown in Figures 8.4.1-1 and 8.4.1-2. The individual transfer functions corresponding to each of the four components (process, measuring device, controller mechanism, and final control element) shown in each of those diagrams are given here.

Steam generator level response:

$$
L(s)=G_{f w}(s) \dot{m}_{f w}(s)+G_{s}(s) \dot{m}_{s}(s)+G_{T}(s) T_{p}(s)
$$

Level measuring device:

$$
L_{m}(s)=G_{m l}(s) L(s)
$$

Controller mechanism:

$$
\begin{array}{ll}
\varepsilon_{1}(s)=L_{\text {tref }}(s)-L_{m}(s) & \text { (Level comparatcr }) \\
c_{l}(s)=G_{c l}(s) \varepsilon_{l}(s) & \text { (Control action) }
\end{array}
$$

Einal control element:

$$
\dot{\mathrm{m}}_{\mathrm{fw}}(\mathrm{s})=\mathrm{G}_{\mathrm{f}}(\mathrm{s}) \mathrm{c}_{\mathrm{l}}(\mathrm{s})
$$




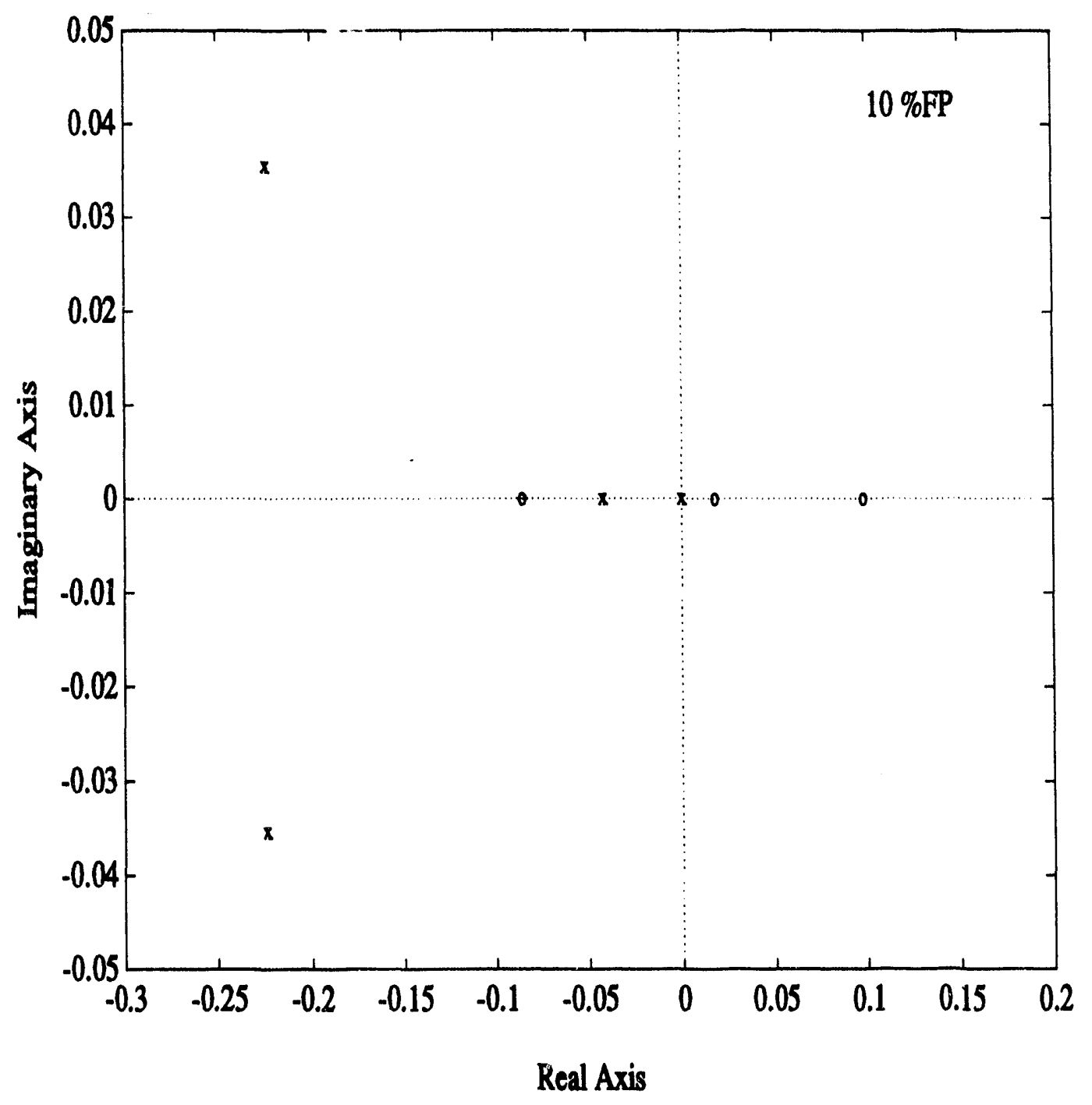

$\begin{array}{cr}\mathrm{x}: \text { poles } & 0: \text { zeroes } \\ 0.0 & 0.0982 \\ -0.2235+0.0354 \mathrm{i} & -0.0859 \\ -0.2235-0.0354 \mathrm{i} & 0.0179 \\ -0.0421 & \end{array}$

Figure 8.4.2.1-1 Pole and Zero Map of the Single-Element Steam Generator Level Transfer Function at $10 \%$ FP. 


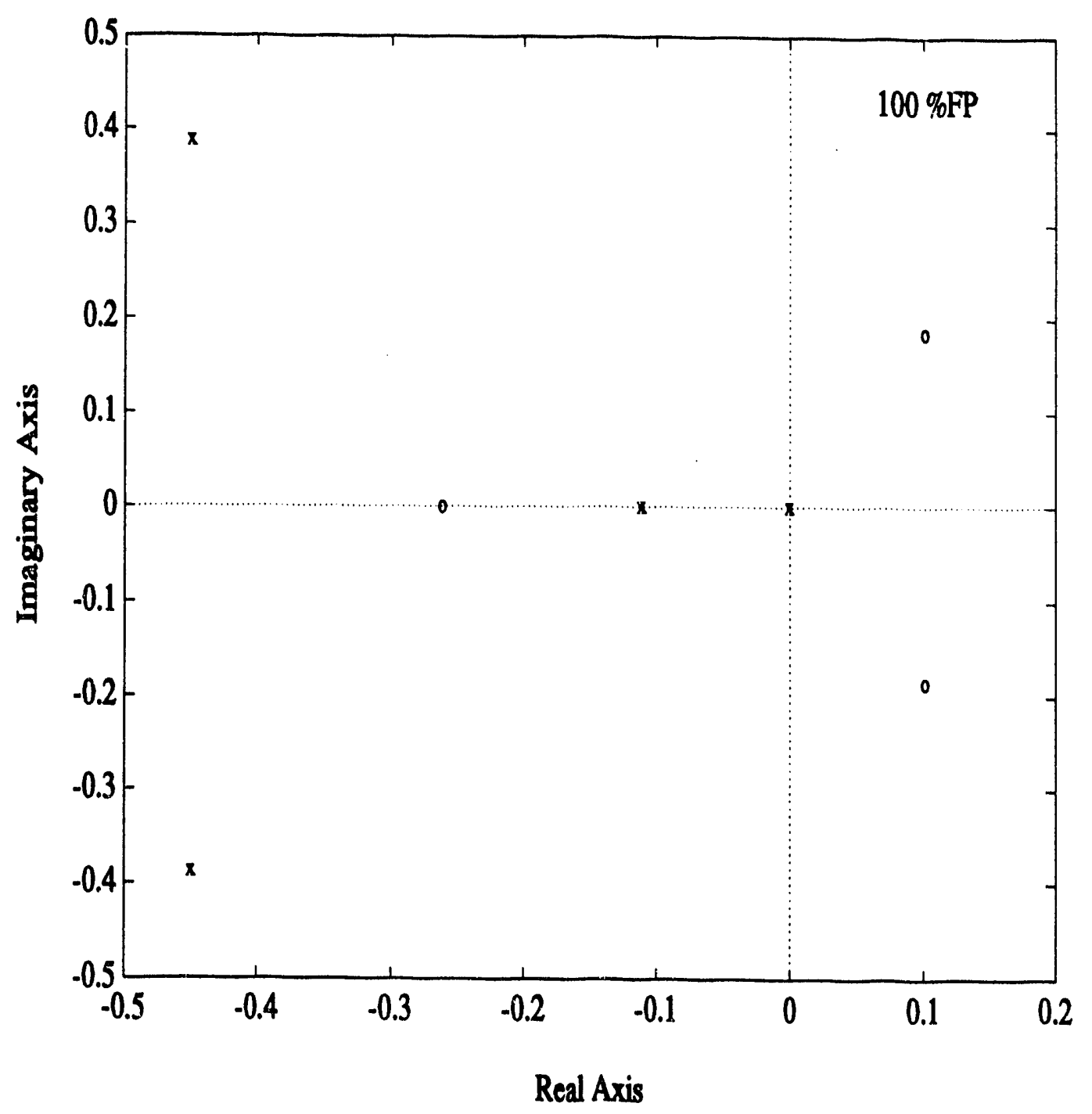

$\begin{array}{lc}\mathrm{x}: \text { poles } & 0: \text { zeroes } \\ 0.0 & -0.2611 \\ -0.4488+0.3876 \mathrm{i} & 0.1009+0.1853 \mathrm{i} \\ -0.4488-0.3876 \mathrm{i} & 0.1009-0.1853 \mathrm{i} \\ -0.1110 & \end{array}$

Figure 8.4.2.1-2 Pole and Zero Map of the Single-Element Steam Generator Level Transfer Function at $100 \% \mathrm{FP}$. 
where $c_{l}(s)$ and $L_{m}(s)$ are Laplace transforms of the controller output and level measurement variations, respectively. Note that the series of blocks between the comparator and the controlled output constitutes the forward path while the block for the measuring device is in the feedback or return path that goes from the controlled levei output to the comparator. The above equations can be combined to give a single expression for the level output. It is:

$$
\begin{aligned}
L(s)= & \frac{G_{f w}(s) G_{f}(s) G_{c l}(s)}{1+G_{f w}(s) G_{f}(s) G_{c l}(s) G_{m l}(s)} L_{r e f}(s) \\
& +\frac{G_{s}(s)}{1+G_{f w}(s) G_{f}(s) G_{c l}(s) G_{m l}(s)} \dot{m}_{s}(s) \\
& +\frac{G_{T}(s)}{1+G_{f w}(s) G_{f}(s) G_{c l}(s) G_{m l}(s)} T_{p}(s) .
\end{aligned}
$$

Equation (8.4.2.1-5) gives the closed-loop response of the process. The first term shows the effect on the output that results from a change in the level setpoint. The second and third terms give the effect on the output of a change in steam flowrate and primary coolant temperature, respectively. Changes in these two quantities are referred to as load disturbances.

If the measuring devices and final control element act perfectly and without any time delay, then the closed-loop transfer functions are:

$$
\begin{aligned}
& G_{s p}(s)=\frac{G_{f w}(s) G_{c l}(s)}{1+G_{f w}(s) G_{c l}(s)} \\
& G_{\text {load,s }}(s)=\frac{G_{s}(s)}{1+G_{f w}(s) G_{c l}(s)} \\
& G_{\text {load, } T}(s)=\frac{G_{T}(s)}{1+G_{f w}(s) G_{c l}(s)}
\end{aligned}
$$

where $G_{s p}(s), G_{\text {load,s }}(s)$, and $G_{\text {load, }}(s)$ are the closed-loop transfer functions for a change in the setpoint, steam flowrate, and primary coolant temperature respectively. It should be noted that each transfer function has the same denominator.

The stability characteristics of the closed-loop response are determined by the poles of the transfer functions $G_{s p}(s), G_{\text {load,s }}(s)$, and $G_{\text {load, }}(s)$. Because all three of these transfer functions have the same denominator, they each have the same poles. These are given by the solution of the equation:

$$
1+G_{f w}(s) G_{c l}(s)=0 \text {. }
$$


Equation (8.4.2.1-9) is called the system's characteristic equation because its roots define the system's dynamic response. The roots of the characteristic equation depend on the controller dynamics and can be modified by the choice of controller type and controller gain. The proper selection of these quantities is the control engineer's responsibility.

The effect of both proportional and proportional-integral controllers was examined with the former being studied first. The proportional gain of this controller is denoted by the symbol K. Root locus plots, which give the roots of the closed-loop characteristic equation as a function of the controller gain, were constructed for several different power levels (10\%FP, $50 \% \mathrm{FP}$, and $100 \% \mathrm{FP}$ ) as shown in Figures 8.4.2.1-3, 8.4.2.1-4 and 8.4.2.1-5 respective'y. Grid lines that show the damping ratio and the undamped natural frequency were also drawn on these plots. The damping ratio lines are drawn from 0 to 1 in increments of 0.1 . Grid lines of the damping coefficient are radial while those of the nacural frequency are circles. The root loci start $(\mathrm{K}=0)$ at the poles of the open-loop transfer function and end $(K=\infty)$ at the zeroes of asymptotes. Because the closed-loop transfer function has positive zeroes, the poles move to the right half of the s-plane and the system becomes :Instable as the feedback gain increases. For each root locus plot, the maximum feedback control gain that does not cause instability is easily obtained by calculating the feedback gain of the root on the imaginary axis. For example, at $100 \% \mathrm{FP}$, the maximum feedback control gain is $65.081 \mathrm{~kg} . \mathrm{s}-1 / \%$. At $10 \% \mathrm{FP}$, it is $4.575 \mathrm{~kg} . \mathrm{s}-1 / \%$. Figure 8.4.2.1-6 shows the maximum feedback control gain at different power levels. The maximum possible value for this gain drops rapidly because of the large inverse response that occurs as the operating power level decreases. Hence, in order to avoid instability, a very low feedback control gain should be used when operating in the low power region. Figure 8.4.2.1-7 shows the undamped natural frequency as a function of power.

The second phase of the study was to examine the effect of proportional-integral (PI) control. With integral action, the maximum feedback control gain decreases as the reset ratio $\left(1 / T_{1}\right.$ where $T_{1}$ is the integral time associated with the level error) increases. The feedback control gain should therefore be set lower than its maximum value. For example, according to accepted controller tuning methods, such as the Ziegler-Nichols method [91] the proper feedback control gain for a PI-type controller is calculated as follows:

$$
\mathrm{K}_{1}=0.45 \mathrm{~K}_{\mathrm{lm}}
$$

where $K_{l}$ is a tuned feedback control gain and $K_{1 m}$ is the maximum feedback control gain. as follows:

Similarly, the Ziegler-Nichols tuning method recommends setting the integral time

$$
T_{1}=\frac{2 \pi}{1.2} \frac{1}{\omega_{n}}
$$

where $\omega_{n}$ is the undamped natural frequency. Therefore, the controller should be tuned to a long integral time (i.e. small reset ratio) for low power operation. 


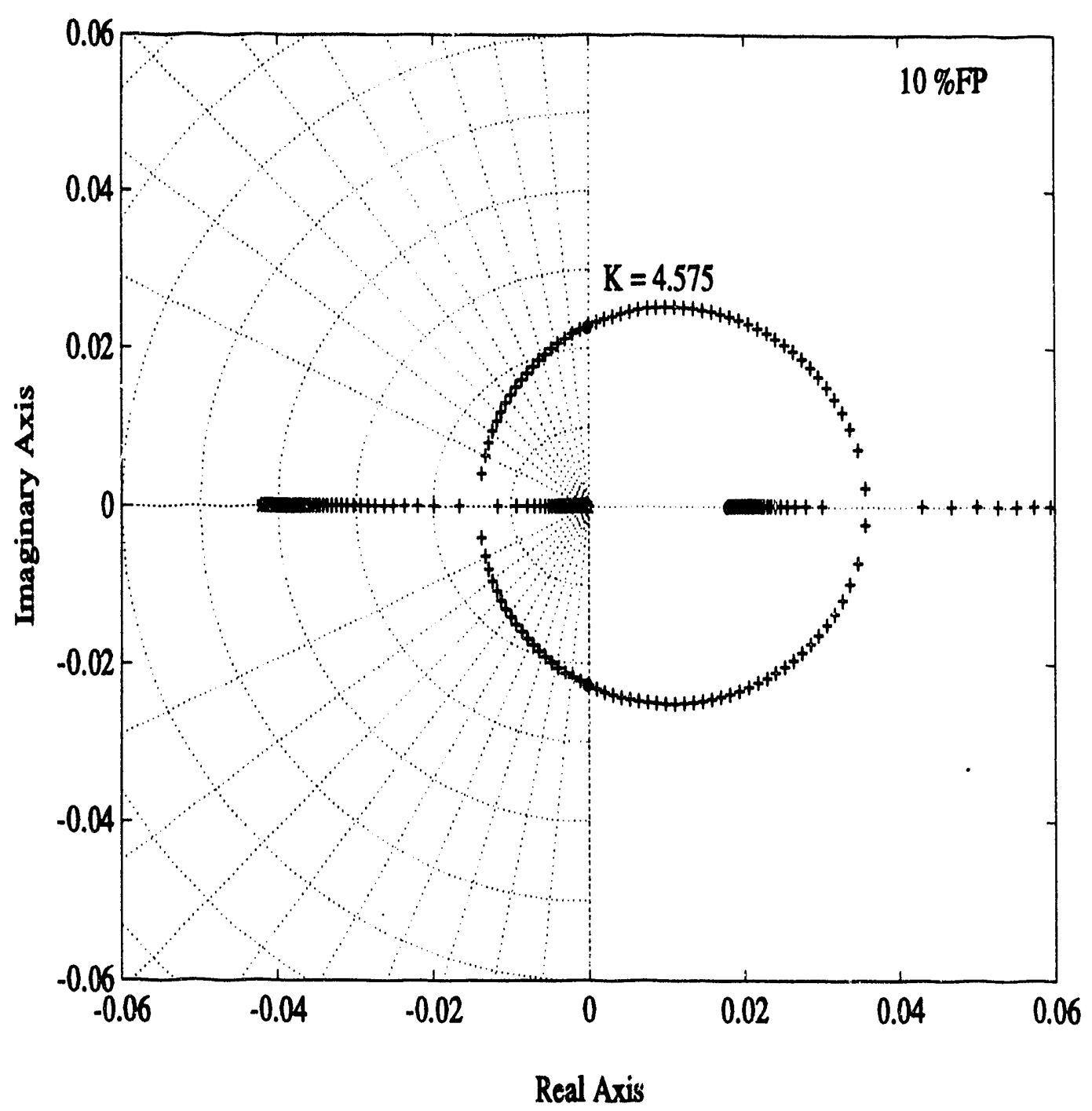

Maximum Proportional Gain : $4.575\left[\mathrm{~kg} \cdot \mathrm{s}^{-1} / \%\right]$ Undamped Natural Frequency : $0.0229\left[\mathrm{~s}^{-1}\right]$

Figure 8.4.2.1-3 Root Locus Plot of the Closed-Loop Transfer Function with a Proportional Controller $\left(\mathrm{T}_{1}=\infty\right)$ at $10 \%$ FP. 


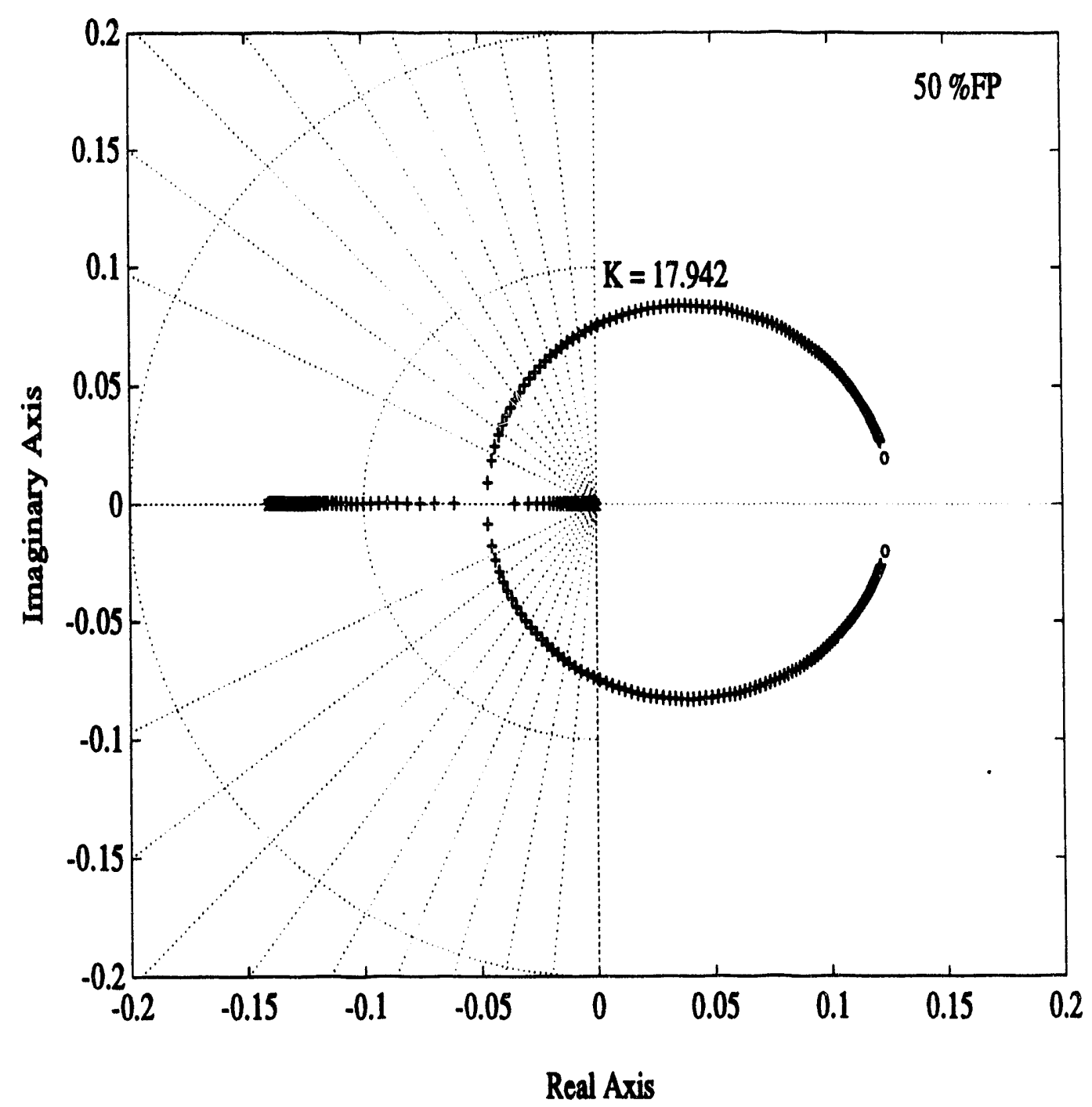

Maximum Proportional Gain : $17.942\left[\mathrm{~kg} \cdot \mathrm{s}^{-1} / \%\right]$

Undamped Natural Frequency : $0.0747\left[\mathrm{~s}^{-1}\right]$

Figure 8.4.2.1-4 Root Locus Plot of the Closed-Loop Transfer Function with a Proportional Controller $\left(\mathrm{T}_{1}=\infty\right)$ at $50 \% \mathrm{FP}$. 


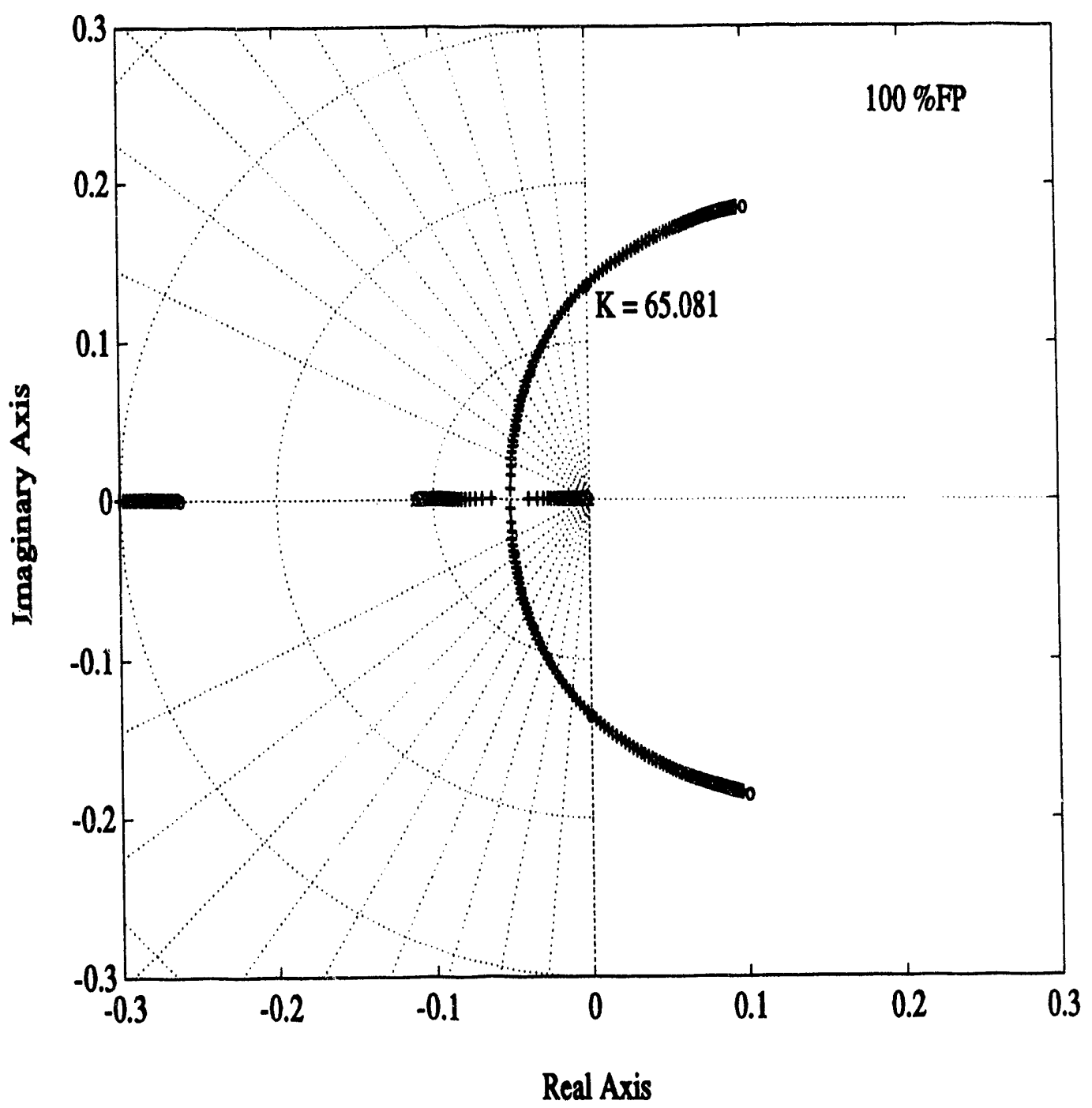

Maximum Proportional Gain : $65.081\left[\mathrm{~kg} \cdot \mathrm{s}^{-1} / \%\right]$
Undamped Natural Frequency : $0.135\left[\mathrm{~s}^{-1}\right]$

Figure 8.4.2.1-5 Root Locus Plot of the Closed-Loop Transfer Function with a Proportional Controller $\left(T_{1}=\infty\right)$ at $100 \%$ FP. 


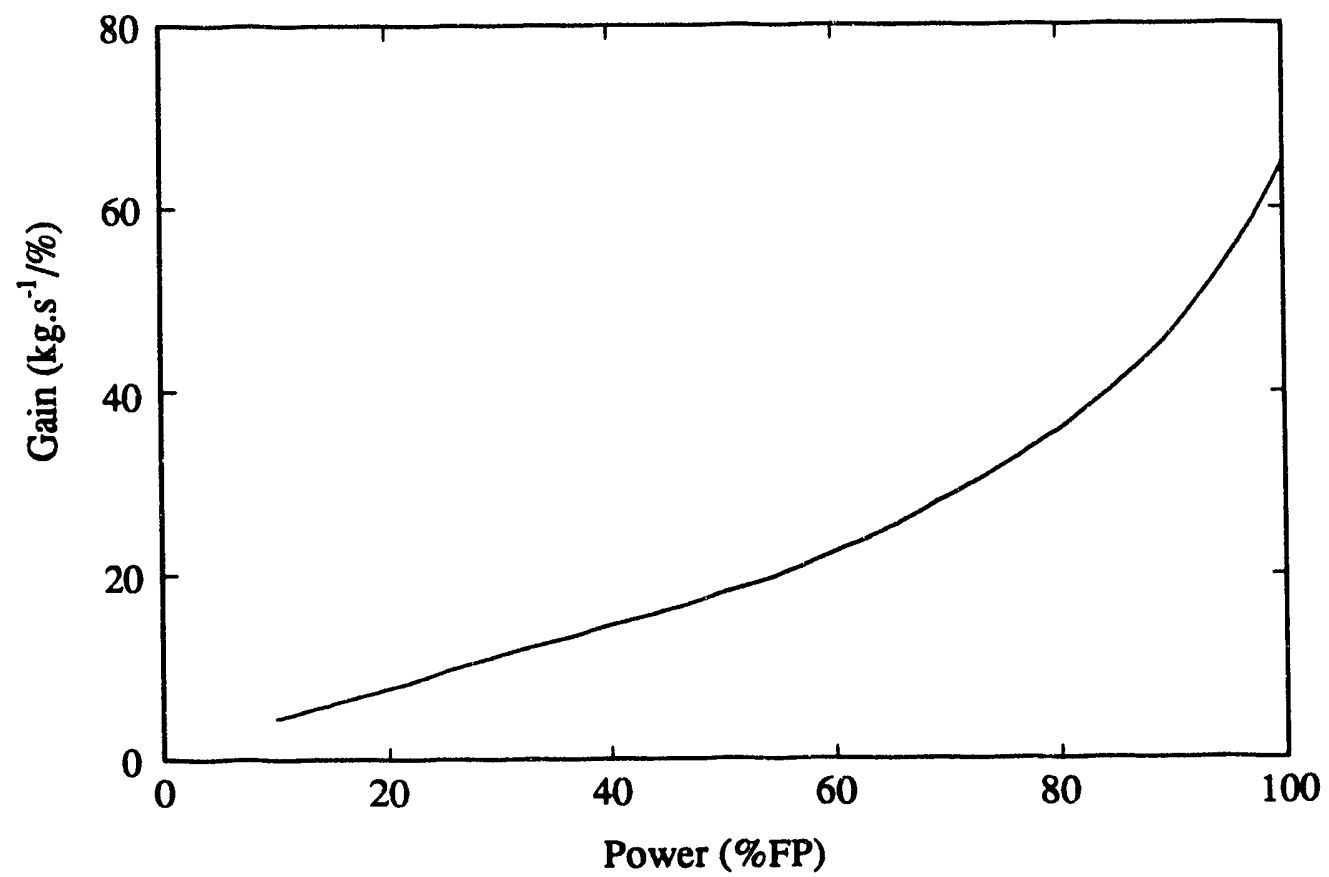

Figure 8.4.2.1-6 Maximum Feedback Control Gain as a Function of Power.

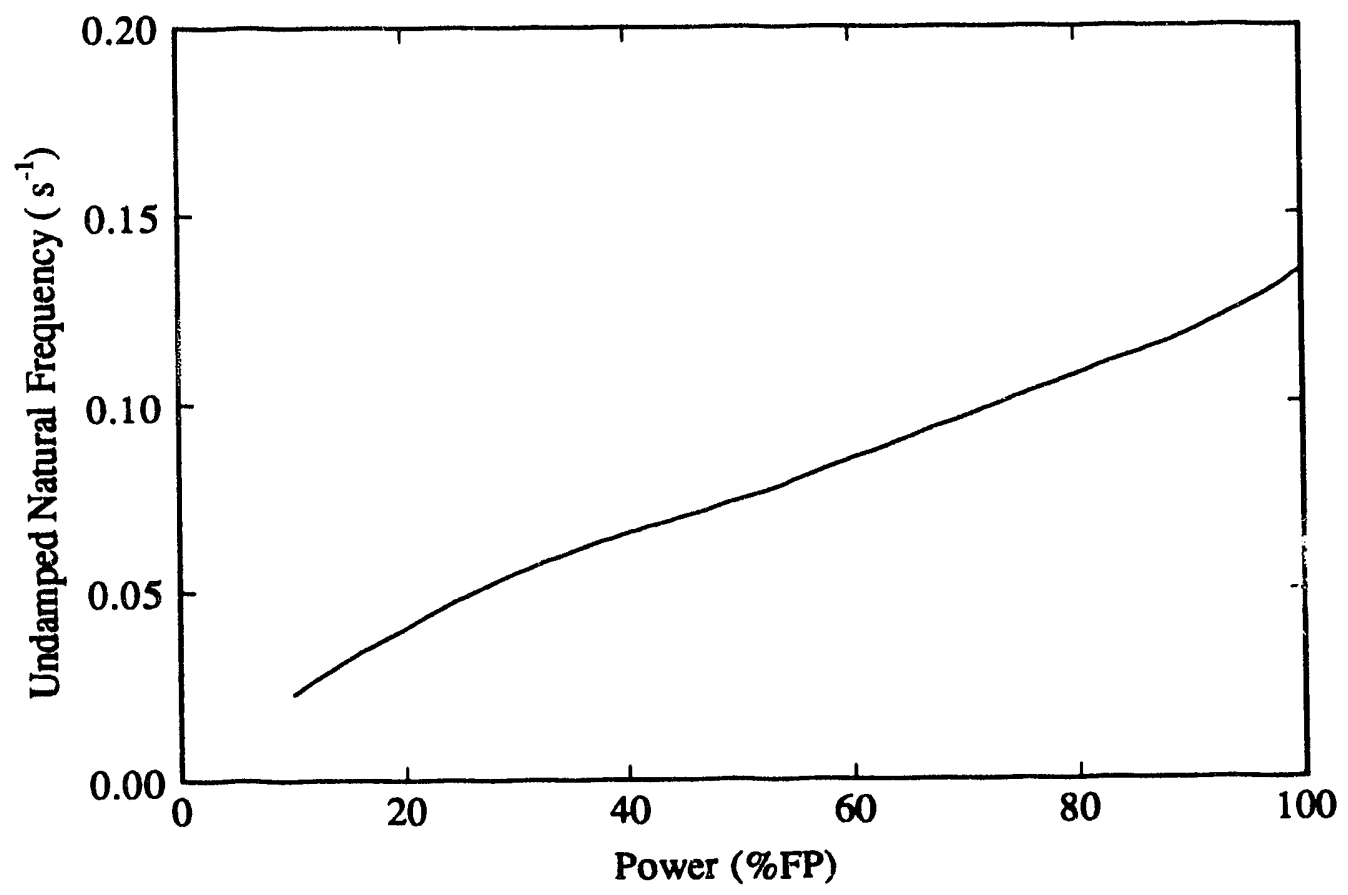

Figure 8.4.2.1-7 Undamped Natural Frequency as a Function of Power. 
Figure 8.4.2.1-8 shows the root locus plot of the closed-loop system at $10 \%$ FP. The controller is of the PI-type with a reset time, selected by the Ziegler-Nichols tuning method, of 228 seconds. Integral control action eliminates any steady-state offset error. However, the elimination of the offset error usually comes at the expense of higher maximum deviations and a long, sluggish oscillatory response. If the feedback control gain is increased to produce a faster response, then the system becomes more oscillatory and perhaps unstable. As shown in this figure, the maximum feedback control gain to avoid system instability decreases to $3.905\left[\mathrm{~kg} . \mathrm{s}^{-1} / \%\right]$. When compared to the maximum feedback control gain of the closed-loop system without integral control action, the maximum gain has decreased by $17.8 \%$.

In summary, the frequency-domain analysis of a current-generation single-element steam generator water level controller shows that the feedback control gain and integral time should be adjusted as a function of the operating power in order for proper control performance to be achieved. Also, at low power, the controller should use a small feedback control gain and a long integral time. These degrade control performance but the alternative is instability.

\subsubsection{Time-Domain Analysis}

Time-domain analyses, which entail the direct solution of the differential equations, were performed to confirm the frequency-domain studies. For this purpose, the detailed non-linear model described in Chapter Seven of this report was used to simulate steam generator water level.

The transient studied involved a perturbation to the feedwater flowrate by a step change of $5.0 \mathrm{~kg} / \mathrm{s}$. Two different sets of tuning constants were used. For high-power operation, $\mathrm{K}_{1}$ was set to $15.69 \mathrm{kg.s}-1 / \%$ and $\mathrm{T}_{1}$ at $200 \mathrm{~s}$. For low-power operation, the values were $2.377 \mathrm{~kg} . \mathrm{s}^{-1} / \%$ and $200 \mathrm{~s}$ respectively. Figure 8.4.2.2-1 shows the steam generator level responses at $10 \%$ FP. For the smaller of the two gains, the level settles at the desired setpoint. However, the response is sluggish and oscillatory because the damping ratio decreases as the feedback control gain increases as was shown in Figure 8.4.2.1-3. For the larger of the two gains, the system response is unstable. (Note: Because some physical property constants were beyond the range of the simulation program's capability, the response with the higher gain was only calculated for 250 seconds after the perturbation.)

Figure 8.4.2.2-2 is from a second study in which the effect of an error in the tuning process was evaluated. When tuned with the Zeigler-Nichols method, the response is adequate. However, when improperly tuned, the response is unstable.

Figure 8.4.2.2-3 shows the level response at $100 \% \mathrm{FP}$ for the two gains. In beth cases, the response is stable. However, the settling time is much longer when the smaller of the two gains is used. Therefore, in order to improve control performance at high power levels, it is betier to tune the controller with the higher gain. 


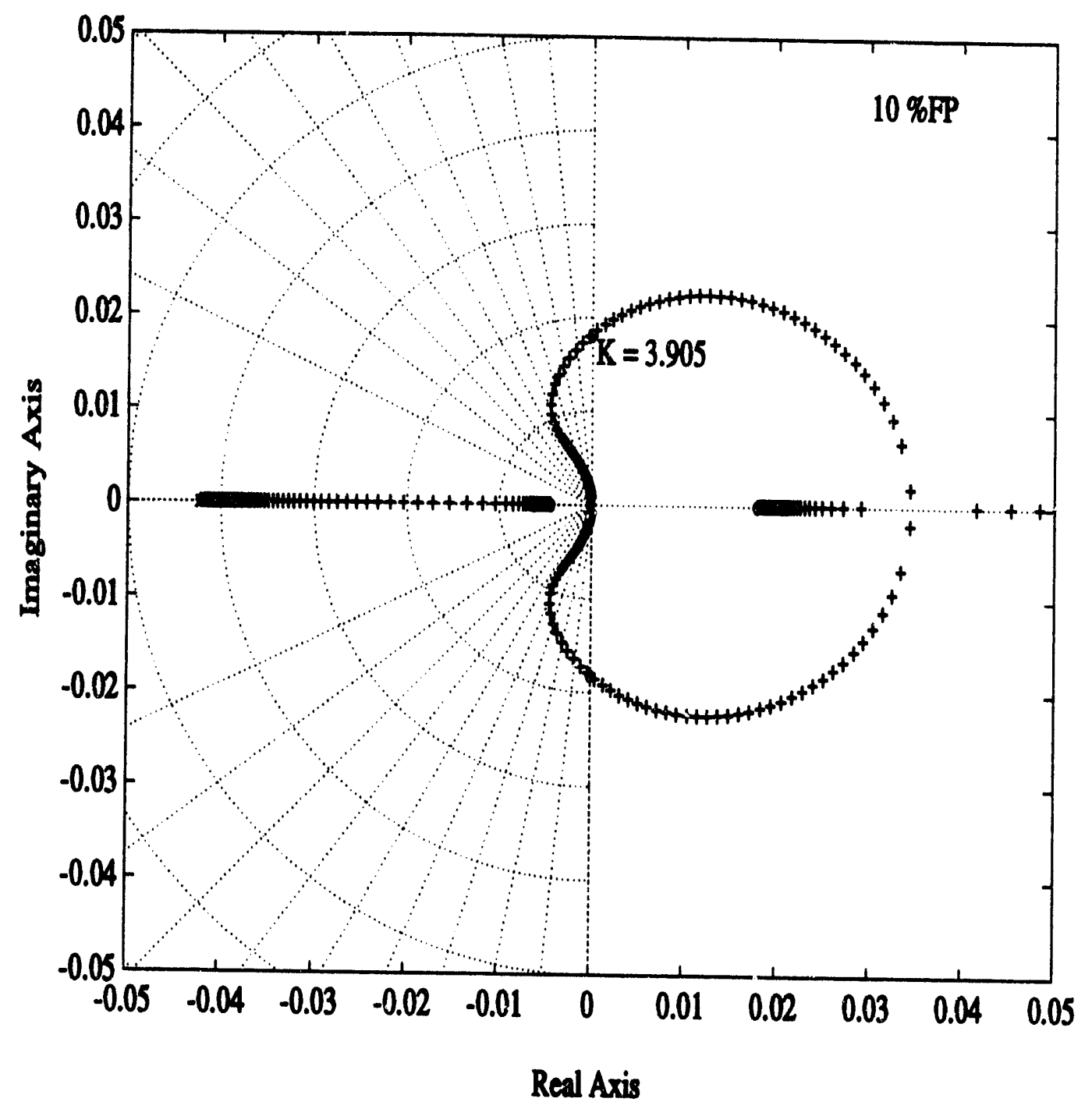

Figure 8.4.2.1-8 Root Locus Plot of the Closed-Loop Transfer Function with a Proportional plus Integral Controller with $\mathrm{T}_{1}=228 \mathrm{~s}$ (Tuned by Ziegler-Nichols Method) at 10 \%FP. 


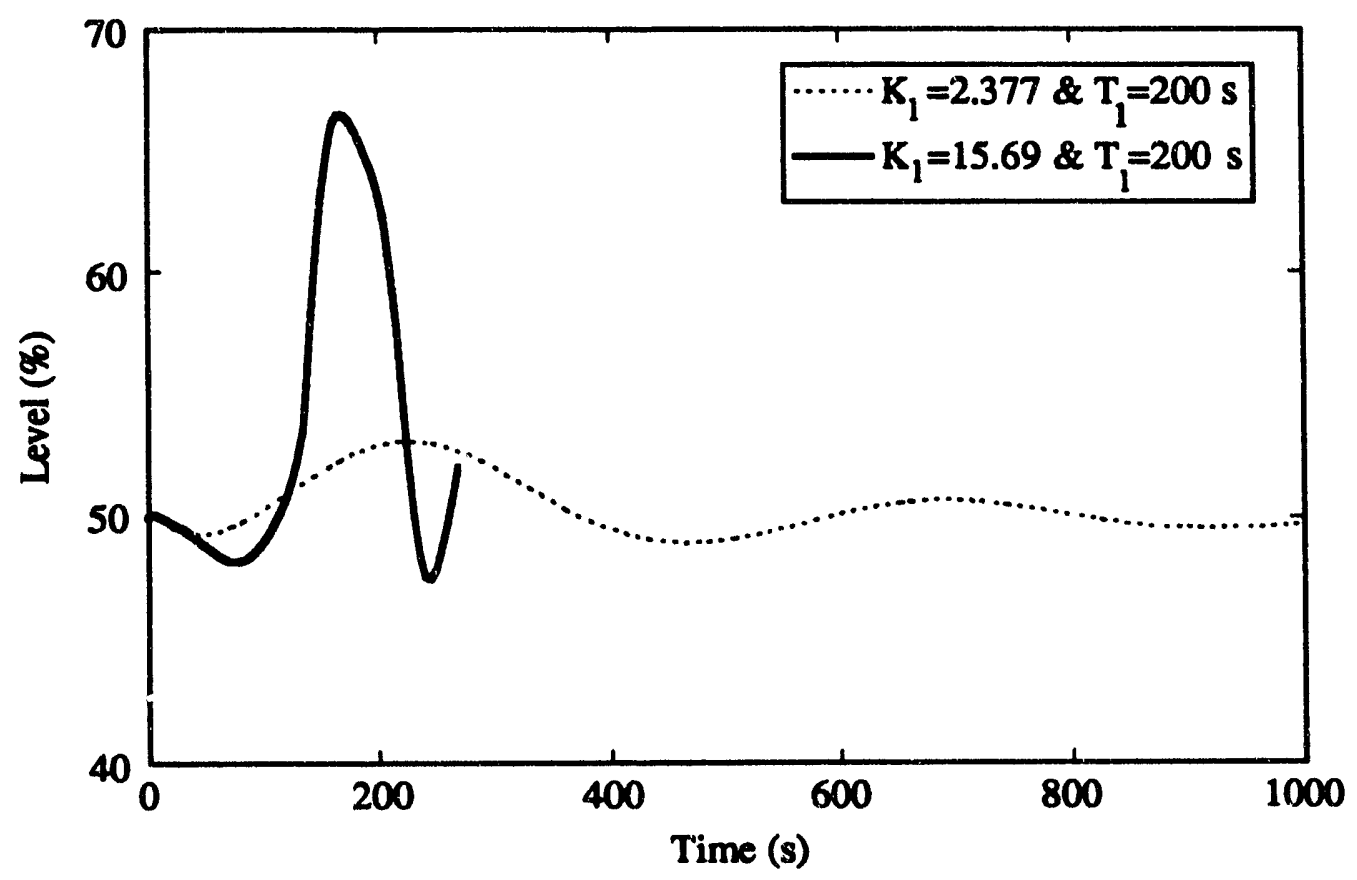

Figure 8.4.2.2-1 Steam Generator Level Response to $5 \mathrm{~kg} / \mathrm{s}$ Step Feedwater Flowrate Perturbation. (Single-Element PI Controller at $10 \% \mathrm{FP}$ )

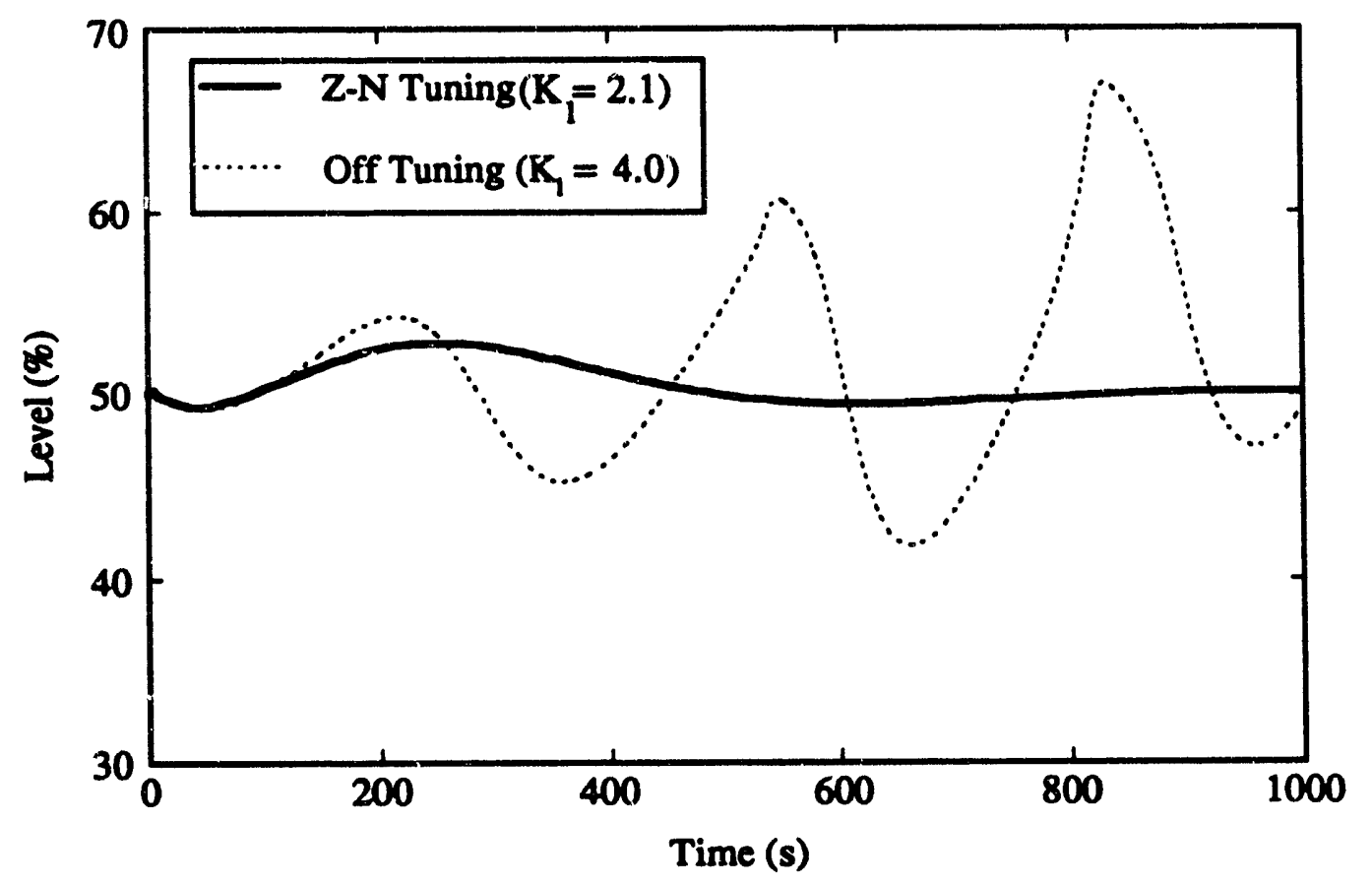

Figure 8.4.2.2-2 Effect of Tuning on Controller Performance During the Feedwater Flowrate Perturbation. (PI Controller at $10 \%$ FP) 


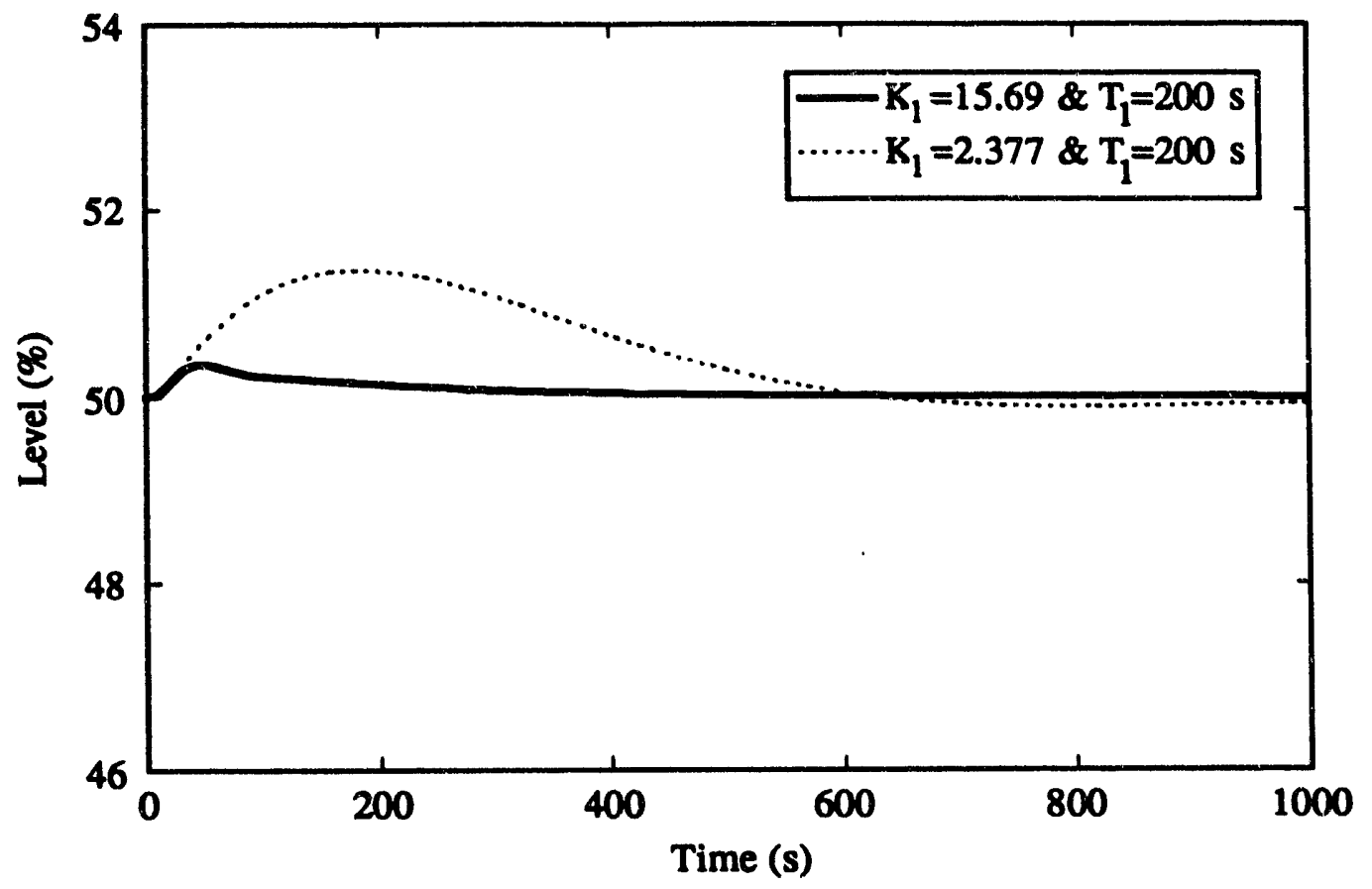

Figure 8.4.2.2-3 Steam Generator Level Response to $5 \mathrm{~kg} / \mathrm{s}$ Step Feedwater

Flowrate Perturbation. (Single-Element PI Controller at $100 \%$ FP)

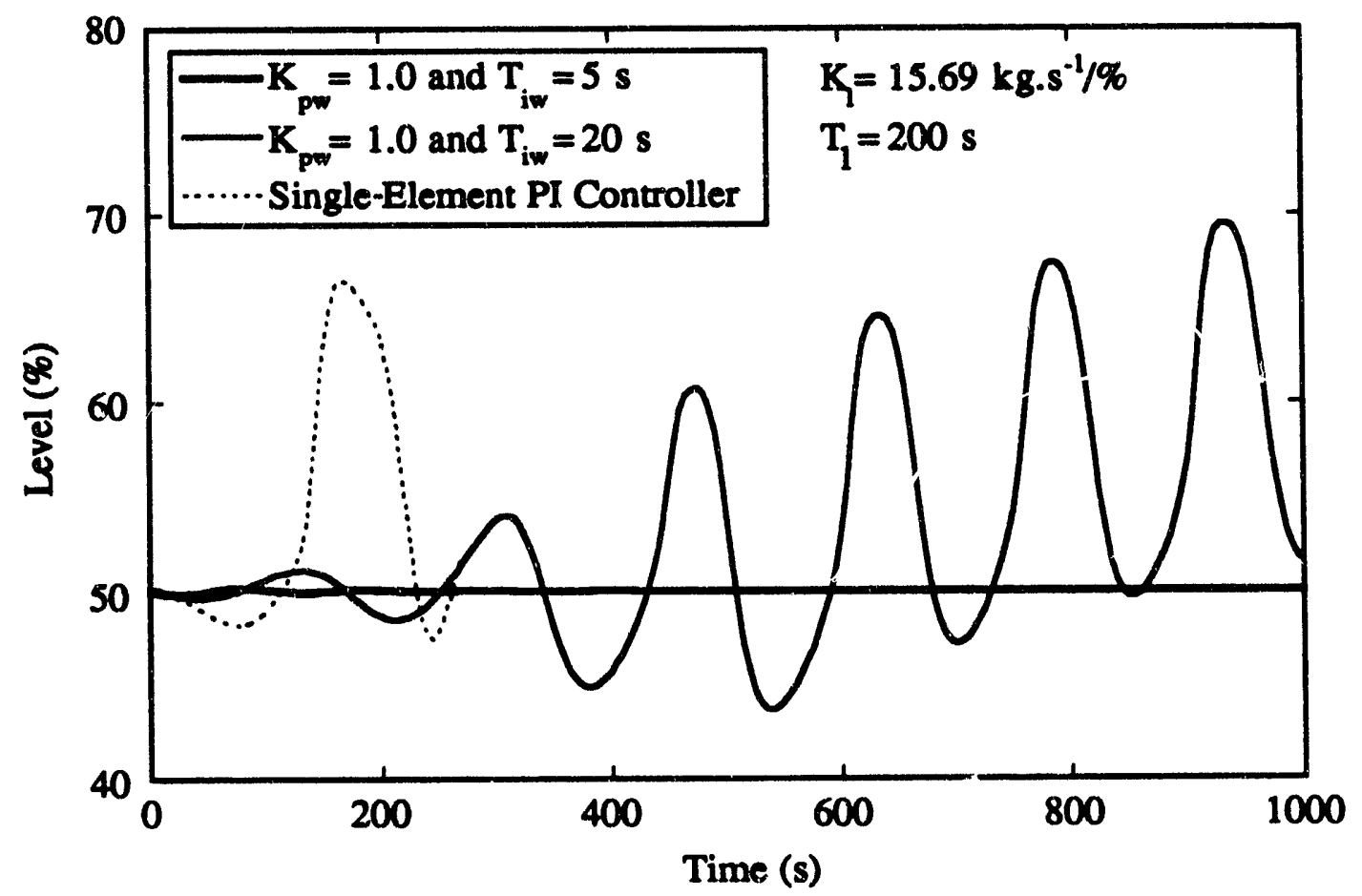

Figure 8.4.2.2-4 Steam Generator Level Response to $5 \mathrm{~kg} / \mathrm{s}$ Step Feedwater Flowrate Perturbation. (Three-Element PI Controller at $10 \%$ FP) 
Figure 8.4.2.2-4 shows the level response at $10 \% \mathrm{FP}$ of the three-element PI controller tuned with the higher gain. The flowrate mismatch error signal gives the correct control action provided that its reset time is set to a small value. Hence, with that parameter set to $5 \mathrm{~s}$, the controller is stable even though tuned with a high gain. However, when the reset time is set to $20 \mathrm{~s}$, the controller becomes unstable because the flowrate mismatch error can no longer immediately provide the correct control action. Note: It should be recalled that uncertainty in flowrate measurements during low-power operation is too large to permit their use. Hence, the option of using a high-gain, three-element controller at low power does not exist.)

\subsubsection{Summary of Control Problems Associated with Current-Generation Steam Generator Level Controllers}

In summary, current-generation steam generator level controllers have two types of problems. The first is to achieve a satisfactory means of control at low power. Specifically, during low-power operation the inverse response associated with shrink and swell effects causes control problems such as an oscillatory level response or instability. The problem is exacerbated by the use of high feedback control gains. A solution is to incorporate feedforwand action by using both the level error and a feedwater/steam flowrate error. This approach works well during high-power opes tion. However, uncertainty in the flowrate measurements precludes the use of this solution during low-power operation.

The second problem is that of tuning. If a level controller is tuned with a low feedback control gain and a long reset time, then the system response to an imposed perturbation will be stable. However, this approach degrades performance by causing a sluggish response. In addition, if the tuning is even slightly in error, the response will be unstable.

\subsection{Design of Proposed Steam Generator Level Controller}

In this section, a new design is proposed with the objective of solving the control problems associated with current-generation steam generator water level controllers. The intent is to achieve both excellent control performance and robustness at all power levels. Specifically, this new design permits the level feedback control gain to be tuned aggressively thereby improving performance and at the same time not incurring instability.

\subsubsection{Solutions Previously Proposed to the Inverse Response Issue}

Several solutions have been previously proposed to overcome the steam generator level control problems that are associated with inverse response. These include:

1. Modify the steam generation process to eliminate or reduce the inverse response phenomenon. The most important modification would be to heat the feedwater during low-power operation This would reduce the vapor collapse that follows sudden increase in feedwater flowrate and hence greatly dissipate the inverse response [92]. This option is usualiy very expensive and often impossible because it requires retrofitting. 
2. Detune the PI feedback controller so that it is characterized by both an extremely low feedback control gain and a long reset time. This will, as described in the previous section of this report, avoid instability. However, it also means a severe degradation in control system performance.

3. Enhance the existing PI control strategy by incorporating additional elements such as a feedforward term that is based on the mismatch between feedwater and steam flowrates. This is done for some curren! three-element controllers and/or four/five-element controllers [93]. In theory, this can yield superior performance, but, in reality it may not because a measurable disturbance variable must be available to implement a feedforward strategy and, as noted earlier, there is much uncertainty in flowrate measurements. Another possibility is to consider use of the wide range level measurement to compensate for shrink and swell effects at low power [94].

4. Employ advanced or alternate control strategies. For example, Raju developed a fuzzy logic controller for drum bciler level [95]. However, this controller requires that the derivative value of the level be measured together with other variables that are linear functions of the evaporator steam exit quality, downcomer flowrate, and evaporator rising inixture flowrate. The steam generator level derivative measurement is often too erratic to use as a control variable.

Another possible solution is the use of compensators. The recent development of microprocessor-based controllers has revived interest in this approach because any compensator, even non-linear ones, can now be designed and easily implemented. For example, Choi suggested the use of a detailed non-linear model as the basis of a compensator [69]. An inverse model that simulates steam generator characteristics given level and pressure measurements, was to be used to avoid the need for uncertain feedwater and steam flowrates measurements as control parameters. The idea was to compensate the measured level by means of the tube bundle mass change which can be estimated on-line from the inverse model. However, the complexity of the model made this idea impractical.

The approach taken in this research was to develop a model-based compensator from the simplified transfer function that was described in Section 8.3 of this report. That model can simulate steam generator level dynamics quite accurately if the system identification process is performed properly. The general concept of the compensator is based on the Smith's dead-time predictor (dead-time compensator) which is described in Appendix B. This compensator is relatively easy to implement as part of a microprocessorbased digital controller.

\subsubsection{Compensator Design and Evaluation}

Compensators were developed for both the feedwater flowrate and the load parameters (steam flowrate and primary temperature). The design and analysis of each is described here. Provided by way of background material is a concise summary of control performance indices. These were used in the design of the load parameter compensators. 
Two important design goals for every controller are to maintain the maximum deviation as small as possible and, when perturbed, to return to the desired operating point in the shortest possible time. Unfortunately, these two performance goals lead to conflicting characteristics in a feedback controller. For example, the settling time increases when the overshoot error decreases. Therefore, controller performance often represents a balance between these conflicting characteristics. Accordingly, in order to compare different controller designs, it is often useful to compare performance indices.

There are two different kinds of control performance indices, simple performance indices and time-integral performance indices. The former represent some characteristic features of the closed-loop response of the system such as overshoot, rise-time, settlingtime, or decay ratio. In contrast, the latter represent the dynamic shape of the complete closed-loop response from perturbation until attainment of a new steady-state. That is, a time-integral performance index is based on the entire response of the process. There are several kinds of time-integral performance indices. In this research, the IAE (Integral of Absolute Value of the Error) and ISE (Integral of Square Value of Error) are used to evaluate the steam generator level controller. IAE and ISE can be calculated as follows:

$$
\begin{aligned}
& \operatorname{IAE}=\int_{0}^{t_{2}}\left|\varepsilon_{1}(t)\right| d t \\
& I S E=\int_{0}^{t_{1}}\left(\varepsilon_{1}(t)\right)^{2} d t
\end{aligned}
$$

where $t_{b}$ is the settling time and $\varepsilon_{l}(t)$ is the level error. In addition to these ilices, the maximum level error is also often examined.

\subsubsection{Design of the Feed water Flowrate Compensator}

The stability of a closed-loop system depends on the system's response to the manipulated variable. In the case of steam generator level control, the manipulated variable is the feedwater flowrate. Hence, the level response to a perturbation in the feedwater flowrate must be stable. However, steam generator level dynamics are very complicated and, as noted earlier, level response to a feedwater flowrate change both exhibits inverse behavior and shows some initial oscillation. Therefore, for a compensator to be of benefit, it must offset these strong non-linear responses and also maintain closed-loop system stability. Compensation for both the inverse response behavior and the initial oscillation term can be obtained by placing a feedback compensator around the existing nominal PI controller. Figure 8.5.2.1-1 is a block diagram of the approach for a feedback-based compensator for feedwater flowrate. Modeling of the final control element is required to generate the compensation signal because the feedwater flowrate is taken from the controller so as to avoid using the measured value. The design of the compensator is based on the Smith principle which has been shown to improve control of many time- 


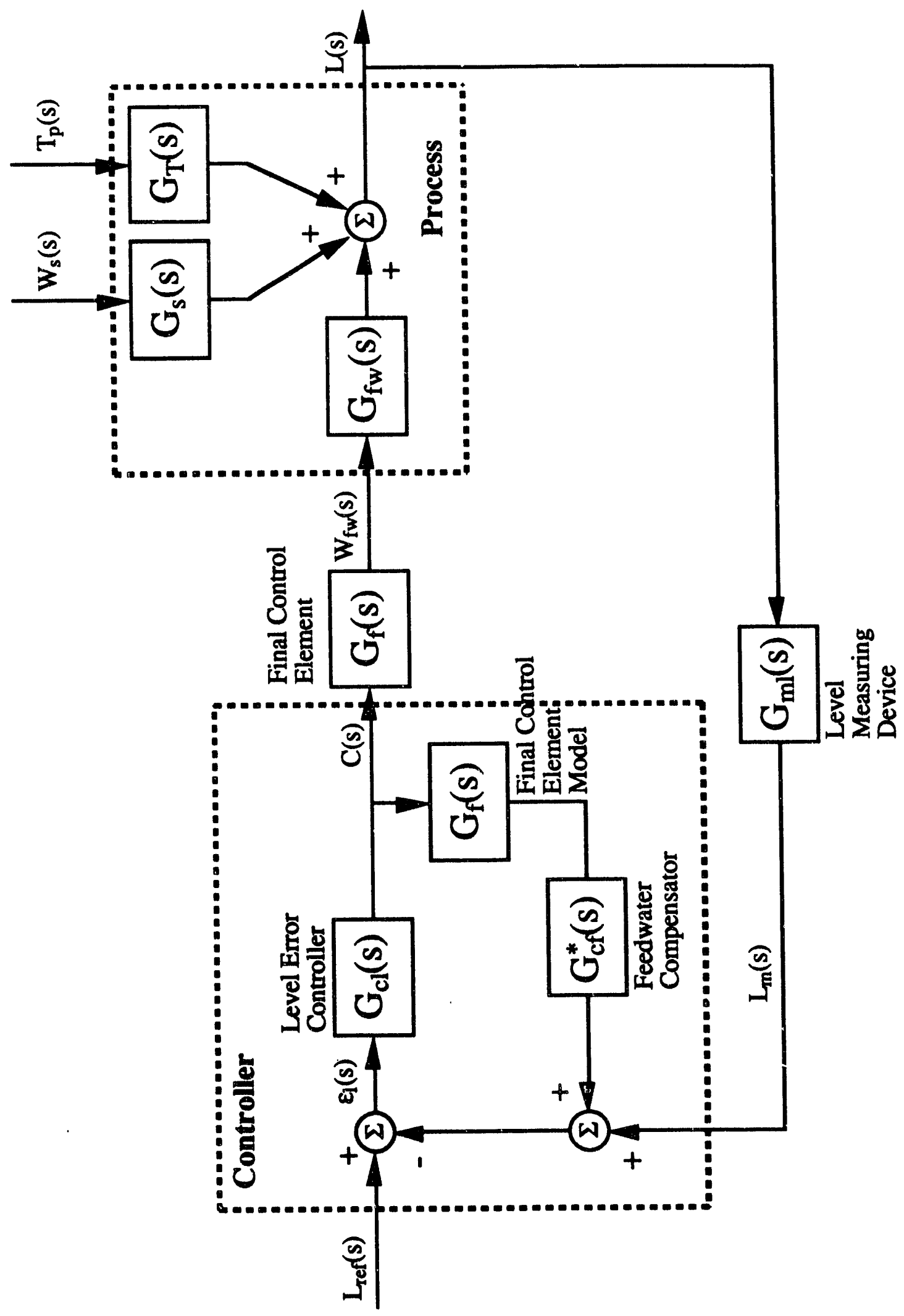


delayed processes [96,97]. A concise summary of Smith's idea is given in Appendix B to this report. The basic idea is to use a system model to generate the information that is not available because of the time delay. The effect of the model is to cancel (or compensate for) time-delay behavior. The extension of the Smith principle to the control of systems that exhibit inverse response was originally proposed by Ilinoya and Altpeter [88]. Several other researchers have applied the concept to drum boiler level control $[98,99]$.

The basic idea is to modify the measured level signal by a compensation signal, $L_{c}(s)$, which is the output of the feedwater flowrate compensator. The control input signal to the PI controller then becomes:

$$
L^{*}(s)=L_{m}(s)+L_{c}(s)
$$

where $\mathrm{L}^{*}(\mathrm{~s})$ is the Laplace transform of the compensated signal that will be used as input to the PI controller, $L_{m}(s)$ is the Laplace transform of the measured level signal, and $L_{c}(s)$ is the Laplace transform of the compensation signal.

The compensation signal is to contain both an inverse response and a mass oscillation term. Hence, the compensator is most readily designed by using the transfer functions for those two terms except with opposite sign. Thus, from Section 8.3, we have:

- For the inverse response term: $\frac{G_{2 f}}{\tau_{2 f} s+1}$

- For the mass oscillation term: $\quad-\frac{G_{3 f} s}{\left(s+1 / \tau_{3 f}\right)^{2}+\omega^{2}}$.

It is important that the compensation signal decay to zero under steady-state conditions. Otherwise, the controlled level would exhibit a steady-state offset. The mass oscillation term does die out at steady-state. However, because the inverse response transfer function consists of a first-order lag term, the compensation signal as a whole will not do likewise. To overcome this difficulty, Surgenor suggested a compensator that consists of an inverse response predictor and an impulse function [98]. The compensator for feedwater flowrate then has the following form:

$$
G_{c f}^{*}=\frac{G_{2 f}}{\tau_{2 f} s+1} \cdot \frac{\alpha_{f} s}{\alpha_{f} s+1}-\frac{G_{3 f} s}{\left(s+1 / \tau_{3 f}\right)^{2}+\omega^{2}}
$$

where $G_{c f}^{*}$ is the transfer function of the feedwater compensator and $\alpha_{f}$ is an adaptive parameter that determines the amount of compensation. If $\alpha_{f}$ is set to zero, no compensating action is taken and the controller is the same as a conventional one. If $\alpha_{\mathrm{f}}$ is set to infinity, the inverse response is completely compensated. However, the difficulty associated with the compensation signal in not going to zero at steady-state, then returns. 
The first term in Equation (8.5.2.1-4) compensates for the inverse response. It consists of the inverse response predictor and the impluse function. The former acts to cancel the inverse response of the process while the latter forces the compensation signal to zero with a time constant of $\alpha_{\mathrm{f}}$. Figure 8.5.2.1-2 shows the time behavior of the compensated level after a $5.0 \mathrm{~kg} / \mathrm{s}$ feedwater flowrate increase in step fashion at $10 \% \mathrm{FP}$. Curves are shown for several values of $\alpha_{f}$. As $\alpha_{f}$ increases, the inverse response is diminished but the steady-state offset error increases. Therefore, the value of the adaptive parameter in the feedwater compensator should be determined from a consideration of both stability and control performance. The detailed procedure for tuning this parameter is addressed Section 8.5.3 of this report.

The compensation signal is then found by taking the product of the feedwater compensator and the feedwater flowrate. Thus, in the frequency domain, it is:

$$
\mathrm{L}_{\mathrm{c}}(\mathrm{s})=\mathrm{G}_{\mathrm{cf}}^{*} \dot{\mathrm{m}}_{\mathrm{f}}(\mathrm{s})
$$

The derivation can also be done in the time domain. Two differential equations are obtained trom Equation (8.5.2.1-4). These are:

and

$$
\tau_{2 f}\left(l_{f} \cdot \frac{d^{2} L_{c i}(t)}{d t^{2}}+\left(\tau_{2 f}+\alpha_{f}\right) \cdot \frac{d L_{c i}(t)}{d t}+L_{c i}(t)=G_{2 f} \alpha_{f} \cdot \frac{d \dot{m}_{f}(t)}{d t}\right.
$$

$$
\frac{d^{2} L_{c m}(t)}{d t^{2}}+\frac{2}{\tau_{3 f}} \frac{d L_{c m}(t)}{d t}+\left(\frac{1}{\tau_{3 f}^{2}}+\omega^{2}\right) L_{c m}(t)=G_{3 f} \frac{d \dot{m}_{f}(t)}{d t}
$$

where $L_{c i}(t)$ and $L_{c m}(t)$ are the compensation level terms for the inverse response and mass oscillation, respectively. The compensation level, $\mathrm{L}_{c}(t)$, is the sum of $\mathrm{L}_{c i}(t)$ and $\mathrm{L}_{c m}(t)$. Equations (8.5.2.1-6) can be rewritten in matrix form as:

$$
\frac{d}{d t}\left[\begin{array}{l}
L_{c i}(t) \\
L_{c i}^{\prime}(t)
\end{array}\right]=\left[\begin{array}{cc}
0 & 1 \\
-\frac{1}{\tau_{2 f} \alpha_{f}} & -\frac{\tau_{2 f}+\alpha_{f}}{\tau_{2 f} \alpha_{f}}
\end{array}\right]\left[\begin{array}{l}
L_{c i}(t) \\
L_{c i}^{\prime}(t)
\end{array}\right]+\left[\begin{array}{c}
0 \\
\frac{G_{2 f}}{\tau_{2 f}}
\end{array}\right] \frac{d \dot{m}_{f}(t)}{d t}
$$

or

$$
\frac{d}{d t}\left[\begin{array}{l}
L_{c m}^{(t)} \\
L_{c m}^{\prime}(t)
\end{array}\right]=\left[\begin{array}{cc}
0 & 1 \\
-\left(\frac{1}{\tau_{3 f}^{2}}+\omega^{2}\right) & -\frac{2}{\tau_{3 f}}
\end{array}\right]\left[\begin{array}{l}
L_{c m}^{(t)} \\
L_{c m}^{\prime}(t)
\end{array}\right]+\left[\begin{array}{c}
0 \\
G_{3 f}
\end{array}\right] \frac{d \dot{m}_{f}(t)}{d t}
$$

where 


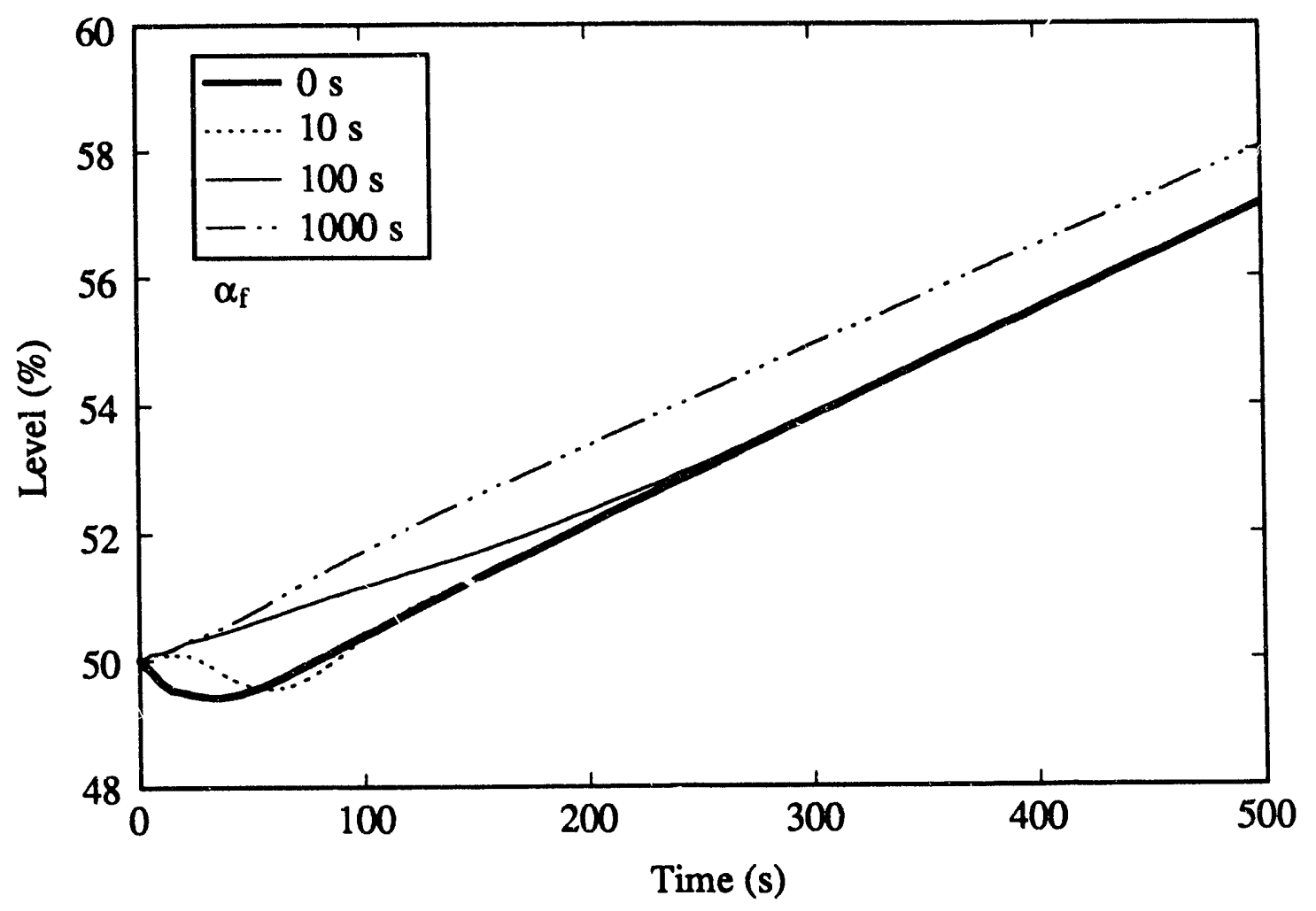

Figure 8.5.2.1-2 Compensated Level After Feedwater Perturbation Without Level Control Action. 


$$
L_{c i}^{\prime}(t)=\frac{d L_{c i}(t)}{d t}
$$

and

$$
L_{c m}^{\prime}(t)=\frac{d L_{c m}(t)}{d t} .
$$

\subsubsection{Stability Analysis of the Closed-Loop System with the Feedwater Flowrate Compensator}

In order to study the stability of the closed-loop system associated with the feedwater flowrate compensator, a frequency domain analysis is again performed. The compensated controller consists of two closed loops. One is for compensation and the other is for feedback. Figure 8.5.2.1-1 with its inner compensator loop can be redrawn to give an equivalent single loop as shown in Figure 8.5.2.2-1. In this figure, $\mathrm{G}_{\mathrm{ser}}^{*}$ represents the equivalent series compensator and is expressed as follows:

$$
\mathrm{G}_{\mathrm{ser}}^{*}(\mathrm{~s})=\frac{1}{1+\mathrm{G}_{\mathrm{cl}}(\mathrm{s}) \mathrm{G}_{\mathrm{cr}(\mathrm{s})}^{*}(\mathrm{~s})} .
$$

From Figure 8.5.2.2-1, the closed-loop transfer function is expressed as:

$$
G_{s p}(s)=\frac{G_{f w}(s) G_{c l}(s) G_{s e r}^{*}(s)}{1+G_{f w}(s) G_{c l}(s) G_{s e r}^{*}(s)}
$$

Upon substitution of Equation (8.5.2.2.-1) into Equation (8.5.2.2-2), the closed-loop transfer function becomes:

$$
G_{s p}(s)=\frac{G_{f w}(s) G_{c l}(s)}{1+G_{f w}(s)\left(G_{f w}(s)+G_{c f}^{*}(s)\right)} .
$$

The response of the closed-loop system is examined through root locus plots when a single proportional feedback controller is incorporated. Shown in Figures 8.5.2.2-2 and 8.5.2.2-3 are plots for two different adaptive parameters ( 10 seconds and 100 seconds) at $10 \%$ FP. When the adaptive parameter is chosen to be 10 seconds, the maximum feedback control gain that does not incur instability is $4.89 \mathrm{~kg} \cdot \mathrm{s}^{-1} / \%$. This is an increase of only $7.0 \%$ as compared to that for existing uncompensated controllers. If the adaptive parameter is chosen to be 100 seconds, all roots of the characteristic equation are located in the left-half plane of the Laplace domain and the closed-loop response associated with the proportional controller is guaranteed to be stable. Hence, it is concluded that a feedwater flowrate compensator based on the simplified transfer function can mitigate the instability 


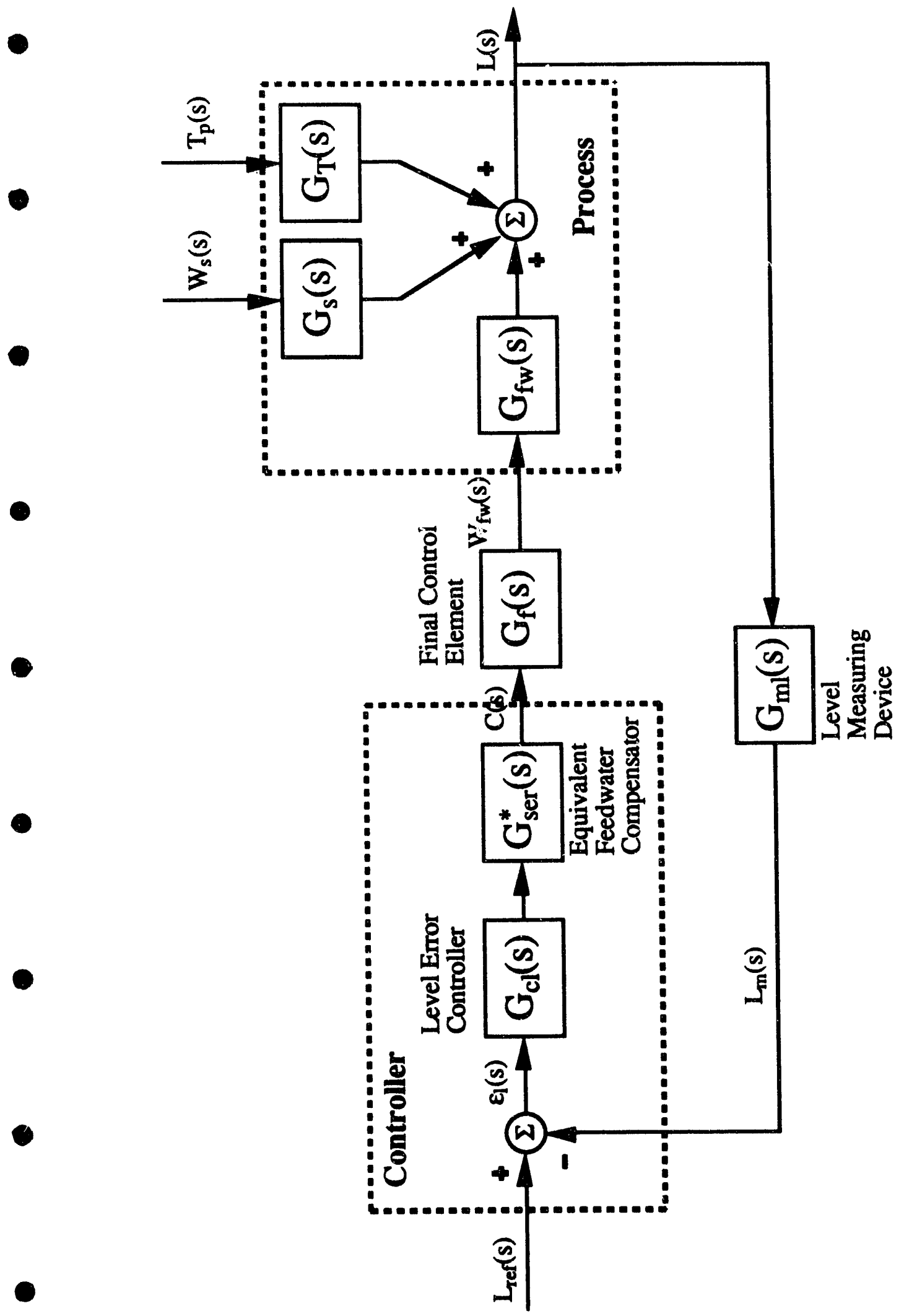

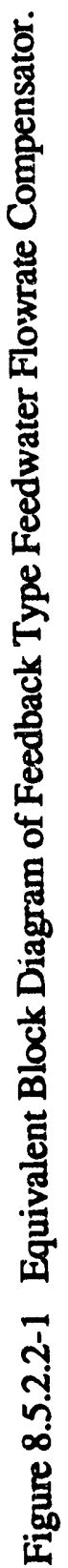




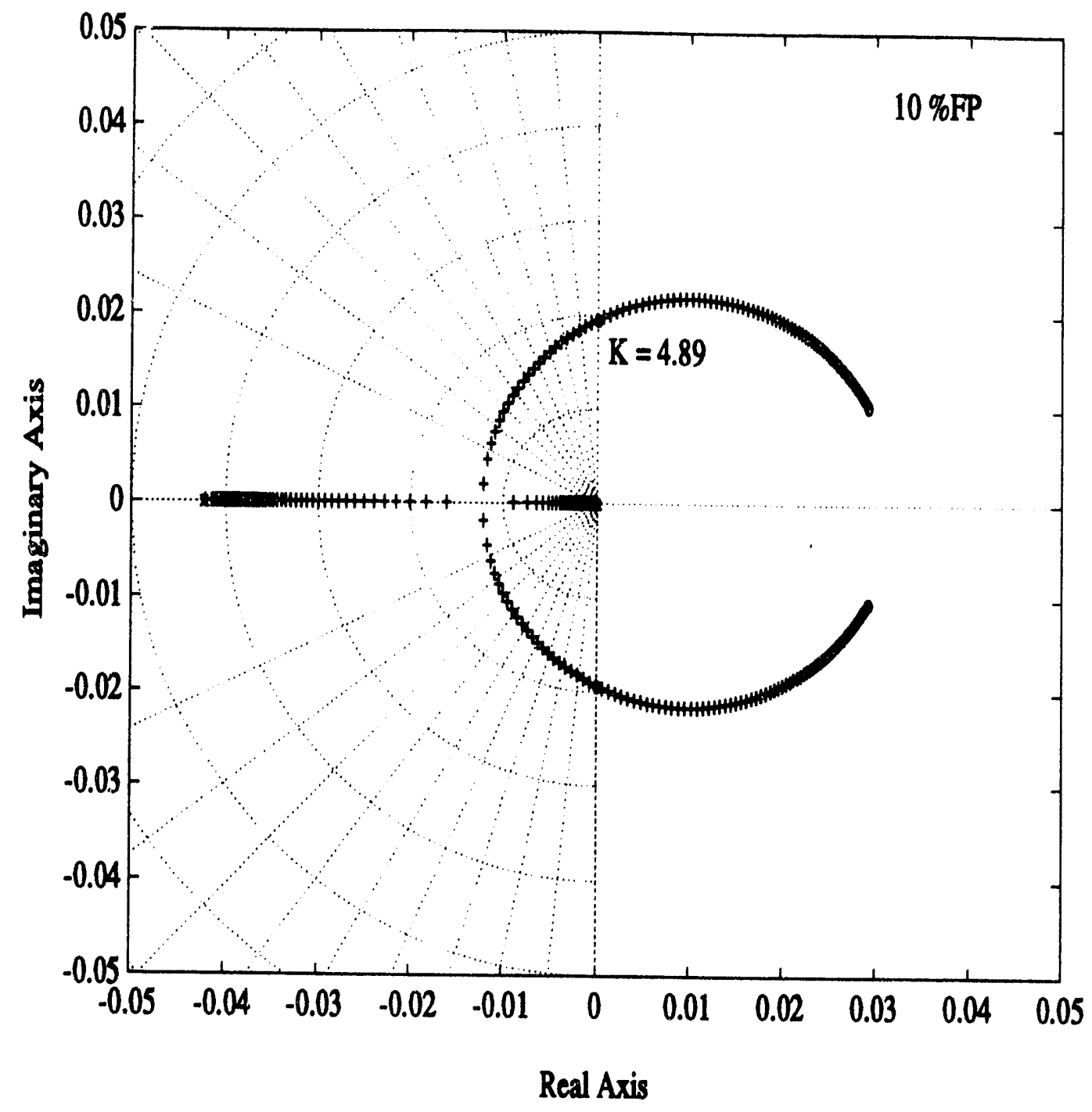

Figure 8.5.2.2-2 Root Locus Plot of the Closed-Loop Transfer Function Associated with Feedwater Flowrate Compensator $\left(\alpha_{\mathrm{f}}=10 \mathrm{~s}\right)$ at $10 \%$ FP. 


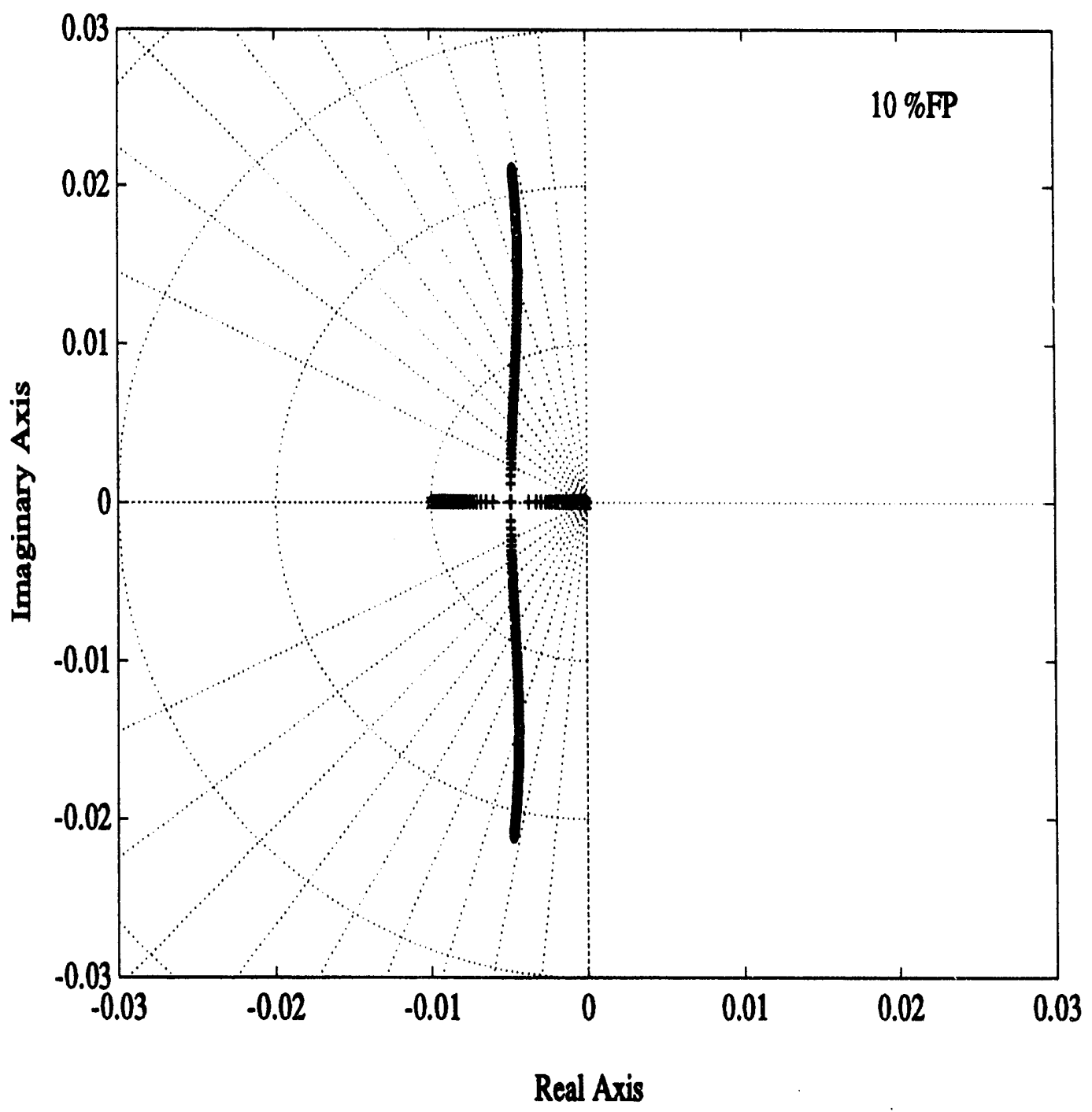

Figure 8.5.2.2-3 Root Locus Plot of the Closed-Loop Transfer Function Associated with Feedwater Flowrate Compensator $\left(\alpha_{\mathrm{f}}=100 \mathrm{~s}\right)$ at $10 \%$ FP. 
problem associated with steam generator level control at low power. Also, the system is stable if the adaptive parameter in the compensator is chosen to be 100 seconds.

\subsubsection{Desien of the Load Parameter Compensators}

Most previous work $[98,99]$ on the control of systems that exhibit inverse response behavior has focused on the design of compensators for the manipulated variable. However, load parameters, such as steam flowrate and primary coolant temperature, also result in an inverse response. This is especially true for steam generators where the level setpoint remains constant while the load changes. The resulting inverse behavior from the change in the load parameters can also lead to a transitory feedback response in the wrong direction. Thus, even though compensation of the manipulated variable (feedwater flowrate) can guarantee stability, it cannot guarantee thai controller performance will be significantly improved.

Figure 8.5.2.3-1 shows the trend in steam generator level after a power increase from $10 \% \mathrm{FP}$ to $15 \% \mathrm{FP}$ on a $5.0 \% \mathrm{FP} /$ minute ramp rate. The steam generator water level controller was compensated by a feedwater flowrate compensator. For purposes of comparison, the time behavior of the steam generator level when regulated by an existing PI controller that was tunea by the Ziegler-Nichols method is also shown in this figure. In the compensated case, the feedback control gain wa: set at $5.0 \mathrm{~kg} \cdot \mathrm{s}^{-1} / \%$ which is a factor of 2.4 greater than that of the existing controller $\left(2.1 \mathrm{~kg} \cdot \mathrm{s}^{-1} / \%\right)$. The process remained stable even when the higher feedback ontrol gain was used. However, in both cases, the control performance was almost the ume. Table 8.5.2.3-1 summarizes the IAE and ISE for 1000 seconds after the start of the power perturbation. The feedwater compensator reduced the IAE and ISE by approximately $40 \%$ and $54 \%$, respectively. However, because a greater feedbeck control gain ivas used, the inverse response effects associated with load disturbances produced a bigger maximum error. Therefore, load parameter compensation is needed to improve control performance during transients that involve changes in demand.

The design of load parameter compensators is similar to that for feedwater flowrate compensation. Figure 8.5.2.3-2 is a block diagram of the load parameter compensators. The inverse response of the measured level is to be offset by using model-based compensators. However, the resulting compensators differ from that developed for feedwater flowrate in two respects. First, load parameter compensation is to be done in an cpen-loop manner. Second, the measureco or estimated magnitude of the load parameter perturbation is required to avoid the need for steam flowrate measurements with their concomitant uncertainty. On the assumption that steam flowrate is proportional to the reactor neutronic power, steam flowrate can be estimated from the neutronic power, the feedwater flowrate, and the feedwater temperature. Thus,

$$
\dot{\mathrm{m}}_{\mathrm{se}}=\frac{\mathrm{Q}_{\mathrm{v}}}{\mathrm{h}_{\mathrm{s}}-\mathrm{h}_{\mathrm{fw}}}
$$


Table 8.5.2.3-1

Control Performance Indices During Power Ramp Transients from $10 \%$ EP to $15 \%$ EP on a $5.0 \%$ EP/minute Ramp Rate

\begin{tabular}{|c|c|c|c|}
\hline Cases & $\begin{array}{l}\text { IAE } \\
{[\% \mathrm{~s}]}\end{array}$ & $\begin{array}{c}\text { ISE } \\
{\left[\%^{2} s\right]}\end{array}$ & $\begin{array}{c}\text { Max Error } \\
{[\%]}\end{array}$ \\
\hline $\begin{array}{l}\text { Without Compensation (A) } \\
\left(\mathrm{K}_{1}=2.1 \mathrm{~kg} / \mathrm{s} / \% \text { and } T_{1}=228 \mathrm{~s}\right)\end{array}$ & 166.2 & 53.4 & 12.7 \\
\hline $\begin{array}{l}\text { With Feedwater Compensation (B) } \\
\left(\mathrm{K}_{1}=5.0 \mathrm{~kg} / \mathrm{s} / \% \text { and } \mathrm{T}_{1}=343 \mathrm{~s}\right)\end{array}$ & 100.2 & 24.6 & 14.6 \\
\hline Difference (B-A)/A [\%] & -39.7 & -53.9 & 15.0 \\
\hline
\end{tabular}

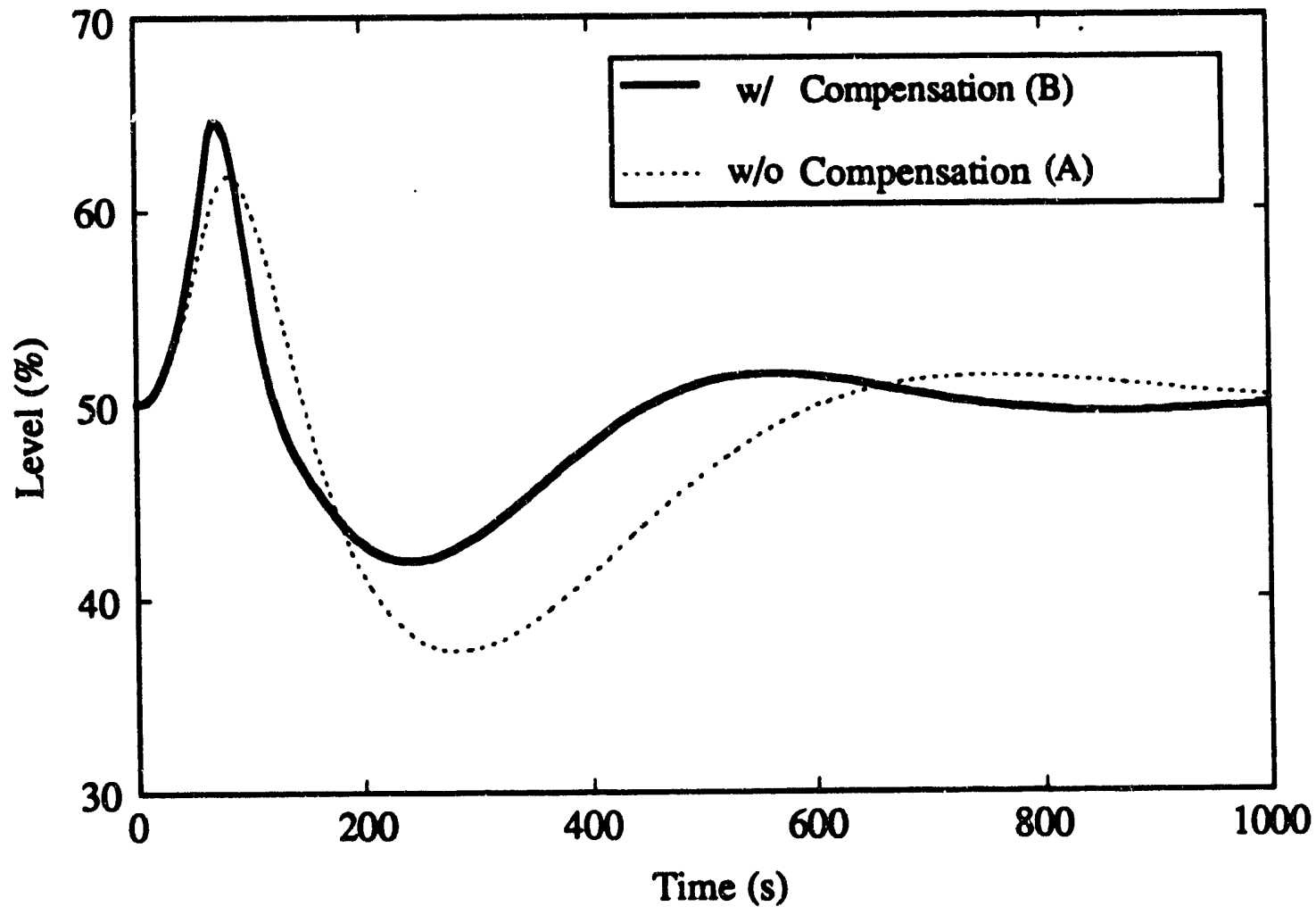

Figure 8.5.2.3-1 Steam Generator Level During Power Ramp Transients from $10 \%$ FP to $15 \% \mathrm{FP}$ at a $5.0 \% \mathrm{FP} /$ minute Ramp Rate. 


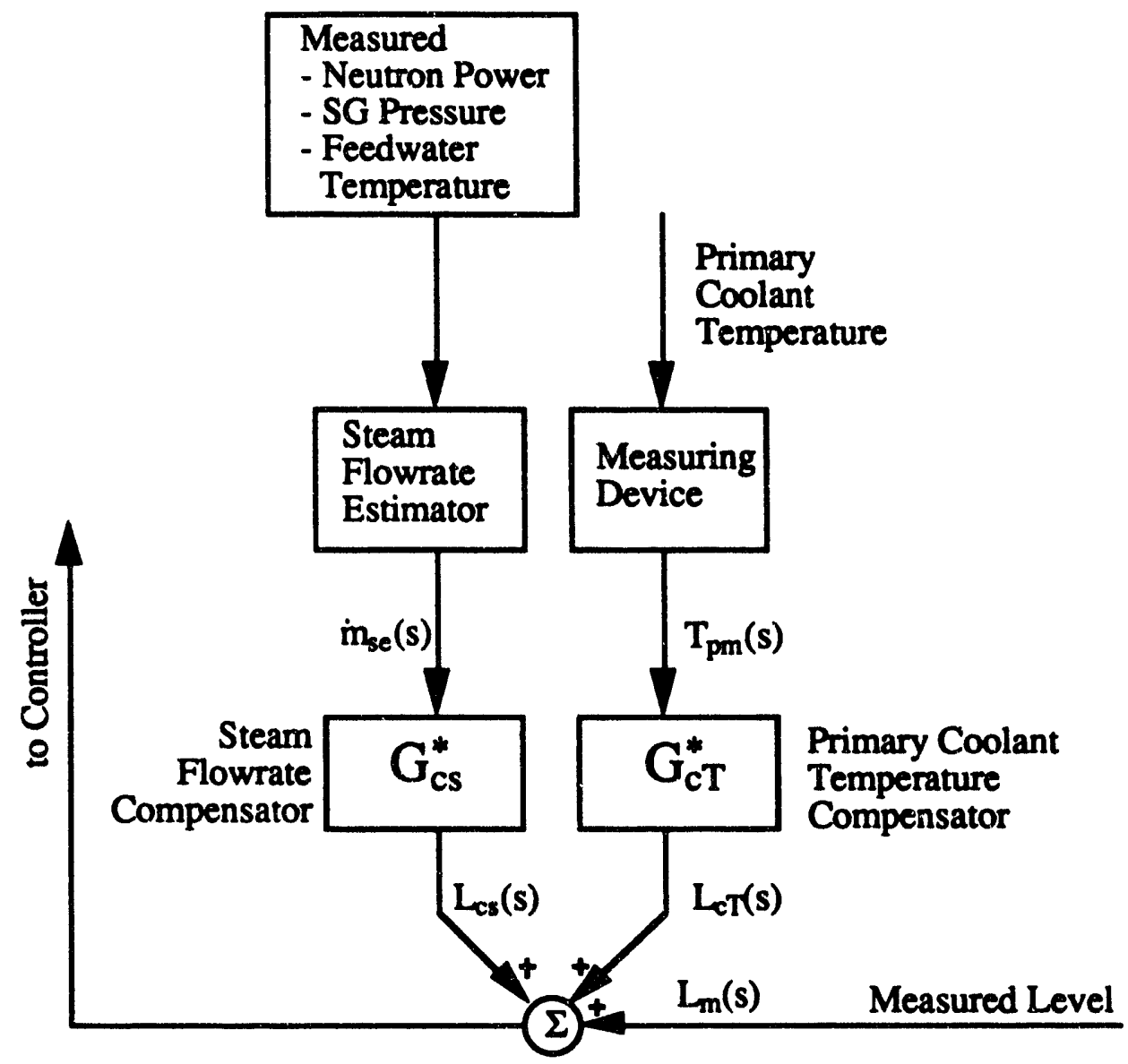

Figure 8.5.2.3-2 Block Diagram of Load Parameter Compensators. 
where $\dot{m}_{2 e}$ is the estimated steam flowrate, $Q_{N}$ is the measured neutronic power, $h_{g}$ is the steam enthalpy, and $h_{f w}$ is the feedwater enthalpy.

For steam flowrate, a compensator based on the simplified transfer function can now be designed in the same form as the feedwater flowrate compensator. Thus,

$$
G_{c s}^{*}=-\frac{G_{2 s}}{\tau_{2 s} s+1} \cdot \frac{\alpha_{s} s}{\alpha_{s} s+1}
$$

where $G_{c z}^{*}$ is the transfer function of the steam flowrate compensator and $\alpha_{3}$ is the adaptive parameter that determines the amount of compensation and precludes an offset error at steady-state. If $\alpha_{3}$ is set to zero, no compensating action is taken. Conversely, if $\alpha_{b}$ is set to infinity, the inverse response is completely offset. This adaptive parameter must, of course, be determined from a tuning procedure.

For the primary coolant temperature compensator, the process transfer function of primary coolant temperature change is modified so that it decays to zero at steady-state. This was done by taking advantage of the fact that $G_{1 T}$ and $G_{2 T}$ are almost equal. Under that assumption, the primary coolant temperature compensator $\left(\mathbf{G}_{\mathrm{cT}}^{*}\right)$ is:

$$
G_{c T}^{*}(s)=-G_{1 T}\left(\frac{1}{\left(1+\tau_{1 T} s\right)}-\frac{1}{\left(1+\tau_{2 T} s\right)}\right) .
$$

Because the compensation factor for primary coolant temperature decays to zero at steadystate, an impulse function that forces such decay was not required. The compensated level signals are then obtained by taking the product of each compensator and its associated load parameter perturbation. Thus, for steam flowrate and primary coolant temperature respectively, we have:

$$
\begin{aligned}
& \mathrm{L}_{c s}(s)=G_{c s}^{*}(s) \dot{m}_{s}(s) \\
& L_{c T}(s)=G_{c T}^{*}(s) T_{P}(s) .
\end{aligned}
$$

The corresponding time-differential equations are:

$$
\begin{aligned}
& \tau_{18} \alpha_{s} \frac{d^{2} L_{c s}(t)}{d t^{2}}+\left(\tau_{1 s}+\alpha_{s}\right) \frac{d L_{c s}(t)}{d t}+L_{c s}(t)=-G_{1 s} \alpha_{s} \frac{d \dot{m}_{s}(t)}{d t} \\
& \tau_{1 T} \tau_{2 T} \frac{d^{2} L_{c T}(t)}{d t^{2}}+\left(\tau_{1 T}+\tau_{2 T}\right) \frac{d L_{c s}(t)}{d t}+L_{c s}(t)=-G_{1 T}\left(\tau_{2 T}-\tau_{1 T}\right) \frac{d T_{p}(t)}{d t}
\end{aligned}
$$


or, in matrix form:

$$
\frac{d}{d t}\left[\begin{array}{l}
L_{c s}(t) \\
L_{c s}^{\prime}(t)
\end{array}\right]=\left[\begin{array}{cc}
0 & 1 \\
-\frac{1}{\tau_{18} \alpha_{s}} & -\frac{\tau_{1 s}+\alpha_{8}}{\tau_{18} \alpha_{s}}
\end{array}\right]\left[\begin{array}{l}
L_{c s}(t) \\
L_{c s}^{\prime}(t)
\end{array}\right]+\left[\begin{array}{c}
0 \\
-\frac{G_{18}}{\tau_{1 s}}
\end{array}\right] \frac{d \dot{m}_{s}(t)}{d t}
$$

and

$$
\frac{d}{d t}\left[\begin{array}{c}
L_{c T}(t) \\
L_{c T}^{\prime}(t)
\end{array}\right]=\left[\begin{array}{cc}
0 & 1 \\
-\frac{1}{\tau_{1 T} \tau_{2 T}} & -\frac{\tau_{1 T}+\tau_{2 T}}{\tau_{1 T} \tau_{2 T}}
\end{array}\right]\left[\begin{array}{l}
L_{c T}(t) \\
L_{c T}(t)
\end{array}\right]+\left[\begin{array}{c}
0 \\
G_{11}\left(\tau_{2 T}-\tau_{11}\right) \\
\tau_{1 T} \tau_{2 T}
\end{array}\right] \frac{\mathrm{dT}_{\mathrm{p}}(t)}{\mathrm{dt}}
$$

where $\mathrm{L}_{c s}^{\prime}(t)$ and $\mathrm{L}_{c T}^{\prime}(t)$ are the time derivatives of the compensated levels for steam flowrate and primary coolant temperature, respectively.

\subsubsection{Incorporation of Feedforward Action}

Feedforward control action is added to improve control system performance. This entails adding the change in the estimated steam flowrate directly to the PI controller's output. Figure 8.5.2.4-1 shows the steady-state mass inventory of a steam generator as a function of power. The mass inventory decreases as power increases because more vapor exists at higher power. Therefore, the mass inventory should be offset when power increases and supplemented when power decreases. Dynamic lag compensation is used for this purpose. The form of the compensator is:

$$
\dot{\mathrm{m}}_{\mathrm{ff}}(s)=\frac{1}{\alpha_{\mathrm{ff}} s+1} \dot{\mathrm{m}}_{\mathrm{se}}(s)
$$

where $\dot{m}_{\mathrm{ff}}(\mathrm{s})$ is the Laplace transform of the feedforward controller output, $\dot{\mathrm{m}}_{\mathrm{se}}(\mathrm{s})$ is that of the estimated steam flowrate, and $\alpha_{\mathrm{ff}}$ is the time constant for the lagged response.

\subsubsection{Controller Synthesis}

Figure 8.5.3-1 shows the schematic of the proposed controller. It consists of a PI controller and three different compensators designed to offset the inverse response associated with feedwater flow, steam flow, and primary coolant temperature. This proposed controller has five adjustable parameters. These are the feedback control gain and the reset time of the PI controller $\left(K_{1}\right.$ and $\left.T_{1}\right)$, the adjustable parameters in the inverse response compensators $\left(\alpha_{f}\right.$ and $\left.\alpha_{8}\right)$, and the time constant of the lag term in the feedforward controller $\left(\alpha_{\mathrm{ff}}\right)$. In order to achieve superior control performance, the proposed controller must br, properly tuned. Optimum tuning for these control parameters 


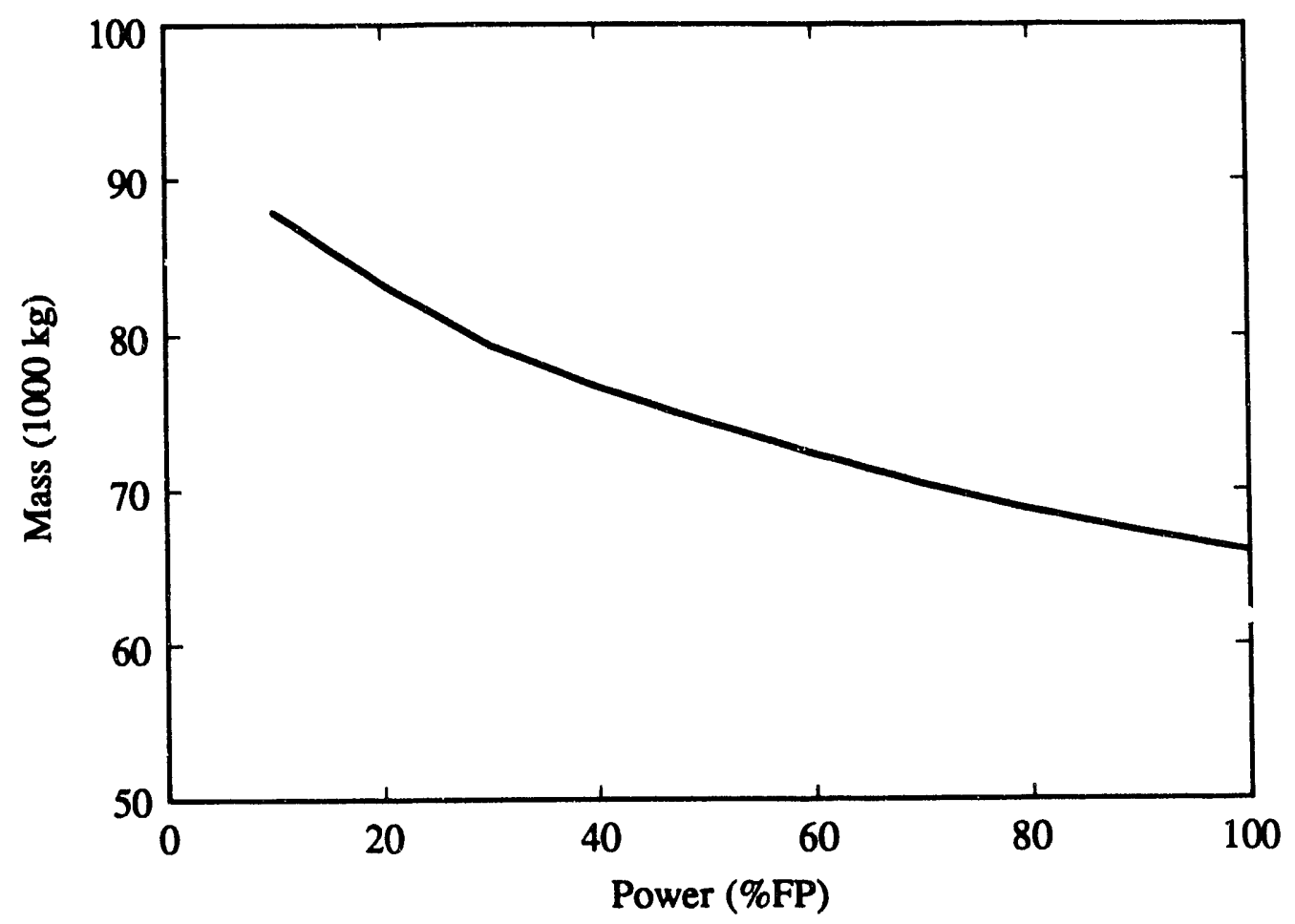

Figure 8.5.2.4-1 Steady-State Steam Generator Mass Inventory. 


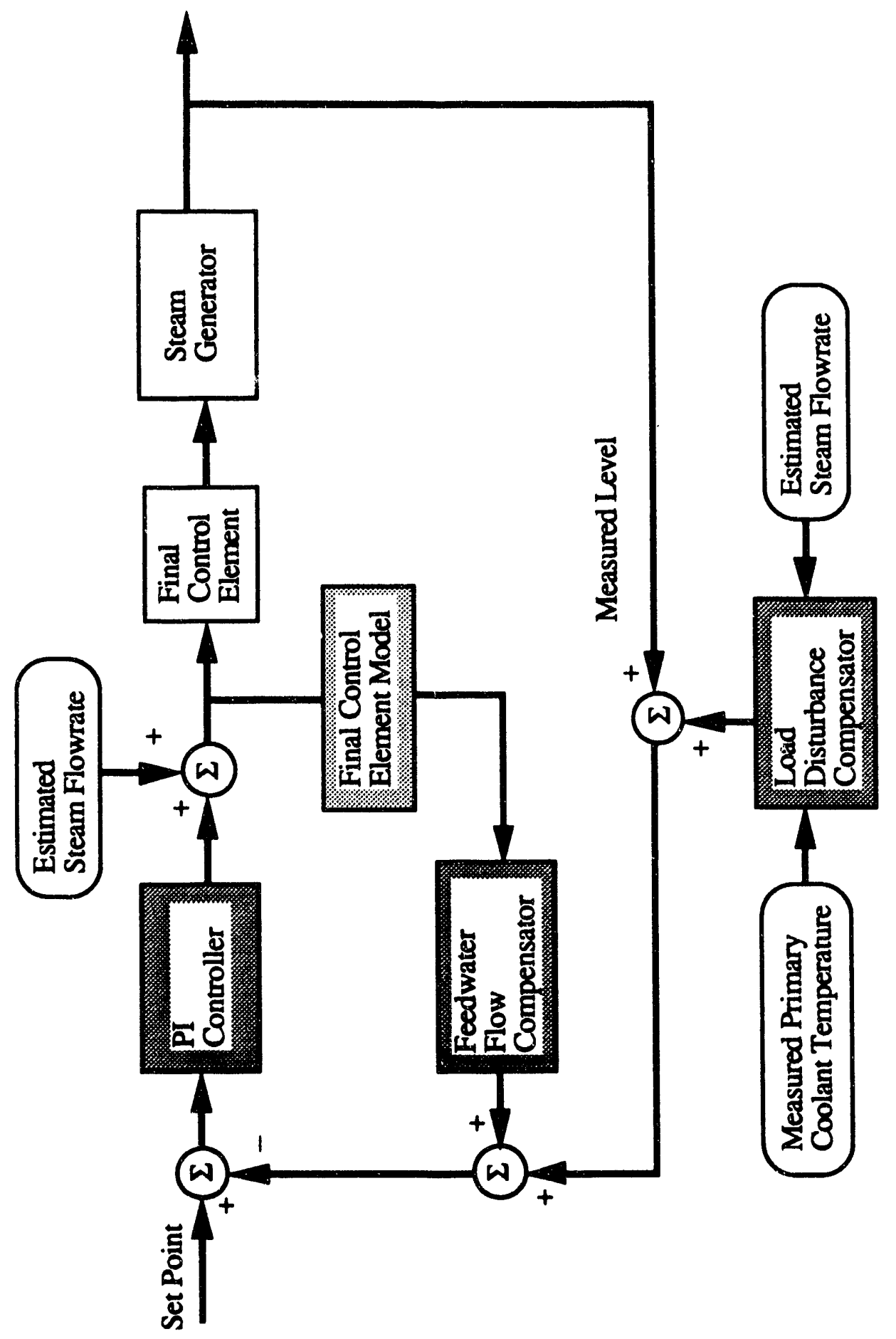

ह્. 
depends not only on control performance criteria but also on the type of disturbance that is encountered. For example, a tuning action that minimizes IAE for a step change in load will not minimize IAE for a ramp load change. Also, a tuning that minimizes IAE for a step change in load will not minimize ISE for the same transient. Optimum tunings also vary with the nature of the process.

In this research, the time-integral performance criteria, IAE and/or ISE, were used to identify optimum tuning parameters. For the feedback control gain and reset time ( $\mathrm{K}_{1}$ and $\left.\mathrm{T}_{1}\right)$, a simple criterion ( 0.316 decay ratio) was used. The feedback control gain was set at $5.0 \mathrm{~kg} \cdot \mathrm{s}^{-1} / \%$ and the reset time at 343 seconds at $10 \% \mathrm{FP}$. These settings were used over the entire power region. In addition, a feedback control gain of $15.69 \mathrm{~kg} \cdot \mathrm{s}^{-1} / \%$ and a reset time of 343 seconds were used to demonstrate stability.

Figure 8.5.3-2 shows the time behavior of steam generator level when $\alpha_{c f}$ and $\alpha_{c s}$ were set at 10,100, and 1000 seconds respectively. As shown in this figure, the best control performance was achieved when both of these adjustable parameters were set at 100 seconds. For the lag time constant in the feedforward controller, a value of 50 seconds was used. It is time-consuming to identify the tuning parameters by trial and error. Therefore, it is important to realize that the values given here for the adjustable parameters may not be the best possible ones.

\subsection{Evaluation of Proposed Steam Generator Level Controller}

Simulation studies in which the proposed controller was evaluated by application to differen: transients are reported here. The detailed non-linear model described in Chapter Seven of this report was used to simulate level response. A single power module with a single steam generator simulation option was used to evaluate the controller's performance for feedwater flowrate perturbations and power transients. Four power modules were used to study the controller's performance for unbalanced load transients in the multi-modular power plants. The performance of the steam generator level controller was evaluated at various power levels by comparing the IAE, ISE, and the maximum level error. The controllers studied included both the detuned, single-element PI controller and the compensated controller that was developed here. The latter was used to control both the single-module plant and the four-module, multi-modular plant.

\subsubsection{Feedwater Elowrate Perturbation Transients}

The reference transient studied was a $5.0 \mathrm{~kg} / \mathrm{s}$ step increase in feedwater flowrate from steady-state with core and turbine power held constant. The objective of the controller was to manipulate the feedwater flowrate so as to maintain the desired steam generator level. The conventional, single-element PI controller was tuned by the ZieglerNichols method while the base case compensated controller developed here was tuned with a 0.316 decay ratio and a reset time of 343 seconds. (Note: Other values for these parameters were also used with the compensated controller to study variations.)

Figure 8.6.1-1 shows the time behavior of the steam generator level for 1000 seconds following the reference transient. The conventional, single-element PI controller 


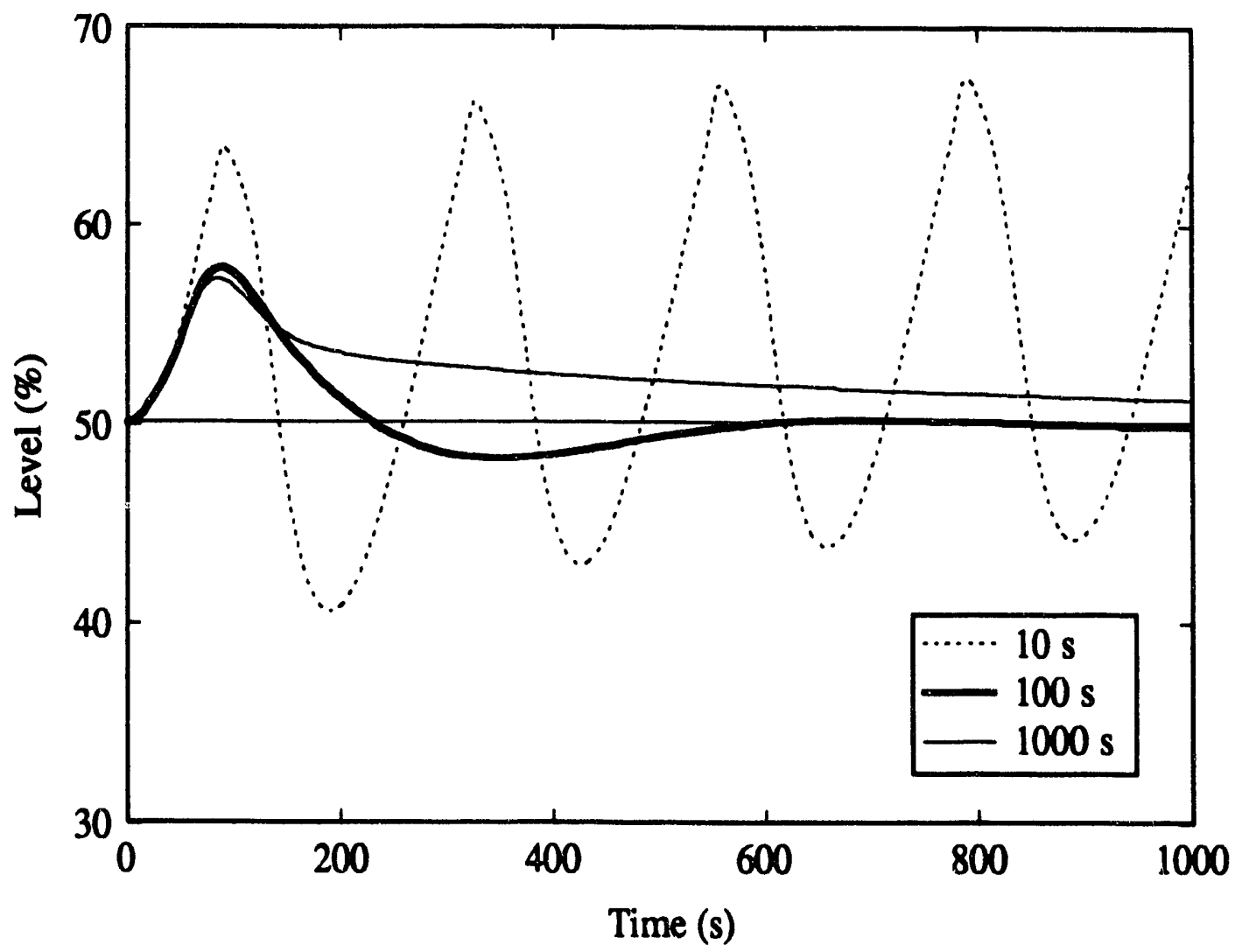

Figure 8.5.3-2 Effect of Inverse Response Compensation During Power Ramp Transients from $10 \% \mathrm{FP}$ to $15 \% \mathrm{FP}$ at a $5.0 \% \mathrm{FP} /$ minute Ramp Rate. 
stabilized the level but its response showed some oscillation. However, the controller designed here (Section 8.5 of this report) both compensated for inverse response effects and provided a less oscillatory response. Also, it kept the level error at a smaller value throughout the transient.

Figure 8.6.1-1 also shows the level response at $10 \% \mathrm{FP}$ when the compensated controller was tuned with a gain of $15.69 \mathrm{~kg} \cdot \mathrm{s}^{-1} / \%$. Reset times of both 200 seconds and 343 seconds were used. Both cases show stable level trends. When tuned with a higher gain, the controller not only guaranteed stability but also performed better. It was decided that, even though a high feedback control gain can improve controller performance during feedwater flowrate perturbations, the feedback control gain should be set at $5.0 \mathrm{~kg} \cdot \mathrm{s}^{-1} / \%$ for power ramp (up or down) transients because an imperfect steam flowrate compensation might degrade performance as a result of small errors in the estimated steam flowrate.

Table 8.6.1-1 summarizes the results of the feedwater flowrate perturbation transients. The compensated controller that was designed here improved control performance in all cases. It reduced the IAE by $54.4 \%$ when the gain was set to $5.0 \mathrm{~kg} \cdot \mathrm{s}^{-1} / \%$ and, when the gain was set at $15.69 \mathrm{~kg} \cdot \mathrm{s}^{-1} / \%$, it reduced the IAEs by $80 \%$ to $85 \%$. The ISE and maximum error were also reduced significantly with the amount ranging from a factor of 2.5 to 50 .

\subsubsection{Power Transients}

Figure 8.6.2-1 shows the steam generator level response during a power increase from $10 \% \mathrm{FP}$ to $15 \% \mathrm{FP}$ at a ramp rate of $5.0 \% \mathrm{FP} /$ minute. The power increase required one minute and the power was then to be maintained at $15 \% \mathrm{FP}$. Both a conventional single-element PI controller tuned by the Ziegler-Nichols method and the compensated controller developed here (Section 8.5 of this report) were used. The latter showed better control performance and a more gradual transition to the new equilibrium. The conventional PI controller did yield a stable response but it was more oscillatory. Table 8.6.2-1 summarizes the time integral control performance indices for the runs. The compensated controller significantly improved control performance in all cases. Specially, it reduced the IAE and ISE by one-third and one-sixth, respectively. The maximum error was also reduced by $40 \%$ when feedback gain was set to $5.0 \mathrm{~kg} \cdot \mathrm{s}^{-1} / \%$. However, higher feedback control gains led to larger maximum level errors.

Figure 8.6.2-2 shows the time behavior of steam and feedwater flowrates. The compensated controller increased the feedwater flowrate gradually until it reached steadystate. In contrast, the conventional controller caused oscillations and at one point decreased the feedwater flowrate to zero because of inverse response effects.

Figure 8.6.2-3 shows the steam generator level response during a power decrease from $20 \% \mathrm{FP}$ to $15 \% \mathrm{FP}$ at a rate of $5.0 \% \mathrm{FP} /$ minute. Power was then to be maintained at $15 \%$ FP. Table 8.6.2-2 summarizes the time integral control performance index. Again, the compensated controller significantly improved performance. 
Table 8.6.1-1

Control Performance Indices During

Eeedwater Flowrate Perturbation Transients at $10 \%$ EP

\begin{tabular}{|c|c|c|c|}
\hline Cases & $\begin{array}{l}\text { IAE } \\
{[\% \mathrm{~s}]}\end{array}$ & $\begin{array}{l}\text { ISE } \\
{\left[\%^{2} s\right]}\end{array}$ & $\begin{array}{c}\text { Max Error } \\
{[\%]}\end{array}$ \\
\hline $\begin{array}{l}\text { a) Without Compensation } \\
\left(\mathrm{K}_{1}=2.1 \mathrm{~kg} / \mathrm{s} / \% \text { and } \mathrm{T}_{1}=228 \mathrm{~s}\right)\end{array}$ & 30.93 & 2.07 & 2.86 \\
\hline $\begin{array}{l}\text { b) With Compensation } \\
\left(\mathrm{K}_{1}=5.0 \mathrm{~kg} / \mathrm{s} / \% \text { and } \mathrm{T}_{1}=343 \mathrm{~s}\right)\end{array}$ & 14.08 & 0.42 & 1.17 \\
\hline $\begin{array}{l}\text { c) With Compensation } \\
\left(\mathrm{K}_{1}=15.69 \mathrm{~kg} / \mathrm{s} / \% \text { and } \mathrm{T}_{1}=343 \mathrm{~s}\right)\end{array}$ & 4.77 & 0.05 & 0.44 \\
\hline $\begin{array}{l}\text { d) With Compensation } \\
\left(\mathrm{K}_{1}=15.69 \mathrm{~kg} / \mathrm{s} / \% \text { and } \mathrm{T}_{1}=200 \mathrm{~s}\right)\end{array}$ & 4.05 & 0.04 & 0.41 \\
\hline
\end{tabular}

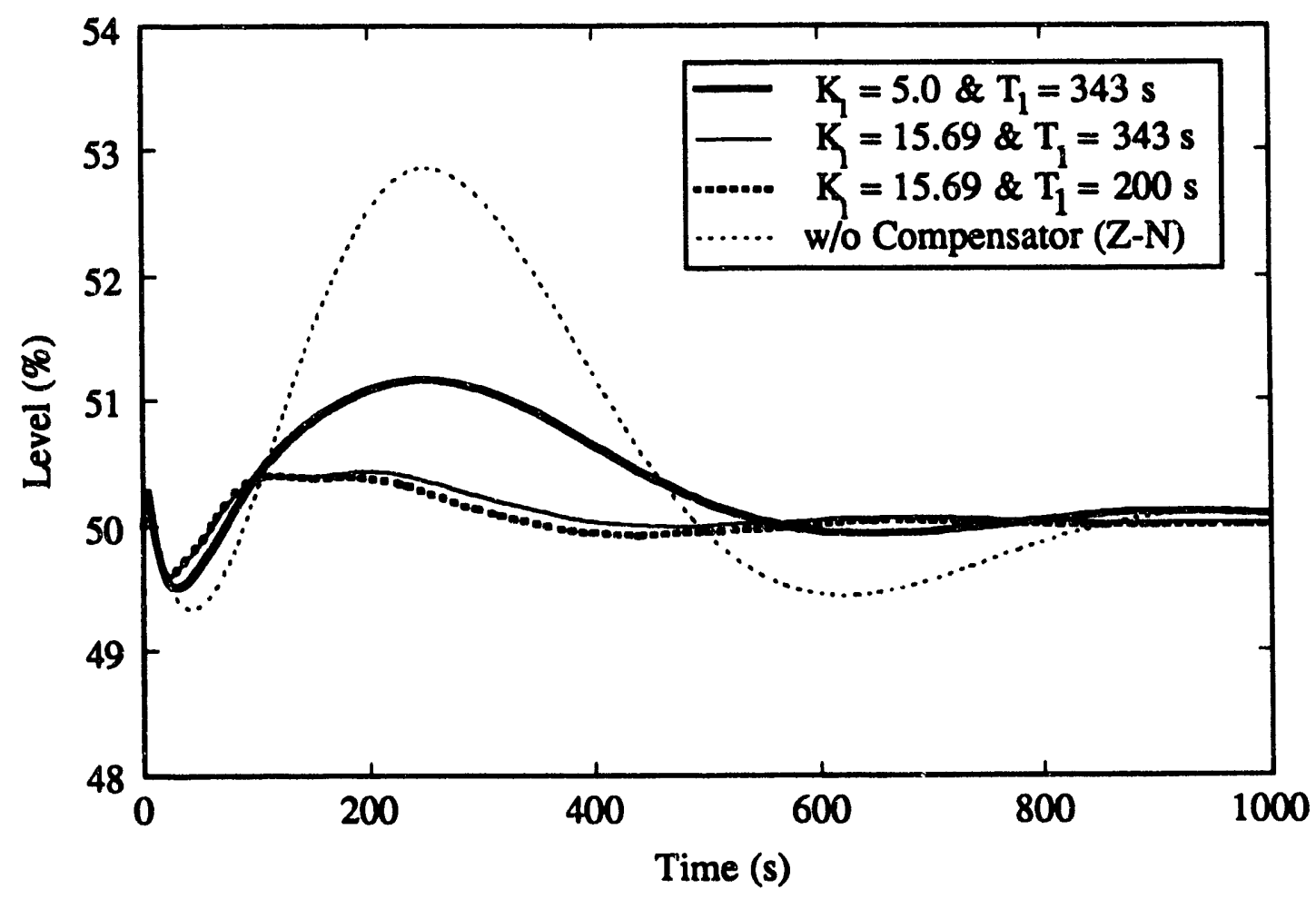

Figure 8.6.1-1 Steam Generator Level During Feedwater Flowrate Perturbation Transients at $10 \% \mathrm{FP}$. 
Table 8.6.2-1

Control Performance Indices During Power Ramp Transients from $10 \% \mathrm{FP}$ to $15 \% \mathrm{FP}$ at a $5.0 \% \mathrm{FP} /$ minute Ramp Rate

\begin{tabular}{|c|r|r|r|}
\hline Cases & \multicolumn{1}{|c|}{$\begin{array}{c}\text { IAE } \\
{[\% \mathrm{~s}]}\end{array}$} & \multicolumn{1}{c|}{$\begin{array}{c}\text { ISE } \\
{\left[\%^{2} \mathrm{~s}\right]}\end{array}$} & \multicolumn{1}{c|}{$\begin{array}{c}\text { Max Error } \\
{[\%]}\end{array}$} \\
\hline $\begin{array}{l}\text { a) Without Compensation, 3 Element PI } \\
\left(\mathrm{K}_{1}=2.1 \mathrm{~kg} / \mathrm{s} / \% \text { and } \mathrm{T}_{1}=228 \mathrm{~s}\right)\end{array}$ & 166.2 & 53.4 & 12.67 \\
$\begin{array}{l}\text { b) With Compensation } \\
\left(\mathrm{K}_{1}=5.0 \mathrm{~kg} / \mathrm{s} / \% \text { and } \mathrm{T}_{1}=343 \mathrm{~s}\right)\end{array}$ & 46.3 & 7.7 & 7.85 \\
$\begin{array}{c}\text { c) With Compensation } \\
\left(\mathrm{K}_{1}=15.69 \mathrm{~kg} / \mathrm{s} / \% \text { and } \mathrm{T}_{1}=343 \mathrm{~s}\right)\end{array}$ & 42.1 & 11.7 & 14.88 \\
\hline
\end{tabular}

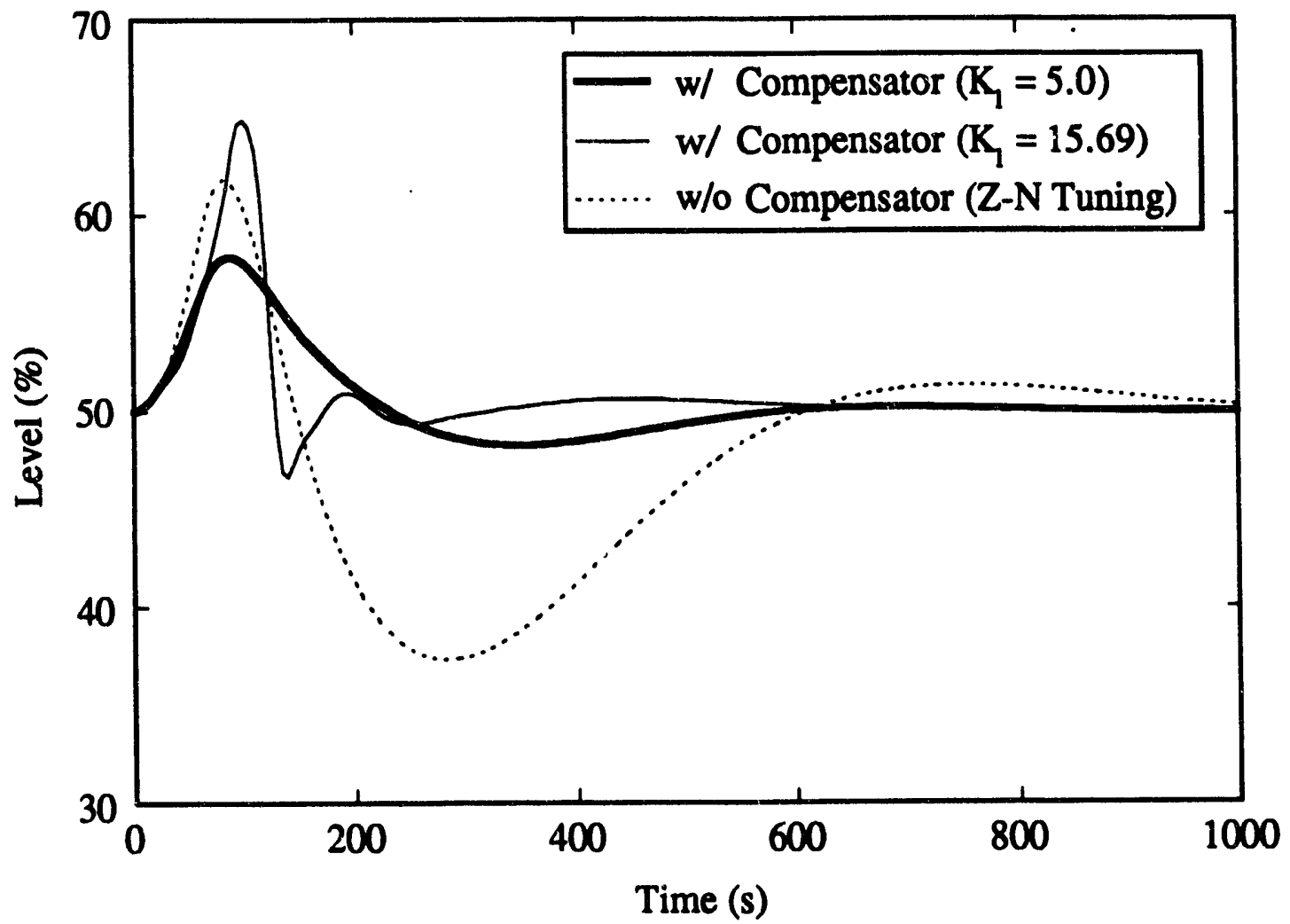

Figure 8.6.2-1 Steam Generator Level During Power Ramp Transients from $10 \% \mathrm{FP}$ to $15 \% \mathrm{FP}$ at a $5.0 \% \mathrm{FP} /$ minute Ramp Rate. 


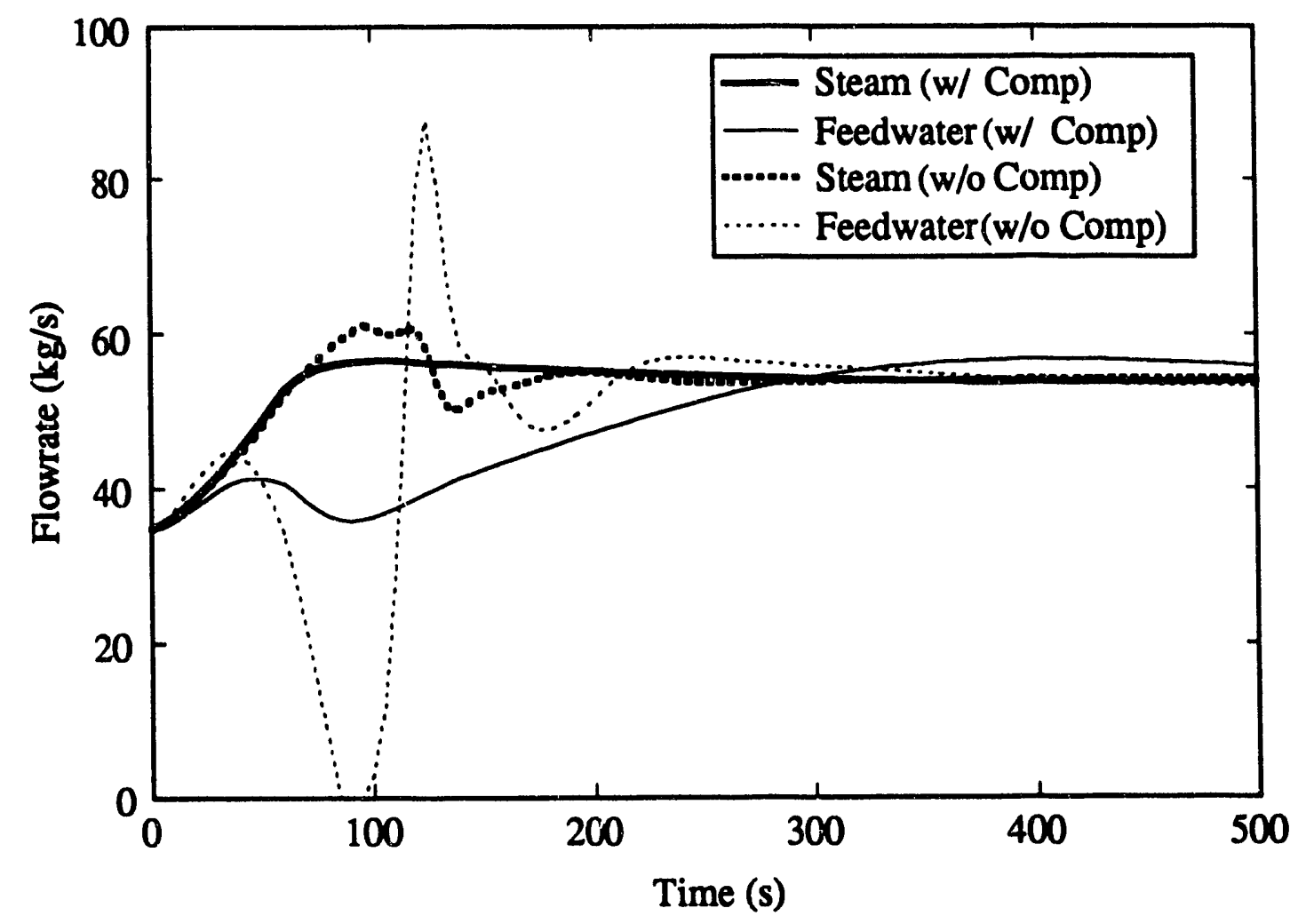

Figure 8.6.2-2 Steam and Feedwater Flowrate Variation During Power Ramp Transients from $10 \% \mathrm{FP}$ to $15 \% \mathrm{FP}$ at a $5.0 \% \mathrm{FP} /$ minute Ramp Rate. 
Table 8.6.2-2

Control Performance Indices During Power Ramp Transients from $15 \% \mathrm{EP}$ to $10 \% \mathrm{EP}$ at a $5.0 \% \mathrm{EP} /$ minute Ramp Rate

\begin{tabular}{|c|c|c|c|}
\hline Cases & $\begin{array}{c}\text { IAE } \\
{[\% \mathrm{~s}]}\end{array}$ & $\begin{array}{c}\text { ISE } \\
{[\% \mathrm{~s}]}\end{array}$ & $\begin{array}{c}\text { Max Error } \\
{[\%]}\end{array}$ \\
\hline $\begin{array}{c}\text { a) Without Compensation } \\
\left(\mathrm{K}_{1}=2.1 \mathrm{~kg} / \mathrm{s} / \% \text { and } \mathrm{T}_{1}=228 \mathrm{~s}\right)\end{array}$ & 173.9 & 59.6 & 14.98 \\
$\begin{array}{l}\text { b) With Compensation } \\
\left(\mathrm{K}_{1}=5.0 \mathrm{~kg} / \mathrm{s} / \% \text { and } \mathrm{T}_{1}=343 \mathrm{~s}\right) \\
\text { c) With Compensation } \\
\left(\mathrm{K}_{1}=15.69 \mathrm{~kg} / \mathrm{s} / \% \text { and } \mathrm{T}_{1}=343 \mathrm{~s}\right)\end{array}$ & 65.2 & 9.1 & 6.88 \\
\hline
\end{tabular}

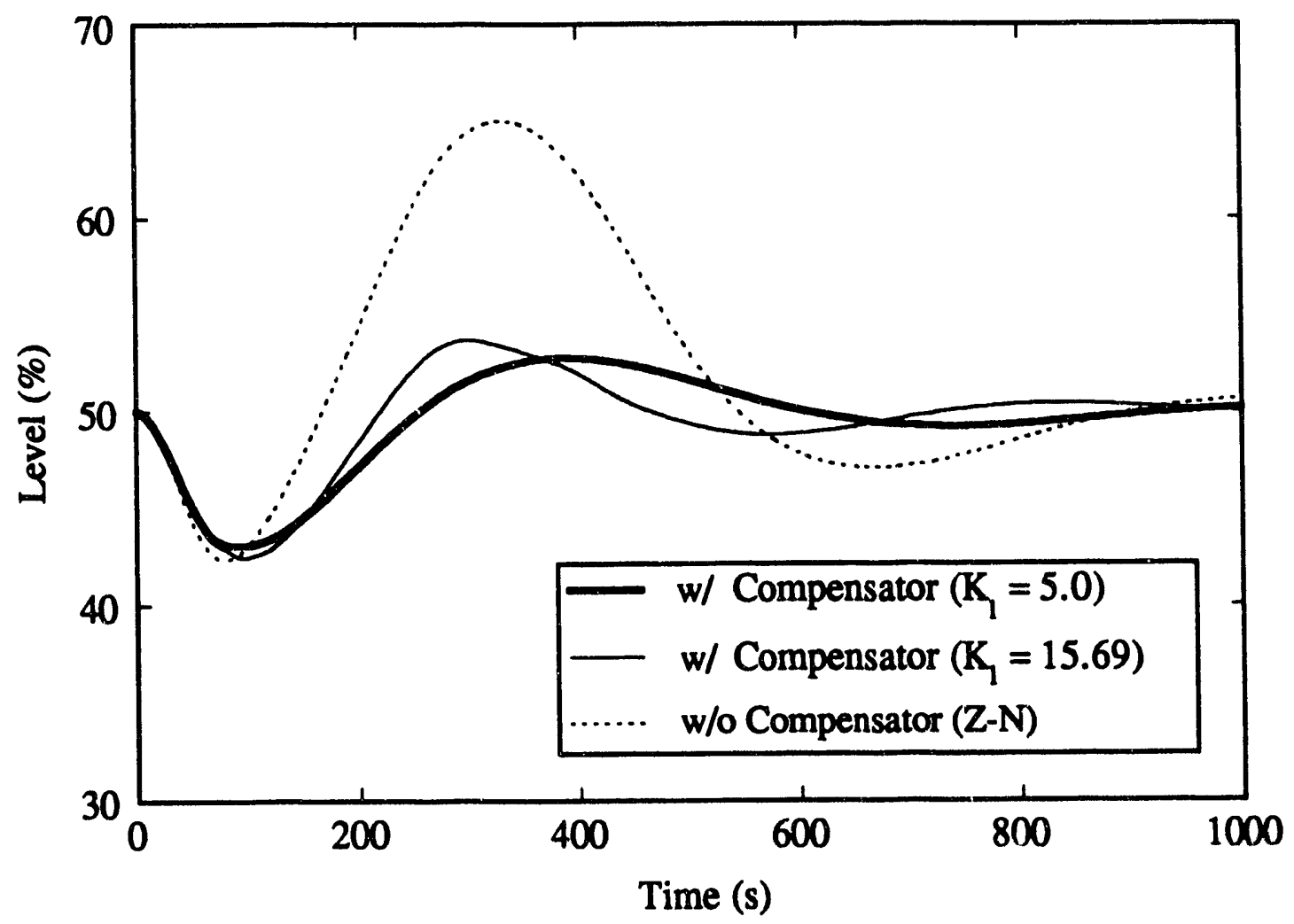

Figure 8.6.2-3 Steam Generator Narrow Range Level During Power Ramp Transients from $15 \% \mathrm{FP}$ to $10 \% \mathrm{FP}$ at a $5.0 \% \mathrm{FP} /$ minute Ramp Rate. 
Figure 8.6.2-4 shows the steam generator level response during a power ramp from $95 \% \mathrm{FP}$ to $100 \% \mathrm{FP}$ at a rate of $5.0 \% \mathrm{FP} /$ minute. Two feedback control gains, 5.0 $\mathrm{k}_{6} \cdot \mathrm{s}^{-1} / \%$ and $15.69 \mathrm{~kg} \cdot \mathrm{s}^{-1} / \%$, were used. A three-element conventional PI controller was also used. As shown in this figure, the proposed controller provided superior performance at this high power. IAE and ISE were only $7.52 \% \cdot s$ and $0.15 \%{ }^{2} \cdot \mathrm{s}$, respectively.

The above simulations showed that, even if the compensated system uses only a single-element PI controller, its performance is adequate for high power operation. Therefore, the proposed compensated controller can be used over the entire power region and flowrate measurements are not needed.

\subsubsection{Aprlication to Multi-Modular Power Plants}

The compensated controller was applied to steam generators configured in a PWRtype multi-modular power plant. The reference transient was to increase the module $\# 1$ power from $10 \%$ FP to $15 \%$ FP while the other modules remained at $10 \%$ FP. Turbine power was increased from 10 \%FP to 11.25 \%FP. The feedback control gain was set at $5.0 \mathrm{~kg} \cdot \mathrm{s}^{-1} / \%$ and the reset time at 343 seconds. Figure 8.6.3-1 shows the level of each steam generator during the reference transient. The module \#1 steam generator level is oscillatory with the amplitude of the oscillations growing in time. Those of the other modules also show slowly growing oscillations. These oscillations in level are the result of the unbalansed power operation. Because the power was increased for only one module, the pressure balance among the steam generators and the main steam line header was disturbed with the result that steam flowrate oscillated as shown in Figure 8.6.3-2. The steam flowrate estimator could not predict the steam flowrate oscillations in a given steam generator because the model that it uses does not allow for time delays and other nonequilibrium effects. Specifically, it assumes that steam flowrate is proportional to the neutronic power. In the case of unbalanced loads, this will not always be true. For example, changes in one module's steam pressure will immediately affect the flowrates in the other modules. However, neutronic power in these modules will not change until the impact of the changes in the flowrates have propagated through the secondary and primary loops. If power in all four modules was increased at the same rate, then the pressure balarce among the steam generators and the main steam line header would be maintained and the time behavior of the steam generator level would be stable as shown in Figure 8.6.3-3. Therefore, in unbalanced power operation, a different and more restrictive steam generator controller tuning is requized. Figure 8.6.3-4 shows each steam generator's level when the feedback control gain is set at $2.0 \mathrm{~kg} \cdot \mathrm{s}^{-1} / \%$. In this case, the levels remain stable. Figure 8.6.3-5 shows the steam flowrates from each steam generator. These are stabilized at the desired point.

Other unbalanced power transients were also simulated. For example, module \# 1 power was increased from 15 \%FP to $20 \%$ FP and module \#2 power was decreased from $15 \%$ FP to $10 \%$ FP both at a ramp rate of $5.0 \% \mathrm{FP} /$ minute. Power in the other two modules was held at $15 \%$ FP. The turbine power was also maintained at $15 \%$ FP. The feedback control gain of the compensated controller was set to $2.0 \mathrm{~kg} \cdot \mathrm{s}^{-1} / \%$. Figure 8.6.36 shows the response of each steam generator's level during the above transient. As would be expected, the module $\# 1$ steam generator shows level swelling and that of module \#2 
Table 8,6,2-3

Control Performance Indices During Power Power Ramap Transients from $95 \% \mathrm{FP}$ to $100 \% \mathrm{EP}$ at a $5.0 \% \mathrm{FP} /$ minute Ramp Rate

\begin{tabular}{|c|c|c|c|}
\hline Cases & $\begin{array}{c}\text { IAE } \\
{[\% \mathrm{~s}]}\end{array}$ & $\begin{array}{c}\text { ISE } \\
{\left[\%^{2} s\right]}\end{array}$ & $\begin{array}{c}\text { Max Error } \\
\text { [\%] }\end{array}$ \\
\hline $\begin{array}{l}\text { a) Without Compensation, } 3 \text { Element PI } \\
\left(K_{1}=15.69 \mathrm{~kg} / \mathrm{s} / \% \text { and } T_{1}=200 \mathrm{~s}\right. \\
\left.K_{w}=1 \mathrm{~kg} / \mathrm{s} / \mathrm{kg} / \mathrm{s} \text { and } T_{1}=200 \mathrm{~s}\right)\end{array}$ & 15.52 & 0.46 & 1.38 \\
\hline $\begin{array}{l}\text { b) With Compensation } \\
\left(\mathrm{K}_{1}=5.0 \mathrm{~kg} / \mathrm{s} / \% \text { and } T_{1}=343 \mathrm{~s}\right)\end{array}$ & 7.52 & 0.15 & 1.40 \\
\hline $\begin{array}{l}\text { c) With Compensation } \\
\left(\mathrm{K}_{1}=15.69 \mathrm{~kg} / \mathrm{s} / \% \text { and } T_{1}=343 \mathrm{~s}\right)\end{array}$ & 7.26 & 0.21 & 1.66 \\
\hline
\end{tabular}

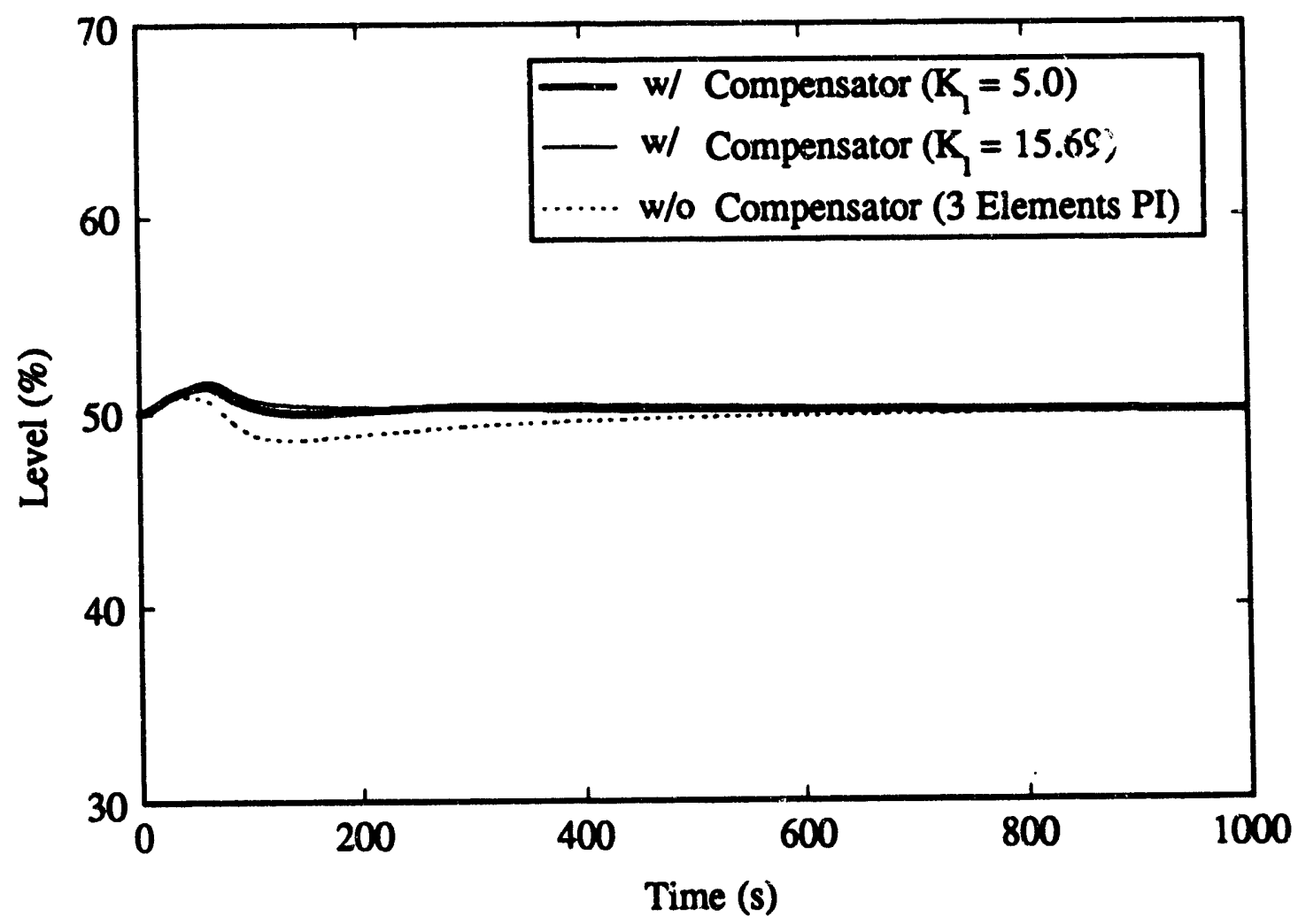

Figure 8.6.2-4 Steam Generator Narrow Range Level During Power Ramp Transients from $95 \%$ FP to $100 \%$ FP at a $5.0 \%$ FP/minute Ramp Rate. 


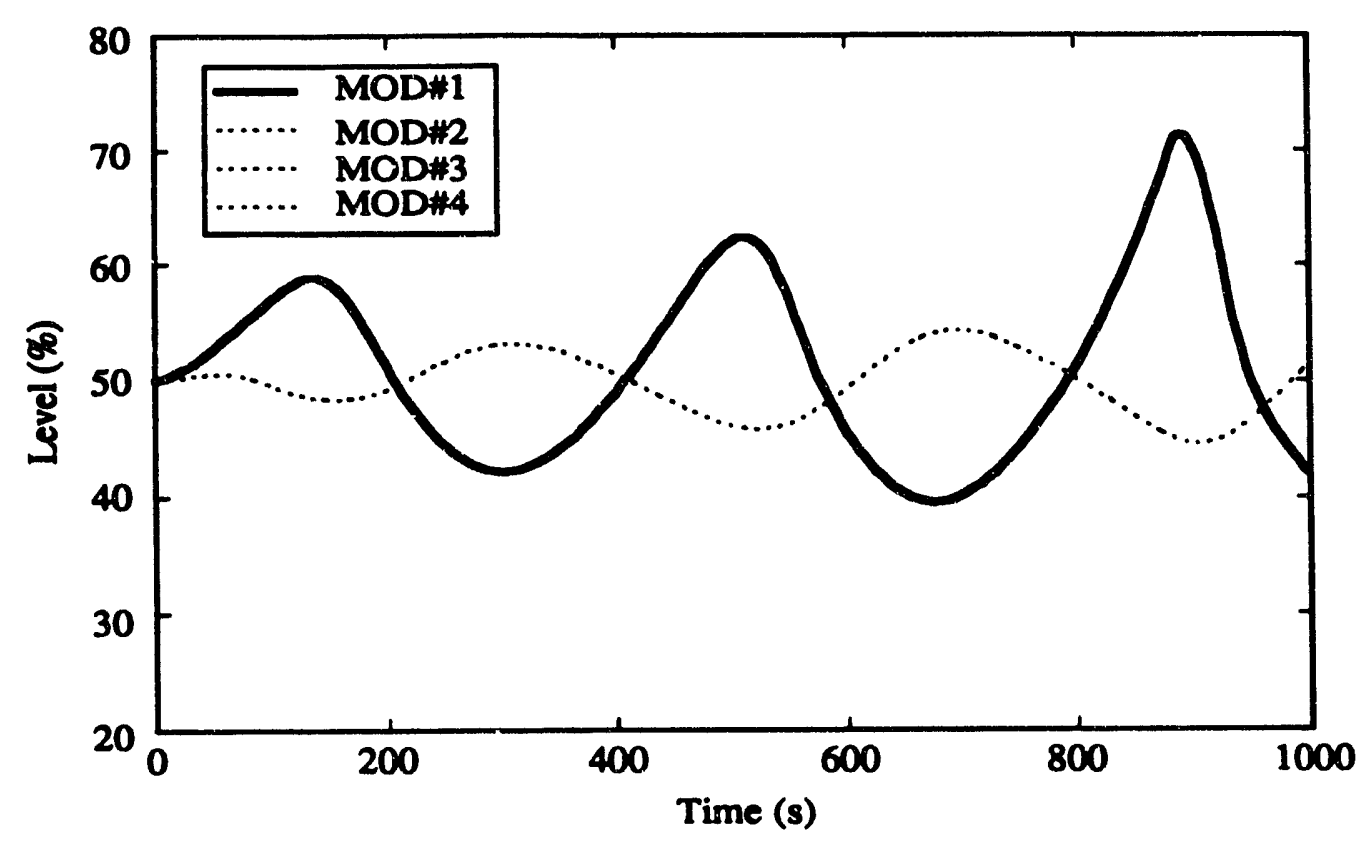

Figure 8.6.3-1 Steam Generator Narrow Range Level During Power Ramp Transients from $10 \% \mathrm{FP}$ to $15 \% \mathrm{FP}$ at a $5.0 \% \mathrm{FP} /$ minute Ramp Rate on Module \#1 Only.

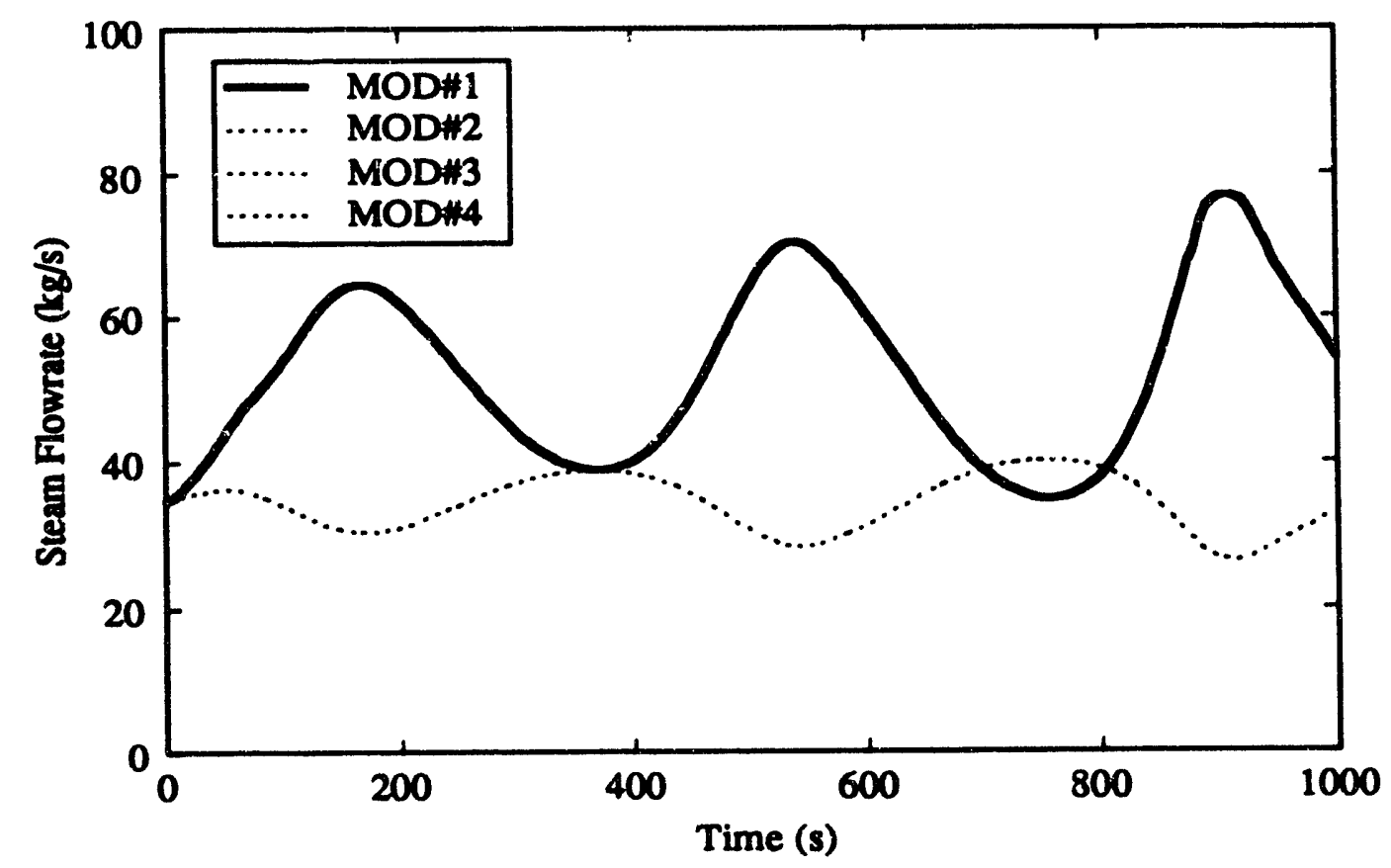

Figure 8.6.3-2 Steam Flowrate During Unbalanced Power Ramp Transients from $10 \% \mathrm{FP}$ to $15 \% \mathrm{FP}$ at a $5.0 \% \mathrm{FP} /$ minute Ramp Rate on Module \#1 Only. 


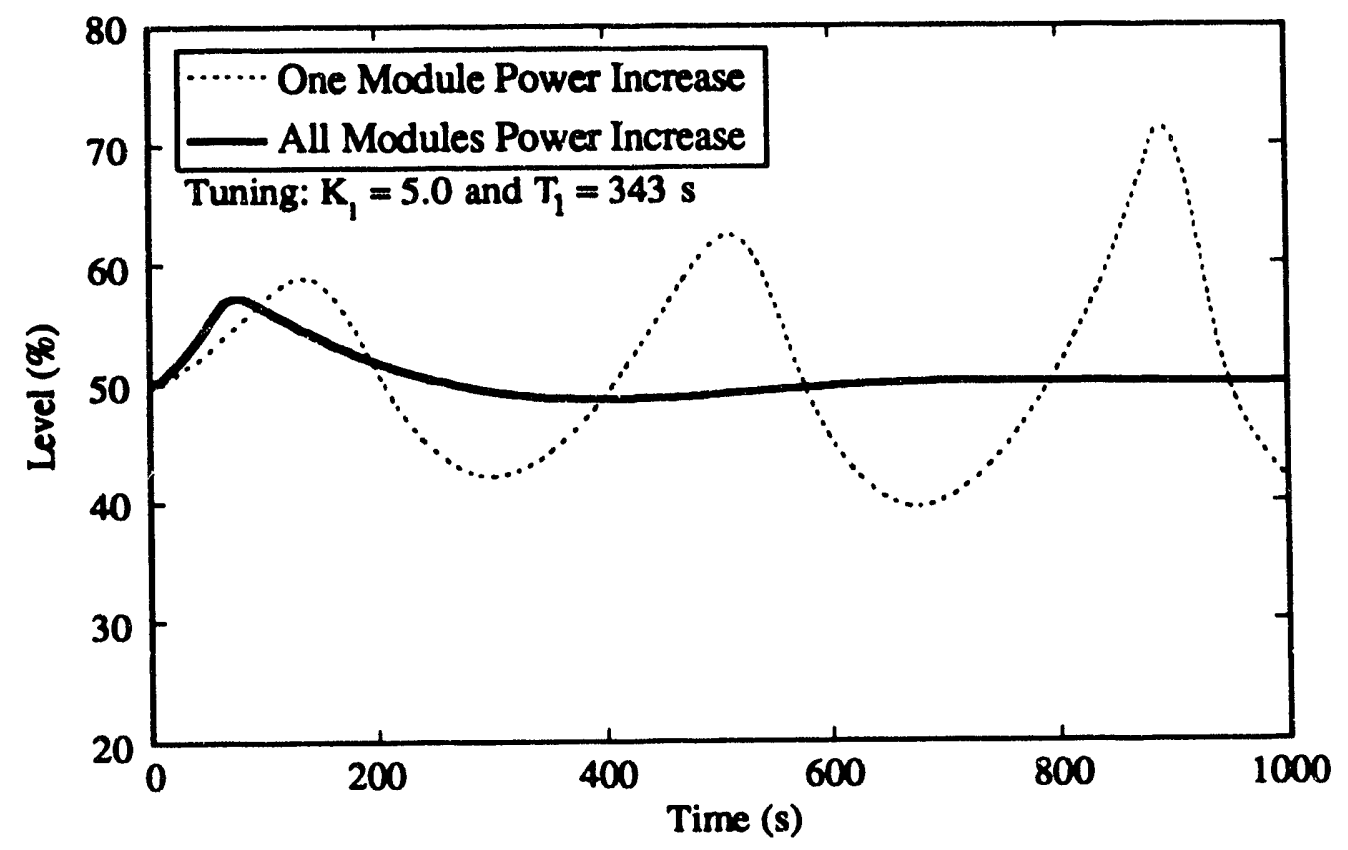

Figure 8.6.3-3 Steam Generator Narrow Range Level During Balanced and Unbalanced Power Ramp Transients.

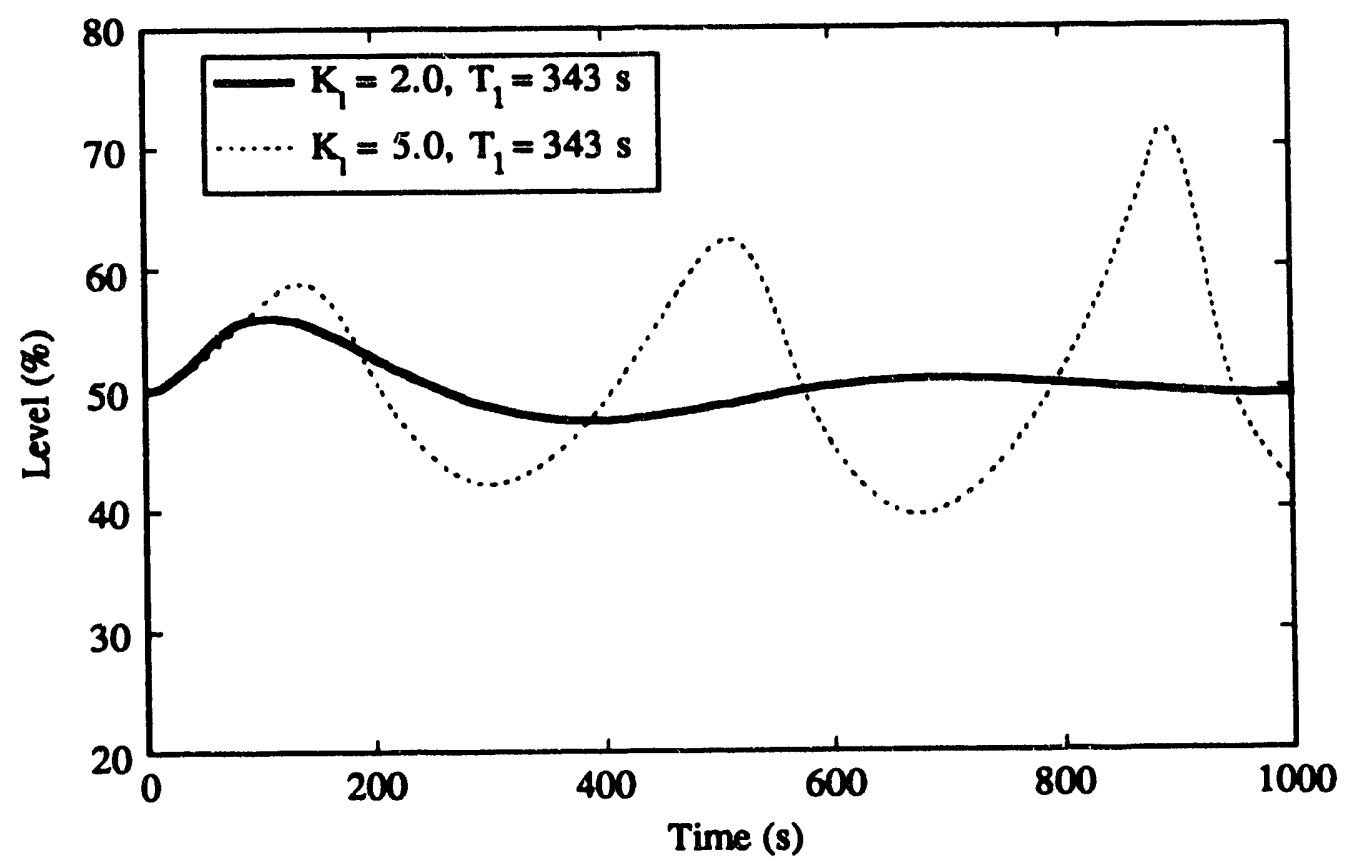

Figure 8.6.3-4 Steam Generator Narrow Range Level During Unbalanced Power Ramp Transients from $10 \% \mathrm{FP}$ to $15 \% \mathrm{FP}$ at a $5.0 \% \mathrm{FP} /$ minute Ramp Rate on Module \#1 Only. 


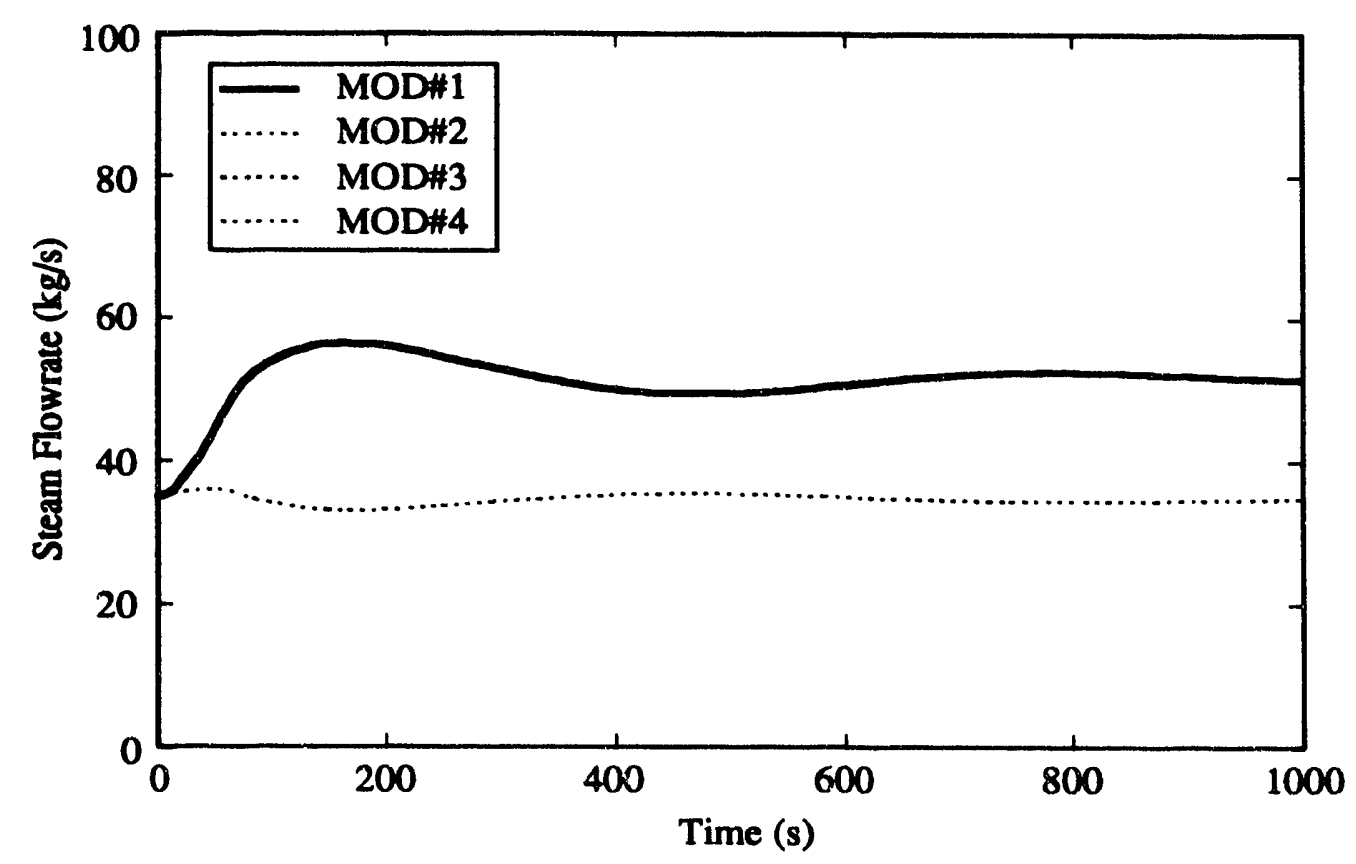

Figure 8.6.3-5 Steam Flowrate During Unbalanced Power Ramp Transients with PI Controller Tuned to Lower Feedback Control Gain $(2.0 \mathrm{~kg} / \mathrm{s} / \%)$.

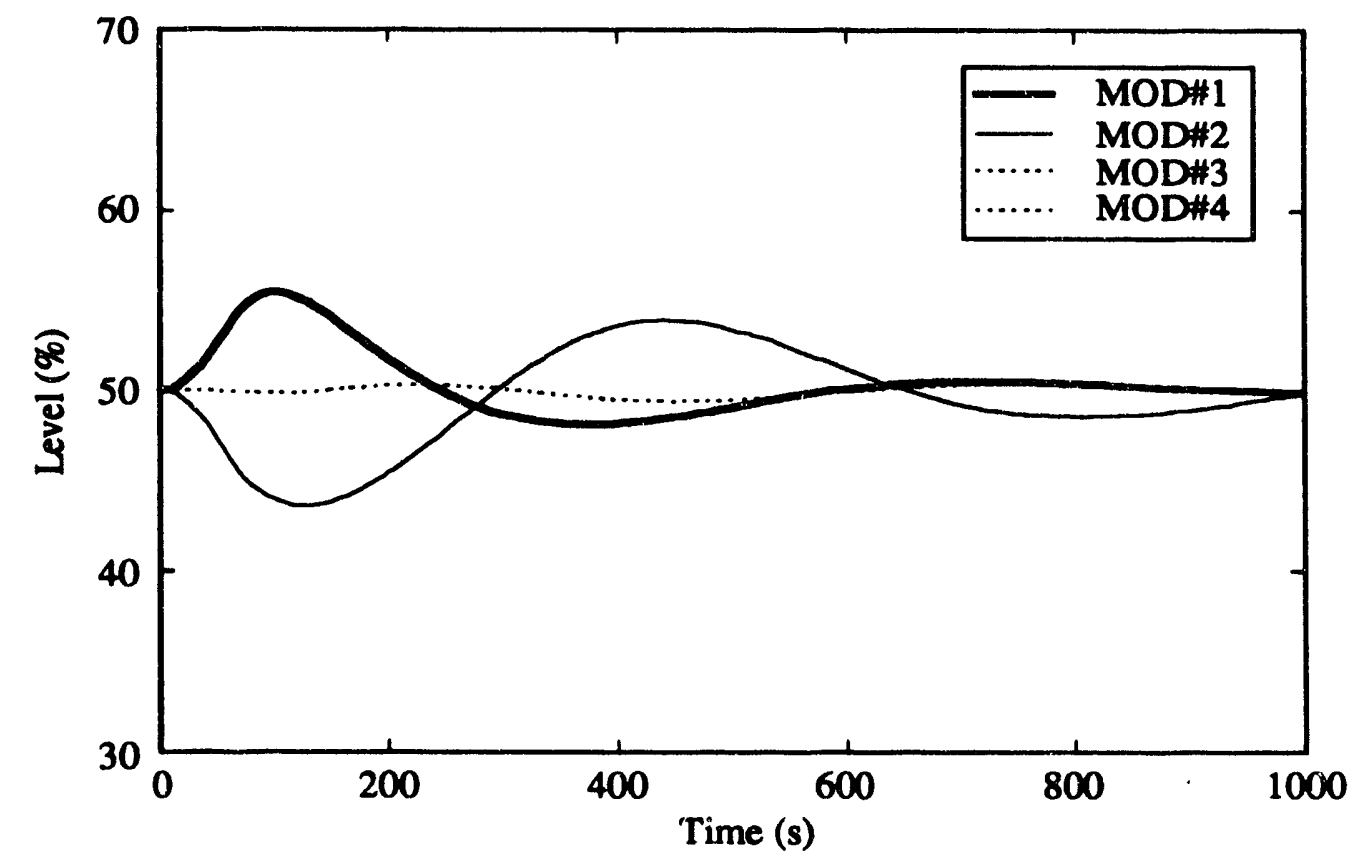

Figure 8.6.3-6 Steam Flowrate During Unbalanced Power Ramp Transients (Module $\# 1$ : from $15 \%$ FP to $20 \% \mathrm{FP}$; Module \#2: from $15 \% \mathrm{FP}$ to $10 \% \mathrm{FP}$; Module \#3 and \#4: held at $15 \%$ FP). 
shows level shrink. However, the compensated controller stabilized the level in both cases.

In summary, the proposed controller was successfully used for simulated steam generator level control in a PWR-type, multi-modular power plant and it showed reasonable performance for unbalanced load operation provided that it was tuned with a low feedback control gain. Additional simulations of multi-modular power plant transients are presented in Chapter Nine of this report.

\subsubsection{Sensitivity Study of the Compensation Parameters}

The controller developed in Section 8.5 of this report adopts inverse-response compensation techniques which are derived from a transfer function model. Even the simplified form of this model has many undetermined parameters which must be identified through numerical experiments. These studies are important because the controller's overall performance depends on the accuracy of its parameters.

For this sensitivity study, it was assumed that the compensator was designed based on an incorrect model. An error of $50 \%$ was either added or subtracted to all the correctly calculated parameters in Equations 8.3.1-4 to 8.3.1-6. The reference case was a balanced power ramp from $10 \% \mathrm{FP}$ to $15 \% \mathrm{FP}$ at a ramp rate of $5.0 \% \mathrm{FP} /$ minute. The feedback gain was set at $5.0 \mathrm{~kg} \cdot \mathrm{s}^{-1} / \%$ and the reset time at 343 seconds. It is important to recall that a conventional, single-element PI controller results in a growing oscillation in the steam generator level response if feedback gain is set greater than $4.0 \mathrm{~kg} \cdot \mathrm{s}^{-1} / \%$.

Figure 8.6.4-1 shows the time behavior of the steam generator level during the reference transient. Use of the controller with the inaccurate parameters resulted in stable control of the steam generator level. However, its performance was, as would be expected, degraded. In particular, when compared to the level response of the correct controller, it was more oscillatory. However, it did settle out quickly when a $-50 \%$ error was imposed on the compensator design. The reason is that, while shorter time constants do result in a more imperfect compensation, the erroneous compensating signal itself dies out more rapidly.

Table 8.6.4-1 summarizes the overall results. The incorrectly designed compensators increased IAE by $12 \%$ to $34 \%$ and ISE by $27 \%$ to $45 \%$. The maximum level error was also increased. This was especially true when a $-50 \%$ error was imposed on the compensator design. In that case, the maximum level error increased by $32 \%$ because of the imperfectness of inverse response compensation. However, the overall level trend is not that of a stable process.

Based on these results, it was concluded that the compensated controller that was designed here was robust to errors that occurred during system identification. Specifically, even if the compensation was based on a somewhat incorrect transfer function model, the controller stabilized level disturbances with its performance being only slightly degraded. 
Table 8.6.4-1

Control Performance Indices During

Power Ramp Transients with Incorrect Parameters

\begin{tabular}{|l|r|r|r|}
\hline Cases & \multicolumn{1}{|c|}{$\begin{array}{c}\text { IAE } \\
{[\% \text { s }]}\end{array}$} & \multicolumn{1}{c|}{$\begin{array}{c}\text { ISE } \\
{\left[\%^{2} \mathrm{~s}\right]}\end{array}$} & \multicolumn{1}{c|}{$\begin{array}{c}\text { Max Error } \\
{[\%]}\end{array}$} \\
\hline a) Exact Parameters (A) & 46.3 & 7.7 & 7.85 \\
b) + 50\% Error on Parameters (B) \\
$\begin{array}{c}\text { Difference (B-A)/A [\%] } \\
\text { c) - 50\% Error on Parameters (C) }\end{array}$ & 61.9 & 9.8 & 7.93 \\
Difference (C-A)/A [\%] & $(34 \%)$ & $(27 \%)$ & $(1 \%)$ \\
\hline
\end{tabular}

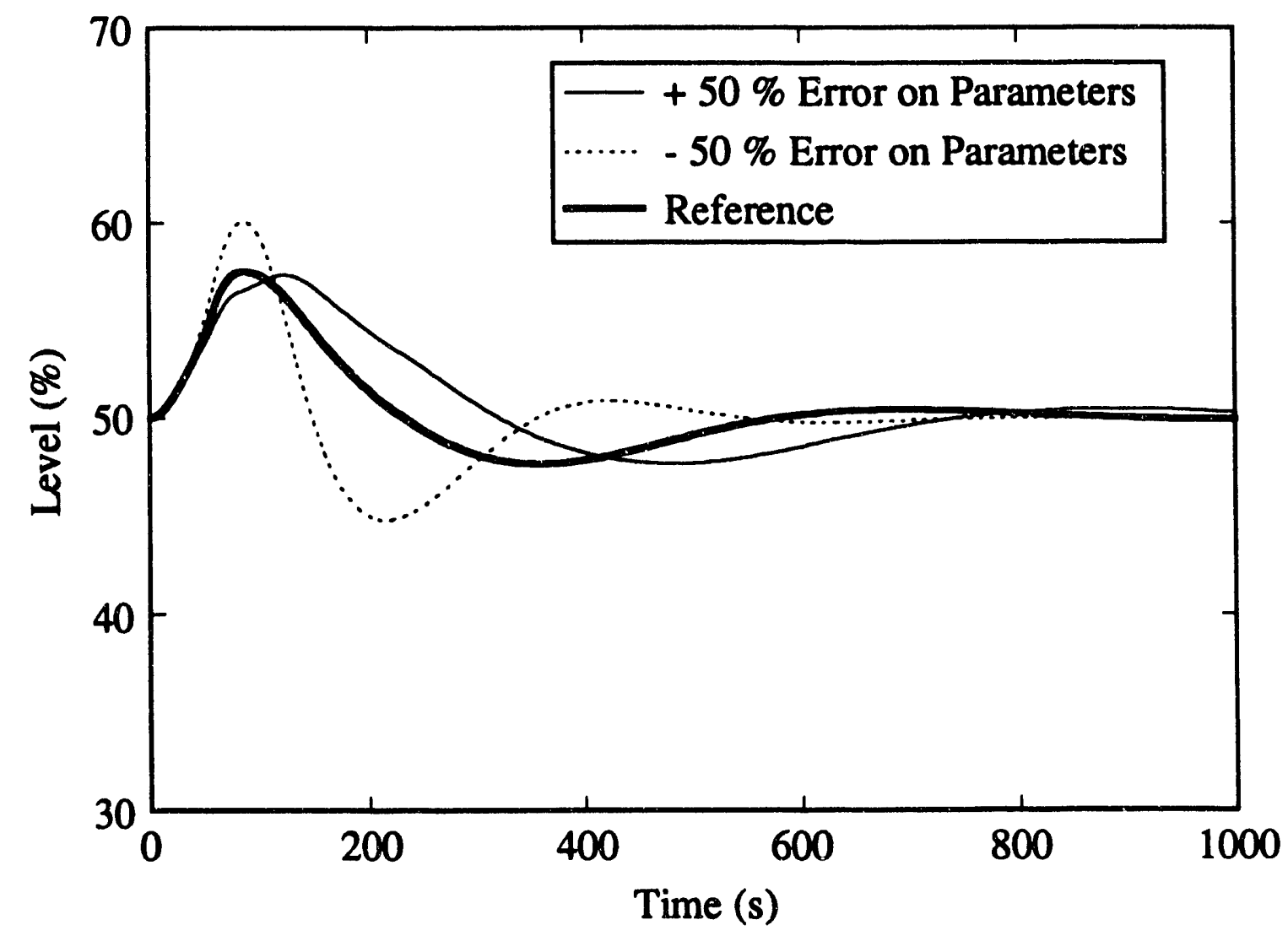

Figure 8.6.4-1 Steam Flowrate During Power Ramp Transients with Incorrect Compensation. 


\subsection{Assessment of Compensator-Based Steam Generator Level Controller}

A robust U-tube steam generator water level control system was proposed to replace current-generation, single-element PI level controllers. The proposed controller should be of benefit in the operation of U-tube steam generators that exhibit 'shrink and swell' effects. Improved steam generator level control performance is obtained through the use of a model-based compensation technique. Compensators for feedwater flowrate, steam flowrate, and primary coolant temperature were developed. These compensators were obtained from a simplified transfer function model for the purpose of offsetting inverse response behavior. Feedforward control was also applied to improve control performance. Finally, in order to avoid the need to use an uncertain measured steam flowrate, a steam flowrate estimator was designed. It uses the measured neutron power, steam generator pressure, and feedwater temperature to approximate the actual steam flowrate.

This compensator-based controller ensures stability at both low and high power even when tuned with a high feedback control gain. Also, because it does not use measured flowrates, it is robust to flowrate measurement errors. Simulation studies of various transients show that the use of this new controller greatly reduces the effect of inverse response and significantly improves the controllability of steam generator level. The proposed controller can be applied to the operation of steam generators in PWR-type, multi-modular power plants. In that case, continued use of a high feedback control gain causes oscillatory behavior because of steam flowrate oscillations between each steam generator and the main steam line header. The compensators do not correct for this because they do not immediately reflect changes in the steam flow split that occur during unbalanced maneuvers. However, if the proposed controller is tuned with a low feedback control gain, it displays acceptable control performance during operation with unbalanced loads. The proposed controller also exhibits robustness to errors in the parameters of the simplified model. 


\section{Control of Power in PWR-Type Multi-Modular Reactors ${ }^{(1)}$}

This chapter reports the development of closed-loop digital strategies for the control of wutronic power in both individual PWR-type multi-modular reactor modules and in the piant as a whole. The goal of these strategies is to achieve a balance between the electric demand as requested by the load dispatcher and the possible need to distribute that load unevenly among the plant's modules. For this purpose, a hierarchical architecture was selected with the plant power controller being the uppermost tier and the individual module controllers being the lower tiers. Included in this chapter are a review of PWR power control methods relevant to multi-modular plants, a description of the proposed controller, and the results of simulation studies in which the controller was evaluated.

\subsection{Statement of Problem}

The operation of a multi-modular power plant should permit unbalanced operation in which each module operates at a different power level. Specifically, should one module be shutdown, the others can continue to supply steam to the turbine generator and therefore maintain a relatively high capacity factor for the plant as a whole. In fact, this mode of operation will probably be used to conduct refuelings without the need for taking the entire plant off-line. This in turn means that each module in a multi-modular plant will be routinely operated at a different load factor so as to stagger the times at which individual cores must be refueled. This approach to facility operation creates a complex control problem because each power module supplies steam to a common header and the steam flowrate from each individual module depends on both the hydraulic conditions in that module and on the pressure in the common steam line header. Thus, conditions in each steam generator and the common steam line header must be coordinated to allow each power module to supply an assigned fraction of the total plant load.

If conventional control methods (PWR-type analog controllers and licensed operator supervisory control) are to be employed, then there will be a significant number of control signals and operating parameters to be monitored and processed. Also, each power module will require its own complete control room with the usual complement of operators. The resulting personnel and training costs could make such an approach to multi-modular power plant operation prohibitively expensive. New control techniques that rely on digital technology can avoid these costs by permitting use of a single centralized control room.

At present, the direct digital control of nuclear power plants is not practiced in the United States. Regulations require that any system that is needed for the shutdown of the plant or that is associated with safety be subject to exhaustive quality control tests. The result is that only proven technologies, usually hard-wired analog devices, are employed. This situation is slowly changing as is illustrated by the installation of digital feedwater systems in several nuclear, llants and by digital control experiments conducted on the Massachusetts Institute of Technology's Research Reactor, MITR-II. Also, as described in Chapter Three of this report, one method for the control of neutronic power via a digital computer has been licensed by the U.S. Nuclear Regulatory Commission for use on the MITR-II. If properly designed and implemented, a digital control system will enhance safe

(1) This chapter is based on work performed by Keung Koo Kim under the supervision of Professor John E. Meyer, Professor David D. Lanning, and Dr. John A. Bernard. 
operation. Nevertheless, concerns exist about the digital option. One of the advantages to multi-modular power plants is that those plants can be made passively safe. This should alleviate concerns about the performance of the control system and hence perhaps make automatic control strategies more acceptable to both licensing authorities and the public.

The research presented here concerns the control of power in PWR-type multimodular plants with efforts focused on the operation of both individual modules and the plant as a whole. The objective is to supply the requisite total steam flowrate while both observing constraints on the use of each module and minimizing perturbations to those modules.

\subsection{Control of Power and Temperature in PWRs}

Control principles and strategies that have proven to be effective in PWRs can be utilized as the basis of a power control system for a PWR-type multi-modular power plant. Presented here is a summary of the approach used for the coordinated control of thermal power and primary coolant temperature in pressurized water reactors.

\subsubsection{PWR Control Strategies}

Control of global (total) reactor thermal power and primary coolant temperature for power operation above $15 \%$ of nominal full power can be accomplished either manually or by an analog automatic controller. The latter uses both the current and desired power as well as temperature dynamic behavior to generate an appropriate control action. The actuator signal is the control rod speed and direction. The analog controller is designed to handle the following PWR operational transients without causing a reactor trip:

1. A ten percent $( \pm 10 \%)$ step change in demanded load,

2. A five percent per minute $( \pm 5 \% / \mathrm{min})$ ramp change in demand, and

3. A one hundred percent (100\%) step electrical load decrease with steam dump operation.

For operation above $15 \%$ of rated power, commercial PWRs are normally controlled using the average primary coolant temperature, $T_{\text {ave }}$. Various reactor coolant temperature programs are used depending on the needs of the plant in question. The two extremes are the 'constant $T_{\text {ave' }}$ and 'constant steam pressure' programs. The merits of each are summarized here.

A constant average temperature is desirable because it keeps the reactor coolant system water volume nearly constant thereby minimizing requirements on pressurizer size. This approach also reduces the need for reactivity control because the changes in moderator temperature are reduced. The major disadvantage to this mode of control is that the secondary plant's steam pressure will vary significantly as reactor neutron power is changed from $15 \%$ to $100 \%$. Figure 9.2.1.1 illustrates a typical constant average temperature program. 


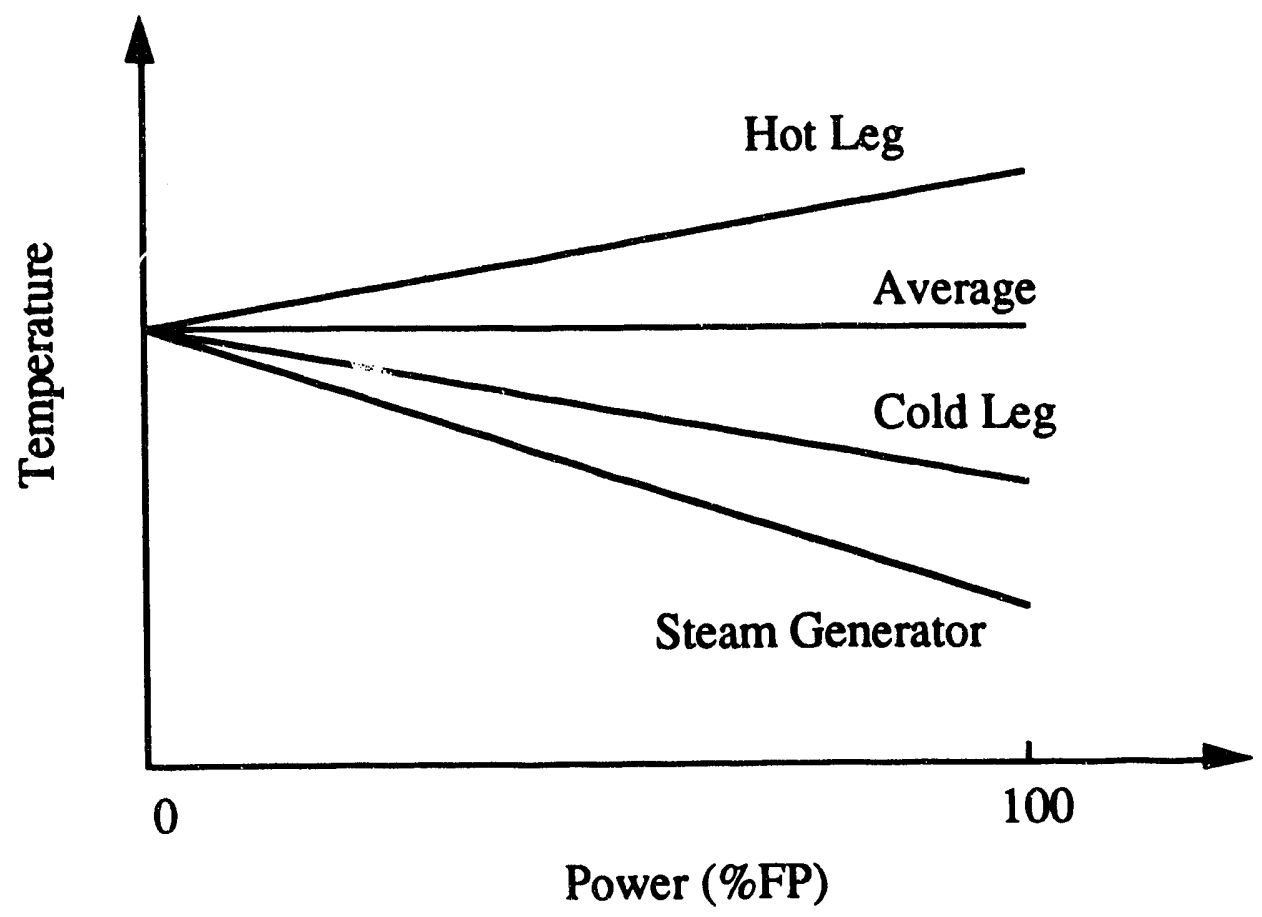

Figure 9.2.1-1 Constant Average Temperature Control Program.

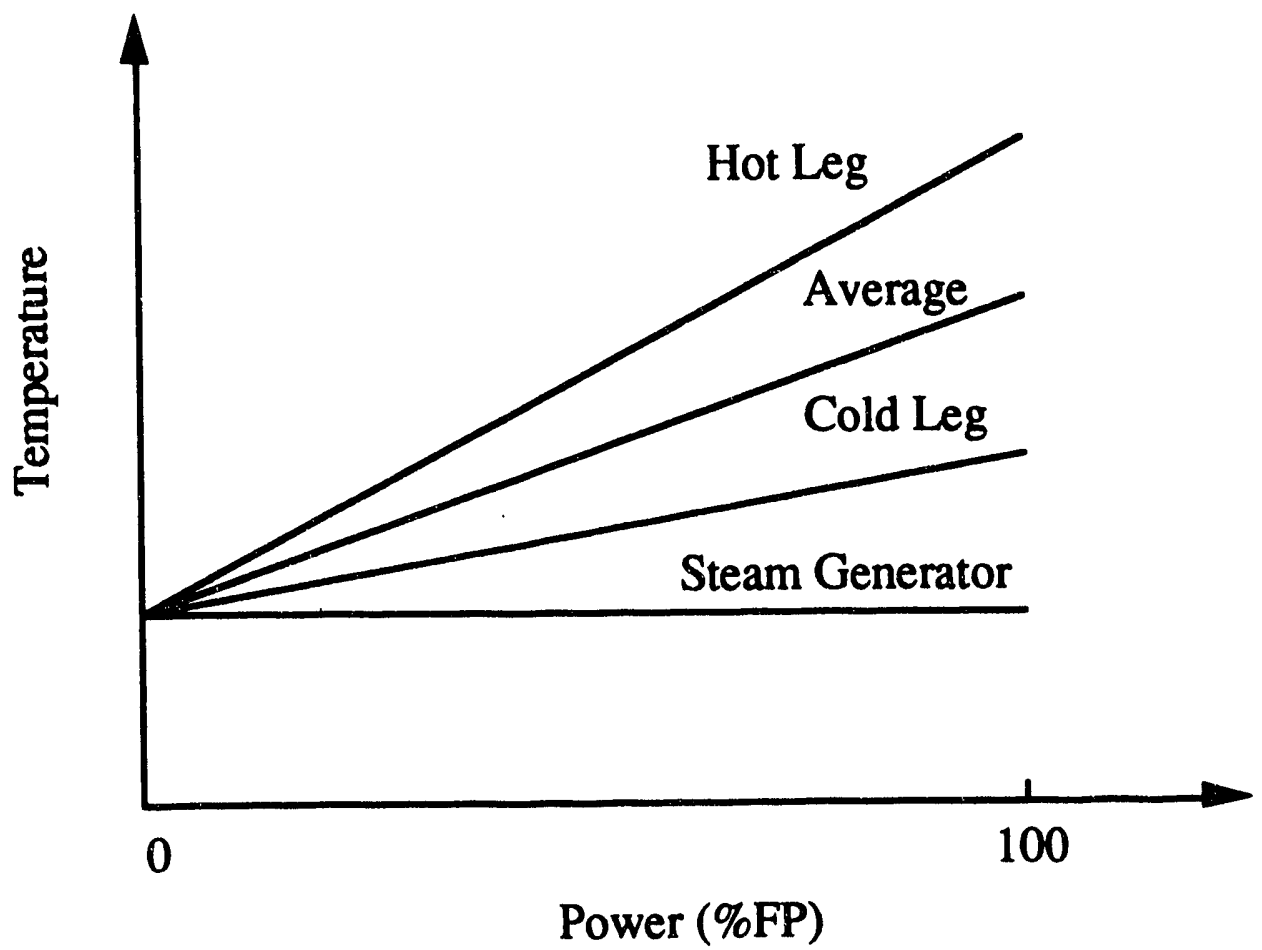

Figure 9.2.1-2 Constant Steam Pressure Control Program. 
A constant steam pressure program optimizes the design of the secondary plant, but results in a large range for the average primary coolant temperature. This in turn means that the control system must be able to handle the large reactivities that result from shifts in the moderator's temperature. Also, sizing of the pressurizer becomes complicated because of the need to compensate for the widely varying reactor coolant system's water volume. Finally, to keep the primary coolant from reaching the undesirable condition of bulk boiling in the core, an upper limit must be placed on the allowed average temperature. Figure 9.2.1-2 depicts a typical constant steam pressure program.

A compromise between the above two extremes in temperature programs is the 'sliding $\mathrm{T}_{\text {ave }}$ ' program which combines the 'constant $\mathrm{T}_{\text {ave' }}$ and the 'constant steam pressure' programs to optimize the pressurizer size, secondary plant design, reactivity control, and the temperature rise across the core. The sliding $T_{\text {ave }}$ program was adopted in this research.

\subsubsection{PWR Analog Controller Control Logic}

Inputs to a PWR's analog controller are a reference primary coolant temperature and the first stage turbine impulse pressure. The latter is linearly proportional to turbine load and is used to determine the appropriate reference temperature. The output from the control system is a desired control rod speed and direction. Figures 9.2.2-1 and 9.2.2-2 are simplified diagrams of the logic that is employed. There are two error signals. The first is a comparison of the auctioneered high $\mathrm{T}_{\mathrm{av}}$ obtained from the narrow range temperature sensors with the reference temperature obtained from the load map. This is inherently slow because of both the finite time for heat transfer and the travel time of the primary coolant through the primary loop. The second error signal is a power rate mismatch that is obtained by comparing the rates-of-change of turbine power and neutronic power. This signal anticipates changes in the average temperature and thereby provides a quicker response to changes in load. The power rate mismatch signal is multiplied by a non-linear or variable gain which converts it to an equivalent temperature error. It is then summed with the average temperature error and sent to the control rod speed and direction program. (Note: The non-linear gain unit causes larger changes in load to have larger effects. Specifically, it imposes a higher gain at low power levels and a lower gain at high power thereby enabling the power rate mismatch error channel to provide adequate control at low power levels as well as stable operation at high power.)

The two error signals complement each other. Because the temperature error is inherently slow, it is desired to have the power rate mismatch signal anticipate changes in average primary coolant temperature. However, the power rate error is based on a rate-ofchange, and therefore will be zero during steady-state operations. Hence, it is possible to have a turbine/core power mismatch with a power rate mismatch of zero. The discrepancy in demanded and actual power would then be controlled by the temperature error. Thus, during steady-state operation, the temperature error signal provides the fine control to maintain the average temperature within the desired band and, during load changes, the power rate mismatch signal provides a quick response.

The rod speed and direction program determines a control rod bank speed and direction based on the magnitude and sign of the total error signal. Rod speed is limited to between 8 and 72 steps per minute, with one step equal to $1.5875 \mathrm{~cm}$. The lock-and-latch 


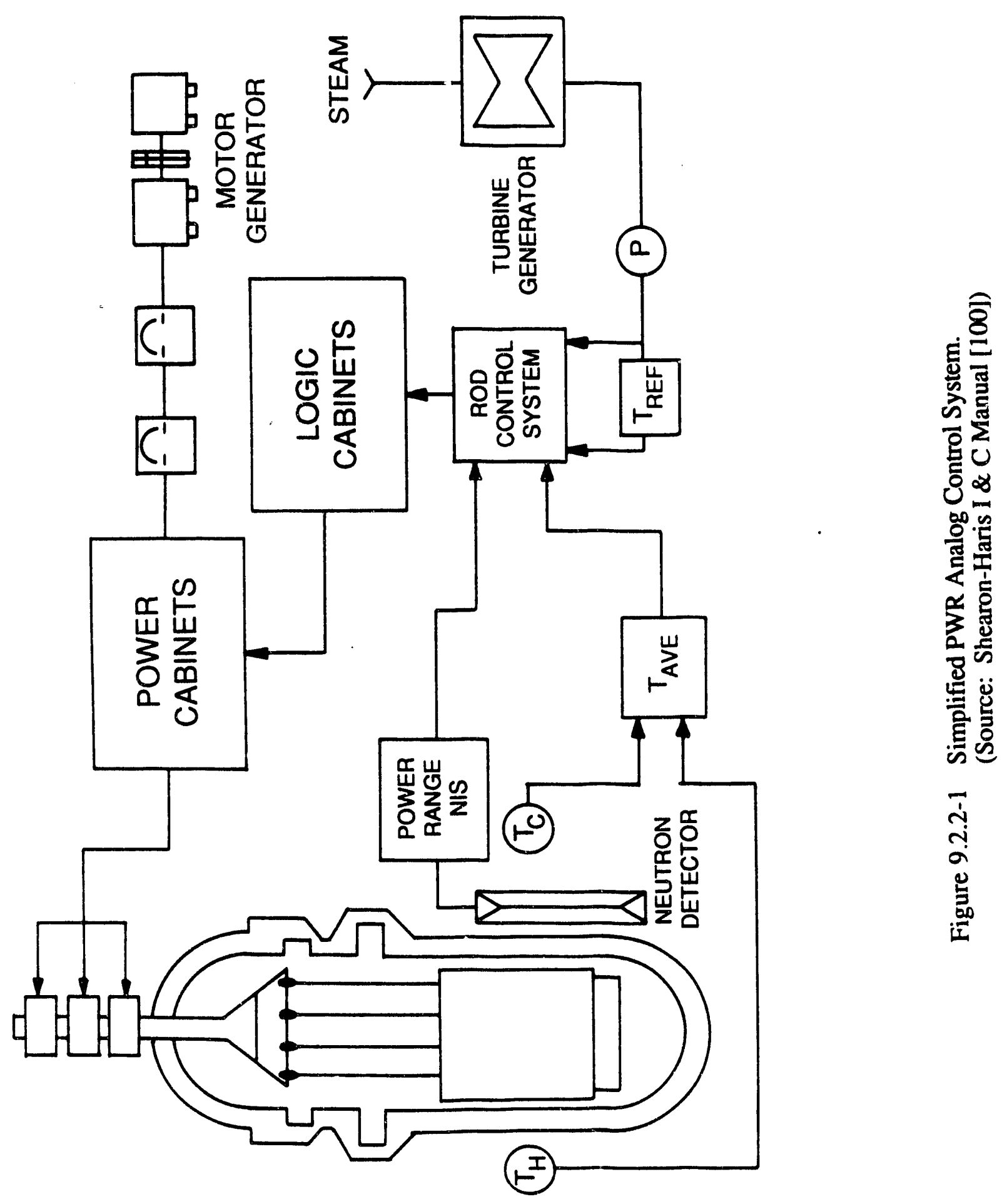




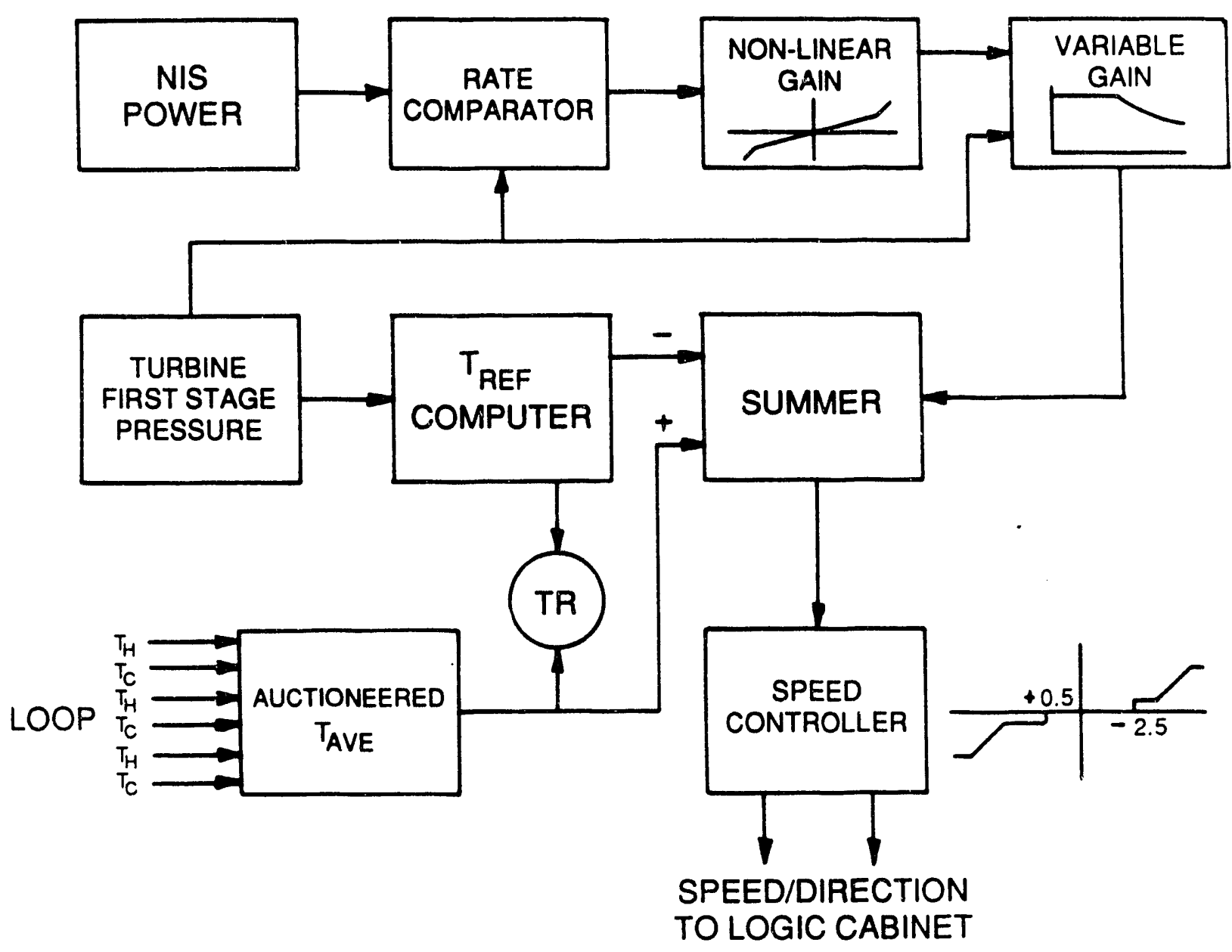

Figure 9.2.2-2 Simplified PWR Rod Control System.

(Source: Shearon-Haris I \& C Manual [100]) 
control rod drive mechanisms for Westinghouse PWRs require approximately 0.8 seconds to move a bank of control rods one step. The rod speed and direction unit also contains a deadband to prevent system oscillation, thereby reducing unnecessary rod motion due to signal noise and prolonging the life of the control rod drive motors. Figure 9.2.2-3 shows a typical PWR control rod speed program. Rod motion begins at $\pm 0.56{ }^{\circ} \mathrm{C}$ at eight steps per minute (spm). The lockup of $\pm 0.28^{\circ} \mathrm{C}$ prevents system oscillation. At an error of $\pm 1.67^{\circ} \mathrm{C}$ error, the speed begins to increase linearly, so that at an error of $\pm 2.83^{\circ} \mathrm{C}$ the rod speed is at its maximum, $72 \mathrm{spm}$. The polarity of the error signal determines the direction of rod motion. If $\mathrm{T}_{\text {ave }}$ is less than $\mathrm{T}_{\mathrm{ref}}$, the rod motion is outward in order to increase power and primary coolant temperature. Conversely, if $T_{\text {ref }}$ is less than $T_{\text {ave, }}$ the rod motion is inward in order to reduce power and primary coolant temperature. Once the required rod movement has been determined, the rod speed and direction signals are sent to the control rod logic cabinet, which selects the proper control rod bank(s) to be moved. Current PWR technical specifications require a fixed overlap between control rod banks to prevent axial flux peaking. This overlap is typically half of the total axial core height. Before the actuator signal is finally implemented, a series of control rod interlocks that block outward rod motion under certain plant conditions are checked. These are designed to prevent an uncontrolled power escalation that could lead to a reactor trip. They include:

1. High (typically 103\%) power level,

2. Low power level to prevent automatic control below $15 \%$ powcr,

3. Control rod bank $D$ full-out limit, and

4. Over-temperature differential temperature and over-power differential temperature limits.

The over-temperature and over-power differential temperature control rod interlocks protect the core from conditions of departure from nucleate boiling (DNB) by blocking outward rod motion before the over-temperature and over-power differential temperature trip setpoints are reached. The - points are calculated from the average temperature, its rate of change, the power level, the primary system pressure, the primary coolant flow, and the measured axial flux difference.

\subsection{Operation of PWR-Type Multi-Modular Power Plants}

The defining characteristic of a multi-modular plant is that of interdependency. This occurs because each module's steam generator supplies steam to a single turbine via a common steam line header. Hence, a change in the power of any given module's reactor affects not only its own coolant temperature, but also those of the other reactors. It is this interdependence that makes it challenging to design a power control system for a multimodular plant.

\subsubsection{Operating Characteristics}

As noted previously, one of the requirements imposed on multi-modular power plants is that it be possible to operate under conditions of unbalanced loads. That is, each 


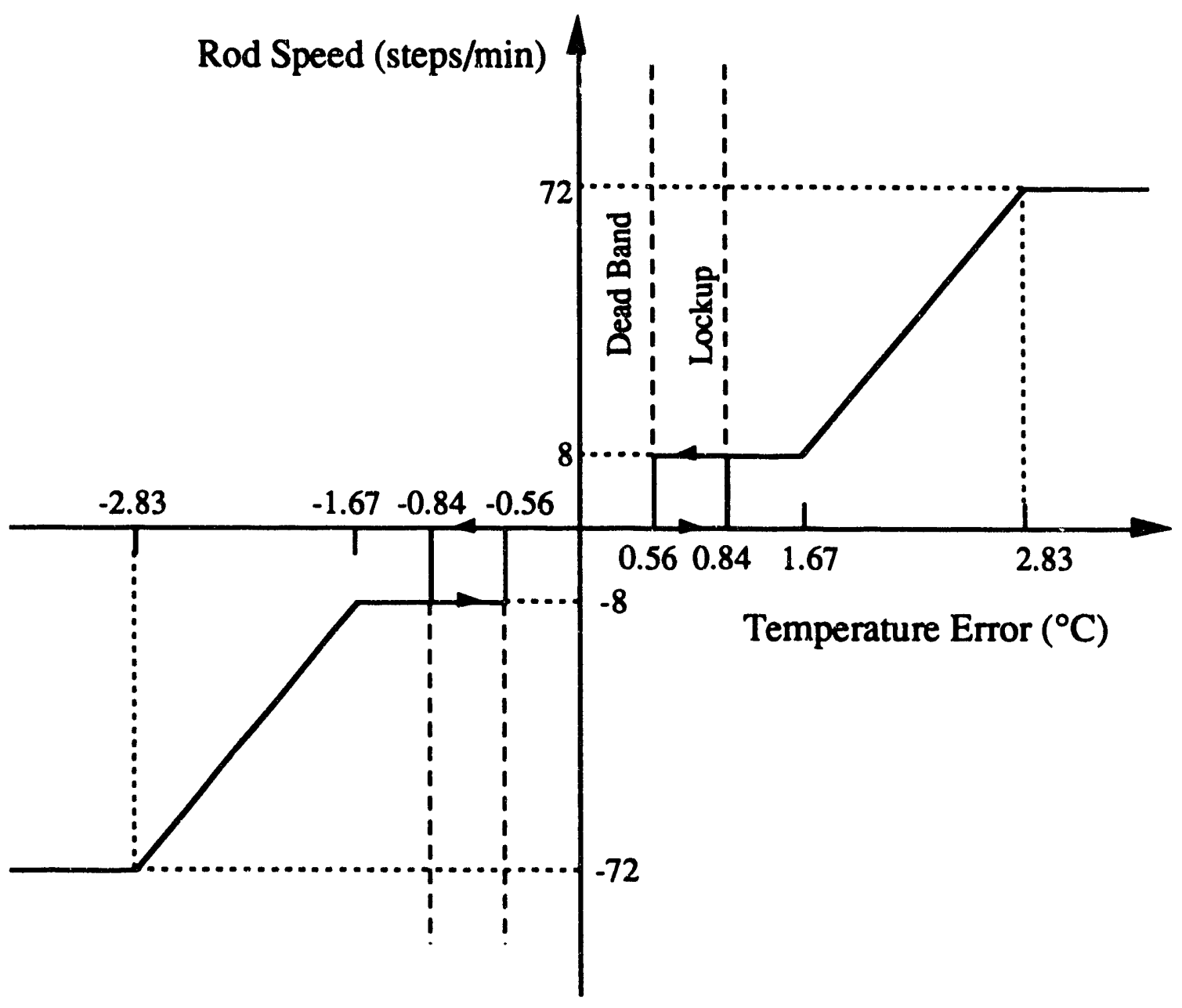

Figure 9.2.2-3 Typical PWR Control Rod Speed Program [101]. 
reactor is at a different power level. For reactors that are ultimately connected to a common steam header, this can only be achieved if each has a different average primary coolant temperature. This is illustrated by the following analysis which assumes ideal heat transfer conditions. The heat produced from fission is given by the expression:

$$
\begin{aligned}
Q & =\dot{\mathrm{m}} c_{\mathrm{p}}\left(\Delta \mathrm{T}_{\text {core }}\right) \\
& =\dot{\mathrm{m}} c_{\mathrm{p}}\left(\mathrm{T}_{\mathrm{H}}-\mathrm{T}_{\mathrm{c}}\right)
\end{aligned}
$$

where $Q$ is the rate of energy production, $\dot{m}$ is the mass flowrate of the primary coolant, $c_{p}$ is the heat capacity of the primary coolant, $\Delta T_{\text {core }}$ is the temperature rise across the core, $\mathrm{T}_{\mathrm{H}}$ is the hot leg temperature, and $\mathrm{T}_{\mathrm{c}}$ is the cold leg temperature. A similar relation can be written for the heat transferred from the primary to the secondary via the steam generator. It is:

$$
\mathrm{Q}=\mathrm{UA}\left(\mathrm{T}_{\mathrm{ave}}-\mathrm{T}_{\mathrm{sg}}\right)
$$

where $U$ is the heat transfer coefficient, $A$ is tube bundle's surface area, $T_{a v e}$ is the average primary coolant temperature, and $\mathrm{T}_{\mathbf{s g}}$ is the saturation temperature of the steam generator's secondary side. Conditions in the steam generator are saturated and steam generator pressure therefore varies directly with $\mathrm{T}_{\mathrm{sg}}$. In order for steam to flow from each module, the pressure in each steam generator must be equal. Hence, because of saturated conditions, the temperature of each on-line steam generator must be the same. This in turn means that it will only be possible for each module to operate at a different power level if the average primary coolant temperatures are all different. That is:

$$
\mathrm{T}_{\mathrm{ave}}=\mathrm{Q} / \mathrm{UA}+\mathrm{T}_{\mathrm{sg}}
$$

The above analysis neglects the effect of losses in the steam piping which are proportional to the square of the steam flowrate. Thus, instead of specifying that each steam generator have the same pressure, a more correct statement would be to require that the pressure at each module's inlet to the main steam header be equal. Pressures in the individual generators will vary slightly depending on the length and curvature of the associated steam line.

As a practical matter, the need to operate each reactor at a different average primary coolant temperature results in a rather complicated temperature-power control program. This is illustrated in the following analysis where it is assumed that each module follows a sliding $\mathrm{T}_{\text {ave }}$ control program. Consider a multi-modular plant that consists of two modules. Figure 9.3.1-1 shows $\mathrm{T}_{\mathrm{ave}}$ and steam generator saturation pressure as a function of module power. If both modules are operated at $Q_{1}$, the pressure in both steam generators is $\mathbf{P}_{\text {sg, }}$ and that of the main steam line header is $\mathbf{P}_{\mathrm{h}, 1}$. However, suppose that modules \# . and $\$ 2$ are operated at two different power levels, $Q_{1}$ and $Q_{2}$ respectively. The situation is now quite different. Module \#1 is operated to follow the original sliding $T_{\text {ave }}$ control program. Hence, its primary coolant temperature is $T_{\text {ave, } 1}$ and its steam generator and main steam line header pressures are $P_{s g, 1}$ and $P_{h, 1}$ respectively. If module \#2 were also operated in accordance with the original program, then its steam generator pressure and coolant temperature would be $\mathrm{P}_{\mathrm{sg}, 2}$ and $\mathrm{T}_{\mathrm{ave}, 2}$ respectively. However, such a situation would be unstable because $P_{\mathrm{sg}, 2}$ exceeds $P_{\mathrm{sg}, 1}$. Hence, steam flowrate from module \#1 


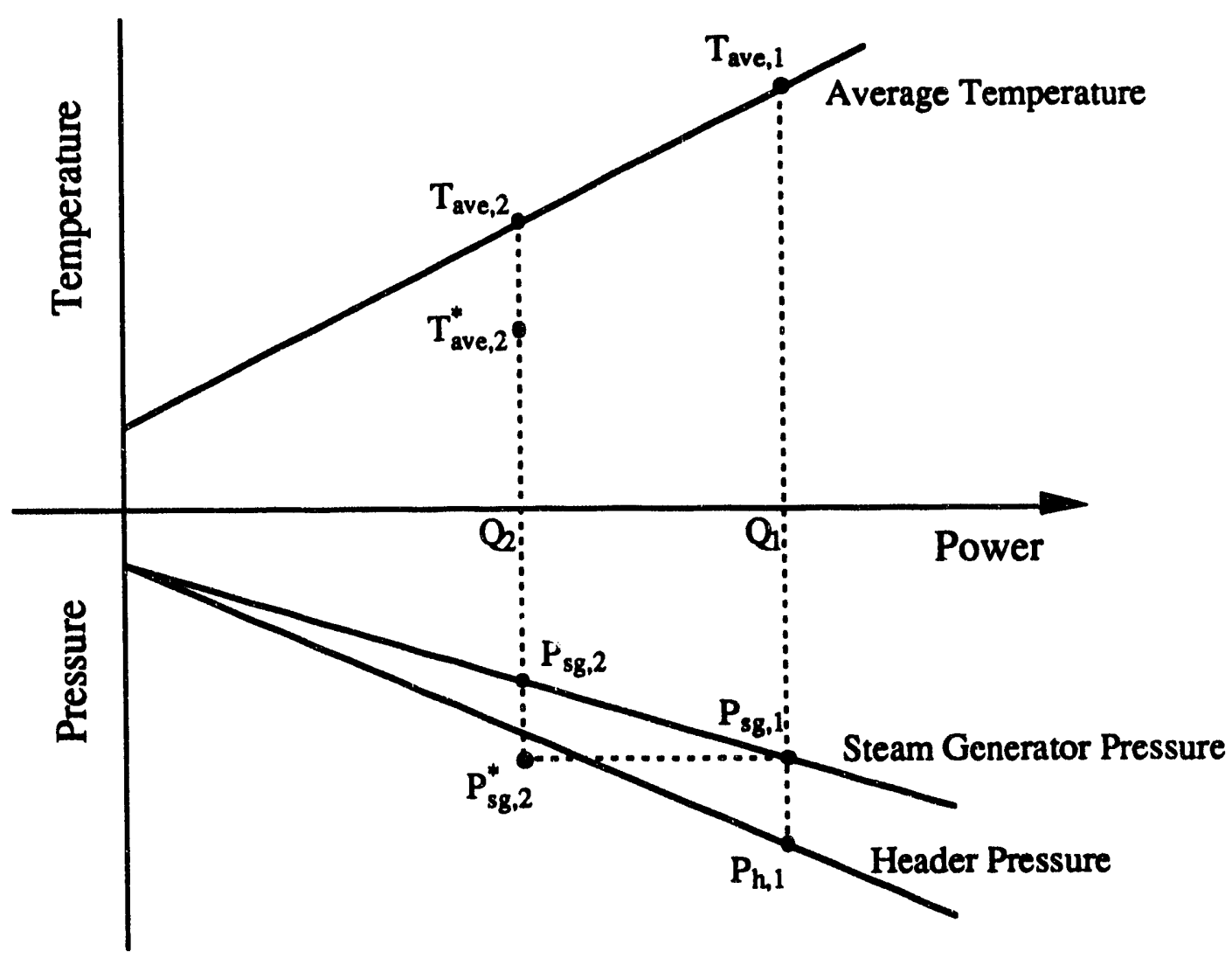

Figure 9.3.1-1 Operation under Sliding Average Temperature Control Program with Unbalanced Loads. 
would be cut off and module \#2's power would rise in response to the load demand. After some oscillatory behavior, it and module \#1 would eventually become equal in power. In order to avoid this situation, the pressure in module \#1's steam generator should be $P_{s g}^{*}$. (Note: Possible differences in piping losses are again ignored.) As a result, module $\$ 2$ 's temperature can not be controlled to maintain $\mathrm{T}_{\mathrm{ave}}, 2$. Rather, as is shown by Equation (9.3.1-3) it should be controlled to some lower value, $T_{\mathrm{ave}, 2}^{*}$ because $\mathrm{P}_{\mathrm{Sg}, 2}^{*}$ and therefore the corresponding steam generator temperature are less than the values specified by the original sliding $\mathrm{T}_{\text {ave }}$ program for a module operated at power level $\mathrm{Q}_{2}$. Thus, three conclusions can be drawn. First, the original sliding $\mathrm{T}_{\text {ave }}$ control program can only be applied to the highest-power reactor in a multi-modular power plant. Second, the primary coolant temperatures of the lower-power modules depend on both their own module's power and on that of the most heavily loaded module. Third, the primary coolant temperature of the highest-power module depends on only that module's power.

Figure 9.3.1-2 is a load map for a reactor in a multi-modular plant. Given module power and the power of the highest-power module, it can be used to determine the appropriate value of the average coolant temperature. Use of the figure requires some practice. The thick black line is that of the highest-power module. The other lines are for the lower-power modules with each representing a power level for the highest-power module. As an illustration, suppose there are two modules in a given plant. The highestpower one is to be operated at $60 \% \mathrm{FP}$ and the lower-power one at $40 \% \mathrm{FP}$. For a module power of $60 \%$, the thick black line gives an average coolant temperature of about $302{ }^{\circ} \mathrm{C}$. In order to determine the temperature for the other reactor, we must first select the appropriate curve. The one with the cross marks $(x)$ is correct because it gives the temperature of the lower-power modules as a function of module power for the condition that the highest-power module is at $60 \% \mathrm{FP}$. Thus, for a $40 \%$ module power, the temperature of the lower-power reactor should be about $298{ }^{\circ} \mathrm{C}$. Figure 9.3.1-3 gives the corresponding information for steam generator pressure.

\subsubsection{Operational Modes and Operating Strategies}

Electric power demand varies over the day with the greatest demand occurring during the daylight hours and the least during the late night and early morning hours. Most electric grids contain a mix of base-loaded and load-following power sources so as to be able to supply this cyclic load with efficiency and at low cost. In general, nuclear plants constitute the base-load component with fossil-fueled ones used for load following. This has made economic sense because nuclear fuel costs are much less than those of their fossil counterparts. However, as the amount of installed nuclear-generating capacity increases, the capability for nuclear plants to operate in a load-following or frequency-control mode will become increasingly important. If a nuclear power plant is assigned to daily loadfollowing, the power demand variation is so slow that plant output can be determined by the operator. This operating approach is therefore almost the same as base-load operation and is referred to as 'reactor-leading.' However, if a nuclear power plant is assigned to a fast-varying type of load-following such as grid frequency control, then plant operation is controlled by the turbine. This approach is termed 'turbine-leading.'

In this research, two operational modes were selected and a requirement was imposed that it be possible to transit smoothly between these modes. Those selected were: 


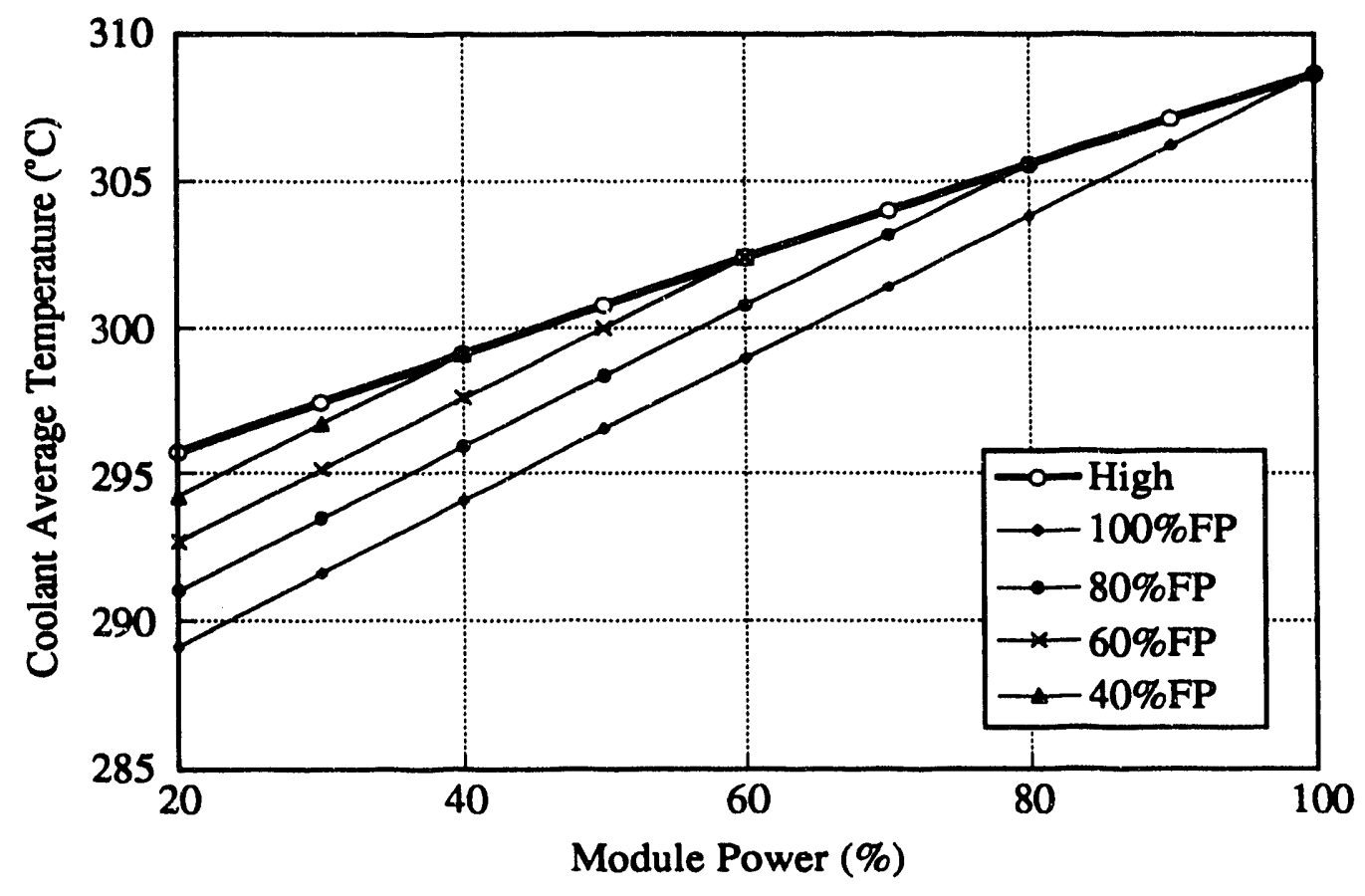

Figure 9.3.1-2 PWR-Type Multi-Modular Power Plant Operation Map. (Steady-State Average Primary Coolant Temperature)

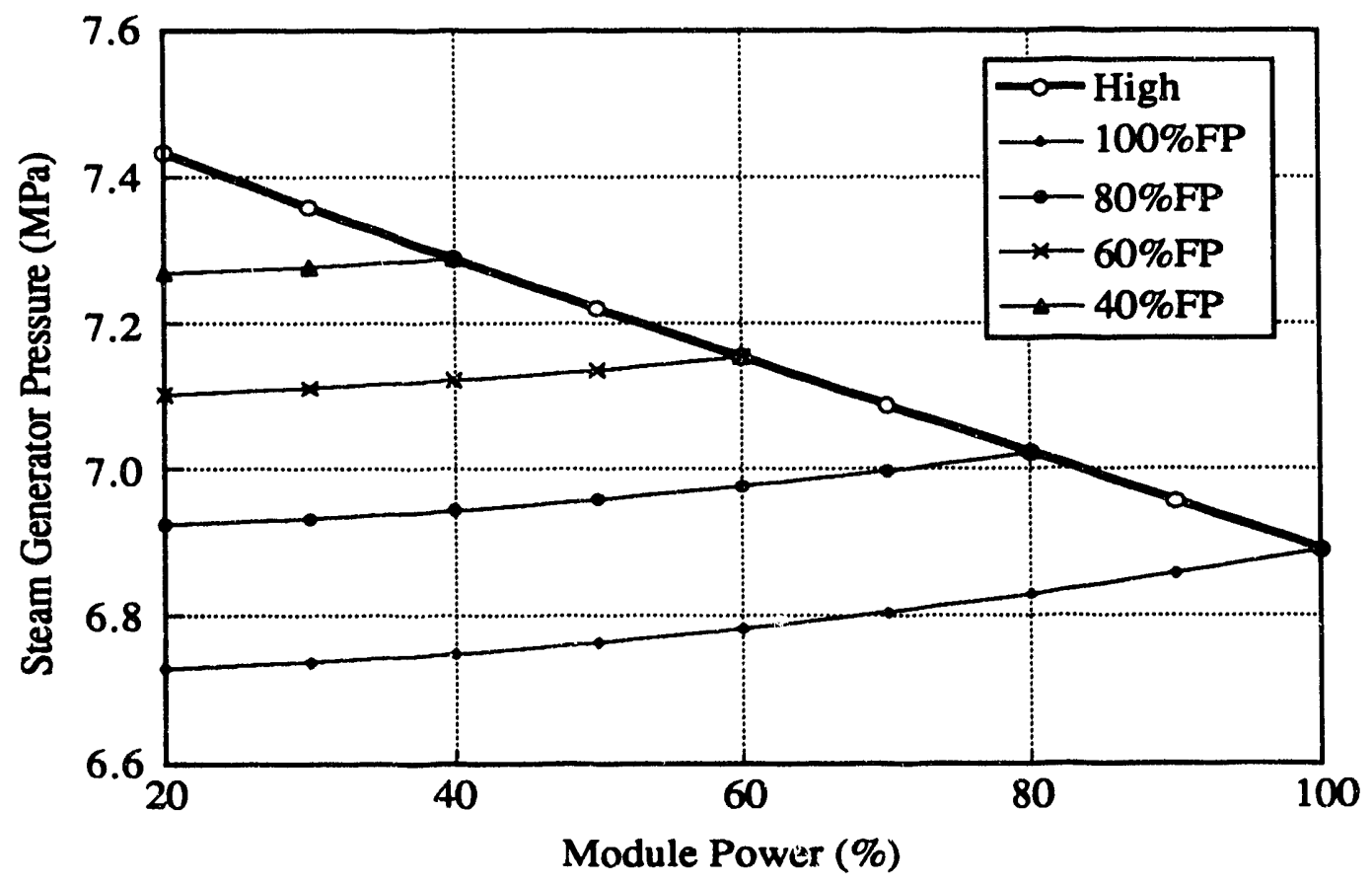

Figure 9.3.1-3 PWR-Type Multi-Modular Power Plant Operation Map. (Steady-State Steam Generator Pressure) 
1. Specified Power Demand Mode: In this mode, the operator inputs a forecasted demand and the power controller adjusts the reactor's output to maintain the operator-specified setpoint. This operational mode is preferred whenever the plant is allocated to base-load operation.

2. Arbitrary Power Demand Mode: In this mode, the turbine power follows the demanded load as specified by the load dispatcher and the power controller adjusts the reactor's output accordingly. This operational mode is preferred whenever the plant is assigned to either grid network frequency-control or fastchanging load-following.

The principal difference between these two operational modes is that future load changes are known if operating in the specified power demand mode but not if in the arbitrary power demand mode. As a result, two different plant power control system designs are needed.

Within each operational mode, several operating strategies are possible. These are used to distribute any change in the demanded load among the various modules. Four such strategies are under consideration for the PRISM multi-modular plant [102] and those four were all examined here. They are:

1. Equal Load: All available modules share the load equally and hence pick up the same fraction of any change in load.

2. Equal Change: The available modules are operating under unbalanced load conditions with each supplying a different fraction of the total demand. Changes in demand are divided by the number of available modules and each picks up the same change in load.

3. Extreme First: The available modules are operating under unbalanced load conditions with each supplying a different fraction of the total demand. The lowest power module in the case of an increase, or the highest in the case of a decrease, is changed first, then the next, etc., until the desired target is reached.

4. Preset Value: The intent here is to permit swing operation in the presence of a constant load demand. The available modules may or may not initially be operating under conditions of unbalanced loads. The reactor operator alters the setpoint of the selected module and the power levels in the other available modules are shifted automatically using either an 'equal change' or 'extreme first' strategy. The result is that the selected module's power is changed to the desired value and the other modules compensate so that there is no net change in the plant's output (i.e., the turbine load remains constant). The preset value strategy also allows changes of power in which a given module is excluded.

The selection of the appropriate operating strategy is done by the reactor operator through the plant power controller. 


\subsubsection{Control of Power and Temperature}

The operation of a multi-modular facility requires the coordinated control of power and temperature in each on-line reactor. Accordingly, it is useful to examine the control of these parameters in current-generation pressurized water reactors. For purposes of illustration, the PWR in question is taken as having the same power rating as a modular reactor and also as having a strong negative temperature coefficient of reactivity, on the order of $1.4 \cdot 10^{-4} \Delta \mathrm{K} / \mathrm{K} / \mathrm{C}$. The examples given in the discussion that follows have been idealized to illustrate the differences that result from a change in turbine valve position as opposed to movement of the control rods.

\subsubsection{Adiustment of Turbine Valve Position - Power Change}

An increase in power can be initiated by opening the valve that admits steam to the turbine. The effect of this action is to lower the secondary side pressure in the steam generators. However, because these steam generators operate under saturated conditions, a decrease in pressure is also reflected as a decrease in temperature. This in turn means that the temperature of the primary coolant that leaves the steam generator (i.e., the cold leg temperature) also drops. The entry of this cooler water into the reactor core inserts positive reactivity because of the negative temperature coefficient. As a result, the fission rate increases and so does the temperature of the coolant exiting the core. That is, the hot leg temperature rises with the result that additional energy is transferred to the secondary system. Steam generator pressure and temperature therefore increase until steam production again equals demand. The net effect is that both reactor power and the difference between the hot and cold leg temperatures have been increased. Also, steam generator pressure and temperature stabilize somewhat below their original values. However, the average primary coolant temperature remains constant. Thus, power has been adjusted without affecting temperature. (Note: In current-generation PWRs, the automatic controller would withdraw the control rods in order to raise temperature in accordance with the sliding $T_{\text {ave }}$ program.) Figure 9.3.3.1-1 illustrates the above response of a PWR to an increase in demanded load.

\subsubsection{Adjustment of Control Rod Position - Temperature Change}

Adjustment of a PWR's average coolant temperature can be achieved through movement of the control rods. Specifically, withdrawal of the control rods will cause an increase in the fission rate and hence a rise in the temperature of the water that exits the core. That is, the hot leg temperature rises with the result that additional energy is transferred to the secondary system. However, unlike the situation described in Section 9.3.3.1 of this report, the steam supply is already equal to that demanded because there has been no change in the position of the valve that admits steam to the turbine. Hence, the effect of the additional energy is to raise steam generator temperature and pressure. This in turn causes the temperature of the primary coolant that leaves the steam generator (i.e., the cold leg temperature) to rise. Upon entering the core, this hotter coolant inserts negative reactivity that offsets the positive reactivity that was the consequence of the rod withdrawal. Reactor power therefore returns to its original value. The net effect is that there has been no change in either the reactor power or the temperature rise across the core. However, both the hot and cold leg temperatures have risen with the result that the reactor is now operating at a higher average coolant temperature. Steam generature temperature has also 

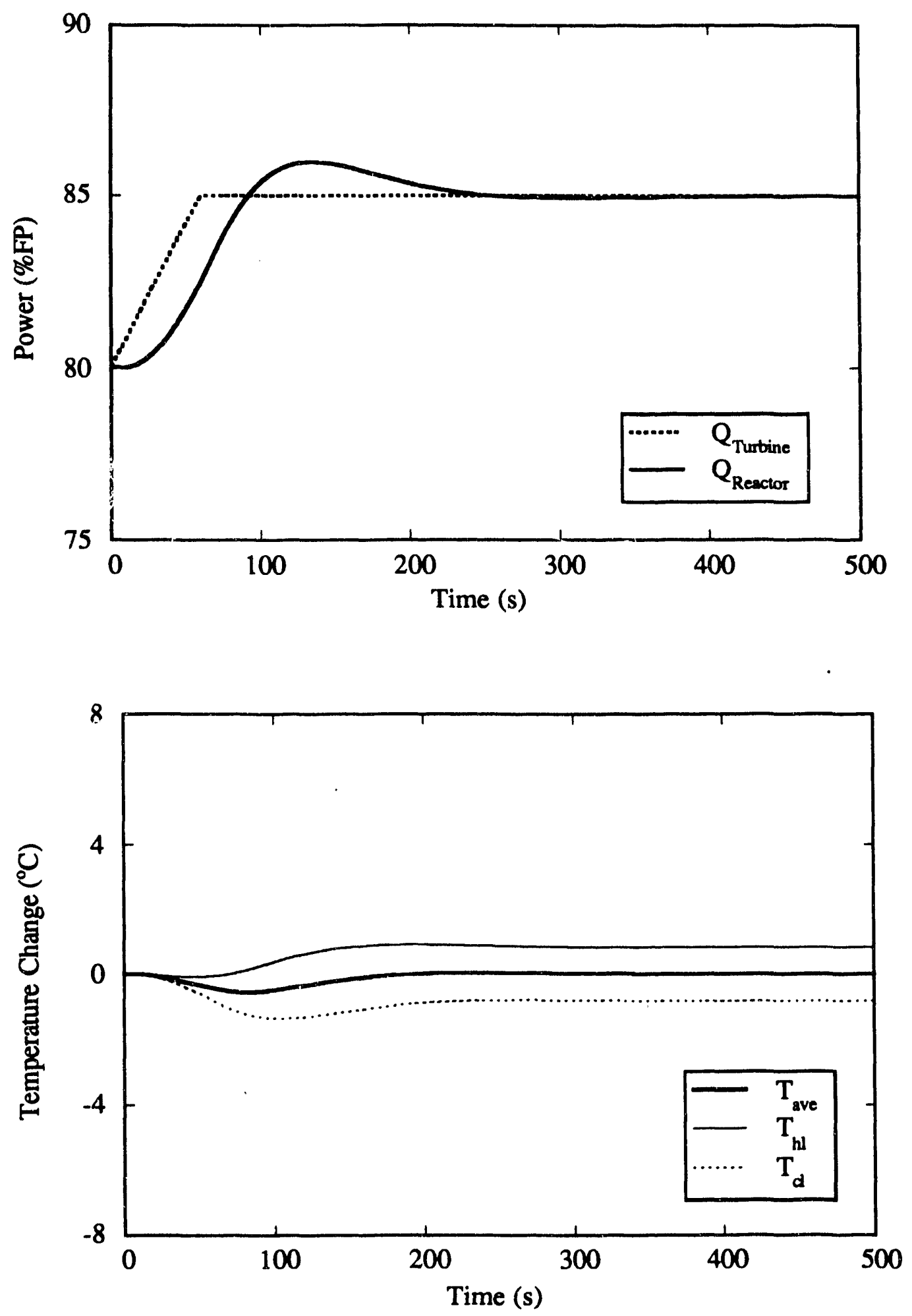

Figure 9.3.3-1 Behavior of PWR Following Increase in Turbine Valve Position. 
$-229-$

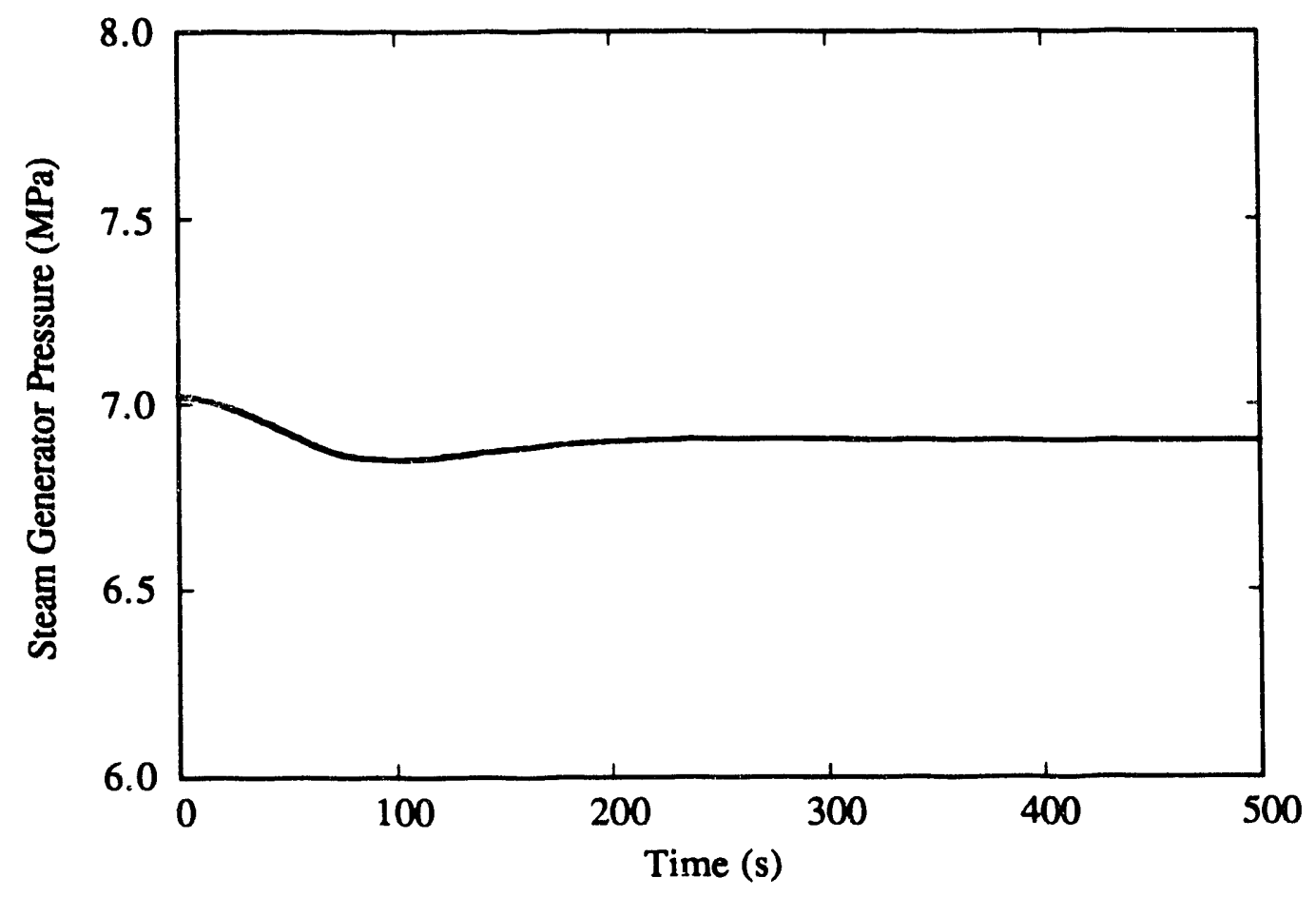

Figure 9.3.3-1 (Continued) 
risen and the control rods remain at their higher position. Figure 9.3.3.2-1 depicts the behavior of a PWR following a rod withdrawal under the conditions described above.

\subsubsection{Adiustment of Multi-Modular Reactor Power and Temperature}

It is likely that one of the most commonly performed operations at a multi-modular facility will be to shift from a condition where all modules are supplying an equal fraction of the load to one where the module with the least fuel burnup is supplying a greater fraction. For purposes of illustrating this maneuver, designate the reactor that is to be operated at the higher power level as module \#A and assume that there are a sufficient number of modules connected to the common steam line header so that no one module can affect conditions in that header. The shift to an unbalanced load condition is initiated by withdrawing the control rods in reactor \#A. This first causes the fission rate and then the hot leg temperature to rise. The net effect is to transfer additional energy to the secondary system. In the case of a PWR (one reactor), the presence of this additional energy causes steam generator temperature and pressure to rise because, in a single reactor system, there will be very little increase in energy removal from the steam generators unless the admission valve to the turbine is opened further. Thus, in a PWR, the increase in steam generator temperature translates to a similar rise in the cold leg temperature of the primary system and this in turn generates the negative reactivity needed to restore the reactor to its original power level. The situation is quite different in a multi-modular plant because in that case an increase in the energy being removed from a given module's steam generator(s) is possible even though total steam flowrate (i.e., that of all modules) is unchanged. This happens because, in the multi-modular plant, conditions in the steam generator are fixed by the common steam line header. Specifically, if the steam generator temperature remains constant while the hot leg temperature rises, then energy conservation dictates that there be an increase in the steam generation rate. Thus, steam flow from module \#A's reactor rises and, because overall demand is unchanged, the flows from the other modules decrease in order to compensate. These decreases result in slight elevations of the cold leg temperatures which in turn create negative reactivity insertions. Thus, power in the other modules is reduced. The net effect is therefore to establish an unbalanced load condition in which one module is at both a higher power and a higher average coolant temperature while the other modules are all at a somewhat lower power level. Each of these other reactors settles out at a slightly higher average coolant temperature with the difference between their hot and cold leg temperatures being lessened. Figures 9.3.3.3-1 and 9.3.3.3-2 illustrate the process.

In reality, changes in module power and temperature may be more complex than in the idealized scenario given above. For example, it may be desired to raise plant power while at the same time shifting the load fraction between the various modules. Also, if a sliding $T_{\text {ave }}$ control program is observed, then it will be necessary to raise temperatures whenever power in increased. These types of changes can be accomplished by the sequential application of reactor power and turbine power adjustments. This is the approach taken in Section 9.4.1.1 of this report where the actual power controller is described. 

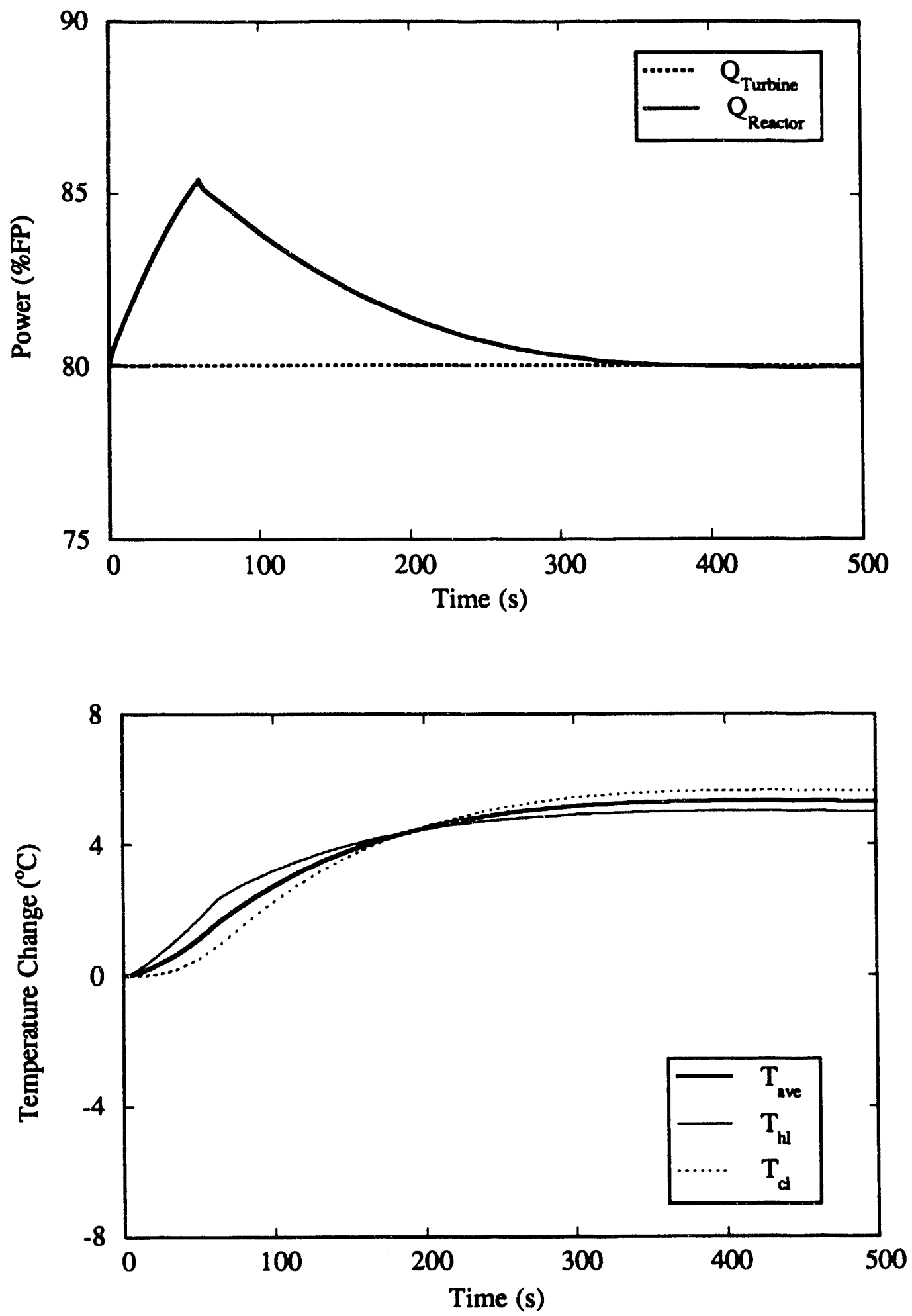

Figure 9.3.3.2-1 Behavior of PWR Following Rod Withdrawal. 


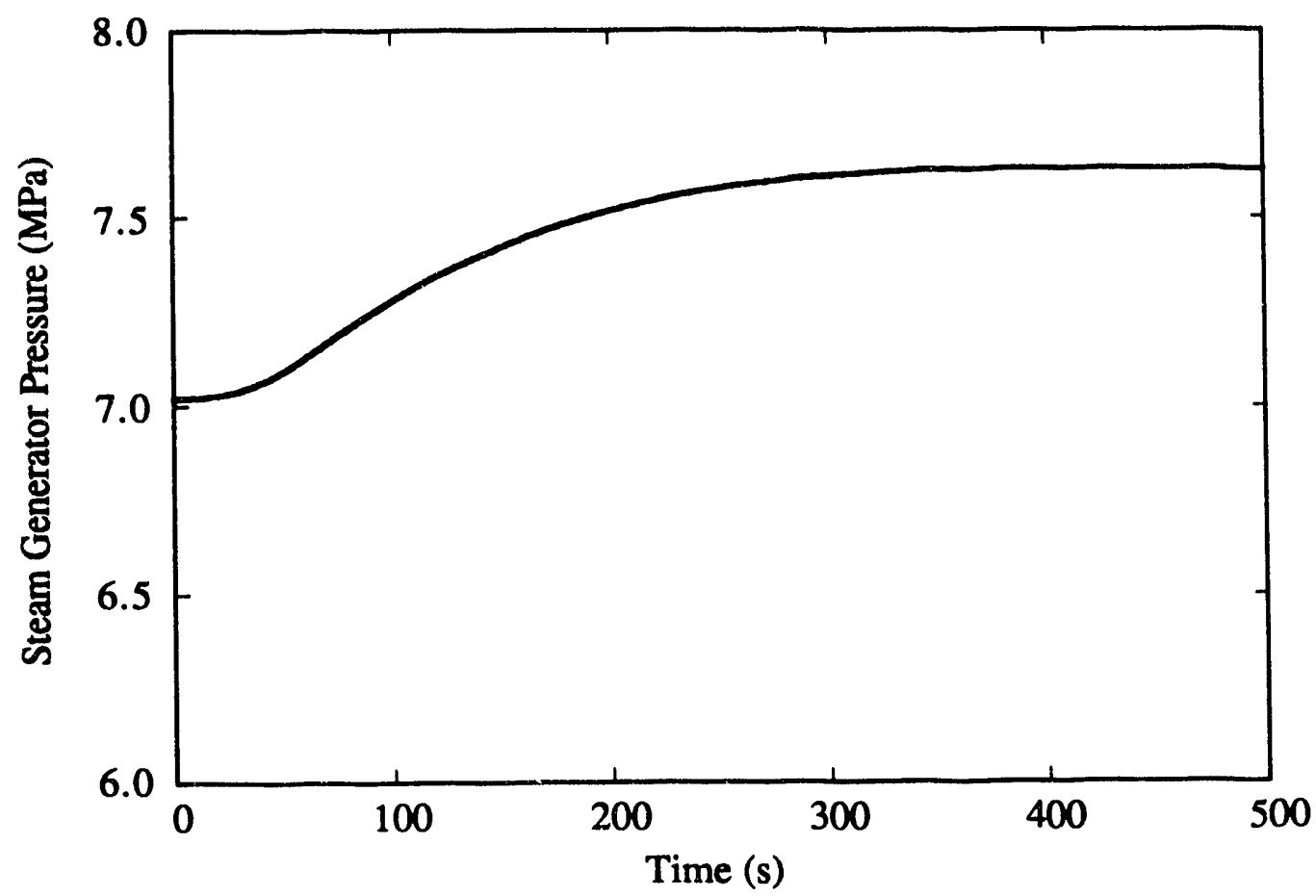

Figure 9.3.3.2-1 (Continued) 

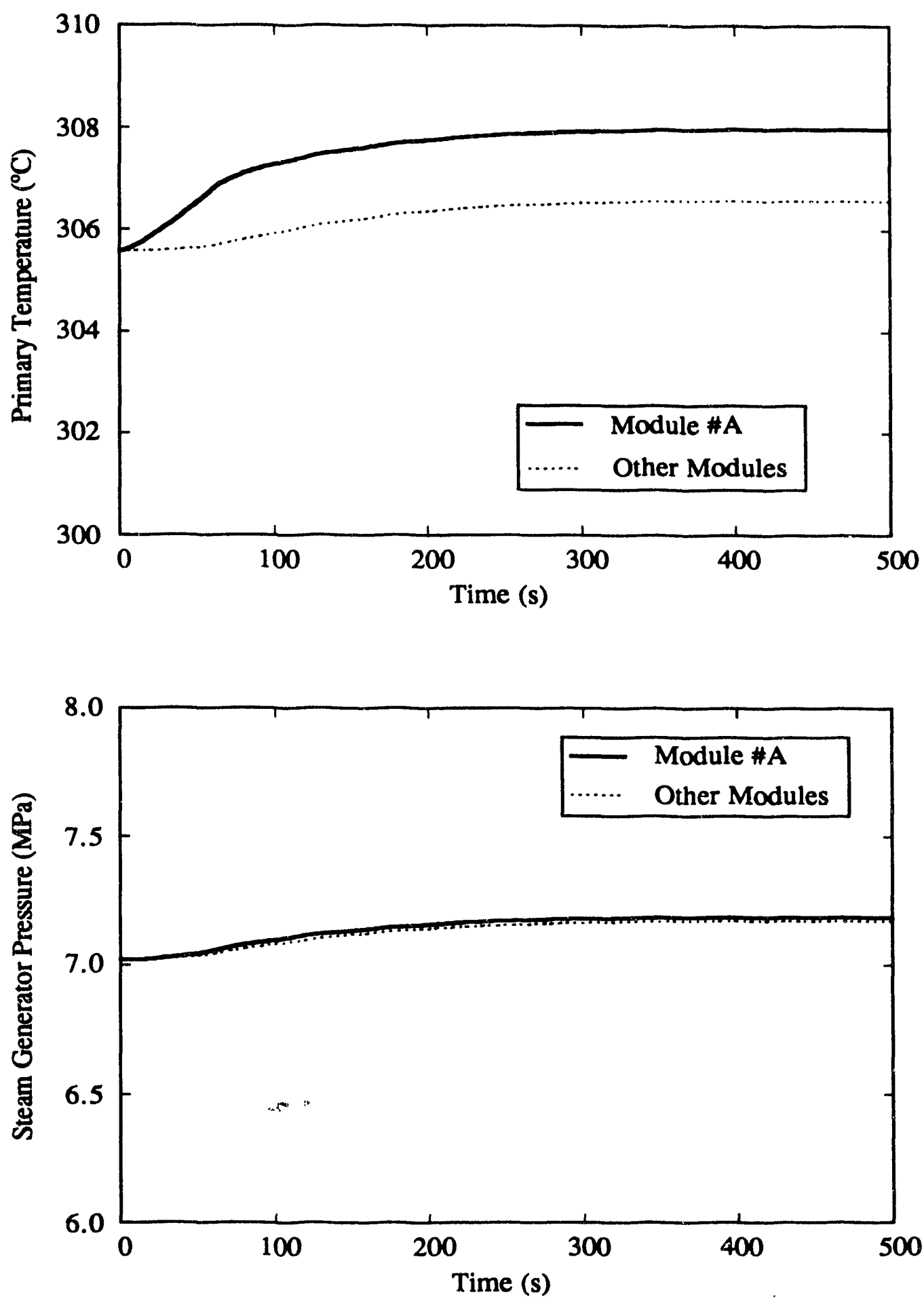

Figure 9.3.3.3-1 Behavior of Primary Temperature and Steam Generator Pressure in Multi-Modular Plant upon Shift to Unbalanced Load Conditions. 

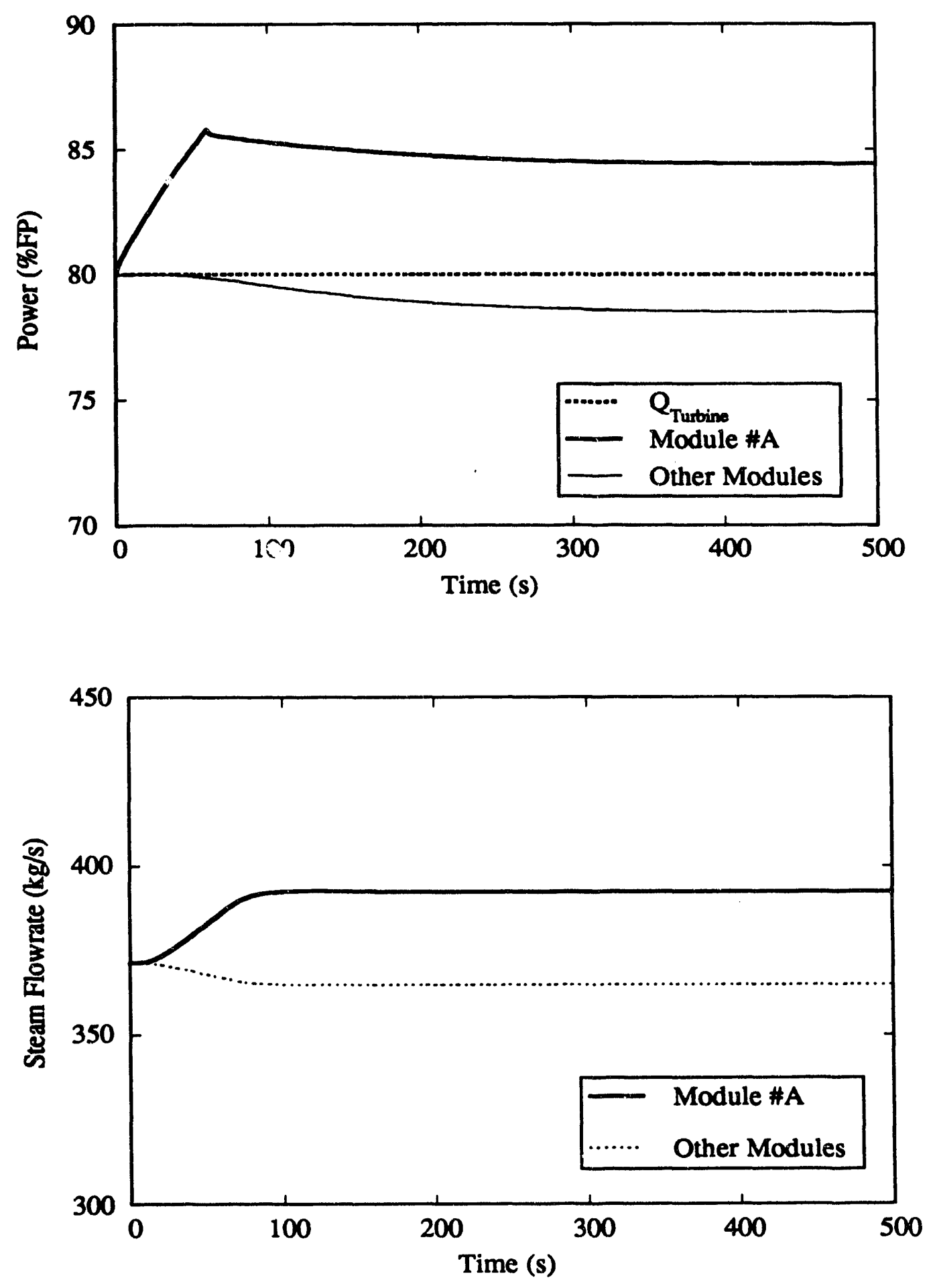

Figure 9.3.3.3-2 Behavior of Power and Steam Flowrate in Multi-Modular Plant upon Shift to Unbalanced Load Conditions. 


\subsection{PWR-Type Multi-Modular Reactor Controller}

The PWR-type multi-modular reactor controller developed as part of the research reported here was designed in accordance with the operational requirements imposed on current-generation PWR analog power controllers. These were given in Section 9.2.1 of this report. In addition, the controller incorporates a supervisory capability such that a challenge would not be made to the safety system as the result of any automatic control action. This capability is particularly important in a multi-modular plant because changes in one module can affect the operation of the other modules. Described here is the architecture of the controller.

\subsubsection{Controller Architecture and Components}

A multi-tiered, hierarchical design was selected for the controller. This choice follows logically from the design of a multi-modular plant because control decisions must be made at the plant, module, and system levels. Developed in the course of this research were plant, module, control rod, and steam generator level controllers. Descriptions of the first three are given here while that for the latter was given in Chapter Eight of this report. Also required, but not covered here, is a turbine controller. Figure 9.4.1-1 shows the hierarchical relationship of these various controllers. (Note: Shaded components are discussed in this chapter.)

\subsubsection{Plant Power Controller}

The principal role of the plant power controller is the allocation of load among the on-line modules. It does this in accordance with the selected operational mode and operating strategy. It also sends the turbine power demand signal to the turbine power controller. In addition to these roles, it may also have other functions such as safeguard and event response.

Figure 9.4.1.1-1 shows the relationship between the plant power controller and the other subsystems. At any time, the operator may select the operational mode and operating strategy that are to be used. The plant power controller receives the load demand from the load dispatcher if in the arbitrary power demand mode or from the operator if in the specified power demand load. It also receives the operational status of each module from the individual module power controllers. Several predetermined rules including ones for demand power allocation under each specific operational mode and operating strategy are encoded in the plant power controller's software. In addition, safeguard rules such as the procedure to be followed should one of the modules suddenly become unavailable would also be encoded.

One of the functions of the plant power controller is to select the appropriate means for effecting both temperature and power adjustments. As noted in Section 9.3.3 of this report, this can be done by making sequential changes in the reactor and turbine power. For example, if temperature and power are both to be increased (as would be the case if a sliding $T_{\text {ave }}$ program is observed during an increase in demand), then it would be appropriate to raise reactor power first and thereby generate the energy needed to heat up the reactor coolant. The turbine power increase, which results in greater energy removal, would then follow. The time interval during which either the reactor or turbine power is 


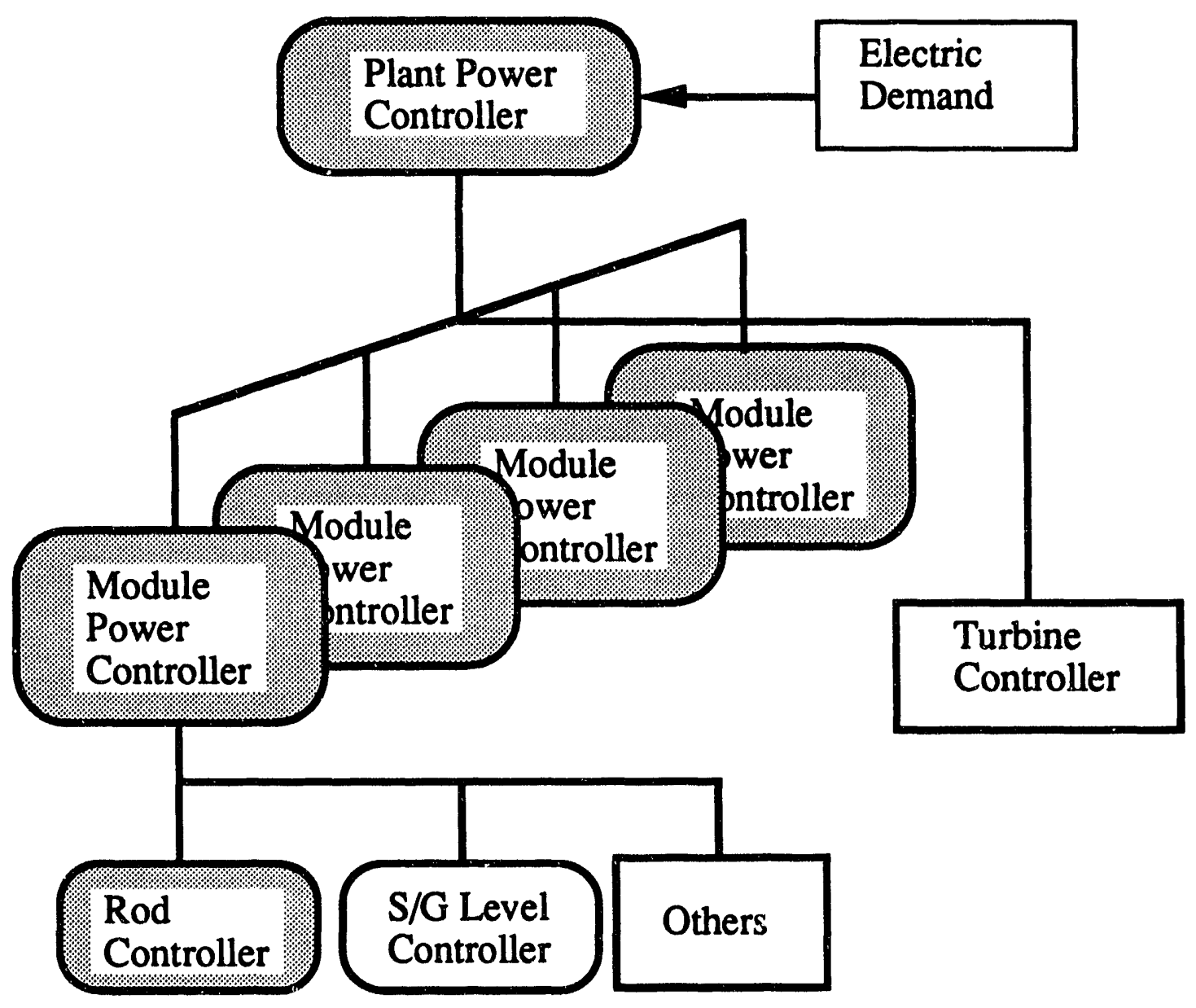

Figure 9.4.1-1 Hierarchical Structure of PWR-Type Multi-Modular Controller. (Source: After ORNL [102]) 


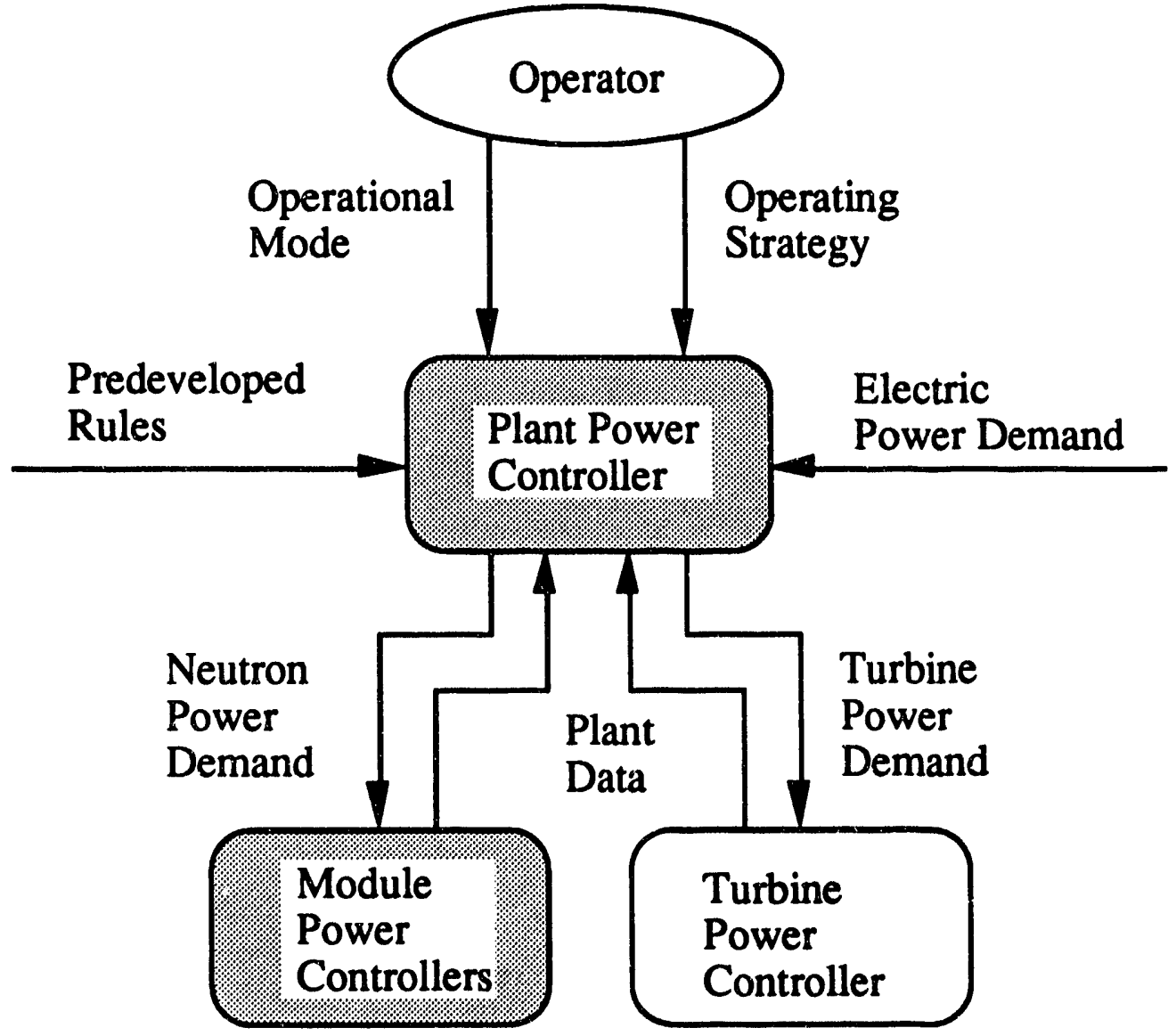

Figure 9.4.1.1-1 Relationship Between Plant Power Controller and Other Systems. (Source: After ORNL [102]) 
held constant while the other is adjusted is referred to here as a 'static time delay.' Table 9.4.1.1-1 lists these delays for certain evolutions. The values shown were selected empirically so as to minimize the deviation in temperature and power from their demanded trajectories.

The static time delay approach works quite well when operating in the specified power demand mode (see Section 9.3.2 of this report) because the demanded power is known in advance. Hence, it is possible to plan a sequence of reactor and turbine power changes. Operation in the arbitrary power demand mode is more difficult because the demanded load can not be anticipated. Hence, the static time delay approach can not be applied. Rather, module and turbine power are both increased at the same time and the module power may overshoot or undershoot its equilibrium value for some time. However, if lead-lag dynamic compensation is applied, the duration of the overshoot or undershoot can be reduced. Values of the lead time constants used here are as given in Table 9.4.1.1-1. A lag time constant of 8.0 seconds was used for all operations. Thus, when the turbine power is delayed (module power increases first), the demanded module power is modified by a positive lead-lag compensator. The result is that module and turbine power are increased at the same time but the module power increases faster because of the lead-lag compensation.

\subsubsection{Module Power Controller}

The module power controller has several important roles including transmission of the demanded module power to the rod controller and supervision of module parameters. The latter entails preventing the rod controller, which forms the lowest tier of the hierarchical controller, from implementing control rod motion that would place the reactor in an undesirable region such that the reactor safety system might be challenged or an operating limit exceeded. Thus, the supervisory controller overrides the rod controller when violation of operating limits might be possible. The basis of the supervisory portion of the module power controller is the reactivity constraint approach that was summarized in Section 3.3 .3 of this report.

\subsubsection{Rod Controller}

The rod controller interfaces directly to the actuators. As such, it forms part of the lowermost tier of the heirarchical controller. Its function is to position the control rods so as to cause module power and temperature to track their demanded trajectories. For this purpose, it combines model-based feedforward and error-based feedback control action. Thus,

$$
u_{r}=u_{m}+u_{f}
$$

where $u_{r}$ is the signal to the control rods, $u_{m}$ is the model-based feedforward action, and $u_{f}$ is the error-based feedback action. The quantity $u_{m}$ is determined from the MIT-SNL. Period-Generated Minimum Time Control Laws with $\omega(t)$ in Equation (3.3.4.1-5) set equal to the inverse period associated with the demanded trajectory. Thus, $\omega(t)$ is a constant and the role of Equation (3.3.4.1-5) is therefore that of a system model rather than that of a control law. The quantity $u_{f}$ is obtained from either of two algorithms. The first is used 
Table 9.4.1.1-1

Static Time Delays for Module Power and Turbine Power Demand Allocation

\begin{tabular}{|l|c|}
\hline \multicolumn{1}{|c|}{ Operation } & Time Delay (s) ${ }^{*}$ \\
\hline $\begin{array}{l}\text { Raise plant power using all } \\
\text { modules (Path \#1) }\end{array}$ & 10 \\
\hline $\begin{array}{l}\text { Raise plant power using the } \\
\text { highest-power module and several } \\
\text { lower-power modules (Path \#2) } \\
-\quad \text { One Module }\end{array}$ & -20 \\
$-\quad$ Two Modules & 0 \\
$-\quad$ Three Modules & 10 \\
\hline $\begin{array}{l}\text { Raise plant power using the } \\
\text { lower-power modules while } \\
\text { maintaining power in the highest- } \\
\text { power module constant (Path \#3) }\end{array}$ & 20 \\
\hline
\end{tabular}

* Note: Positive time delay implies that module power is increased first and turbine power later. 
for the highest-power module and entails computing the module power and primary coolant temperature errors. The latter is converted to an equivalent module power error as follows:

$$
\varepsilon_{\mathrm{e}}=\varepsilon_{\mathrm{p}}+\mathrm{K}_{\mathrm{Tp}} \varepsilon_{\mathrm{T}}
$$

where $\varepsilon_{e}$ is the equivalent total error, $\varepsilon_{\mathrm{p}}$ is the module power error, $\varepsilon_{\mathrm{T}}$ is the primary coolant temperature error, and $\mathrm{K}_{\mathrm{Tp}}$ is the conversion constant (e.g., $1.0 \% \mathrm{FP} /{ }^{\circ} \mathrm{C}$ ). The second is used for the lower-power modules. The erros in module power ratio to the highest-power module is computed for feedback control. This error is:

$$
\varepsilon_{e}=\frac{N_{d}}{N_{d}^{*}} N_{m}^{*}-N_{m}
$$

where $N_{m}$ and $N_{d}$ are the measured and demanded module power and $N_{m}^{*}$ and $N_{d}^{*}$ are the measured and demanded power of the highest-power module.

Figure 9.4.1.3-1 shows the program that is used to convert the above errors to a rod velocity. Figure 9.4.1.3-2 is a schematic of proposed the rod controller.

\subsection{Evaluation of PWR-Type Multi-Modular Reactor Controller}

The performance of the proposed controller was demonstrated through the simulation of transients that are associated with the normal operation of a PWR-type multimodular plant. For this purpose, the model described in Chapter Seven of this report was used as the simulation tool. The simulation studies were done by working upwards from the lowest tier of the hierarchical controller. Thus, the rod controller was tested first, then the module power controller, and finally the plant power controller. All transients assumed a maximum allowable maneuvering rate of $5 \% \mathrm{FP}$ per minute for both module and turbine power. Other conditions imposed on the simulations were:

1. The primary coolant flowrate in each module was taken as a constant $4453 \mathrm{~kg} / \mathrm{s}$.

2. The primary coolant pressure in each module was considered constant at $\mathbf{1 5 . 5 1}$ $\mathrm{MPa}$.

3. Feedwater temperature was assumed to vary with turbine power as was shown in Figure 7.3.4-2.

4. The charging and letdown flowrates were taken as zero so as to separate the controller's performance from the effects of a changing boron concentration. Also, the boron concentration in the makeup flow was taken as zero for the same reason.

The simulation program's secondary plant model does not include the turbine, condenser, or steam admission valve. As a result, it is not possible to calculate the steam flowrate in the main steam line header from a specification of the demanded load. However, this quantity is needed as a boundary condition in the main steam line header 


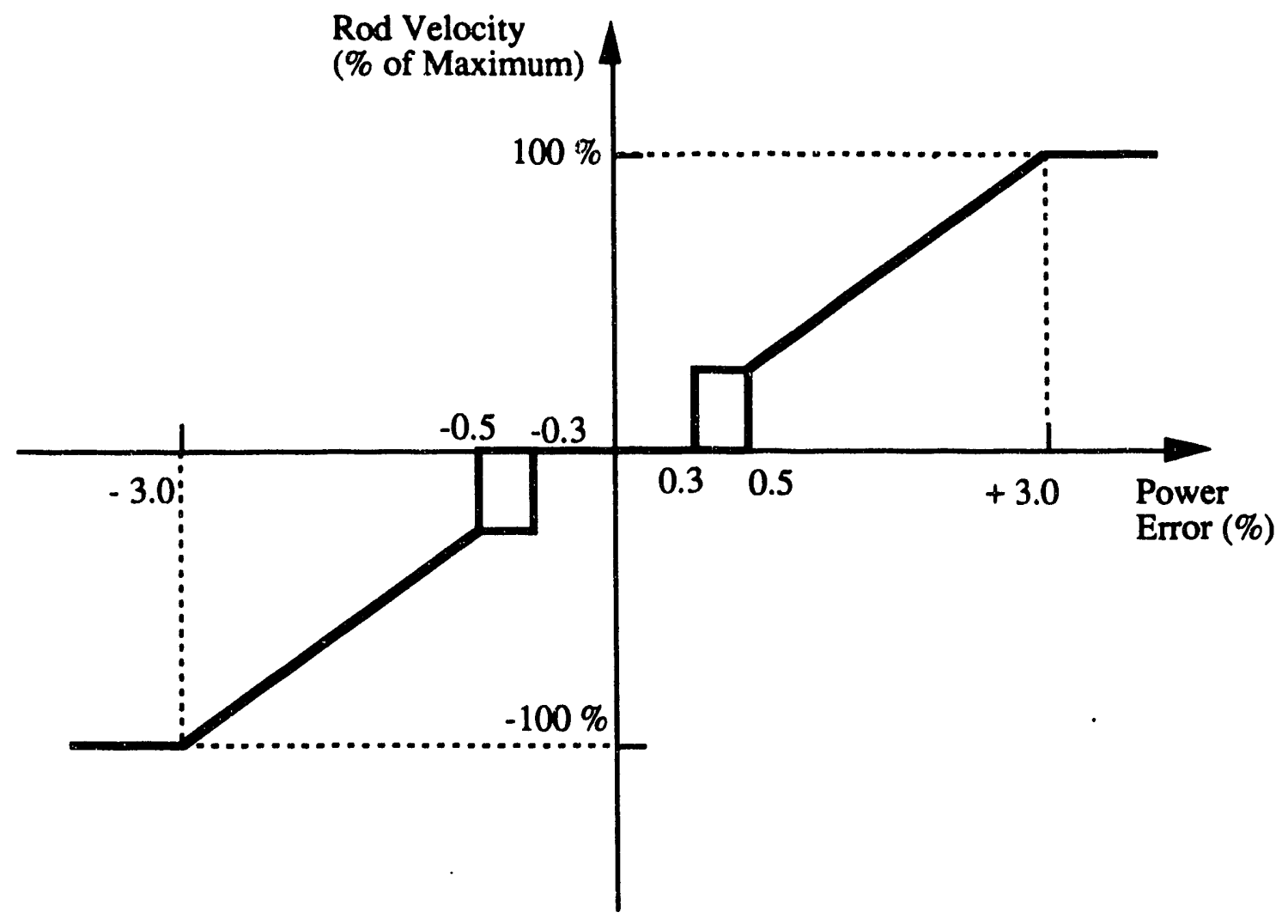

Figure 9.4.1.3-1 Rod Speed and Direction Control Program. 


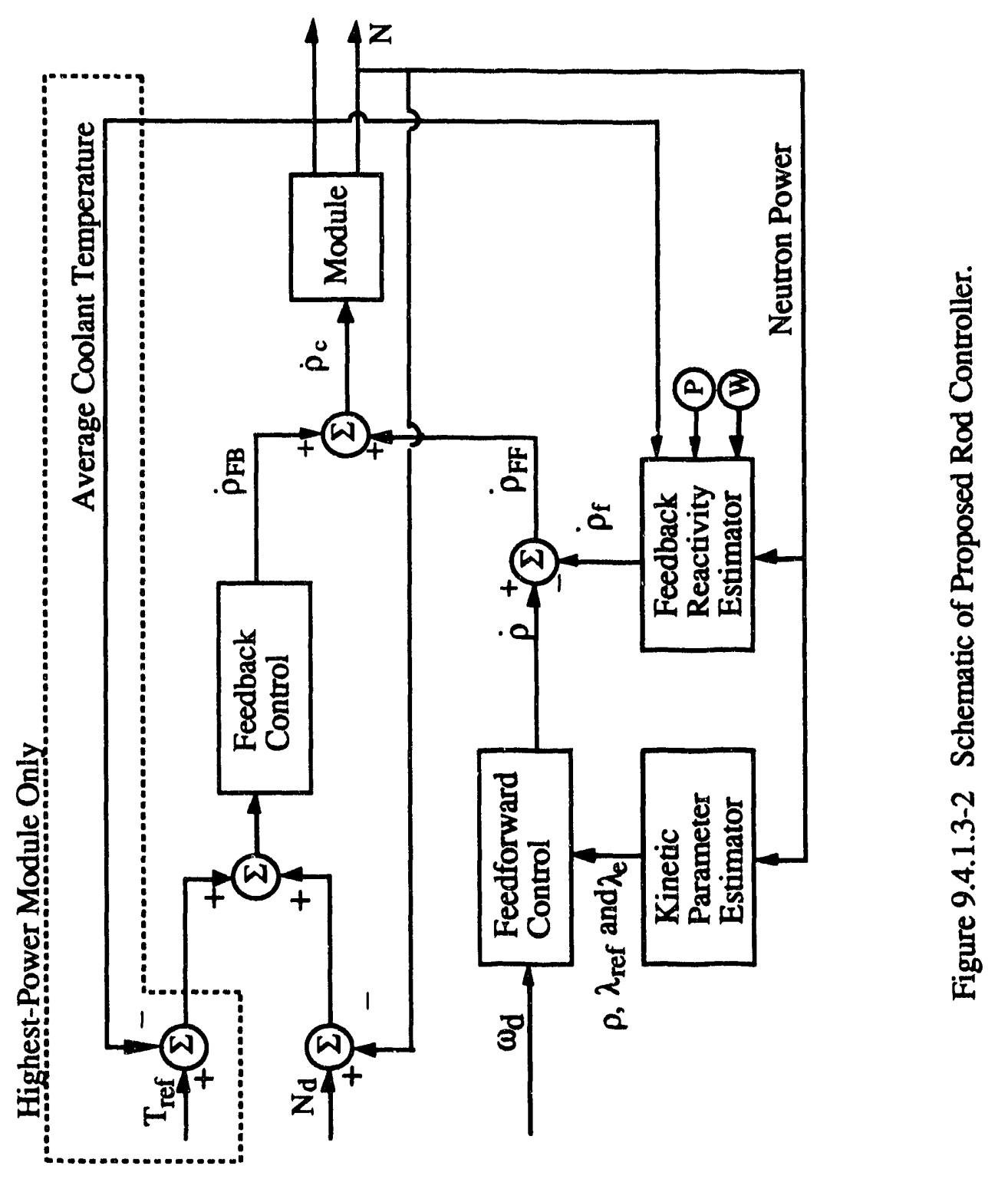


model. Accordingly, it is obtained by taking the power withdrawn from the main steam line header as always being equal to the turbine power. Under this assumption the steam flowrate is:

$$
\dot{\mathrm{m}}_{\mathrm{B}}=\frac{\mathrm{Q}_{\mathrm{IB}}}{\mathrm{h}_{\mathrm{g}}-\mathrm{h}_{\mathrm{fW}}}
$$

where $Q_{\text {TB }}$ is the turbine power, $h_{g}$ is the saturated vapor enthalpy of the steam generator inventory, and $h_{f w}$ is the enthalpy of the feedwater.

Another assumption that had to be made for the purpose of conducting the simulation studies was that each reactor's control rods could be moved both continuously and at variable speed. In reality, the rods of current-generation PWRs are moved in discrete steps. (Note: Although not included in this report, simulations were done in which the plant power controller was evaluated with discrete rod movement. The results showed it to be effective [103].)

A time step size of $0.1 \mathrm{~s}$ was used for both the numerical solution of the plant simulation models and the controller's sampling interval.

Finally, it should be noted that the overall objective of the proposed controller is to maintain the desired primary coolant temperature and hence, the desired steam flowrate fraction as specified. To this end, the composite controller generates demanded control signals for each module that will result in proper control of both module power and temperature. However, if these two objectives are in conflict, the control of temperature has precedence. For example, in the arbitrary demand mode, module power is allowed to overshoot the targeted power so as to maintain temperature and hence steam flowrate. Under these circumstances, the supervisory control action of each module's power controller becomes important because it is that supervisory action that restricts module power to a safe envelope of conditions.

\subsubsection{Evaluation of the Rod Controller}

The purpose of the rod controller is, as discussed in Section 9.4.1.3 of this report, to move the reactivity control devices so that module power will follow a demanded trajectory. The rod controller consists of a model-based feedforward control law and a feedback control law. In the highest-power module, the feedback control signal is computed from a combination of the power and primary coolant temperature errors while in the low-power modules, it is computed from the ratio of power error to that of the module with the highest power. The rod control algorithm was tested by demanding an increase in module power from $95 \%$ FP to $100 \% \mathrm{FP}$ at a ramp rate of $5.0 \% \mathrm{FP}$ per minute. Noise levels of $\pm 0.1 \% \mathrm{FP}$ and $\pm 0.1^{\circ} \mathrm{C}$ were randomly imposed on the power and temperature signals obtained from the model. Those signals were then smoothed using the techniques described in [61]. Figure 9.5.1-1 shows the results. As is evident from this figure, the rod controller caused the simulated or 'observed' power to track that which was demanded. 


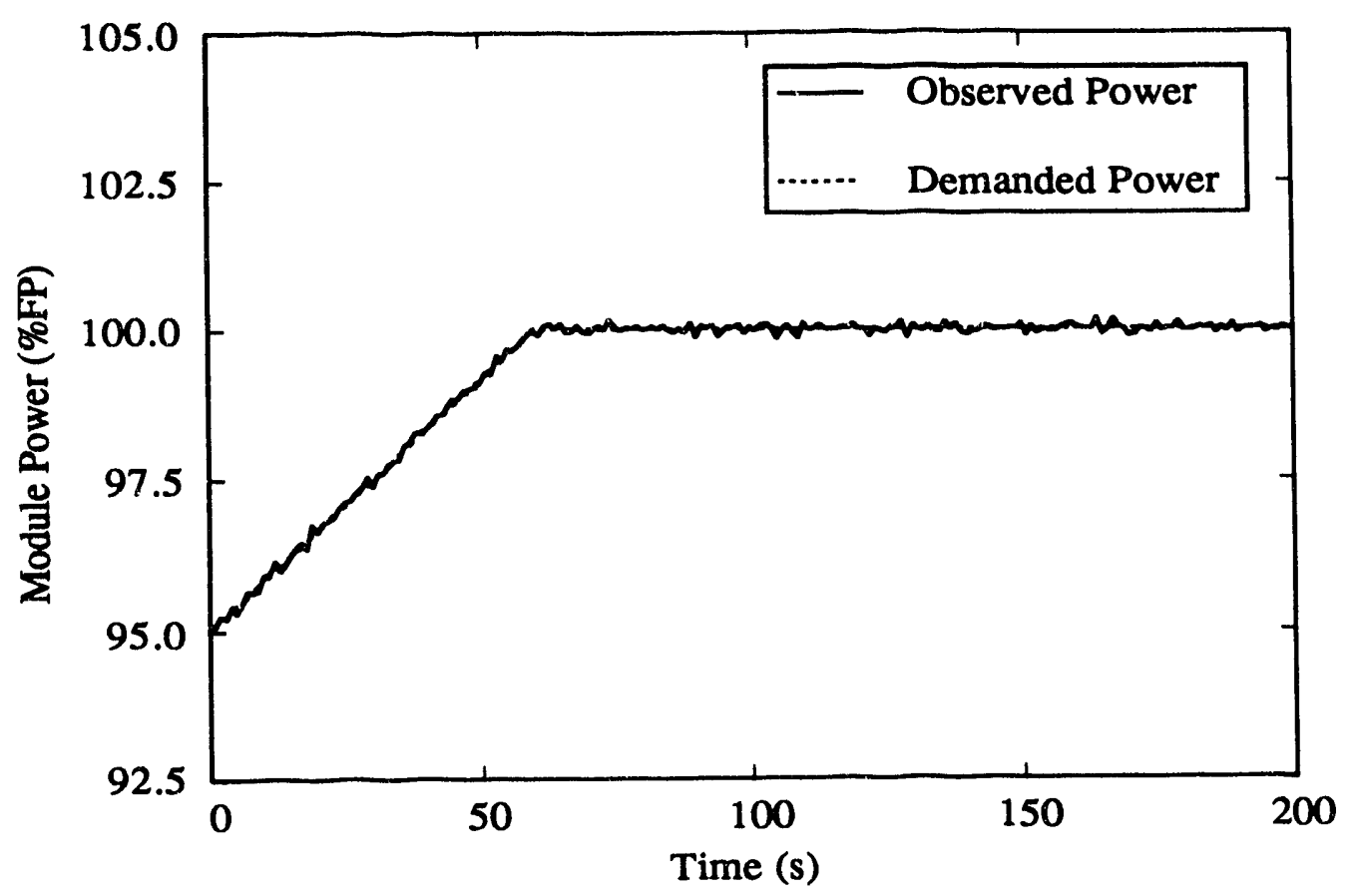

Figure 9.5.1-1 Simulation Study of Rod Controller. 


\subsubsection{Evaluation of Module Power Controller}

The purpose of the module power controller is to preclude a challenge to the reactor's safety system. It does this by using reactivity constraints to monitor control law decisions. This type of supervisory control is particularly important when operating in the arbitrary power demanded mode because, in that case, it may be necessary to allow power to exceed its targeted value temporarily in order to maintain temperature on its demanded trajectory. Simulation tests of the module power controller were conducted by directing the rod controller to withdraw a rod bank continuously at maximum speed. The supervisory component of the module power controller intervened and prevented power from exceeding the operational limit of $103 \%$ FP. Demonstrations of this type have been previously conducted on both the MIT Research Reactor and the Annular Core Research Reactor. These are described in Section 3.3.3.3 of this report and in several previous reports $[1,19,27]$.

\subsubsection{Evaluation of Plant Power Controller}

The purpose of the plant power controller is to coordinate changes in module power and temperature given a demanded turbine power and/or a proposed fuel depletion strategy. The controller assumes a maximumi allowable ramp rate of $\pm 5.0 \%$ FP per minute with the actual rate depending on the number of active modules. Presented here are the results of five simulation studies in which the controller's capabilities were demonstrated for power increases and decreases assuming either a specified or arbitrary power demand mode. Emphasis was placed on operation with unbalanced loads. Note: The initial conditions chosen for each simulation were selected so as to necessitate frequent changes of the operating strategy. This was done in order to illustrate flexibility. Time delays were often imposed arbitrarily between such changes so as to provide visual separation of each strategy in the figures. These delays are noted in the tables that describe each simulation. Plant conditions were held constant during these delays. Thus, they are purely cosmetic and would not be required in an actual plant.)

\subsubsection{Case One: Power Increase in the Specified Power Demand Mode}

Case One is a power increase in the specified power demand mode. Initially, each module's power level is different and the objective is to raise the power of ach to $100 \%$ FP. The power increase sequences, the operating strategies, and the demand allocation methods are all shown in Table 9.5.3.1-1. At the outset, module \#1 is the highest-power module and the others are all at different power levels. During the first phase, the active operating strategy is 'equal change' and all modules are active. Hence, all module powers are increased by $5 \% \mathrm{FP}$ at a $5.0 \% \mathrm{FP} /$ minute ramp rate. Note: The choice of the 'equal change' strategy was arbitrary. Other strategies could have been selected.) Module \#1 then reaches 100 \%FP and this module cannot remain active any longer. The operating strategy is then changed to 'extreme first.' Under this strategy, only module \#4 is active because it is currently the lowest-power module. The maximum turbine power ramp rate is now reduced to $1.25 \% \mathrm{FP} /$ minute because only one module is active. If a higher ramp change in load is demanded, the plant power controller must either reject it or change the operating strategy. For example, the 'equal change' strategy with module \#1's power held constant would yield a maximum turbine power ramp rate of $3.75 \% \mathrm{FP} /$ minute. The second phase continues until module \#4 attains $90 \%$ FP. Both it and module \#3 are 
Table 9.5.3.1-1

Sequence for Power Increase in Specified Power Demand Mode

\begin{tabular}{|c|c|c|c|c|c|c|c|c|}
\hline \multirow{2}{*}{ Phase } & \multicolumn{4}{|c|}{$\begin{array}{c}\text { Module Power } \\
\text { (\%FP) }\end{array}$} & $\begin{array}{c}\text { Turbine } \\
\text { Power } \\
\text { (\%FP) }\end{array}$ & $\begin{array}{c}\text { Operating } \\
\text { Strategy }\end{array}$ & $\begin{array}{c}\text { Demand } \\
\text { Allocation }\end{array}$ & $\begin{array}{c}\text { Initiation } \\
\text { Time }\end{array}$ \\
\cline { 2 - 5 } & $\# 1$ & $\# 2$ & $\# 3$ & $\# 4$ & & & \\
\hline Initial & 95 & 90 & 85 & 80 & 87.5 & Equal Change & Path 1 & $0 \mathrm{~s}$ \\
\hline 1 & 100 & 95 & 90 & 85 & 92.5 & Extreme First & Path 3 & $200 \mathrm{~s}$ \\
\hline 2 & 100 & 95 & 90 & 90 & 93.75 & Extreme First & Path 3 & $400 \mathrm{~s}$ \\
\hline 3 & 100 & 95 & 95 & 95 & 96.25 & Extreme First & Path 3 & $500 \mathrm{~s}$ \\
\hline 4 & 100 & 100 & 100 & 100 & 100 & -- & -- & - \\
\hline
\end{tabular}

Notes: (1) As discussed in Section 9.4.1.1 of this report, the demand allocation method depends on the operational mode and operating strategy. The path designations given in the table refer to:

Path \#1 - Use all modules.

Path \#2 - Use highest-power module and one or more of the lowerpower ones.

Path \#3 - Use lower-power modules.

(2) The 'initiation time' denotes the start of each operating strategy. If the action taken under the previous strategy was complete prior to the initiation of the next phase, then a time delay was arbitrarily imposed. Plant conditions were held fixed during these delays. Their only effect is to provide visual separation of the phases in the figures. 
now equal in power and they therefore both become active. This portion of the transient is designated as the third phase. It continues until these two low-power modules artain 95 \%FP. Module \#2 is already at $95 \%$ FP so the fourth phase starts with all three modules being used to increase power.

Figure 9.5.3.1-1 shows the module and turbine powers. In each phase of the simulation, the increase in turbine power is delayed relative to that of the module powers. The delays, which are specified in Table 9.4.1.1-1, are ten seconds for the initial phase and twenty seconds for the others. This is done in order to generate the energy needed to raise the temperature of each module. Figure 9.5.3.1-2 gives the average primary coolant temperature of each module and the reference temperature (i.e., the demanded trajectory) for the average temperature of the highest-power module. That the latter is tracked quite closely shows that the strategy of sequencing changes in module and turbine power is effective. In particular, even though the average prima y coolant temperature is only a controlled variable in the highest-power module, all average primary coolant temperatures track their desired trajectories within the allowed error band $\left.\pm 0.5^{\circ} \mathrm{C}\right)$. Figure 9.5.3.1-3 shows the equivalent control rod positions. These remain constant after the power increase. This means that no further control action is needed to adjust the primary coolant temperatures. Therefore, it can be concluded that the plant power controller achieves the demanded average primary coolant temperature trajectories through proper sequencing of module and turbine power changes.

Figure 9.5.3.1-4 shows the steam flowrates from the individual steam generators. The load is shared without any oscillations during either transient or steady-state conditions. Figure 9.5.3.1-5 shows the steam generator water levels. As expected, the level of each module shows the effects of swell when module power is increased. However, the proposed model-based steam generator level controller maintains level successfully. Figure 9.5.3.1-6 shows the steam generator pressures. 


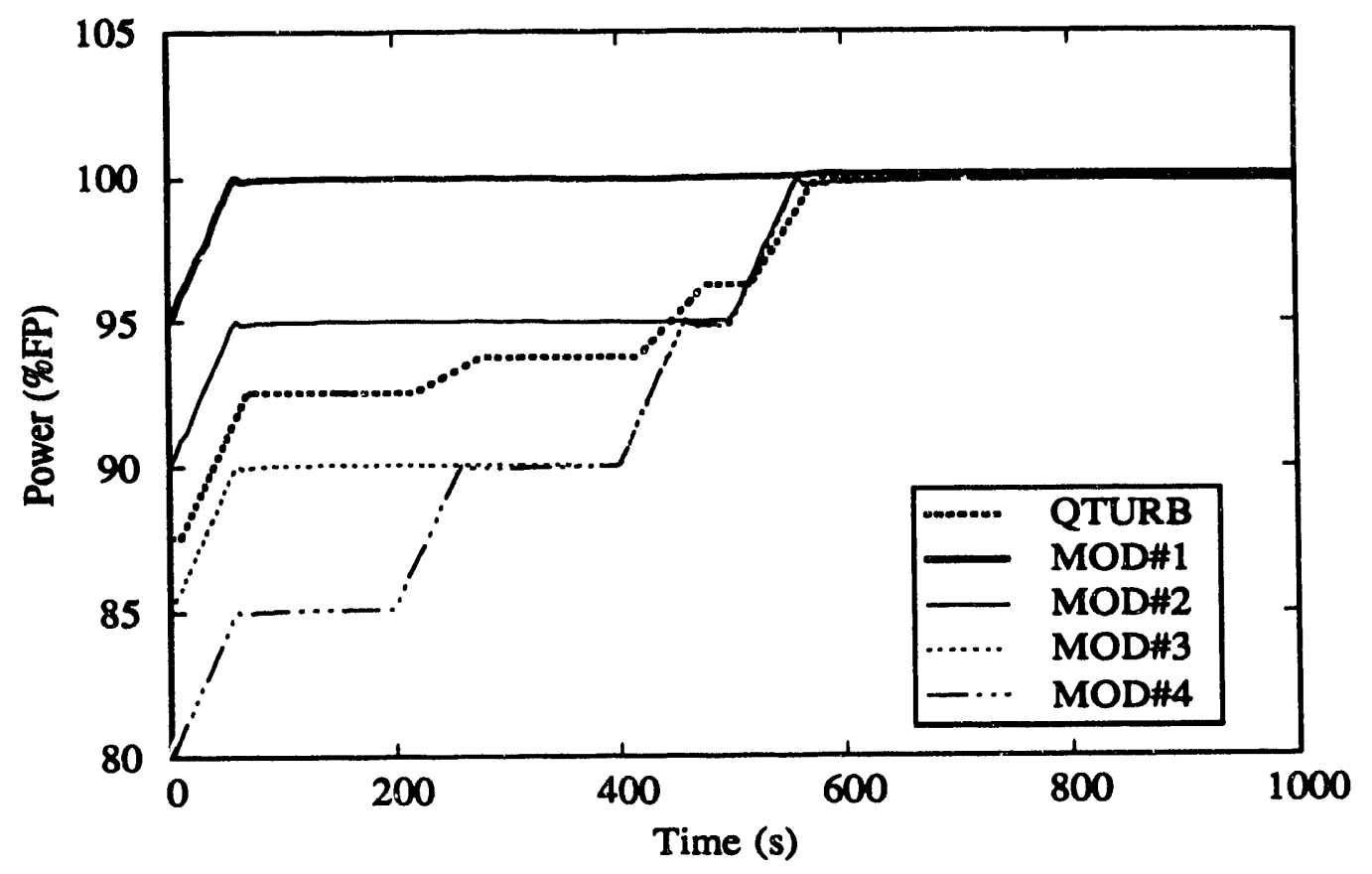

Figure 9.5.3.1-1 Module and Turbine Power. (Case 1 Transient)

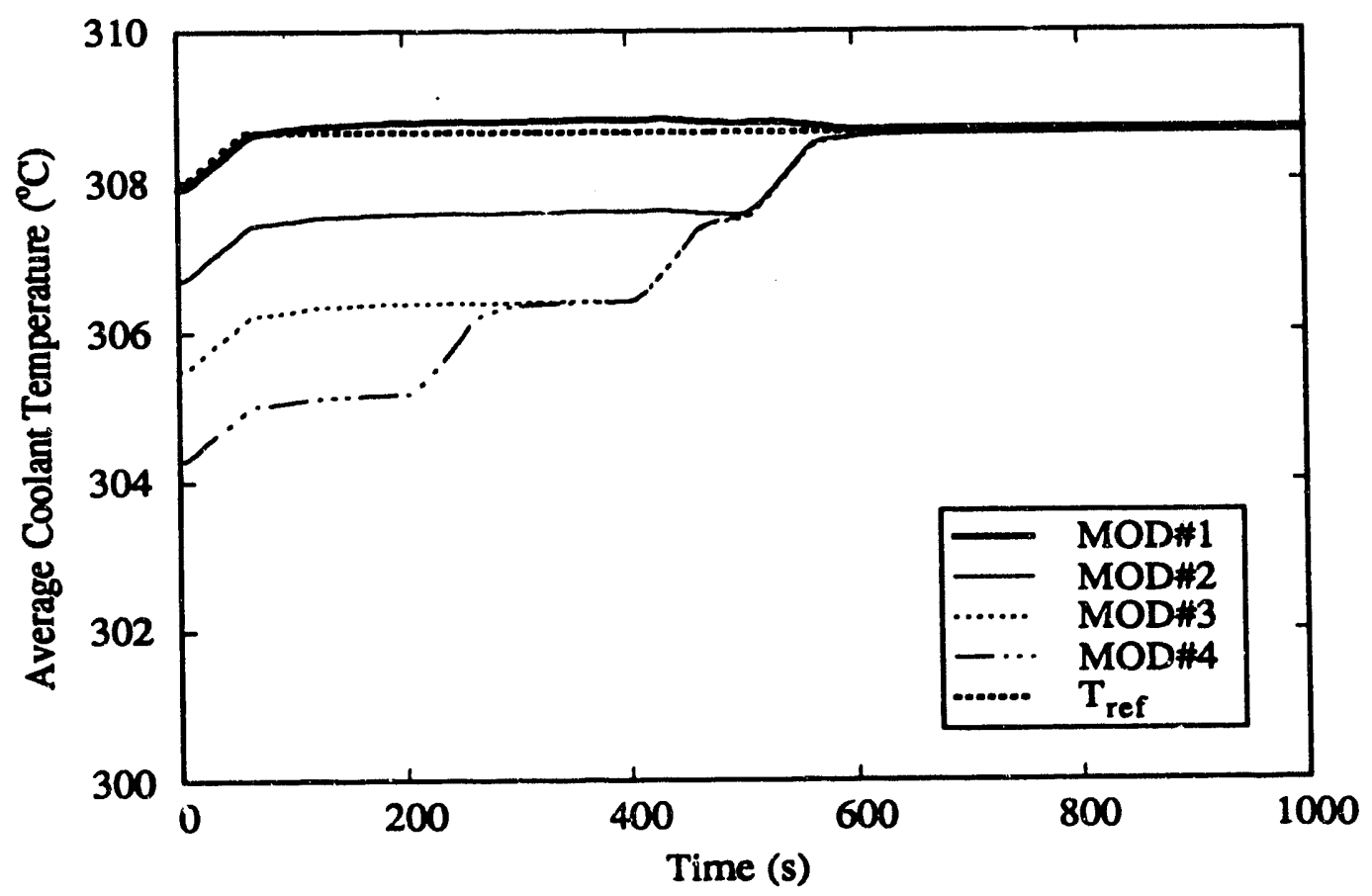

Figure 9.5.3.1-2 Average Primary Coolant Temperatures. (Case 1 Transient) 


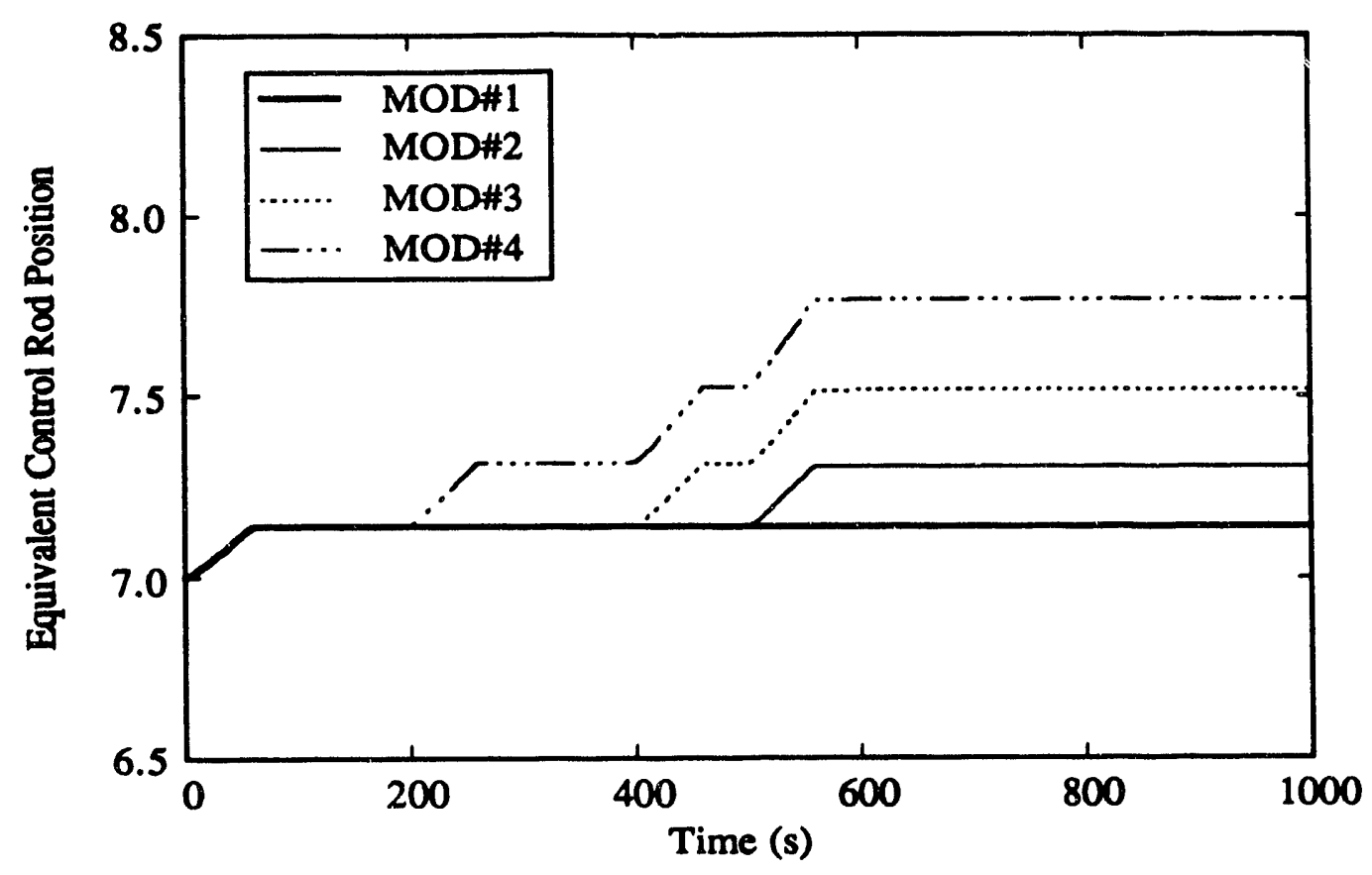

Figure 9.5.3.1-3 Equivalent Control Rod Positions. (Case 1 Transient)

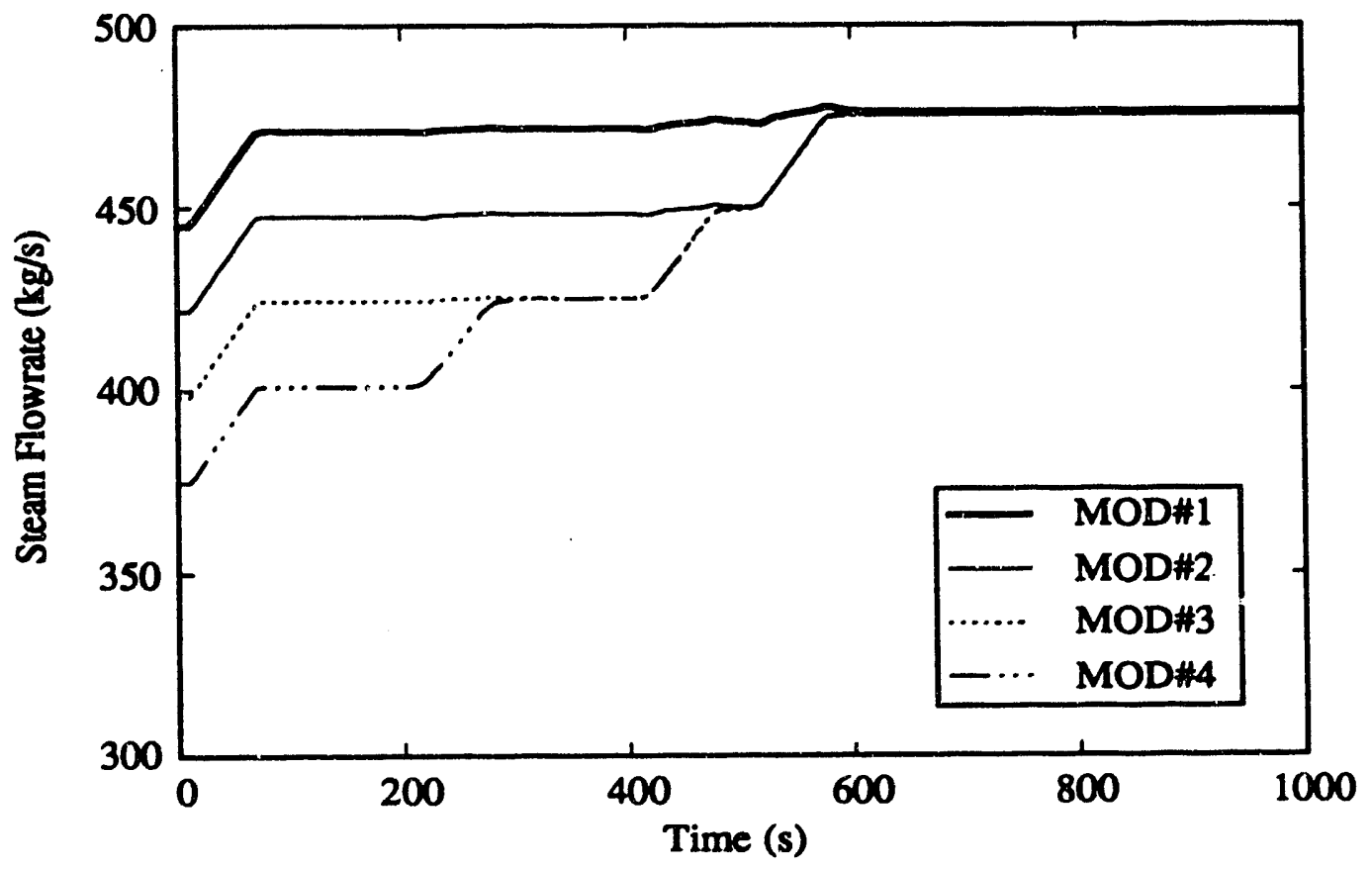

Figure 9.5.3.1-4 Steam Flowrates from Individual Steam Generators. (Case 1 Transient) 


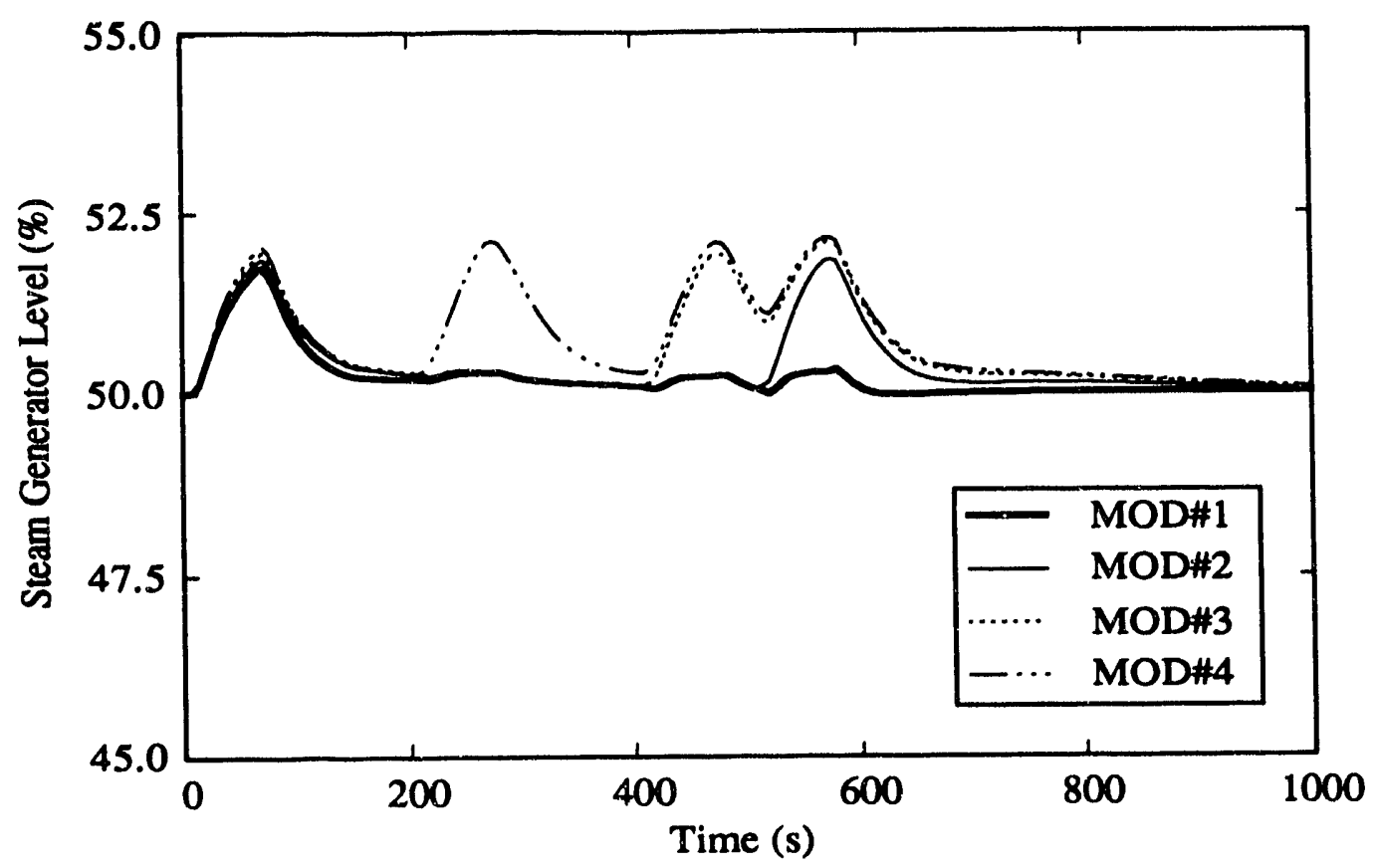

Figure 9.5.3.1-5 Steam Generator Levels. (Case 1 Transient)

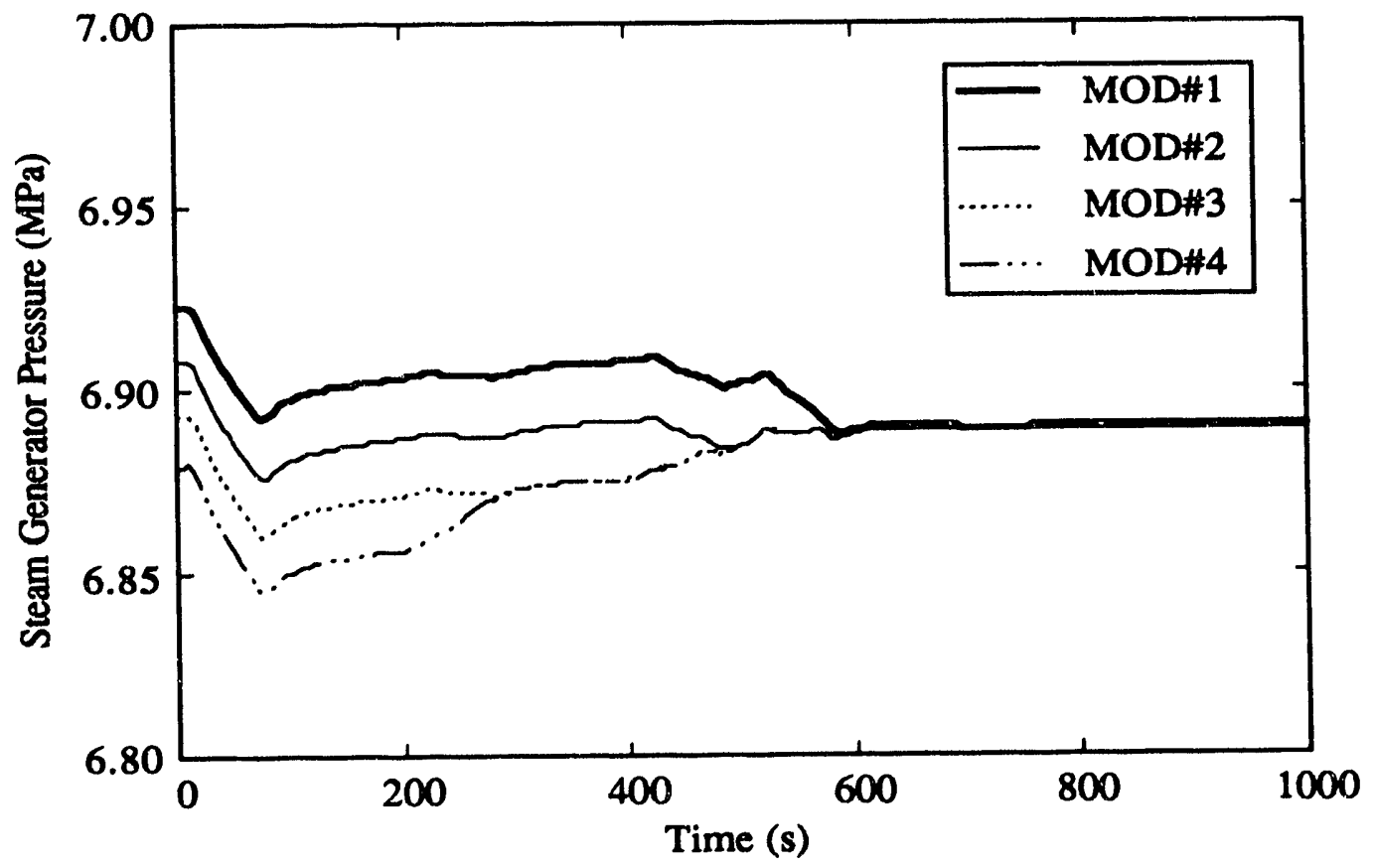

Figure 9.5.3.1-6 Steam Generator Pressures. (Case 1 Transient) 


\subsubsection{Case Two: Power Increase in the Arbitrary Power Demand Mode}

Case Two is the same as the first case except that the arbitrary power demand mode is used for both module and turbine power demand allocation. Figures 9.5.3.2-1 through 9.5.3.2-6 present the results of this simulation.

Figure 9.5.3.2-1 shows the module and turbine powers. In this operational mode, the demanded module power is adjusted by a lead-lag dynamic compensator so that more energy is generated by the module than is extracted by the turbine. Thus, on a temporary basis, the module power is allowed to overshoot the desired power. However, because module power is supervised by the module power controller, it is guaranteed that module power will not exceed any safety limit. Figure 9.5.3.2-2 shows the average primary coolant temperature of each module and the reference temperature of the highest-power module. As before, the average primary coolant temperatures remain within the allowed error band of $\pm 0.5^{\circ} \mathrm{C}$. The other simulation results that are given include control rod position (Figure 9.5.3.2-3), steam flowrate from each steam generator (Figure 9.5.3.2-4), generator level (Figure 9.5.3.2-5), and steam generator pressure (Figure 9.5.3.2-6). All parameters follow their desired trajectories and remain stable upon completion of the transient. 


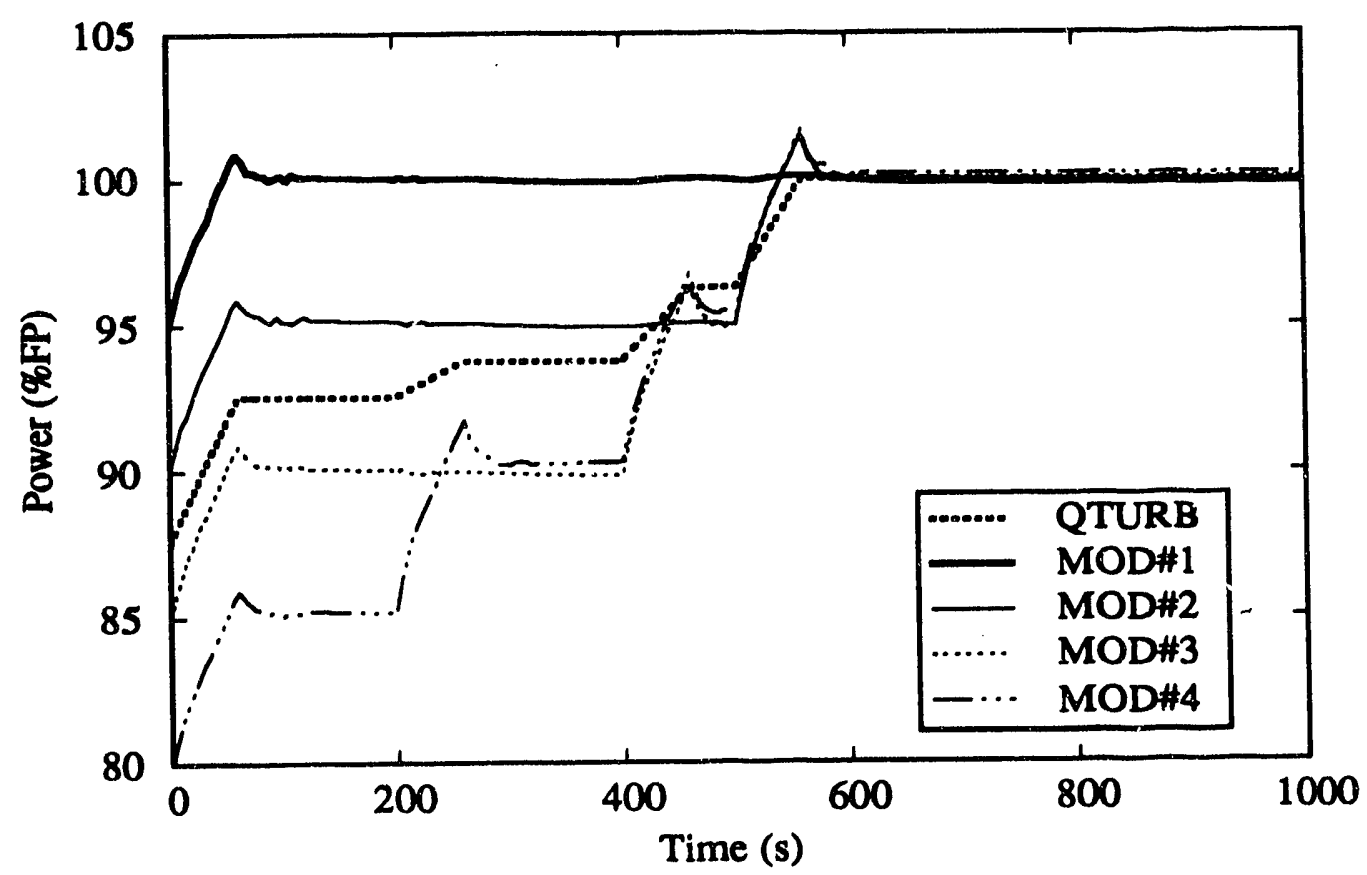

Figure 9.5.3.2-1 Module and Turbine Power. (Case 2 Transient)

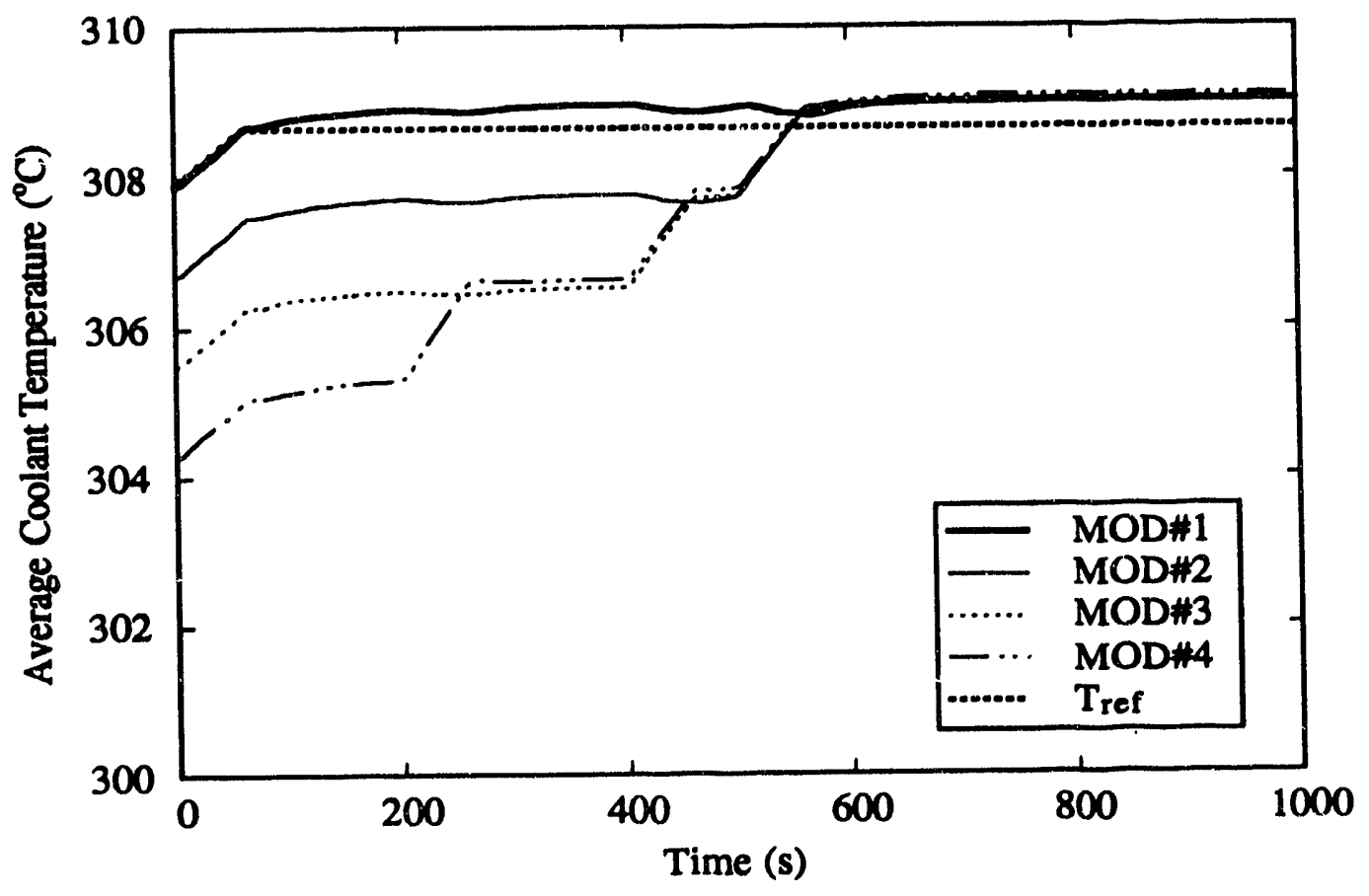

Figure 9.5.3.2-2 Average Primary Coolant Temperatures. (Case 2 Transient) 


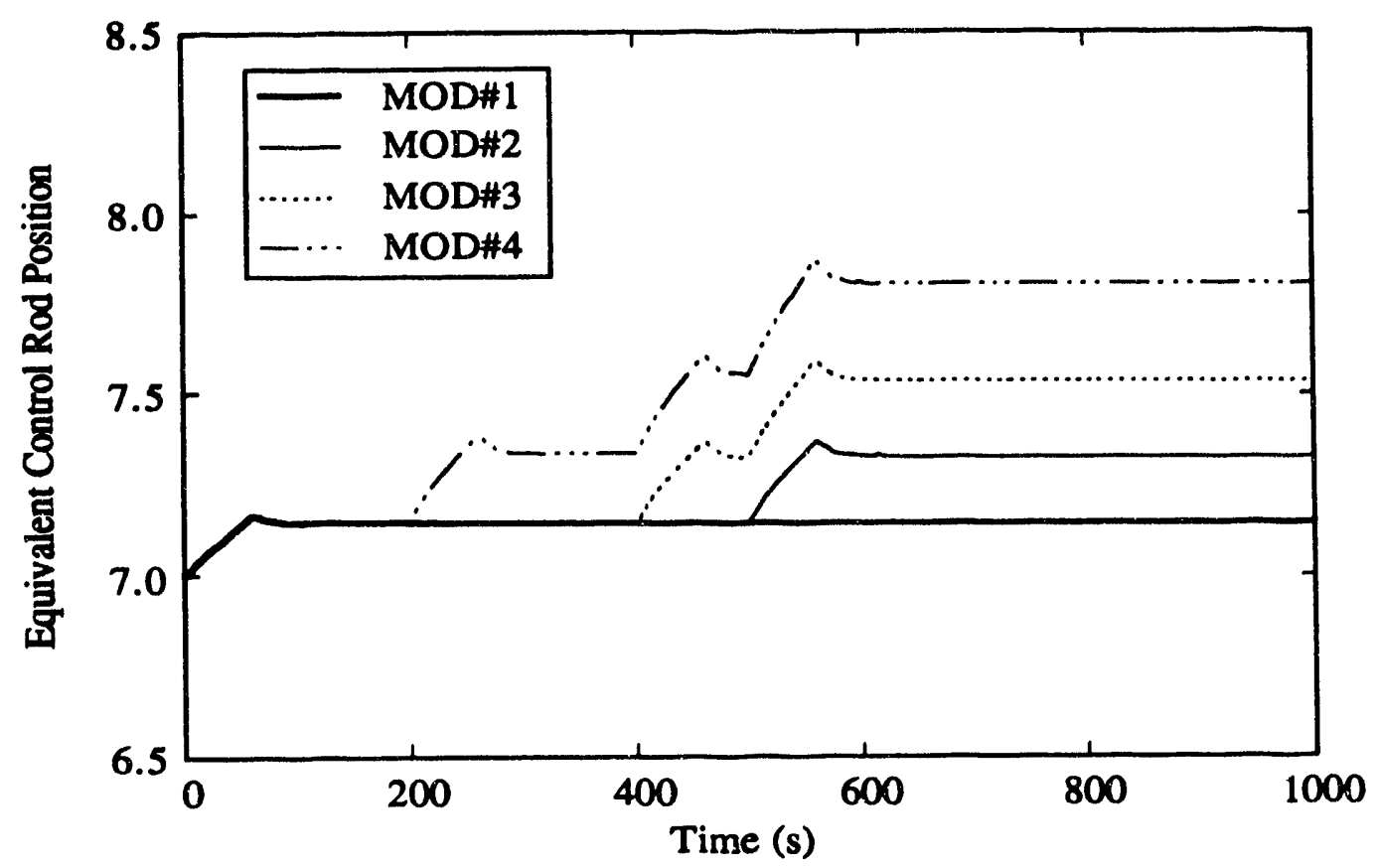

Figure 9.5.3.2-3 Control Rod Positions. (Case 2 Transient)

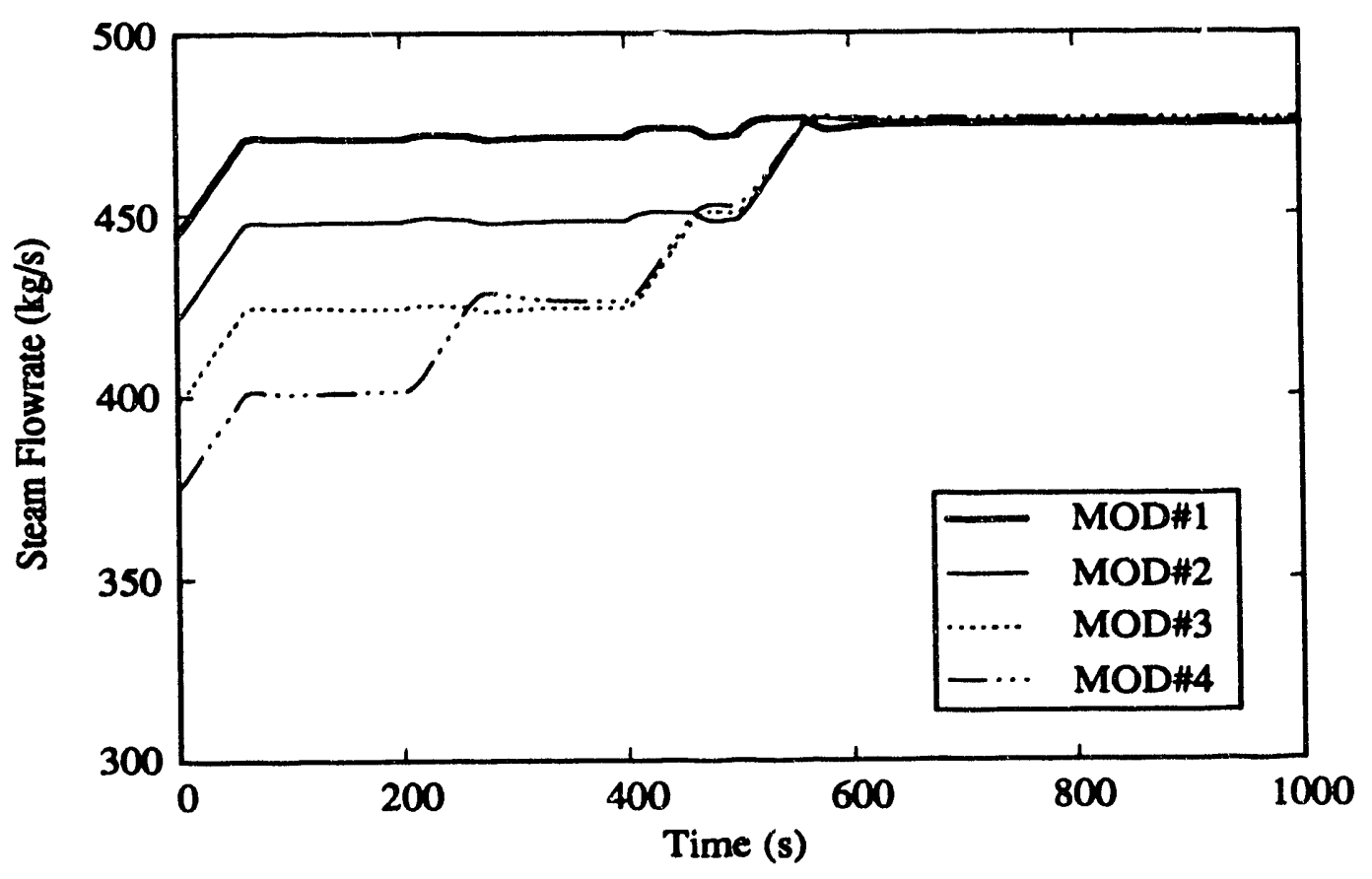

Figure 9.5.3.2-4 Steam Flowrates from Individual Steam Generators. (Case 2 Transient) 


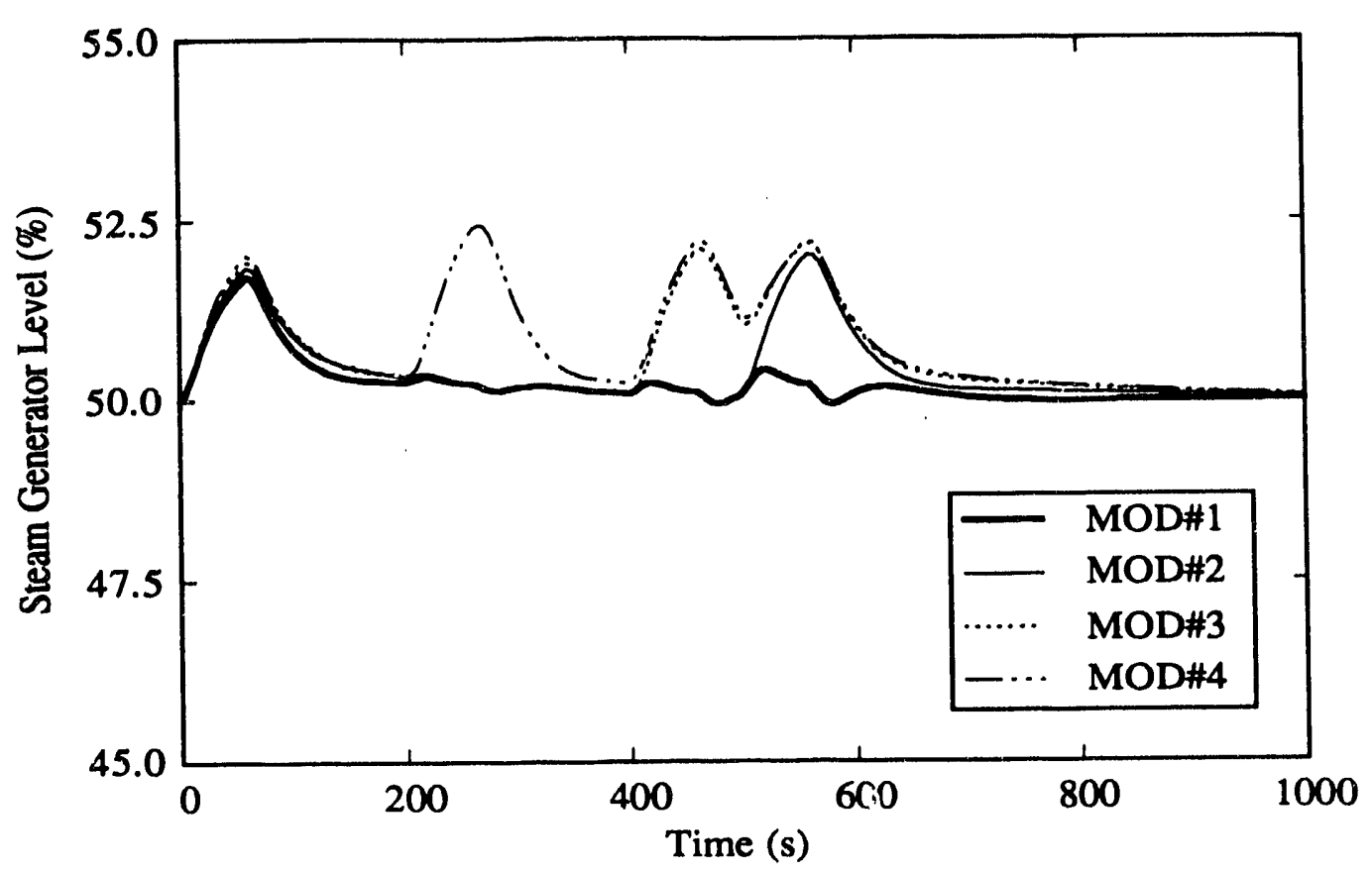

Figure 9.5.3.2-5 Steam Generator Levels. (Case 2 Transient)

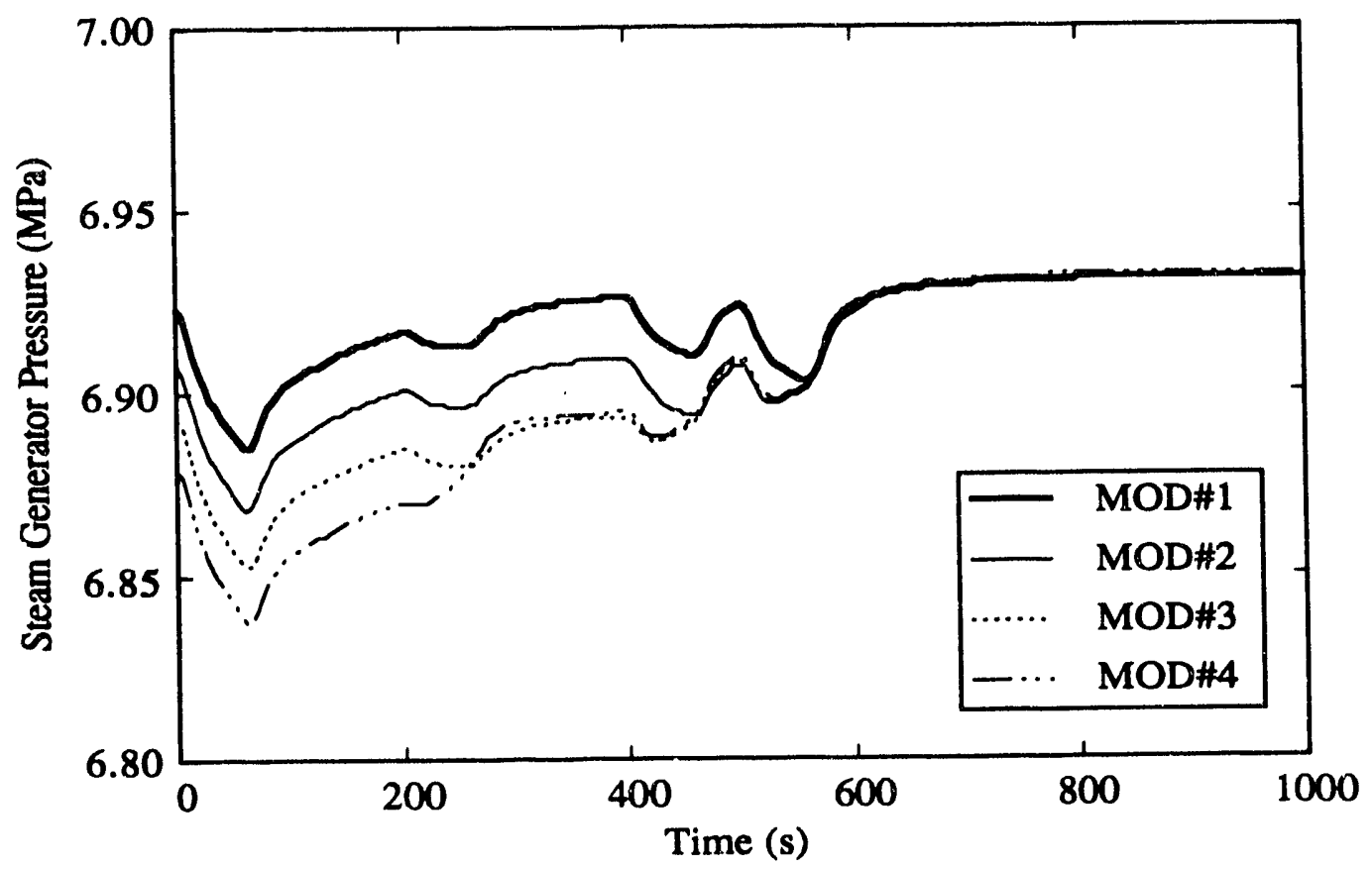

Figure 9.5.3.2-6 Steam Generator Pressures. (Case 2 Transient) 


\subsubsection{Case Three: Power Decrease in the Specified Power Demand Mode}

Case Three involves a power decrease in the specified power demand mode. Initially, each module is at a different power level. The transient entails lowerinis all module powers to $80 \% \mathrm{FP}$ with varying operating strategies. The power decrease sequences, the operating strategies, and the demanded allocation methods are given in Table 9.5.3.3-1. During the first phase, the operating strategy is 'extreme first.' Hence, power in the highest-power module, in this case module \#1, is decreased first because only that module is active in an 'extreme first' strategy. Once module \#1's power reaches 95 \%FP, module \#2 is also activated because its power has become equal to that of module \#1. This approach continues until all modules have attained the same power level. An 'equal change' strategy is then adopted. Figures 9.5.3.3-1 through 9.5.3.3-4 present the results of this evaluation. 
Table 9.5.3.3-1

Sequence for Power Decrease in Specified Power Demand Mode

\begin{tabular}{|c|c|c|c|c|c|c|c|c|}
\hline \multirow{2}{*}{ Phase } & \multicolumn{4}{|c|}{ Module Power } & $\begin{array}{c}\text { Turbine } \\
\text { Power } \\
(\% \mathrm{FP})\end{array}$ & $\begin{array}{c}\text { Operating } \\
\text { Strategy }\end{array}$ & $\begin{array}{c}\text { Demand } \\
\text { Allocation }\end{array}$ & $\begin{array}{c}\text { Initiation } \\
\text { Time }\end{array}$ \\
\cline { 2 - 8 }$\# 1$ & $\# 2$ & $\# 3$ & $\# 4$ & & & \\
\hline Initial & 100 & 95 & 90 & 85 & 92.5 & Extreme First & Path 3 & $0 \mathrm{~s}$ \\
\hline 1 & 95 & 95 & 90 & 85 & 91.25 & Extreme First & Path 3 & $100 \mathrm{~s}$ \\
\hline 2 & 90 & 90 & 90 & 85 & 88.75 & Extreme First & Path 3 & $300 \mathrm{~s}$ \\
\hline 3 & 85 & 85 & 85 & 85 & 85.0 & Equal Change & Path 1 & $500 \mathrm{~s}$ \\
\hline 4 & 80 & 80 & 80 & 80 & 80.0 & -- & -- & -- \\
\hline
\end{tabular}

Notes: (1) As discussed in Section 9.4.1.1 of this report, the demand allocation method depends on the operational mode and operating strategy. The path designations given in the table refer to:

Path \#1 - Use all modules.

Path \#2 - Use highest-power module and one or more of the lowerpower ones.

Path \#3 - Use lower-power modules.

(2) The 'initiation time' denotes the start of each operating strategy. If the action taken under the previous strategy was complete prior to the initiation of the next phase, then ₹ ime delay was arbitrarily imposed. Plant conditions were held fixed during these delays. Their only effect is to provide visual separation of the phases in the figures. 


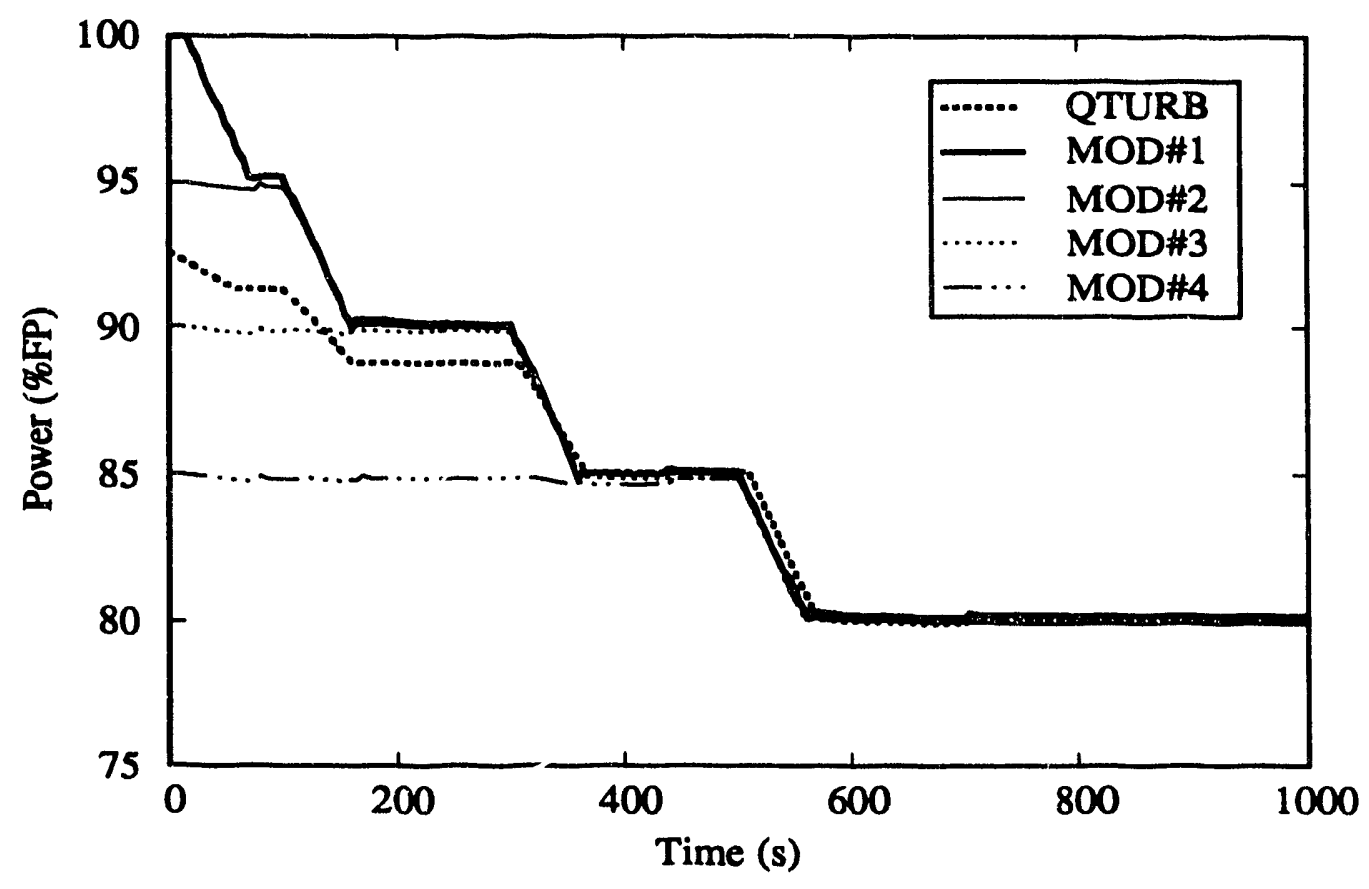

Figure 9.5.3.3-1 Module and Turbine Power. (Case 3 Transient)

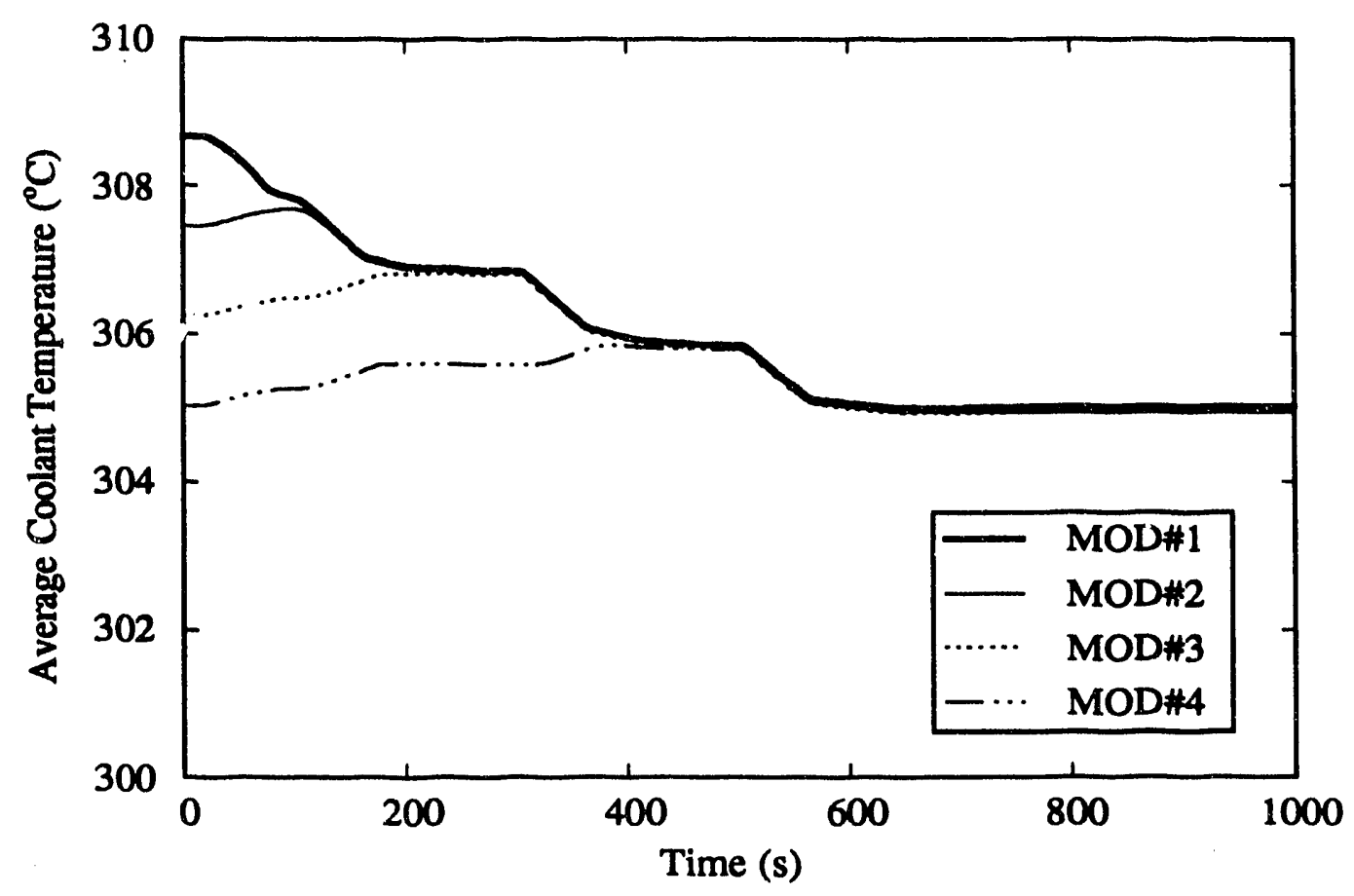

Figure 9.5.3.3-2 Average Primary Coolant Temperatures. (Case 3 Transient) 


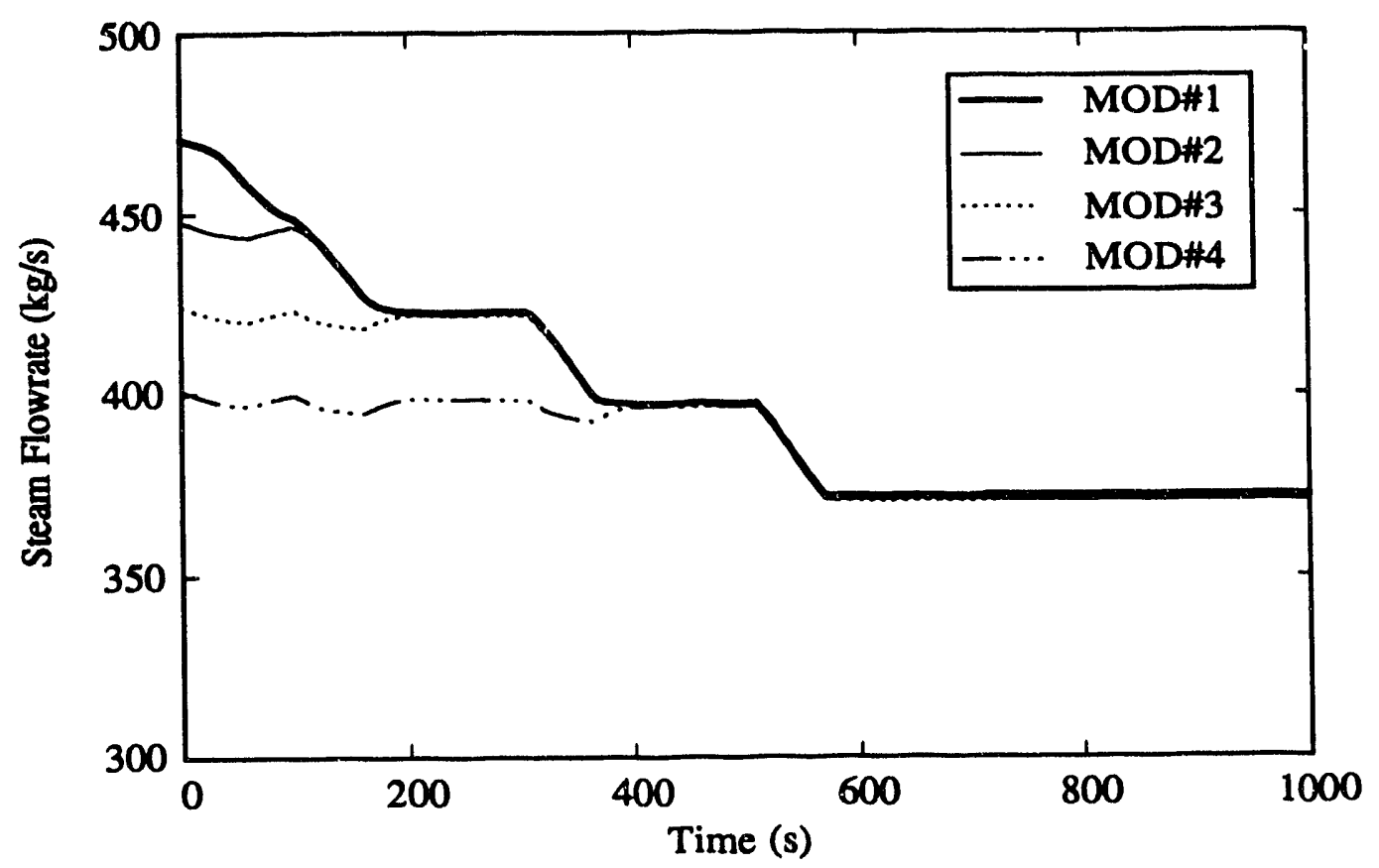

Figure 9.5.3.3-3 Steam Flowrates from Individual Steam Generators. (Case 3 Transient)

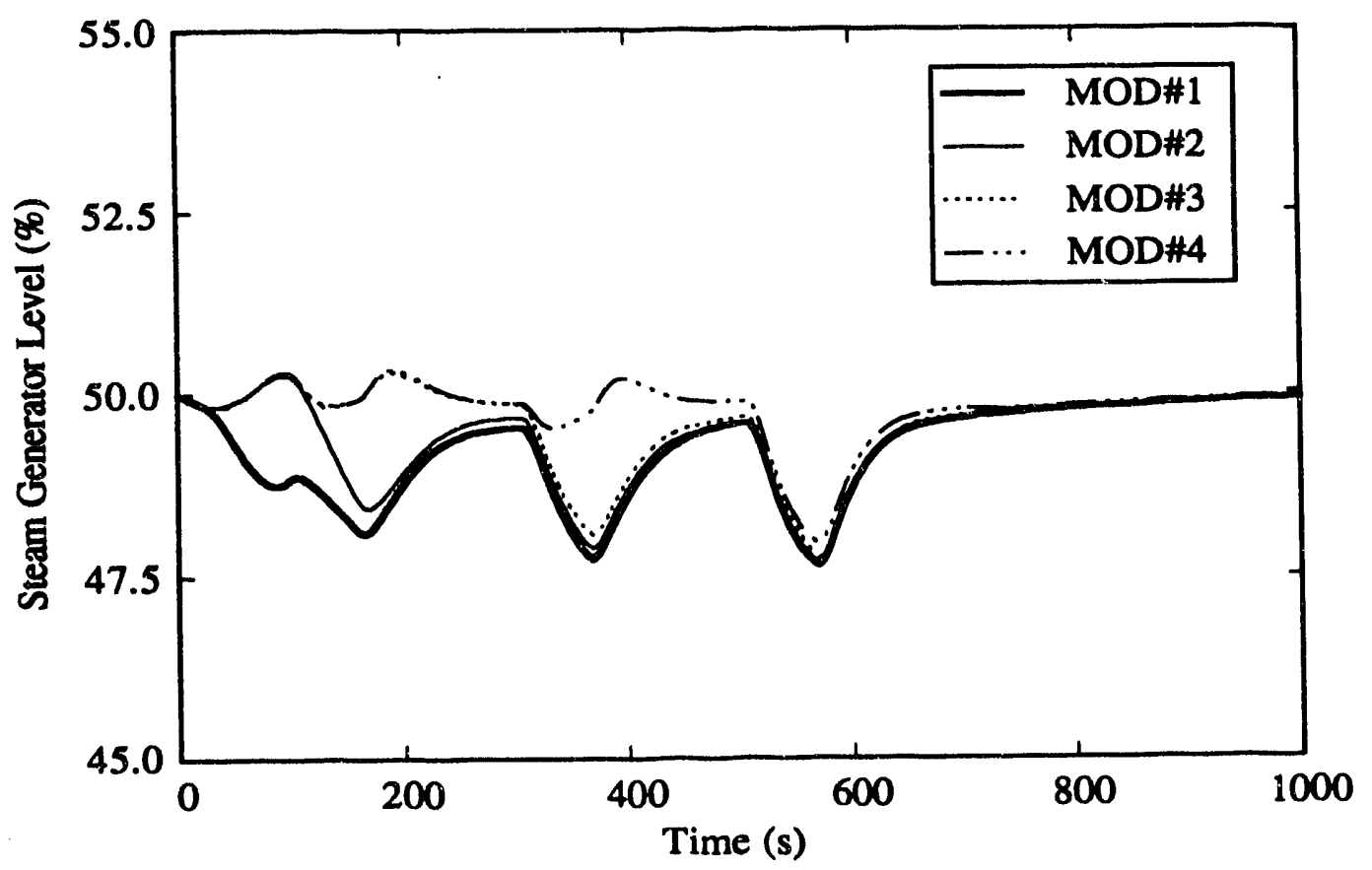

Figure 9.5.3.3-4 Steam Generaior Levels. (Case 3 Transient) 


\subsubsection{Case Four: Power Decrease in the Arbitrary Power Demand Mode}

Case Four is identical to the third case except that the arbitrary power demand mode is used for both module and turbine power demand allocation. Figures 9.5.3.4-1 through 9.5.3.4-4 present the results of this evaluation. 


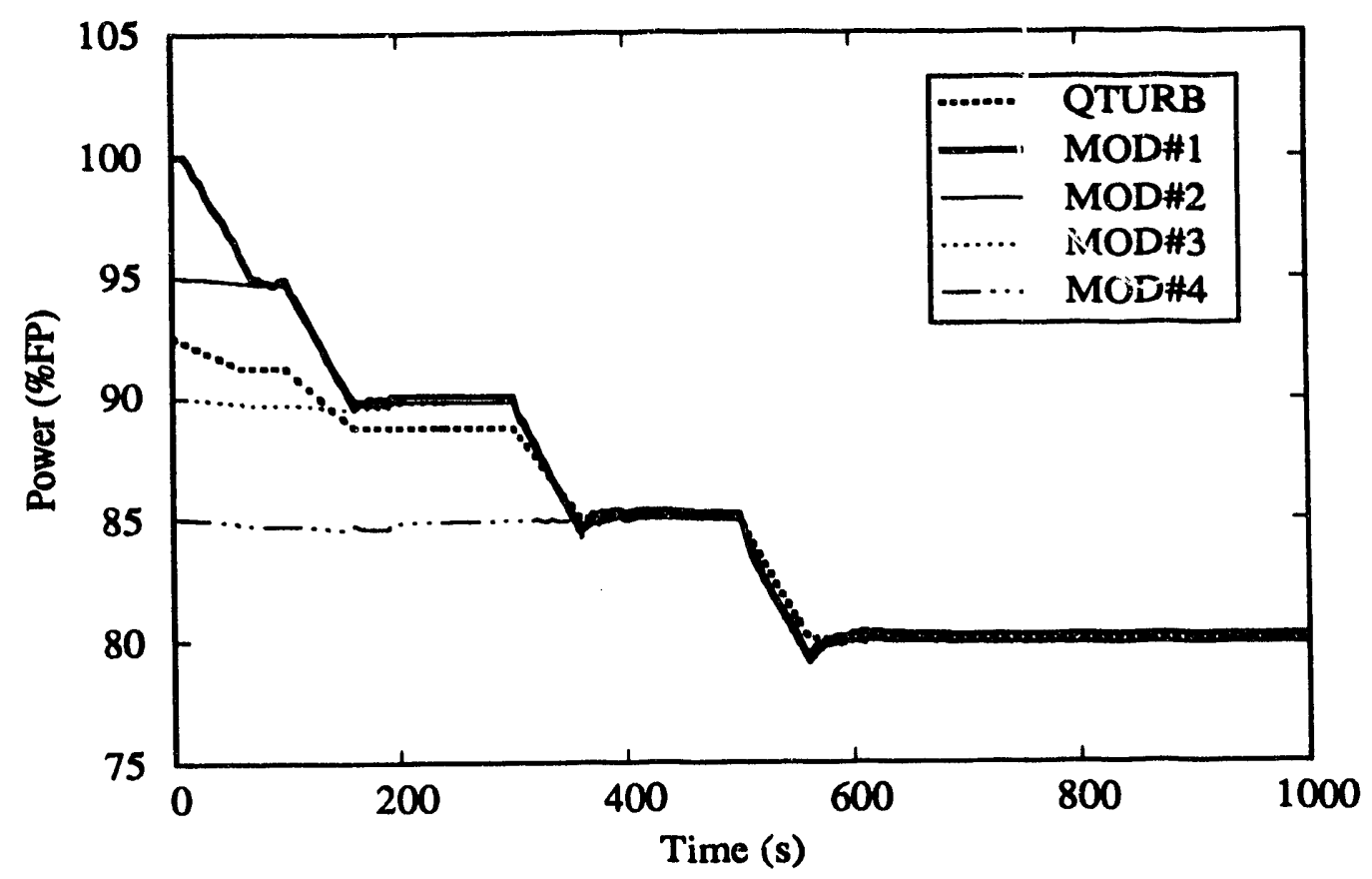

Figure 9.5.3.4-1 Module and Turbine Power. (Case 4 Transient)

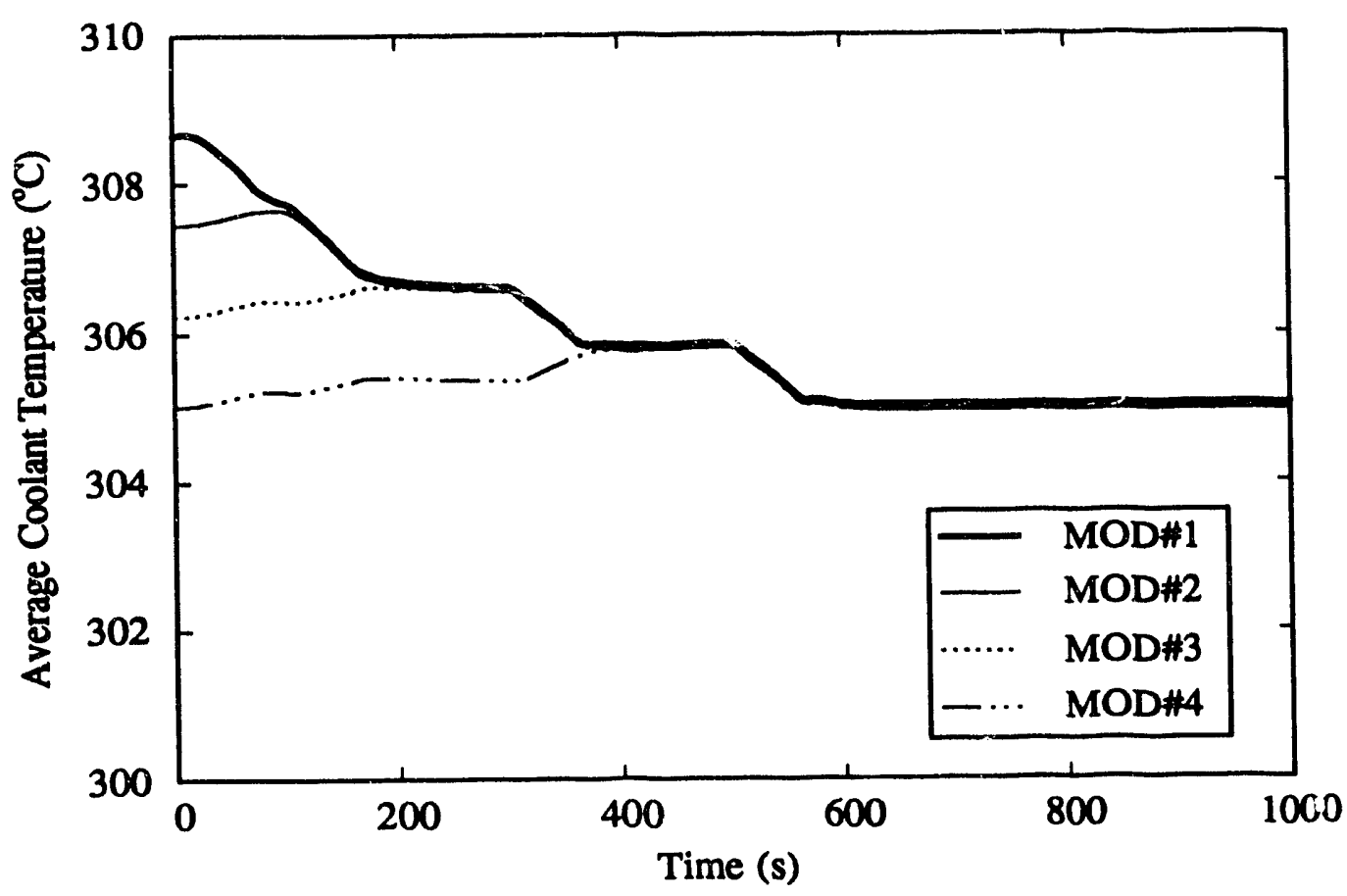

Figure 9.5.3.4-2 Average Primary Coolant Temperatures. (Case 4 Transient) 


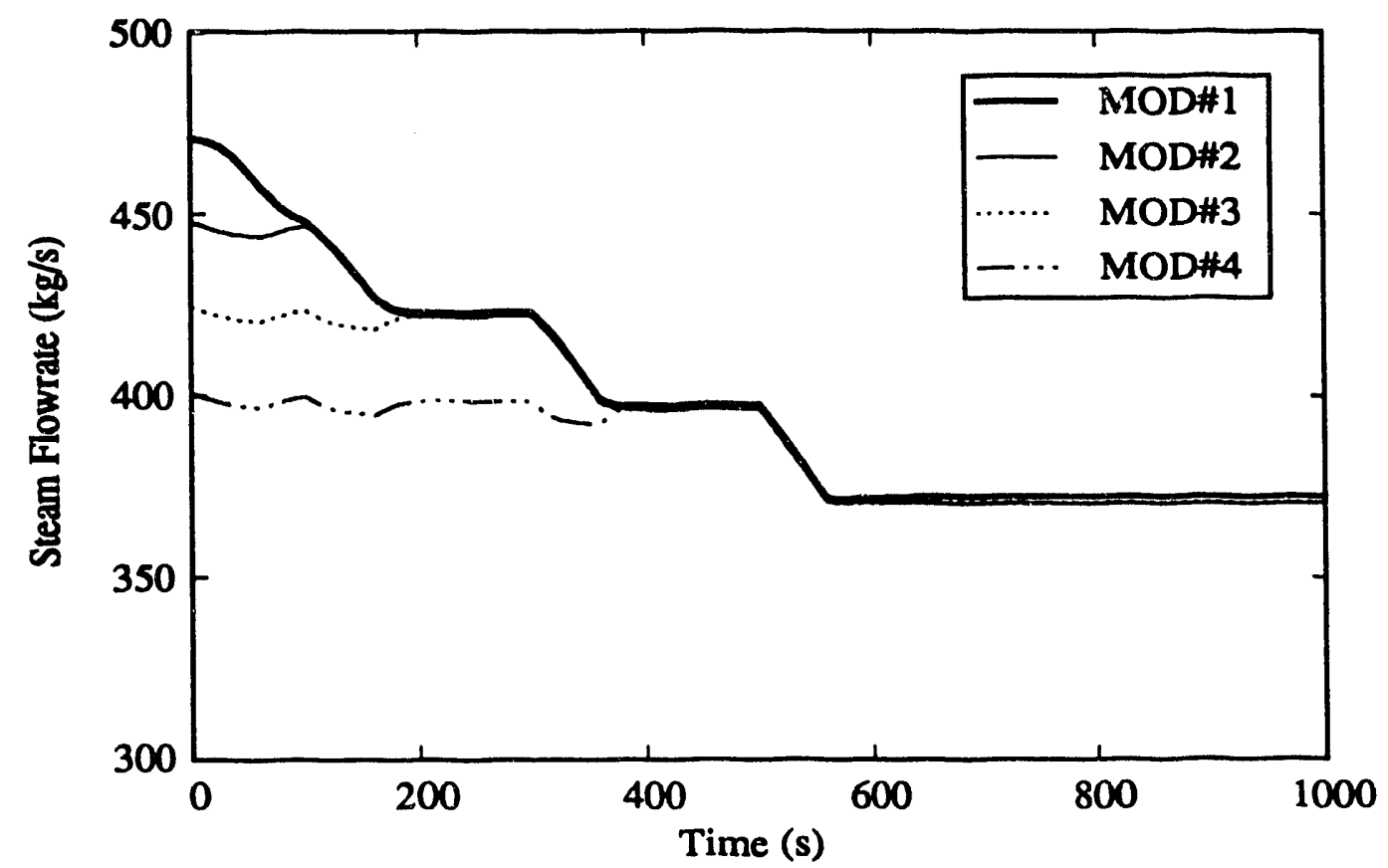

Figure 9.5.3.4-3 Steam Flowrates from Individual Steam Generators. (Case 4 iransient)

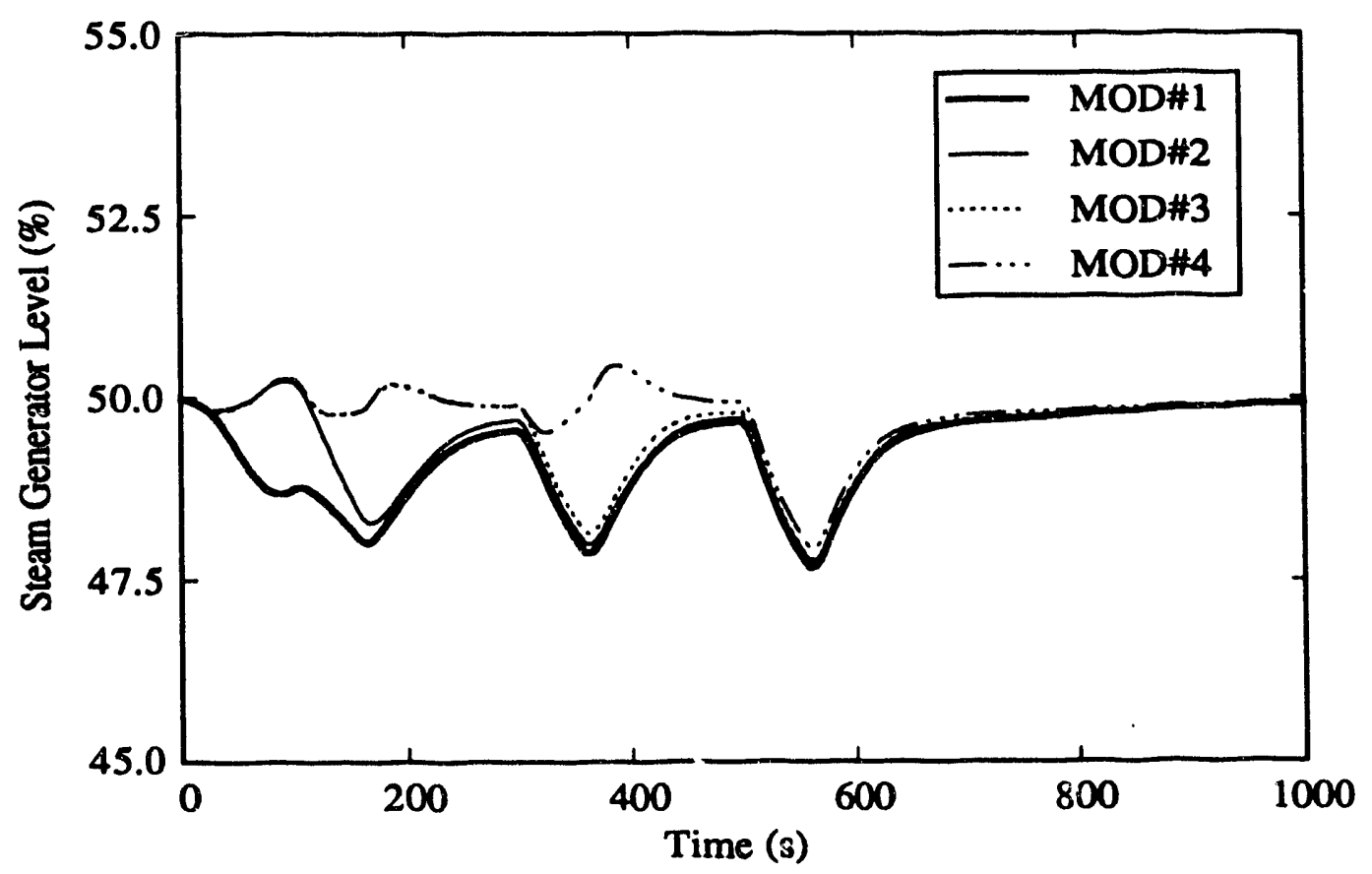

Figure 9.5.3.4-4 Steam Generator Levels. (Case 4 Transient) 


\subsubsection{Case Five: Power Transient When Demand Allocation Method is Changed}

Cases One through Four involved power increases or decreases under various operational modes and operating strategies. In these cases, the designated highest-power module did not change during the power ramp and hence the demand allocation method was not changed either. Case Five involves a transient in which the highest-power module is kept at the initial power and the lower-power modules are active. During the power ramp, the designated highest-power module is changed. This in turn necessitates a change in the demand allocation method. Table 9.5.3.5-1 gives the power increase sequences, the operating strategies, and the demand allocation methods for this transient.

Initially, the plant is at steady-state and module \#1 is the highest-power module. Hence, demand allocation path three is followed and the power of modules \#2, \#3, and \#4 is increased. Thirty seconds into the power increase, module \#4's power reaches $95 \% \mathrm{FP}$ and it is then designated as the highest-power module. Therefore, the demand allocation rule is changed from path three to path two for the remainder of the power ramp. Under the new rule, three modules are active. These are the highest-power one, which is now module \#4, and the two lower-power ones, modules \#2 and \#3. Module \#1's power is still held constant. As given in Table 9.4.1.1-1, the magnitude of the time delay of path three is +20 seconds while that for path two with three active modules is only +10 seconds. Hence, upon changing the demand allocation rule, the plant power controller holds module power at current levels for ten seconds so that instead of the original twenty second delay between the start of the module and turbine power ramps, turbine power is now delayed only ten seconds relative to that of the module power. During the latter stages of this simulation, a similar change again occurs. Figures $9.5 .3 .5-1$ through $9.5 .3 .5-4$ present the results of this evaluation.

Figures 9.5.3.5-5 through 9.5.3.5-8 show the simulation results for the same transient except that the arbitrary power demand mode is used. In this case, only the value of the leading time constant is changed when the demand allocation rule is switched. 
Table 9.5.3.5-1

Sequence for Power Transient with Change of Demand Allocation Method

\begin{tabular}{|c|c|c|c|c|c|c|c|c|}
\hline \multirow[t]{2}{*}{ Phase } & \multicolumn{4}{|c|}{$\begin{array}{l}\text { Module Power } \\
\text { (\%FP) }\end{array}$} & \multirow[t]{2}{*}{$\begin{array}{l}\text { Turbine } \\
\text { Power } \\
\text { (\%FP) }\end{array}$} & \multirow[t]{2}{*}{$\begin{array}{l}\text { Operating } \\
\text { Strategy }\end{array}$} & \multirow[t]{2}{*}{$\begin{array}{l}\text { Demand } \\
\text { Allocation }\end{array}$} & \multirow[t]{2}{*}{$\begin{array}{l}\text { Initiation } \\
\text { Time }\end{array}$} \\
\hline & $\# 1$ & $\# 2$ & $\# 3$ & $\# 4$ & & & & \\
\hline Initial & 95 & 90 & 85 & 92.5 & 90.625 & Preset Value & $\begin{array}{c}\text { Path 3 } \\
\text { w/Shift to } \\
\text { Path 2 }\end{array}$ & $0 \mathrm{~s}$ \\
\hline 1 & 95 & 95 & 90 & 97.5 & 94.375 & Preset Value & $\begin{array}{l}\text { Path 3 } \\
\text { w/Shift to } \\
\text { Path 2 }\end{array}$ & $200 \mathrm{~s}$ \\
\hline 2 & 100 & 100 & 95 & 97.5 & 98.125 & -- & -- & \\
\hline
\end{tabular}

Notes: (1) As discussed in Section 9.4.1.1 of this report, the demand allocation method depends on the operational mode and operating strategy. The path designations given in the table refer to:

Path \#1 - Use all modules.

Path \#2 - Use highest-power module and one or more of the lowerpower ones.

Path \#3 - Use lower-power modules.

(2) The 'initiation time' denotes the start of each operating strategy. If the action taken under the previous strategy was complete prior to the initiation of the next phase, then a time delay was arbitrarily imposed. Plant conditions were held fixed during these delays. Their only effect is to provide visual separation of the phases in the figures. 


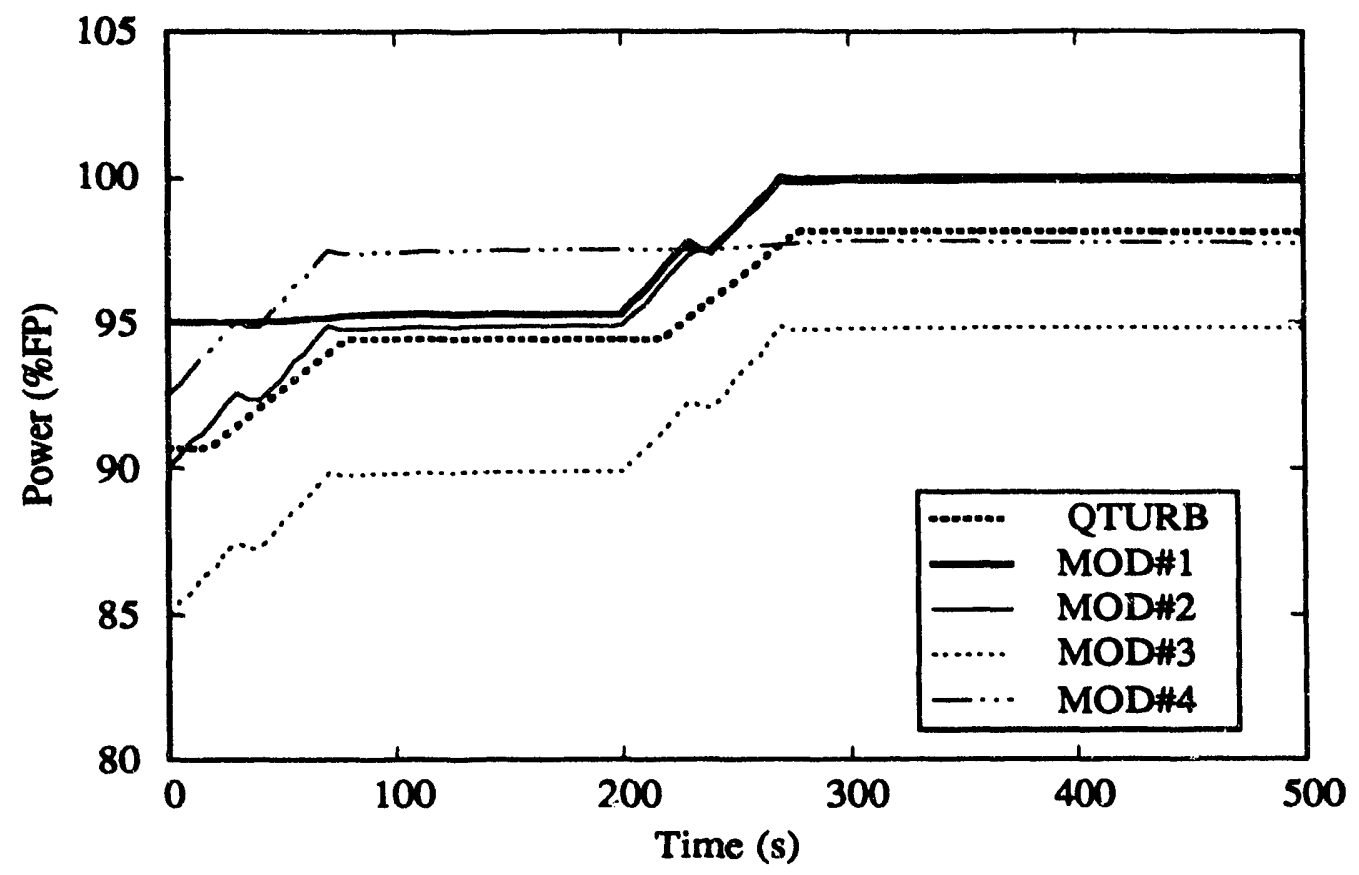

Figure 9.5.3.5-1 Module and Turbine Power in Specified Power Demand Mode. (Case 5 Transient)

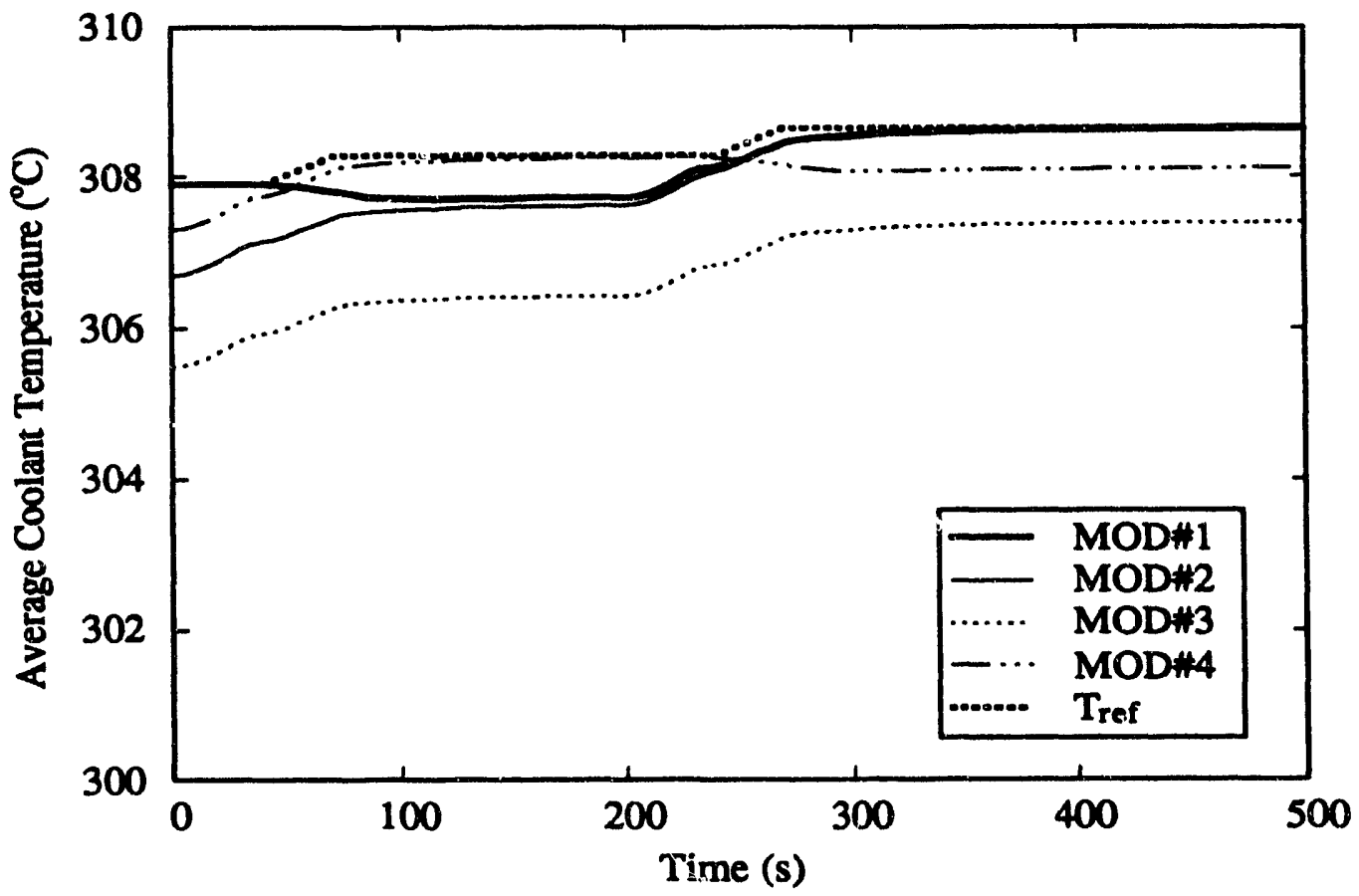

Figure 9.5.3.5-2 Average Primary Coolant Temperatures in Specified Power Demand Mode. (Case 5 Transient) 


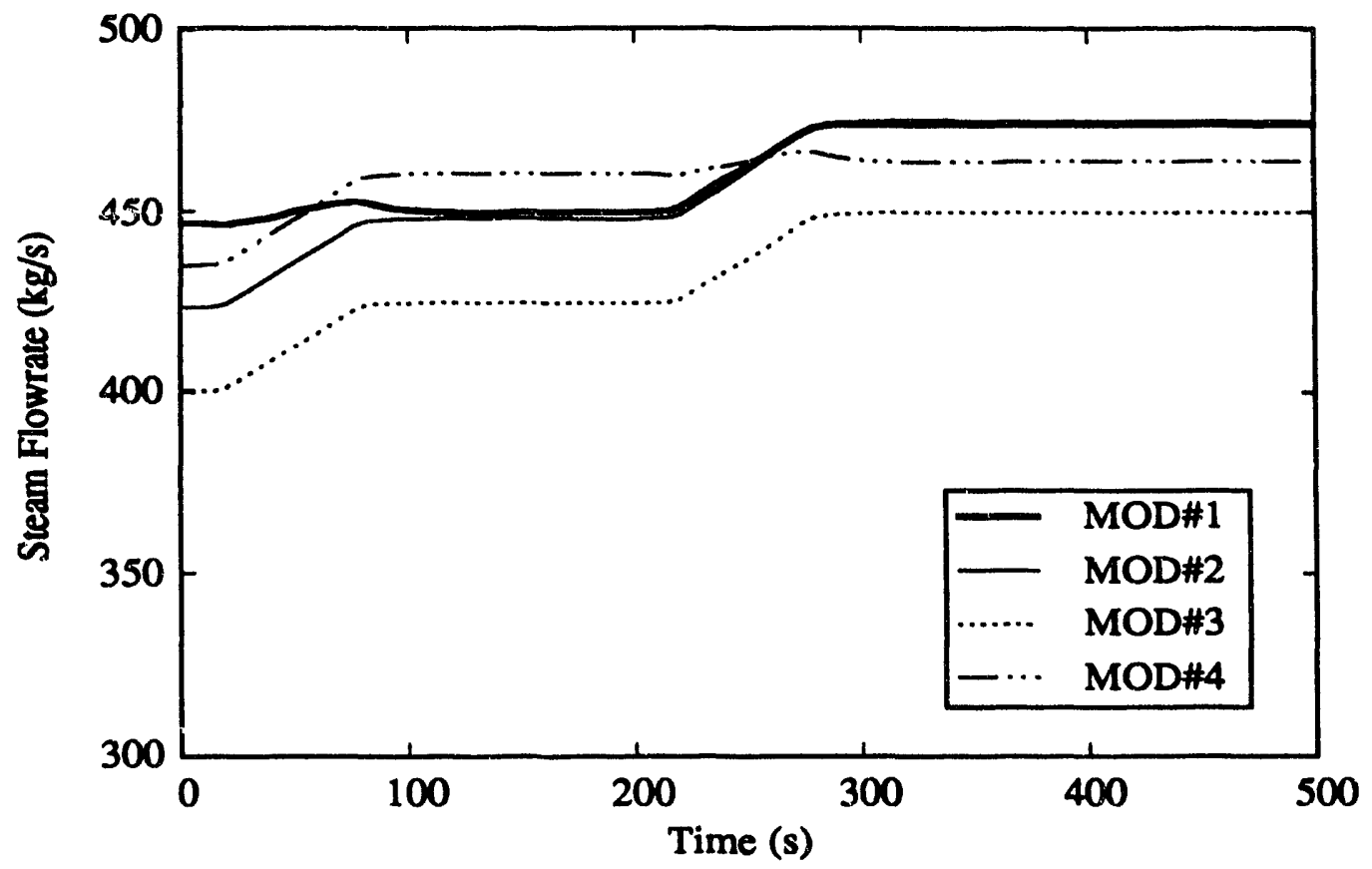

Figure 9.5.3.5-3 Steam Flowrates from Individual Steam Generators in Specified Power Demand Operational Mode. (Case 5 Transient)

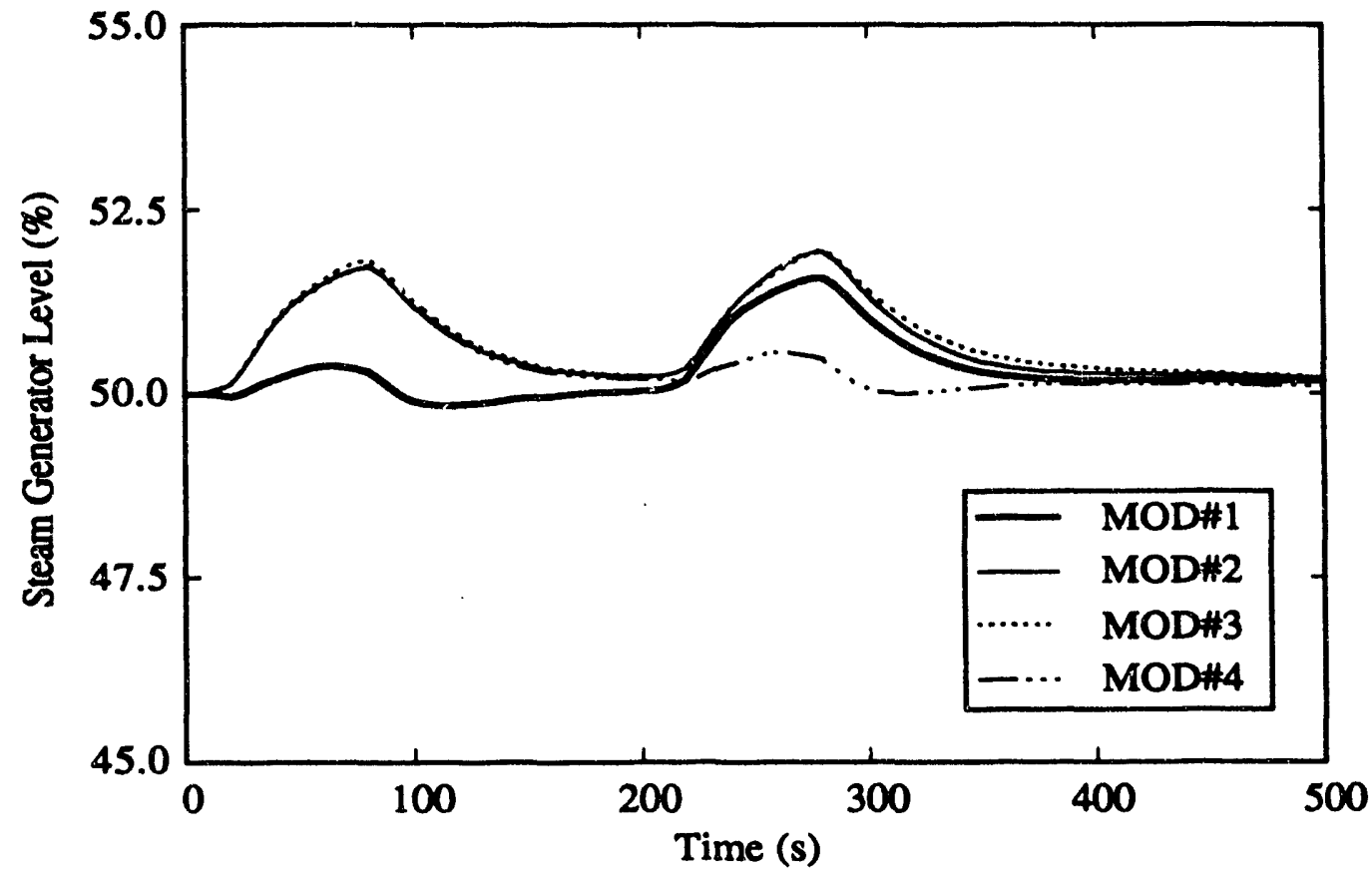

Figure 9.5.3.5-4 Steam Generator Levels in Specified Power Demand Operational Mode. (Case 5 Transient) 


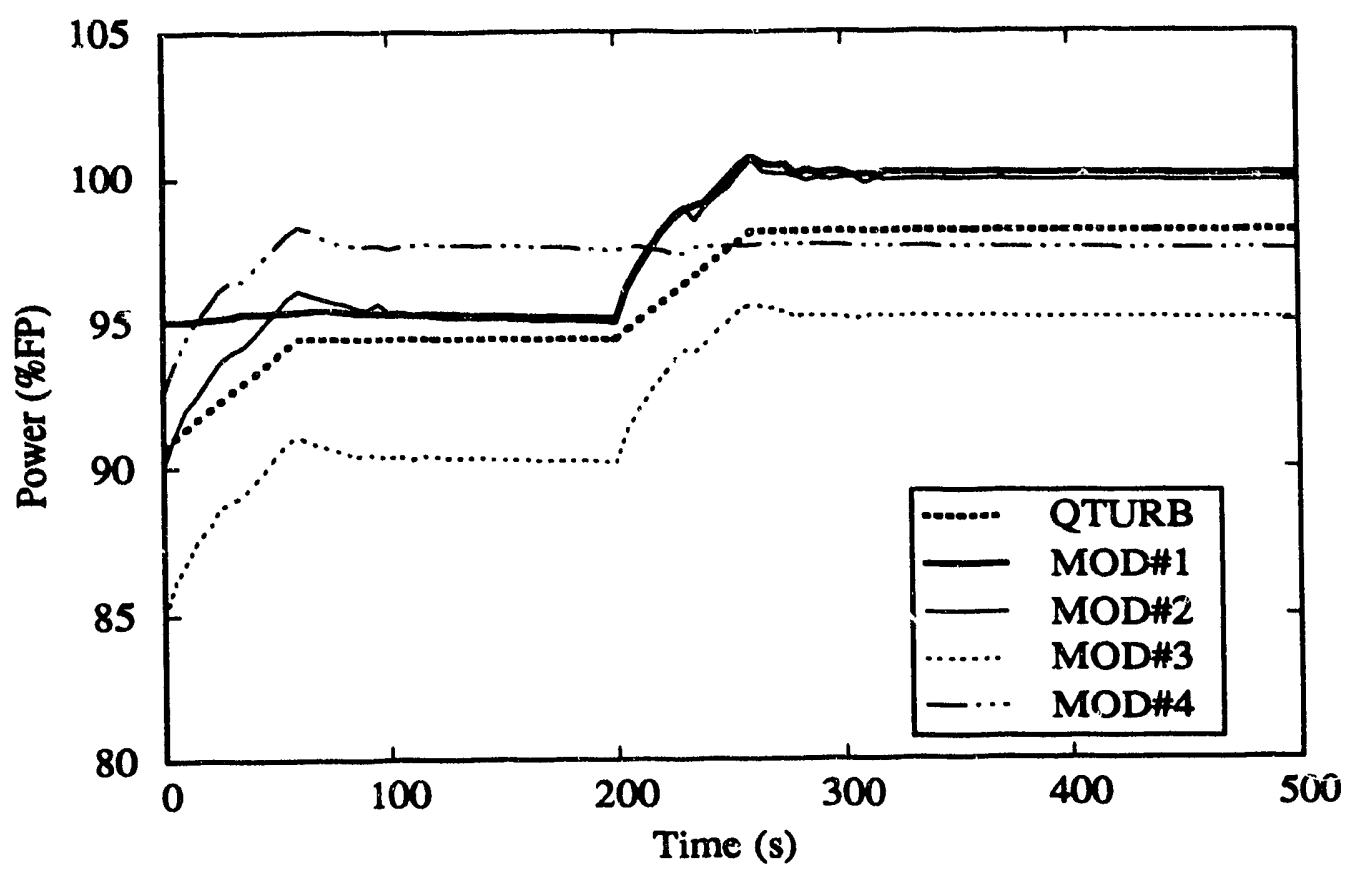

Figure 9.5.3.5-5 Module and Turbine Power in Arbitrary Power Demand Mode. (Case 5 Transient)

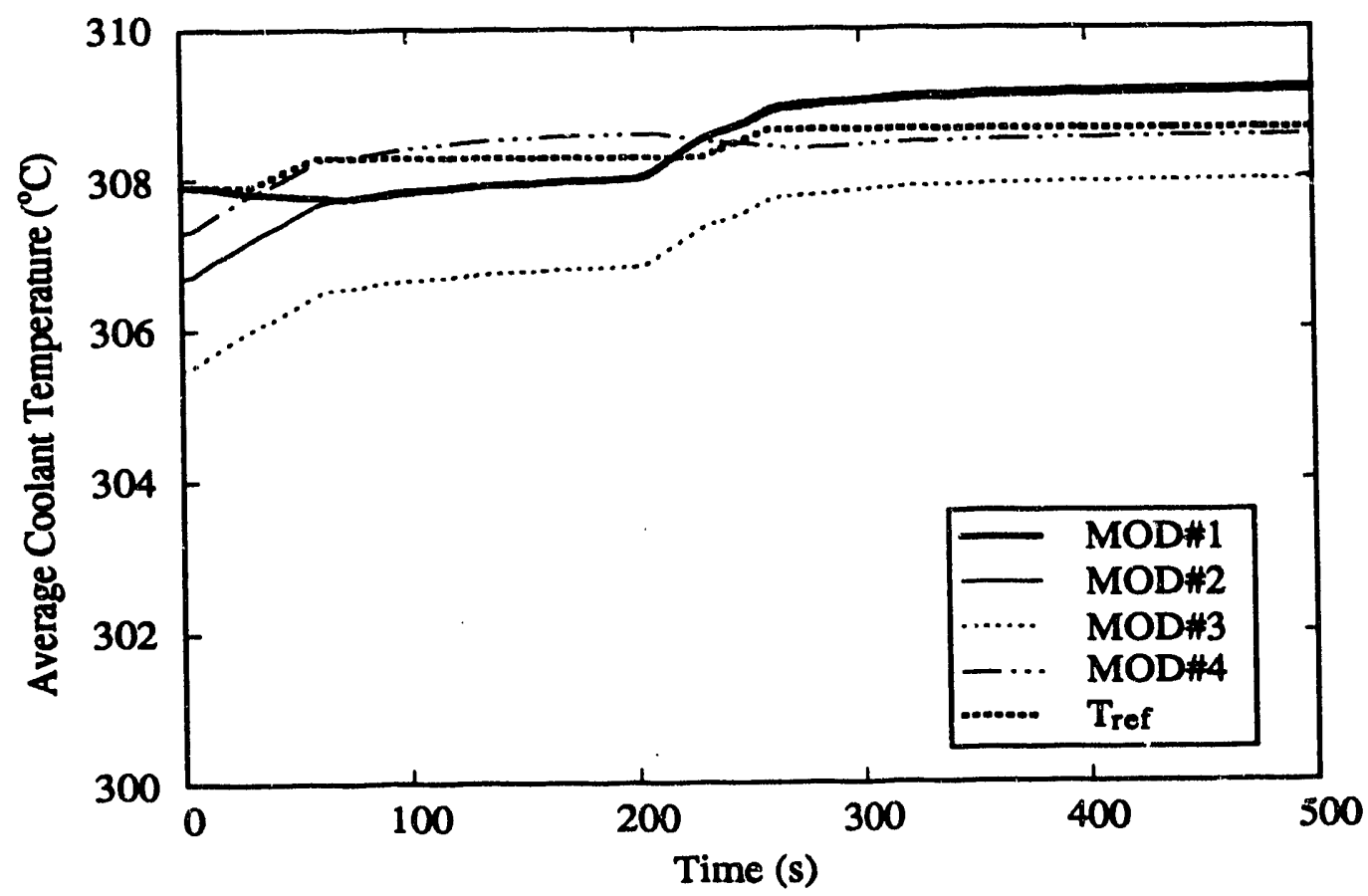

Figure 9.5.3.5-6 Average Primary Coolant Temperatures in Arbitrary Power Demand Mode. (Case 5 Transient) 


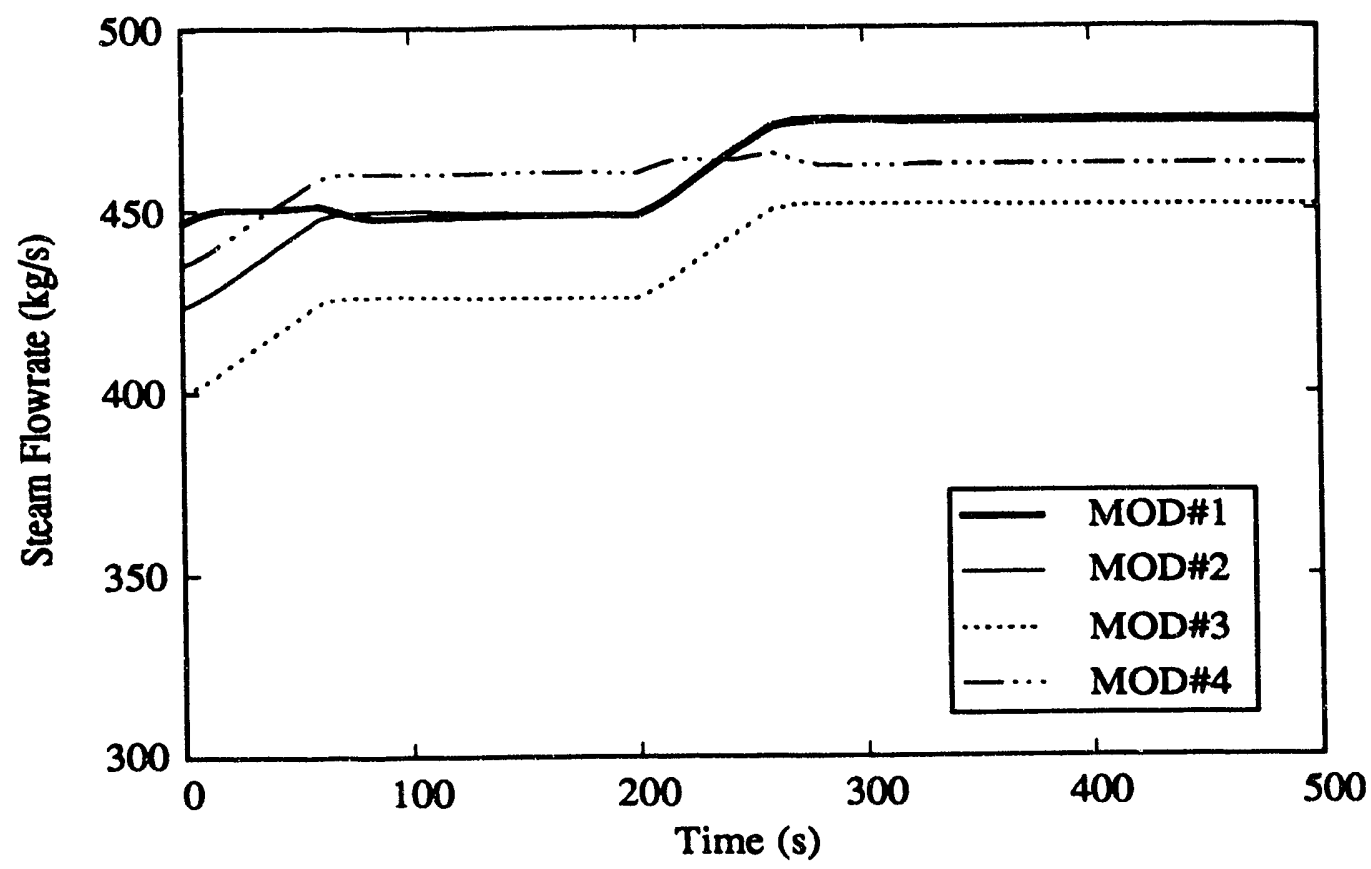

Figure 9.5.3.5-7 Steam Flowrates from Individual Steam Generators in Arbitrary Power Demand Operational Mode. (Case 5 Transient)

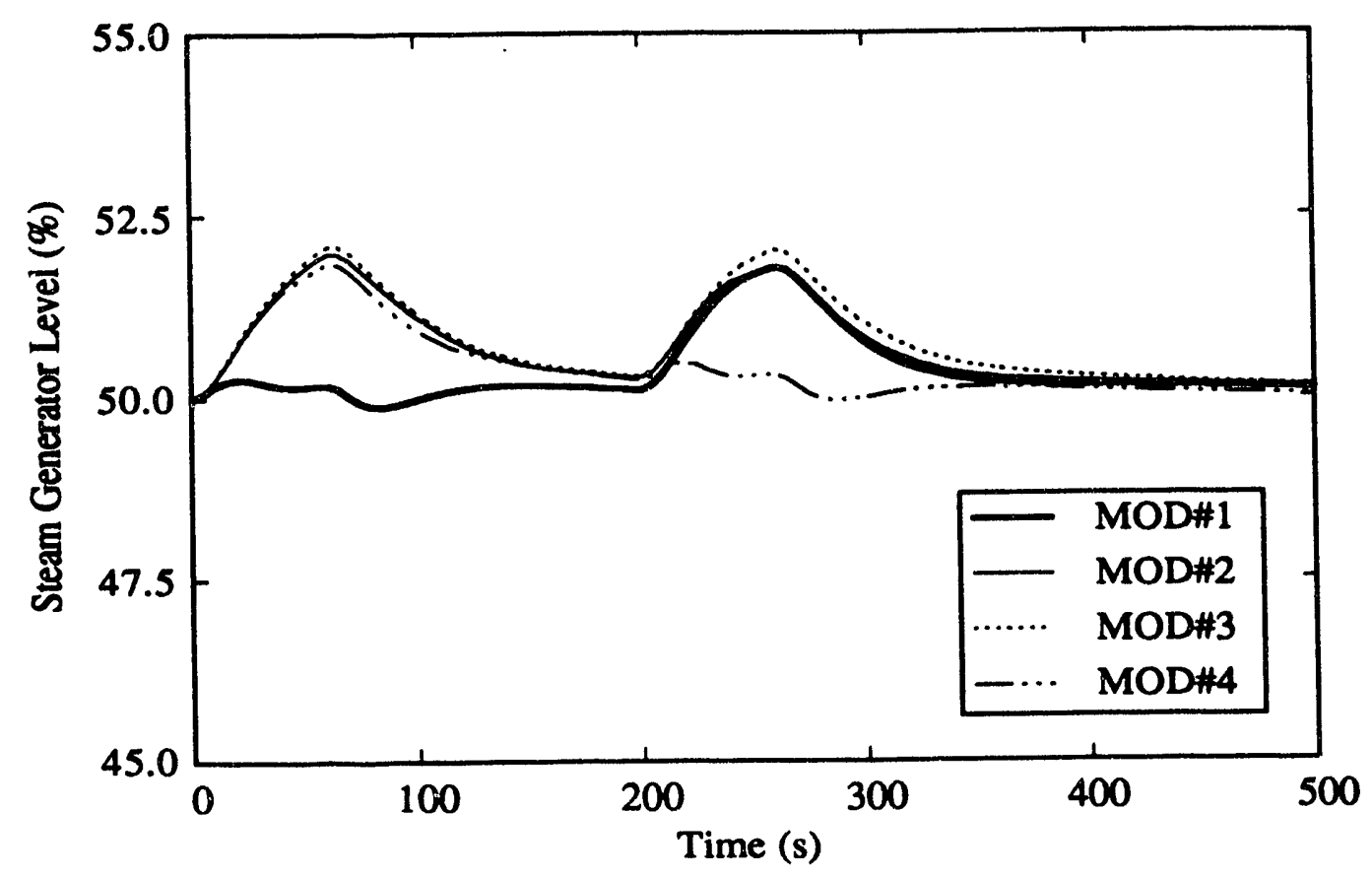

Figure 9.5.3.5-8 Steam Generator Levels in Arbitrary Power Demand Mode. (Case 5 Transient) 


\subsection{Assessment of PWR-Type Multi-Modular Power Plant Controller}

A system for the control of power and primary temperature in a PWR-type multimodular power plant was developed and evaluated through simulation studies. This controller functions under both transient and steady-state conditions without violating any safety limits. Also, it gives satisfactory control performance under a variety of operational modes and operating strategies. The control system consists of three different controllers arranged in a hierarchical architecture. The three levels are, in descending order, the plant power controller, the module power controller, and the rod controller. Also needed, but not addressed in this research, is a turbine steam controller and a primary system pressurizer controller for each module. Each controller has its own unique purpose. The plant power controller supervises the overall plant and allocates demand based on predeveloped rules that depend on the active operational mode and operating strategy. Several demand allocation rules were developed, all with the objective of achieving proper control of primary coolant temperatures during power transients. This controller would also provide the man-machine interface in that operators could select the operational mode and preferred operating strategy. The module power controller supervises transients so as to ensure that module power always remains within a predefined envelope of safe conditions. For this role, the reactivity constraint approach is applied. The rod controller interfaces directly to the plant signal and control elements. Its most important role is to position the control rods so as to cause module power to follow a demanded trajectory. Model-based feedforward action was combined with feedback to accomplish this objective.

The performance of the proposed power control system was demonstrated by simulating various operational transients on a PWR-type multi-modular power plant. The simulation program was PMSIM which was developed in this research and which was described in Chapter Seven of this report. The major concerns in these evaluations were the automatic control of module power and primary coolant temperature and the proper division of steam flowrate among the individual steam generators when operating with unbalanced loads. All evaluation results showed satisfactory control performance. In particular, the primary coolant temperature followed the desired trajectory within allowed error bands and the steam flowrates shared their desired fractions without any unsatisfactory oscillations among the modules. 
10. Issues Regarding the Design and Acceptance of Inielligent Support Systems for Reactor Operators $(1)$

One of the objectives of the MIT program on the operation and control of multimodular power plants was to investigate the potential for the use of expert systems. Accordingly, factors relevant to the design and acceptance of intelligent support systems for the operation of nuclear power plants are enumerated and discussed in this chapter. The central premise is that conventional expert systems which encode experiential knowledge in production rules are not a suitable vehicle for the creation of practical operator support systems. The principal difficulty is the need for real-time operation. This in turn means that intelligent support systems will have knowledge bases derived from temporallyaccurate plant models, inference engines that permit revisions in the search process so as to accommodate revised or new data, and man-machine interfaces that do not require any human input. Such systems will have to be heavily instrumented and the associated knowledge bases will require a hierarchical organization so as to emulate human approaches to analysis. Arguments to support the above findings are made by first examining the needs of licensed reactor operators and then reviewing the capabilities of conventional expert systems. Differences between existing expert systems and proposed intelligent support tools are then identified by comparing certain design features including the source and content of the knowledge base, the mode of interaction with the user, knowledge organization, the inference engine, and the man-machine interface. Issues related to operator acceptance of intelligent support tools are then reviewed. Possible applications are described and the relative merits of the machine- and human-centered approaches to the implementation of intelligent support systems are enumerated. The chapter concludes with a plea for additional experimental evaluations.

\subsection{Statement of Problem}

In 1989, Bernard and Washio published a state-of-the-art review on the utilization of expert systems in the nuclear industry [5]. Some 287 expert systems were identified as either under development or in use within the nuclear industry. These included systems for use as engineering tools, the capturing of human expertise, plant design, facility management, maintenance planning, interactive diagnostics, real-time diagnostics, decision support, emergency response, cognitive models, and control. The numerical breakdown of these systems by application is shown in Figure 10.1-1. In addition to enumerating expert systems activities, the study sought to identify major trends and to reveal unresolved issues. Three findings stood out. First, about twenty-five percent of the identified expert systems were being developed to assist humans with managerial responsibilities in the areas of plant design, management, and maintenance. Examples included the scheduling of work permits, regulatory compliance, repair of complex equipment, and the interpretation of water chemistry analyses. None of these tasks required real-time performance and few were done by licensed reactor operators. A second trend was that enormous resources were being devoted to the development of expert systems that would assist licensed reactor

(1) This material was originally presented by Dr. Bernard as a keynote address at the 2nd International Forum on Expert Systems and Computer Simulation in Energy Engineering held 17-20 March 1992 in Erlangen, Germany under the auspices of the International Centre for Heat and Mass Transfer, Belgrade, Yugoslavia. It was subsequently published in IEEE Transactions on Nuclear Science, V'ol. NS-39, No. 5, Oct. 1992. 


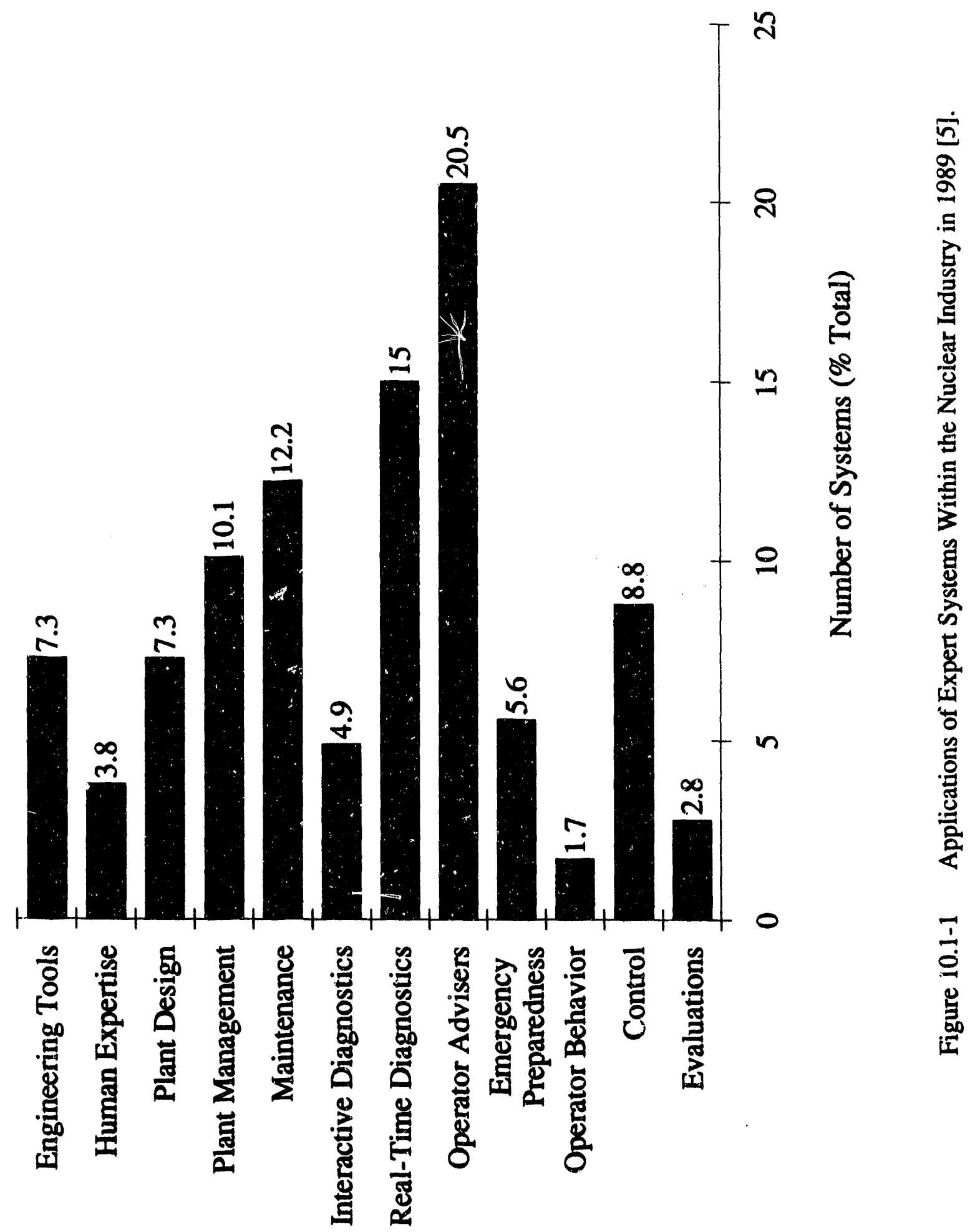


operators in the discharge of their responsibilities, especially emergency response. This is evident from the figure which shows that forty percent of the total number of identified expert systems were for real-time diagnostics, operator advisers, or emergency preparedness. A third, and very disturbing trend, was that little had been done in the way of experimental work to assess operator response to expert systems. A further discussion of these three trends as well as other findings has previously been given [38].

More than two years have passed since the completion of the original state-of-theart review and it is now appropriate to revisit the findings of that study. The material presented here is one result of that reexamination effort. Its focus is on the progress made in developing intelligent technologies for the direct support of reactor operators in the discharge of their licensed duties.

The most gratifying change that has occurred during the past two years is that several operator-support expert systems have either become operational or are very close to that goal. In the United States, the most prominent is the Reactor Emergen " Action Level Monitor or REALM which was developed for the Indian Point Unit 2 plant and is now also under consideration for San Onofre [104,105]. In Asia, the Emergency Operating Procedures Tracking System (EOPTS) has undergone extensive and very successful testing at the Taiwan Power Company's Kuo Sheng plant [106]. Also, the Japanese national effort on the Advanced Man-Machine System for Nuclear Power Plants (MMSNPP) continues to progress [107]. In Europe the very impressive SAS-II system is undergoing testing at Sweders s Forsmark plant where it will be used to assist shift supervisors in following emergency operating procedures [108]. The success of these and other systems is proof that witt: proper attention to the issues of validation and verification, expert systems technology cart make a contribution to control room operation. However, other less-positive trends that bear on the utilization of operator-support expert systems are also apparent. First, despite the enormous effort that was mounted in the late 1980s, most of the nuclear expert systems that are now becoming operational are not for the direct support of reactor operators. Rather, they are to assist non-licensed personnel in such areas as safety review analysis, water chemistry control, maintenance scheduling, core refueling, compliance with proper welding procedures, and plant life extension. Second, expert systems are more readily being used in a support role in the non-nuclear aspects of the electric power industry such as fossil plant operation and especially load dispatching. Third, there is a growing realization that the traditional rule-based expert system will not suffice for the development of a useful operator-support tool. Rather a combination of analytic methods, primarily system models, and A/I programming techniques is needed.

This Chapter examines issues that bear on the design and acceptance of intelligent support systems. Its specific objectives are to: (1) discuss the needs of reactor operators in the performance of their licensed duties, (2) review the use of rule-based expert systems with emphasis on the types of application to which they are suited, (3) enumerate the unique design requirements of real-time intelligent support systems, and (4) identify factors relevant to operator acceptance $c$ ! these systems. 


\subsection{Operator Needs}

As an illustration of an operator's needs, consider the following sequence of events. A nuclear plant is operating under steady-state conditions when the on-duty operator notes that condenser vacuum is a little low for the reactor's current power level. No setpoint has been violated and the indicator on the vacuum gauge is oscillating slightly with the expected value being occasionally attained. Unless additional information in the form of redundant measurements is available, no decision can be made as to whether the problem is a failing instrument or an actual incipient loss of vacuum. The operator focuses his or her attention on the condensate system and, over the next few minutes, observes a small but definite decrease in vacuum. What might be the cause? Perhaps the air ejector, which is a steam-driven jet pump that removes non-condensable gases, is not operating at its design pressure. Or perhaps one of the plant's many drain tanks that collect fresh water from pump seals and other components and direct it to the condenser has become uncovered. Or perhaps the condenser's pneumatically-operated hot well level control valve is maintaining the water level improperly. The corrective actions for each of these possibilities are obvious. A second air ejector can be placed on line, the offending tank can be secured, or the hot well level can (with difficulty) be maintained manually. Unfortunately, each of these actions imposes a penalty on plant operation. Thus, the challenge to the operator is to decide which of the possible initiating events is in fact causing the loss of vacuum. To continue the scenario, suppose that while the operator is gathering information to make an informed decision, the hot well level control valve fails open. The sudden surge of comparatively cool condensate may effect operation of the feedwater pumps and hence jeopardize heat removal from the reactor via the steam generators. The operator is suddenly faced with a potentially major crisis.

What can we conclude about the needs of licensed reactor operators? First, as a result of their extensive training and operating experience, most operators have excellent pattern recognition skills and can readily distinguish between expected and abnormal plant behavior. For example, the operator in the illustration realized that the vacuum gauge reading was inappropriate for the plant's operating state. Second, operators know the appropriate response for a given casualty. When difficulties do arise, they are most often the result of inability to identify the nature of the problem. This was the case in the scenario given above. The operator initially lacked sufficient information to distinguish between a failing sensor and an actual casualty. Later, after it had become evident that the loss of vacuum was real, he couldn't determine which of three possible initiating events was the true cause. An operator's principal need is therefore for timely, accurate analyses of actual plant conditions.

The nuclear industry has, of course, long recognized the difficulties associated with accurate, real-time diagnostics. During the 1960 s, the approach taken was to provide licensed operators with abnormal operating procedures (AOPs) for every conceivable casualty. Each AOP was characterized by a unique set, or in some cases a time-dependent sequence, of alarms. This event-oriented approach was challenging even to those who operated plants of only a few hundred megawatts and, as plant sizes grew, it became totally impractical. One alternative was to increase the number of instrument readouts available to the operator and thereby reduce the number of initiating events that could only be 
distinguished by a different sequence of the same set of alarms. However, this merely swamped the operator with information. In the early 1980 s, the industry introduced symptom.based procedures which direct the operator to treat the consequences of a malfunction without necessarily identifying it as to initiating event. For example, instead of rectifying the cause of a plant upset, the operator is to focus his activities on ensuring that certain safety functions, such as heat removal and containment integrity, are maintained. While this symptom-based approach is an improvement, it results in lengthy procedures that have multiple entry points. The result is that operators must often do many tasks in parallel. Expert systems, such as the Emergency Operating Procedures Tracking System [106], have been developed to assist with this problem. Another deficiency of symptom-based procedures is that, while they are very useful in mitigating the consequences of major problems, they may not prevent the propagation of minor difficulties such as an incipient loss of condenser vacuum. For that, a definitive identification of the initiating event is needed. Hence, despite improvements such as symptom-based procedures, the need still exists for a real-time diagnostic capability. This is one of the major challenges to the designers of intelligent support systems for reactor operators.

\subsection{Expert System Capabilities}

Expert systems are a special type of comp w sor tware for which the objective is to reproduce the capabilities of an exceptionally talented ivman or group of humans. This is achieved by encoding human experience in various knowledge representation schemes. Although many such schemes are available, most expert systems are rule-based. That is, the knowledge is represented as a set of production rules that are in the form "if condition A and condition B are present, then the following regulation applies." The function of the expert system is first to identify the current plant condition and then, via its inference mechanism, to compare the antecedent clauses of each production rule against the observed plant status. If a match exists, the rule is considered to be applicable. A characteristic feature of expert systems is that the experience of the human experts (the knowledge base) is kept separate from the method by which that experience and information is accessed (the inference engine). Expert systems differ from conventional algorithmic programming in two respects. First, as new information is obtained, it can be added to the knowledge base without revising the inference engine. That is, no reprogramming is needed. Second, an expert system can at any time provide the rationale for its conclusions. It does this by keeping track of the chain of deductions that support each particular conclusion. These two features account for much of the appeal of expert systems technology to the nuclear industry. Separation of the knowledge base and inference mechanism means that, as the plant's layout is changed or as new regulations are imposed, the knowledge base can be updated without incurring the need to revise the inference mechanism. If a conventional programming technique were used, the entire program would require revision because the knowledge and the method for its interpretation would be intertwined and dependent on each other. Similarly, the capability to explain the logic used in arriving at a conclusion is of great benefit in an industry where virtually every decision must be documented to show proof of regulatory compliance. 
The nuclear industry embraced expert systems with great enthusiasm in the mid1980s. Among the systems described at a recent conference were the Component Degradation Assessment Tool for use by Yankee Atomic, the Licensing Review Assistant for use by EPRi-member utilities, the Safety Review Advisor developed by Sargent and Lundy, the Knowledge Assisted Tag-Out system for Consolidated Edison, and the Welding Manual Advisor for Northern States Power [109]. Other specific applications for which expert systems have been successfully developed include technical specification monitoring, plant life extension, the planning of crane movements during refuelings, steam generator tube bundle inspection, and analysis of demineralizer performance. Systems of this type exhibit the following traits:

1. The intended users of the expert system are generally not reactor operators. Rather, they are plant managers, welders, chemists, quality assurance supervisors, or startup engineers. This may be an advantage in that, unlike reactor operators, these user groups are highiy defined. Hence, the design of the man/machine interface may be simpler.

2. The systems being developed are for the purpose of assisting, not replacing or supplanting, a human. The objective is to improve productivity by giving the user more immediate access to necessary information.

3. Many areas of application are highly focused. This limits the extent of the knowledge base needed to support the expert system. That means that many issues related to the system's construction and implementation are simplified.

4. The expert system is accessed in an interactive manner with the system periodically querying the human user for additional information.

5. The content of the knowledge base is, in some cases, derived from a human expert. In others, it is a set of regulations or a piping diagram. However, in all cases it is static.

The above features are in many ways responsible for the success achieved in applying expert systems technology to the areas of plant management, maintenance, and interactive diagnostics. Hence, it is ironic that some of these same characteristics are the stumbling blocks that preclude extension of the technology to real-time diagnustics. For example, a narrowly-focused knowledge base would be inappropriate because of the interdependency of plant components. If real-time diagnostic expert systems are to avoid being both incomplete and brittle, then they must have knowledge bases that encompass the entire plant. Similarly, interactive interfaces would only distract a licensed reactor operator from monitoring the plant at the very time that his full attention was needed most. Alse, a static knowledge base would be of little benefit. Real-time diagnosties requires information on the temporal sequence of events. For example, a pressurizer high pressure alarm followed by one of low pressure might mean that a relief valve had opened and become stuck. In contrast, the reverse sequence (low pressure followed by high) suggests that the normal bank of pressurizer heaters failed to energize, the backup bank therefore came on, and for some reason remained on. 
Many of those seeking to develop an expert diagnostic capability have concluded that the traditional concept of a rile-based system with experiential knowledge is flawed. One of the drawbacks to rules is that they proliferate rapidly and hence are not a practical means for organizing large knowledge bases. Also, there are no human experts when it comes to diagnostics. It is not a case where a few reactor operators are proficient and the rest are not. Rather most, if not all, son-etimes experience difficulty because of a lack of information. Thus, the creation of expert systems for real-time diagnostics can not be achieved through the extension of the concepts observed in the development of off-line systems. Rather, the entire process needs to be rethought and new approaches idersified.

\subsection{Desien of Real-Time Intelligent Support Systems}

Real-time intelligent support systems for reactor operators are under development at several centers of nuclear engineering research. The material presented heie is drawn from reports issued by these centers as well as from on-going research at the Massachusetts Institute of Technology (MIT).

\subsubsection{Source of Knowledge}

The traditional method for the construction of an expert system is for a knowledge engineer to identify a human expert and then, through a series of interviews, to extract the relevant information. This approach is not recommended for the design of support systems that offer a real-time diagnostic capability. One issue has already been noted. Namely, there may not be sufficient information, in the form of sensor readings, available to the operator. Thus, no matter how talented the individual may be, he might not be capable of rendering a complete diagnosis and therefore rules derived from his understanding of the plant might prove inadequate. A second issue is that diagnosis is often a multi-disciplinary problem requiring several types of knowledge including that of the operators regarding procedures and system interactions, that of engineers concerning thermodynamics and fluid behavior, and that of plant designers relative to component specifications and layout. The nuclear industry addressed this by assigning shift technical advisers to each operating crew. Knowledge engineers are now also recognizing the issue. For example, Mah and Damon who are developing the Alarm Processing and Diagnostic System (APDS) for the Bechtel Corporation found that four distinct but related domairis had to be surveyed [110]. These were operations, training, licensing, and design. Others have broken even further with convention and complately abandoned interviews with human experts as a means of obtaining a knowledge base. For example, Suddeth notes that rule bases derived from humans may have many deficiencies including lack of organization, incompleteness, and little relation to physical laws [111]. He advocates the use of process models for the construction of a knowledge base. Reliance on such models as a sounce of knowledge is an increasingly common characteristic of intelligent support systems.

\subsubsection{Content of Knowledge Base}

Physical models of plant systems are used in many of the intelligent support systems that are now under development. The information derived from these models is 
supplemented by that obtained from written procedures, physical laws such as those of mass and energy conservation, and diagrams of plant systems. One advantage of incorporating models in a system for automated diagnostics is that expected plant responses can be determined for any set of initial conditions. Thus, alarms can be anticipated with any mismatch between the sequences of actual and predicted alarms being cause for further investigation. Models are also essential to the establishment of the temporal relations that are so necessary to diagnostics. In this regard, it is important that there be some provision in the knowledge base for recognizing the passage of time. For example, suppose that primary flow is established in a shutdown reactor that has been in the natural circulation mode for decay heat removal. The initiation of forced convection should result in changes in the coolant's temperature sensors within so many seconds. One indication that all valves are properly aligned would be that these changes occur when expected. Thus, both an event (temperature change) and its timing are important to diagnosing valve status.

Unfortunately, tre model-based approach to the construction of a knowledge base engenders many difficulties. First, the model must function in real time. That is, for a given change in plant conditions, the time required to compute the effects of those changes by means of the model must be substantially less than the time for the change to occur in the actual system. Many developers of intelligent diagnostic systems have established a goal of one second or less for the execution speed of the morlel. This is a major challenge. For example, a three-dirinensional, near real-time, reactor physics and thermal-hydraulic model of a pressurized water reactor (core and primary loop) was recently developed and benchmarked at MIT [4]. This effort took many man-years and does not yet include the secondary systems. In addition to the problem of real-time execution, it is necessary that the model be periodically calibrated. The model should compute certain measurable quantities (temperatures, pressures, flowrates) and these calculated values should be compared to actual data from plant sensors. Unless both the model and the plant sensors are maintained in calibration, it will be very difficult to determine if a difference between the model's prediction and a sensor is the result of a modeling error, a sensor failure, or an actual perturbation. A third challenge is to transform a model so that the information generated is of use for automated diagnostics. Felkel has examined this issue in depth $[112,113]$. Among his findings are the following:

1. The computer-based model should be directly coupled to the actual physical process in the sense that it both operates in real time ands is accurate.

2. The model should be structured so that parameter variations are readily performed. This is necessary in order to simulate both unanticipated system configurations and plant upset conditions.

3. The model should be constructed on a modular basis with modules grouped in a hierarchical organization. This will allow detailed study of specific components of a given plant configuration.

4. The model's software should be structured to accommodate binary changes of state such as those that occur upon valve closure, or the tripping of an interlock. Also, the model should accept user actions as input. 
Other issues that are often raised relative to system models are those of breadth and depth. As for the first, models incorporated in an automated diagnostic system should encompass the entire plant because a disturbance in one system may propagate to others. Unfortunately, the coupling of this need for extreme breadth with the difficulties noted earlier relative to the creation of models with mathematical rigor makes it quite challenging to achieve depth. As a result, some have advocated a less demanding approach in which only qualitative behavior is predicted [114]. This technique, which is known as qualitative physics, would presumably allow cause and effect to be determined but not the actual magnitudes of the physical quantities involved. Such an approach may possibly be of benefit either by itself or as a preliminary scoping analysis. However, it should be noted that there is one area in which compromise cannot be allowed. Specifically, the model must be temporally correct. Accurate sequencing of a given series of occurrences is essential to the determination of the initiating event. Thus, rates of change, durations, and time delays must be modeled and/or calculated carefully.

\subsubsection{Mlode of Interaction}

In conventional expert systems, such as those developed for water chemistry analysis, the user supplies the various test results and is queried $a_{\text {a. }}$ necessary for additional information. 'This mode of interaction is not acceptable for intelligent support systems because, in the first place, it couldn't be done in real time and, more importantly, it would distract the reactor operators from their licensed duties. The alternative is for all of the information needed by the intelligent support system to be provided directly from the plant instrumentation. However, many existing plants were not instrumented for this purpose and would require retrofitting. For example, existing diagnostic expert systems such as those for turbine-generators, have required the installation of significant numbers of additional sensors [5].

\subsubsection{Organization of Knowledge Base}

One of the features that distinguishes existing interactive expert systems from those under development for on-line use is the method selected for organization of the knowledge base. The former almost overwhelmingly rely on production rules while the latter utilize object-oriented programming. This is a major difference because the use of object-oriented programming in lieu of production rules implies the need for internal organization of the knowledge base. The conventional ideas of simply adding knowledge in the form of a new rule and of searching through those rules without regard to order will not suffice for realtime intelligent support systems. Instead, the knowledge itself must be structured in order to facilitate model-based reasoning, diagnostics, and possibly control functions. The benefits of the object-oriented approach have been enumerated by Moore who designed the very successful G2 real-time expert system [115]. His observations include:

1. Knowledge can be expressed in a compact manner through the use of object classes. In particular, a single statement can apply to many objects. Moore gives the following example: 
"If the standard deviation of any level sensor over the last 10 seconds >...."

In contrast, there is no convenient way to identify an entire group of instruments as level sensors when using the rule-based approach. Hence, a separate rule would have to be written for each.

2. Attributes are associated with each object. For example, sensors could be categorized as being for level, pressure, temperature, or flow. This facilitates a hierarchical organization of the kno ledge base with some attributes applying to all sensors and others to only one subclass. Moore points out that this mode of representation "fits well with the way we (humans) organize our thinking ..., and it is much more powerful than having separate rules or models for each instance of an object."

3. The object-oriented approach facilitates the representation of factors that relate or "connect" one object to another. This feature is particularly useful in a power plant where components such as pumps, valves, and heat exchangers are connected to form systems. Two benefits result. First, this mode of representation lends itself to a graphical depiction similar to the piping and wiring diagrams that exist for power plants. Second, reasoning is facilitated because, if a given component is malfunctioning, a directed search can be made either upstream or downstream as appropriate for causes and effects.

Another advantage of the object-oriented approach is that it provides a means for recognizing the state of a system. Felkel has emphasized this point [112]. He notes that rules deal with events which are changes of state. An example would be the cycling of a valve. However, the expert system should also store information on the state itself. Otherwise, directives might be issued to close valves that are already shut.

\subsubsection{Inference Engine}

There are a number of special requirements imposed on the inference engine of a real-time, intelligent support system. These include:

1. The search must be accomplished rapidly so as to maintain real-time performance. Alternatively, the expert system should be programmed to provide the best possible answer within a fixed time interval [115].

2. The system should be capable of validating sensor information. Moreover, provisions should exist whereby the system can continue to function even if information is judged to be inaccurate or is missing [106].

3. The system should be capable of iteration because the receipt of new or updated information may alter conclusions that have already been reached [109]. 
4. Conclusions should be periodically reverified as part of a "truth-maintenance" program that ensures the validity of all prior reasoning [115].

5. The order of the reasoning process may have implications for the manner in which the knowledge base is organized. This is particularly true for control applications [115].

6. The system should be capable of reasoning about multiple problems at the same time. Also, an interrupt provision should exist so as to permit rapid response to high priority events [115].

7. The reasoning that supports a given conclusion should not be routinely displayed to the plant operator. However, it should be available for review upon demand [5].

\subsubsection{Man-Machine Interface}

The proper design of the man-machine interface is recognized as the single-most important factor in determining user acceptance of an expert system. Displays should be uncluttered and use high quality, easy-to-read, color graphics. In addition, displays should be designed to reflect the educational level and computer capabilities of the user. Systems intended for multiple groups (operators, shift supervisors, engineers) should be equipped with separate interfaces, each for the skill level of the group in question. Finally, and perhaps most important, the display should reinforce an operator's existing cognitive approach to the plant. As noted earlier, most operators have excellent pattern-recognition skills. The man-machine interface should therefore use graphics rather than text to convey information. Several of the intelligent support systems that are currently under development have been devised with interfaces that are both innovative and informative. For example, Beltracchi has advocated the development of displays that combine thermodynamic information with a block diagram of the plant in a manner that allows the operator to monitor the production and flow of energy from the reactor to the turbine [116]. This approach is being incorporated in the production-goal displays of the Japanese MMSNPP Project [117]. Also, researchers at MIT have developed a predictive display that allows reactor operators to visualize the consequences of a control action before implementing it [30]. This technology has been successfully demonstrated on MIT's 5-MWt Research Reactor. These disp' ${ }^{1}$ vys, as well as those designed by researchers at other organizations such as the OECD-Fi.jen Project, have demonstrated the feasibility of creating effective man-machine interfaces. Unfortunately, little actual use has been made of this technology. In particular, the man-machine interfaces of many of the expert systems that are currently commercially available are of very poor quality. Should such practice be continued for the design of intelligent support systems, the reaction of reactor operators will be quite negative.

\subsection{Acceptance of Real-Time Intelligent Support Systems}

One of the findings of the original review of nuclear expert systems by Bernard and Washio was that there had been few studies in which the impact of providing a reactor 
operator with expert advice had been evaluated under controlled conditions [5]. Studies had been performed at the Idaho National Engineering Laboratory in the United States and at the OECD-Halden Project in Europe $[118,119]$. However, the results were mixed. Since that time, additional evaluations have been performed with positive results. For example, there have been comparative studies of operator actions both with and without access to the Emergency Operating Procedures Tracking System (EOPTS) during simulated emergencies [120]. Thus, there is no longer any question that artificial intelligence methods can contribute to the safe, effective use of nuclear energy. However, the issue remains as to the proper role of man and machine and as to whether or not operators will accept intelligent support tools. Those questions are explored here in conjunction with a discussion of possible applications for intelligent support systems.

\subsubsection{Training}

Several expert systems which were originally designed for the purpose of assisting either maintenance workers or licensed reactor operators are now also being used in a training capacity. One of the first systems to be employed in this mode was EXPERT-GV which was developed by Framatome to assist in the analysis of steam generator tube cracking [121]. EXPERT-GV is now also used as a training tool for inspection and repair crews. The developers of both REALM and EOPTS have reported a similar result. REALM has been found to be of use both for a training aid and for the preparation of emergency plan exercise scenarios. As for EOPTS, its original purpose was to assist plant operators in the handling of emergencies. However, it was so useful in explaining emergency operating procedures (EOPs) that its intended role, at least at the Kuo Sheng Nuclear Power Station, is now that of a simulator-based training aid [106].

\subsubsection{Signal Validation}

Signal validation is, as discussed earlier, an area where operators may require assistance. The validation process itself can be done entirely by analytic methods. However, if data is missing or if a judgment has to be made as to the reliability of a particular sensor reading, then an expert system may be of use. For example, the Finnish power company Imairan Voima Oy and the OECD-Halden Reactor Project are jointly developing the Early Fault Detection (EFD) system for use on the Loviisa pressurized water reactor [122]. The istent is to provide reactor operators with indications of incipient failures and thus facilitate corrective action before an actual alarm condition is created. EFD has been under test since July 1989 on the high pressure preheaters of the Unit No. 1 plant.

\subsubsection{Alarm Filtering and Prioritization}

Alarm diagnosis and filtering is of particular concern to the nuclear industry because of the need to avoid cognitive overload. Hence, methods for suppressing irrelevant alarms and for the automatic identification of root cause are being actively researched. Such techniques must be flexible because an alarm that is of no significance at one point in time may be of extreme importance at a later stage of a malfunction. Also, such systems must be accurate. A number of alarm filtering systems have been developed 
including EXTRA by Electricité de France, HALO (Handling Alarms with Logic) at the OECD-Halden Reactor Project, and the Alarm Filtering System by EG\&G Idaho, Inc. [5]. One of the more interesting approaches to alarm filtration is the "hybrid" expert system that has been advocated by Westinghouse as a means for the appropriate division of tasks between man and machine [123]. Under the hybrid approach, the machine is used both to provide continuous monitoring and to transform or structure information so that it will be of direct use to a human. Tasks that require common sense or deep knowledge of the plant's dynamics are left to the operator. Contrast this with a conventional expert system where an inference engine is used to sort through the knowledge base and then, via an interface, provide the system's conclusions to the operator. There, the system does the actual problem solving. In a hybrid expert system, the human retains that role. The machine contains the knowledge base but its task is merely to organize information so as to best support human problem-solving capabilities. This issue is further discussed by Mah and Damon in their paper on Bechtel's Alarm Processing and Diagnostic System [109].

\subsubsection{Predictive Displays}

The Massachusetts Institute of Technology has explored the idea of providing reactor operators with predictive displays that permit the consequences of a given control action to be visualized prior to its implementation. Thus far, displays have been developed for the control of both reactor power and steam generator level [31]. In each case, an accurate model that can be executed at a rate much faster than real time is coupled to a custom-designed graphics package. For example, the steam generator display predicts the effect of closing, holding constant, or opening the feedwater regulating valve. The duration of the projection is selectable. This type of intelligent technology is particularly beneficial when, as is the case with steam generator level, the process dynamics are counterintuitive.

\subsubsection{Operator Assistance}

The most ambitious task for which intelligent support tools are presently being considered is the real-time assistance of reactor operators. Systems under development for this purpose include Japan's MMS-NPP project [107], the OECD-Halden efforts on SASII, which is an extension of the German STAR project [108], Atomic Energy of Canada Limited's Operator Companion [124], and the French systems KSE and GRADIENT. KSE addresses the proper operation of electric power supplies in pressurized water reactors [125], while GRADIENT, which stands for 'Graphics and Knowledge-Based Dialogue for Dynamic Systems,' is a graphical expert system that serves as an intelligent man-machine interface [126]. Many other examples are summarized in the review by Bernard and Washio [5].

The degree to which intelligent support systems should be used to assist reactor operators is the subject of much debate. The possibilities range from manual operation to complete automation. The disadvantage of the former is that it places too great a burden on the reactor operator in terms of system monitoring, diagnosis, and the rapid implementation of corrective actions. The drawback to the latter is that, while machines can do those tasks in which humans are deficient, they can not reason, at least not reliably, about situations 
that were not anticipated when their knowledge bases were developed. While neither extreme is today viewed as desirable, there is no consensus as to an appropriate compromise. American and French designers are developing computer-based systems to provide central displays of safety-related information, to prioritize alarms, and to track procedures. However, the decision to implement a given action still rests with the licensed operator. In contrast, Canadian, Japanese, and German plants are increasingly being built to incorporate a high degree of automated control.

All of the designers of nuclear power plants are developing intelligent support tools. These can be classified as either 'machine-centered' or 'human-centered' depending on the division of responsibilities between man and machine. lin the former, functions that have been traditionally performed by the licensed operator are automated and the human's role becomes one of supervision. Thus, the machine is used for process inonitoring, alarm diagnostics, and procedure selection. The responsibility of the licensed ope:ntor is to verify the machine's reasoning. This approach has the advantage that the operator is relieved of many tedious duties, that diagnostic and response actions will always be consistent, and that human indecision and/or error is reduced. However, this approach also brings with it certain limitations. For example, humans might lose their learned skills and possibly tecome overly dependent on the machine's availability. Such a situation might also make the human operator feel that he is superfluous and his job of no consequence. An alternative is a human-centered approach in which the licensed operators continue to perform their normal duties but they are both monitored and assisted by the digital system. 'This concept is really an extension of existing practice under which an independent safety system places the plant in a safe condition should the operator fail to do so. Only now, all of the operator's actions are monitored and, if necessary corrected, so as to avoid the possibility of even an incipient challenge to the safety system. For example, should an operator fail to note some alarm or not implement a given step in a procedure within the expected time frame, the intelligent system will provide a prompt. This relation between man and machine has the advantage that the licensed operator continues to perform his or her normal duties and is therefore always cognizant of plant status. Hence, should an emergency occur, he or she can act at once. In contrast, the machine-centered philosophy might result in an operator's not being mentally alert. The human-centered approach also has the advantage that it may foster operator acceptance of intelligent tools because, rather than perceiving of them as a threat, licensed personnel may view them as a means of improving performance. This will be especially true if the intelligent support systems provide operators with some capability that they presently lack. For example, acceptance of intelligent tools by load dispatchers is reported to be high because real-time information on the comparative costs of each power generation source is now available [109].

While the human-centered approach offers much that is commendable, it nevertheless remains essential that the operator fully understand the capabilities and limitations of the machine. In this respect, experience gained in the aerospace industry is worth noting. Some aircraft are now being constructed with a 'fly-by-wire' philosophy. The pilot retains nominal control but all actions are processed through an intelligent computer system. This has resulted in some difficulties. For example, the A320 jetliner, which is manufactured by the European consortium Airbus Industrie, is computer- 
controlled and it offers a protective envelope. On 26 June 1988, an A320 crashed during a French air show killing three and injuring fifty. At the time of the crash, the pilot was making a low-speed pass at an altitude of only 15 meters. The intent was to fully display the A320 to those attending the air show. The pilot may have assumed that the computerbased protective system would automatically intervene if the plane's speed reached the stall point. But, the software was programmed to assume that a landing was in progress if the plane was being flown manually below 30 meters. Hence, there was no automatic protective action. The plane did stall and then crash [127]. The implication for the designers $\boldsymbol{\alpha}$ intelligent support systems is that extreme care must be exercised not only to design an intelligent tool that is both reliable and accurate but also to create one that is fully understool by its prospective users.

A further difficulty associated with the design and use of intelligent support tools for reactor operators is that there is very little guidance available on the subject. Studies such as the aforementioned evaluation of the Emergency Operating Procedures Tracking System [106] have established that the use of an expert system can improve operator performance. However, it remains to perform comparative assessments of the machineand human-centered approaches and, at an even more fundamental level, experimental work is needed to define the optinal division of responsibility between man and machine. The studies that have been completed in this area suggest that the required experiments will be both complex and costly. For example, one of the conclusions reached during the evaluation of the HALO system was that results relevant to commercial reactors would be obtained only if experiments were done on full-scale simulators [108]. In addition to simulator studies, there should be an extensive operating history in which intelligent support tools and other similar computer-based technologies are demonstrated over periods of several years at the research and test reactor level. This combination of simulator evaluations and on-line testing will gradually establish the confidence necessary for industry to adopt and regulators to accept intelligent support tools. A further discussion of this and other factors relevant to the realization of advanced digital technologies for nuclear reactors is given in the report, "European Nuclear Instrumentation and Controls" [128].

\subsection{Assessment of Potential for the Use of Expert Systems in Multi-Modular Plants}

Artificial intelligence techniques have the potential to make a significant contribution to the reliable operation of nuclear power stations. Moreover, that potential is currently being realized through the application of expert systems to such tasks as plant design, facility management, maintenance planning, and interactive diagnostics. However, it remains an open question as to whether intelligent support systems can be successfully developed for real-time diagnosis and operator guidance. It is evident that the needed technology will not result from the mere extension of conventional, expert systems which encode experiential knowledge in production rules. The need for real-time performance imposes too many difficulties. Intelligent support systems will have knowledge bases derived from temporally-correct plant models, inference engines that permit revisions in the search process as information is updated, and non-interactive man-machine interfaces Also, their knowledge bases will require internal organization so as to facilitate diagnostics and possibly control activities. Many quality research efforts are in progress and it seems certain that these will ultimately be successful. However, much supporting work needs to 
be done in the area of real-time model development and calibration. Also, the needs of the reactor operator must always be kept in focus and the appropriate role of the human and the machine needs to be defined. Finally, it is imperative that each incremental advance in the technology be tested in an operational setting. Experimentation is the key to maintaining the technology both realistic and of benefit. 


\section{Issues Relevant to the Digital Control of Multi-Modular Reactors}

The significance of the work reported here is that it may ultimately facilitate the automated operation of multi-modular reactors and thereby (1) minimize the number of required personnel and thus contain both operating and personnel costs, (2) allow each module to be operated at a different power level thereby staggering the times at which refuelings would be needed, and (3) maintain the competitiveness of U.S. industry relative to foreign vendors who are developing and applying advanced control concepts. Issues related to the digital control of multi-modular reactors are discussed here including the relation of the completed MIT work to the overall automation of a multi-modular facility, practical considerations in the operation of two or more modules under condition of unbalanced loads, and suggestions for further research.

\subsection{Automated Operation of Multi-Modular Plants}

The objectives of the research that has been described in this report were to investigate, develop, and where possible experimentally demonstrate generic techniques for the closed-loop digital control of power, temperature, and steam generator level in multimodular plants. Specific topics that were addressed included the design of a computer architecture in which invariant supervisory constraints are kepi separate from plantdependent control laws, the identification of a method for automated startups with the online estimation of subcriticality, a comparison of trajectory tracking techniques, the development of a multi-modular plant model, the design and evaluation of compensators for the proper control of steam generator level despite shrink and swell effects, the enumeration and evaluation of strategies for the adjustment of reactor power under conditions of unbalanced loads, and an assessment of the challenges involved in the provision of intelligent support tools to reactor operators.

Table 11.1-1 lists the major functions associated with the startup and operation of a multi-modular plant. Also shown are actions that could possibly be taken to automate each task. Those actions that were addressed during the course of the research reported here are denoted by an asterisk $\left({ }^{*}\right)$. If these techniques were to be combined with others including existing methods for automating turbine run-ups and yet-to-be developed methods for automated diagnostics, then it should be possible to operate each module of a multimodular plant from a manned central control room.

\subsection{Suggestions on the Oyeration of Multi-Modular Plants}

As was noted at the outset of this report, the economical operation of a multimodular reactor plant may require that each module be run at a different power level. This is necessary so that each module's core will be depleted at a different rate thus allowing refuelings to be staggered. Unless this approach is adopted, all modules will have to be refueled at the same time and that will impact an economic performance. The inference to be drawn from the above argument is that multi-modular plants will have to be run under conditions of unbalanced loads. This in turn is challenging because the commercial nuclear industry lacks experience with such operation. In reality, operation with unbalanced loads should not be difficult provided that the plant is designed with this objective in mind. The following observations may be of use: 
Table 11.1-1

\section{Major Functions Associated with the Startup and Operation of}

a Multi-Modular Reactor Power Plant

Task

1. Precritical checks

2. Reactor startup

3. Startup of balance of plant

4. Power increase to point of adding heat

5. Turbine warm-up (main steam stops closed, bypasses open)

6. Placement of plant on-line

7. Turbine run-up

8. Operation of multiple reactors with unbalanced loads

9. Assessment of control actions

\section{Possible Action}

1. Remain manual but use computerized aids for monitoring system status.

*2. Automate using period-generated control laws in conjunction with perturbed reactivity method.

3. Perform manually with aid of procedure tracking system and computerized information for checkout and diagnosis.

*4. Automate using period-generated control laws.

*5a. Automate control of steam generator level.

5b. Perform remainder of process manually with a procedure tracking system.

*6a. Automate control of steam generator level.

*6b. Develop predictive displays to assist operation if manual control is desired.

7. Automated systems available.

*8. Automate using hierarchical controller that combines supervisory control with control laws appropriate to the selected operating strategy and mode.

9a. Identify abnormal behavior using neural nets.

9b. Diagnose cause of malfunctions using automated reasoning.

Note: An asterisk $\left({ }^{*}\right)$ denotes actions which could be implemented given the research results contained in this report. 
1. A long-term strategy for operating each module so as to create staggered refuelings should be prepared whenever a multi-modular plant is placed in (or returned to) service.

2. Unanticipated maintenance outages will probably make it impractical to implement the original operating plan exactly. Accordingly, as is now the case with both commercial PWRs and BWRs, flexibility will be essential.

3. The time required for both refueling and most plant maintenance is short compared to the lifetime of current-generation reactor cores. This means that small differences in load can have large effects. For example, suppose that a given fuel loading is rated for eighteen months at $100 \%$ of full power (FP). If one module is run at $100 \%$ FP and another at $90 \%$ FP, then after eighteen months, the depletion of the second module's core will lag by 7.2 weeks. This is enough time to refuel a reactor of several hundred megawatts. The point is that extreme imbalances in load are not necessary in order to achieve staggered refuelings.

4. Small differences in the power distribution between modules can be achieved without significant deviation from normal operating practice. For example: most reactors are operated so that the average coolant temperature is maintained within a band of a few degrees. Operation of one module at the high end of this band and another at the low end will, over an interval of several months, yield a significant difference in fuel burnup.

5. It is a relatively simple matter either to establish unbalanced operation or to return to balanced conditions. The reactor console operator merely has to withdraw or insert the control devices so as to establish the desired primary coolant temperature (refer to Section 9.3.3.3 of this report). This process requires only a few minutes. Hence, while methods for adjusting plant power during an unbalanced load condition were demonstrated in Chapter Nine of this report, such operation may not be necessary. If a power change is requested by the load dispatcher, the plant can first be returned to balanced operation. This is desirable because, as discussed in Section 8.6.3 of this report, it will facilitate control of steam generator level.

6. One challenge in the operation of a multi-modular plant is to establish unbalanced load conditions. Another is to bring a module on-line when the other modules are already at power. This problem will be especially acute if, as is likely to be the case, the other modules are at or close to $100 \%$ FP. Specifically, difficulties may arise in the control of steam generator level because the potential exists for severe swell in the on-coming unit as well as substantial shrink in the others. To control this situation, the main steam valves that connect each module to the common header should be designed to permit precise control of the steam flowrate. This in turn will enable the operator to transfer the load to the on-coming module slowly.

7. The paralleling of modules requires that several precautions be observed. First, the oncoming module should be critical at normal operating temperature before 
any attempt at paralleling is made. Moreover, its power level should be sufficiently ahove the point of adding heat so that all its systems and instruments are ready for full-power operation. (Note: The 'point of adding heat' is the power level above which an increase in temperature is observed upon an increase in power.) Second, if variable speed pumps are used, then primary flow should, of course, be increased prior to initiating a transfer of load. Third, the average primary coolant temperature of the on-coming module should be above that of the on-line modules. This will ensure that the on-coming unit does in fact pick up part of the load. Fourth, the main steam stop bypass valves for the on-coming unit should be open thus ensuring that the module being brought on-line is functioning at some small percent of full power. The transfer of load is affected by orening the main steam stops for the on-coming module, withdrawing that module's control blades as necessary to maintain temperature and, at the same time, inserti. o the control blades in the on-line modules. Fifth, whenever a module is brought on line, it is preferable that an equal distribution of the load be achieved first Then, as part of a second maneuver, conditions of unbalanced load can be established if desired.

\subsection{Suggestions for Further Research}

Reports have been issued since 1988 on the MIT program on the development and experimental evaluation of advanced control concepts for nu-lear reactors at a frequency of more than one per year. As a result, many of the suggestions made in previous reports remain valid. These are therefore not repeated here except to emphasize the extreme importance of demonstration projects. Control technology is advancing rapidly on several fronts. In particular, enormous progress, has been achieved during the past decade in the capability to model complex, non-linear systems in real time. The nuclear industry could use these models to advantage by making them the basis of on-line diagnostic systems, by incorporating them in optimal control strategies, and by structuring them to form manmachine interfaces. A transition in technology of this magnitude can not be accomplished in a single step. Rather, a series of small, incremental advances is desired. Otherwise, neither utiliiy executives nor regulatory authorities will develop confidence in the new concepts. This sicuation is particularly acute for those nations, the United States among them, that are currently experiencing a hiatus in new plant orders. An alternative would be either to conduct long-term demonstrations of advanced control concepts on some of the university and government-owned test and research reactors or to build a dedicated demonstration plant. If this is not done, then when new plant orders do finally occur, the barrier to implementing the new theories will iv insurmountable. Engineering advances occur by coupling theory to practice. Neither suffices by itself. 


\section{References}

1. Bernard, J. A., and D. D. Lanning, Eault-Tolerant Systems Approach Toward Closed-Loop Digital Control of Nuclear Power Reactors, CPE-8317878, National Science Foundation, Washington, D.C., Jan. 1988.

2. Bernard, J. A., Formulation and Experimental Evaluation of Closed Form Control Laws for the Rapid Maneuvering of Reactor Neutronic Power, Report No. MITNRL-030, Massachusetts Institute of Technology, Cambridge, MA, Sept. 1989.

3. Bernard, J. A., Startup and Control of Nuclear Reactors Characterized by SpaceIndependent Kinetics, SAND90-7082, Sandia National Laboratories, Albuquerque, NM.

4. Bernard, J. A., Henry, A. F., Lanning, D. D., and J. E. Meyer, Closed-Loop Digital Control of Nuclear Reactors Characterized by Spatial Dynamics, Report No. MITNRL-041, Massachusetts Institute of Technology, Cambridge, MA, Mar. 1991.

5. Bernard, J. A., and T. Washio, Expert Systems Applications Within the Nuclear Industry, American Nuclear Society, La Grange Park, IL, Oct. 1989.

6. World Nuclear Industry Handbook 1992, Varley, J., Ed., supplement to November 1991 issue of Nuclear Engineering International, Reed Business Publishing, Surrey, England, 1991.

7. The New Reactors, Payne, J., ed., Special Section in September 1992 issue of Nuclear News, American Nuclear Society, La Grange Park, II, 1992.

8. Mears, L. D., and M. L. Brown, "America Considers the Modular HTGR," Nuclear Engineering International, Vol. 30, No. 376, Oct. 1985, pp 36-38.

9. Lanning, D. D., "Modularized High-Temperature Gas-Cooled Reactor Systems," Nuclear Technology, Vol. 88, No. 2, Nov. 1989, pp 139-156.

10. Staudt, J. E., "Design Study of an MGR Direct Brayton Cycle Power Plant," Ph.D. Thesis, Department of Mechanical Engineering, Massachusetts Institute of Technology, Cambridge, MA, Aug. 1987.

11. Bernard, J. A., Lanning, D. D., and A. Ray, "The Nuclear Industry and Digital Control: An Unrealized Opportunity," INTECH, Vol. 32, No. 9, Sept. 1985, pp 61-64.

12. Ray, A., Desai, M.N., and J. Deyst, "Fault Detection and Isolation in a Nuclear Reactor," Joumal of Energy, Vol. 7, No. 1, Jan.-Feb. 1983, pp 79-85. 
13. Bernard, J. A., Ray, A., and D. D. Lanning, "Digital Control of Power Transients in a Nuclear Reactor," IEEE Transactions on Nuclear Science, Vol. NS-31, No. 1, Feb. 1984, pp 701-705.

14. Bernard, J. A., Lanning, D. D., and A. Ray, "Experimental Evaluation of Reactivity Constraints for the Closed-Loop Control of Reactor Power," Proceedings of the NRC-EPRI Symposium on New Technologies in Nuclear Power Plant Instrumentation and Control, Washington, D.C., Nov. 1984, pp 99 111. (Published by Instrument Society of America.)

15. Bernard, J. A., Lanning, D. D., and A. Ray, "Use of Reactivity Constraints for the Automatic Control of Reactor Power," IEEE Transactions on Nuclear Science, Vol. NS-32, No. 1, Feb. 1985, pp 1036-1040.

16. Bernard, J. A., and D. D. Lanning, "Issues in the Closed-Loop Digital Control of Reactor Power: The MIT Experience," IEEE Transactions on Nuclear Science, Vol. NS-33, No. 1, Feb. 1986, pp 992-997.

17. Bernard, J. A., "Use of a Rule-Based System for Process Control," IEEE Control Systams Magazine, Vol. 8, No. 5, Oct. 1988, pp 3-13.

18. Ornedo, R. S., Bernard, J. A., Lanning, D. D., and J. H. Hopps, "Design and Experimental Evaluation of an Automatically Reconfigurable Controller for Process Plants," Proceedings of the American Control Conference, Minneapolis, MN, Vol. 3, June 1987, pp 1662-1668.

19. Bernard, J. A., Henry, A. F., and D. D. Lanning, "Application of the 'Reactivity Constraint Approach' to Automatic Reactor Control," Nuclear Science and Engineering, Vol. 98, No. 2, Feb. 1988, pp 87-95.

20. Bernard, J. A., "An Experimental Comparison of Reactor Power Controllers Based on the Standard and Alternate Formulations of the Dynamic Period Equation," IEEE Transactions on Nuclear Science, Vol. NS-34, No. 1, Feb. 1987, pp 548-552.

21. Bernard, J. A., "Time-Optimal Control of Reactor Power," Proceedings of the American Nuclear Society International Topical Meetine on Artificial Intelligence and Other Innovative Computer Applications in the Nuclear Industry, Snowbird, UT, Sept. 1987, pp 461-470.

22. Bernard, J. A., "Evaluation of 'Period-Generated' Control Laws for the TimeOptimal Control of Reactor Power," IEEE Transactions on Nuclear Science, Vol. NS-35, No. 1, Feb. 1988, pp 888-893.

23. Bernard, J. A., Kwok, K. S., Lanning, D. D., Henry, A. F., and J. E. Meyer, "Transient Control of Reactor Power Generation for Rapid Maneuvering," in Space Nuclear Power Systems 1987, El-Genk, M. S., and M. D. Hoover, eds., Orbit Book Co., Malabar, FL, Feb. 1989, pp 299-309. 
24. Bernard, J. A., Kwok, K. S., Menadier, P. T., Thome, F. V., and F. J. Wyant, "Experimental Evaluation of the MTT-SNL Period-Generated Minimum Time Control Laws for the Rapid Adjustment of Reactor Power," in Space Nuclear Power Systems 1988, El-Genk, M. S., and M. D. Hoover, eds., Orbit Book Co., Malabar, FL, pp 495-508, Feb. 1990.

25. Bernard, J. A., Kwok, K. S., Thome, F. V., and F. J. Wyant, "The MIT-SNL Experiments Concerning the Closed-Loop Digital Control of Reactor Power," Proceedings of the ANS International Reactor Physics Conference, Jackson Hole, Wyoming, Vol. IV, Sept. 1988, pp 193-206.

26. Bernard, J. A., Kwok, K. S., and T. Washio, "Autonomous Control of Spacecraft Nuclear Reactors," Proceedings of the SPIE Conference: Space Station Automation IV, SPIE Vol. 1006, Nov. 1988, pp 28-45.

27. Bernard, J. A., "Progress Toward a Generic Methodology for the Closed-Loop Digital Control of Nuclear Reactor Power," Proceedings of the ANS/NIST Conference "Fifty Years with Nuclear Fission", Behrens, J. W., and A. D. Carlson, eds., American Nuclear Society, La Grange Park, Il, Apr. 1989, pp 262 273.

28. Bernard, J. A., and D. D. Lanning, "Considerations in the Design and Implementation of Control Laws for the Digital Operation of Research Reactors," Nuclear Science and Engineering, Vol. 110, No. 4, Apr. 1992, pp 425-444.

29. Bernard, J. A., "Non-Linear Control of Neutronic Power in Reactors Described by Space-Independent Kinetics," International Journal Control - Theory and Advanced Technology (C-TAT), Vol. 8, No. 3, Sept. 1992, pp 495-511.

30. Lau, S. H., Bernard, J. A., Kwok, K. S., and D. D. Lanning, "Experimental Evaluation of Predictive Information as an Operator Aid in the Control of Research Reactor Power," Proceedings of the American Control Conference, Atlanta, GA, Vol. 1, June 1988, pp 214-220.

31. Bernard, J. A. Lau, S. H., Kwok, K. S., Kim, K.K., and D. D. Lanning "Design, Installation, and Initial Use of a Smart Operator Aid," Proceedings of the Conference on Expert System Applications for the Electric Power Industry, Boston, MA, Sept. 9-11, 1991.

32. Kelley, C. R., Manual and Automatic Control, John Wiley and Sons, New York, NY, 1968.

33. Sheridan T. B., and W. R. Ferrell, Man-Machine Systems: Information. Control. and Decision Models of Human Performance, MIT Press, Cambridge, MA, 1974.

34. Bernard, J. A., Ornedo, R. S., and D. D. Lanning, "Human Approach to Process Control and the Role of Digital Technology," Proceedings of the American Control Conference, Minneapolis, MN, Vol. 2, June 1987, pp 934-940. 
35. Proceedings of the American Nuclear Society International Topical Meeting on Artificial Intelligence and Other Innovative Computer Applications in the Nuclear Industry, Snowbird, UT, Sept. 1987, Plenum Press, New York, NY, 1988.

36. Proceedings of the EPRI Conference on Expert Systems Applications in Power Plants, Electric Power Research Institute, Palo Alto, CA, 1987.

37. Proceedings of the EPRI Conference on Expert Systems Applications for the Electric Power Industry, Electric Power Research Institute, Palo Alto, CA, 1989.

38. Bernard, J. A., and T. Washio, "The Utilization of Expert Systems Within the Nuclear Industry," Proceedings of the American Control Conference, Pittsburgh, PA, Vol. 1, June 1989, pp 373-378.

39. Bernard, J. A., "Derivation of the Standard Dynamic Period Equation," Transactions of the American Nuclear Society, Vol. 55, Nov. 1987, pp 598-600.

40. Bernard, J. A., "The Relation of the Inhour and the Dynamic Period Equations," Transactions of the American Nuclear Society, Vol. 55, Nov. 1987, pp 69-71.

41. Washio, T., and J. A. Bernard, "Stability Considerations Concerning the Implementation of the MIT-SNL. Period-Generated Minimum Time Control Laws," in Space Nuclear Power Systems 1989, Orbit Book Co., Malabar, FL, 1992.

42. Bernard, J. A., "Development and Experimental Demonstration of Digital ClosedLoop Control Strategies for Nuclear Reactors," Ph.D. Thesis, Department of Nuclear Engineering, Massachusetts Institute of Technology, Cambridge, MA, June 1988.

43. Ray, A., Bernard, J. A., and D. D. Lanning, "On-Line Signal Validation and Feedback Control in a Nuclear Reactor," Proceedings of the Fifth Power Plant Dynamics. Control, and Testing Symposium, Knoxville, TN, March 1983, Paper No. 38.

44. Ray, A., Lanning, D. D., and J. A. Bemard, "Computer-Aided Feedback Control of Power in a Fission Reactor," Proceedings of the International Association of Science and Technology for Development (IASTED), Cambridge, MA, July 1982 , pp 119-122.

45. Ray, A., Bernard, J. A., and D. D. Lanning, "Computer Control of Power in a Nuclear Reactor," IEEE Transactions on Nuclear Science, Vol. NS-30, No. 1, Feb. 1983, pp 820-824.

46. Kwok, K. S., "Experimental Implementation of the MIT-SNL Period-Generated Minimum Time Control Laws," M.S. Thesis, Department of Nuclear Engineering, Massachusetts Institute of Technology, Cambridge, MA, Jan. 1989. 
47. Bernard, J. A., "Experiments Demonstrating the Efficacy of Period-Generated Control for the Trajectory Tracking of Reactor Neutronic Power," Proceedings of the Eighth Power Plant Dynamics. Control \& Testing Symposium, Knoxville, TN, May 27-29, 1992, Vol. 1, pp 29.01-29.14.

48. Tuddenham, R. S., Lau, S.H., Washio, T., Bernard, J. A., and D. D. Lanning, "Experimental Demonstration of Proportional-Integral-Derivative Feedback in the Closed-Loop Digital Control of Reactor Neutronic Power," Proceedings of the Seventh Power Plant Dynamics, Control, and Testing Symposium, Knoxville, TN, May 15-17, 1989, Vol. 2, pp 72.01-72.16.

49. Bernard, J. A., "Demonstration of Feedback Using the MIT-SNL Minimum Time Control Laws for the Rapid Maneuvering of Reactor Power," IEEE Transactions on Nuclear Science, Vol. NS-38, No. 2, Apr. 1991.

50. Washio, T., and J. A. Bernard, "Development and Experimental Demonstration of a Noise Reduction Technique for a Non-Linear Dynamic System," Transactions of the American Nuclear Society, Vol. 57, Oct.-Nov. 1988, pp 96-97.

51. Bernard, J. A., "The Measurement of Reactivity Using Algorithms Derived from the Dynamic Period Equation," IEEE Transactions on Nuclear Science, Vol. NS36, No. 1, Feb. 1989, pp 1270-1275.

52. Bernard, J. A., Kwok, K. S., Washio, T., Wyant, F. J., and F. V. Thome, "The Automated Startup of Spacecraft Nuclear Reactors," Transactions of the Sixth Symposium on Space Nuclear Power Systems, CONF-890103-Summs., Albuquerque, NM, Jan. 1989, pp 466-469.

53. Bernard, J. A., "Power Cutbacks as an Alternative to Scrams: An Application of the MIT-SNL Period-Generated Minimum Time Control Laws," IEEE Transactions on Nuclear Science, Vol. NS-38, No. 2, Apr. 1991, pp 838-844.

54. Lau, S. H., Washio, T., Kwok, K. S., Bernard, J. A., Lanning, D. D., and F. J. Wyant, "A Methodology for the Control of Core Average Temperature in Spacecraft Nuclear Reactors," Transactions of the Seventh Symposium on Space Nuclear Power Systems, CONF-900109, Albuquerque, NM, Jan. 1990, pp 956961.

55. Bernard, J. A., "Period-Generated Control for Trajectory Tracking," Proceedings of the American Control Conference, Boston, MA, Vol. 3, June 1991, pp 30363037 .

56. Lau, S. H., "Experimental Evaluation of Trajectory Control Techniques for Reactor Neutronic Power," N.E. Thesis, Department of Nuclear Engineering, Massachusetts Institute of Technology, Cambridge, MA, May 1991. 
57. Kim, M. H., "The Use of Bilinearly Weighted Cross Sections for Few-Group Transient Analysis," Ph.D. Thesis, Department of Nuclear Engineering, Massachusetts Institute of Technology, Cambridge, MA, Apr. 1988.

58. Kao, P. W., "Application of Supernodal Methods to Transient Analysis," Ph.D. Thesis, Department of Nuclear Engineering, Massachusetts Institute of Technology, Cambridge, MA, Aug. 1988.

59. Cabral, E. L., "Real-Time Three-Dimensional Thermal-Hydraulic Model and NonLinear Controller for Large PWR Cores," Ph.D. Thesis, Depariment of Nuclear Eng., Massachusetts Institute of Technology, Nov. 1988.

60. Aviles, B. N., "Digital Control Strategies for Spatially-Dependent Cores with Thermal-Hydraulic Feedback," Ph.D. Thesis, Department of Nuclear Engineering, Massachusetts Institute of Technology, Cambridge, MA, Feb. 1990.

61. Kwok, K. S., "Automated Startup of Nuclear Reactors: Reactivity Estimation, Computer System Development, and Experimental Evaluation," Ph.D. Thesis, Department of Nuclear Engineering, Massachusetts Institute of Technology, Cambridge, MA, June 1991.

62. Sastre, C. A., "The Measurement of Reactivity," Nuclear Science and Engineering, Vol. 8, Nov. 1960, pp 443-447.

63. Kwok, K. S., Bernard, J. A., and D. D. Lanning, "Design, Assembly, and Initial Use of a Digital System for the Closed-Loop Control of a Nuclear Research Reactor," Proceedings of the 26th Intersociety Energy Conversion Engineering Conference (IECEC), Boston, MA, Vol. 5, Aug. 1991, pp 7-12.

64. Henry, A. F., Nuclear Reactor Analysis, MIT Press, Cambridge, MA, 1975.

65. An, C. H., Atkeson, C. G., and J. M. Hollerback, Model-Based Control of a Robot Manipulator, MIT Press, Cambridge, MA, 1988.

66. Shinskey, F. G., Process Control Systems: Application. Design. and Tuning, McGraw-Hill, New York, NY, 1988.

67. Bernard, J. A., "Period-Generated Control: A Space-Spinoff Technology," Proceedings of the Ninth Symposium on Space Nuclear Power Systems, CONF920104, Albuquerque, NM, Jan. 1992, pp 583-593.

68. Strohmayer, W. H., "Dynamic Modeling of Vertical U-Tube Steam Generators for Operational Safety System," Ph.D. Thesis, Department of Nuclear Engineering, Massachusetts Institute of Technology, Cambridge, MA, Aug. 1982.

69. Choi, J. I., "Non-Linear Digital Computer Control for the Steam Generator System in a Pressurized Water Reactor Plant," Ph.D. Thesis, Department of Nuclear Engineering, Massachusetts Institute of Technology, Cambridge, MA, Aug. 1987. 
70. Auh, G. S., et al., "Development of Transient Simulation Code for Pressurized Water Reactors," Journal of the Korean Nuclear Society, Vol. 19, No. 3, Sept. 1987.

71. Kao, S. P., "A Multiple-Loop System Model for Pressurized Water Reactor Plant Sensor Validation," Ph.D. Thesis, Department of Nuclear Engineering, Massachusetts Institute of Technology, Cambridge, MA, July 1984.

72. Kao, S. P., "PRISM (Pressurized Reactor Interactive Simulation Model) User's Guide," V3.3, Simulation Expert System 1991.

73. Gofuku, A., et al., "Diagnostic Techniques of a Small-Break Loss-of-Coolant Accident at a Pressurized Water Reactor Plant," Nuclear Technology, Vol. 81, June 1988, pp 313-332.

74. Waltrip, M. K., "Multi-Modular Nuclear Reactor Plant Simulation and Control," M.S. Thesis, Department of Nuclear Engineering, Massachusetts Institute of Technology, Cambridge, MA, Nov. 1988.

75. Rovere, L. A., et al., "Implementation of a Multimodular LMR Nuclear Plant Simulation on a Parallel Computer," Proceedings of the South Eastern Simulation Conference, Pensacola, Fl, Oct. 1989.

76. Choi, J. I., "SGLCP (Steam Generator Level Controller Program) Manual," Department of Nuclear Engineering, Massachusetts Institute of Technology, Cambridge, MA, 1987.

77. "Curve Book, Training Material (Shearon Harris Nuclear Power Plant)," Carolina Power \& Light Co., Raleigh, NC, 1978.

78. "Millstone Nuclear Power Station - Unit 3; Final Safety Report," Northeast Utilities, Hartford, CT.

79. Lewis, E. E., Nuclear Power Reactor Safety, John Wiley and Sons, New York, NY, 1977.

80. Kelly, J. E. Jr., et al., "User's Guide for Thermit-2: A version for THERMIT for Both Core-Wide and Subchannel Analysis of Light Water Reactors," MIT-EL-81029, Energy Laboratory, Massachusetts Institute of Technology, Cambridge, MA, Aug. 1981.

81. Singh, G., et al., "Design and Operation of a Digital Low-Power Feedwater Control System for PWRs;" Report No. EPRI/NP-6149, Electric Power Research Institute, Palo Alto, CA, Jan. 1989. 
82. Proceedings of the EPRI Conference on Advanced Digital Computers, Controls. and Automation Technologies for Power Plants, Electric Power Research Institute, Palo Alto, CA, 1991.

83. "Steam Generator Water Level Control," Seabrook Training Center, Detailed System Text, 1986.

84. Shinskey, F. G., Process Control System, Third Edition, McGraw-Hill Book Company, New York, NY, 1988.

85. Stephanopoulos, G., Chernical Process Control: An Introduction to Theory and Practice, Prentice-Hall, Inc., Englewood-Cliffs, NJ, 1984.

86. Ogata, K., Modern Control Engineering, Prentice-Hall, Inc., Englewood-Cliffs, NJ, 1970.

87. Yocum, F. H., and J. M. Zimmerman, "Real Time Control of Inverse Response Using Dynamic Matrix Control," Proceedings of the American Control Conference, Vol. 1, 1988, pp 266-269.

88. Ilinoya, K., and R. J. Altpeter, "Inverse Response in Process Control," Industrial and Engineering Chemistry, Vol. 54, No. 7, July 1962, pp 39-43.

89. Irving, E., and C. Bihoreaux, "Adaptive Control of No-Minimum Phase Systems Application to the PWR Steam Generator Water Level Control," Proceedings of the 19th IEEE Conference. Control and Decision, Albuquerque, NM, Dec. 1980, Vol. 1, pp 274-279.

90. Grace, A., et al., Control System Tool Box for Use with Matlab, The MathWorks, Inc., Natick, MA, 1990.

91. Luyben, W. L., Process Modeling Simulation and Control for Chemical Engineers, McGraw-Hill Book Company, New York, NY, 1973.

92. Outwater, J. O., "Operator Error in Control of The Steam Generator Liquid Level of a Pressurized Water Reactor," M.S. Thesis, Department of Nuclear Engineering, Massachusetts Institute of Technology, Cambridge, MA, Feb. 1989.

93. Thomasson, F. Y., "Improved Control of Drum Level for Boilers with 'Shrink and Swell' Problems," Process Control, Vol. 71, No. 6, June 1988, pp 65-71.

94. Baeyens, R., and J. Prevot, "Fully Automated Steam Generator Level Control: Belgium Shows How," Nuclear Engineering International, Vol. 34, No. 418, May 1989, pp 22-23. 
95. Raju, G. V. S., Zhou, J., and R. A. Kinser, "Fuzzy Logic Controller to a Steam Generator Feedwater Flow," Proceedings of the American Control Conference, San Diego, CA, Vol. 2, June 1990, pp 1491-1498.

96. Marshall, J. E., Control of Time Delay Systems, The Institute of Electrical Engineers, London, 1979.

97. Smith, O.J.M., "Closer Control of Loops with Dead Time," Chemical Engineering Progress, Vol. 53, No. 5, May 1957, pp 217-219.

98. Surgenor, S. W., and G. Jie, "Evaluation of a Digitally Compensated Drum Water Level Controller," Proceedings of the 30th Power Instrumentation Symposium, Rochester, NY, Vol. 30, May 1987, pp 85-92.

99. Tucker, T. W., "A Compensator Technique for Inverse Response Using a Multiloop Distributed Controller," Transactions of the Instrumentation Society of America, Vol. 25, No. 1, 1986, pp 33-40.

100. Shearon Harris Nuclear Power Plant Instrumentation and Control System Description, Carolina Power and Light.

101. Masche, G., "System Summary of a Westinghouse Pressurized Water Reactor Nuclear Power Plant," Second Edition, Westinghouse Electric Corporation, Dec. 1972.

102. Otaduy, P. J., et al., "Supervisory Control for Multimodular ALMRs," ORNLCTM11698, Oak Ridge National Laboratory, Sept. 1990.

103. Kim, K. K., "Design and Simulation of a Digital Control System for a MultiModular Power Plant," Ph.D. Thesis, Department of Nuclear Engineering, Massachusetts Institute of Technology, Cambridge, MA, Aug. 1992.

104. Travato, S., Touchton, R. A., and S. D. Rausch, "What Happens After Development? Getting an Expert System Tested and Deployed," Proceedings of the EPRI Seminar Expert System Applications for the Electric Power Industry, Lauby, M., Divakaruni, M., and J. Naser, eds., Electric Power Research Institute, Palo Alto, CA, Sept. 1991.

105. Grime, D. B., Anderson, C., Zenker, M., and F. Liu, "The Second Deployment of REALM at Southern California Edison's San Onofre Nuclear Generating Station," Proceedings of the ERRI Seminar Expert System Applications for the Electric Power Industry, Lauby, M., Divakaruni, M., and J. Naser, eds., Electric Power Research Institute, Palo Alto, CA, Sept. 1991. 
106. Petrick, W., Sun, Bill K. H., and J. A. Naser, "Knowledge Acquired During the Implementation of the BWR Emergency Procedures Tracking System," Proceedings of the EPRI Seminar Expert System Applications for the Electric Power Industry, Lauby, M., Divakaruni, M., and J. Naser, eds., Electric Power Research Institute, Palo Alto, CA, Sept. 1991.

107. Monta, K., et al., "Development of Knowledge-Based Decision Support System for Nuclear Power Plants," Proceedings of the IAEA International Symposium on Balancing Automation and Human Action in Nuclear Power Plants, Munich, July 1990.

108. Owre, F., Nilsen, S., Forsman, T., and J. E. Stenmark, "Experiences Gained from Developing and Integrating an Expert System and a Modern Graphic Display System for a Swedish Nuclear Power Plant Control Room," Broceedings of the AI 91: Frontiers in Innovative Computing for the Nuclear Industry, Lindsey, R., and G. Cordes, eds., American Nuclear Society, La Grange Park, IL, pp 393-402, Sept. 1991.

109. Proceedings of the EPRI Seminar Expert System Applications for the Electric Power Industry, Lauby, M., Divakaruni, M., and J. Naser, eds., Electric Power Research Institute, Palo Alto, CA, Sept. 1991.

110. Mah E., and L. Damon, "An Engineering Approach to Knowledge-Based Systems, the Alarm Processing and Diagnostic System," Proceedings of the EPRI Seminar Expert System Applications for the Electric Power Industry, Lauby, M., Divakaruni, M., and J. Naser, eds., Electric Power Research Institute, Palo Alto, CA, Sept. 1991.

111. Suddeth, A. L., "Diagnostic Reasoning Using Qualitative Causal Models," Proceedings of the EPRI Seminar Expert System Applications for the Electric Power Industry, Lauby M., Divakaruni M. and J. Naser, eds., Electric Power Restearch Institute, Palo Alto, CA, Sept. 1991.

112. Felkel, L., "Process Monitoring, Diagnosis, and Prediction: The Integration of Expert System and Simulation Technology," Broceedings of the Al 21: Frontiers in Innovative Computing for the Nuclear Industry, Lindsey, R., and G. Cordes, eds., American Nuclear Society, La Grange Park, IL, pp 62-71, Sept. 1991.

113. Felkel, L., "Modeling Techniques for On-Line Expert Systems in Nuclear Power Plants," Proceedings of the Advances in Human Factors Research on Man/Computer Interactions: Nuclear and Beyond, Schurman, D. L., and H. E. Knee, eds., American Nuclear Society, La Grange Park, II, pp 11-18, June 1990.

114. de Kleer J., and J. S. Brown, "A Qualitative Physics Based on Confluences," Qualitative Reasoning about Physical Systems, Bobrow, D. G., ed., MIT Press, Cambridge, MA, 1985. 
115. Moore, R. L., "The G2 Real-Time Expert System," Proceedings of the EPRI Seminar Expert System Applications for the Electric Power Industry, Lauby, M., Divakaruni, M., and J. Naser, eds., Electric Power Research Institute, Palo Alto, CA, Sept. 1991.

116. Beltracchi, E.L., "A Direct Manipulation Interface for Heat Engines Based upon the Rankine Cycle," IEEE Trans. Systems, Man. and Cybernetics, Vol. SMC-17, No. 3, pp 478-487, May-June 1987.

117. Monta, X., et al., "An Intelligent Man-Machine System for BWR Nuclear Power Plants," Proceedings of the AI 91: Frontiers in Innovative Computing for the Nuclear Industry, Lindsey, R., and G. Cordes, eds., American Nuclear Society, La Grange Park, IL, pp 383-392, Sept. 1991.

118. Nelson, W. R., "Response Trees and Expert Systems for Nuclear Operations," NUREG/CR-3631, U.S. Nuclear Regulatory Commission, Washington, D.C., 1984.

119. Marshall, E. C., Reiersen, C. S., and F. Owre, "Operator Performance With the HALO II Advanced Alarm System for Nuclear Power Plants: A Comparative Study," Proceedings of the ANS International Topical Meeting Artificial Intelligence and Other Innovative Computer Applications in the Nuclear Industry, Majumdar, D., and J. Sackett, eds., Plenum Press, New York, NY, pp 353-360, 1988.

120. Spurgin, A. J., et al., "The BWR Emergency Operating Procedures Tracking System (EOPTS) Evaluation by Control-Room Operating Crews," Report No. NP6846, Electric Power Research Institute, Palo Alto, CA, May 1990.

121. Delaigue, D., and M. Grundstein, "A Survey of Framatome's Expert Systems Activity," Proceedings of the ANS International Topical Meeting Artificial Intelligence and Other Innovative Computer Applications in the Nuclear Industry, Majumdar, D., and J. Sackett, eds., Plenum Press, New York , NY, pp 29-35, 1988.

122. Berg, O., Bye, A., Sorenssen, A., and H. Jokinera, "Early Fault Detection and Signal Validation at the Loviisa Nuclear Power Plant," Proceedings of the AI 91: Frontiers in Innovative Computing for the Nuclear Industry, Lindsey, R., and G. Cordes, eds., American Nuclear Society, La Grange Park, IL, pp. 111-119, Sept. 1991.

123. Elm, W. C., "How Artificial Intelligence Can Help," Nuclear Engineering International, Vol. 33, No. 406, pp 36-40, 1988.

124. Popovic, J. R., Anderson, J. W. D., and H. E. Sills, "The Roles of Expert Systems for Supporting Nuclear Plant Operations In Canada," Proceedings of the Third International Topical Meeting Nuclear Power Plant Thermal Hydraulics and Operations, Seoul, Korea, p B6-41, Nov. 1988. 
125. Ancelin, J., Chériaux, F., Gaussot, J. P., Legaud, P., Pichot, D., Sancerni, G., and G. Voisin, "KSE: Expert System Mon toring Electric Power Supplies in a PWR Power Plant," Proceedings of the AI 91: Frontiers in Innovative Computing for the Nuclear Industry, Lindsey, R., and G. Cordes, eds., American Nuclear Society, La Grange Park, IL, pp 80-90, Sept. 1991.

126. Hollnagel, E., "Knowledge-Based Dialogue in Intelligent Decision Support Systems," Proceedings of the ANS International Topical Meeting Artificial Intelligence and Other Innovative Computer Applications in the Nuclear Industry, Majumdar, D., and J. Sackett, eds., Plenum Press, New York, NY, pp 321-329, 1988.

127. "Flying the Electric Skies," Science, Vol. 244, pp 1532-1533.

128. White, J. D., Lanning, D. D., Beltracchi, L., Best, F. R., Easter, J. R., Oakes, L. C., and A. L. Suddeth, "European Nuclear Instrumentation and Controls," NTIS Report No. PB92-100197, National Science Foundation, Washington, D.C., Dec. 1991.

129. Kelly, C.R., "Manual and Automatic Control," John Wiley and Sons, New York, 1968.

130. Ellis, S., and W. McGreevy, "Perspective Traffic Display Format and Airline Pilot Traffic Avoidance," Journal of the Human Factors Society, Vol. 29, No. 4, Aug. 1987.

131. Hinchley, E. M., et. al., "The CANDU Man-Machine Interface and Simulator Training," AECL-7768, CRNL, Ontario, Sept. 1982.

132. Venhuiizen, J. R., and J. M. Griffith, "Predictive Display Concepts for Use in Nuclear Plant Control," NUREG/CR-3103, U.S. Nuclear Regulatory Commission, Washington, D.C., Jan. 1983. 
Appendix A

Steam Generator Water Level Predictive Display Program

\section{A.1 Statement of Problem}

Unplanned reactor trips that are the result of steam generator level instability have contributed significantly to the unavailability of PWRs [81]. The problem, which was discussed in Chapter Eight of this report is that steam generator water level control is complicated by inverse response effects known as 'shrink and swell.' Current generation level controllers can correct for these effects provided that both flow and level signals are available. Unfortunately, flow measurements are too uncertain for use as a controller input at low power. Hence, operators sometimes assume manual control during low power operation. However, such action may not in itself be without difficulty because system interactions, the delay between control demand and response, and the counterintuitive steam generator water level dynamics combine to complicate the operator's task. To deal with these complexities, an operator should understand how steam generator parameters respond to a planned control action. In this regard, a model-based predictor with appropriate displays may improve an operator's capability to control level by revealing the consequences of a particular control action prior to its actual implementation.

The concept of providing predictive displays as an aid to those responsible for controlling complex processes is not new. Early applications of the approach concerned the diving controls for submarines and the landing system for Apollo spacecrafts [129]. More recently, the technique has been utilized for air and railroad traffic control $[130,131]$. Relative to the nuclear industry, the use of predictive display technology as an operator aid for steam generator level in PWRs has been previously suggested though not implemented [132]. The motivation for the work reported here on the design of a predictive display for steam generator level was a successful study that had been previously performed at MIT on the provision of predictive information on neutronic power to reactor operators [30]. Five displays that provided the operator with various combinations of derivative, current, and predictive information were developed and evaluated through actual use on the 5-MWt MIT Research Reactor, MITR-II. Operators had to be trained to use the predictive information. However, once they understood it, most reported its availability to be of benefit. This was especially true if a power maneuver was to be performed with the control rods configured in other than their normal pattern. Given this success, it was decided to develop a similar display for steam generator level.

Currently, a steam generator operator's primary indications of the water inventory are the narrow- and wide-range level indicators located in the steam generator downcomer. However, because these indications are susceptible to the counterintuitive effects of shrink and swell, it is often difficult for the operator to make the correct decision about how he or she should respond to keep the mass inventory of the steam generator within the prescribed limits. A predictive display might ameliorate this situation by enabling an operator to visualize the consequences of adjustments to the feedwater flow some thirty seconds to a few minutes into the future. Such displays might reduce the frequency of operator-related trips by forewarning operators of the effects of shrink and swell, and hence curbing their 
natural response to overcompensate for level changes caused by transient phenomena such as bubble formation and collapse. In this Appendix, a program that was developed for this purpose is reported. The program has been designated as the Steam Generator Level Display Program (SGLDP).

\section{A.2 Predictive Display Program}

The SGLDP is a FORTRAN program that combines a steam generator water level simulation routine, a level display routine, and an operator interaction program as shown in Figure A-1. This program runs on any IBM-compatible personal computer that is equipped with a VGA or Hercules graphic board.

The steam generator water level simulation routine uses the mathematical model that was developed in Chapter Seven of this report. The program projects the narrow-range steam generator level signal for three cases: control valve being closed, control valve position maintained constant, and control valve being opened. The operator can select the speed at which the valve is to be opened or closed. The demanded reactor power is also displayed so that the operator can observe the correlation (or lack thereof) between power demand and change in anticipated level in the steam generator. The model gives the narrow-range steam generator level in the downcomer as a function of the steam and feedwater flowrates. Four terms are included. The first is a mass capacity term that reflects the net difference between the steam and feedwater flowrates. The second allows for shrink/swell effects associated with changes in feedwater flowrate. The third is similar except that it is for changes in steam flowrate. The fourth allows for short-lived mechanical oscillations that can be caused by the addition of feedwater to the generator. As was reported in Chapter Eight, this model's accuracy was verified by comparison with a much larger and more rigorous model that had been benchmarked against plant data. Projections of up to $200 \mathrm{~s}$ are possible with a display update frequency of one second.

Figure A-2 shows the predictive display for steam generator level. The upper portion shows the reactor power and the lower portion depicts steam generator level. Reactor power was initially at $10 \%$ of rated and it is being raised $8 t 2.5 \%$ of rated per minute. Derivative information, the steam generator level for the previous $100 \mathrm{~s}$, is shown together with the current level. Emanating from the current level are the three projections, each corresponding to a possible control option (valve opened at selected rate, held constarit, or closed at selected rate). In the actual display, each option is shown in a different color. The advantage of this display is that an operator can visualize the effects of adjusting the position of the feedwater control valve before doing so. This capability should result in more reliable operation because even though operators are trained to and do understand the counterintuitive nature of shrink and swell, they may have difficulty quantifying those effects. Thus far, no trials of this display have been conducted either by simliation or in an actual plant. Additional information is given in [31].

It is recognized that much remains to be done before the use of predictive information will become routine in the nuclear industry. In particular, regulatory issues remain to be explored. These have not yet been broached as part of the MIT program because all use of the neutronic power display has been under an approved experimental protocol [1]. Issues such as operators becoming overly dependent on a display or the 


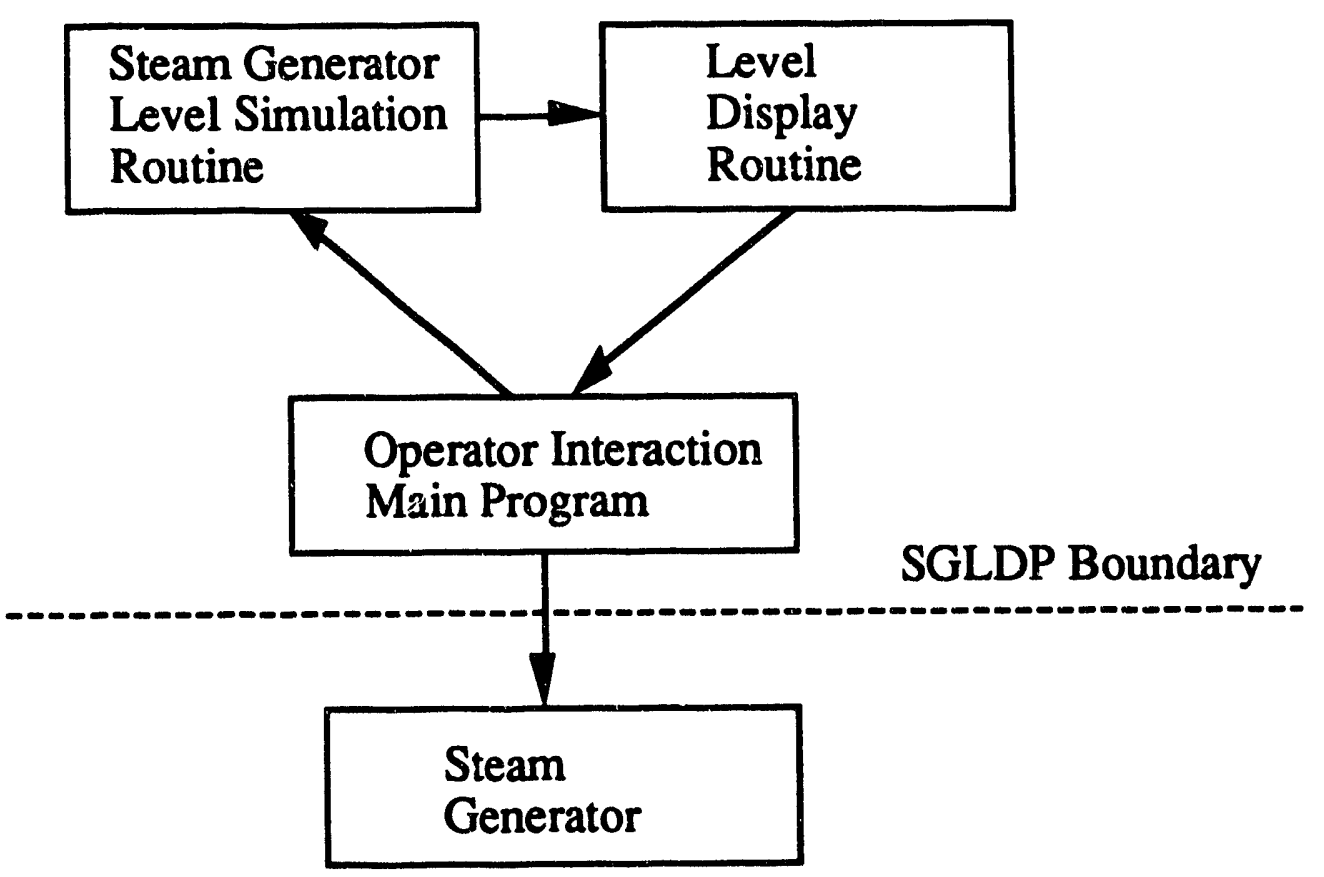

Figure A-1 Block Diagram of SGLDP. 

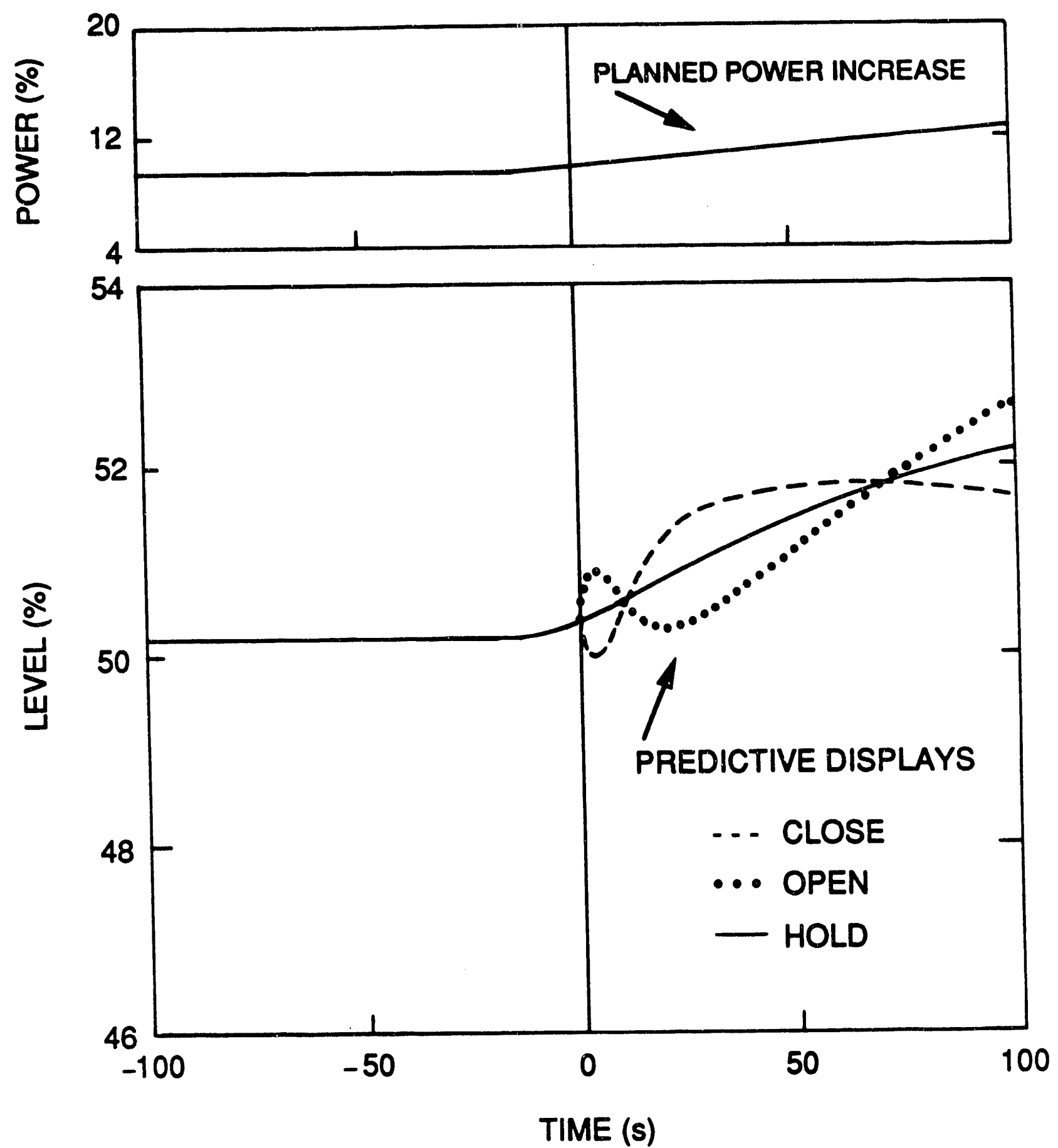

Figure A-2 Steam Generator Level Display. 
consequences of inaccuracy in a projection remain to be addressed. While not minimizing those challenges, predictive displays may offer a means of gradually incorporating digital technology in reactor control rooms and thereby bridging the gulf that now exists between manual and fully automated control of nuclear power facilities. 
Appendix B

\author{
Smith's Dead-Time Compensator
}

\title{
B.1 Statement of Problem
}

If a physical process involves significant dead time such as a transport lag, a conventional feedback controller may provide a quite unsatisfactory closed-loop response. The problem is that a change in an input does not produce an immediate, corresponding change in an output. Hence, it is not possible to obtain any information for use as feedback. Control action may be incorrect because such actions will be based on previous instead of current measurements of the output. Therefore, the presence of dead time may be an important source of instability in a closed-loop response. In order to improve control of such processes, Smith suggested a compensation element connected around the primary controller [97].

\section{B.2 Compensator Function and Design}

Smith's compensator is based on the Smith principle which states that if a response satisfies the design criteria for the delay-free case, then the response to be designed for the delayed case should be the same except that it is delayed by whatever dead time is involved. In order to obtain the delay-free signal, a model-based dead-time compensator that predicts the delayed effect is used. Figure B-1 is a schematic of the Smith dead-time compensator. The measured signal, $y(s)$, is compensated by the signal, $y^{\prime}(s)$. This compensating signal is obtained from a simple local feedback loop that goes arc und the controller. It is called the Smith dead-time compensator. The resulting control input signal, $y^{*}(s)$, can be expressed as:

$$
\begin{aligned}
y^{*}(s) & =y(s)+y^{\prime}(s) \\
& =\left[G_{c} G e^{-\iota_{d} s}+\left(1-e^{-t_{s} s}\right) G G_{c}\right] y_{s p}(s) \\
& =G_{c} y_{s p}(s)
\end{aligned}
$$

As shown by Equation (B-1), the strength of the Smith approach is that the delayed effect is canceled by the model. Thus, the controller's input signal contains current and not delayed information. Figure B-2 shows the equivalent block diagram of the control system associated with the Smith dead-time compensator. It is possible to eliminate entirely the undesired effect of dead-time provided that a perfect model of the process dynamics can be obtained. The model-based compensator of fsets the deficient performance of the original system by altering its overall behavior so that the system behaves as desired. 


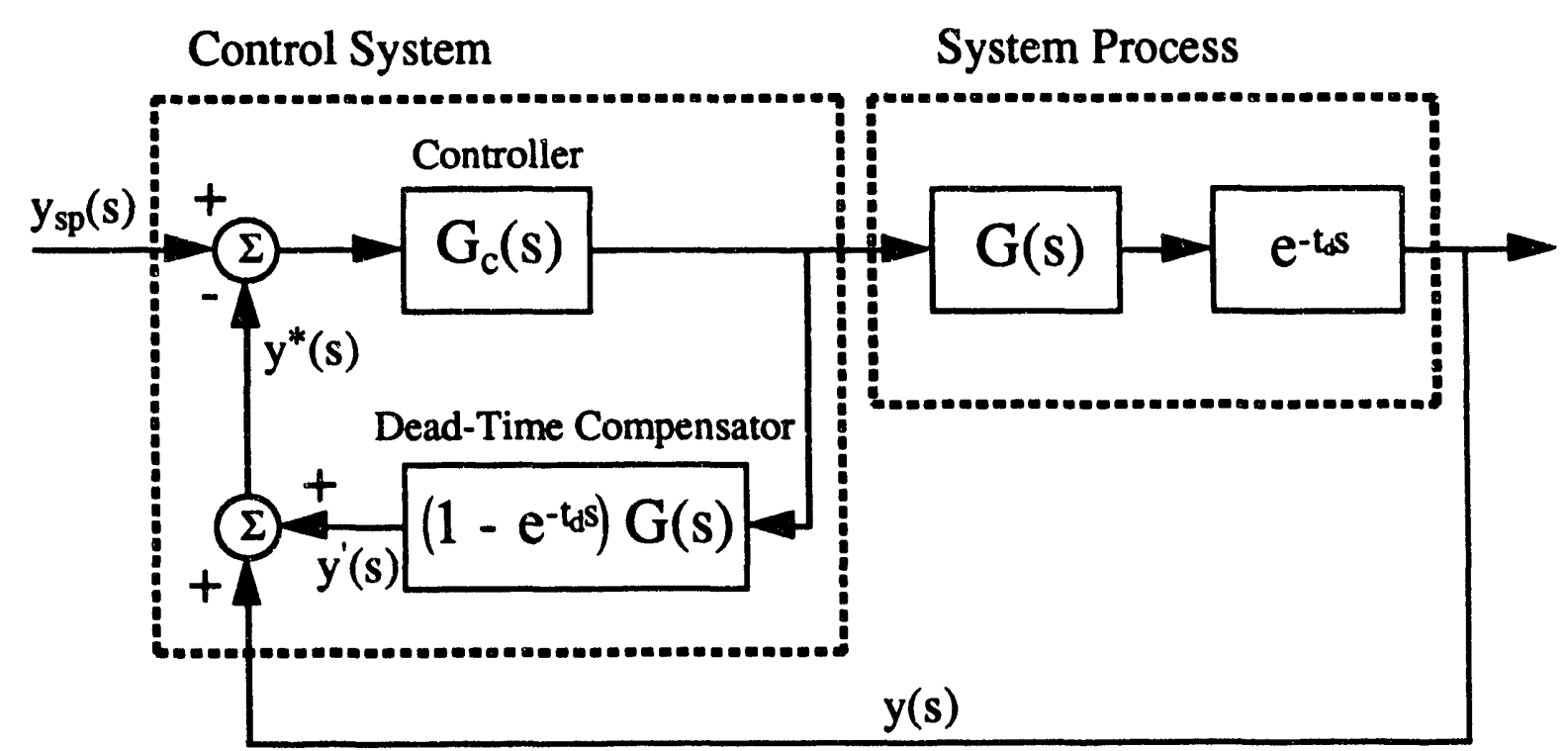

Figure B-1 Schematic of Control System with Smith's Dead-Time Compensator.

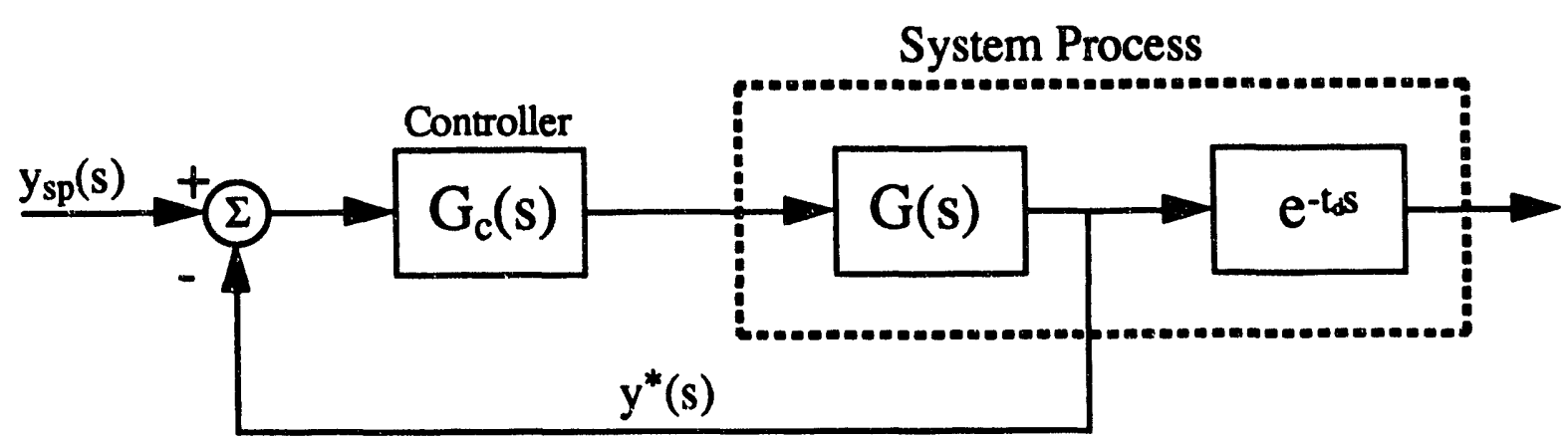

Figure B-2 Equivalent Block Diagram of the Control System with Smith's Dead-Time Compensator. 
DATE FILMED $1 / 20 / 93$ 


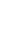

